\title{
IR-anregungsmodulierte Photoionisationsspektroskopie an natriumdotierten Wasserclustern
}

\author{
Dissertation \\ zur Erlangung des mathematisch-naturwissenschaftlichen Doktorgrades \\ „Doctor rerum naturalium“" \\ der Georg-August-Universität Göttingen \\ im Promotionsprogramm Chemie \\ der Georg-August University School of Science (GAUSS)
}

vorgelegt von

Florian Zurheide

aus Herford

Göttingen, 2021 


\section{Betreuungsausschuss}

Prof. Dr. Thomas Zeuch

Abteilung Physikalische Chemie II, Institut für Physikalische Chemie, Georg-AugustUniversität Göttingen

Prof. Dr. Burkhard Geil

Abteilung Biophysikalische Chemie, Institut für Physikalische Chemie, Georg-AugustUniversität Göttingen

\section{Mitglieder der Prüfungskommission}

Referent: Prof. Dr. Thomas Zeuch

Abteilung Physikalische Chemie II, Institut für Physikalische Chemie, Georg-AugustUniversität Göttingen

Korreferent: Prof. Dr. Dietmar Stalke

Abteilung Anorganische Chemie I, Institut für Anorganische Chemie, Georg-AugustUniversität Göttingen

\section{Weitere Mitglieder der Prüfungskommission}

Prof. Dr. Burkhard Geil

Abteilung Biophysikalische Chemie, Institut für Physikalische Chemie, Georg-AugustUniversität Göttingen

Dr. Tim Schäfer

Abteilung Physikalische Chemie I, Institut für Physikalische Chemie, Georg-AugustUniversität Göttingen

Prof. Dr. Phillipp Vana

Abteilung Makromolekulare Chemie, Institut für Physikalische Chemie, Georg-AugustUniversität Göttingen

Prof. Dr. Thomas Waitz

Abteilung Fachdidaktik Chemie, Institut für Anorganische Chemie, Georg-AugustUniversität Göttingen 
„Das Prinzip aller Dinge ist Wasser; aus Wasser ist alles, und ins Wasser kehrt alles zurück."

- Thales von Milet - 



\section{Inhaltsverzeichnis}

$\begin{array}{ll}1 . \text { Einleitung } & 1\end{array}$

2. Wissenschaftlicher Kontext $\quad 7$

2.1. Cluster als Bindeglied zwischen Gasphase und kondensierter Materie. . . . 8

2.2. Phasenänderungen in begrenzten Systemen . . . . . . . . . . . . . . . . 11

2.2.1. Der Beitrag der Phasengrenzflächen zur freien Enthalpie . . . . . . 12

2.2.2. Statistische Beschreibung von Phasenänderungen . . . . . . . . . . 20

2.3. Wasser und Wassercluster . . . . . . . . . . . . . . . . 26

2.3.1. Struktur und Eigenschaften des Wassermoleküls . . . . . . . . . 26

2.3.2. Wechselwirkungen zwischen Wassermolekülen . . . . . . . . . . . 26

2.3.3. Kristallisation in wässrigen Nanotröpfchen . . . . . . . . . . . . . . 27

$2.3 .4 . \quad$ Eis 1 . . . . . . . . . . . . . . . . . . . . . . . . . . . . . 29

2.3.5. Bisherige Studien an ungeladenen Wasserclustern . . . . . . . . . . 31

3. Erzeugung und IR-spektroskopische Untersuchung natriumdotierter Was-

sercluster

3.1. Erzeugung ladungsfreier Wassercluster in adiabatischen Überschallexpan-

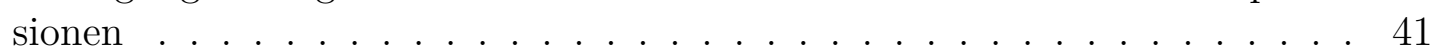

3.1.1. Das Strömungsfeld . . . . . . . . . . . . . . . . . . . . . . 42

3.1.2. Umwandlung thermischer Energie in kinetische Energie . . . . . . . 44

3.1.3. Kondensationsprozesse in freien Expansionen . . . . . . . . . . . . . 47

3.1.4. Die Größenverteilung der gebildeten Partikel . . . . . . . . . . . . . . . 49

3.1.5. Einfluss der Expansionsparameter auf die Kühlraten. . . . . . . . . 52

3.2. Größenselektion und IR-Spektroskopie . . . . . . . . . . . . . . . . 54

3.2.1. Natriumdotierung . . . . . . . . . . . . . . . . . . . . 55

3.2.2. Photoionisation . . . . . . . . . . . . . . . . . . 59

3.2.3. Wirkungsspektroskopie mittels IR-Anregung . . . . . . . . . . . . 65

3.2.4. Flugzeitbasierte Massenselektion. . . . . . . . . . . . . . . . . . . 74

3.2.5. Detektion . . . . . . . . . . . . . . . . . 77

\begin{tabular}{|l|l|}
\hline 4. Versuchsaufbau und experimentelle Methoden & 79
\end{tabular}

4.1. Übersicht . . . . . . . . . . . . . . . . . . . . . . . . . . . 79

4.2. Molekularstrahlquelle . . . . . . . . . . . . . . . . . . . . . 80

$4.3 . \quad$ Pickup-Zelle . . . . . . . . . . . . . . . . . . . . . . . . . . . . . . . . 82

4.4. Reflektron-Flugzeitmassenspektrometer . . . . . . . . . . . . . . . . . . . . 83

4.4.1. Aufbau der Ionenquelle . . . . . . . . . . . . . . . . . . . . . . . 84

4.4.2. Aufbau des Reflektrons . . . . . . . . . . . . . . . . . . . 85

4.4.3. Aufbau der Detektoreinheit . . . . . . . . . . . . . . . . 86

4.5. Vakuumsystem . . . . . . . . . . . . . . . . . . . . . . . . . . . . . . . . . 88

4.5.1. Aufbau des Vakuumsystems . . . . . . . . . . . . . . . 88 
4.5.2. Vakuumpumpen. . . . . . . . . . . . . . . . . . . 88

4.5.3. Druckmessung . . . . . . . . . . . . . . . . . . . . . . . . . . 90

4.5.4. Betriebsparameter . . . . . . . . . . . . . . . . . . . 90 90

4.6. Infrarot-Lasersystem . . . . . . . . . . . . . . . . . . . . . . . . . . 92

4.6.1. Nd:YAG-Pumplaser Powerlite Precision II 8000 . . . . . . . . . . . 92

4.6.2. Seeding-Laser . . . . . . . . . . . . . . . . . . . . . . . . 94

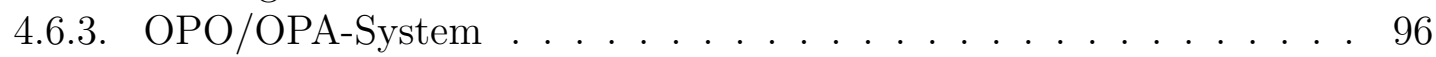

4.7. UV/VIS-Lasersystem . . . . . . . . . . . . . . . . . . . . . 100

4.7.1. Nd:YAG-Pumplaser Powerlite 9010 . . . . . . . . . . . . . . . . . . 100

4.7.2. Farbstofflaser Cobra-Stretch . . . . . . . . . . . . . . . . . . . 102

4.7.3. Relay Imaging Teleskop . . . . . . . . . . . . . . . . . . . . . . 105

4.8. Steuerung des Messablaufs . . . . . . . . . . . . . . . 106

4.8.1. Synchronisation der Lasersysteme und des Massenspektrometers . . 107

4.8.2. Automatisierung des Messablaufs . . . . . . . . . . . . . . . 112

\begin{tabular}{ll}
\hline 5. Datenverarbeitung & 119
\end{tabular}

5.1. Erhebung und Speicherung von Rohdaten . . . . . . . . . . . . . . . . . . 119

5.2. Automatisierte Datenauswertung . . . . . . . . . . . . . . . . 120

5.2.1. Bedienung der Auswertungssoftware . . . . . . . . . . . . . . . . . 121

5.2.2. Funktionsweise des Algorithmus . . . . . . . . . . . . . . . . . . . . 123

6. Ergebnisse und Diskussion 135

6.1. Wahl und Beschreibung der Expansionsbedingungen . . . . . . . . . . . . 136

6.1.1. Clustererzeugung unter optimalen Kristallisationsbedingungen . . . 137

6.1.2. Unterdrückung der Kristallisation . . . . . . . . . . . . . . . . . . . 140

6.2. Die gebundene OH-Streckschwingung als Kriterium der Phasenänderung . 141

6.3. Der Einfluss der Kristallisation auf die Bande der freien OH-Oszillatoren . 145

\begin{tabular}{ll}
\hline 7. Zusammenfassung & 159
\end{tabular}

\begin{tabular}{ll}
\hline 8. Ausblick & 161
\end{tabular}

A. Experimentelle Parameter der vorgestellten Messungen I

B. Liste verwendeter Chemikalien III

C. Schaltplan der Triggerbox $\quad$ V

\begin{tabular}{ll}
\hline Q. Quelltext zur Automatisierung & VII
\end{tabular}

E. Auswertungsskript XXIII

Liste der verwendeten Symbole LIII

\begin{tabular}{|ll}
\hline Abkürzungsverzeichnis & LIX
\end{tabular}

Literaturverzeichnis LXXIII 


\section{Einleitung}

$\mathrm{H}_{2} \mathrm{O}$ - Trotz seines einfachen molekularen Aufbaus ist Wasser eine hochkomplexe Substanz, die eine Vielzahl ungewöhnlicher Eigenschaften besitzt. Das Verhalten flüssigen Wassers weicht in vielen Aspekten von dem anderer Flüssigkeiten ab 1]: So nimmt die Dichte flüssigen Wassers bei Temperaturen unterhalb von $4^{\circ} \mathrm{C}$ bei weiterer Abkühlung zu; auf Druckerhöhung reagiert Wasser mit einer Abnahme der Viskosität und einer steigenden Diffusionskonstante144. Dieses außergewöhnliche Verhalten des Wassers als Flüssigkeit sowie seine Fähigkeit, mannigfaltige Eismodifikationen auszubilden 15 sind nicht allein aus den Eigenschaften eines isolierten Wassermoleküls heraus erklärbar. Sie entspringen vielmehr den Wechselwirkungen, die in der kondensierten Phase zwischen einer großen Zahl interagierender Moleküle auftreten - genauer gesagt, aus ihrer Fähigkeit, ein ausgedehntes Netzwerk von Wasserstoffbrückenbindungen auszubilden, dessen Topologie sich in der flüssigen Phase innerhalb von Pikosekunden verändert $2[3]$.

In der Biosphäre spielt Wasser eine Schlüsselrolle als ubiquitäres Lösungsmittel, als vielfältiger Reaktionspartner und -promotor in der belebten [6] und unbelebten 7 Natur, als Hauptbestandteil von Wolken und allgegenwärtiger Akteur in den vielfältigen chemischen und physikalischen Prozessen, die ursächlich für unser Wetter ${ }^{89}$ und unser Klima sind 7. Die ungewöhnlichen thermodynamischen Eigenschaften des Wassers sind z. B. verantwortlich dafür, dass Gewässer von der Oberfläche und nicht vom Grund her gefrieren und für die Zirkulation der Ozeane, die ebenfalls einen großen Einfluss auf das lokale und globale Klimageschehen ausübt 1 .

In der Atmosphäre können sich Eispartikel durch heterogene 10 und homogene $11 \mathrm{Nu}$ kleation innerhalb bereits bestehender, flüssiger Aerosole bilden, deren Tröpfchen Größen im Nanometerbereich besitzen und die variierende Mischungen von Schwefelsäure, Salpetersäure, Ammoniak und Wasser sowie eine große Zahl organischer Verbindungen enthalten können 12]. Diese Partikel sind in der Lage, auf ihrer Oberfläche andere Moleküle zu adsorbieren und zu akkumulieren 13|14, sie zu aktivieren 15 , chemische Reaktionen zu begünstigen und teilweise sogar aktiv zu katalysieren 16.

Bei der experimentellen Charakterisierung atmosphärischer Aerosole in Höhen zwischen 5 und $20 \mathrm{~km}$ entsteht das Problem, dass Partikel mit einem Durchmesser unterhalb von $20 \mathrm{~nm}$ mit den bisher angewandten Methoden der Größenbestimmung nur schwer charakterisiert werden können. So sind die meisten derzeit zur Verfügung stehenden Verfahren ungeeignet, da sie entweder in diesem Größenbereich nicht anwendbar sind, schwach gebundene Aggregate dabei zerstört werden, oder sie das Vorliegen geladener Partikel erfordern. So ermöglicht das weit verbreitete Scanning Mobility Particle Sizing (SMPS) als einziges Verfahren die Größenbestimmung neutraler Aerosolpartikel im unteren, einstelligen Nanometerbereich $(r \gtrsim 1-2 \mathrm{~nm})$. Diese Methode liefert allerdings erstens keine Informationen über die Beschaffenheit der Partikel und ist zweitens nicht geeignet zur Untersuchung schwach gebundener Aggregate wie Wassercluster, da diese während des 
Detektionsprozesses verdampfen 17/18. Ausgerechnet diesen kleinen Partikeln mit ihrer vergleichsweise großen relativen Oberfläche wird allerdings ein besonders großer Einfluss zugeschrieben 11|19|20. Umso größere Bedeutung kommt der selektiven Erzeugung kristalliner Nanopartikel im Labor ${ }^{17}$ sowie der Entwicklung von Methoden und Indikatoren zu, die eine Übertragbarkeit der Ergebnisse zwischen beiden Feldern ermöglichen 20|21].

Der Abbau von Ozon durch Chlorverbindungen auf den Oberflächen nanometergroßer Eiskristalle $22[24$ ist sicherlich das bekannteste Beispiel für den großen Einfluss, den nanometergroße Eispartikel auf das Atmosphärengeschehen ausüben können. Diese Entdeckung war verbunden mit der Einsicht, dass den Prozessen auf der Oberfläche dieser Partikel ein komplexes, nur ansatzweise verstandenes Wechselspiel aus Evaporations- und Kondensationsprozessen zugrunde liegt und ist daher außerdem ein eindrucksvolles Beispiel dafür, wie ein praxisnahes Problem eine ausgesprochen stimulierende Wirkung auf den Fortschritt der Grundlagenforschung in Theorie und Experiment entfalten kann.

In lebenden Zellen ist Wasser imstande, durch seine ausgedehnten, variablen Brückennetzwerke Wechselwirkungen zwischen funktionellen Gruppen organischer Moleküle zu vermitteln, die in der Folge Wasserstoffbrückenbindungen untereinander ausbilden können. Es ermöglicht somit die Molekülerkennung über Distanzen von bis zu einem Nanometer hinweg. Damit dürfte dem Wasser als Lösungsmittel u. a. eine zentrale Rolle in der Proteinfaltung zukommen - einem Feld, das bisher weitgehend im Dunkeln liegt 2 .

Lange Zeit hat sich die Wissenschaft darauf beschränkt, das Verhalten zweier unterschiedlicher Materieformen getrennt zu beschreiben - es wurden entweder isolierte Atome bzw. Moleküle in der Gasphase betrachtet, oder das Verhalten von Feststoffen und Flüssigkeiten in der kondensierten Phase war Gegenstand der Untersuchung 25 . In den 1980er Jahren wurde damit begonnen, die Lücke zwischen diesen beiden Betrachtungsweisen zu schließen, indem ein Fokus auf die Wechselwirkungen zwischen einer kleinen Zahl von Atomen bzw. Molekülen gelegt wurde. Ein besonders gut geeigneter Weg, um diese Wechselwirkungen zu verstehen, besteht in der Untersuchung kleiner Aggregate, sogenannter Cluster. Aufgrund ihrer geringen Größe im Nanometer- bzw. Subnanometerbereich besitzen derartige Aggregate einzigartige Eigenschaften, die sich weder an isolierten Atomen bzw. Molekülen noch an der kondensierten Materie beobachten lassen, die sich aber mit zunehmender Größe (bzw. wachsender Konstituentenzahl $N$ ) denen kondensierter Phasen annähern. Daher können Cluster als Bindeglied zwischen dem isolierten Atom bzw. Molekül und der kondensierten Materie betrachtet werden (siehe Abschnitt 2.1) 25 28. Gelingt es, die Eigenschaften von Clustern und Nanopartikeln einer bestimmten Substanz in Abhängigkeit von deren Größe zu untersuchen, können die so erhobenen Daten dazu dienen, die physikalischen Ursachen für das Verhalten der kondensierten Materie aufzuklären. Die Erforschung der Struktur- und Bindungseigenschaften kleiner Wassercluster stellt somit einen Schlüssel für das Verständnis des Verhaltens von festem und flüssigem Wasser sowie für das Verständnis von Solvatationsphänomenen dar 1129. In der Vergangenheit haben die Bestrebungen, durch Untersuchungen an Wasserclustern die Entwicklung observabler Eigenschaften in der kondensierten Phase zu verfolgen, regelmäßig neue, überraschende Einblicke in die fundamentalen Eigenschaften dieser außergewöhnlichen Substanz gewährt 30 .

Eine unbedingte Voraussetzung für die Gewinnung belastbarer Daten ist die Untersuchung neutraler, d.h. elektrisch ungeladener Cluster, da aufgebrachte Ladungen die 
Struktur des Clusters stark beeinflussen 3134. Die Erzeugung von ungeladenen Clustern beliebiger Größe aller chemischer Elemente sowie der weitaus meisten Verbindungen stellt heutzutage kein Problem dar 35 37. Die hierfür zur Verfügung stehenden Verfahren (Expansionstechniken 38 42, Kollisionskühlungszellen 43/44 sowie Gasaggregationsquellen 45 47]), erlauben jedoch keine strenge Größenkontrolle der erzeugten Partikel. Vielmehr wird stets eine Größenverteilung erhalten, deren Maximum beeinflusst werden kann $36 \mid 37$. Die Präparation und größenselektive Charakterisierung solcher Aggregate bringt allerdings große Schwierigkeiten mit sich, die erst in den letzten Jahren in zunehmendem Maße überwunden wurden (vgl. Abschnitt 2.3.5).

Ein universell anwendbares Verfahren zur Größenselektion ungeladener Cluster existiert bisher nicht. Daher besteht ein großes Interesse an der Entwicklung von Methoden, die zumindest in einem begrenzten Anwendungsbereich eine Lösung für dieses Problem bieten 48 . So besteht ein Ansatz zur Überwindung dieser Schwierigkeit in der Anlagerung eines Chromophors auf der Oberfläche eines ungeladenen Clusters, in der Hoffnung, dessen Topologie möglichst wenig zu beeinflussen. Durch selektive Photoionisation des Chromophors soll die Struktur des Clusters selbst erhalten bleiben. Anwendbar wird dieses Verfahren, sofern die ionisierende Strahlung keinen nennenswerten Energieeintrag in den Cluster selbst bewirkt, da ansonsten eine Dissoziation von Konstituenten auftreten könnte. Als Chromophore werden z. B. aromatische Verbindungen wie Benzol 49 und Phenol $32|50| 51]$ verwendet. Eine akkurate Größenselektion konnte hiermit allerdings nur bis zur Clustergröße $N\left(\mathrm{H}_{2} \mathrm{O}\right)=10$ erzielt werden. Mit moderater Größenselektion $\left(\Delta N\left(\mathrm{H}_{2} \mathrm{O}\right) \leq 6\right)$ wurde der Bereich bis $N\left(\mathrm{H}_{2} \mathrm{O}\right)=50$ vermessen 52 .

Ein mächtiges Werkzeug, das in den letzten Jahren in unserem Arbeitskreis weiterentwickelt wurde, basiert auf der Verwendung von Natrium als Chromophor 41|53. Im Kern besteht dieses Verfahren aus der Anlagerung eines einzelnen Natriumatoms auf der Oberfläche eines sehr kalten Clusters. Experimentelle Studien an Eis- und Methanoloberflächen legen nahe, dass Natriumatome bei Temperaturen unterhalb von $95 \mathrm{~K}$ auf diesen Oberflächen kinetisch stabilisiert sind $54 \sqrt[56]{5}$. Die anschließende Photoionisation erfolgt durch ultraviolette (UV-)Strahlung in Form eines Laserpulses. Wird diese mit einer vorausgehenden vibronischen Anregung durch ebenfalls gepulste Infrarot- (IR-)Strahlung kombiniert, wird eine Intensitätsmodulation des Photoionisationssignals beobachtet. Der Grund hierfür ist die Erhöhung der Clustertemperatur als Folge der vibronischen Anregung. Durch Wiederholung dieses Experiments unter Variation der Wellenlänge des IR-Lasers lassen sich somit Schwingungsspektren generieren. Eine eingehende Beschreibung dieses Verfahrens findet sich in Abschnitt 3.2,

Durch systematische Variation der verschiedenen experimentellen Parameter und Interpretation der Beobachtungen in enger Kooperation mit theoretisch arbeitenden Forschungsgruppen ist es in den letzten Jahren gelungen, die diesem Verfahren zugrundeliegenden Mechanismen aufzuklären 5760 .

Einen Durchbruch brachten die in Kapitel 4 beschriebenen apparativen Neuerungen: Die Implementierung eines neuen UV-Lasersystems und die Entwicklung einer neuen elektronischen Steuerung ermöglichen erstmals die Einbeziehung der Zeitdifferenz zwischen beiden Laserpulsen als Versuchsparameter. Es zeigte sich, dass hierdurch eine gezielte Selektion vorwiegend amorpher bzw. vorwiegend kristalliner Wasseraggregate er- 
reicht werden kann, die im gleichen Experiment unter identischen Bedingungen erzeugt und nunmehr separat untersucht werden können 61. Zudem gelang es in diesem Zuge, den Messmodus zu verändern und damit die Qualität der erhaltenen Daten erheblich zu verbessern. Komplettiert werden die Neuerungen durch die Entwicklung zweier neuer Softwareanwendungen: Die Erste (basierend auf der Programmiersprache $C++$ ) ermöglicht eine weitgehende Automatisierung des Messbetriebs. Damit verbunden ist eine Erhöhung der erhobenen Datenmenge pro Zeiteinheit. Die zweite Anwendung basiert auf der quelloffenen Skriptsprache Python 3. Sie ermöglicht die automatisierte Auswertung der im neuen Messmodus erhaltenen Rohdaten und kann leicht an zukünftige Fragestellungen angepasst werden (siehe Kapitel 5).

Als Folge dieser Verbesserungen konnte die gut separierte, aber schwache Bande der ungebundenen OH-Gruppen auf der Clusteroberfläche erstmals mit hoher Genauigkeit vermessen werden. Diese Gruppen stellen nicht nur eine äußerst reaktive Bindungsstelle für organische ${ }^{1462}$ und anorganische ${ }^{63}$ Moleküle in der Atmosphäre dar, sondern können auch als empfindlicher Indikator für die Stärke der Wasserstoffbrücken in ihrer Umgebung und damit für Strukturmotive 51 sowie für Ionen und Ladungen dienen 32.34.

Zwei wichtige Fragen konnten bisher nicht endgültig geklärt werden 6465: Nach welchem Mechanismus läuft die Bildung von Eis ab? Und wie viele Wassermoleküle muss ein Wassercluster enthalten, um eine kristallartige Struktur ausbilden zu können? Diese Fragen besitzen hohe Relevanz im Hinblick auf das Verständnis von Nukleationsprozessen 39. Auch hier besteht ein direkter Bezug zur Atmosphärenforschung, da mikrophysikalische Prozesse wie die Nukleation, die z. B. zur Bildung von Cirruswolken und anderen Eispartikelwolken führen, bisher nur unzureichend verstanden sind und daher einen großen Unsicherheitsfaktor bei der Ermittlung der globalen Strahlungsbilanz und der Vorhersage der Klimaentwicklung darstellen 66. Die physikalischen Eigenschaften solcher Wolken hängen stark von den vorherrschenden Nukleationsmechanismen und -raten und der resultierenden Größenverteilung der gebildeten Eispartikel ab. Besonders die Nukleation kleiner Eispartikel aus der Flüssigkeit kann die Modulation der Sonnenstrahlung und des hydrologischen Fluxes in der Atmosphäre beeinflussen 67 .

Bisherige experimentelle Arbeiten zur minimalen (kritischen) Clustergröße $N^{*}$ der Nukleation in wässrigen Nanotröpfchen lieferten stark variierende Ergebnisse für $N^{*}$ von $2 0 0 \leq N ( \mathrm { H } _ { 2 } \mathrm { O } ) \leq 1 0 0 0 \longdiv { 3 8 }$ bis hin $\mathrm{zu} N \approx 275 \underline{41}$. Diese Zahlen stellen jeweils einen oberen Grenzwert für $N^{*}$ unter den im Experiment herrschenden Erzeugungsbedingungen dar. Besonderen Einfluss auf die experimentell bestimmte Größe von $N^{*}$ besitzt die Kühlrate in dem Temperaturbereich, in dem der Phasenübergang auftritt 68 .

Auf Basis numerischer Simulationen wurden unterschiedliche Vorhersagen getroffen, die von $123<N\left(\mathrm{H}_{2} \mathrm{O}\right) \leq 293\left[62\left[69\right.\right.$, über $100 \leq N\left(\mathrm{H}_{2} \mathrm{O}\right) \leq 137 \underline{68}$ bis hin zu $N\left(\mathrm{H}_{2} \mathrm{O}\right)=90[70$ reichen. Zur Klärung dieser Frage ist ein kombinierter Ansatz aus Experiment und Theorie notwendig:

Zum einen sind experimentell arbeitende Forschungsgruppen auf die Unterstützung theoretisch arbeitender Gruppen angewiesen. Strukturinformationen lassen sich aus Schwingungsspektren von Wasserclustern nur indirekt entnehmen. Die Topologie der zugrundeliegenden Strukturen (meistens sind es mehrere) lässt sich daraus nicht direkt ablesen, sondern muss durch den Vergleich mit berechneten Spektren simulierter Strukturen ermittelt werden $48|62| 71$. 
Zum anderen sind theoretisch arbeitende Gruppen auf experimentelle Daten als Grundlage angewiesen. Die Modellierung ausreichend großer Systeme, um die Nukleation von Wasser beobachten zu können (ca. 100 Wassermoleküle), erfordert Vereinfachungen, deren Rechtfertigung nur experimentell überprüft werden kann. Die Beobachtung eines stochastischen Ereignisses wie der Nukleation in einem derart großen System, das sämtliche Wechselwirkungen mit der größtmöglichen Genauigkeit erfasst, erfordert einen rechnerischen Aufwand, der die derzeitigen Möglichkeiten übersteigt. Der Grund ist, dass die homogene Nukleation verglichen mit der Zeitskala molekulardynamischer Simulationen einen sehr langsamen Prozess darstellt 44. Daraus erwachsen zwei konkurrierende Anforderungen an die verwendeten in-silico-Modelle: Einerseits sollten sie die Wechselwirkungen zwischen Wassermolekülen mit ausreichender Genauigkeit beschreiben, um deren Verhalten realitätsnah wiederzugeben, andererseits sollten sie effizient genug sein, um die erforderlichen langen Simulationszeiten mit vertretbarem Rechenaufwand zu ermöglichen 68. Sowohl die theoretische als auch die experimentelle Forschung konnten in den letzten Jahren deutliche Fortschritte verzeichnen:

Auf Seiten des Experiments ermöglichte das oben erwähnte bessere Verständnis das Auffinden geeigneter Expansionsbedingungen mit niedrigen Kühlraten im Temperaturbereich knapp unterhalb von $200 \mathrm{~K}$, in dem molekulardynamische Simulationen eine kritische Clustergröße von wenigen hundert Molekülen vorhersagen 62 68.

Auf theoretischer Seite wird dieser Fortschritt durch die Entwicklung neuer Simulationstechniken flankiert: Insbesondere das neue flexible, atomistische Vielkörperpotential (genannt $M B-p o l)^{72}$ sagt Schwingungsspektren vorher, die ausnehmend gut mit experimentell ermittelten Spektren übereinstimmen $73[74$.

Buch et al.62 sagten als IR-spektroskopisches Kriterium zur Unterscheidung zwischen amorphen Clustern und solchen, die einen kristallinen Kern aufweisen, einen Übergang der maximalen Bandenintensität der gebundenen $\mathrm{OH}-$ Oszillatoren von $3400 \mathrm{~cm}^{-1} \mathrm{zu}$ $3200 \mathrm{~cm}^{-1}$ voraus, der in bisherigen experimentellen Studien vielfach als Kriterium zur Unterscheidung amorpher und kristalliner Cluster herangezogen wurde $32|41| 48|75| 76$. Diese Beobachtung entspricht auch dem Verhalten der kondensierten Phase in makroskopischen Wassertropfen und Eiskristallen sowie großen Nanopartikeln 77 79. Das komplexe Kopplungsverhalten der zahlreichen intramolekularen und intermolekularen Schwingungsmoden in großen Wasserclustern akkurat vorherzusagen, setzt jedoch eine hohe Qualität der angewandten in Rechenmodelle voraus 48 . Wenngleich auch aus der Kopplung verschiedener Schwingungsmoden neue Signalmaxima resultieren können ${ }^{62}$, waren Buch et al. gezwungen, ihr Modell an dieser Stelle einzuschränken 62.

Methoden aus dem Kreis der Wirkungsspektroskopie, die zur simultanen Gewinnung von Größen- und Strukturinformation angewendet werden, erlauben nur eine indirekte Bestimmung der IR-Absorption der untersuchten Aggregate. Dies macht sie anfällig für spektrale Artefakte, die in der Größen- oder Strukturabhängigkeit der ausgenutzten Wirkungseffekte oder durch Fragmentierungsprozesse begründet sein können.

Um letzte, berechtigte Zweifel an diesem bisher einzigen bekannten Kriterium auszuräumen, wäre es daher zu begrüßen, auf weitere Unterschiede in den Schwingungsspektren amorpher und kristalliner Cluster zurückgreifen zu können, die als unabhängiger, komplementärer Indikator für das Aufkommen kristallartiger Strukturen dienen können. 
Das Ziel der vorliegenden Arbeit war die präzise, größenselektive Vermessung der Bande der ungebundenen $\mathrm{OH}-$ Oszillatoren auf der Clusteroberfläche. Auch deren Verhalten in Abhängigkeit vom Phasenzustand der gebildeten Partikel sollte näher untersucht werden. In Abschnitt 2.2 werden daher zunächst die thermodynamischen Grundlagen von Phasenübergängen umrissen. Weiterhin werden die Gründe für das Auftreten der kritischen Clustergröße $N^{*}$ erläutert, bei deren Unterschreiten kristalline Strukturen thermodynamisch instabil werden. Aus einer statistischen Betrachtung geht hervor, dass der in makroskopischem Wasser beobachtete, sprunghafte Phasenübergang zu kristallinem Eis seinen diskontinuierlichen Charakter in kleinen Aggregaten verliert 27/80|81. Dieser Umstand ist bei der Interpretation der experimentellen Ergebnisse zu berücksichtigen. Daher werden die zugrunde liegenden Überlegungen in Abschnitt 2.2.2 kurz skizziert. Der Fokus all dieser Ausführungen liegt auf der Substanz Wasser sowie den in wässrigen Clustern und Nanopartikeln mutmaßlich vorliegenden Phasenzustände.

Gegenstand der hier vorgenommenen experimentellen Untersuchung sind amorphe und kristallartige Cluster und Nanopartikel, die aus Wassermolekülen zusammengesetzt sind. In Abschnitt 2.3 ist daher der bisherige Forschungsstand zur Bildung von kristallinem Eis sowie über die Strukturen dieser Aggregate zusammengefasst.

Die theoretischen Grundlagen der hier verwendeten experimentellen Methoden sind in Kapitel 3 dargelegt. Voraussetzung für die Durchführung der in dieser Arbeit vorgestellten Messreihen war die Weiterentwicklung der vorhandenen Molekularstrahlapparatur. Daher werden deren Aufbau sowie die daran vorgenommenen Änderungen in Kapitel 4 ausführlich dargelegt und dokumentiert. Die Verarbeitung der Messdaten zur Gewinnung von IR-Spektren mit Hilfe der neu entwickelten Auswertungssoftware ist in Kapitel 5 erläutert.

Eine Auswahl der vorgenommenen Messungen sowie deren Ergebnisse werden in Kapitel 6 vorgestellt, diskutiert und mit den Resultaten theoretisch ausgerichteter Forschungsgruppen verglichen. Es zeigte sich, dass die Entwicklung der Schwingungsbande freier OH-Oszillatoren auf der Partikeloberfläche als Funktion der Teilchengröße abhängig von deren Phasenzustand unterschiedlich verläuft. Diese Bande kann daher als komplementärer Indikator zur Unterscheidung amorpher und kristallartiger Strukturen herangezogen werden. Die gewonnenen Ergebnisse weisen deutlich darauf hin, dass in kleinen Aggregaten amorphe und kristalline Strukturen unter bestimmten Bedingungen nebeneinander im thermodynamischen Gleichgewicht vorliegen könnten und der aus der kondensierten Materie bekannte Schmelzübergang in solchen Systemen seinen diskontinuierlichen Charakter verlieren könnte. 


\section{Wissenschaftlicher Kontext}

Am Beginn dieser Arbeit soll der Versuch einer Definition des Forschungsgegenstands Stehen. Während die Verbindung Wasser durch ihre chemische Formel $\mathrm{H}_{2} \mathrm{O}$ mit hinreichender Genauigkeit beschrieben ist, bedarf der Begriff Cluster einer genaueren Eingrenzung. Eugen Illenberger verwendet sinngemäß folgende Definition:

Cluster sind eine Gruppe (oder eine Ansammlung) von Atomen oder Molekülen, die durch schwache, zwischenmolekulare Kräfte zusammengehalten werden. 25

Diese gute Beschreibung der qualitativen Eigenschaften von Clustern ermangelt allerdings einer Eingrenzung bezüglich ihrer Größe: Sie trifft ebenfalls auf die kondensierte Materie zu, von der der Begriff des Clusters abgegrenzt werden soll. Jortner schlägt vor, Cluster als endliche Aggregate mit $N=2-10^{4}$ Konstituenten einzugrenzen [26] und bietet an anderer Stelle ${ }^{82}$ eine Klassifikation anhand der Größe an:

- Mikrocluster $(N \lesssim 10)$ : Diese besitzen weitgehend diskrete Eigenschaften. Es existieren definierte Strukturisomere, die getrennte Banden im Vibrationsspektrum aufweisen.

- Kleine Cluster $(10 \lesssim N \lesssim 100)$ : Diese zeichnen sich durch eine größere Isomerenvielfalt aus. Ihre Infrarotspektren sind durch ihr komplexes Schwingungsverhalten sowie durch Beiträge zahlreicher Isomere mit diskreten, aber nah beieinanderliegenden Energiezuständen gekennzeichnet. Cluster dieser Größe besitzen häufig amorphe Strukturen.

- Große Cluster $(N>100)$ : In diesem Größenbereich findet eine graduelle Entwicklung der Festkörperstruktur mit zunehmender Clustergröße statt. Sowohl ihre elektronische als auch die phononische Zustandsdichte nehmen mit wachsender Konstituentenzahl weiter zu und entsprechen letztlich einem Quasikontinuum. Dennoch bestehen Unterschiede im Vergleich zu den Eigenschaften der kondensierten Materie. Die Gründe werden in Abschnitt 2.1 erläutert.

Pauly übernimmt diese Klassifikation, fügt ihr allerdings zwei weitere Klassen an, indem er zudem zwischen Nanoclustern $\left(10^{3}<N<10^{6}\right)$ Nanopartikeln $\left(N>10^{6}\right)$ unterscheidet 83 . Diese Definition ist insofern besonders willkürlich, als sie sich allein auf die Konstituentenzahl bezieht und nicht an qualitative Unterschiede gebunden ist.

Eine für die Zwecke dieser Arbeit brauchbare Abgrenzung zwischen Clustern und Nanopartikeln schlugen Landes et al. vor, die als Bedingung für die Einordnung eines Aggregats als Nanopartikel einen ausgeprägten Kern fordern, dessen Konstituenten sich in ihrer Umgebung und in ihrem Verhalten von denen der Oberfläche unterscheiden 84 . Victoria Buch 
und J. Paul Devlin 79 folgend, auf deren umfangreichen Vorarbeiten zum Verständnis der Substanz Wasser diese Arbeit aufbaut, soll diese Abgrenzung übernommen werden, da sich zeigt, dass die Ausbildung eines von der Oberfläche abzugrenzenden Clusterkerns in den Größenbereich fällt, in dem der Clusterdurchmesser den einstelligen Nanometerbereich erreicht. Daher soll im Folgenden der Begriff Nanopartikel für Aggregate verwendet werden, die aufgrund ihrer Größe sicher in der Lage sind, einen Kernbereich auszubilden. Für kleinere Aggregate und im Zweifelsfall wird der Begriff Cluster gewählt.

\subsection{Cluster als Bindeglied zwischen Gasphase und kondensierter Materie}

Seit den Arbeiten von Johannes van der Waals 55 ist bekannt, dass die makroskopischen Eigenschaften der Materie auf Kräften beruhen, die zwischen den Atomen bzw. Molekülen auftreten, aus denen sie aufgebaut ist. Neben den dispersiven und elektrostatischen Wechselwirkungen, von denen die sogenannten Van-der-Waals-Cluster (Edelgase, aber auch organische Moleküle, $\mathrm{I}_{2}$ ) zusammengehalten werden, können als attraktive Wechselwirkungen auch Wasserstoffbrücken (z. B. $\left.\left(\mathrm{H}_{2} \mathrm{O}\right)_{N},\left(\mathrm{CH}_{3} \mathrm{OH}\right)_{N}\right)$, ionische (z. B. $\left.(\mathrm{NaCl})_{N}\right)$, kovalente (z. B. $\left.\mathrm{S}_{8}\right)$ oder metallische (z. B. $\mathrm{Na}_{N}$ ) Bindungen in Clustern auftreten 82 .

Cluster besitzen neben charakteristischen Eigenschaften isolierter Moleküle und Eigenschaften der kondensierten Phase auch Charakteristika, die sich in keiner der beiden vorgenannten Materieformen finden 86 . In diesem Sinne stellen sie eine Brücke zwischen der Gasphase und der kondensierten Materie dar 25|26|28. Dabei lassen sich weitreichende Unterschiede ausmachen, die wohl die meisten Bereiche observabler Eigenschaften umfassen 26|87]:

- Struktureffekte: Eine Abhängigkeit ausgebildeter Strukturmotive und Strukturparameter (z. B. Koordinationszahlen) ist nur unterhalb einer bestimmten, substanzabhängigen 88 Größe zu beobachten. In größeren Aggregaten bilden sich die regulären Strukturmerkmale der kondensierten Phase aus. Die minimale Clustergröße, ab der die Struktur des makroskopischen Feststoffs auftritt, ist abhängig von der Stärke und der Reichweite der zwischenmolekularen Wechselwirkungen. Die Größenabhängigkeit des Isomerieverhaltens von Übergängen zwischen starren und mobilen Zuständen in begrenzten Temperaturbereichen kennzeichnen Phasenänderungen ${ }^{1}$ in Clustern.

- Quanteneffekte: So sind z. B. die Beträge der endlichen Energielücken zwischen elektronischen Zuständen oft vergleichbar mit der Energie thermischer Anregungen. Weiterhin rückt die effektive Wellenlänge von Elementaranregungen in die Größenordnung der Dimensionen des Clusters.

- Größenabhängigkeit der elektronischen Struktur: Einige Substanzen bilden in kleinen Clustern ausschließlich Van-der-Waals-Wechselwirkungen aus, während sich mit zunehmender Größe eine metallische Bindung ausbilden kann. Mit der zunehmender

${ }^{1}$ Der Begriff Phasenänderung wird in Abgrenzung zum Phasenübergang der kondensierten Materie gewählt, siehe Abschnitt 2.2 .2 
Größe kommt es zur Ausbildung von Leitungs- und Valenzbändern, auch Übergänge zwischen metallischen und nichtmetallischen Zuständen sind möglich.

- Thermodynamische Größeneffekte: Die thermodynamischen Eigenschaften eines Clusters wie Oberflächenspannung und Dampfdruck sind stark größenabhängig, weiterhin zeigt sich abweichendes Verhalten in Bezug auf Phasenänderungen und Isomerie, die Folge der endlichen Konstituentenzahl sind (siehe Abschnitt 2.2)

- Dynamische Effekte: Die Mechanismen und Zeitskalen der Energiespeicherung, Umverteilung und Abgabe (Dissoziation und Evaporation, Relaxation, vibronische Energieumverteilung (IVR) können ebenfalls mit der Clustergröße variieren.

Dabei lässt sich die größenabhängige Entwicklung der Clustereigenschaften in zwei Bereiche unterteilen: In kleineren Clustern nimmt diese Entwicklung einen stark nichtmonotonen Verlauf. Hier tritt aufgrund von Quanten- und Grenzflächeneffekten häufig ungewöhnliches Verhalten bei spezifischen Clustergrößen auf. Auch der Abschluss von Schalen und die Möglichkeit zur Ausbildung von Strukturen mit hoher Stabilität (sog. magische Größen) tragen zum vordergründig erratischen Verhalten kleiner Cluster bei. $\mathrm{Ab}$ einer bestimmten, substanzabhängigen Konstituentenzahl folgt ein Bereich, in dem die Messgrößen sich nicht mehr sprunghaft, sondern zunehmend kontinuierlich und skalierbar verändern, wobei sie einem Potenzgesetz der Form $\alpha N^{\beta}$ gehorchen und gegen den Grenzwert der kondensierten Materie konvergieren $28 \mid 88]$.

Der Größenbereich, in dem die Eigenschaften amorpher Wassercluster größenabhängige, diskontinuierliche Änderungen zeigen, erstreckt sich bis $N \approx 65$. Dies zeigt sich in sprunghaften Änderungen der IR-Bandenpositionen natriumdotierter Cluster sowie in Unregelmäßigkeiten bezüglich der Neigung, ein auf der Oberfläche adsorbiertes Natriumatom zu solvatisieren, die unterhalb dieser Clustergröße auftreten [54]. Mehrere Gruppen beobachteten im Größenbereich $50 \lesssim N \lesssim 60$ Anomalien des Photoelektronenspektrums sowie in der Lebensdauer thermalisierter, ionischer Wassercluster $31|89| 90]$. Diese experimentellen Resultate deuten darauf hin, dass in diesem Bereich einige Konstituentenzahlen die Ausbildung von Wasserstoffbrückentopologien mit ausnehmend hoher thermodynamischer Stabilität ermöglichen. Kazachenko und Thakkar fanden in Übereinstimmung mit diesen Resultaten unter den Minimumstrukturen der Clustergröße $N=51$ solche, die ein Strukturmotiv mit hoher Symmetrie und hoher Bindungsenergie aufweisen (vgl. Abschnitt 2.3.5) 91 .

Neben Struktur- und Quanteneffekten, die auf die geringe Größe zurückzuführen sind, ist ein wesentlicher Grund für die besonderen Eigenschaften von Clustern und Nanopartikeln ihre große relative Oberfäche. Für dicht gepackte metallische und Van-der-WaalsCluster lässt sich leicht ein Ausdruck für den größenabhängigen Anteil der Atome herleiten, die die Clusteroberfläche bilden 25|26]: Nimmt man sowohl die Konstituenten des Clusters als auch den Cluster selbst als sphärisch an, und weiter, dass dem sich das Volumen des Clusters additiv aus den Volumina $v_{\text {mono }}$ der Monomere zusammensetzt, so beträgt das Volumen $V$ eines Clusters aus $N$ Monomeren:

$$
V=\frac{4}{3} \pi r^{3}=N v_{\text {mono }}=N \frac{4}{3} \pi r_{\text {mono }}^{3}
$$


Der effektive Radius eines Monomers $r_{\text {mono }}$ lässt sich z. B. aus der Dichte der kondensierten Phase abschätzen 92 . Der Radius $r$ des Clusters hängt somit linear vom effektiven Monomerradius und invers von der dritten Potenz der Konstituentenzahl ab:

$$
\begin{gathered}
V=\frac{4}{3} \pi r^{3}=N \frac{4}{3} \pi r_{\text {mono }}^{3} \\
r=r_{\text {mono }} \cdot N^{1 / 3}
\end{gathered}
$$

Durch Einsetzen dieses Ausdrucks in die Formel für die Oberfläche eines sphärischen Clusters erhält man für die Oberfläche $A$ des Partikels:

$$
A=4 \pi r^{2}=4 \pi N^{2 / 3} \cdot r_{\text {mono }}^{2}
$$

Aus Glg. 2.2 und Glg. 2.4 wird ersichtlich, dass die relative Oberfläche kleiner Partikel sich invers proportional zu deren Radius $r$ sowie zur dritten Wurzel der Konstituentenzahl $N$ verhält:

$$
\frac{A}{V} \propto \frac{r^{2}}{r^{3}} \equiv r^{-1} \propto N^{-1 / 3}
$$

Die Anzahl $N_{\mathrm{O}}$ der Monomere auf der Clusteroberfläche ergibt sich mit dem effektiven Querschnitt des Monomers $\pi r_{\text {mono }}^{2}$ zu:

$$
N_{O}=\frac{A}{\pi \cdot r_{\text {mono }}^{2}}=4 N^{2 / 3}
$$

Der Anteil von $N_{\mathrm{O}}$ an der Konstituentenzahl $N$ des Clusters beträgt folglich:

$$
\frac{N_{\mathrm{O}}}{N}=\frac{4 N^{2 / 3}}{N}=4 N^{-1 / 3}
$$

Durch ihre große relative Oberfläche eröffnen Cluster einen experimentellen Zugang zu mikrophysikalischen Oberflächenprozessen 26. Im Gegensatz zu makroskopischen Eiskristallen, die seit Jahrhunderten Gegenstand der Forschung sind, ist die Eisoberfläche erst seit Kurzem in den Fokus des wissenschaftlichen Interesses Gerückt. Der Hauptgrund hierfür ist die schlechte experimentelle Zugänglichkeit der Oberfläche, die in makroskopischen Kristallen nur einen verschwindend geringen Anteil der untersuchten Probe ausmacht 79.

Wie oben erwähnt, lässt sich zwischen der Konstituentenzahl eines Partikels und der Ausprägung seiner quantifizierbaren Eigenschaften ein empirischer Zusammenhang ableiten, der einem Potenzgesetz entspricht. Mit Blick auf Gleichung 2.5 wäre zu erwarten, dass alle Messgrößen, die in kausalem Zusammenhang zum Oberfläche-VolumenVerhältnis kleiner Cluster stehen, ein entsprechendes Skalierungsverhalten zeigen. Im Falle von Wasserclustern gilt dies z. B. für den Quotienten der Bandenintensitäten freier $\mathrm{OH}-\mathrm{Gruppen}$ auf der Oberfläche und gebundener OH-Gruppen im Clusterinneren 76 . In der Literatur finden sich jedoch zahlreiche weitere Beispiele energetischer Eigenschaften, die nicht in direktem Zusammenhang mit der relativen Clusteroberfläche stehen und dennoch gemäß Gleichung 2.5 skalieren: Ein Skalierungsverhalten mit $N^{-1 / 3}$ wurde an adiabatischen und vertikalen Ionisationsenergien neutraler und anionischer Wasserund Ammoniakcluster 92 sowie an Solvatationsenergien von Anionen, Kationen und Elektronen $26|30| 99]$ beobachtet. Das vertikale Ionisationspotential (VIP) natriumdotier- 
ter Wassercluster fällt im Bereich $N \leq 4$ linear mit $(N+1)^{-1 / 3}$ ab 100 , obgleich die sphärische Näherung hier sicherlich nicht angebracht ist. Eine Voraussetzung für solches Verhalten ist, dass die betreffende Messgröße nicht ausschließlich auf kurzreichweitigen Wechselwirkungen mit den nächsten Clusterkonstituenten beruht, sondern langreichweitige Wechselwirkungen mit jedem Atom bzw. Molekül im Cluster einen wesentlichen Beitrag leisten 93|95|101|102]. So können Elektronen, die auf der Oberfläche eines Clusters lokalisiert sind, eine abweichende IP-Entwicklung zeigen 94 . Gleiches gilt, wenn der Gyrationsradius des Elektrons bzw. der Radius des Anions nicht deutlich kleiner ist, als der Radius des Clusters. Unter Annahme der sphärischen Näherung lässt sich die mittlere Entwicklung einer energetischen Messgröße $E(N)$, deren Wert im makroskopischen Grenzfall gegen einen endlichen Wert konvergiert, entsprechend des Liquid-Drop-Modells als Summe von Energiebeiträgen $E_{\mathrm{V}}$ (des Volumens), $E_{\mathrm{O}}$ (der Oberfläche) und $E_{\mathrm{Kr}}$ (der Oberflächenkrümmung) ausdrücken 26[88.

$$
E(N) / N=E_{\mathrm{V}}+E_{\mathrm{O}} N^{-1 / 3}+E_{\mathrm{Kr}} N^{-2 / 3}=\varphi r_{\text {mono }}^{3}+\vartheta r_{\text {mono }}^{2} N^{-1 / 3}+\varsigma r_{\text {mono }} N^{-2 / 3}
$$

Ein diesem Potenzgesetz entsprechendes Skalierungsverhalten ist folglich durchaus auf die sphärische Symmetrie eines Clusters zurückzuführen, in dem Konstituenten mit verschiedener Koordinationsumgebung in unterschiedlichem Ausmaß zur Ausprägung einer Eigenschaft beitragen.

\subsection{Phasenänderungen in begrenzten Systemen}

In einem makroskopischen System findet ein Phasenübergang dann statt, wenn hierdurch eine Minimierung der freien Energie $F(T, V, N)$ bzw. der freien Enthalpie $G(T, p, N)$ erreicht werden kann. Dieses Prinzip der Minimierung stellt die Bedingung für den Verlauf der Phasengrenzlinien im $(p, T)$-Diagramm dar. Da es sich bei den oben genannten $\mathrm{Zu}$ standsgrößen um extensive Größen handelt, setzen diese sich aus den anteiligen Beiträgen der flüssigen (l) und der festen Phase (s) zusammen:

$$
G=G_{1}+G_{\mathrm{s}}
$$

Unter den unabhängigen Variablen, von denen die Funktion $G_{\mathrm{s}}\left(T, p, N_{\mathrm{s}}\right)$ abhängt, ist nur $N_{\mathrm{s}}$ ebenfalls eine extensive Größe. Daher skaliert $G_{\mathrm{s}}$ linear mit $N_{\mathrm{s}}$ :

$$
G_{\mathrm{s}}=N_{\mathrm{s}} g_{\mathrm{s}}(T, p)
$$

Hierbei stellt $g_{\mathrm{s}}$ die spezifische freie Enthalpie der festen Phase dar. Gleichung 2.10 gilt analog auch für die flüssige Phase. Die spezifische freie Enthalpie einer Substanz bzw. Phase i, d.h. die Änderung der freien Enthalpie mit deren Teilchenzahl, wird als chemisches Potential $\mu_{\mathrm{i}}$ von i bezeichnet:

$$
\mu_{\mathrm{i}}(T, p)=\left(\frac{\partial G}{\partial N_{\mathrm{i}}}\right)_{T, p}=g_{\mathrm{i}}(T, p)
$$

Gleichungen 2.10 und 2.11 gelten in analoger Weise auch für die freie Energie $F$. Die freie Enthalpie des Gesamtsystems setzt sich additiv aus diesen Beiträgen Zusammen: 


$$
G\left(T, p, N_{\mathrm{s}}, N_{\mathrm{l}}\right)=N_{\mathrm{s}} \mu_{\mathrm{s}}(T, p)+N_{\mathrm{l}} \mu_{\mathrm{l}}(T, p)
$$

Geht nun ein Teilchen von der festen in die flüssige Phase über, gilt bei $N=$ konst:

$$
\begin{aligned}
\mathrm{d} N_{\mathrm{l}} & =-\mathrm{d} N_{\mathrm{s}} \\
\mathrm{d} G & =\left(\mu_{\mathrm{l}}-\mu_{\mathrm{s}}\right) \mathrm{d} N_{\mathrm{s}}
\end{aligned}
$$

Das Gleichgewicht zwischen beiden Phasen hat sich eingestellt, sofern der Übergang weiterer Teilchen zwischen den beiden Phasen die gesamte freie Enthalpie nicht weiter vermindert. Die Bedingung für die in diesem Falle vorliegende Koexistenz zweier Phasen lautet folglich 103 :

$$
\begin{aligned}
\mathrm{d} G & =0 \\
G_{\mathrm{S}}(T, p) & =G_{\mathrm{l}}(T, p)
\end{aligned}
$$

Bezogen auf das chemische Potential gilt Ausdruck 2.17

$$
\mu_{\mathrm{s}}(T, p)=\mu_{\mathrm{l}}(T, p)
$$

Diese Bedingungen sind im $(p, T)$-Diagramm auf der Phasengrenzlinie erfüllt (vgl. Abb. 2.1). Nähert sich der durch Druck und Temperatur definierte Zustand einer thermodynamisch stabilen Phase 1 der Phasengrenzlinie an, steigt deren freie Enthalpie (bzw. deren chemisches Potential) mit zunehmender Nähe zur Phasengrenzlinie an. Jenseits der Phasengrenzlinie übertrifft das chemische Potential von Phase 1 das Potential einer Phase 2, die in diesem Zustand thermodynamisch begünstigt ist. Eine Phasengrenzlinie im $(p, T)$-Diagramm markiert somit die Schar von Punkten, in denen die Werte der Freien Enthalpien zweier Phasen als Funktion der Kontrollparameter $T$ und $p$ den gleichen Wert besitzen. Auf dieser Linie liegen jedoch keine differenzierbaren Maxima vor. Vielmehr sind die Punkte auf der Phasengrenzlinie Singularitäten bezogen auf die freie Enthalpie des gesamten Systems, da sich die Werte dieser Größe als Funktion von Druck und Temperatur für die jeweiligen Phasen in der Phasengrenzlinie schneiden. Es ist daher nicht möglich, ein System in einem auf dieser Linie liegenden Zustand zu äquilibrieren, obwohl hier formal die Bedingungen für die Koexistenz beider Phasen erfüllt sind $\stackrel{2}{2}^{2}$

\subsubsection{Der Beitrag der Phasengrenzflächen zur freien Enthalpie}

Die Koexistenz zweier räumlich getrennter Phasen in einem System geht zwangsläufig mit der Ausbildung einer Grenzfläche $A$ zwischen diesen Bereichen einher. Mikroskopische Systeme zeichnen sich dadurch aus, dass die Anzahl der Konstituenten im Inneren dieser Grenzfläche und damit deren Beitrag zur Energie des Systems im Gegensatz zu makroskopischen Systemen nicht mehr vernachlässigbar ist (vgl. Abschnitt 2.1). Der Beitrag der Grenzfläche dürfte sich daher entsprechend Gleichung 2.7 näherungsweise proportional

${ }^{2}$ Diese Aussage gilt nur in dem Fall, dass das Volumen konstant gehalten wird. Sofern zwei unabhängige Kontrollparameter unbestimmt sind, ist das System in der Lage, seinen Gleichgewichtszustand zu finden und zwei Phasen auszubilden. 


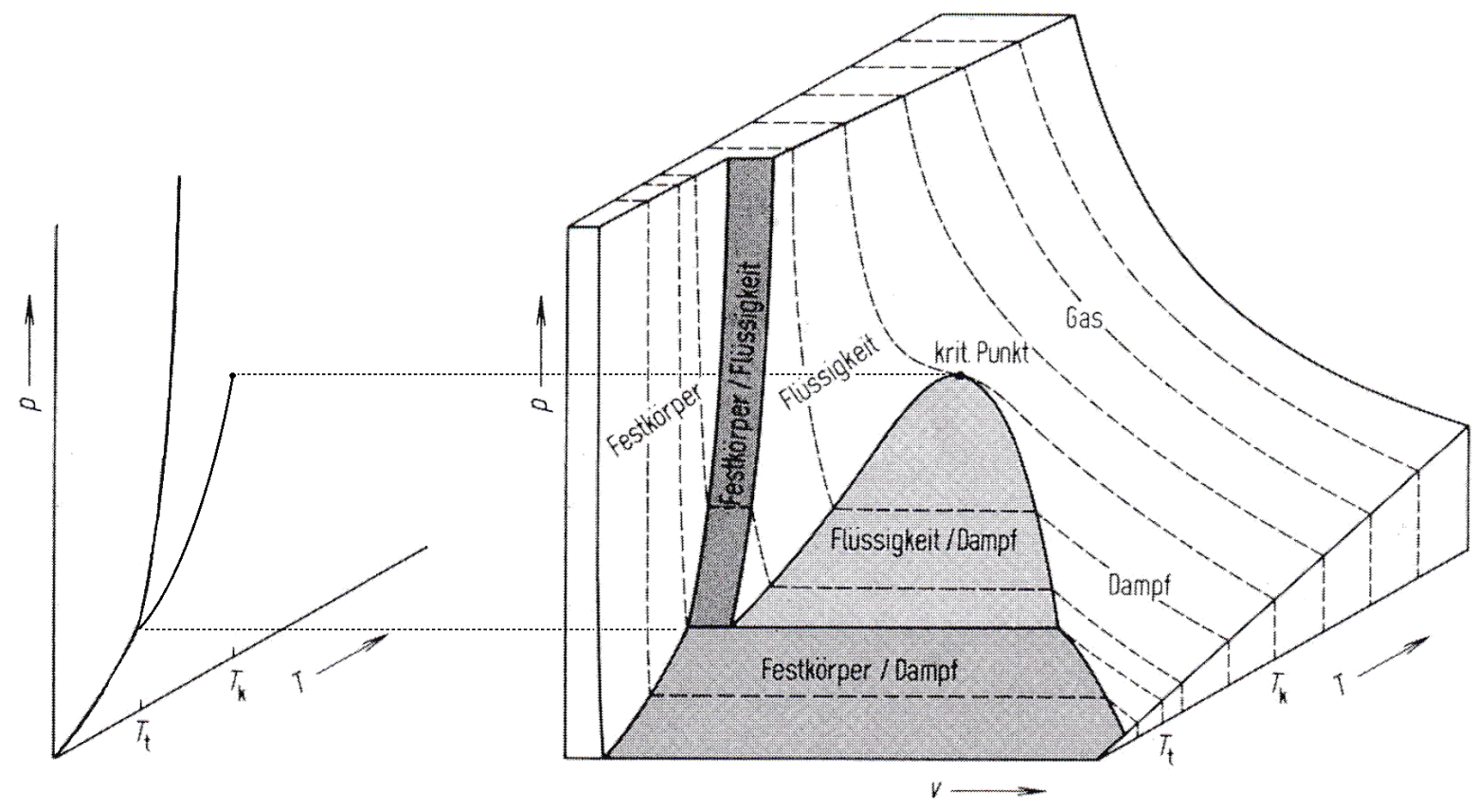

Abbildung 2.1.: $(p, V, T)$-Diagramm eines Einkomponentensystems; verändert nach Referenz 104.

zum Anteil der in der Grenzfläche enthaltenen Monomere und zum Quotienten $A / V$ verhalten.

Im thermodynamischen Formalismus tritt diese Grenzfläche $A$ als zusätzliche Variable auf, die in Form reversibler Arbeit $W_{\text {rev }}$ einen Beitrag zum Gesamtenergiegehalt eines Systems leistet:

$$
\mathrm{d} G=-S d T+V \mathrm{~d} p+\sum_{\mathrm{i}} \mu_{\mathrm{i}} \mathrm{d} N_{\mathrm{i}}+\mathrm{d} W_{\text {rev }}
$$

Dieser Größe wird als Formelzeichen der griechische Buchstabe $\sigma$ zugeordnet:

$$
\sigma \equiv{\frac{\partial W_{\text {rev }}}{\partial A}}_{S, V, p, T, N}=\frac{\partial U}{\partial A}_{S, V, N}=\frac{\partial G}{\partial A}_{T, p, N}=\frac{\partial F}{\partial A}_{T, V, N}
$$

Damit lässt sich die freie Enthalpie in Glg. 2.18 als Funktion der Oberfläche ausdrücken. Im einfachsten Falle sind keine geometrischen Faktoren zu berücksichtigen:

$$
\mathrm{d} G=-S d T+V \mathrm{~d} p+\sum_{\mathrm{i}} \mu_{\mathrm{i}} \mathrm{d} N_{\mathrm{i}}+\sigma \mathrm{d} A
$$

Die physikalische Bedeutung, die den in Gleichung 2.19 aufgeführten Differentialquotienten und somit der Größe $\sigma$ zukommt, lässt sich aus einer Dimensionsbetrachtung ableiten: Der Ausdruck beschreibt das Verhalten einer zweidimensionalen Teilchenschicht und hat die Dimension Arbeit pro Fläche bzw. Kraft pro Länge. Im dreidimensionalen Fall (sofern $A$ die Dimension eines Volumens hätte) würde ihm die Dimension einer Spannung (Kraft pro Fläche) zufallen. Daher wird $\sigma$ als Oberflächenspannung bezeichnet 104 . 
Der Flächeninhalt $A$ der Grenzfläche kann nur eingeschränkt als extensive Variable betrachtet werden. Das Ausmaß ihrer Änderung mit der Größe des Systems hängt von dessen Geometrie ab. Im Falle eines sphärischen Systems, dem näherungsweise die im Folgenden behandelten, wässrigen Nanotröpfchen entsprechen, skaliert sie nach Gleichung 2.7 mit $N^{1 / 3}$. Dieses Skalierungsverhalten hat Auswirkungen auf den fest-flüssig-Phasenübergang in solchen Partikeln sowie auf deren Kondensations- und Evaporationsverhalten.

\section{Der Einfluss des Krümmungsradius}

Im Inneren eines sphärischen Tropfens mit dem Radius $r$, der mit der ihn umgebenden Gasphase bei gegebener Temperatur unter dem Druck $p_{\text {eq }}$ im Gleichgewicht steht, herrscht aufgrund der Grenzflächenspannung ein höherer Druck mit dem Wert $p_{1}$. Dieser Druck ist eine auf die Tropfenoberfläche normierte Gegenkraft, die das Tropfeninnere dem Bestreben der Oberfläche entgegensetzt, sich zu kontrahieren. Wird dem Tropfen eine Substanzmenge hinzugefügt, die dessen Radius um den Betrag $\mathrm{d} r$ vergrößert, so erfordert dies das Aufbringen einer Volumenarbeit. Diese setzt sich zusammen aus einem Beitrag $p_{\text {eq }} \mathrm{d} V$, die gegen den Umgebungsdruck verrichtet wird, und einem Beitrag $\left(p_{1}-p_{\text {eq }}\right) \mathrm{d} V$ gegen den im Tropfeninneren herrschenden Druck, die der aufzubringenden Oberflächenarbeit $\sigma \mathrm{d} A$ äquivalent ist. Bezogen auf den Radius ändert sich die freie Enthalpie des Tropfens somit entsprechend Gleichung 2.21

$$
p \mathrm{~d} V=4 \pi r^{2}\left(p_{1}-p_{\text {eq }}\right) \mathrm{d} r=8 \pi r \sigma \mathrm{d} r=\sigma \mathrm{d} A
$$

Umformung nach $p_{1}$ liefert die Young-Laplace-Gleichung:

$$
p_{\mathrm{l}}=p_{\mathrm{eq}}+\frac{2 \sigma}{r}
$$

Der zweite Term auf der rechten Seite von Gleichung 2.22 skaliert invers proportional zum Radius des Partikels und wird als Laplace-Druck bezeichnet. Hierbei handelt es sich um den Betrag, um den der Druck im Inneren eines kleinen, sphärischen Partikels durch dessen Oberflächenspannung erhöht wird ${ }^{9}$. Sollen beide Phasen im Gleichgewicht koexistieren, müssen wiederum die chemischen Potentiale beider Phasen identisch sein (vgl. Glg. 2.20). Da die Stoffmenge konstant bleibt, gilt Gleichung 2.13 analog. Damit lässt sich die Gleichgewichtsbedingung wie folgt ausdrücken:

$$
\mathrm{d} G_{p, T}=N\left(\mu_{\mathrm{l}}-\mu_{\mathrm{v}}\right)+\sigma \mathrm{d} A
$$

Aus Gleichung 2.23 geht hervor, dass der Übergang eines Monomers von der Gasphase in die flüssige Phase mit zweierlei Beiträgen zur freien Enthalpie einher geht: Der erste Beitrag ist der Energiegewinn, der aus dem Übergang eines Monomers aus der Gasphase in die flüssige Phase resultiert, sofern die flüssige Phase die Stabilere ist. Dieser Beitrag lässt sich ausdrücken als $N\left(\mu_{1}-\mu_{\mathrm{v}}\right)$ und ist bei höherer Stabilität der Flüssigkeit negativ, da das chemische Potential der flüssigen Phase hier den niedrigeren Wert besitzt. Der zweite Beitrag besteht in der Oberflächenarbeit $\sigma \mathrm{d} A$, die bei der Vergrößerung des Tropfens aufgewendet werden muss. Letztere leistet in jedem Fall einen positiven Beitrag und setzt somit die thermodynamische Triebkraft zur Vergrößerung des Tropfens herab. Im Falle eines sphärischen Tropfens entspricht die zu leistende Oberflächenarbeit dem Betrag $4 \pi r^{2} \sigma$. Unter Zuhilfenahme eines Ausdrucks für die Anzahl $N$ der Monomere eines 
Tropfens mit dem Radius $r$, der sich aus Gleichung 2.1 ableiten lässt,

$$
N=\frac{4 \pi r^{3}}{3 v_{\text {mono }}}
$$

und Einsetzen dieses Quotienten sowie des oben angegebenen Ausdrucks für die Oberflächenarbeit in Gleichung 2.23 erhält man:

$$
\Delta G_{p, T}=\frac{4 \pi r^{3}}{3 v_{\text {mono }}}\left(\mu_{1}-\mu_{\mathrm{v}}\right)+4 \pi r^{2} \sigma
$$

Die Konstanz der freien Enthalpie als Gleichgewichtsbedingung muss nun allerdings bei den verschiedenen Drücken erfüllt sein, die in der jeweiligen Phase herrschen. Die Druckabhängigkeit der chemischen Potentiale $\mu_{1}$ und $\mu_{\mathrm{v}}$ muss berücksichtigt werden. Ein Ausdruck für $\mu_{\mathrm{l}}-\mu_{\mathrm{v}}$ ergibt sich aus Glg. 2.20 nach $\mathrm{d} \mu_{\mathrm{i}}=\mathrm{d} g_{\mathrm{i}}=v_{\mathrm{i}} \mathrm{d} p$ bzw. $\mathrm{d} g_{\mathrm{i}}=\left(v_{\mathrm{l}, \mathrm{i}}-v_{\mathrm{g}, \mathrm{i}}\right) \mathrm{d} p$. Unter Vernachlässigung von $v_{\mathrm{l}, \mathrm{i}}$ und mit Hilfe des idealen Gasgesetzes lässt sich Gleichung 2.26 ableiten.

$$
\mu_{p_{2}}-\mu_{p_{1}}=k_{\mathrm{B}} T \int_{p_{1}}^{p_{2}} \frac{\mathrm{d} p}{p}=k_{\mathrm{B}} T \ln \left(\frac{p_{2}}{p_{1}}\right)
$$

Durch Einsetzen in Gleichung 2.25 ergibt sich Gleichung 2.27.

$$
\Delta G_{p, T}=-\frac{4 \pi r^{3}}{3 v_{\text {mono }}} k_{\mathrm{B}} T \ln \Phi+4 \pi r^{2} \sigma
$$

Der Ausdruck $\Phi$ steht hierbei für den Quotienten aus dem Gleichgewichtsdampfdruck $p_{\mathrm{v}}$, der sich bei einer gegebenen Temperatur in der Gasphase über einer makroskopischen kondensierten Phase einstellt, und dem Druck $p_{\text {eq }}$, der notwendig ist, damit ein Tropfen des Radius $r$ mit einer ihn umgebenden Gasphase im Gleichgewicht steht. Dieser Quotient wird auch als Übersättigungsverhältnis bezeichnet:

$$
\Phi \equiv \frac{p_{\mathrm{eq}}}{p_{\mathrm{v}}}
$$

Bei $\Phi<1$ tragen beide Terme positiv zu $\Delta G$ bei, sodass sich keine neue Phase bilden kann. Bei $\Phi>1$ leistet der erste Term einen negativen Beitrag, während der Zweite weiterhin positiv bleibt und die Triebkraft für die Ausbildung der neuen Phase vermindert. Dieser Term überwiegt bei kleinen Radien $r$, sodass die Bildung eines sehr kleinen Partikels aus einer homogenen Gasphase thermodynamisch ungünstig wird. Erst, wenn der Radius einen kritischen Wert $r^{*}$ überschreitet, wird die freie Enthalpie beim Übergang eines Monomers in die neu gebildete Phase negativ, sodass effektiv eine Barriere entsteht, die die Bildung einer neuen Phase verhindert (vgl. Abb. 2.21 105. Der kritische Radius $r^{*}$ ist somit durch ein Maximum sowie eine verschwindende erste Ableitung von $\Delta G_{p, T}(r)$ gekennzeichnet. Mit $\Delta G_{p, T}(r)=0$ lässt sich aus Gleichung 2.27 der kritische Radius bei gegebener Temperatur und gegebenem Übersättigungsverhältnis ermitteln:

$$
r^{*}=\frac{3 \sigma v_{\text {mono }}}{k_{\mathrm{B}} T \ln \Phi}
$$

Mit der Definition des Übersättigungsverhältnisses $\Phi$ aus Gleichung 2.28 erhält man durch Umformen nach $p_{\text {eq }} 103$ : 


$$
p_{\text {eq }}=p_{\mathrm{v}} \exp \left(\frac{3 \sigma v_{\text {mono }}}{k_{\mathrm{B}} T \cdot r}\right)
$$

Gleichung 2.30 wird als Kelvin-Gleichung für sphärische Tropfen bezeichnet 105 . Aus ihr geht hervor, dass der Dampfdruck über einer gekrümmten Flüssigkeitsoberfläche, d. h. der Druck, bei dem die Flüssigkeit mit der daran angrenzenden Gasphase im Gleichgewicht steht, sich umso stärker erhöht, je kleiner der Krümmungsradius wird. Dieser KelvinEffekt tritt allgemein über gekrümmten Oberflächen auf, die z. B. von Flüssigkeiten in Kapillargefäßen ausgebildet werden 104 und ist $\mathrm{u}$. a. auch verantwortlich für die OstwaldReifung, die darin besteht, dass in einem aus unterschiedlich großen Tropfen bestehenden Aerosol sich die kleineren Tropfen auflösen, während die Größeren an Masse gewinnen 104.

Der Kelvin-Effekt tritt allgemein bei diskontinuierlichen Phasenübergängen auf, die mit einem Volumensprung verbunden sind. So bewirkt er ebenfalls eine Absenkung der Nukleationstemperatur mit abnehmendem Tropfenradius, d.h. der Temperatur, unterhalb der ein flüssiger Tropfen gefrieren kann 39 . Weiterhin verhindert er nicht nur die Kondensation aus der Gasphase, sondern auch die homogene Nukleation einer festen oder gasförmigen Phase aus der flüssigen Phase heraus. Formuliert man die freie Enthalpie der flüssigen Phase analog zu Glg. 2.23 als Summe eines Volumen- und eines Oberflächenterms, die unter den unterschiedlichen, im Tropfeninneren und der umgebenden Gasphase herrschenden Drücken im Gleichgewicht stehen müssen, lautet die Gleichgewichtsbedingung in Glg. 2.16 mit der Druckabhängigkeit von $\Delta G$ nach Glg. 2.26.

$$
G_{1}^{\circ}+W_{\text {rev }}=G_{\mathrm{v}}^{\circ}+R T \ln \left(\frac{p_{\mathrm{eq}}}{p^{*}}\right)
$$

Der Druck $p^{*}$ bezeichnet hierbei den Standarddruck von 1 bar, der Kreis ${ }^{\circ}$ bezeichnet eine thermodynamische Größe unter Standardbedingungen. Der Gleichgewichtsdampfdruck $p_{\text {eq }}$ einer kondensierten Phase mit vernachlässigbarem Oberflächenbeitrag (entspricht dem Standarddampfdruck $p^{\circ}$ ) ergibt sich durch Umformen von Glg. 2.31 zu:

$$
p^{\circ}=p^{*} \exp \left(\frac{G_{1}^{\circ}-G_{\mathrm{v}}^{\circ}}{R T}\right)
$$

Durch Umstellen von Gleichungen 2.31 und 2.32 nach dem Ausdruck $\left(G_{1}^{\circ}-G_{\mathrm{v}}^{\circ}\right)$ und Gleichsetzen erhält man für den Dampfdruck $p_{\text {eq }}$ über einer gekrümmten Oberfläche:

$$
p_{\text {eq }}=p^{\circ} \exp \left(\frac{W_{\text {rev }}}{R T}\right)
$$

Ein Vergleich von Gleichung 2.33 und Gleichung 2.30 liefert für den Oberflächenbeitrag $W_{\text {rev }}$ der freien Enthalpie Gleichung 2.34, wobei der in der Gasphase herrschende Dampfdruck $p_{\mathrm{v}}$ als Dampfdruck unter Standardbedingungen $p^{\circ}$ interpretiert wird:

$$
W_{\mathrm{rev}}=\frac{3 \sigma_{\mathrm{l, \textrm {v }}} \cdot v_{\mathrm{mono}, 1}}{r}
$$

Unter Verwendung dieses Ausdrucks für $W_{\text {rev }}$ soll nun in Form der Gibbs-ThomsonGleichung ein Ausdruck für die Änderung der Gleichgewichtsschmelztemperatur kleiner Tröpfchen hergeleitet werden: Für eine sphärische feste Phase im Inneren einer makroskopischen Flüssigkeit (d. h. bei vernachlässigbarem Laplace-Druck, sodass die freie Enthal- 


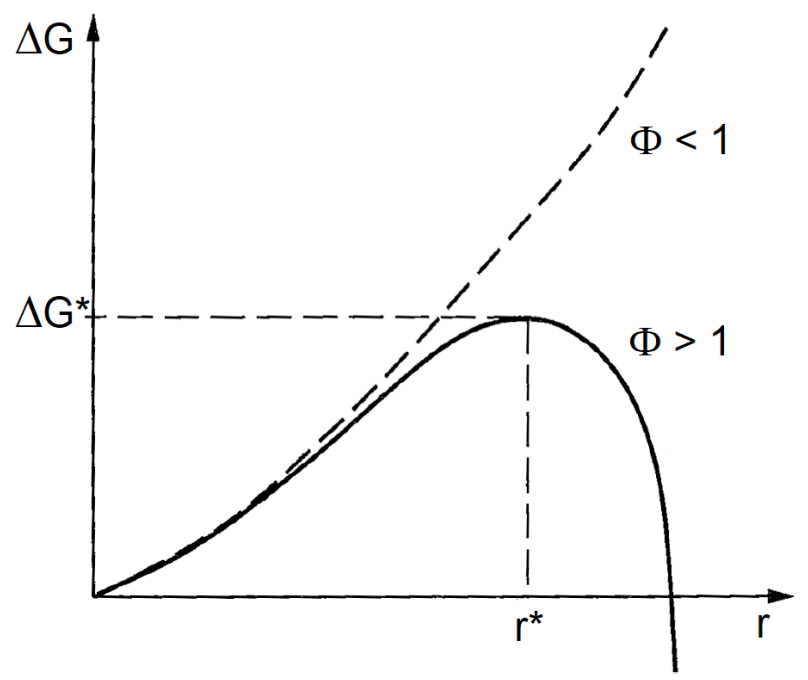

Abbildung 2.2.: $\Delta G$ bei homogener Nukleation als Funktion des Partikelradius $r$ bei $\Phi<1$ und $\Phi>1$; verändert nach 105

pie dem Wert unter Standardbedingungen entspricht) lässt sich Gleichung 2.16 analog als Summe von Beiträgen der Enthalpie $H$, der Entropie $S$ und der Oberflächenarbeit $W_{\text {rev }}$ formulieren,

$$
H_{\mathrm{l}}^{\circ}-T S_{\mathrm{l}}^{\circ}=H_{\mathrm{s}}^{\circ}-T S_{\mathrm{s}}^{\circ}+\frac{3 \sigma_{\mathrm{s}, 1} v_{\mathrm{mono}, \mathrm{s}}}{r}
$$

wobei $\sigma_{\mathrm{s}, 1}$ nun die Oberflächenspannung zwischen fester und flüssiger Phase darstellt. Nun wird Glg. 2.35 nach der Temperatur umgestellt, wobei die Differenzen der Enthalpie bzw. der Entropie zwischen beiden Phasen als Schmelzenthalpie $\Delta_{\mathrm{m}} H^{\circ} \equiv H_{\mathrm{l}}^{\circ}-H_{\mathrm{s}}^{\circ}$ bzw. Schmelzentropie $\Delta_{\mathrm{m}} S^{\circ} \equiv S_{1}^{\circ}-S_{\mathrm{s}}^{\circ}$ definiert werden:

$$
\Delta_{\mathrm{m}} H^{\circ}=T \cdot \Delta_{\mathrm{m}} S^{\circ}+\frac{3 \sigma_{\mathrm{s}, 1} \cdot v_{\mathrm{mono}, \mathrm{s}}}{r}
$$

Sofern der Radius $r$ groß genug ist, kann der Oberflächenterm von Gleichung 2.36 vernachlässigt werden, sodass am Schmelzpunkt unter Gleichgewichtsbedingungen der bekannte Zusammenhang zwischen den thermodynamischen Größen unter Standardbedingungen gilt: $\Delta_{\mathrm{m}} H^{\circ}=T_{\mathrm{m}}^{\circ} \cdot \Delta_{\mathrm{m}} S^{\circ}$. Einsetzen in Gleichung 2.36 liefert die Gibbs-ThomsonGleichung, die das aufgrund des Oberflächenbeitrags abweichende Schmelzverhalten eines kleinen, sphärischen Tropfens beschreibt 103.

$$
T_{\mathrm{m}}=T_{\mathrm{m}}^{\circ}-\frac{3 \sigma_{\mathrm{s}, 1} \cdot v_{\mathrm{mono}, \mathrm{s}}}{r \cdot \Delta_{\mathrm{m}} S^{\circ}}
$$

Mit der Definition des molaren Volumens $v_{\mathrm{m}} \equiv V / n$ lässt sich Gleichung 2.37 auch in Abhängigkeit von den molaren Größen ausdrücken. Bei genauer Betrachtung müsste an dieser Stelle anstatt des molaren Volumens die relative Änderung des molaren Volumens eingesetzt werden. Damit wird deutlich, dass der im Inneren der neuen Phase herrschende Laplace-Druck zu einer Verschiebung der Phasenkoexistenzlinie zu höheren Drücken führt, sofern der Phasenübergang mit einer Volumenzunahme verbunden ist. Da im Falle von Wasser sowohl die Evaporation als auch die Kristallisation mit einer Zunahme des molaren 
Volumens einhergehen, werden diese Phasenübergänge bei gegebener Temperatur erst bei höherem Druck, bzw. bei gegebenem Druck schon bei niedrigerer Temperatur stattfinden.

Wenngleich die Gibbs-Thomson-Gleichung und die Kelvin-Gleichung auf den ersten Blick unterschiedlich aussehen, stellen sie analoge Beschreibungen des gleichen Phänomens dar 103. Es ist zu beachten, dass die Gibbs-Thomson-Gleichung unter der Annahme von Gleichgewichtsbedingungen hergeleitet wurde und somit die Veränderung der Phasenübergangstemperatur im thermodynamischen Gleichgewicht beschreibt 106.

Im Falle wässriger Tropfen erhält der Kelvin-Effekt eine signifikante Bedeutung, sofern der Tropfenradius einen Wert von ca. $50 \mathrm{~nm}$ unterschreitet ${ }^{105}$. Nimmt der Radius weiter ab, kommt ein weiterer Effekt zum Tragen: Sinkt der Radius auf molekulare Dimensionen, kann die Dicke der Grenzschicht nicht länger vernachlässigt werden. Die Oberflächenspannung beginnt, sich in Abhängigkeit vom Radius zu verändern. Tolman schlug daher im Jahre 1949 die Einführung eines Korrekturterms vor, der diesen Effekt durch einen empirischen Parameter, die sogenannte Tolman-Länge $\delta_{\mathrm{T}}$ quantifiziert 107:

$$
\sigma_{\mathrm{T}}=\frac{\sigma}{1+\frac{2 \delta_{\mathrm{T}}}{r}}
$$

Übliche Werte für $\delta_{\mathrm{T}}$ betragen ca. $10-20 \%$ des Moleküldurchmessers. Negative Werte bedeuten eine steigende Oberflächenspannung mit abnehmendem Radius. Für Wasser wurde kürzlich der Wert $\delta_{\mathrm{T}} \approx 0,3 \mathrm{~nm}$ angegeben 108 . Mit $\sigma \approx 72 \mathrm{mN} \mathrm{m}^{-2}$ bei einer Temperatur von $298 \mathrm{~K}[105$ ergibt sich für einen Tropfen mit einem Durchmesser von $2 \mathrm{~nm}$ ein Laplace-Druck von ca. $110 \mathrm{MPa}$ (ca. 1110 bar), der gut mit den Ergebnissen der in Referenz [108] durchgeführten Simulationen übereinstimmt. Dabei ist zu berücksichtigen, dass die Oberflächenspannung flüssigen Wassers mit sinkender Temperatur zunimmt.

\section{Premelting - Mobilere Phasenzustände der Oberfläche}

Nicht jeder diskontinuierliche Phasenübergang setzt das Eintreten eines Nukleationsereignisses voraus. So lässt sich der Zustand einer metastabilen Flüssigkeit oberhalb der Siedetemperatur insbesondere im Falle von Wasser leicht herstellen. Hingegen ist es nicht möglich, den entsprechenden Festkörper zu überhitzen, ohne ihn zu verflüssigen. Gustav Tammann postulierte daher im Jahr 1910 die Existenz eines Flüssigkeitsfilms auf der Festkörperoberfläche bei Temperaturen unterhalb der Phasenübergangstemperatur ${ }^{109}$, die als Nukleationskeim der flüssigen Phase dienen kann. Eine direkte Beobachtung einer solchen Schicht gelang erstmals durch Frenken et al. im Jahr 1985 auf einer Bleioberfläche110. Im Falle von Wasser gilt die Existenz einer Schicht aus ungeordneten Molekülen auf der Oberfläche kristallinen Eises mittlerweile als gesichert 39|68|111|112]. Der Grad der Mobilität dieser Moleküle hängt naturgemäß von der Temperatur ab. Devlin et al. fanden in den äußersten Wassermolekülen bereits bei Temperaturen oberhalb von $60 \mathrm{~K}$ populierte Rotationszustände $[79$.

Zur freien Enthalpie eines festen Körpers, der von einer flüssigen Schicht umgeben ist (oder einer Flüssigkeit, auf deren Oberfläche sich eine feste Phase befindet), tragen zwei gespannte Oberflächen bei, deren Beitrag jeweils positiv ist. Die doppelte Grenzfläche kann jedoch nur stabil sein, wenn dadurch die freie Enthalpie des Systems insgesamt nicht erhöht wird, d.h. sofern die mit dem Vorliegen einer zweiten kondensierten Phase verbundene Erhöhung der freien Enthalpie durch die Herabsetzung der Grenzflächenener- 
gie kompensiert wird. Für einen Feststoff, der von einem Flüssigkeitsfilm überzogen ist, lässt sich dieser Beitrag als Differenz der Oberflächenspannungen ausdrücken, sofern die Dicke des Films den Durchmesser eines Moleküls deutlich übersteigt:

$$
\Delta \sigma \equiv \sigma_{\mathrm{s}, \mathrm{l}}+\sigma_{\mathrm{l}, \mathrm{v}}-\sigma_{\mathrm{s}, \mathrm{v}} \leq 0
$$

Die Ursache für die Oberflächenspannung besteht darin, dass die Summe der Kräfte, die auf die Moleküle in der Oberfläche wirken, sich von der Summe der Kräfte im Inneren einer Phase unterscheidet 113. Vermindert sich die Dicke $d$ der Grenzschicht soweit, dass sie molekulare Dimensionen erreicht, wird sich daher auch das Kräftegleichgewicht und somit auch die Spannung beider Oberflächen verändern111. Der Gesamtbeitrag der Oberflächenspannung ist daher eine Funktion der Dicke $d$ der Grenzschicht. Dash et al. definierten daher einen Grenzflächenkoeffizienten $\sigma(d)$, um dieses Verhalten zu beschreiben:

$$
\sigma(d)=\Delta \sigma \cdot f(d)+\sigma_{\mathrm{s}, \mathrm{v}}
$$

Der Wert des Oberflächenpotentials $f(d)$ nimmt bei $d=0$ den Wert 0 an und konvergiert bei $d \rightarrow \infty$ gegen den Wert 1. Der Wert des Grenzflächenkoeffizienten ändert sich entsprechend vom Wert der unbenetzten Oberfläche $\sigma_{\mathrm{s}, \mathrm{v}}$ bei $d=0$ auf $\sigma_{\mathrm{s}, 1}+\sigma_{\mathrm{l, \textrm {v }}}$ bei $d \rightarrow \infty$. Die exakte funktionelle Abhängigkeit von der Filmstärke $d$, insbesondere bei sehr kleinem $d$, ist substanzabhängig und entzieht sich der analytischen Beschreibung, sodass hier auf empirisch erhaltene Werte oder Modellrechnungen zurückgegriffen werden muss.

In den gebogenen Grenzflächen kleiner Partikel verkomplizieren sich die Verhältnisse weiter, da hier eine Änderung der Filmdicke $d$ stets mit einer Änderung des Krümmungsradius $r$ sowie des Flächeninhalts $A$ der Grenzfläche verbunden ist. An dieser Stelle soll daher ein einfaches Modell beschrieben werden, das die Verhältnisse sphärischer Nanopartikel beschreibt 114 :

Ein sphärischer Partikel mit dem Radius $r$ besitze einen festen Kern mit dem Radius $r_{\mathrm{K}}$. Dieser Partikel befinde sich im Gleichgewicht mit der umgebenden Gasphase. Der Beitrag der Grenzfläche zur Energie dieses Systems beträgt dann:

$$
W_{\mathrm{rev}}=4 \pi r^{2}\left[\sigma_{\mathrm{s}, 1}\left(\frac{r_{\mathrm{K}}}{r}\right)^{2}+\sigma_{\mathrm{l}, \mathrm{v}}+\Delta \sigma \cdot f\left(r-r_{\mathrm{K}}\right)\right]
$$

Der Gewinn der freien Enthalpie durch die Bildung der flüssigen Phase auf der Oberfläche hängt dabei von den unterschiedlichen Krümmungsradien der beiden Oberflächen ab:

$$
\Delta \sigma=\sigma_{\mathrm{s}, \mathrm{v}}-\left[\sigma_{\mathrm{l}, \mathrm{v}}+\sigma_{\mathrm{s}, \mathrm{l}}\left(\frac{r_{\mathrm{K}}}{r}\right)^{2}\right]
$$

Das Oberflächenpotential $f\left(r-r_{\mathrm{K}}\right)$ nimmt bei $r=r_{\mathrm{K}}$ den Wert 1 und bei $r_{\mathrm{K}}=0$ den Wert 0 an. Im Falle eines komplett flüssigen Tropfens $\left(r_{\mathrm{K}}=0\right)$ wird $W_{\text {rev }}$ zu:

$$
W_{\text {rev }}\left(r_{\mathrm{K}}=0\right)=4 \pi r^{2} \sigma_{\mathrm{l}, \mathrm{v}}
$$


Im Falle eine komplett festen Partikels wird $W_{\text {rev }}$ zu:

$$
W_{\text {rev }}\left(r_{\mathrm{K}}=r\right)=4 \pi r^{2} \sigma_{\mathrm{s}, \mathrm{v}}
$$

Falls nur kurzreichweitige Wechselwirkungen zwischen den Atomen oder Molekülen an der Oberfläche vorliegen (wie es bei kleinen Metall- oder Van-der-Waals-Clustern der Fall ist), lässt sich $f(d)$ durch einen Exponentialterm der Form $f(d)=\exp ^{-d / \xi}$ annähern 114 . Im Falle von Wasser dürfte zur Beschreibung der Wechselwirkungen eine komplexere Funktion notwendig sein $62[108$.

\subsubsection{Statistische Beschreibung von Phasenänderungen}

Auf der Theorieebene stellt die statistische Mechanik die eingangs erwähnte Brücke zwischen der mikroskopischen und der makroskopischen Physik dar. Die unter diesen Begriff fallenden Modelle basieren auf der Idee, dass die Kenntnis des Zustandes eines jeden Atoms bzw. Moleküls nicht notwendig ist, um das mittlere Verhalten eines aus diesen Teilchen bestehenden Systems zu beschreiben, das sich im thermischen Gleichgewicht befindet. Die mittleren Eigenschaften eines solchen Systems konvergieren im Grenzfall unendlicher Teilchenzahl gegen die Eigenschaften der kondensierten Materie. Abweichungen einzelner Teilchen von diesen Mittelwerten fallen aufgrund der hohen Teilchenzahl in makroskopischen Systemen (sie liegt in der Größenordnung $10^{20}$ ) nicht ins Gewicht. Eine wesentliche Anwendung der statistischen Mechanik ist die Ableitung thermodynamischer Zustandsgrößen makroskopischer Systeme wie dem Druck $p$, der Temperatur $T$ oder der Entropie $S$, die nunmehr einer direkten mechanistischen Interpretation zugänglich werden 104 .

Die charakteristischen Eigenschaften eines Phasenübergangs erster Ordnung in der kondensierten Materie, d. h. ein unstetiges Verhalten der thermodynamischen Funktionen, die den Zustand eines aus $N$ Konstituenten bestehenden Systems beschreiben (insbesondere der spezifischen Freien Energie sowie der spezifischen Entropie), sind die Folge des asymptotischen Grenzfalls eines unendlich großen Systems $(N \rightarrow \infty)$.

Van Hove zeigte erstmals, dass die Singularitäten dieser Zustandsgrößen als Funktion der Temperatur verschwinden, sofern die Konstituentenzahl $N$ und das Volumen $V$ eines Systems als endlich betrachtet werden, und dass alle thermodynamischen Funktionen eines endlich großen Systems sich stetig verhalten und stetige Ableitungen besitzen müssen 115. Die Singularitäten, die im Grenzfall $N \rightarrow \infty$ am Punkt des Phasenübergangs auftreten, verändern sich demnach in endlich großen Systemen zu integrierbaren Maxima, die sich über einen endlichen Bereich des variierten Kontrollparameters erstrecken 116.

Das Verhalten von Clustern $(N \lesssim 100)$ lässt sich nicht mehr durch eine klassische thermodynamische Beschreibung erfassen, da sich ihr Verhalten nicht länger zuverlässig auf das Vorliegen eines einzigen Makrozustands reduzieren lässt, dessen statistisches Gewicht das aller anderen möglichen Makrozustände bei weitem übersteigt. R. Stephen Berry führte eine quantenstatistische Argumentationslinie ein $27|80| 81 \mid 86]$, die das abweichende Phasenverhalten kleiner Cluster und Nanopartikel in der Nähe der Phasenübergangstemperatur (im Folgenden $T_{\text {eq }}$ ) im Vergleich zur kondensierten Materie qualitativ beschreibt. Diese soll im Folgenden möglichst anschaulich skizziert werden: 
Im ersten Schritt werden die Zustandsdichten des feststoffartigen sowie des flüssigkeitsartigen Phasenzustands abgeschätzt. Mit dieser Information lassen sich die jeweiligen Zustandssummen $Z(T)$ als Funktion der Temperatur $T$ aufstellen, aus denen wiederum nach Gleichung 2.45 deren Freie Energie $F(T)$ ermittelt werden kann 104.

$$
F=-k_{\mathrm{B}} T \ln (Z)
$$

Der feststoffartige Phasenzustand wird als fast gänzlich starres, strukturiertes und konventionelles Molekül angenommen. Der flüssigkeitsartige Phasenzustand sei ein mobiles Gebilde, ähnlich einem beweglichen Molekül. Ist eine dieser Formen thermodynamisch stabil, so bildet sie (sowohl bei quantenmechanischer als auch bei klassischer Betrachtung) einen wohldefinierten Zustand aus. So kann die feststoffartige Phase z. B. durch ein Einstein-Modell 104 beschrieben werden, in dem sämtliche internen Bewegungsfreiheitsgrade als harmonische Oszillatoren mit gleicher Grundfrequenz beschrieben werden. Diesem lässt sich die Annahme hinzufügen, dass diese Frequenz von der Größe des Systems abhängt. Als stark vereinfachtes Modell zur Beschreibung flüssigkeitsartiger Cluster eignet sich z. B. das Gartenhaus-Schwartz-Modell117, das alle internen Freiheitsgrade als harmonische Oszillatoren mit gemeinsamer Grundfrequenz behandelt, wobei im Gegensatz zu Einsteins Modell keine ausgewiesenen Rotationsfreiheitsgrade angenommen werden. Auch komplexere, realitätsnähere Modelle sind denkbar, die z. B. die für Flüssigkeiten charakteristischen, niederfrequenten und hochamplitudigen Bewegungsmoden einbeziehen.

Nach Berry lassen sich die Zustandssummen sowie die freien Energien dieser beiden Zustände korrelieren, sodass deren Änderung beim Übergang zwischen beiden Zuständen quantifiziert werden kann 27. In der mathematischen Beschreibung der Zustandsänderung wird daher die freie Energie (bzw. die freie Enthalpie, sofern der Druck konstant gehalten wird) nicht nur als Funktion der Temperatur $T$ und der Teilchenzahl $N$, sondern auch als Funktion eines Mobilitätsparameters $\gamma$ ausgedrückt, der als Maß für die jeweiligen Anteile beider Zustände am Gesamtbild aufgefasst werden kann. Dieser Parameter kann auf unterschiedlichen Wegen formuliert werden. Eine mikroskopische Definition von $\gamma$ stellt die normierte Defektdichte dar: Stillinger und Weber gelang es, die Zustandssumme eines Kristalls als Funktion der Anzahl an Kristallgitterdefekten auszudrücken 118. Dieses Resultat wurde Von Wales und Berry generalisiert, sodass der Wert von $\gamma$ sich nach Normierung auf die Anzahl der Gitterplätze im Intervall $[0 \leq \gamma \leq 1]$ bewegt 27 .

Besitzt das System eine niedrige innere Energie, so weist die feststoffartige Form eine höher Zustandsdichte auf, als die flüssigkeitsartige Form: Der Wert ihrer Zustandssumme ist größer und die freie Energie damit niedriger, als die Werte der flüssigkeitsartigen Phase. Die feststoffartige Phase stellt somit den thermodynamisch stabilen Zustand dar. Wird dem System Energie zugeführt, erhöhen sich die Zustandsdichten beider Formen. Erfordert die Erzeugung von Defekten in der flüssigartigen Form einen Energieeintrag, wird deren Zustandsdichte den Wert 0 allerdings auch beibehalten, sofern das System bezogen auf die Nullpunktsenergien beider Formen bereits eine geringfügig höhere innere Energie besitzt, und erst später ansteigen, als die Zustandsdichte der feststoffartigen Form. Die Anzahl der zugänglichen Zustände und damit die Zustandsdichte der flüssigkeitsartigen Phase nimmt allerdings deutlich schneller zu, als die der feststoffartigen Phase. Als Folge der unterschiedlich schnell ansteigenden Entropiebeiträge zur jeweiligen freien Energie beider phasenartiger Zustände wird schließlich die freie Energie der feststoffartigen Phase die freie Energie der flüssigkeitsartigen Phase übersteigen. 
Die Anzahl der möglichen Zustände, die ein aus $N$ Konstituenten bestehender Cluster einnehmen kann, steigt näherungsweise faktoriell mit dessen Konstituentenzahl 104 . Für ein makroskopisches System mit $N \rightarrow \infty$ bedeutet dies, dass die Anzahl der in beiden Aggregatzuständen erreichbarer Zustände und damit ebenfalls die Beträge der freien Energie $F(T)$ in den jeweiligen Aggregatzuständen divergieren. Bei der Temperatur des Phasenübergangs $T_{\text {eq }}$ ändert sich deren Verhältnis nahezu unstetig: Das statistische Gewicht der einen Phase wird das statistische Gewicht der anderen Phase schlagartig um einen Betrag übersteigen, der ebenfalls gegen unendlich strebt. Ein Cluster mit einer endlichen Konstituentenzahl kann hingegen nur eine endliche Anzahl möglicher Zustände einnehmen. Dies führt zu einer endlichen Differenz der freien Energien beider möglicher Zustände $F_{1}-F_{\mathrm{s}}$.

Abbildung 2.3 a) zeigt die Konsequenzen, die sich daraus für den Verlauf der freien Energie eines sehr kleinen, endlichen Systems als Funktion von $T$ und $\gamma$ ergeben: Bei niedrigen Temperaturen zeigt die freie Energie $F(T, N, \gamma)$ zwischen einem Wert nahe $\gamma \approx 0$ und einem Wert $\gamma \approx 1$ ein monoton ansteigendes Verhalten.

Bei steigender Temperatur wird der Beitrag des Energieterms beider Formen linear (falls $C_{\mathrm{v}}=$ konst.) bzw. schneller (falls $C_{\mathrm{v}}$ ebenfalls zunimmt) ansteigen. Sowohl in feststoffartigen als auch in flüssigkeitsartigen Clustern wird der Anstieg des Entropieterms diesen Anstieg überkompensieren, sodass die freie Energie in beiden Fällen mit steigender Temperatur abnimmt. Der Abfall der freien Energie wird im flüssigkeitsartigen Cluster signifikant stärker ausfallen, da hier die Zustandsdichte mit zunehmender Energie schneller wächst, als die des feststoffartigen Clusters. Daher wird die zuvor monoton ansteigende Kurve der freien Energie als Funktion von $\gamma$ zusehends abflachen, bis ihre erste Ableitung bei einer Temperatur $T_{\mathrm{f}}$ für $\gamma \rightarrow 1$ den Wert 0 annimmt.

Ein weiterer Temperaturanstieg führt zur Ausbildung zweier Minima. Dieser Umstand impliziert das Vorliegen zweier lokal stabiler Zustände, die der Cluster einnehmen kann. Er stellt allerdings lediglich ein notwendiges und kein hinreichendes Kriterium für das vorliegen dieses Sachverhaltes dar. Eine weitere Voraussetzung ist, dass diese Formen lang genug existieren, um für den jeweiligen phasenartigen Zustand charakteristische Gleichgewichtseigenschaften auszubilden. Die Frage, ob diese Voraussetzung in einem System erfüllt ist, lässt sich ausschließlich durch Untersuchung seines dynamischen Verhaltens beantworten: Wechselt das System zu schnell zwischen den verschiedenen Minima, (d. h. auf einer Zeitskala, die nicht ausreichend lang im Vergleich zu den Schwingungsperioden der Konstituenten ist), ist es nicht in der Lage, sich in einem der beiden Zustände zu äquilibrieren.

Bei der Temperatur $T_{\text {eq }}$ hat die Zustandsdichte der flüssigkeitsartigen Phase infolge weiterer Temperaturerhöhung den gleichen Wert wie die der feststoffartigen Phase eingenommen. Wird schließlich die Temperatur $T_{\mathrm{m}}$ erreicht, verschwindet das Minimum in der Nähe von $\gamma=0$ und wird zu einem Wendepunkt. Diese Temperatur stellt die obere Grenze für die thermodynamische Stabilität des feststoffartigen Zustands dar. Bei höheren Temperaturen weist die Freie Energie nur ein einziges Minimum als Funktion von $\gamma$ auf, das auf Seiten der flüssigartigen Phase liegt.

Nach diesem Modell existiert für ein kanonisches Ensemble von Clustern mit einer definierten und konstanten Konstituentenzahl $N$ ein Temperaturfenster zwischen $T_{\mathrm{m}}$ und $T_{\mathrm{f}}$, in dem die Koexistenz feststoffartiger und flüssigkeitsartiger Zustände thermodynamisch möglich ist. Innerhalb dieses Koexistenzbereiches lässt sich der Anteil des jeweiligen Zu- 
a)

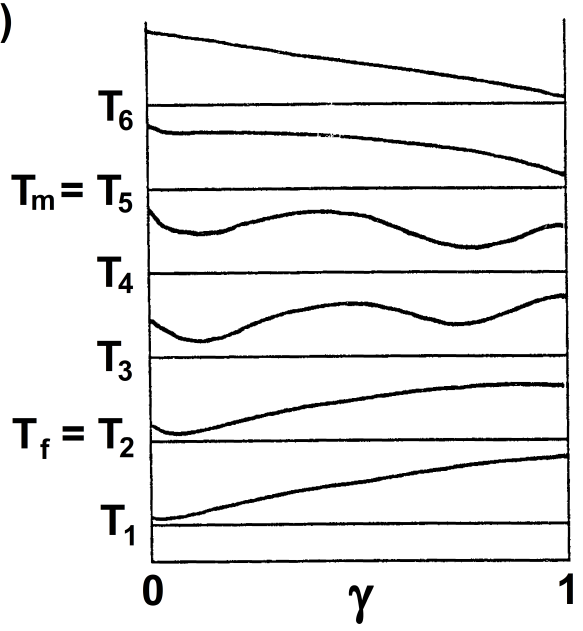

b)

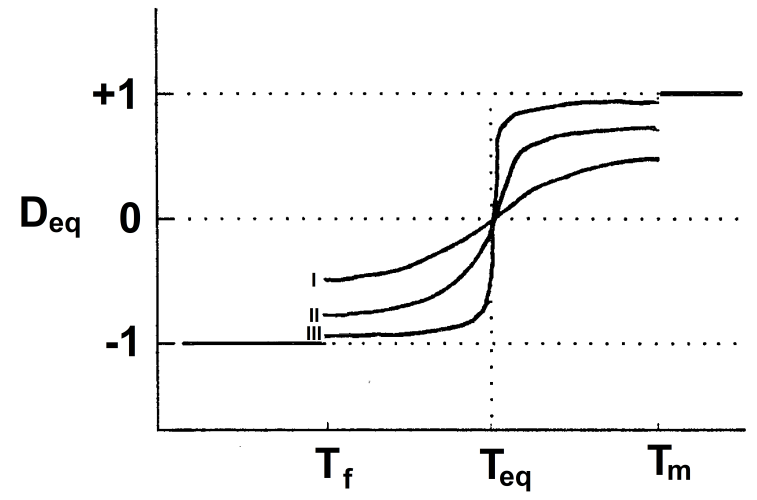

Abbildung 2.3.: Teil a): Qualitative Visualisierung der freien Energie als Funktion des Parameters $\gamma$ bei unterschiedlichen Temperaturen; Teil b): Qualitativer Verlauf der Gleichgewichtsverteilungsfunktion $D_{\text {eq }}$ für einen kleinen (I), einen mittleren (II) sowie einen großen Cluster (III), verändert nach Referenz 81 .

stands am Gesamtbild bezogen auf die Anzahl $N$ der Clusterkonstituenten durch die wohlbekannte Gleichgewichtskonstante $K_{\text {eq }}$ ausdrücken:

$$
K_{\text {eq }} \equiv \frac{[\text { flüssig }]}{[\text { fest }]}=e^{\frac{-\left(F_{1}-F_{\mathrm{s}}\right)}{k_{\mathrm{B}} T}}=e^{-\frac{N \Delta \mu}{k_{\mathrm{B}} T}}
$$

Hierbei stellt $\Delta \mu$ die Differenz der mittleren chemischen Potentiale der beiden Zustände dar. Am Punkt der Gleichgewichtstemperatur $T=T_{\text {eq }}$ ändert diese Größe entsprechend Gleichung 2.17 ihr Vorzeichen. Unter Bedingungen, die eine höhere thermodynamische Stabilität der flüssigkeitsartigen Phase bewirken, gilt somit:

$$
\mu_{\mathrm{l}}<\mu_{\mathrm{s}}
$$

Aus der obigen Argumentation folgt, dass der Wert von $K_{\text {eq }}$ bei $T<T_{\mathrm{f}}$ den Wert 0 annehmen und gleichsam bei $T>T_{\mathrm{m}}$ gegen unendlich streben muss. Im Intervall dazwischen wird $K_{\text {eq }}$ kontinuierlich ansteigen. Sofern das betrachtete System als ergodisch betrachtet werden kann, wird ein einzelner Cluster im Laufe seiner zeitlichen Entwicklung sämtliche Zustände mit der gleichen mittleren Wahrscheinlichkeit einnehmen, die dem über ein entsprechendes Ensemble gemittelten Durchschnittswert entsprechen würde. Folglich würde die Beobachtung bzw. die Simulation eines einzigen Clusters über einen ausreichend langen Zeitraum genügen, um den Wert von $K_{\text {eq }}$ näherungsweise zu ermitteln.

Das Erreichen einer näherungsweise als ergodisch zu betrachtenden Simulation birgt im Fall von Wasser allerdings eine Reihe von Problemen: Erstens ist der Phasenraum aufgrund der starken intermolekularen Wechselwirkungen durch hohe Barrieren gekennzeichnet, zwischen denen das System wechseln muss 62|91|119. Zweitens erweitert sich der Phasenraum, dessen Dimensionalität bereits durch die Orts- und Impulskoordinaten der Monomere exponentiell mit deren Anzahl $N$ anwächst, aufgrund der 
Anisotropie der Wechselwirkungen um eine ebenfalls exponentiell anwachsende Anzahl von Orientierungskoordinaten 48|120]. Während dem ersten Problem eine Erhöhung der Temperatur in den Simulationen entgegengesetzt werden kann 62, kann das zweite Problem dazu führen, dass die relative Wahrscheinlichkeit für das Auffinden eines naheliegenden Minimums aufgrund der großen Anzahl von Konfigurationsmöglichkeiten stark herabgesetzt wird. Zerfällt der Phasenraum aus diesen Gründen in mehrere Bereiche, zwischen denen das System quasi nicht wechseln kann, bezeichnet man die berechnete Trajektorie als quasiergodisch. Innerhalb eines solchen Bereiches kann das Kriterium der Ergodizität gut erfüllt sein, während sich das System bezogen auf den gesamten Phasenraum ausgesprochen nicht-ergodisch verhält.

Insbesondere in der Nähe diskontinuierlicher Phasenübergänge wird dieses Problem akut, da die beiden Phasen unterschiedlichen Bereichen des Zustandsraumes (bzw. Phasenraumes) zugeordnet werden müssen, zwischen denen relativ wenige Verbindungskanäle existieren. So erfordert die in-silico-Beobachtung von Nukleationsereignissen in Wasserclustern äußerst lange Simulationszeiten und daher hohe Rechenkapazitäten. Eine Beschneidung des Phasenraumes durch Implementierung eines starken elektrischen Feldes oder durch Reduktion des Systems auf eine Doppelschicht aus Wassermolekülen erhöht die Wahrscheinlichkeit eines Phasenübergangs deutlich 4 .

Dennoch konnte die von Berry vorgebrachte, oben beschriebene analytische Argumentationslinie durch eine Reihe von Simulationen gestützt werden (vgl. Ref. [81] und Referenzen darin). Johnston et al. beobachteten die Auflösung und Neubildung eines kristallinen Kernes im Inneren eines Wasserclusters aus 137 Monomeren in auf dem mW-Wassermodell basierenden MD-Simulationen 68. Kastelowitz et al. wiederum beobachteten in ihren MDSimulationen zahlreiche Oszillationen des Phasenzustands in einer Doppelschicht aus Wassermolekülen, die ebenfalls durch das mW-Modell repräsentiert waren 121. Beide Arbeiten bestärken nicht nur die These, dass der fest-flüssig-Phasenübergang in kleinen Wasserclustern seinen diskontinuierlichen Charakter verliert, sondern legen auch nahe, dass die Koexistenz beider Phasen nicht räumlich, sondern durch zeitliche Oszillationen realisiert ist. Als Grund wird von Kastelowitz et al. unter Anderem explizit der Beitrag der Grenzfläche zwischen beiden Phasen angegeben, der das gleichzeitige Vorliegen beider Phasen ungünstig macht und somit die von Berry geforderte Barriere darstellt. Eine weitere Barriere, die geeignet ist, um einen Phasenzustand über einen ausreichend langen Zeitraum zu stabilisieren, könnte sich aus der oben beschriebenen geringen Wahrscheinlichkeit für einen Phasenübergang ergeben.

\section{Extrapolation auf große Systeme}

Um die Entwicklung des oben beschriebenen Phasengleichgewichtes mit steigender Konstituentenzahl $N$ zu betrachten, ist der Übergang zu einer Größe $D_{\text {eq }}$ hilfreich, die nach Gleichung 2.48 aus der Gleichgewichtskonstanten $K_{\text {eq }}$ abgeleitet werden kann und einer Verteilung der Konstituenten auf die beiden betrachteten Zustände entspricht. Im Temperaturintervall zwischen $T_{\mathrm{f}}$ und $T_{\mathrm{m}}$ wird $D_{\text {eq }}$ wie folgt definiert 81 :

$$
D_{\text {eq }} \equiv \frac{K_{\text {eq }}-1}{K_{\text {eq }}+1}=\frac{[\text { flüssig }]-[\text { fest }]}{\text { Gesamtmenge }}
$$


Im Gegensatz zu $K_{\text {eq }}$ besitzt die Größe $D_{\text {eq }}$ einen eingeschränkten Wertebereich zwischen $D_{\text {eq }}=-1$ (feststoffartiges System) und $D_{\text {eq }}=+1$ (flüssigkeitsartiges System) und ist neben der Temperatur $T$ und der Konstituentenzahl $N$ auch eine Funktion des im System herrschenden Druckes $p$, da die Temperaturwerte $T_{\mathrm{f}}$ und $T_{\mathrm{m}}$ druckabhängig sind. Der Druck soll daher als konstant angenommen werden. In einem kleinen System wird $D_{\text {eq }}$ bei Temperaturen unterhalb von $T_{\mathrm{f}}$ den Wert von -1 annehmen (siehe Abbildung 2.3 b)). Wird die Temperatur $T_{\mathrm{f}}$ erreicht, ändert sich der Wert von $D_{\text {eq }}$ unstetig auf einen Wert zwischen 0 und -1 . Ein weiterer Temperaturanstieg führt wiederum zu stetigen zu Änderungen von $D_{\text {eq }}$, die dem Verlauf einer Sigmoidalfunktion entsprechen. Bei $T=T_{\mathrm{m}}$ zeigt sich eine weitere Unstetigkeit, da $D_{\text {eq }}$ auf den Wert von +1 springt. Die Frage, ob unterkühlte Flüssigkeiten bzw. überhitzte Feststoffe an den Temperaturen $T_{\mathrm{f}}$ bzw. $T_{\mathrm{m}}$ durch thermische Fluktuationen soweit destabilisiert werden, dass diese Unstetigkeiten in kleinen Systemen beobachtet werden können, ist noch nicht endgültig beantwortet 81 .

Innerhalb des Koexistenzbereiches ändern sich die Werte von $K_{\text {eq }}$ und $D_{\text {eq }}$ für einen kleinen Cluster $(10 \lesssim N \lesssim 100)$ kontinuierlich. Die Sigmoidalkurve wird dabei umso steiler, je mehr Teilchen das betrachtete System enthält. Im Grenzfall eines sehr großen Systems ändert sich der Wert von $D_{\text {eq }}$ innerhalb eines unmessbar kleinen Temperaturintervalls, das im Bereich von $T_{\text {eq }}$ liegt, von einem Wert, der mit steigender Clustergröße gegen -1 konvergiert, auf einen Wert, der ebenso nah an +1 liegt. Die beiden Unstetigkeiten, die in kleinen Clustern an den Temperaturen $T_{\mathrm{f}}$ und $T_{\mathrm{m}}$ auftreten, verbinden sich zu einer einzigen Unstetigkeit bei $T=T_{\text {eq }}$. Der Bereich zwischen $T_{\mathrm{f}}$ und $T_{\mathrm{m}}$, der sich im $(p, T)$-Diagramm eines mikroskopischen Systems als Phasenkoexistenzfläche darstellen würde, geht in die bekannte, unendlich schmale Phasengrenzlinie über, auf der ein System nicht äquilibriert werden kann (vgl. S. 12). Nach dieser Betrachtung stellt der fest-flüssig-Phasenübergang eines makroskopischen Systems als Phasenübergang erster Ordnung (d.h. mit unstetiger Ableitung bei $T=T_{\text {eq }}$ ) den Grenzfall des Verhaltens eines großen Systems mit $N \rightarrow \infty$ dar.

Die stetigen Phasenänderungen kleiner Nanopartikel sind hingegen keine Phasenübergänge erster Ordnung, sondern vielmehr der mikroskopische Grenzfall eines auf quantenstatistischen Effekten beruhenden Phänomens, das sich in makroskopischen Systemen als diskontinuierlicher Phasenübergang äußert. In ausreichend kleinen Systemen sollte demnach ein scharf abgrenzbarer Temperaturbereich bestehen, dessen Grenzen $T_{\mathrm{f}}$ und $T_{\mathrm{m}}$ unterscheidbar werden und Funktionen des Drucks sind. Innerhalb dieses Temperaturbereiches können zwei phasenartige Zustände koexistieren. Außerhalb dieses Bereiches ist jeweils nur eine einzige Phase thermodynamisch stabil. 


\subsection{Wasser und Wassercluster}

\subsubsection{Struktur und Eigenschaften des Wassermoleküls}

Das Wassermolekül (siehe Abb. 2.4) mit der chemischen Formel $\mathrm{H}_{2} \mathrm{O}$ gilt als besonders einfach aufgebaute und thermodynamisch stabile Verbindung. Es hat sich jedoch gezeigt, dass die Substanz Wasser weit davon entfernt ist, als inert gelten zu können22. Die ungewöhnlichen Eigenschaften von Wasser können qualitativ bereits durch Betrachtung der Elektronenverteilung im Wassermolekül verstanden werden: Die Ladungsdichte ist hauptsächlich in vier Bereichen lokalisiert, die sich tetraedrisch um das Sauerstoffatom verteilen: Die beiden Wasserstoffatome (im Fol-
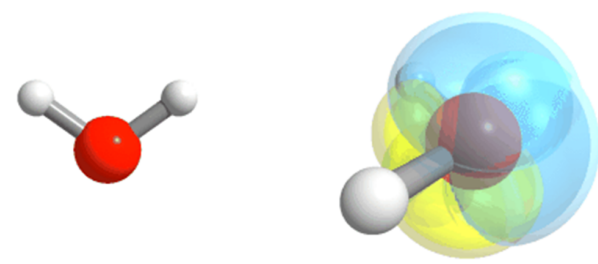

Abbildung 2.4.: Struktur (l) und Elektronendichteverteilung (r) des $\mathrm{H}_{2} \mathrm{O}$-Moleküls; aus Ref. [1] genden kurz H-Atome) bilden zwei Bereiche mit positiver Partialladung, während sich die negative Partialladung am Sauerstoffatom (im Folgenden kurz O-Atom) auf die beiden Molekülorbitale der nicht-bindenden Elektronenpaare verteilt. Diese Elektronenverteilung führt zu einem $\mathrm{H}-\mathrm{O}-\mathrm{H}$-Gleichgewichtsbindungswinkel von 104, $5^{\circ}$, einem Dipolmoment von 1,83 D, das sich entlang der Symmetrieachse erstreckt, und einer nahezu tetraedrischen Koordinationsgeometrie des $\mathrm{H}_{2} \mathrm{O}$-Moleküls in der kondensierten Phase 122 .

\subsubsection{Wechselwirkungen zwischen Wassermolekülen}

Die dominierende Wechselwirkung zwischen Wassermolekülen stellen Wasserstoffbrückenbindungen (im Folgenden kurz H-Brücken) dar 1]. Diese setzen sich aus mehreren Beiträgen unterschiedlichen physikalischen Ursprungs zusammen, die nur schwer voneinander $\mathrm{zu}$ trennen sind und deren Anteile in Abhängigkeit von der chemischen Umgebung sowie vom Abstand der Bindungspartner variieren $1|122| 123]$ : Im Einzelnen sind dies die Delokalisationsenergie durch Ladungstransfer (engl. Charge Transfer, CT), elektrostatische Wechselwirkungen, Dispersionswechselwirkungen und Austauschrepulsion 124. Als Folge entsteht eine Bindung mit Kovalenten Anteilen, die ihrer Stärke nach zwischen der kovalenten Bindung und den

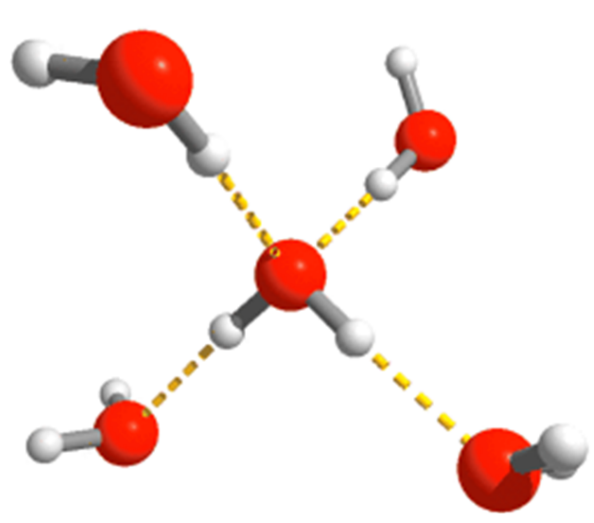

Abbildung 2.5.: Tetraedrisches Walrafen-Pentamer; aus Ref. [1] Van-der-Waals-Wechselwirkungen einzuordnen ist. Die Bildungsenthalpie einer H-Brücke variiert zwischen ca. $0,1 \mathrm{eV}$ und ca. 0,7 eV 22. Die IUPAC empfiehlt seit 2011 folgende Definition einer Wasserstoffbrückenbindung 123 :

"The hydrogen bond is an attractive interaction between a hydrogen atom from a molecule or a molecular fragment $X-H$ in which $X$ is more electronegative than $H$, and an atom or a group of atoms in the same or a different molecule, in which there is evidence of bond formation." 
Die übliche Darstellung erfolgt in der Form X-H. . Y - Z, wobei X ein Atom mit hoher Elektronenaffinät darstellt. X-H wird als Wasserstoffbrückendonor bezeichnet, während Y-Z Akzeptor genannt wird. Der Akzeptor Y kann ein polares Molekül, eine funktionelle Gruppe oder ein Anion sein, besitzt aber in jedem Fall eine hohe Elektronendichte bzw. ein elektronenreiches, nicht-bindendes Orbital oder auch ein $\pi$-Elektronensystem 2[123]125.

Unter zwei Wassermolekülen bilden sich H-Brücken zwischen einem H-Atom des Donors und einem freien Elektronenpaar am O-Atom des Akzeptors aus, sodass jedes Wassermolekül maximal vier H-Brücken ausbilden kann. Abbildung 2.5 zeigt das sogenannte Walrafen-Pentamer, das prototypisch für die tetraedrische Koordination eines Wassermoleküls in der kondensierten Phase ist. Die Van-der-Waals-Radien der beteiligten Gruppen

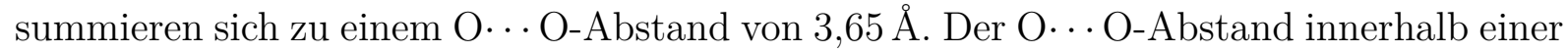
H-Brücke variiert hingegen je nach Stärke der Bindung von ca. 2,98 $\AA$ (im Dimer), über ca. $2,85 \AA$ (in der Flüssigkeit) bis auf ca. 2,74 $\AA$ (in Eis $\mathrm{I}_{\mathrm{h}}$ ), das als raumerfüllende Anordnung von Walrafen-Pentameren aufgefasst werden kann ${ }^{1]}$. Diese Verkürzung des O $\cdots$ OAbstands in der kondensierten Phase beruht auf kooperativen Effekten, die zwischen mehreren H-Brücken auftreten und deren gegenseitige Verstärkung bewirken 51. Hierbei wird die Ladungsverteilung in zwei beteiligten Wassermolekülen derart verschoben, dass die Donorwirkung des Protonendonors sowie die Akzeptorwirkung des Protonenakzeptors verstärkt werden. Folge dieser sich gegenseitig verstärkenden CT-Wechselwirkungen ist eine Aufweitung der kovalenten $\mathrm{OH}-$ Bindung bei gleichzeitiger Verkürzung der intermolekularen $\mathrm{O}-\mathrm{H} \cdots$ O-Brücke und daher einem verkürzten $\mathrm{O} \cdots$ O-Bindungsabstand 1 .

Seine elektronische Struktur befähigt das Wassermolekül somit, Netzwerke aus sich gegenseitig verstärkenden H-Brücken auszubilden, die für die hohe Schmelz- und Siedetemperatur, Viskosität, Oberflächenspannung und die gesamte makroskopische Erscheinung der kondensierten Phase verantwortlich ist 11126. Die H-Brücken, die zwischen Wassermolekülen und Proteinen sowie DNA-Molekülen ausgebildet werden, sind Voraussetzung für deren strukturelle Stabilität 2 .

\subsubsection{Kristallisation in wässrigen Nanotröpfchen}

In sämtlichen Experimenten zur Erzeugung und Untersuchung kristalliner Nanopartikel wurde zunächst die Bildung eines unterkühlten, flüssigen Partikels beobachtet, der im Anschluss kristallisierte $39|65| 75 \mid 78$.

Flüssiges Wasser besteht aus einem dynamisch fluktuierenden Netzwerk von H-Brücken, das sich auf einer Pikosekundenzeitskala ständig von neuem arrangiert. In diesem Zuge treten neben der vierfachen, zur Bildung von Sechsringen führenden Koordinationsgeometrie auch dreifach koordinierte Wassermoleküle sowie Fünfringe auf 48 . Dabei bilden in flüssigem Wasser zu jedem Zeitpunkt weit mehr als $99 \%$ aller OH-Gruppen eine HBrücke aus. Dennoch führt die im Rahmen der hochamplitudigen Librationsbewegungen der Flüssigkeit auftretende Verzerrung der Koordination oberhalb des Gefrierpunktes zu einer deutlich dichteren Struktur, als dies in kristallinem Eis der Fall ist 2 .

Bei Temperaturen unterhalb von $0^{\circ} \mathrm{C}$ wird die flüssige Phase zum metastabilen Zustand 2]. Aus den in Abschnitt 2.2.1 erläuterten Gründen lässt sich die Temperatur extrem reinen Wassers weiter senken, ohne dass ein Phasenübergang stattfindet. Die Amplituden der Librationsbewegungen sowie die Dichte nehmen dabei kontinuierlich ab, bis 
der Phasenübergang durch homogene Nukleation bei $T_{\mathrm{H}} \approx 232 \mathrm{~K}[64-235 \mathrm{~K}[75$ unweigerlich eintritt. $T_{\mathrm{H}}$ wird daher als homogene Nukleationstemperatur bezeichnet. Fällt die Temperatur auf einen Wert knapp oberhalb von $T_{\mathrm{H}}$, so nehmen die Abweichungen von der idealen, tetraedrischen Koordinationsgeometrie sowie die Anzahl dreifach koordinierter Wassermoleküle stark ab, sodass eine vierfach koordinierte Flüssigkeit vorliegt, die große strukturelle Ähnlichkeit mit Eis I aufweist. Daraus wiederum folgt eine erhebliche Herabsetzung der Nukleationsbarriere, sodass sich die Flüssigkeit in diesem Zustand nicht äquilibrieren kann, da die Kristallisation schneller voranschreitet. Die Kristallisationsgeschwindigkeit erreicht bei dieser Temperatur ein Maximum. Bei höheren Temperaturen stellt die Bildung kritischer Nuclei den geschwindigkeitsbestimmenden Schritt dar, bei niedrigeren Temperaturen ist die Geschwindigkeit des Kristallwachstums der limitierende Faktor 64 .

Speedy und Angell schlugen daher vor, dass die Eisbildung bei Temperaturen unterhalb von $T_{\mathrm{H}}$ das Ergebnis einer spinodalen Entmischung sein könnte 127 . Kennzeichnend für diesen Mechanismus ist die komplette Abwesenheit einer thermodynamischen Barriere für den Phasenübergang. Dennoch setzt in Nanotröpfchen, die durch Überschallexpansionen in Laval-Düsen erzeugt wurden, die Kristallisation erst bei Temperaturen knapp oberhalb von $200 \mathrm{~K}$ ein $75 \mid 128$. In diesem Zusammenhang ist zu berücksichtigen, dass der hohe Laplace-Druck die homogene Nukleationstemperatur absenken kann (vgl. Abschnitt 2.2.13967/75. Manka et al. beobachteten den Beginn der Kristallisation bei Temperaturen zwischen $215 \mathrm{~K}(\langle r\rangle=5,8 \mathrm{~nm})$ und $202 \mathrm{~K}(\langle r\rangle=3,2 \mathrm{~nm})$. Die homogene Nukleationstemperatur $T_{\mathrm{H}}$ der kleinsten Tropfen wurde von ihnen hingegen $\mathrm{zu} T_{\mathrm{H}}=228 \mathrm{~K}$ berechnet. Der Anteil wässriger Nanotröpfchen, der bei einer gegebenen Temperatur unterhalb von $T_{\mathrm{H}}$ in einer bestimmten Zeit kristallisiert, ist dabei sowohl von der Temperatur als auch von der Partikelgröße abhängig 67/75. Wird die Nukleation gehindert, findet ein Glasübergang statt, der größenabhängig bei $T_{\mathrm{G}} \gtrsim 115 \mathrm{~K}$ auftritt 129 .

Die kombinierten experimentellen Ergebnisse sprechen damit für einen stochastischen Prozess als Nukleationsmechanismus bei Temperaturen um 200 K: Je weniger Moleküle in einer Probe vorhanden sind, desto geringer ist die Wahrscheinlichkeit, dass sich eine ausreichende Anzahl von Wassermolekülen zu einem kritischen Nukleus zusammenfindet. Die Nukleationswahrscheinlichkeit wird dabei nicht allein durch den Laplace-Druck, sondern auch durch die geringe Größe der Tropfen herabgesetzt. Hinzu kommt, dass die Anzahl der Moleküle $N_{\text {krit }}$, die zur Bildung eines kritischen Nukleus erforderlich ist, stark von der thermodynamischen Triebkraft abhängt. So nimmt sie mit steigender Temperatur zu 64. Liegt die Temperatur des Partikels nur knapp oberhalb von $T_{\mathrm{H}}$, so kann durch die oben beschriebene strukturelle Reorganisation hin zu stärkerer tetraedrischer Koordination ein kritischer Nukleus stabilisiert werden 64130. Auch mit abnehmendem Partikelradius steigt der Wert von $N_{\text {krit }}$ an 67. Die in einer Überschallexpansion beobachtete kritische Clustergröße $N_{\text {krit }}$, d.h. die kleinste Größe, die zur Bildung eines kristallinen Kerns in der Lage ist, stellt daher jeweils sowohl eine neue Obergrenze für die Konstituentenzahl $N^{*}$ als auch für den größen- und temperaturabhängigen Wert von $N_{\text {krit }}$ dar.

Weiterhin hängt seine Größe von der Geometrie ab: Je kompakter die Anordnung ist, desto weniger Moleküle sind erforderlich, um die Barriere zu überwinden 130 .

Die homogene Nukleation einer unterkühlten Flüssigkeit stellt keinen Gleichgewichtsprozess dar. Dementsprechend ist die sich bildende Eismodifikation durch kinetische Einflüsse determiniert(vgl. Abschnitt 2.3.4). 
a)

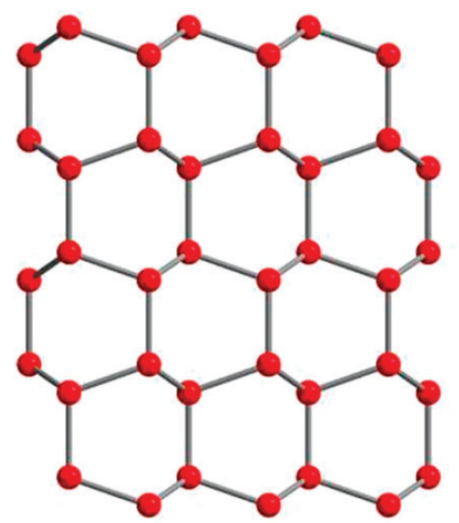

b)

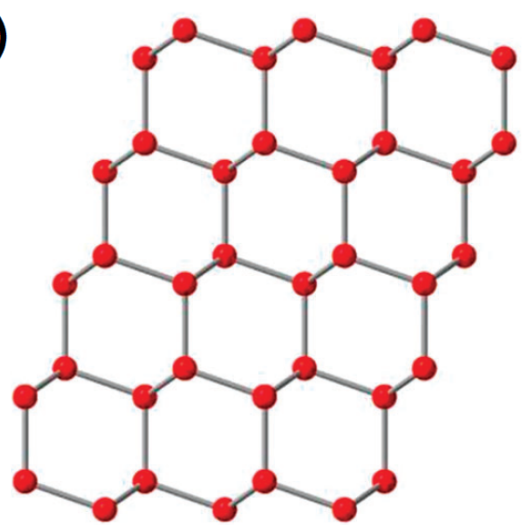

Abbildung 2.6.: Stapelfolge von Eis $\mathrm{I}_{\mathrm{h}}$ (Teil a)) und Eis $\mathrm{I}_{\mathrm{c}}$ (Teil b)), H-Atome sind nicht dargestellt; entnommen aus Referenz [131].

\subsubsection{Eis I}

Derzeit sind sechzehn Eismodifikationen (Eis I bis Eis XVI) bekannt [5, von denen als Einzige Eis I durch Kristallisation von Wasser unter den relativ niedrigen, in der Atmosphäre herrschenden Drücken gebildet wird 79 .

Makroskopisches, flüssiges Wasser kristallisiert unter Gleichgewichtsbedingungen zur weitverbreiteten, bei niedrigen Drücken thermodynamisch stabileren hexagonalen Form Eis $\mathrm{I}_{\mathrm{h}}{ }^{62}$. Im Jahr 1943 wurde jedoch erstmals eine weitere kubische Eismodifikation $\left(\right.$ Eis $\mathrm{I}_{\mathrm{c}}$ ) beschrieben ${ }^{132}$, die der hexagonalen Form stark ähnelt: Sowohl kubisches als auch hexagonales Eis I bestehen aus Stapeln von Doppelschichten, die aus gefalteten Sechsringen bestehen 1112. Im Eis $\mathrm{I}_{\mathrm{h}}$ weisen die Ringe eine Bootkonformation auf [62. Jede Schicht ist dabei spiegelbildlich zu den ihr benachbarten Schichten orientiert 133 . Im Eis $\mathrm{I}_{\mathrm{c}}$ liegen die Sechsringe in Sesselkonformation vor ${ }^{62}$, wobei die Schichten jeweils um einen halben Durchmesser eines Sechsringes versetzt sind(vgl. Abb. 2.6. 133.

Ideal strukturierte Kristalle beider Formen folgen den Eisregeln (Bernal-FowlerRegeln): Jedes O-Atom weist vier nächstgelegene O-Atome auf, von denen es in tetraedrischer Geometrie umgeben ist. Zwischen den Sauerstoffatomen befindet sich jeweils ein Wasserstoffatom. Jedes Sauerstoffatom ist an zwei der vier Wasserstoffatome, von denen es umgeben ist, chemisch gebunden, zu den zwei übrigen Wasserstoffatomen bildet es eine Wasserstoffbrückenbindung aus. Die molekulare Integrität des Wassermoleküls bleibt dabei folglich erhalten 79 . Die lokale Koordinationsgeometrie eines einzelnen Wassermoleküls ist in beiden Formen identisch, entsprechend lassen sie sich durch Methoden der Infrarotspektroskopie nicht unterscheiden 62 .

Im Rahmen dieser Eisregeln bilden allerdings nur die Sauerstoffatome ein periodisches Kristallgitter aus, während die Orientierung der Wassermoleküle und damit die Lage der Wasserstoffatome keiner periodischen Gesetzmäßigkeit unterliegt. Diese Abwesenheit einer weitreichenden Korrelation der Orientierungen wird als Protonenunordnung bezeichnet ${ }^{79 \mid 134}$. Innerhalb eines makroskopischen Eiskristalls ergibt sich somit eine Vielzahl möglicher Strukturen mit unterschiedlichen Orientierungen der darin enthaltenen Wassermoleküle, die einen annähernd identischen Energiegehalt besitzen $62[112$. 
Bis vor wenigen Jahren herrschte Konsens darüber, dass Eis I ausschließlich in Form zweier periodischer Modifikationen (Eis $\mathrm{I}_{\mathrm{h}}$ und Eis $\mathrm{I}_{\mathrm{c}}$ ) auftritt. Dementsprechend wurde in zahlreichen experimentellen Studien, die die Untersuchung der gebildeten Eismodifikation einbezogen, nur zwischen diesen beiden Formen entschieden. Das Vorliegen von Eis $I_{c}$ wurde berichtet, sofern die erhobenen Beugungsdaten eher den für Eis $I_{c}$ Erwarteten als denen von Eis $I_{h}$ entsprachen $38|39| 78 \mid 135$. Malkin et al. wiesen im Jahr 2012 darauf hin, dass die erhaltenen Beugungsmuster nicht vollständig durch die Struktur von Eis $I_{c}$ erklärbar sind 136 . Sie zeigten, dass die Ergebnisse vielmehr im Einklang mit einer Hybridstruktur stehen, die aus einer zufälligen Abfolge kubisch und hexagonal strukturierter Doppelschichten besteht, deren Anordnung von den Bildungsbedingungen bzw. der Temperaturhistorie des Eiskristalls abhängt (vgl. Abb. 2.7). Entsprechend schlugen sie die Bezeichnung stacking disordered ice bzw. Eis $\mathrm{I}_{\mathrm{sd}}$ für alle Eisphasen vor, die mehr als 1\% der weniger häufigen Schichten enthalten 131|136.

Die homogene Nukleation einer unterkühlten Flüssigkeit findet grundsätzlich unter Vorliegen eines großen thermodynamischen Ungleichgewichts statt. Daher ist das Kristallwachstum stark durch kinetische Einflüsse geprägt 39 : Eis $\mathrm{I}_{\mathrm{h}}$ weist im Vergleich mit Eis $I_{c}$ und Eis $I_{s d}$ eine relativ hohe Oberflächenenergie auf, sodass die Energiebarriere für die Bildung eines Eis $\mathrm{I}_{\mathrm{h}}$-Nukleus höher liegt, als die der Bildung der beiden übrigen Eis I-Modifikationen. Hingegen besitzt Eis $\mathrm{I}_{\mathrm{h}}$ ebenfalls eine hohe Oberflächenentropie, was die

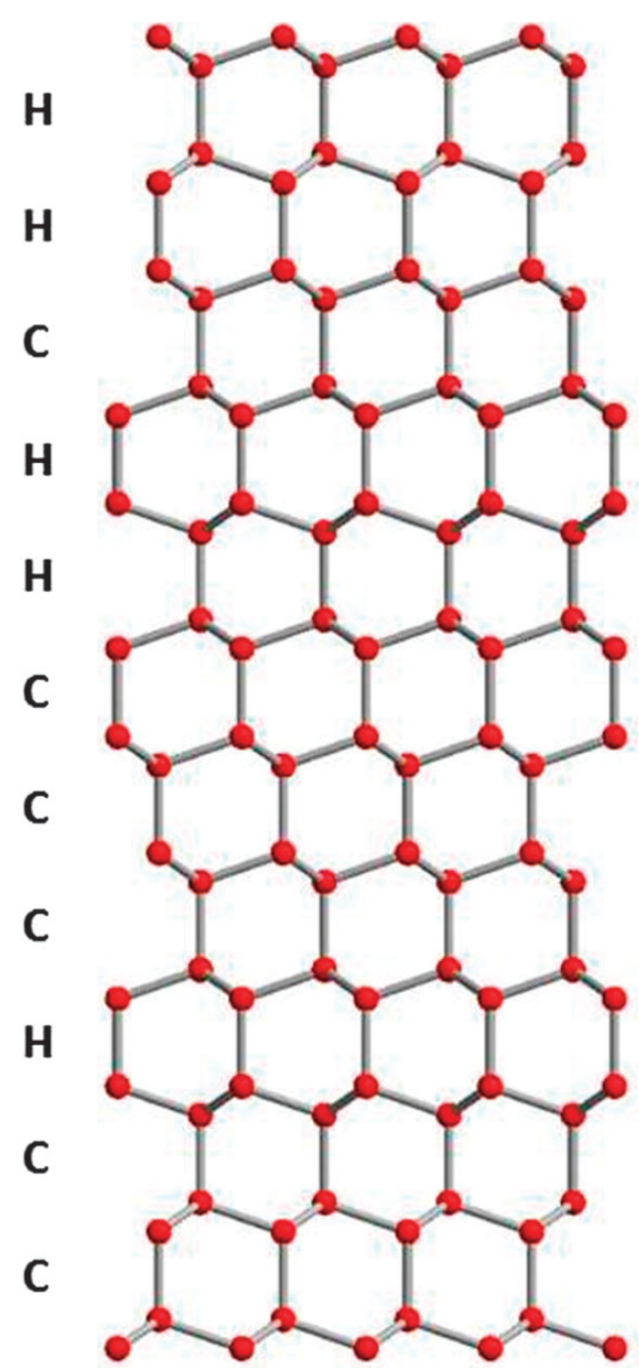

Abbildung 2.7.: Stapelfolge von Eis $\mathrm{I}_{\mathrm{sd}}, \mathrm{H}$-Atome sind nicht dargestellt; entnommen aus Ref. 131

Ausbildung einer entsprechenden Oberfläche bei hohen Temperaturen begünstigt $137[138]$. Dementsprechend treten Abweichungen von der Eis $\mathrm{I}_{\mathrm{h}}$-Struktur immer bei der Kristallisation unterkühlter flüssiger Phasen auf. Bereits Eis I, das bei Temperaturen $\lesssim 257 \mathrm{~K}$ entstanden ist, besitzt eine messbare, wenn auch transiente, Stapelunordnung 131.

Auch in Nanotröpfchen, die naturgemäß erst bei niedrigeren Temperaturen gefrieren (vgl. Abschnitt 2.2.1), weicht die Struktur des gebildeten Eises von Eis $\mathrm{I}_{\mathrm{h}}$ ab. Johari berechnete auf Basis der klassischen Nukleationstheorie (d.h. unter Nutzung der Grenzflächenspannungen von Eis $I_{h}$ und Eis $I_{c}$ ) den Radius, unterhalb dessen die Bildung von Eis $\mathrm{I}_{\mathrm{c}}$ gegenüber der Bildung von Eis $\mathrm{I}_{\mathrm{h}}$ begünstigt ist, auf $r=15 \mathrm{~nm} 68 \mid 139$. Wenngleich diese scharfe Unterscheidung im Lichte der oben beschriebenen, neueren Erkenntnisse nicht aufrechterhalten werden kann, lässt sie doch erwarten, dass wässrige Nanopartikel, wie sie im Rahmen dieser Arbeit untersucht werden, nicht zu Eis $\mathrm{I}_{\mathrm{h}}$ kristallisieren, sondern entsprechend der oben beschriebenen experimentellen Resultate und der numerischen Simulationen von Johnston et al. 68 stattdessen eine Form von Eis $\mathrm{I}_{\text {sd }}$ gebildet wird. 
In der Atmosphäre scheint Eis $\mathrm{I}_{\mathrm{c}}$ wenn überhaupt nur sehr selten vorzukommen: Murray et al. führen aus, dass Kristalle von Eis $\mathrm{I}_{\mathrm{sd}}$ im Gegensatz zu den hexagonalen Kristallen von Eis $\mathrm{I}_{\mathrm{h}}$ und den oktaedrischen Kristallen von Eis $\mathrm{I}_{\mathrm{c}}$ eine trigonale Symmetrie aufweisen und sich bei niedrigen Temperaturen vielerorts in der Atmosphäre nachweisen lassen. Bei Temperaturen $\gtrsim 368 \mathrm{~K}$ beginnen diese, sich zu Eis $\mathrm{I}_{\mathrm{h}}$ umzulagern 133 . In der tropischen Tropopause bei Temperatur von $193 \mathrm{~K}$ besaßen $50 \%$ aller beobachteten Kristalle eine dreizählige Symmetrie. Es ist daher zu erwarten, dass kalte Eiswolken in der Troposphäre, Stratosphäre und Mesosphäre hauptsächlich aus Eis $\mathrm{I}_{\mathrm{sd}}$ bestehen. Auch in polaren stratosphärischen Wolken (Polar Stratospheric Clouds, PSC) vom Typ II, die nur bei $T<188 \mathrm{~K}$ entstehen, dürfte Eis $\mathrm{I}_{\text {sd }}$ häufig auftreten. Grund ist, dass die Geschwindigkeit der Umwandlung in Eis $\mathrm{I}_{\mathrm{h}}$ bei diesen Temperaturen nicht ins Gewicht fällt und Eis $\mathrm{I}_{\mathrm{sd}}$ als kinetisch stabil betrachtet werden kann 133|140. Eines der wenigen Indizien für die das Auftreten von Eis $\mathrm{I}_{\mathrm{c}}$ in der Atmosphäre stellt ein seltenes Phänomen namens Scheiners Halo dar, das durch Reflektion des Lichtes der Sonne bzw. des Mondes in einem Winkel von $28^{\circ}$ entsteht, der für das Vorliegen oktaedrischer Kristalle spricht 131|137.

\subsubsection{Bisherige Studien an ungeladenen Wasserclustern}

Ladungsfreie Wasseraggregate vom Dimer 141 über kleine Cluster 142 bis hin zu Filmen 77 und mikrometergroßen Eiskristallen 78 waren in den letzten vierzig Jahren Gegenstand zahlreicher experimenteller und theoretischer Forschungsarbeiten. An dieser Stelle soll der bisherige Forschungsstand kurz vom experimentellen Standpunkt aus zusammengefasst werden. Motivation all dieser Studien war unter anderem der Wunsch, Informationen über die zwischenmolekularen Wechselwirkungen zwischen Wassermolekülen zu erhalten. Kleine Aggregate mit definierter Konstituentenzahl sollen als größenbegrenztes und damit vereinfachtes Modell dienen, um beim Dimer beginnend und sukzessive zu größeren Clustern fortschreitend ein genaues Bild der Wasserstoffbrückenbindung, ihres Einflusses auf das einzelne Wassermolekül und über das Zusammenwirken mehrerer Wasserstoffbrückenbindungen im Verbund zu erhalten. Erklärtes Ziel ist dabei, ein besseres Verständnis von der kondensierten Phase zu erhalten 162|134. Eine Voraussetzung für die Eignung eines Clusters als Modell für die kondensierte Phase ist das Vorliegen der Spezies darin, aus der diese in kristallinem und flüssigem Aggregatzustand fast ausschließlich aufgebaut ist eines vierfach koordinierten Wassermoleküls in annähernd tetraedrischer Koordinationsgeometrie (vgl. Abb. 2.5 in Abschnitt 2.3.2 2134. Kleine Cluster, die hauptsächlich aus drei- bis fünfzähligen Ringen bestehen, sind weder als Modell für Eis I noch als Modell für flüssiges Wasser geeignet 134. Dennoch bildet das Wissen über die Eigenschaften dieser Systeme von geringerer Komplexität die Basis, um auch größere Cluster und Nanopartikel in den Fokus nehmen zu können.

\section{Kleine Cluster $\mathrm{N}\left(\mathrm{H}_{2} \mathrm{O}\right) \leq 10$}

Die Cluster im Größenbereich $2 \leq N\left(\mathrm{H}_{2} \mathrm{O}\right) \leq 10$ sind vergleichsweise leicht zugänglich und wurden unter Anwendung einer Vielzahl spektroskopischer Methoden untersucht, sodass mittlerweile ein konsistentes Bild aus experimentellen und theoretischen Ergebnissen entstanden ist $19143 \quad 145$. Ein Überblick über die wichtigsten, experimentell nachgewiesenen Strukturen ist in Abbildung 2.8 dargestellt. 
Die Struktur des Wasserdimers wurde erstmals 1974 durch Dyke et al. experimentell vermessen. Methode der Wahl war hier die Mikrowellenspektroskopie von Dimeren, die in Überschallexpansionen erzeugt worden waren 141|146. Die Spektren der kleinsten Cluster weisen erwartungsgemäß die stärksten größenabhängigen Änderungen auf 147 . Sie überschneiden sich nicht, sodass hier auch ohne strikte Größenselektion bei entsprechend hoher Auflösung konsistente Ergebnisse erhalten werden konnten. Saykally et al. nutzten die gute Unterscheidbarkeit der Rotationskonstanten kleiner Cluster aus und untersuchten das Dimer sowie die Oligomere bis $N=6$ mit Hilfe der von ihnen entwickelten Schwingungs-Rotations-Tunnelspektroskopie (engl. vibration-rotation tunneling spectroscopy, VRT) 148 152. Diese Daten belegten erstmals, dass die Clustergrößen $3 \leq N \leq 5$ in Form quasiplanarer Ringe vorliegen 153 . Weiterhin gestatteten sie eine akkurate Bestimmung der O-H...O-Abstände in diesen Spezies. Sämtliche Untersuchungen deuten auf eine exponentielle Abnahme dieses Abstands mit zunehmender Konstituentenzahl hin. Diese Verkürzung kann als Maß für die Stärke der kooperativen Wechselwirkungen zwischen mehreren H-Brücken im Verbund betrachtet werden. In großen Clustern konvergiert der $\mathrm{O}-\mathrm{H} \cdots \mathrm{O}$-Abstand gegen den vom Eis $\mathrm{I}_{\mathrm{h}}$ bekannten Wert von 2,74 $\AA^{1}$. Huisken et al. veröffentlichten im Jahr 1996 erstmals vollständig größenaufgelöste Schwingungsspektren der Clustergrößen $2 \leq N \leq 5 \underline{29}$. Die Größenselektion war durch die von Buck und Meyer entwickelte kinematische Methode 154 erreicht worden. Diese Spektren belegten ebenfalls die ringförmige Struktur dieser Cluster 143.

Suhm et al. publizierten im Jahr 2014 erstmalig die Raman-Spektren der Spezies $2 \leq N\left(\mathrm{H}_{2} \mathrm{O}\right) \leq 5$, die eine zunehmende Fermi-Resonanz mit steigender Clustergröße belegten 155 .

Das Wasserhexamer gilt als Prüfstein für theoretische Methoden, da es die kleinste Clustergröße darstellt, die ein Wasserstoffbrückennetzwerk mit dreidimensionaler Struktur und dreifach koordinierten Wassermolekülen auszubilden vermag. Zudem sind bei dieser Clustergröße mehrere Isomere unterschiedlicher Topologie im Bereich des Möglichen, deren statistisches Gewicht von der Clustertemperatur abhängt: Bei niedrigen Temperaturen werden aus enthalpischen Gründen Strukturen bevorzugt, in denen die Summe der Bindungsenergien der Wasserstoffbrücken maximiert ist. Bei höheren Temperaturen nimmt das Gewicht der entropischen Beiträge aller möglichen Strukturen zu, sodass eine Reihe energetisch ungünstigerer Strukturen mit verzerrter Geometrie zum Gesamtbild beiträgt 156. Das Hexamer stellt somit das kleinste System dar, an dem sich die Modellierung dieser Eigenschaften, die auch für die Beschreibung größerer Cluster relevant ist, erproben lässt 157 . Bei Temperaturen von ca. $40 \mathrm{~K}$ zeigten Schwingungsspektren von Steinbach et al., die durch eine Kombination aus kinematischer Größenselektion und vibronischer Prädissoziation erhalten worden waren, konsistent mit den Arbeiten von Saykally et al. $152[153$ eine käfigartige Struktur mit acht Wasserstoffbrücken. Bei einer Temperatur von ca. $60 \mathrm{~K}$ waren hingegen Beiträge eines weiteren Isomers nachweisbar, das als buchartig beschrieben wird und sieben Wasserstoffbrücken aufweist 158|159]. Diese Resultate zeigen gute Übereinstimmung mit kürzlich veröffentlichten MD-Simulationen von Brown et al. auf Basis des MB-pol-Potentials, die das statistische Gewicht verschiedener Isomere des Hexamers als Funktion der Temperatur untersuchten: Hier zeigte sich ein erstes Aufkommen des buchartigen Isomers im Temperaturintervall zwischen $40 \mathrm{~K}$ und 60 K 156. Die zu den planaren Ringen kleinerer Cluster analoge Sechsringstruktur mit nur sechs Wasserstoffbrücken ist hingegen trotz der für kooperative Verstärkung günstigen 


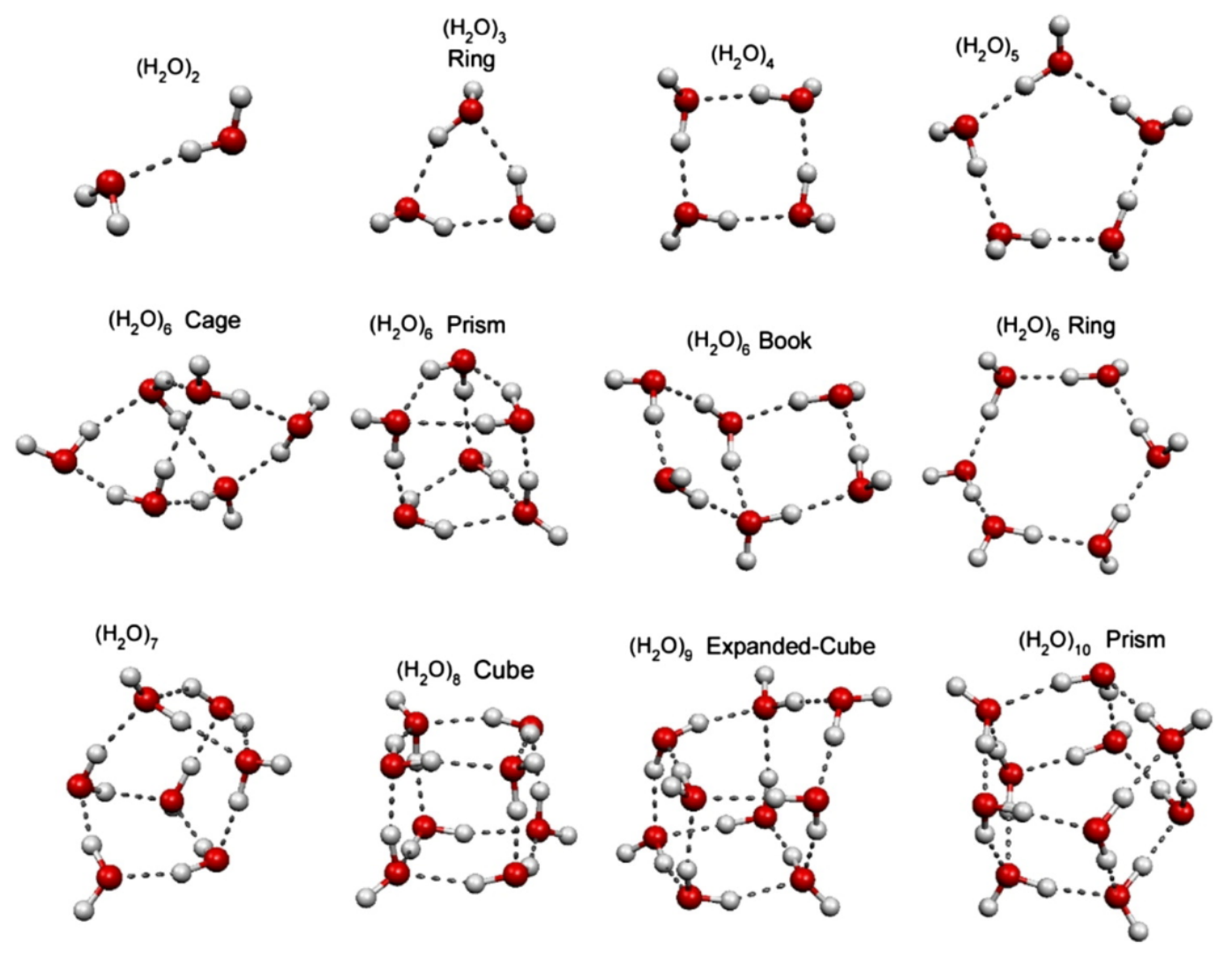

Abbildung 2.8.: Strukturen experimentell nachgewiesener, kleiner Wassercluster mit $N\left(\mathrm{H}_{2} \mathrm{O}\right) \leq 10$; entnommen aus Referenz [51].

Geometrie energetisch ungünstiger und wurde lediglich in flüssigen Heliumtröpfchen beobachtet, in denen sie durch Insertion von Wassermolekülen in kleinere Ringstrukturen gebildet wird 160 .

Spektrale Informationen über ladungsfreie, undotierte Cluster mit $7 \leq N \leq 10$ wurden erstmals von Buck und Mitarbeitern unter Anwendung der oben erwähnten kinematischen Größenselektion in Kombination mit vibronischer Prädissoziation erhalten 161|162. Hierbei wurden die Cluster in einer Überschallexpansion erzeugt und ihre Flugbahnen in Abhängigkeit von ihrer Masse (genauer: ihrem Impuls) durch einen im rechten Winkel auftreffenden Molekularstrahl aus Heliumatomen um einen charakteristischen Winkel abgelenkt, sodass sie anschließend größenselektiv untersucht werden konnten 29|143|163. Als Methode kam hier die vibronische Prädissoziation zum Einsatz $40|142| 143$. Dieses Verfahren stellt bisher den einzigen experimentellen Ansatz dar, der geeignet ist, um größenselektive Strukturinformationen ladungsfreier, undotierter Cluster mit $N>6$ zu erhalten 51.

Bei niedrigen Temperaturen werden Strukturen gebildet, die sich formal durch Hinzufügen bzw. Entfernen von Monomereinheiten von der würfelförmigen Struktur des Oktamers ableiten lassen $40|134| 143$. Bei der Clustergröße $N=9$ konnte erstmals der temperaturabhängige Übergang von einer starren Konformation mit klar strukturiertem Schwingungsspektrum bei $T \approx 70 \mathrm{~K}$ zu einer beweglichen Konformation mit einem relativ strukturlosen Schwingungsspektrum bei $T \approx 190 \mathrm{~K}$ nachgewiesen werden 164 . Letztere besitzt genug innere Energie, um zwischen verschiedenen energetisch ungünstigeren, lokalen Minima zu wechseln. 
Auch die Dotierung von Wasserclustern mit aromatischen Chromophoren wie Benzol 49 oder Phenol 50|51|165 und anschließende Zweiphotonenionisation lieferte im Bereich $N \leq 10$ Schwingungsspektren bei scharfer Größenselektion.

Zusammenfassend lässt sich sagen, dass bei kleinen Clustern die Größenselektion entweder über die unterschiedlichen Rotationskonstanten $(2 \leq N \leq 6)$, durch Strahlablenkung $(2 \leq N \leq 10)$ oder durch Dotierung mit aromatischen Chromophoren $(1 \leq N \leq 10)$ erreicht wurde. Allen Strukturen in diesem Größenbereich ist gemeinsam, dass sie Käfige aus mehr oder weniger gespannten Ringen darstellen, die maximal dreifach koordinierte Wassermoleküle enthalten. Eine ausgeprägte Protonenunordnung, wie in Abschnitt 2.3.4 beschrieben, existiert nicht, da unterschiedliche Orientierungen der Wassermoleküle in diesen kleinen Clustern zu relativ großen Energieunterschieden führen 134.

\section{Mittelgroße Cluster $10<N\left(\mathrm{H}_{2} \mathrm{O}\right)<90$}

Während im Größenbereich bis $N\left(\mathrm{H}_{2} \mathrm{O}\right)=10$ mehrere unterschiedliche experimentelle Ansätze zu gesicherten Ergebnissen geführt haben, war es lange Zeit nicht möglich, größenselektive Strukturinformationen für größere Cluster zu erhalten, da die Größenabhängigkeit der Merkmale, anhand derer die Größenselektion für Cluster mit $N\left(\mathrm{H}_{2} \mathrm{O}\right) \leq 10$ vollzogen wurde (Rotationskonstanten, Photoionisationswellenlängen bzw. Ablenkwinkel) mit wachsender Konstituentenzahl abnimmt. Hamashima et al. untersuchten die Schwingungsspektren phenoldotierter Cluster im Bereich $20 \lesssim N \lesssim 50$ mit moderater Größenselektion $(0 \leq \Delta N \leq 6)$ und fanden eine relative Abnahme freier OH-Gruppen auf der Oberfläche, die einer relativen Zunahme vierfach koordinierter Moleküle im Clusterinneren entspricht. Die kleinste Clustergröße, die ein vierfach koordiniertes Wassermolekül im Clusterinneren aufweist, verorteten sie im Bereich $20 \leq N \leq 30 \underline{51 \mid 52}$. Weitere experimentelle Arbeiten, die ungeladene Wassercluster bzw. Nanopartikel zum Gegenstand haben $38|40| 44|78| 79|143| 166]$, beschränken sich darauf, die gemittelten Eigenschaften der erzeugten Größenverteilung zu behandeln.

Kennzeichnend für diesen Größenbereich ist die wachsende Isomerenvielfalt mit zunehmender Clustergröße, die spätestens ab dem mittleren, zweistelligen Bereich die Ermittlung globaler Minimumstrukturen obsolet macht: Numerische Simulationen von Buch et al. ergaben, dass die Struktur dreidimensionaler H-Brückennetzwerke formal als Ergebnis eines zweistufigen Prozesses aufgefasst werden kann. Die erste Stufe besteht in der Festlegung der Netzwerktopologie bezüglich der Sauerstoffatome. Diese bestimmt die Form des Clusters und die Anzahl der gebildeten H-Brücken. In der zweiten Stufe wird die Orientierung der Wassermoleküle festgelegt. Hier entscheiden sich die Positionen der HAtome innerhalb der H-Brücken. Auf jeder der beiden Stufen ergeben sich bereits bei $N=6$ mehrere Variationsmöglichkeiten, deren Anzahl mit steigender Konstituentenzahl in beiden Fällen exponentiell ansteigt 62/69|120]. Die Energiedifferenzen zwischen den isomeren Strukturen vermindern sich mit steigender Clustergröße, sodass zunehmend mehr Strukturen zu einem Spektrum beitragen 48 .

Die akkurate quantenchemische Behandlung H-brückengebundener Netzwerke gestaltet sich äußerst schwierig, da einerseits die Bindungsenergien eine Größenordnung kleiner sind, als die kovalenter Bindungen, aber sie andererseits die Form der Molekülorbitale beeinflussen, sodass sie nicht als reine Van-der-Waals-Wechselwirkungen aufgefasst werden können 22. Weitere Fehlerquellen entstehen beim Einbezug der Schwingungsnullpunktsenergie und des temperaturabhängigen Entropieterms, sodass die Frage nach der energe- 
tischen Staffelung und nach dem statistischen Gewicht der möglichen isomeren Strukturen ab einer bestimmten Clustergröße nicht mehr mit vertretbarem Aufwand zuverlässig beantwortet werden kann. Bei der Suche nach Minimumstrukturen zeigt sich jedoch, dass diese zum Teil hohe Ähnlichkeit aufweisen, sodass die Suche nach Familien aus nahezu identischen Strukturen oder Strukturmotiven in diesem Größenbereich mehr Erfolg verspricht, als die Suche nach einer globalen Minimumstruktur 48 .

Auf theoretischer Seite wurde dennoch eine Reihe von Studien durchgeführt, die zum Teil in sehr überzeugenden Strukturvorhersagen mündeten: Erste Vorhersagen wurden von Whales und Hodges im Bereich bis $N=21$ auf Basis des TIP4P-Potentials getroffen, das allerdings nur im Bereich $N \leq 12$ verlässliche Strukturvorhersagen ermöglicht. Bandow und Hartke verwendeten komplexere Potentiale der TTM-Serie [167, um Minimumstrukturen bis $N=100 \mathrm{zu}$ ermitteln, die sie im Bereich $N \leq 34$ als zuverlässig einstuften. Diese Vorhersagen wurden im Bereich $N \leq 21$ auf MP2-Niveau bestätigt 168 . Später erweiterten Kazachenko und Thakkar den Bereich ernstzunehmender Vorhersagen für globale Minimumstrukturen auf $N \leq 55$ 91.

Auch einige experimentelle Studien an ionischen Clustern, die nicht die Gewinnung von Strukturinformation sondern die relative Stabilität und die thermodynamischen Eigenschaften der Cluster in den Fokus nahmen, lieferten Ergebnisse, die möglicherweise auf ladungsfreie Cluster übertragbar sind. Ein Aspekt, der sowohl experimentell als auch theoretisch arbeitende Gruppen beschäftigt, ist die mögliche Existenz von Konstituentenzahlen, die zur Bildung von Strukturen mit ausnehmend hoher thermodynamischer Stabilität führen (sogenannte magische Größen). Beide Seiten fanden in den letzten Jahren zahlreiche Hinweise auf solche Strukturen:

Knapp et al. publizierten im Jahr 1987 erstmals die Beobachtung ausnehmend hoher Signalabundanzen im Massenspektrum anionischer Wassercluster der Größen $N=51$, 53, 55 und 57. Die Cluster waren in einer ungeseedeten Überschallexpansion gasförmigen Wassers erzeugt und durch Anlagerung thermischer Elektronen ionisiert worden 89 . Anlagerung von Elektronen höherer kinetischer Energie ergab keine derartige Beobachtung. Auch Massenspektren in Koexpansion mit Argon ( $p=9$ bar) erzeugter, in gleicher Weise ionisierter Wassercluster zeigten keine Auffälligkeiten 169. Schindler et al. fanden erhöhte thermische Stabilitäten der Größen $N=53$ und $N=55$ bei der Beobachtung der durch die thermische Hintergrundstrahlung in ihrem Ion Cyclotron Resonance (ICR)Massenspektrometer induzierten Evaporation protonierter Wassercluster. Als mögliche Erklärungen führten sie entweder eine erhöhte thermodynamische Stabilität oder eine verminderte IR-Absorbanz durch hohe Symmetrie dieser Strukturen (oder eine Kombination beider Faktoren) an 90 . Zurheide et al. fanden später in ladungsfreien, natriumdotierten Clustern keine im Ganzen herabgesetzte IR-Absorbanz der fraglichen Clustergrößen, vielmehr manifestierte sich deren abweichendes Verhalten in einer erhöhten Absorbanz der freien OH-Gruppe bei kleineren Wellenzahlen. Weiterhin fanden sie eine erhöhte Absorbanz der freien OH-Streckschwingung der ungeraden Clustergrößen im Bereich zwischen $N=49$ und $N=5554$. Eine derartige Rotverschiebung bedeutet eine herabgesetzte Elektronendichte in der OH-Bindung, die durch stärkere H-Brücken in der unmittelbaren Umgebung dieser Gruppe verursacht wird51. Ma et al. fanden erhöhte Abundanzen der geradzahligen Clustergrößen im Bereich von $N=50$ bis $N=58$ an anionischen Wasserclustern, die durch Kollisionen auf eine Temperatur von $121 \mathrm{~K}$ thermalisiert worden waren 31. Diese Temperatur führt zu Evaporation von Konstituenten auf der Zeitskala 
des Experiments 170 . Weiterhin zeichnen sich die betreffenden (hier die geradzahligen) Clustergrößen durch ein erhöhtes vertikales IP aus 31. Kazachenko und Thakkar sagten für die Clustergröße $N=51$ die Existenz einer Minimumstruktur mit ausnehmend hoher Symmetrie und thermodynamischer Stabilität voraus ${ }^{91}$. Zusammenfassend lässt sich daher folgendes Bild zeichnen: Die Existenz thermodynamisch besonders stabiler Strukturen ist durch mehrere unabhängige Arbeiten belegt. Ihre Stabilität ist ausreichend hoch, um eine Protonierung des Clusters zu überleben. Eine erhöhte Abundanz lässt sich jedoch nur beobachten, wenn im Cluster erstens genug thermische Energie vorhanden ist, um auf der Zeitskala des Experiments Evaporationsereignisse auszulösen und zweitens die Detektion der Cluster nicht durch Fragmentierung gestört ist. In den Cluster eingebrachte Elektronen verändern dessen Struktur bei ausreichend hoher Clustertemperatur (z. B. in IRPD-Experimenten) insofern, als sie ein Wassermolekül ersetzen, sodass die Strukturen erhöhter Stabilität bei um den Wert 1 verminderten Clustergrößen auftreten 31 .

Die IR-anregungsmodulierte Photoionisationsspektroskopie natriumdotierter Cluster ermöglicht eine zuverlässige Separation von Clustern in diesem Größenbereich (vgl. Abschnitt 3.2). Auf Basis der mit dieser Methode erhaltenen Schwingungsspektren konnten einigen Clustergrößen versuchsweise Strukturen zugeordnet werden. So scheinen für die Clustergröße $N=20$ zwei nahezu isoenergetische Minimumstrukturen zu existieren - ein trigonales Pentaprisma, in dem alle Wassermoleküle auf der Oberfläche lokalisiert sind, und eine tropfenartige Struktur, die ein nahezu tetraedrisch koordiniertes Molekül im Zentrum aufweist (vgl. Abb. 2.9 71]. Der Cluster $N=25$ scheint bevorzugt eine einzige Struktur auszubilden. Die experimentellen Spektren der Größen $N=24$ und $N=32$ konnten nur durch Überlagerung simulierter Spektren mehrerer Strukturen zufriedenstellend reproduziert werden 48 . Die Auswahl und Gewichtung der simulierten Spektren für diese Zuordnung beinhaltet viele Freiheitsgrade, die nur durch hohe Qualität der Messdaten und der simulierten Spektren eliminiert werden können. Daher können bisherige Strukturzuordnungen, die auf einer Kombination mehrerer simulierter Spektren beruhen, nicht als gesichert betrachtet werden.

\section{Große Cluster und Nanopartikel}

Der Fokus bisheriger Studien an mikroskopischen Proben kristallinen Eises im Labor 15|21|22|44|78|79|125] und in der Atmosphäre 12|19|20|133| lag meist auf dünnen Filmen oder Partikeln mit Größen im Mikrometerbereich oder im dreistelligen Nanometerbereich. Der Grund hierfür ist die schlechte experimentelle Zugänglichkeit kleinerer Partikel $17[20]$.

Ein erster Anlauf zur Gewinnung größenabhängiger, spektraler Informationen an wässrigen Nanopartikeln im Bereich oberhalb von $N=10$ wurde von Devlin et al. unternommen. Zur Erzeugung der Partikel kam eine von Bauerecker et al. entwickelte, heliumgekühlte Kollisionskühlungszelle 43 zum Einsatz, die für die Erzeugung möglichst kleiner Aggregate optimiert war. Die Größenbestimmung wurde durch Adsorption einer Monolage von $\mathrm{CF}_{4}$-Molekülen auf der Partikeloberfläche und anschließende Vermessung der relativen Bandenintensität im Vergleich zur Bande der gebundenen OH-Oszillatoren erreicht. Wie einleitend bereits erwähnt, war eine Größenselektion lediglich durch Variation der mittleren Partikelgröße möglich. Die kleinste, in diesen Experimenten erreichbare Clustergröße war $\langle N\rangle=130446279$. Andersson et al. publizierten Schwingungsspektren ladungsfreier Aggregate im Größenbereich $\langle N\rangle=20$ bis $\langle N\rangle=2000$, die mit Hilfe der Photofragmentspektroskopie erhalten worden waren. Diese Methode, die auf der Detektion von Dissozi- 

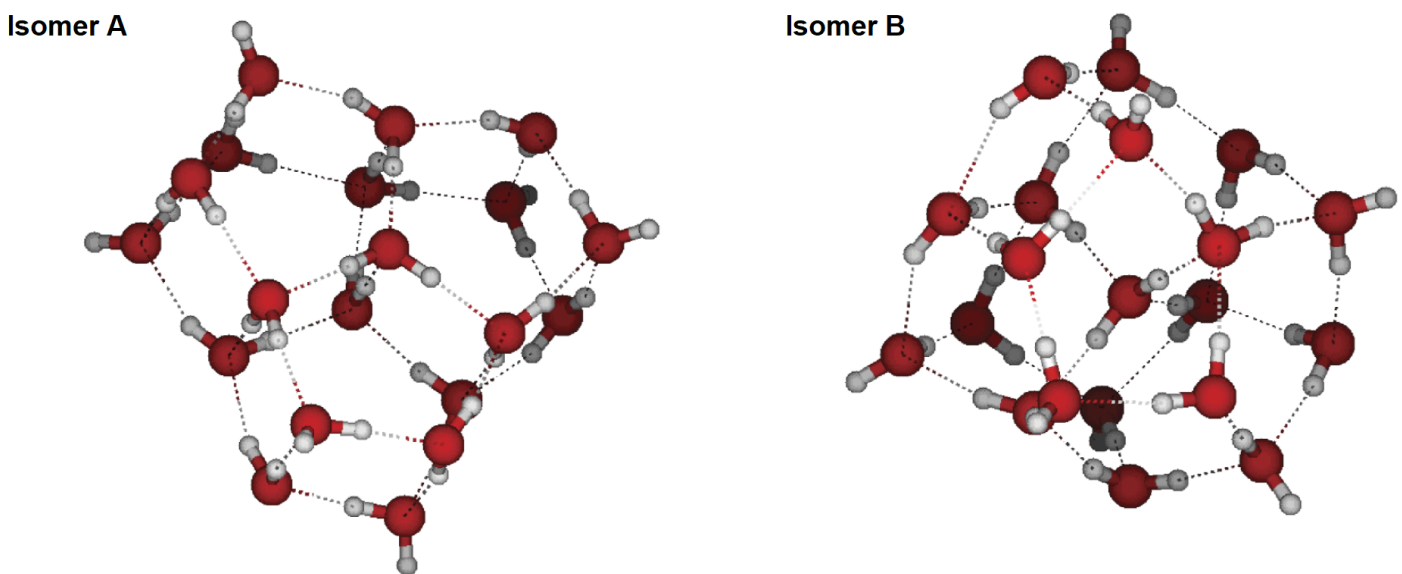

Abbildung 2.9.: Minimumstrukturen von $\left(\mathrm{H}_{2} \mathrm{O}\right)_{20}$; entnommen aus Ref. [59].

ierten Oligomeren nach IR-Anregung basiert, liefert allerdings selektiv Spektren amorpher Aggregate, da Partikel mit kristallinem Kern eine deutlich geringere Neigung zur Abspaltung von Oligomeren zeigen $40[166$. Neben der in dieser Arbeit verwendeten Methode der Größenselektion sind die in diesen Veröffentlichungen beschriebenen Verfahren die einzigen geeigneten Ansätze, um größenabhängige, spektrale Informationen über ladungsfreie Wassercluster im Größenbereich $N \lesssim 50 \lesssim 1000$ zu erhalten 5278 .

Wasseraggregate dieser Größe lassen sich in zwei Gruppen unterteilen: Einerseits in Partikel mit kristallinem Kern, andererseits in große amorphe Cluster, deren Kern keine ausgeprägten Unterschiede zu den äußeren Schichten aufweist ${ }^{79}$. Die in amorphen Clustern gebildeten H-Brückennetzwerke bestehen aus drei- bis fünfzähligen Ringen, sodass deren Dichte über der kristalliner Partikel liegt 134 . Letztere besitzen im Kern ein Kristallgitter entsprechend Abschnitt 2.3.4, das in den kleinsten Partikeln nicht ideal relaxiert ist, sondern von der optimalen Geometrie abweicht und daher unter Spannung steht 79 .

Der Grund für diese Abweichung liegt in einer energetischen Konkurrenz zwischen Kern und Oberfläche: Simulationen von Buch et al. zeigten, dass die Fortsetzung der für das Kristallgitter charakteristischen Ringstruktur bis zur Partikeloberfläche dort zu zahlreichen freien OH-Gruppen führt, die nicht in eine H-Brücke eingebunden sind. Diese Situation ist energetisch und entropisch ungünstig, sodass die Moleküle der Oberfläche eine amorphe Struktur aus verzerrten Ringen unterschiedlicher Größe ausbilden, die einen Kompromiss zwischen einer maximalen Anzahl ausgebildeter H-Brücken und einer optimalen Koordinationsgeometrie möglichst vieler Wassermoleküle darstellt. Daher weisen die Oberflächen kristalliner Partikel ebenso wie die amorpher Cluster keine periodische Ordnung der Sauerstoffatome auf. Auch die Oberflächen größerer Nanokristalle, in denen eine ungeordnete Sauerstoffstruktur nahezu isoenergetisch mit einer geordneten Struktur zu sein scheint, dürften aufgrund der höheren Entropie bei Abwesenheit einer periodischen Ordnung amorph sein 62[68/79. Laut Buch und Devlin setzt auch eine vollständig geordnete, durch eine Doppelschicht von Wassermolekülen abgeschlossene Kristalloberfläche mindestens eine Doppelschicht darunter unter Spannung. Die Unordnung einer amorphen Oberfläche setzt sich ein bis zwei Doppelschichten ins Partikelinnere fort, sodass sich als Summe von Oberfläche und Suboberfläche eine Schicht mit einer Stärke von ca. $5 \AA$ ausbildet $62 \mid 79$. Johnston et al. fanden in neueren Rechnungen lediglich eine Stärke von ca. 2,6 A, entsprechend einer Monolage von Wassermolekülen 68. 


\begin{tabular}{|c|c|c|c|c|}
\hline JAHR & $N^{*}$ & SPEZIES & ANSATZ & REFERENZ \\
\hline 1983 & $200-1000$ & $\left(\mathrm{H}_{2} \mathrm{O}\right)_{N}$ & experimentell & Torchet et al. 38 \\
\hline 2000 & $\approx 500$ & $\left(\mathrm{H}_{2} \mathrm{O}\right)_{N}$ & experimentell & Devlin et al. $\underline{44}$ \\
\hline 2003 & $123-293$ & $\left(\mathrm{H}_{2} \mathrm{O}\right)_{N}$ & theoretisch & Kazimirski; Buch 69. \\
\hline 2006 & $\approx 90$ & $\left(\mathrm{H}_{2} \mathrm{O}\right)_{N}$ & theor & Bandow; Hartke 70 \\
\hline 2010 & $\approx 100$ & $\mathrm{H}^{+}\left(\mathrm{H}_{2} \mathrm{O}\right)_{N}$ & experimentell & Mizuse et al.32 \\
\hline 2012 & $100-137$ & $\left(\mathrm{H}_{2} \mathrm{O}\right)_{N}$ & the & Johnston; Molinero 68 \\
\hline 2012 & $275 \pm 25$ & $\mathrm{Na}\left(\mathrm{H}_{2} \mathrm{O}\right)_{N}$ & experimentell & Pradzynski et al. ${ }^{41]}$ \\
\hline 2014 & $<200$ & $\mathrm{Na}\left(\mathrm{H}_{2} \mathrm{O}\right)_{N}$ & experimentell & Buck et al. 48 \\
\hline 2015 & $<150$ & $\mathrm{Na}\left(\mathrm{H}_{2} \mathrm{O}\right)_{N}$ & experimentell & Gimelshein et al.59 \\
\hline 2016 & $\approx 375$ & $\mathrm{La}^{3+}\left(\mathrm{H}_{2} \mathrm{O}\right)_{N}$ & experimentell & Cooper et al. 76 \\
\hline 2019 & $90 \pm 10$ & $\mathrm{Na}\left(\mathrm{H}_{2} \mathrm{O}\right)_{N}$ & experimentell & Dierking 61 \\
\hline
\end{tabular}

Tabelle 2.1.: Übersicht über bisherige experimentelle und theoretische Arbeiten zur kritischen Clustergröße für die Ausbildung eines kristallartigen Kerns. Der Vollständigkeit halber sind auch ionische Spezies aufgeführt.

Ein Nanotropfen muss folglich eine bestimmte Mindestzahl $N^{*}$ von Wassermolekülen enthalten, um sowohl einen kristallartigen Kern als auch eine amorphe Oberfläche ausbilden zu können, die die Kristallstruktur energetisch stabilisiert. Abbildung 2.10 visualisiert qualitativ die größenabhängige Entwicklung der Clusterstrukturen bis hin zur Ausbildung einer Kristallstruktur. Der genaue Wert von $N^{*}$ war bisher Gegenstand zahlreicher experimenteller und theoretischer Arbeiten an elektrisch neutralen und ionischen Spezies.

Tabelle 2.1 enthält (ohne Anspruch auf Vollständigkeit zu erheben) einen Überblick über den bisherigen Forschungsstand. Dabei ist zu beachten, dass jeder der publizierten, experimentellen Werte von $N^{*}$ einen oberen Grenzwert darstellt.

Abgesehen von den Arbeiten von Cooper et al. und Devlin et al., die ihre Partikel durch Elektrosprayionisation 76 bzw. in einer Kollisionskühlungszelle 44 erzeugten, kamen in sämtlichen experimentellen Arbeiten Expansionstechniken zum Einsatz. Sämtliche Werte für $N^{*}$, die an natriumdotierten Clustern ermittelt wurden, kamen unter Verwendung der adiabatischen Überschallexpansion (vgl. Kap. 3) und unter Nutzung derselben Versuchsapparatur (vgl. Kap. 4) zustande.

Es ist ersichtlich, dass die in Tabelle 2.1 zusammengefassten Werte für die kritische Clustergröße $N^{*}$ stark variieren, sodass die zugrundeliegenden Arbeiten sich nur schlecht miteinander vergleichen lassen. Die Teilchengröße, oberhalb der im Experiment die Bildung eines kristallartigen Kerns nachweisbar ist, hängt stark von den Bedingungen ab, unter denen die Cluster erzeugt wurden 48/59]. Der Grund ist, dass die Relaxation möglichst vieler Partikel in ihre globale Minimumstruktur gewährleistet sein sollte, um auch den kleinsten Aggregaten, in denen ein Phasenübergang thermodynamisch möglich ist, ausreichend Zeit hierfür einzuräumen. Da die Länge des Zeitfensters, das einem Cluster zum Auffinden seines globalen Minimums zur Verfügung steht, von der Geschwindigkeit seiner Abkühlung abhängt 68 und die Wahrscheinlichkeit eines Nukleationsereignisses mit der Partikelgröße abnimmt 9 , besitzt die Kühlrate einen besonders großen Einfluss auf den experimentell beobachteten Wert von $N^{*}$. Der hauptsächliche Grund für die schlechte Vergleichbarkeit verschiedener experimenteller Studien liegt folglich in den variierenden 


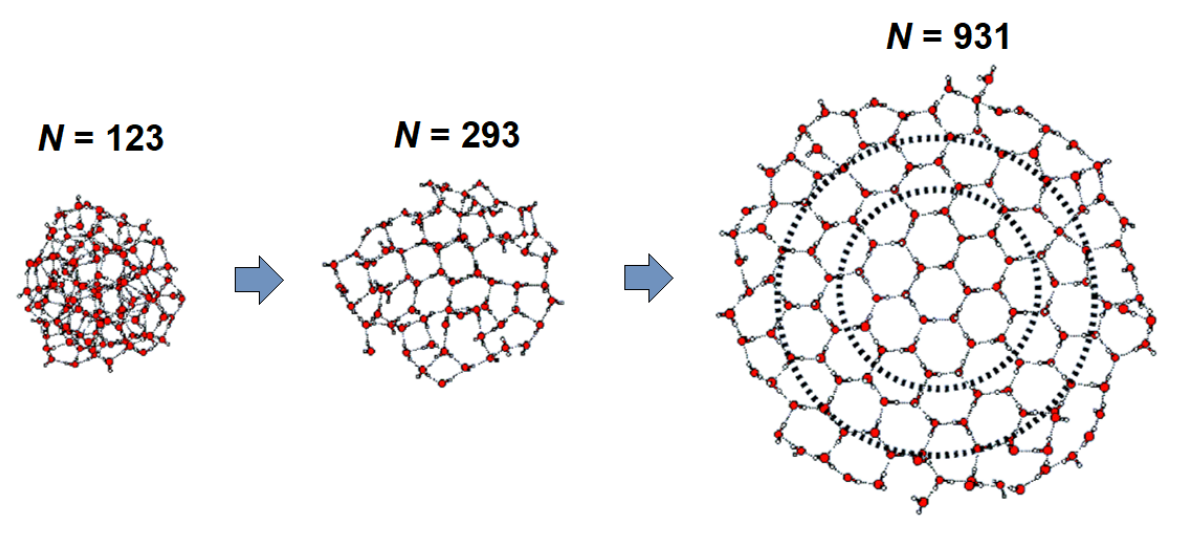

Abbildung 2.10.: Größenabhängige Strukturentwicklung ladungsfreier Wassercluster nach Buch et al. 62 ; bei $N=293$ und $N=931$ ist jeweils nur ein zentraler Schnitt gezeigt, der den Blick auf eine kristalline Schicht ermöglicht. Im Kern des Clusters mit $N=293$ deutet sich die Herausbildung einer kristallinen Struktur in Form verzerrter Sechsringe an. Die konzentrischen Kreise bei $N=931$ kennzeichnen die Bereiche der Oberfläche, Suboberfläche und des kristallinen Kerns; verändert nach Referenz [48].

Kühlraten (vgl. Kap. 3.1) 59. Ein weiterer Grund ist die limitierte Größenselektion früherer Arbeiten. In diesen wurde die Größe publiziert, ab der sich die experimentelle Signatur kristalliner Partikel eindeutig von der Signatur ebenfalls in der erzeugten Größenverteilung vorhandener amorpher Partikel unterscheiden ließ.

Eine parallele Argumentationslinie lässt sich auf Basis der Ausführungen in Abschnitt 2.2.2 für die theoretische Seite aufstellen: Voraussetzung für die verlässliche Vorhersage einer Minimumstruktur ist zunächst eine akkurate Parametrisierung des Modells, sodass das subtile energetische Gleichgewicht zwischen optimaler Geometrie und maximaler H-Brückenzahl korrekt wiedergegeben wird. Diese Anforderung bedingt einen hohen Rechenaufwand für das Auffinden und die Optimierung möglicher Minimumstrukturen. Die Potentialhyperfläche, die aus einer Vielzahl lokaler Minima getrennt von hohen Barrieren besteht $62[69]$, sowie der hochdimensionale Phasenraum 48|120|171] verlängern hier analog zum Experiment die zur optimalen Relaxation eines Clusters benötigte Zeit.

Die Ausbildung eines kristallinen Kerns in Abgrenzung zur amorphen Oberfläche stellt dabei längst nicht den Endpunkt der größenabhängigen Strukturmodulation wässriger Nanopartikel dar. Zahlreiche Arbeiten belegen, dass die Eigenschaften der kleinsten kristallinen Eispartikel sich mit steigendem Durchmesser stark verändern, bis sie im Größenbereich um $20 \mathrm{~nm}$ gegen konstante Werte streben $15|20| 44|48| 62 \mid 77$ :

Es zeigt sich eine größenabhängige Verschiebung IR-Absorptionsbanden, weiterhin ändert sich deren Form. Mit zunehmendem Verlust des kristallinen Charakters bei Durchmessern unterhalb von $4 \mathrm{~nm}$ verbreitert sich die Bande der gebundenen $\mathrm{OH}-$ Streckschwingung und das Maximum bei $3220 \mathrm{~cm}^{-1}$ beginnt $\mathrm{zu}$ verschwinden. Die kleinsten kristallartigen Aggregate besitzen IR-Spektren mit ausgeprägter Bandenstruktur 48 . Buch et al. fanden in diesen Partikeln eine verminderte Oszillatorstärke der freien $\mathrm{OH}-$ Gruppen im Vergleich zu ihrer Neigung, $\mathrm{CF}_{4} \mathrm{zu}$ adsorbieren 62 . Beide Befunde deuten darauf hin, dass diese Partikel eine charakteristische Oberflächenbeschaffenheit besitzen, die sich sowohl von der Oberfläche amorpher Cluster als auch von der größerer, kristalliner Nanopartikel unterscheidet. 


\section{Erzeugung und IR-spektroskopische Untersuchung natriumdotierter Wassercluster}

Es existiert eine Reihe experimenteller Studien über die Kristallisation ungeladener Wassertropfen (vgl. z. B. [38, 39, 44, 62, 65, 67, 75, 78, 106, 139, 147, 172, 173]). Die meisten dieser Arbeiten befassen sich mit Kristallen im Mikrometer- bzw. im dreistelligen Nanometerbereich. Der Grund hierfür sind die Schwierigkeiten, die bei der Präparation und der Charakterisierung kleinerer Partikel entstehen 170. Auf den folgenden Seiten soll dargelegt werden, wie diese Hürden in der vorliegenden Arbeit überwunden wurden.

\subsection{Erzeugung ladungsfreier Wassercluster in adiabatischen Überschallexpansionen}

Becker et al. beobachteten im Jahr 1956 in der Ausstoßfahne einer Lavaldüse erstmals die Phänomene, die eine adiabatische Überschallexpansion auszeichnen: Die Einengung der Geschwindigkeitsverteilung, homogene Kondensationsvorgänge aus der Gasphase und eine z. T. bimodale Größenverteilung 174. Seither haben Überschallexpansionen in der Erzeugung von Clustern und Nanopartikeln weite Verbreitung gefunden 42 |83|175| 177.

Partikel, die durch Expansionsmethoden erzeugt wurden, bewegen sich frei im Vakuum. Im Vergleich zu anderen experimentellen Zugängen wie der Bildung von Emulsionen 106 oder der Größenbegrenzung durch Nanoporen (vgl. Ref. [139] und Referenzen darin) bieten diese Techniken den Vorteil, dass die gebildeten Partikel keine Wechselwirkungen mit einer Oberfläche eingehen, die den Nukleationsprozess beeinflussen könnte 39 .

Bei Verwendung einer Laval-Düse findet die Expansion kontrolliert statt, sodass die darin auftretenden Phasenübergänge unter Gleichgewichtsbedingungen und bei gleichzeitiger Bestimmung von Nukleationsraten und Temperatur beobachtet werden können 75 . Der große Nachteil der Laval-Düsen besteht in deren hohem Gasdurchsatz, weshalb sie erstens gepulst betrieben werden müssen 59 und sich zweitens Schwierigkeiten bei der Kombination mit der Pickup-Technik (vgl. Abschnitt 3.2.1) ergeben.

Demgegenüber kann die Expansion bei Verwendung einer sehr kleinen, konischen Düse kontinuierlich betrieben werden. Derartige Expansionen werden in der Literatur in Abgrenzung zu Laval-Düsen als freie Expansionen (engl. free jet expansions) bezeichnet 178 . Nachteilig wirkt sich hier der schlecht definierte thermodynamische Zustand der gebildeten Partikel aus 59|87. Da die Kondensation aus einer stark übersättigten Gasphase im thermodynamischen Ungleichgewicht stattfindet, stellt die Bestimmung der Temperatur eine nicht-triviale Aufgabe dar: Eine nachträgliche Thermalisierung ist nur mit geladenen Partikeln in Ionenfallen möglich 31|129. Die in Abschnitt 2.3.5 erwähnte VRT 
zur Charakterisierung der kleinsten, ringförmigen Cluster eröffnete in diesen Experimenten einen eleganten, spektroskopischen Zugang zur Temperatur über die Messung der Rotationskonstanten 148 [150|152 . Im Falle größerer Aggregate muss die Temperatur hingegen aus den Expansionsbedingungen abgeschätzt werden 164 . Aufgrund der damit verbundenen Unsicherheit wurden in der Vergangenheit meist allenfalls relative Temperaturangaben publiziert $59 \mid 166$.

Da die im vorliegenden Experiment verwendeten, konischen Düsen eine kontinuierliche Expansion ermöglichen, lässt sich der Nachteil des schlecht definierten thermodynamischen Zustands ausgleichen, sofern (wie im vorliegenden Fall) eine akkurate Vermessung der Größenverteilung möglich ist. Diese Daten wurden von Gimelshein et al. als Referenz zur Konstruktion eines numerischen Modells verwendet, mit dessen Hilfe die Temperatur präzise ermittelt werden kann $36|59| 92 \mid 179]$.

Das oben erwähnte Ungleichgewicht manifestiert sich erstens darin, dass in der Expansion ablaufende, reversible Prozesse wie die Addition und Evaporation von Monomeren während des gesamten Expansionsvorgangs nicht ihren Gleichgewichtszustand erreichen und zweitens im Temperaturunterschied zwischen dem Trägergas und den gebildeten Aggregaten, der durch die Kollisionsprozesse nicht vollständig ausgeglichen wird 59. Dieses Ungleichgewicht ist einer der Gründe dafür, dass die klassische Nukleationstheorie auf freie Überschallexpansionen nicht angewandt werden kann: Die Nukleationsrate, die zentrale Größe dieser Theorie, wurde unter Annahme von Gleichgewichtsbedingungen definiert. Ein weiterer Grund ist die zunehmende Größenabhängigkeit der Oberflächenspannung in sehr kleinen Clustern und bei niedrigen Temperaturen. Weiterhin verlieren unter Annahme eines Kontinuums definierte physikalische Größen bei der Beschreibung kleinster Cluster mit Konstituentenzahlen im unteren zweistelligen Bereich ihre Gültigkeit 180 .

\subsubsection{Das Strömungsfeld}

Die komplexen Prozesse in wenig kontrollierten, freien Expansionen lassen sich nur näherungsweise analytisch behandeln. Eine exakte Beschreibung ist nur durch Monte Carlobzw. MD-Simulationen möglich 42/59. Unter Annahme einiger Näherungen lässt sich jedoch ein grundlegendes Verständnis über die in solchen Strömungsfeldern ablaufenden Vorgänge erlangen. Diese Näherungen sind im Einzelnen zunächst ein ideales Verhalten der beteiligten Gase sowie die Abwesenheit von Impuls- und Wärmeleitung, d.h. es wird ein adiabatischer Prozess angenommen. Diese Annahmen sind bei den in der Expansion auftretenden, hohen Flussgeschwindigkeiten und auf der kurzen Zeitskala des Experiments gut erfüllt175. Hinzu kommt die Näherung eines kontinuierlichen (d.h. kollisionskontrollierten 181 ) Flusses, die weniger gut erfüllt sein dürfte ${ }^{175}$. Weiterhin wird eine konstante und (bei Expansionen von Gasmischungen) annähernd gleiche Wärmekapazität aller Komponenten im fraglichen Temperaturbereich angenommen 175.

Abbildung 3.1 zeigt einen Querschnitt durch das komplexe Strömungsfeld einer axialsymmetrischen, kontinuierlichen, freien Überschallexpansion. Die Strömungsfelder dieses Expansionstyps weisen eine hohe symmetrische Ähnlichkeit auf 182 , was die Ableitung verallgemeinerter Aussagen ermöglicht.

Das Gas strömt mit zunächst niedriger Geschwindigkeit von einem Bereich hohen Drucks und hoher Temperatur $\left(p_{0}, T_{0}\right.$, den Stagnationsbedingungen) und angetrieben durch die Druckdifferenz $\left(p_{0}-p_{1}\right)$ zum Hintergrunddruck $p_{1}$ in die äußere Kammer, wo- 


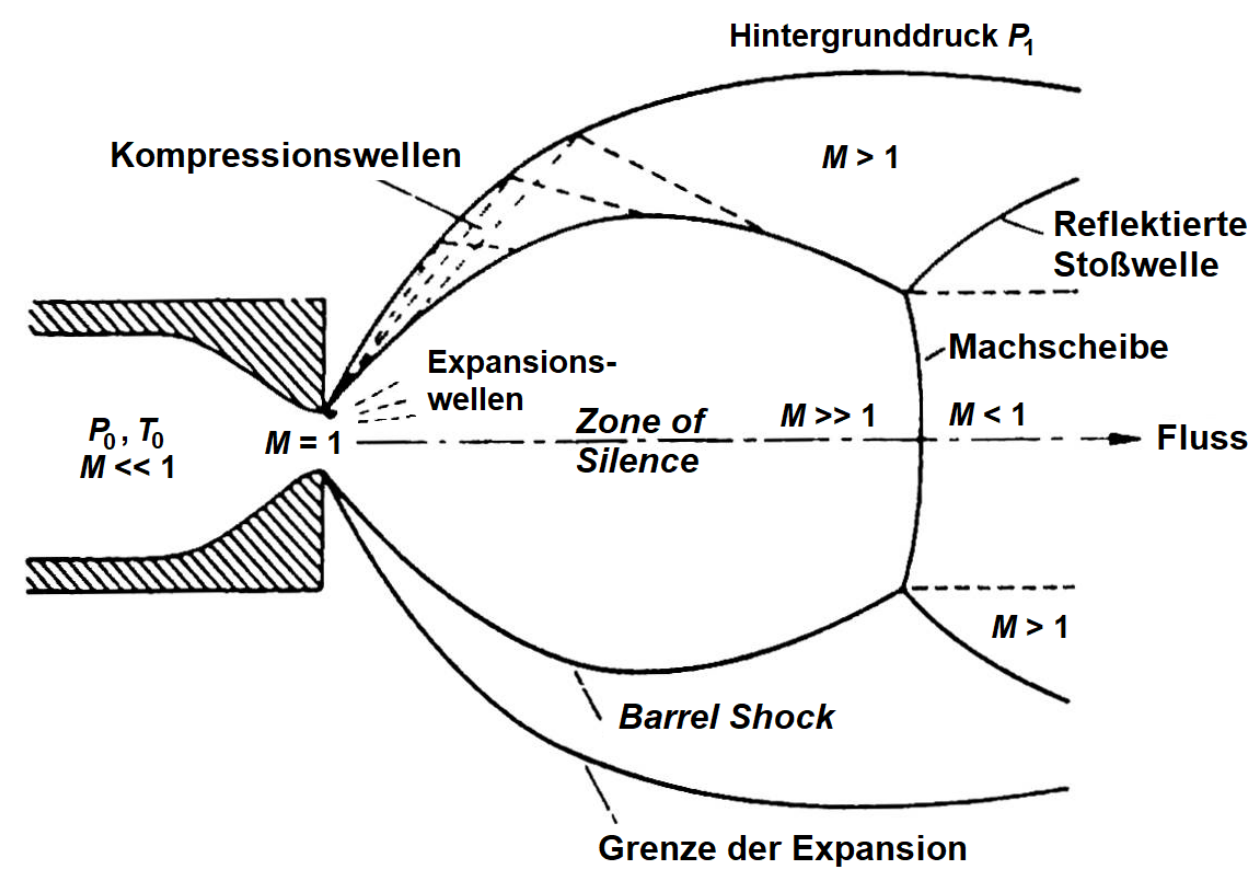

Abbildung 3.1.: Kontinuierliche, freie Überschallexpansion; verändert nach Ref. 175.

bei sich die Flussgeschwindigkeit $v_{\mathrm{F}}$ erhöht. Überschreitet der Quotient $\left(p_{0} / p_{1}\right)$ einen nach Gleichung 3.1 zu berechnenden Wert, der für alle Gase den Betrag von 2,1 unterschreitet, entspricht die Flussgeschwindigkeit $v_{\mathrm{F}}$ des Gases in der engsten Stelle der Düse der lokalen Schallgeschwindigkeit $a$ und wird diese nach dem Durchtritt durch diesen Düsenhals überschreiten, da es nach dessen Passage weiter expandieren kann.

$$
p_{0} / p_{1}=\frac{\gamma+1}{2}^{\gamma / \gamma-1}
$$

Die Größe $\gamma=C_{p} / C_{V}$ bezeichnet hier den Quotienten aus den Wärmekapazitäten bei konstantem Druck $C_{p}$ und bei konstantem Volumen $C_{V}$. Tritt das Gas nach der Passage einer beliebig geformten Düse in das Vakuum der Expansionskammer mit dem Druck $p_{1}$ ein, reduziert sich sein Druck zunächst auf ca. $p_{0} / 2$. Bei ausreichend großem Verhältnis von $p_{0} / p_{1}$ übersteigt dieser Wert weiterhin den Hintergrunddruck $p_{1}$, sodass das Gas weiter expandieren und seine Flussgeschwindigkeit erhöhen kann. Diese übersteigt nunmehr die lokale Schallgeschwindigkeit $a$. Der Quotient aus der Flussgeschwindigkeit $v_{\mathrm{F}}$ und der lokalen Schallgeschwindigkeit $a$ wird als Machzahl $M$ bezeichnet:

$$
M=v_{\mathrm{F}} / a
$$

Die Machzahl ist ein universelles Charakteristikum des Expansionsexperiments und ist allein Funktion des reduzierten Düsenabstands $x_{\text {red }}=x / d_{\mathrm{D}}$ sowie des Quotienten $\gamma$, d.h. einer Eigenschaft des Strömungsfeldes und einer Eigenschaft des expandierenden Gases $175 \mid 181]$. Hierbei bezeichnet $x$ den absoluten Abstand von der Düse, $d_{\mathrm{D}}$ bezeichnet deren Durchmesser. Bei Kenntnis der lokalen Machzahl werden sämtliche weiteren thermodynamischen Größen des expandierenden Gases an diesem Ort zugänglich (vgl. Abschnitt 3.1.2. 
Im Zuge der Expansion nimmt die Machzahl weiter zu. Diese Eigenschaft ist der entscheidende Vorteil einer Überschallexpansion gegenüber einer effusiven Expansion $(M<1)$ : Ein expandierendes Gas wird unweigerlich auf eine Barriere treffen (die Behälterwand oder, wie in Abb. 3.1, das Hintergrundgas in der Expansionskammer mit dem Druck $p_{1}$, sobald der Druck im Inneren der zone of silence sich diesem Wert annähert). Die Information über die durch den Wert von $p_{1}$ vorgegebenen Randbedingungen des Strömungsfeldes kann sich maximal mit Schallgeschwindigkeit darin ausbreiten. Sofern die Gasmoleküle sich mit einer Geschwindigkeit $v_{\mathrm{F}}<a$ fortbewegen, wird deren Ausbreitung beeinflusst, da das Strömungsfeld sich den durch den Hintergrunddruck $p_{1}$ vorgegebenen Grenzen anpassen muss. Im Falle von $v_{\mathrm{F}}>a(M>1)$ wird das expandierende Gas nicht durch eine stromabwärts liegende Barriere beeinträchtigt. Sinkt der Druck des expandierenden Gases auf den Wert von $p_{1}$ ab, treten stationäre Schockwellen (schmale, nicht-adiabatische Regionen mit hohen Werten von Temperatur, Druck und Dichte sowie hohen Geschwindigkeitsgradienten) auf, die eine Anpassung des Strömungsfeldes erzwingen: Die Expansionswellen werden an einer durch $p_{1}$ vorgegebenen Fläche reflektiert. Es folgt eine Änderung der Strömungsrichtung (und z. T. auch der Machzahl, sofern die Richtungsänderung zur Anpassung an die Randbedingungen nicht ausreicht), sodass sich das Strömungsfeld den durch den Hintergrunddruck $p_{1}$ vorgegebenen Randbedingungen anpasst 175. Der Winkel, in dem das expandierende Gas anfangs divergieren kann, ist dabei umso größer, je kleiner der Wert von $p_{1}$ wird. Da sich innerhalb der zone of silence keine Informationen über die Randbedingungen fortsetzen, kann das Gas über-expandieren, bis sein Druck unter den Wert von $p_{1}$ abfällt. Als Folge wird das Gas durch die reflektierten Expansionswellen rekomprimiert 177. Diese Wellen verringern den Expansionsquerschnitt von neuem und führen zu einer gebogenen, lateralen Grenzfläche der Expansion, die aufgrund ihrer fassförmigen Anmutung als Barrel Shock bezeichnet wird. Am Schnittpunkt der Kompressionswellen $x_{\mathrm{M}}$ wird der anfängliche Druck durch eine senkrecht zur Zentralachse stehende Schockwelle, die sogenannte Machscheibe, wieder hergestellt 177. Deren Position ist unabhängig von den Eigenschaften des expandierten Gases und lässt sich aus der empirischen Gleichung $x_{\mathrm{M}} / d_{\mathrm{D}}=0,67^{p_{0} / p_{1}}$ ableiten $175 \mid 177$. Um zu verhindern, dass sich in der Expansion gebildete Aggregate bei der Passage der Machscheibe aufheizen, wird innerhalb der Distanz $x_{\mathrm{M}}$ ein Skimmer platziert, der den Aggregaten den Übergang in eine weitere Kammer niedrigeren Drucks ermöglicht.

\subsubsection{Umwandlung thermischer Energie in kinetische Energie}

Unter adiabatischen Bedingungen bleibt die Enthalpie $\rrbracket^{1} H$ des expandierenden Gases auf einer Strömungslinie konstant 175. Unter Annahme konstanter Wärmekapazität folgt daraus ein Zusammenhang zwischen der lokalen Temperatur und der Flussgeschwindigkeit 42 :

$$
H_{0}\left(p_{0}, T_{0}\right)=H+\frac{1}{2} m v_{\mathrm{F}}^{2}=C_{p} T_{0}
$$

Nach Einführung der spezifischen Enthalpie $h=H / m$ und der spezifischen Wärmekapazität $c_{p}=C_{p} / m$ ergibt sich für die Flussgeschwindigkeit Gleichung 3.4 .

\footnotetext{
${ }^{1}$ Die Enthalpie ist deshalb die relevante Größe, da das expandierende Gas Strömungsarbeit (bzw. Druckänderungsarbeit $p / \rho$ ) verrichtet 175
} 


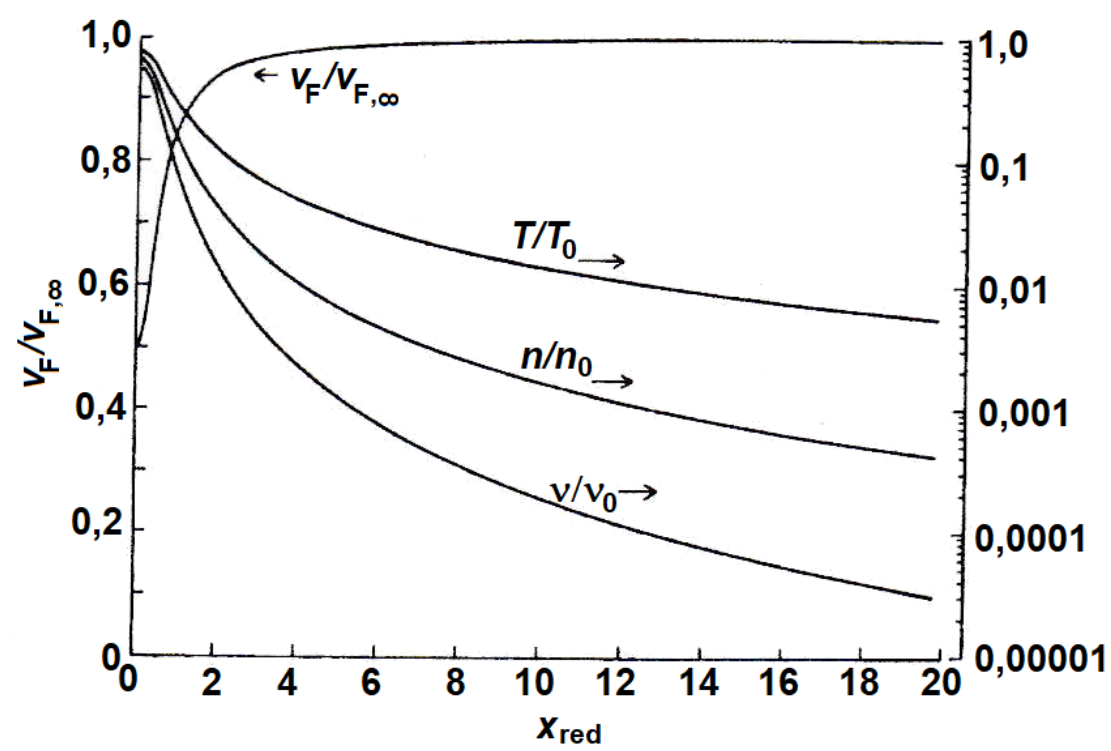

Abbildung 3.2.: Eigenschaften des frei expandierten Gases auf der Zentralachse: Normierte Parameter Flussgeschwindigkeit $v / v_{\mathrm{F}, \infty}$, Temperatur $T / T_{0}$, Dichte $n / n_{0}$ und Stoßfrequenz $\nu / \nu_{0}$ für $\gamma=5 / 3$; verändert nach Referenz 175 .

$$
v_{\mathrm{F}}^{2}=2\left(h_{0}-h\right)=2 \int_{T}^{T_{0}} c_{p} \mathrm{~d} T \approx 2 c_{p}\left(T_{0}-T\right)
$$

Unter Annahme einer konstanten Wärmekapazität im fraglichen Temperaturbereich ergibt sich:

$$
v_{\mathrm{F}}=\sqrt{2 c_{p}\left(T_{0}-T\right)}
$$

Unter der Annahme einer effektiven Kühlung während der Expansion $\left(T \ll T_{0}\right)$ lässt sich ein Ausdruck für die endgültige Expansionsgeschwindigkeit $v_{\mathrm{F}, \infty}$ ableiten. Für ideale Gase gilt der Ausdruck $c_{p}=k_{\mathrm{B}} / m[\gamma /(\gamma-1)]$. Gleichzeitiges Einsetzen dieses Ausdrucks für $c_{p}$ liefert eine Abschätzung des oberen Grenzwertes für die Flussgeschwindigkeit, wenn sämtliche thermische Energie in Flussenergie des Molekularstrahls umgewandelt worden ist:

$$
v_{\mathrm{F}, \infty}=\sqrt{\frac{2 k_{\mathrm{B}}}{m} \frac{\gamma}{\gamma-1} T_{0}}
$$

Durch Einsetzen der Formel für die lokale Schallgeschwindigkeit $a$

$$
a=\sqrt{\frac{\gamma k_{\mathrm{B}} T_{1}}{m}}
$$

und mit der Definition der Machzahl aus Gleichung 3.2 lässt sich Gleichung 3.4 umstellen, sodass die thermodynamischen Größen des Gases als alleinige Funktion der Machzahl ausgedrückt werden können (vgl. Abbildung 3.2 175]:

$$
\frac{T}{T_{0}}=\left(1+\frac{\gamma-1}{2} M^{2}\right)^{-1}
$$




$$
v_{\mathrm{F}}=\sqrt{\frac{\gamma k_{\mathrm{B}} T}{m}} M\left(1+\frac{\gamma-1}{2} M^{2}\right)^{-1 / 2}
$$

Für adiabatische Zustandsänderungen eines idealen Gases gilt die Adiabatengleichung 104:

$$
p \cdot V^{\gamma}=p_{0} \cdot V_{0}^{\gamma}
$$

Unter Nutzung von Gleichung 3.10 sowie des Idealen Gasgesetzes $p V=n R T$ lassen sich weitere nützliche Ausdrücke für den Druck und die Dichte des expandierenden Gases formulieren:

$$
\begin{aligned}
\frac{p}{p_{0}} & ={\frac{T}{T_{0}}}^{\gamma / \gamma-1}=\left(1+\frac{\gamma-1}{2} M^{2}\right)^{-\gamma / \gamma-1} \\
\frac{\rho}{\rho_{0}}=\frac{n}{n_{0}} & ={\frac{T}{T_{0}}}^{1 / \gamma-1}=\left(1+\frac{\gamma-1}{2} M^{2}\right)^{-1 / \gamma-1}
\end{aligned}
$$

Auf mikroskopischer Ebene wird die aus Gleichung 3.6 und Abbildung 3.2 ersichtliche, unvollständige Umwandlung thermischer Energie in die Expansionsenergie eines gerichteten Molekularstrahls durch Kollisionen ermöglicht. Im Bereich hoher Teilchendichte kurz nach der Düsenengstelle können die Teilchen durch Drei- oder Mehrfachstöße ihre Geschwindigkeiten angleichen. Die Translationstemperatur $T_{\|}$im Bezugssystem, das sich mit der Flussgeschwindigkeit $v_{\mathrm{F}}$ bewegt, wird reduziert. Solange Kollisionen auftreten können, kann ein Teil der in den Rotations- und Schwingungsfreiheitsgraden der Moleküle gespeicherten Energie an dieses durch Reduktion der Translationsenergie entstandene Kältebad abgegeben werden. Als Maß für die Effektivität der Depopulierung verschiedener angeregter Freiheitsgrade dient der Wirkungsquerschnitt $\sigma_{\mathrm{S}}$ des Energietransfers. Dieser nimmt i. A. in der Reihenfolge $\sigma_{\mathrm{S}}\left(E_{\text {trans }} \rightarrow E_{\text {trans }}\right)>\sigma_{\mathrm{S}}\left(E_{\text {rot }} \rightarrow E_{\text {trans }}\right)>\sigma_{\mathrm{S}}\left(E_{\mathrm{vib}} \rightarrow E_{\text {trans }}\right)$ ab 183 . Diese Abstufung ist eine Folge der Zustandsdichten der jeweiligen Freiheitsgrade: Während die bei einem Stoß übertragbare Translationsenergie jeden beliebigen Wert annehmen kann, ist die Depopulierung angeregter Rotations- und Schwingungszustände nur durch die Abfuhr diskreter Energiebeträge möglich. Die Wahrscheinlichkeit für die Depopulierung eines Zustands während eines Stoßes steigt dabei mit der Zustandsdichte bzw. mit einem geringeren Abstand zwischen den Energieniveaus 184 . Daher sind für die Ableitung einer bestimmten Energiemenge aus großen Molekülen bzw. für die Relaxation von Rotationsfreiheitsgraden mit höherer Zustandsdichte durchschnittlich weniger Stöße notwendig, als für die Energieabfuhr aus kleinen Molekülen bzw. die Relaxation von Schwingungsfreiheitsgraden, zwischen deren Energieniveaus größere Abstände vorliegen $175 \mid 184$.

Sobald die Dichte, die mit dem Quadrat von $x$ abnimmt, soweit abgesunken ist, dass keine Stöße mehr auftreten, enden die Angleichung der Geschwindigkeiten und der Temperaturabfall 183. Diese Angleichung erfolgt aufgrund der rasch abnehmenden Kollisionswahrscheinlichkeit nicht vollständig [59]185], sodass im Strahl eine thermische Restgeschwindigkeit $\alpha$ verbleibt, die der Strömungsgeschwindigkeit $v_{\mathrm{F}}$ überlagert ist 185. 


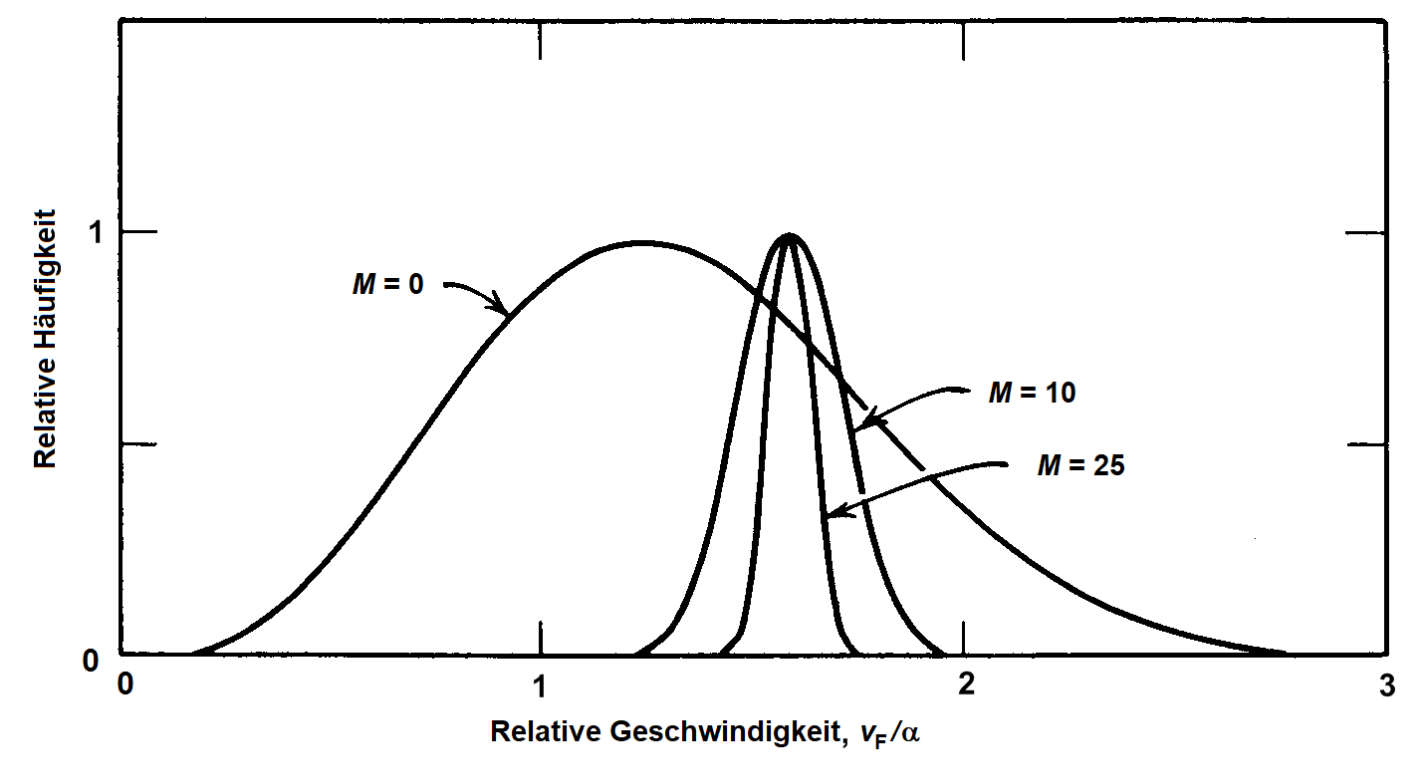

Abbildung 3.3.: Geschwindigkeitsverteilungen monoatomarer Gase mit zugehörigen Machzahlen $M$; verändert nach [185].

$$
\alpha=\sqrt{\frac{2 k_{\mathrm{B}} T_{\|}}{m}}
$$

$T_{\|}$stellt die kinetische Resttemperatur nach der Expansion dar. Es handelt sich hierbei um die Translationstemperatur, die in einem Bezugssystem herrscht, das sich mit der mittleren Flussgeschwindigkeit $\bar{v}_{\mathrm{F}}$ parallel zur Strömungsrichtung bewegt. Sie ergibt sich mit $T_{1}=T_{\|}$nach Gleichung $3.11^{185 \mid 186}$. Die resultierende Geschwindigkeitsverteilung ist abhängig von der Machzahl (vgl. Abb. 3.3) und wird gewöhnlich als eine der mittleren Flussgeschwindigkeit $\bar{v}_{\mathrm{F}}$ überlagerte Maxwell-Verteilung beschrieben $\underline{42}$ :

$$
f(v)=\left(\frac{v_{\mathrm{F}}}{\alpha}\right)^{3} \exp \left[-\left(\frac{v_{\mathrm{F}}-\bar{v}_{\mathrm{F}}}{\alpha}\right)^{2}\right]
$$

Experimentell ist die Machzahl somit über die Bestimmung der Flussgeschwindigkeit $\bar{v}_{\mathrm{F}}$ des Strahles sowie der Translationstemperatur $T_{1}=T_{\|}$aus dessen Geschwindigkeitsverteilung zugänglich 187.

\subsubsection{Kondensationsprozesse in freien Expansionen}

Sämtliche bisherigen Betrachtungen gelten bei Expansion einer einzelnen Gaskomponente ohne Einbeziehung von Kondensationsprozessen. Sie lassen sich allerdings auf Bedingungen ausweiten, solche Prozesse möglich sind. Wie bereits erwähnt, ist die klassische Nukleationstheorie zur Beschreibung der in freien Expansionen stattfindenden Prozesse nur bedingt geeignet, da der Phasenübergang hier nicht im thermodynamischen Gleichgewicht erfolgt. Die Prinzipien, auf denen diese Theorie fußt, behalten jedoch auch in freien Expansionen ihre Gültigkeit: 
Voraussetzung für die Stabilität einer kondensierten Phase ist das Vorliegen einer Übersättigung $\Phi \geq 1$. An diesem Punkt entspricht der Dampfdruck der kondensierten Phase einer Substanz definitionsgemäß ihrem Partialdruck in der umgebenden Gasphase. Dieser Dampfdruck ist jedoch abhängig vom Krümmungsradius der Oberfläche (vgl. Abschnitt 2.2.1). Die Lage der Kurve mit $\Phi=1$ in Abbildung 3.4 entspricht der Übersättigung gegenüber dem Dampfdruck einer planaren Oberfläche 42 . Ist der Zustand des expandierten Gases auf dieser Kurve zu verorten, werden Kondensationsprozesse aus thermodynamischer Sicht möglich. Aufgrund der in Abschnitt 2.2.1 beschriebenen Barriere sind sie bei $\Phi=1$ allerdings derart unwahrscheinlich, dass sie unter diesen Bedingungen auf der Zeitskala des Experiments nicht auftreten. Im weiteren Verlauf der Überschallexpansion sinkt nicht nur der Druck des expandierenden Gases, sondern als Folge der Geschwindigkeitsangleichung auch die Translationstemperatur $T_{\|}$ab. Der Zustand des Gases bewegt sich entlang der in Abb. 3.4 eingezeichneten Adiabaten. Diese besitzt eine geringere Steigung als die ebenfalls eingezeichneten Dampfdruckkurven, sodass das Übersättigungsverhältnis $\Phi$, nachdem es im Punkt A den Wert $\Phi=1$ erreicht hat, weiter ansteigt und schließlich einen Wert $\Phi_{\mathrm{k}}$ (Punkt B) erreicht, an dem die Wahrscheinlichkeit zur Bildung von Kondensationskeimen soweit angestiegen ist, dass auf der Zeitskala des Experiments die Bildung von Partikeln stattfinden kann. Die Lage von Punkt B hängt von thermodynamischen (z. B. Temperatur und Druck in Punkt A) sowie kinetischen Aspekten (Düsenform, Kühlrate, Stoßzahl) ab 188 . Durch die freigesetzte Kondensationswärme erfolgt ein Temperaturanstieg. Der in Abbildung 3.4 dargestellte Temperatursprung zu Punkt $\mathrm{C}$ bei $\Phi \gtrsim 1$ entspricht einem Kondensationsprozess, der sich so lange fortsetzt, bis sich ein Gleichgewicht von Kondensations- und Evaporationsereignissen zwischen der Gasphase und einer Anzahl von Partikeln hinreichender Größe einstellt, sodass deren Dampfdruck durch den Kelvin-Effekt nur geringfügig beeinflusst wird. Der weitere Kurvenverlauf in Abb. 3.4 parallel zu den Dampfdruckkurven visualisiert den Fortbestand dieses dynamischen Gleichgewichtes.

In einer freien Expansion wird dieses idealisierte Verhalten nicht vollständig eintreten. Das Zeitfenster, in dem die Gasdichte ausreichend hoch ist, um durch Kollisionen eine Angleichung der Temperaturen der Gasphase und der gebildeten Aggregate zu ermöglichen, reicht nicht aus, um einen Gleichgewichtszustand herzustellen. Die gebildeten Partikel verzeichnen einen starken Temperaturanstieg, der deren Zustand bei der Koagulation größerer Tröpfchen auch in den Bereich mit $\Phi<1$ versetzen kann. Im Gegensatz dazu verzeichnet die Gasphase lediglich einen geringeren Temperaturanstieg, der durch die teilweise Aufnahme dieser Kondensationswärme begründet ist, bevor die Aggregate in den stoßfreien Bereich eintreten. Die Kühlrate, d. h. das Ausmaß, in dem das Trägergas die Kondensationswärme aufnehmen kann, hängt von den Expansionsbedingungen ab. Im stoßfreien Bereich kann eine weitere Abkühlung der gebildeten Aggregate ausschließlich durch evaporative Kühlung erfolgen $39|59| 189$ 191]. Übersteigt die Temperatur eines frisch gebildeten Partikels einen bestimmten, substanz- und größenabhängigen Wert $T_{\text {evap }}$, stellt dieser ein evaporatives Ensemble ${ }^{192}$ dar, das in signifikantem Ausmaß Monomereinheiten verlieren wird, bis seine Temperatur auf einen Wert von ca. $20 \mathrm{~K}$ unterhalb von $T_{\text {evap }}$ abgesunken ist 170 . 


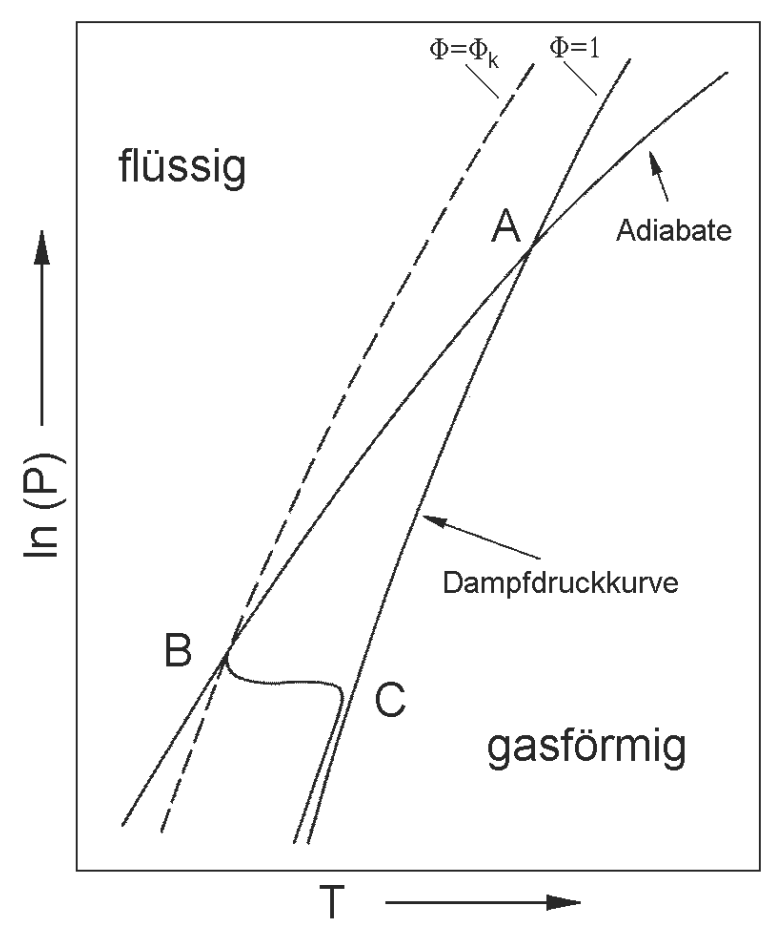

Abbildung 3.4.: Zustandsänderung einer gasförmigen Substanz während der Überschallexpansion; entnommen aus Referenz [42].

\subsubsection{Die Größenverteilung der gebildeten Partikel}

Aus Gleichungen 3.10 und 3.11 wird ersichtlich, dass verschiedene Kombinationen der Stagnationsparameter $p_{0}$ und $T_{0}$ zu Zuständen führen, die im $(p, T)$-Diagramm (vgl. Abb. 3.4) auf der gleichen Adiabaten verortet sind, sodass sich die Dichte, die Relativgeschwindigkeit und das Übersättigungsverhältnis $\Phi$ mit der Temperatur in gleicher Weise ändern. Wird ein höherer Stagnationsdruck eingestellt, lässt sich folglich das Übersättigungsverhältnis bei gegebener Temperatur konstant halten, wenn die Stagnationstemperatur soweit abgesenkt wird, dass sich der Wert des Produktes $p_{0} T_{0}^{\gamma /(1-\gamma)}$ nicht ändert. Auf diese Beobachtung stützt sich das Prinzip der übereinstimmenden Strahlen. Dieses kombiniert das thermodynamisch vergleichbare Verhalten bestimmter Gase in übereinstimmenden Zuständen mit der gaskinetischen Ähnlichkeit von Strömungsfeldern, die durch Düsen mit vergleichbarem äquivalentem Durchmesser erzeugt wurden. Eine Folge dieses Prinzips ist, dass in Gasen mit ähnlichen thermodynamischen Eigenschaften (Wärmekapazität, Wechselwirkungspotentiale) ähnliche Größenverteilungen der gebildeten Partikel auftreten, sofern diese unter identischen Stagnationsbedingungen expandiert wurden. Der Wert des oben genannten Produktes kann dabei als Spezialfall eines sogenannten Skalierungsparameters aufgefasst werden. Derartige Parameter stellen Produkte mehrerer experimenteller Größen dar, die jeweils mit substanzspezifischer Potenz in das Ergebnis einfließen. Die veränderte Kombination aus Temperatur und Druck führt allerdings zu einem veränderten Temperaturgradienten und einer veränderten Flussgeschwindigkeit 193. Diese Diskrepanz eröffnet die Möglichkeit, die für die Nukleation entscheidende Kühlrate (vgl. Abschnitt 3.1.5) bei gegebener Temperatur zu variieren. 
Auf Basis dieses Prinzips erarbeitete Otto Hagena ein Skalierungsgesetz, das einen Zusammenhang zwischen der mittleren Konstituentenzahl der in der Expansion gebildeten Aggregate und den Stagnationsparametern aufzeigt. Dieses Gesetz wurde zunächst für Edelgascluster entwickelt 188 und später auf kondensierende Metalldämpfe erweitert 180.

Bobbert et al. erweiterten dessen Gültigkeitsbereich abermals auf mehratomige Moleküle, indem sie deren größere Anzahl innerer Freiheitsgrade berücksichtigten, sodass auf dieser Grundlage nunmehr auch Größenverteilungen von Ammoniak- und Wasserclustern vorhergesagt werden können $36|40| 62]$.

In der ursprünglichen Version wird ein Parameter $\psi$ definiert. Dieser ist ein Maß für den Temperaturabfall, den ein expandierendes Gas bei gegebenen Ausgangswerten für Temperatur und Teilchendichte $\left(T_{0}, n_{0}\right)$ sowie gegebenem Düsendurchmesser $d_{\mathrm{D}}$ erfährt. Er verknüpft unter der Näherung, dass nur bimolekulare Stöße stattfinden und keine Kondensationsprozesse auftreten, die final erreichbare Temperatur $T_{\infty}$ mit den Expansionsbedingungen. Der genaue Zusammenhang zwischen $T_{\infty}$ und $\psi$ ist vom Wechselwirkungspotential der Monomere abhängig. In Gleichungen 3.15 und 3.16 wird als Näherung ein Hartkugelpotential angenommen. In diesem Fall ist das Produkt aus mittlerer freier Weglänge $\lambda_{\mathrm{M}}$ und Teilchendichte $n$ unabhängig von der Temperatur, sodass die Anzahl der Stöße allein Funktion von $n$ ist. Damit ergibt sich ein einfacher Zusammenhang zwischen $\psi$ und $T_{\infty}$ über eine substanzspezifische Konstante $K{ }^{180}$ :

$$
\begin{aligned}
\psi & =n_{0} d T_{0}^{-1.25} \\
T_{\infty} & =K \psi^{-0.8}
\end{aligned}
$$

Der erweiterte Parameter $\Gamma$ beschreibt das Expansionsverhalten bei gleichzeitig stattfindenden Kondensationsprozessen. Er entspricht einer auf die Partikeloberfläche normierten Anzahl kollidierender Monomere und ist unabhängig von der Masse der Gasmoleküle, jedoch nicht von deren Durchmesser. Alle Expansionen eines gegebenen Gases, deren Expansionsparameter zum gleichen Wert von $\Gamma$ führen, liefern eine vergleichbare Clustergrößenverteilung $\underline{36[188}$ :

$$
\Gamma=n_{0} d^{q} \cdot T_{0}^{s q-f / 2} \quad 0<q \leq 1
$$

Der Exponent $q$ ist eine empirisch ermittelte Größe, die dem langsameren Fluss bei größerem Düsendurchmesser Rechnung tragen soll. Er wird durch wiederholte Messungen unter Variation des Düsendurchmessers bei ansonsten identischen Bedingungen bestimmt 36 . Der Exponent der Temperatur setzt sich aus der Anzahl der zugänglichen Freiheitsgrade $f$ und der Konstanten $s$ zusammen, die für axialsymmetrische Strömungsfelder durch den Ausdruck $s=(f-2) / 4$ definiert ist 188. Im Falle eines einatomigen Gases ist $f=3$, für lineare bzw. nichtlineare mehratomige Gase ergibt sich die Anzahl der Freiheitsgrade unter Berücksichtigung der Rotationsfreiheitsgrade zu $f=5$ bzw. $f=6$.

Auch zwischen Düsen mit unterschiedlicher Geometrie lässt sich einer Vergleichbarkeit herstellen, indem statt des absoluten Durchmessers $d_{\mathrm{D}}$ der äquivalente Durchmesser $d_{\mathrm{D} \text {, eq }}$ verwendet wird, der sich nach Gleichung 3.18 berechnen lässt. Dieser Größe liegt die Idee zugrunde, dass die Strömungsfelder einer Lochdüse und einer konisch divergenten Düse genau dann identische Ausmaße annehmen, wenn die Innenwand der konischen Düse mit dem äquivalenten Durchmesser $d_{\mathrm{D}}$, eq parallel zu einer Strömungslinie der aus einer Loch- 
düse mit äquivalentem, absolutem Durchmesser $d_{\mathrm{D}}$ austretenden Gasfahne verläuft 194 .

$$
d_{\mathrm{D}, \text { eq }} \equiv K(\gamma) \cdot d \cdot \cot \Theta
$$

Die Konstante $K(\gamma)$ ist abhängig von der Geometrie des Strömungsfeldes (axial oder planar) und den inneren Freiheitsgraden des expandierenden Gases. Im Falle axialer Symmetrie nimmt sie für einatomige Gase den Wert $K\left(\gamma=\frac{5}{3}\right)=0.736$ und für zweiatomige Moleküle den Wert $K\left(\gamma=\frac{7}{5}\right)=0.866$ an 188|194. Die Kotangensfunktion besitzt im Definitionsbereich des halben Öffnungswinkels $\Theta\left(0<\Theta \leq \frac{\pi}{2}\right)$ positive Funktionswerte. Daher wird sie den kleinen Wert der Konstanten $K(\gamma)$ ausgleichen, sofern der Winkel $\Theta$ im Bereich $\Theta \leq 36.13^{\circ}$ bzw. $\Theta \leq 40.69^{\circ}$ liegt. In diesem Falle überschreitet der äquivalente Düsendurchmesser den absoluten Durchmesser.

Diese beiden anhand von einatomigen Gasen abgeleiteten Skalierungsgesetze können auf weitere Substanzen übertragen werden. Voraussetzung hierfür ist die Normierung der Skalierungsparameter $\psi$ und $\Gamma$ auf die Eigenschaften der untersuchten Substanzen und somit die Ermittlung dem Theorem übereinstimmender Zustände entsprechender, dimensionsloser, reduzierter Größen $\psi^{*}$ und $\Gamma^{*}$. Zu diesem Zwecke wurden von Hagena die charakteristische Temperatur $T_{\mathrm{ch}}=\Delta h_{0}^{0} / k_{\mathrm{B}}$, der charakteristische Radius $r_{\mathrm{ch}}=(\mathrm{m} / \rho)^{\frac{1}{3}}$, sowie der charakteristische Druck $p_{\mathrm{ch}}=k_{\mathrm{B}} T_{\mathrm{ch}} / r_{\mathrm{ch}}^{3}$ eingeführt. Der Parameter $\rho$ bezeichnet hier die Dichte des Festkörpers, $\Delta h_{0}^{0}$ steht für die atomare Sublimationsenthalpie bei $0 \mathrm{~K}[180$. Für nichtlineare Moleküle mit sechs aktiven Freiheitsgraden ergibt sich der reduzierte Skalierungsparameter $\Gamma^{*}$ nach folgender Gleichung $\underline{36}$ :

$$
\Gamma^{*}=\Gamma / \Gamma_{\mathrm{ch}}=\Gamma \cdot r_{\mathrm{ch}}^{3-q} \cdot T_{\mathrm{ch}}^{3-q}
$$

Aus dem Wert des reduzierten Skalierungsparameters $\Gamma^{*}$ kann nach Gleichung 3.20 der Mittelwert $\langle N\rangle$ der resultierenden Größenverteilung abgeleitet werden:

$$
\langle N\rangle=D\left(\frac{\Gamma^{*}}{1000}\right)^{a}
$$

Die beiden Regressionsparameter $D$ und $a$ in Gleichung 3.20 sowie der Parameter $q$ in Gleichung 3.19 (bzw. wahlweise ein Parameter $\alpha=q-3,3$ ) wurden von Bobbert et al. durch experimentelle Bestimmung der mittleren Clustergröße $\langle N\rangle$ unter variierenden Expansionsbedingungen und anschließende Regressionsrechnung empirisch ermittelt 36 .

Die beobachteten Größenverteilungen lassen sich anhand ihrer Form und ihres Entstehungsmechanismus in drei Klassen einteilen:

- Unter Bedingungen, die die Produktion kleiner Partikel $(\langle N\rangle \approx 80)$ begünstigen (niedriger Stagnationsdruck, hohe Düsentemperatur), wird die Größenverteilung durch exponentiell abfallendes Verhalten dominiert.

- Expansionsbedingungen, die zur Bildung sehr großer Partikel $(\langle N\rangle \approx 700)$ führen (niedrige Temperatur, hoher Druck), ergeben eine Verteilung, die durch eine logNormalkurve beschrieben werden kann.

- Verminderung des Druckes bzw. Erhöhung der Temperatur ausgehend von diesem Zustand führt zur Ausbildung einer bimodalen Verteilung, deren zwei Maxima jeweils für sich durch eine log-Normalverteilung beschrieben werden können. 
Die Ursache für dieses Verhalten liegt in zwei parallel ablaufenden Mechanismen der Clusterbildung: Ein Maximum bei kleineren Clustergrößen kommt durch die Addition von Monomeren und kleineren Oligomeren (Dimeren, Trimeren) an den Cluster zustande (vgl. Abbildung 6.1 a). Ein weiteres Maximum bei größeren Werten kann bei ausreichender Teilchendichte durch spätere Koagulation größerer Partikel entstehen (vgl. Abbildung 6.1 b). Die hierbei freiwerdende Kondensationswärme kann zur zwischenzeitlichen Verflüssigung führen, bis sie durch evaporative Kühlung weitgehend wieder abgegeben wurde $36[40$.

\subsubsection{Einfluss der Expansionsparameter auf die Kühlraten}

Wasser ist eine der wenigen Substanzen, die eine Kontrolle des Aggregatzustandes von in Überschallexpansionen gebildeten Partikeln erlauben ${ }^{39}$. Sofern die Partikelgröße die Unterscheidung verschiedener Phasenzustände zulässt (vgl. Abschnitt 2.3.5), hängt der finale Zustand von der Verweilzeit in dem Temperaturfenster ab, in dem der Phasenübergang sowohl thermodynamisch als auch kinetisch möglich ist, da mit der Länge dieser Zeitspanne die Wahrscheinlichkeit für ein Nukleationsereignis steigt (vgl. Abschnitt 2.3.3). Die Kristallisation der zuvor gebildeten, flüssigen Nanotröpfchen kann dabei die Temperatur der Partikel nachträglich erhöhen 48 . Die Dauer dieses Zeitfensters lässt sich im Experiment durch Variation der Kühlrate im fraglichen Temperaturintervall beeinflussen 59 .

Abbildung 3.5 visualisiert den Einfluss verschiedener Parameter auf die Kühlrate einkomponentiger Expansionen: Die höchsten Kühlraten treten in nahezu allen Fällen direkt nach der Düsenengstelle auf, wo die relative Geschwindigkeit und die Teilchendichte am höchsten sind. Dabei verhält sich die initiale Kühlrate invers proportional zum äquivalenten Düsendurchmesser und kann für die kleinsten, gebräuchlichen Düsen Werte in der Größenordnung von $10^{11} \mathrm{~K} \mathrm{~s}^{-1}$ erreichen. Sie steigt annähernd exponentiell mit der Stagnationstemperatur $T_{0}$. Gase mit niedrigerem Molekulargewicht führen dabei zu höheren Kühlraten 181] (vgl. Abb. 3.5 a). Die Wegstrecke, die das Gas während der Passage eines gegebenen Temperaturintervalls $\Delta T$ zurücklegen muss, erhöht sich allerdings ebenfalls mit der Stagnationstemperatur (vgl. Abb. 3.5 b). Die durch die Temperaturerhöhung ebenfalls erhöhte Strömungsgeschwindigkeit kompensiert diesen Effekt nicht vollständig, sodass in der Summe eine geringfügig erhöhte Transitzeit durch das Temperaturintervall $\Delta T$ mit $T_{0}^{1 / 4}$ für Edelgase bzw. $T_{0}^{3 / 4}$ für zweiatomige Gase mit $\gamma=7 / 5$ die Folge ist 193 .

Unter Annahme einer sich ideal verhaltenden Mischung können in Gleichung 3.5 bzw. 3.6 die Masse $m$ bzw. die Wärmekapazität $c_{p}$ durch molar gemittelte Größen entsprechend der Stoffmengenanteile $X_{\mathrm{i}}$ der verschiedenen Komponenten i eines expandierten Gasgemisches ersetzt werden: Man erhält $\bar{c}_{p}=\sum X_{\mathrm{i}} \cdot c_{p, \mathrm{i}}=\sum X_{\mathrm{i}} \cdot R\left[\gamma_{\mathrm{i}} /\left(\gamma_{\mathrm{i}}-1\right)\right] m_{\mathrm{i}}{ }^{-1}$ bzw. $\bar{m}=\sum X_{\mathrm{i}} m_{\mathrm{i}}$. Die sich aus Gleichung 3.3 ergebende, maximal mögliche kinetische Energie ist in Wahrheit Durchschnittswert, von dem einzelne Teilchen in der Expansion in Abhängigkeit von ihrer Masse abweichen können. Können die Werte von $c_{p, \mathrm{i}}$ bzw. $\gamma_{\mathrm{i}}$ aller Komponenten als annähernd gleich und konstant angenommen werden, ergibt sich aus Gleichung 3.6 ein invers proportionaler Zusammenhang zwischen der mittleren Strömungsgeschwindigkeit $\bar{v}_{\mathrm{F}}$ und der über die Stoffmengenanteile aller Komponenten gemittelten Masse der Teilchen in der Expansion. Da sich deren Geschwindigkeiten in einer Überschallexpansion wie in Abschnitt 3.1.2 beschrieben angleichen, ist die Geschwindigkeit aller Teilchen vom Molekulargewicht der Hauptkomponente in der Expansion abhängig: Schwerere Teilchen können durch Verdünnung in einem leichteren Trägergas beschleu- 
a)

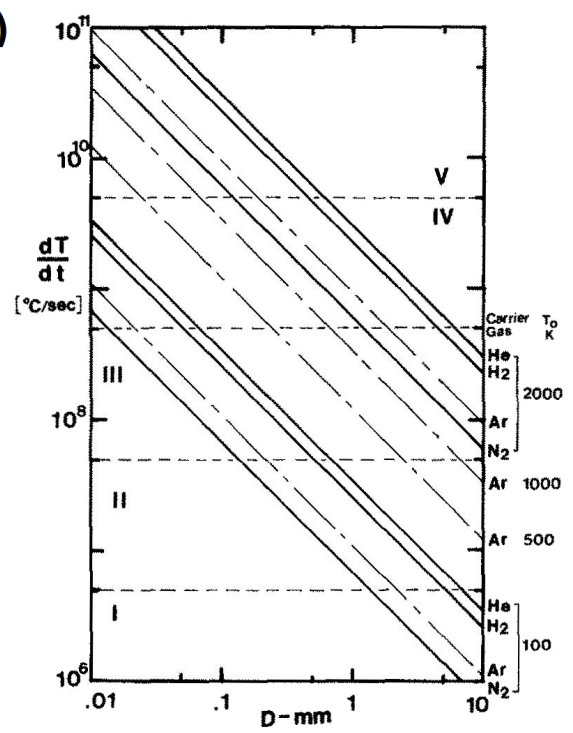

b)

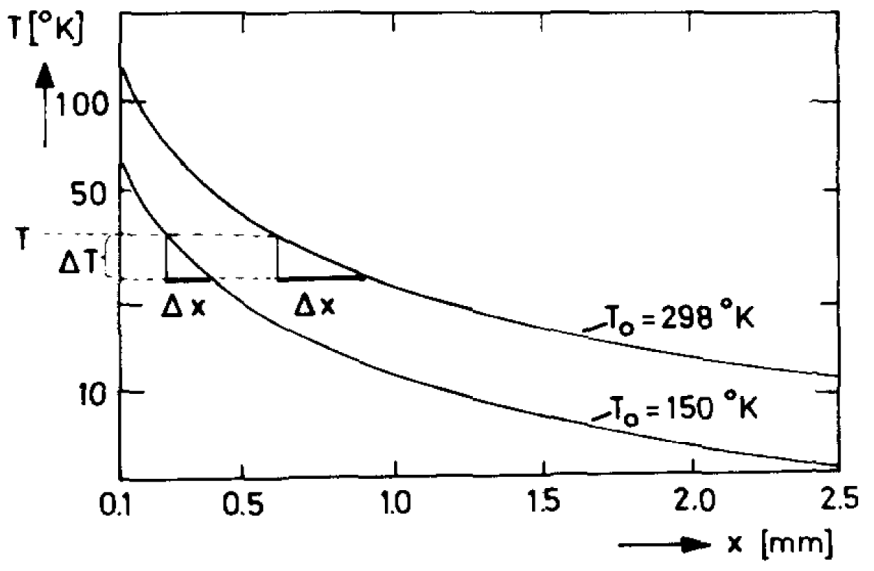

Abbildung 3.5.: a): Berechnete, maximale Kühlraten $\frac{\mathrm{d} T}{\mathrm{~d} t}$ verschiedener expandierter Gase als Funktion des Düsendurchmessers $d_{\mathrm{D}}$, bei verschiedenen Stagnationstemperaturen $T_{0}$; entnommen aus Ref. [181]. b): Gastemperaturen als Funktion des absoluten Abstands $x$ vom Düsenhals, berechnet für eine konische Düse und ein Gas mit $\gamma=7 / 5$ bei verschiedenen Temperaturen; entnommen aus Ref. [193].

nigt werden 175 . Somit erhöht sich die Zeit, die das Gas für die Passage eine Strecke $\Delta x$ benötigt, mit dem Molekulargewicht des Trägergases. Die auf ein Zeitintervall bezogene Kühlrate nimmt dementsprechend ab.

Die aus Abbildung 3.5 a) ersichtlichen, höheren anfänglichen Kühlraten in Gasen mit geringerem Molekulargewicht sind daher hauptsächlich auf deren höhere Strömungsgeschwindigkeit (vgl. Glg. 3.6) zurückzuführen. Aus diesen Werten lässt sich keine Aussage über die endgültige Temperatur der gebildeten Cluster ableiten. So zeigt sich, dass Argon als Trägergas bei vergleichbaren Stagnationsbedingungen zu kälteren Wasserclustern führt, als Helium bzw. Neon $48 \mid 195$. Dabei verhalten sich der Anteil des Trägergases und die Endtemperatur der gebildeten Cluster umgekehrt proportional. Die Gründe sind erstens eine effektivere Kühlung, da mehr Stöße mit dem Trägergas erfolgen, und zweitens eine Verminderung der freiwerdenden Kondensationswärme bei geringerem Stoffmengenanteil der aggregierenden Substanz $59[176$.

Die Kühlrate eines Clusters verhält sich proportional zum Produkt aus dem Stoßquerschnitt $\sigma_{\mathrm{S}}$, der thermischen Relativgeschwindigkeit $v_{\mathrm{F} \text {, rel }}$ und der Teilchendichte $n$ 59. Der Stoßquerschnitt wiederum ist proportional zu $r^{2}$. Da die innere Energie eines Clusters bei gegebener Temperatur mit dessen Volumen und daher mit $r^{3}$ skaliert, fällt die Temperatur kleiner Cluster anfangs schneller ab. Nach Gimelshein et al. gleichen sich die Temperaturen der Cluster allerdings innerhalb von $50 \mu \mathrm{m}$ nach dem Durchtritt durch den Düsenhals an, sodass sie letztendlich nahezu unabhängig von der Clustergröße werden. Die Gasphase wird hingegen entsprechend den obigen Ausführungen auf eine deutlich niedrigere Temperatur abgekühlt 59 . 


\subsection{Größenselektion und IR-Spektroskopie}

Wie in der Einleitung erwähnt, ist die Erzeugung ungeladener Cluster heutzutage problemlos möglich 35 37. Soll anschließend eine größenselektive Untersuchung stattfinden, gilt es eine Reihe von Herausforderungen zu überwinden.

Die erste Herausforderung betrifft die Grenzen der verfügbaren Methoden: Sämtliche Verfahren, die zur Erzeugung neutraler Cluster zur Verfügung stehen, insbesondere auch diejenigen, die zur Produktion neutraler Wassercluster verwendet werden (Expansionstechniken ${ }^{42}$, Kollisionskühlungszellen ${ }^{44}$ sowie Gasaggregationsquellen ${ }^{45}$ ), erlauben keine streng größenselektive Clustererzeugung. Vielmehr wird stets eine Größenverteilung erhalten, deren Maximum kontrolliert werden kann 36|37.

Eine allgemeine Methode zur Massenselektion neutraler Cluster wurde erstmals im Jahr 1984 von Buck et al. vorgestellt: Die kinematische Größenselektion durch Streuung an Heliumatomen stellte einen ersten Durchbruch in der Charakterisierung neutraler, schwach gebundener Spezies dar. Hierbei werden die Cluster in einer Überschallexpansion erzeugt. Anschließend werden sie an einem gekreuzten Strahl aus Heliumatomen gestreut und in Abhängigkeit von ihrem Impuls (und somit ihrer Masse) in unterschiedlichen Winkeln abgelenkt, sodass sie separat vermessen werden können 154. Auf Basis dieser Methode konnten die Schwingungsspektren neutraler, undotierter Wassercluster bis $N=10$ erhalten und die Strukturen im Bereich $7 \leq N \leq 10$ aufgeklärt werden (vgl. Abschnitt 2.3.5) 40[143].

Spätestens ab einer Clustergröße von $N=10$ wird zur Größenselektion die Anwendung massenspektrometrischer Verfahren unter Ionisation der Cluster notwendig 41|51|143|161], die zwei weitere Herausforderungen mit sich bringt:

Erstens führt der naheliegende Ansatz, wasserstoffbrückengebundene Cluster zu ionisieren, zwangsläufig zu Fragmentierungsprozessen. Grund ist die thermische Energie, die auf das System übertragen wird, während der Cluster von seiner neutralen Gleichgewichtsgeometrie zu der des ionischen Systems übergeht. Diese Überschussenergie führt zur Dissoziation von Konstituenten $42|163| 196$.

Das zweite Problem ist der Einfluss, den die Ladung während des Experiments auf den Cluster ausübt: Eine aufgebrachte Ladung führt zu einer Änderung der Clusterstruktur, sofern diese nicht durch eine ausnehmend niedrige Temperatur kinetisch gehindert ist $31 \mid 34$. Soll im Experiment ein Schwingungsspektrum durch IR-induzierte Photodissoziation (IRPD) aufgenommen werden, ist hierfür eine Temperaturerhöhung bis hin zur Evaporationsschwelle erforderlich 31|51|197. Dies stellt auch für Verfahren, die geladene Cluster durch Nanosprayionisation $33 \mid 34$ oder durch Anlagerung langsamer Elektronen an ausreichend kalte Cluster ${ }^{89}$ herstellen, ein Hindernis dar, da deren Ergebnisse im GröBenbereich $N\left(\mathrm{H}_{2} \mathrm{O}\right) \lesssim 50$ durch ladungsinduzierte Strukturumlagerungen beeinträchtigt werden können 31 und durch weitere experimentelle Befunde an möglichst ungestörten Clustern abgesichert werden müssen: Werden Cluster von Beginn an mit einer Ladung versehen, so ist nicht klar, ob die an den geladenen Spezies beobachteten Eigenschaften auch denen der neutralen Spezies entsprechen 189.

Eine ausreichende Vergleichbarkeit von auf diese Weise erhaltenen IR-Spektren mit denen neutraler Cluster, die durch die weiter unten beschriebenen Methoden erhalten wurden, konnte bisher ab $N\left(\mathrm{H}_{2} \mathrm{O}\right)=250$ gezeigt werden 71 . Die Schaffung eines experimentellen Zugangs zu neutralen Clustern im Größenbereich zwischen $N\left(\mathrm{H}_{2} \mathrm{O}\right)=10$ und $N\left(\mathrm{H}_{2} \mathrm{O}\right)=250$ ist unbedingt wünschenswert, um valide Ergebnisse zu erhalten. 
Zur Untersuchung ungeladener Wassercluster braucht es daher geeignete Verfahren der Erzeugung, Größenselektion und Detektion, die darüber hinaus miteinander kombinierbar sein müssen: Als naheliegende, struktursensitive Detektionsverfahren kommen Beugungsmethoden unter Verwendung von Elektronen, Neutronen oder Röntgenstrahlen in Betracht. Diese scheiden jedoch aus zwei Gründen aus: Erstens ist hier keine kombinierte Größenselektion möglich. Zweitens können im Falle von Röntgen- oder Neutronenbeugung aufgrund der geringen Teilchendichte der in der Gasphase vorliegenden Cluster keine Messdaten in ausreichender Qualität erhalten werden 37 .

Die Methode der Wahl zur Strukturbestimmung ist daher die Schwingungsspektroskopie: Die OH-Bindungen des Wassermoleküls erfahren durch die Einbindung in eine Wasserstoffbrückenbindung in Abhängigkeit von deren Stärke eine enorme Rotverschiebung ihrer Schwingungswellenzahl um bis zu $800 \mathrm{~cm}^{-1} 143$. Die Bindungsstärke einer Wasserstoffbrücke wiederum hängt empfindlich von der eigenen (lokalen) Geometrie sowie von der Stärke benachbarter Wasserstoffbrücken ab 51|62. Somit stellt das Schwingungsspektrum eines Wasserclusters eine wertvolle Quelle indirekter Strukturinformation dar.

\subsubsection{Natriumdotierung}

Schulz et al. berichteten im Jahr 1986 erstmals die Erzeugung ungeladener Wassercluster, an die in einem Kreuzstrahlexperiment mit einem Strahl aus Natriumatomen 198 je ein einzelnes Na-Atom angelagert worden war. Nachdem bekannt war, dass das adiabatische IP dieser Spezies nach Übertragung des 3s-Elektrons vom Na-Atom auf den Wassercluster abfällt, im Größenbereich $N \geq 4$ einen konstant niedrigen Wert bei 3,17 eV annimmt und somit eine weitgehend fragmentierungsfreie Ionisation dieser Cluster möglich ist (vgl. Abschn. 3.2.2), schlugen sie im Jahr 1988 Natrium als Chromophor für die Untersuchung neutraler Wassercluster vor 199 . In der Folge wurde die Methode neben Wasserclustern auch zur fragmentierungsfreien Größenbestimmung von Ammoniak- 36 und Edelgasclustern 179 angewandt.

Wenngleich auch andere Alkali- $198 \mid 200$ und Erdalkalimetalle 201 ähnlich reagieren, sind unter diesen Elementen nur Natrium, Cäsium und Beryllium von Natur aus isotopenrein 202 und daher als Chromophor in der Massenspektrometrie gut geeignet. Die Verwendung von Cäsium wird durch dessen hohe Reaktivität erschwert. Beryllium scheidet aufgrund seiner hohen Schmelztemperatur von über $1200^{\circ} \mathrm{C}$ aus $\underline{202 \mid 203}$.

Um Natrium als Chromophor anwenden zu können, müssen chemische Reaktionen mit Bestandteilen des Clusters ausgeschlossen sein (vgl. Abschnitt 3.2.1). Diese Voraussetzung bedeutet eine starke Einschränkung des Anwendungsbereiches, da ein einzelnes Molekül einer unverträglichen Substanz ausreicht, um die Detektion zu verhindern 204. Zusammenfassend lassen sich folgende Voraussetzungen für die Anwendbarkeit dieser Methode formulieren:

1. Es muss sichergestellt sein, dass im Zuge des Pickup-Prozesses die chemische Zusammensetzung des Clusters nicht durch Folgereaktionen verändert wird. Gleiches gilt für die anschließende Photoionisation. Chemische Reaktionen in Folge der Natriumdotierung könnten erstens zu einem Energieeintrag und anschließender Fragmentierung führen und zweitens die bezweckte Absenkung des Ionisationspotentials verhindern 204|205. 
2. Auf der Zeitskala des Experiments darf es dem Elektron nicht möglich sein, einen molekularen Bindungszustand einzunehmen, der thermodynamisch günstiger ist, als die Bindung im 3s-Orbital des Natriumatoms, da andernfalls das IP des Clusters ebenfalls erhöht wird 206|207.

Sofern die obigen Voraussetzungen erfüllt sind, bietet die Methode folgende Vorteile:

1. Das Ionisationspotential natriumdotierter Cluster liegt mit einem Wert von $\leq 5,14 \mathrm{eV}$ sehr niedrig und weit unterhalb der Energie, die zur Ionisation der meisten kovalent gebundenen Moleküle aufgebracht werden muss 3692.

2. Strahlung dieser Energie ist durch kommerziell erhältliche UV-Lasersysteme leicht zugänglich (vgl. Abschnitt 4.7).

3. Aufgrund der strukturellen Besonderheiten natriumdotierter Cluster wird die ansonsten omnipräsente Fragmentierung weitgehend vermieden (vgl. Abschnitt 3.2.2).

4. Natrium ist als Chromophor über mehrere Größenordnungen vom Dimer bis hin zu Aggregaten mit Radien von $20 \mathrm{~nm}$ anwendbar 208.

\section{Der Pickup-Prozess}

Christof Steinbach konstruierte im Rahmen seiner Promotion 209 eine Kollisionszelle, die eine effizientere Dotierung bei niedrigerem Druck ermöglichte, als ein gekreuzter Molekularstrahl 36|210|211], und die bis dato Anwendung findet (vgl. Abschnitt 4.3). Soll die Methode der Natriumdotierung zur Vermessung der Größenverteilung, d.h. zur Ermittlung relativer Clusterabundanzen eingesetzt werden, muss die Größenabhängigkeit der zugrundeliegenden Prozesse berücksichtigt werden: Falls die Wahrscheinlichkeit für die Aufnahme eines Natriumatoms nicht von der Anzahl bereits aufgenommener Atome abhängt, lässt sich die Wahrscheinlichkeit für die Aufnahme von $k$ Na-Atomen durch eine Poisson-Verteilung ausdrücken 212 :

$$
P(k, n)=\frac{l \cdot n \cdot \sigma_{\mathrm{s}}}{k !} \exp -l \cdot n \cdot \sigma_{\mathrm{s}}
$$

Hierbei steht $n$ für die Teilchendichte in der Gasphase, $l$ bezeichnet die Länge der Pickup-Zelle und $\sigma_{\mathrm{s}}$ den Stoßquerschnitt des Clusters. Letzterer kann für sehr kleine Cluster $(r \lesssim 0,5 \mathrm{~nm})$ durch ein Langevin-Modell beschrieben werden, während oberhalb dieses Wertes ein Hartkugelmodell angewandt werden sollte. Die Clustergröße, bei der der Übergang zwischen beiden Modellen stattfindet, hängt von der Polarisierbarkeit und den resultierenden Dispersionswechselwirkungen ab und ist folglich substanzabhängig 208 .

Während des Pickup-Prozesses können weiterhin Intensitätsverluste auftreten, da die Cluster durch elastische Stöße mit Natriumatomen gestreut oder im Zuge des Natriumeinfangs durch den zusätzlichen Impuls des Natriumatoms abgelenkt werden können213. Diese Effekte können die Detektionswahrscheinlichkeit leichter Cluster herabsetzen.

Die Adsorptionseffizienz, d.h. die Wahrscheinlichkeit, mit der das gebildete Natriumaddukt auf der Zeitskala des Experiments intakt bleibt, ist hingegen unabhängig von der Clustergröße: Um den gebildeten Komplex zu stabilisieren, ist die Umverteilung der Kollisionsenergie erforderlich. Der langsamste Schritt dieses Vorgangs ist die intramolekulare 
Energieumverteilung in den unmittelbar an der Kollision beteiligten Clusterkonstituenten, während die anschließende Umverteilung der Energie auf die übrigen Moleküle im Cluster deutlich schneller verläuft. Da der geschwindigkeitsbestimmende Schritt ein lokaler Prozess ist, kann er in guter Näherung als größenunabhängig angenommen werden 208. Im Falle von Wasser sind darüber hinaus starke, nicht-monotone Änderungen der Detektionswahrscheinlichkeit im Größenbereich $N \lesssim 65$ zu berücksichtigen 54 .

Die oben genannten Prozesse spielen im Rahmen der vorliegenden Arbeit nur eine untergeordnete Rolle, da im Rahmen der Auswertung die Abundanzen unterschiedlicher Clustergrößen niemals miteinander verrechnet werden. Relevant hingegen ist die Frage, inwiefern die in den Cluster eingetragene Kollisionsenergie zur Evaporation führen könnte.

Bei Annahme einer Strahlgeschwindigkeit von $1400 \mathrm{~m} \mathrm{~s}^{-1}$ und unter Vernachlässigung der thermischen Geschwindigkeit des Natriumatoms wird durch die Kollision zwischen Cluster und Natriumatom eine Energie von $0,23 \mathrm{eV}$ übertragen. Da die Dissoziationsenergie eines Monomers mindestens $0,42 \mathrm{eV}$ beträgt 170|214, kann davon ausgegangen werden, dass die Kollision im Rahmen des Pickup-Prozesses nur in wenigen Fällen die Dissoziation eines Monomers bewirkt.

\section{Chemische Reaktionen des Natriums mit Wasser und anderen Clusterkonstituenten}

Metallisches Natrium geht als Alkalimetall eine stark exotherme Reaktion mit Wasser ein, die nach Gleichung 3.22 abläuft 203|215. Diese Reaktion tritt im Experiment nach Addition eines einzelnen Na-Atoms oder -Dimers an einen Wassercluster nicht auf, da sie aus zwei Gründen kinetisch gehindert ist: Der Erste ist die niedrige Temperatur der Cluster. Der Zweite ist die hohe Energiebarriere der Reaktion von $\mathrm{Na}$ oder $\mathrm{Na}_{2}$ mit den Molekülen des Clusters 40|211|216: MD-Simulationen von Mercuri, Mundy et al. aus den Jahren 2000 und 2001 legen nahe, dass die Reaktion nach Gleichung 3.22 aus entropischen Gründen unwahrscheinlich ist, da hierfür eine Polarisation des Na-Dimers erfolgen muss 217 . Wurde zuvor ein Na-Atom vom Cluster aufgenommen, das bereits sein 3s-Elektron auf die Wassermoleküle übertragen hat (vgl. hierzu Abschn. 3.2.1), liegt im Cluster eine asymmetrische Ladungsverteilung vor, die die Reaktionswahrscheinlichkeit erhöht 204|218.

$$
\mathrm{Na}_{2}+\mathrm{H}_{2} \mathrm{O}_{N} \longrightarrow(\mathrm{NaOH})_{2}\left(\mathrm{H}_{2} \mathrm{O}\right)_{M}+\mathrm{H}_{2}+(N-M-2) \mathrm{H}_{2} \mathrm{O}
$$

Folglich ist für diese Reaktion die sequentielle Addition eines Na-Monomers und eines $\mathrm{Na}_{2}$-Dimers erforderlich. Ein entsprechender Mechanismus nach Gleichungen 3.23 und 3.24 war bereits im Jahr 1998 von Buck und Steinbach vorgeschlagen worden 210:

$$
\begin{aligned}
\mathrm{Na}+\mathrm{H}_{2} \mathrm{O}_{N} & \longrightarrow \mathrm{Na}\left(\mathrm{H}_{2} \mathrm{O}\right)_{M}+\mathrm{X} \mathrm{H}_{2} \mathrm{O}, X=2,3 \\
\mathrm{Na}_{2}+\mathrm{Na}\left(\mathrm{H}_{2} \mathrm{O}\right)_{M} & \longrightarrow(\mathrm{NaOH})_{2}\left(\mathrm{H}_{2} \mathrm{O}\right)_{J}+\mathrm{H}_{2}+(M-J-2) \mathrm{H}_{2} \mathrm{O}
\end{aligned}
$$

Die zweite Reaktion lässt sich als Abfolge dreier Teilschritte auffassen 217:

$$
\begin{aligned}
\mathrm{Na}_{2}+\mathrm{Na}_{\left(\mathrm{H}_{2} \mathrm{O}\right)_{M}} & \longrightarrow \mathrm{Na}_{3}\left(\mathrm{H}_{2} \mathrm{O}\right)_{M} \\
\mathrm{Na}_{3}\left(\mathrm{H}_{2} \mathrm{O}\right)_{M} & \longrightarrow \mathrm{Na}(\mathrm{NaH})(\mathrm{NaOH})\left(\mathrm{H}_{2} \mathrm{O}\right)_{M-1} \\
\mathrm{Na}(\mathrm{NaH})(\mathrm{NaOH})\left(\mathrm{H}_{2} \mathrm{O}\right)_{M-1} & \longrightarrow(\mathrm{NaOH})_{2}\left(\mathrm{H}_{2} \mathrm{O}\right)_{M-2}+\mathrm{H}_{2}
\end{aligned}
$$


Nach diesem Mechanismus wird das Na-Dimer zunächst polarisiert. Das Natriumatom, das in diesem Zuge eine negative Partialladung erhält, abstrahiert ein Proton eines Wassermoleküls, sodass ein Hydroxid-Anion gebildet wird. Dieses bildet ein NaOH-Addukt mit dem positiv geladenen Natriumion aus. Das reaktive Natriumhydrid bewirkt die Spaltung eines weiteren Wassermoleküls. Als Folge bildet sich ein zweites Äquivalent $\mathrm{NaOH}$. Im gleichen Schritt wird ein $\mathrm{H}_{2}$-Molekül freigesetzt. Die Spaltung des ersten Wassermoleküls stellt hierbei den geschwindigkeitsbestimmenden Reaktionsschritt dar $40|211| 217 \mid 218$. Die Reaktion ist insgesamt stark exotherm. Die Umsetzung zweier Natriumatome zu Natriumhydroxid setzt eine Energiemenge von $8,9 \mathrm{eV}$ frei, sodass sie die Evaporation zahlreicher Wassermoleküle zur Folge haben dürfte 204

Enthält der Cluster ein Molekül wie z. B. $\mathrm{HNO}_{3} 206$ oder $\mathrm{NO}_{2} 207$, das ein unvollständig besetztes, energetisch niedrig liegendes Orbital aufweist, wird das Elektron diesen Zustand besetzen. Einige organische Moleküle führen zur Bildung radikalischer Spezies. Entstehen im ersten Schritt derart reaktive Intermediate durch Aufnahme des vom Natriumatom freigesetzten Elektrons, wird hierdurch eine komplexe Folgechemie initiiert, die nicht nur die Zusammensetzung des Clusters grundlegend verändert, sondern ebenfalls eine beträchtliche Reaktionsenergie freisetzt und somit dessen Fragmentierung bewirkt 205.

\section{Die chemische Beschaffenheit natriumdotierter Wassercluster}

Ein einzelnes angelagertes Natriumatom kann bei ausreichend hoher Temperatur von den Molekülen eines Wasserclusters solvatisiert werden 199 , wobei das 3s-Elektron des NaAtoms auf das H-Brückennetzwerk übertragen wird. Hier kann das Elektron zunächst einen schwach gebundenen Zustand einnehmen, sodass das Ionisationspotential (IP) eines $\mathrm{Na}\left(\mathrm{H}_{2} \mathrm{O}\right)_{N^{-}}$Adduktes im Vergleich zu den Reinsubstanzen $\left(\mathrm{IP}\left(\mathrm{H}_{2} \mathrm{O}\right)=12,61 \mathrm{eV} 219\right.$; $\mathrm{IP}(\mathrm{Na})=5,139 \mathrm{eV}[220]$ ) herabgesetzt ist: So sinkt das adiabatische IP (vgl. Abschnitt 3.2.2 mit zunehmender Konstituentenzahl mit $(N+1)^{-1 / 3}$ bis es bei $N=4$ einen zunächst konstanten Wert von $3,17 \mathrm{eV}$ erreicht 100 . Eine vergleichbare IP-Entwicklung wurde bei anderen Alkalimetallen beobachtet 200|221. Als Grund für die Konstanz der Ionisationspotentiale solcher Cluster im Größenbereich $N \geq 4$ wird die Vervollständigung der Solvatationshülle des resultierenden Alkali-Kations angenommen $57 \mid 199$ 201|221.

Diese Beobachtung steht im Gegensatz zu den adiabatischen und vertikalen Ionisationspotentialen anionischer Wassercluster, in denen dem solvatisierten Elektron kein Kation gegenübersteht: Diese Werte skalieren (soweit bekannt) linear mit $N^{-1 / 3}$, wobei die Proportionalitätskonstante vom Bindungszustand des Elektrons (Oberfläche vs. Kern) abhängig ist $23|94| 97|102|$ und konvergieren gegen die entsprechenden Werte an der Oberfläche bzw. im Volumen lokalisierter, solvatisierter Elektronen in der kondensierten Materie $222[223,22$ Aus den Erläuterungen in Abschnitt 2.1 ergibt sich, dass die mit $N^{-1 / 3}$ bzw. $r^{-1}$ abnehmende Ionisationsenergie anionischer Wassercluster eine Folge der Wechselwirkungen des solvatisierten Elektrons mit allen Konstituenten des Clusters ist, während dessen Bindungszustand in $\mathrm{Na}\left(\mathrm{H}_{2} \mathrm{O}\right)_{N^{-}}$Clustern durch lokale Wechselwirkungen dominiert wird, bzw. beide Beiträge sich ausgleichen. Im Falle anionischer Cluster konnte gezeigt werden, dass der schwächer gebundene Zustand entsprechend den thermodynamischen GesetzmäBigkeiten dem stärker gebundenen Zustand gegenüber metastabil ist $\underline{31|99| 225 \mid 226}$.

${ }^{2}$ Der exakte Wert der Bindungsenergie eines solvatisierten Elektrons in makroskopischem Wasser ist Gegenstand eines andauernden Diskurses 224 
An $\mathrm{Na}\left(\mathrm{H}_{2} \mathrm{O}\right)_{N}$-Clustern beobachteten Forck et al. im Größenbereich $11<N<15$ eine weitere Absenkung der Erscheinungsionisationsenergie auf einen Wert von 2,8 eV. Als Erklärung führten sie das Aufkommen eines weiteren, schwächer gebundenen Zustands des solvatisierten Elektrons in diesen Clustern an, der bei $N \geq 15$ vollständig ausgebildet ist. Dieser Zustand wird nicht in allen beobachteten Clustern erreicht, sondern koexistiert mit Clustern gleicher Größe, die ein höheres Ionisationspotential aufweisen. Als Ursache für dieses unterschiedliche Verhalten führten sie Unterschiede in der räumlichen Trennung zwischen der Ladungsverteilung des Elektrons und dem Natriumion an, die von variierenden Positionen des Na-Ions im Cluster herrührten. Sie klassifizierten dieses Bindungsmotiv als solvenssepariertes Ionenpaar, in Abgrenzung von den zuvor beobachteten, fester gebundenen Zuständen, die als Kontaktionenpaar eingeordnet wurden. Die breiten Flanken der aufgenommenen Photoionisationskurven deuteten auf das Vorliegen einer Vielzahl ähnlicher Bindungsmotive hin, deren geringfügig verschiedene Ionisationspotentiale sich im Photoionisationsspektrum als nicht auflösbares Kontinuum darstellten. Daher wurden die zwei verschiedenen Bindungsmotive als Prototypen zweier verschiedener Isomerklassen aufgefasst, wobei die Cluster mit fester gebundenen Elektronen als Isomerklasse I bezeichnet wurden, während die leichter ionisierbaren Cluster als Isomerklasse II eingeordnet wurden 57 . Dierking et al. führten erstmals eine vollständige und größenabhängige Untersuchung der Photoionisationskurven im Größenbereich $2 \leq N \leq 90$ durch. Aus den Stufen und Wendepunkten der Kurven ergaben sich Hinweise auf zahlreiche Isomere, in denen das solvatisierte Elektron in unterschiedlich fest gebundenen Zuständen vorliegt. MD-Simulationen ergaben eine starke Korrelation zwischen dem Ionisationspotential des Clusters und dem Gyrationsradius des solvatisierten Elektrons. Demnach liegt ein schwach gebundenes Elektron in einem stark delokalisierten Zustand vor, während ein fest gebundenes Elektron einen lokalen Bindungszustand einnimmt 60. Dabei verläuft die Solvatation des Natriumions keinesfalls immer vollständig, sondern ist kinetisch gehindert: In Abhängigkeit von der Clustertemperatur kann ein großer Teil der natriumdotierten Cluster Konfigurationen einnehmen, in denen das Natrium unvollständig solvatisiert ist 60 oder auf der Oberfläche des Clusters verbleibt, ohne dessen Wasserstoffbrückentopologie zu verändern 41]. In den letztgenannten Strukturen ist das Natriumion einfach oder zweifach koordiniert. Sie besitzen die höchste Ionisationsenergie mit Werten im Bereich $\gtrsim 3,9 \mathrm{eV} \underline{60}$. Solche Cluster stellen bei ausreichend niedrigen Temperaturen die größte Fraktion des Molekularstrahls dar41|60|71. Im Größenbereich $N \geq 9$ tritt die Sättigung des Ionensignals größenunabhängig bei $4,1 \mathrm{eV}$ ein 60 .

\subsubsection{Photoionisation}

\section{Das Franck-Condon-Prinzip: Vertikale vs. adiabatische Ionisation}

Der Begriff Photoionisation beschreibt das Herauslösen von Elektronen aus Atomen oder Molekülen (bzw. in diesem Falle aus Clustern) durch elektromagnetische Strahlung ausreichend hoher Frequenz 202 . Die Energie des absorbierten Photons (bzw. der absorbierten Photonen) wird dabei aufgewendet, um die Bindungsenergie des Elektrons zu überwinden. Übersteigt die auf das Elektron übertragene Energie die aufzuwendende Austrittsarbeit, entspricht die Differenz dieser Energiebeiträge der kinetischen Energie des emittierten Elektrons 104 . 
Die ionisierte Spezies geht folglich in einen Bindungszustand über, in dem sie eine höhere innere Energie besitzt, als vor der Ionisation. Die Elektronenstruktur ist verändert, sodass auf die Atomkerne nunmehr ein verändertes Kraftfeld wirkt. Dieses muss nicht notwendigerweise entlang jeder Bindungsachse ein Minimum aufweisen: Das neue Kraftfeld kann ein repulsives Potential darstellen, in dem es zur Spaltung einer (oder mehrerer) Bindungen kommt 104 .

Liegt auch nach der Ionisation ein bindendes Potential vor, so sind die Gleichgewichtspositionen der Atomkerne in der Regel gegenüber dem neutralen Zustand verschoben. In diesem Falle ergeben sich aus dem neuen, ionischen Zustand veränderte Randbedingungen für die Schwingungseigenfunktionen $\Psi$ des Systems. Wie im elektronischen Grundzustand $\Psi_{v^{\prime \prime}}$ kann das System im elektronisch angeregten Zustand $\Psi_{v^{\prime}}$ nur diskrete Schwingungszustände einnehmen. Die Frage, in welchen Schwingungszustand des angeregten elektronischen Zustands das System nach der Ionisation am wahrscheinlichsten übergehen wird, lässt sich anhand des Franck-Condon-Prinzips beantworten. Dieses besagt, dass die Wahrscheinlichkeit für den Übergang zwischen zwei Zuständen proportional zum Quadrat des Überlappungsintegrals der zugehörigen Schwingungseigenfunktionen ist 227.

$$
S_{\mathrm{FC}, v^{\prime \prime}, v^{\prime}}^{2}=\left(\int \Psi_{v^{\prime \prime}}(\vec{R}) \Psi_{v^{\prime}}(\vec{R}) \mathrm{d} \tau\right)^{2}
$$

Die Größe $\vec{R}$ bezeichnet hier die generalisierte Ortskoordinate. Die Größe $S_{\mathrm{FC}, v^{\prime \prime}, v^{\prime}}^{2}$ wird Franck-Condon-Faktor genannt. Die Überlegungen von Edward Condon aus dem Jahr 1928 beruhen auf der Born-Oppenheimer-Näherung, die besagt, dass die Zeitskala, auf der die elektronische Anregung stattfindet, kurz gegenüber einer Schwingungsperiode der Atomkerne ist ${ }^{228}$. Daher können für Gleichung 3.28 die Schwingungseigenfunktionen ausgehend von den Gleichgewichtsgeometrien beider Zustände herangezogen werden. Nimmt man weiter an, dass die elektronische Anregung aus dem Schwingungsgrundzustand heraus erfolgt $\left(v^{\prime \prime}=0\right)$, lassen sich bei Kenntnis der Potentialhyperflächen beider elektronischer Zustände und der zugehörigen Schwingungseigenfunktionen die Übergangswahrscheinlichkeiten $S_{\mathrm{FC}, 0, v^{\prime}}^{2}$ berechnen 104 . Ein beispielhaftes Resultat für ein zweiatomiges Molekül ist in Abbildung 3.6 dargestellt, wobei das Potential entlang der Bindungskoordinate als harmonischer Oszillator angenähert wurde 229 . Demnach erfolgt der wahrscheinlichste Übergang bei stark unterschiedlichen Potentialhyperflächen in einen schwingungsangeregten Zustand des oberen elektronischen Zustands (Paneel c in Abb. 3.6).

Ein reales, anharmonisches Potential weist im Gegensatz hierzu einen Bereich in $\vec{R}$ auf, in dem es keinen bindenden Charakter besitzt, da sein Wert der potentiellen Energie $E_{\text {pot }}(\vec{R})$ bei $|\vec{R}| \rightarrow \infty$ gegen einen endlichen Wert konvergiert. Oberhalb dieses Energiewertes liegen in Ermangelung limitierender Randbedingungen keine diskreten Eigenfunktionen mehr vor: Das System kann ein Kontinuum möglicher Zustände annehmen. Besitzt die Schwingungseigenfunktion des elektronischen Grundzustands $\Psi_{v^{\prime \prime}}(\vec{R})$ Intensität in einem Bereich von $\vec{R}$, in dem der Wert von $E_{\text {pot }, v^{\prime}}(\vec{R})$ den Wert von $E_{\text {pot }, v^{\prime}}(|\vec{R}| \rightarrow \infty)$ übersteigt (und ist dieser Bereich durch die eingebrachte Photonenenergie erreichbar), kann die Wahrscheinlichkeit für einen Übergang in diesen Bereich folglich nicht mehr durch die Berechnung von Überlappungsintegralen gelöst werden. Gleiches gilt für ein repulsives Potential, das kein Minimum besitzt 35 . In diesem Falle behilft man sich mit der "Reflektionsnäherung “230]: Die Funktion $\Psi_{v^{\prime \prime}}^{2}(\vec{R})$ wird orthogonal zu allen Komponenten 
a)
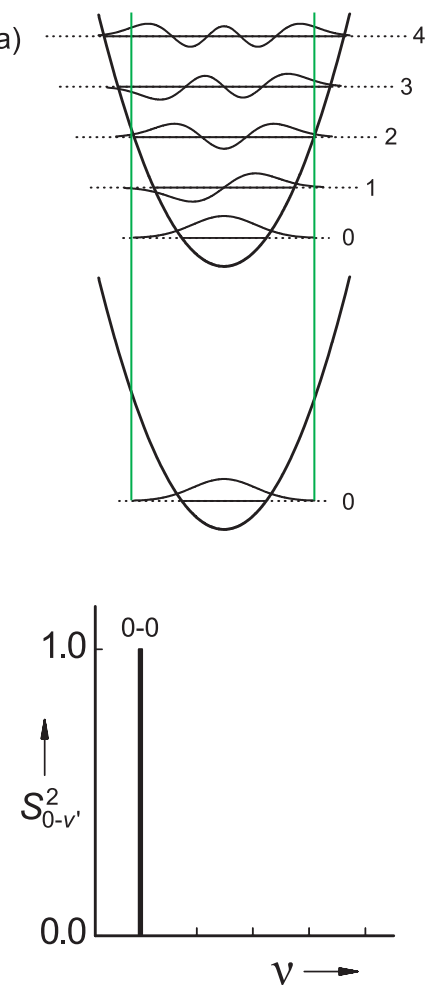

b)
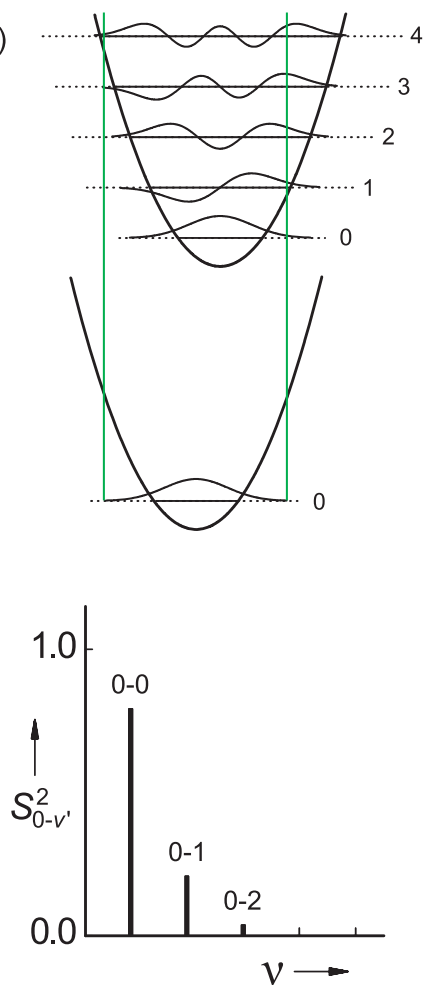

c)
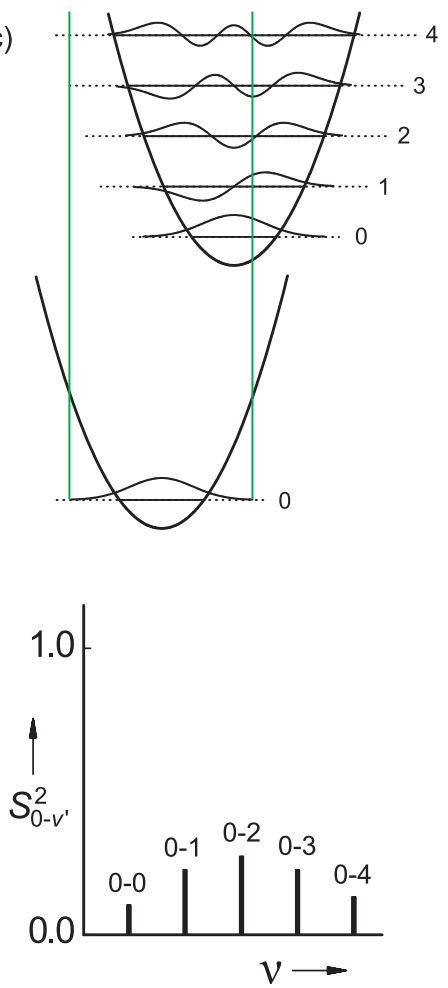

Abbildung 3.6.: Qualitative Visualisierung des Franck-Condon-Prinzips anhand der Potentialkurven eines zweiatomigen Moleküls bei harmonischer Näherung bei a) identischen, b) leicht verschiedenen und c) stark verschiedenen Gleichgewichtsabständen der Atomkerne. Im unteren Teil sind die Franck-Condon-Faktoren der einzelnen vibronischen Übergänge dargestellt; verändert nach Referenz [229].

von $\vec{R}$ auf den Graphen von $E_{\mathrm{pot}, v^{\prime}}(\vec{R})$ projiziert. Die erneute Projektion dieses Bildes auf die Energiekoordinate liefert relative Ionisationsquerschnitte. Diese Näherung wird auch zur Vorhersage der Photoionisationsspektren von Spezies mit flachen Potentialkurven und einer entsprechend hohen Zahl erreichbarer Schwingungszustände im ionisierten Zustand angewandt, obwohl das ionische Potential bindend ist. Beispiele sind durch Vander-Waals-Wechselwirkungen oder Wasserstoffbrücken gebundene, flexible Systeme wie z. B. Wassercluster 98. Dieses Vorgehen ist in Abbildung 3.7 graphisch dargestellt.

Da die oben beschriebenen Prozesse zu einer Verbreiterung der gemessenen Ionisationsenergie führen, werden zur Interpretation von Photoionisationsprozessen isolierter Spezies in der Gasphase vier verschiedene energetische Größen herangezogen $60|87| 93]$ :

- Adiabatische Ionisationsenergie (AIE): Diese Größe bezeichnet die Energiedifferenz zwischen den Schwingungsgrundzuständen des elektronischen Grundzustands und des ionischen Zustands. Ein Übergang zwischen diesen beiden Zuständen führt nicht zur vibronischen Anregung der ionisierten Spezies.

- Vertikale Ionisationsenergie (VIE): Der Begriff „vertikal“ ist der (semi-)klassischen (und nicht korrekten) Betrachtung des Photoionisationsvorgangs entlehnt, bei der die Atomkerne explizit als Teilchen betrachtet werden. Er entspringt der Vorstellung, dass sich die Atomkerne nach der Ionisation an dem Punkt der Potentialkurve wie- 
derfinden, der im Energiediagramm vertikal über ihrer Ausgangsposition liegt 229. Dieser Punkt wird zum neuen Umkehrpunkt ihrer Schwingung. In der Literatur wird die ,vertikale“ IE häufig dem Übergang mit der höchsten Wahrscheinlichkeit nach Gleichung 3.28 gleichgesetzt. Dieser findet zwischen den beiden Zuständen statt, die bei vertikaler Ionisation (d.h. bei konstant gehaltenen Positionen der Atomkerne) das größte Überlappungsintegral der Schwingungseigenfunktionen in den Gleichgewichtsgeometrien der beiden elektronischen Zustände aufweisen. Die VIE ist die Größe, die üblicherweise als Maximum eines Photoelektronenspektrums erhalten wird. Die Differenz zwischen AIE und VIE wird als Reorganisationsenergie bezeichnet. Bei klassischer Betrachtung ist dies die Energie, die aufgebracht werden muss, um von der Gleichgewichtsgeometrie der ionisierten Spezies die räumliche Anordnung der Atomkerne herzustellen, in der sie nach der vertikalen Ionisation vorliegen. In der quantenmechanischen Betrachtungsweise entspricht sie bei Ionisation aus dem Grundzustand der durch das Photon eingetragenen Energiemenge, die aufgrund der vibronischen Anregung im Cluster verbleibt.

- Erscheinungsionisationsenergie (EIE): Diese ist die niedrigste Energie, die im Experiment ausreicht, um eine Ionisation herbeizuführen. Sofern die Ionisation ausschließlich aus dem Schwingungsgrundzustand heraus erfolgt und das Überlappungsintegral der Schwingungseigenfunktionen beider Schwingungsgrundzustände nicht verschwindet (d.h. die Gleichgewichtsabstände nicht zu sehr variieren und ein Übergang zwischen diesen Zuständen möglich ist, vgl. Abb. 3.6), entspricht sie in guter Näherung der AIE.

- Sättigungsionisationsenergie (SIE): Die Energie, die maximal notwendig ist, um sämtliche Vertreter einer Spezies zu ionisieren. Weitere Erhöhung der Energie führt nicht zur Steigerung der Anzahl gebildeter Ionen.

\section{Experimentelle Ermittlung von Ionisationsenergien}

Zur experimentellen Bestimmung von Ionisationsenergien und -wahrscheinlichkeiten dieser Spezies haben sich zwei Verfahren etabliert. Eine häufig angewandte Methode ist die Photoelektronenspektroskopie, bei der die kinetische Energie des emittierten Elektrons gemessen wird. Dessen Bindungsenergie bzw. die Arbeit, die zur Herauslösung dieses Elektrons aufzuwenden ist, ergibt sich aus dem Einstein'schen Frequenzgesetz. Aus der Verteilung der kinetischen Energie der emittierten Elektronen nach Photoionisation durch monochromatische Laserstrahlung einer einzelnen Frequenz ergibt sich somit direkt die Verteilung der Bindungsenergien der untersuchten Spezies 104.

Ein zweiter Ansatz besteht in der Ermittlung der Ionisationseffizienz: Hier erfolgt die Ionisation mit Hilfe eines durchstimmbaren UV/VIS-Lasersystems. Nach der Ionisation findet eine Massenselektion der gebildeten Ionen statt. Da nicht die Elektronen, sondern die gebildeten Ionen detektiert werden, ermöglicht dieses Verfahren die gleichzeitige, größenselektive Vermessung von Clustern unterschiedlicher Masse. Durch Messung der (auf die Strahlungsintensität normierten) Ionenausbeute als Funktion der verwendeten Photonenenergie lassen sich die Ionisationsquerschnitte der untersuchten Spezies berechnen 87 . Das adiabatische Ionisationspotential wird durch Extrapolation des ersten linearen Anstiegs der Ionenausbeute und Bestimmung von dessen Schnittpunkt mit der Achse der Photo- 


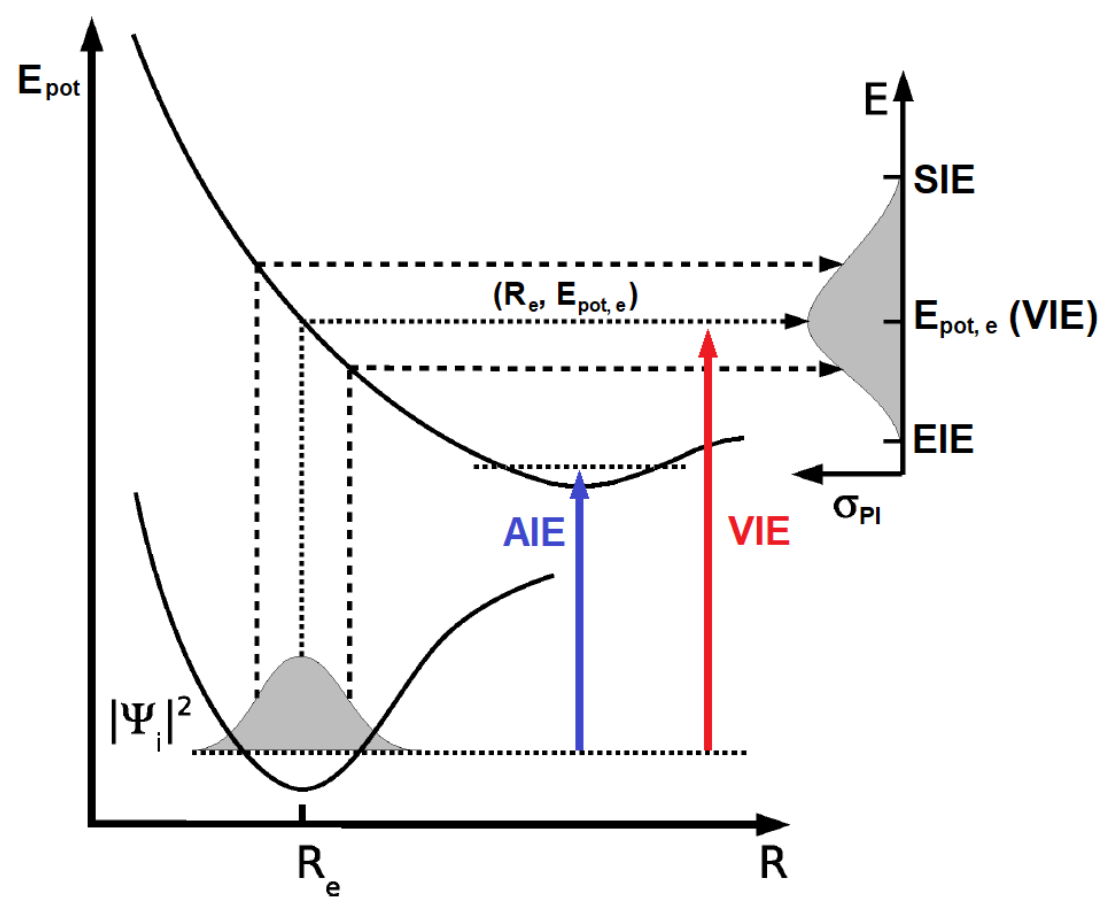

Abbildung 3.7.: Schematische Visualisierung der Reflektionsnäherung: Das Betragsquadrat der Eigenfunktion des elektronischen Grundzustands wird unter der Annahme einer vertikalen Ionisation auf die Potentialkurve des elektronisch angeregten (bzw. ionischen) Zustands projiziert. Erneute Projektion dieses Bildes auf die Energieachse liefert eine genäherte Verteilung der Ionisationsenergien, die sich als Funktion der tatsächlich im Moment der Ionisation vorliegenden Geometrien ergeben. $E_{\text {pot }}$ bezeichnet die potentielle Energie, $E$ die Photonenenergien, R steht für die Ortskoordinate; der tiefgestellte Index e bezeichnet Werte in der Gleichgewichtsgeometrie, $\sigma_{\mathrm{PI}}$ bezeichnet den relativen Photoionisationsquerschnitt; die Abkürzungen AIE, VIE, EIE und SIE bezeichnen die adiabatische, die vertikale, die Erscheinungs- bzw. die Sättigungsionisationsenergie; verändert nach Referenz $[98$.

nenenergie ermittelt $57 \mid 87$. Die resultierende Kurve kann näherungsweise als integriertes Photoelektronenspektrum betrachtet werden, da Maxima eines solchen Spektrums hier durch Stufen bzw. Wendepunkte erkennbar werden 60 .

\section{Photoionisation natriumdotierter Wassercluster}

Cluster, die durch Wasserstoffbrücken, Van-der-Waals- oder ionische Wechselwirkungen gebunden sind, weisen nach der Ionisation gewöhnlich deutlich stärkere Bindungen auf, als im neutralen Zustand. Daher sind die Gleichgewichtsabstände der Atomkerne nach der Ionisation zu signifikant kleineren Werten verschoben 35536|53|231]. Die Anwendung eines gängigen Ionisationsverfahrens auf solche Systeme führt daher zwangsläufig zu ausgeprägter Fragmentierung, die im Falle von Wasserclustern zudem von schnell ablaufenden chemischen Reaktionen begleitet ist $40|143| 232$. 
Aus den Ausführungen in Abschnitt 3.2 .2 folgt, dass eine vibronische Anregung und eventuell dadurch bedingte Fragmentierungsprozesse vermieden werden können, wenn sichergestellt ist, dass die Ionisation in den Schwingungsgrundzustand des ionischen Niveaus erfolgt $58 \mid 92$. Voraussetzung hierfür ist allerdings, dass die Änderung der Gleichgewichtskernpositionen so gering ausfällt, dass die bei der vertikal stattfindenden Ionisation in den Cluster eingetragene Energie nicht zur Auslösung von Fragmentierungsereignissen ausreicht ${ }^{143}$. Sofern die adiabatische Ionisationsenergie und die vertikale Ionisationsenergie nahe beieinander liegen, ist diese Bedingung mit hoher Wahrscheinlichkeit erfüllt. Eine Reihe quantenchemischer Rechnungen sagt eine vollständige Delokalisation des solvatisierten Elek-

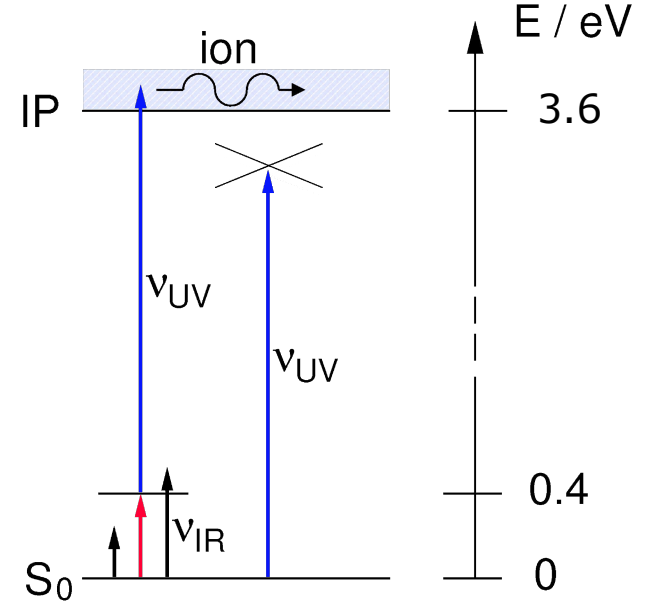

Abbildung 3.8.: Absenkung der EIE in vibronisch angeregten Clustern; verändert nach Ref. 53 trons und (unter dieser Voraussetzung) nahezu identische Bindungslängen in kleinen $(1 \leq N \leq 6)$, neutralen und kationischen, natriumdotierten Wasserclustern vorher $95|198| 199|217| 233 \mid$. Allerdings deuten breite Flanken in gemessenen Photoionisationsspektren darauf hin, dass dieses einfache Bild die realen Verhältnisse nicht korrekt wiedergibt. Diese Ergebnisse legen vielmehr Beiträge von Strukturen nahe, deren Franck-Condon-Faktoren die Wahrscheinlichkeit einer adiabatischen Ionisation vermindern 60[199]. Eine Reihe neuerer theoretischer Arbeiten legen nahe, dass diese Beobachtung das Resultat einer Vielzahl unterschiedlich fest gebundener Elektronen mit unterschiedlichen Gyrationsradien in den untersuchten Clustern ist (vgl. Abschnitt 3.2.1). Die geringsten Änderungen der Gleichgewichtsabstände und damit die niedrigsten Reorganisationsenergien treten in solchen Clustern auf, in denen das Elektron größtmöglich delokalisiert ist, sodass diese Cluster besonders leicht ionisiert werden können $58|60| 225 \mid 234$. Eine weitgehend fragmentierungsfreie Ionisation lässt sich somit durch Wahl von Ionisationsbedingungen sicherstellen, unter denen ausschließlich Cluster ionisiert werden, die stark delokalisierte, schwach gebundene Elektronen aufweisen 17 .

Als Obergrenze der Photonenenergie für eine sicher fragmentierungsfreie Ionisation wurden bisher Werte von 3,7 eV (Dierking et al. $\sqrt{60}$ ) bzw. 3,9 eV (Huang et al..$\underline{196}$ ) gefunden. Einige Autoren argumentieren, dass auch die überschüssige Energie noch energiereicherer Photonen nahezu vollständig in kinetische Energie der freigesetzten Elektronen umgewandelt wird und daher nicht zur thermischen Anregung der Cluster führt 205|208|235.

Die im Experiment beobachtete EIE kann auch niedriger als die AIE ausfallen, sofern die Ionisation aus einem vibronisch angeregten Zustand heraus erfolgt. Da die Geometrien der Grundzustände neutraler und ionischer Cluster sich gewöhnlich stark unterscheiden, kann eine höhere thermische Energie der Cluster somit zur Absenkung der EIE unter den Wert der AIE führen 53|87. Grund ist eine stärkere Überlappung der Wellenfunktion des kationischen Grundstands mit der eines neutralen, vibronisch angeregten Zustands. Die Intensität Letzterer ist in jedem Falle über einen weiteren Bereich von $\vec{R}$ verteilt, da die Potentialminima sich zu höheren Energien weiten. Sinkt die beobachtete EIE unter den Wert der AIE, wurde die im Cluster vorhandene thermische Energie aufgewendet, um das solvatisierte Elektron zu dissoziieren. Abbildung 3.8 stellt diesen Sachverhalt graphisch 
dar. Im klassischen bzw. semi-klassischen Bild des Photoionisationsprozesses befähigt die zusätzliche thermische Energie den neutralen Cluster, im Zuge seiner Schwingungsdynamik eine Geometrie einzunehmen, die der Geometrie des kationischen Grundzustands ähnlicher ist als die Gleichgewichtsgeometrie seines vibronischen Grundzustands, wodurch die zusätzlich für die Reorganisation der Atomkerne aufzuwendende Energiemenge sinkt. Der Cluster liegt nach der Ionisation in einem niedrigeren Schwingungszustand vor.

\subsubsection{Wirkungsspektroskopie mittels IR-Anregung}

\section{IR-anregungsmodulierte Photoionisation}

Buck und Steinbach beobachteten im Jahr 2006, dass die Natriumdotierung sich nicht allein zur Größenbestimmung von Clustern bestimmter Substanzen durch fragmentierungsfreie Photoionisation eignet, sondern die Ionenausbeute solcher Cluster auch durch deren vibronische Anregung moduliert werden kann 53. Wird die vibronische Anregung kontrolliert mit Hilfe eines durchstimmbaren IR-Lasers durchgeführt, kann diese Signalmodulation zur Aufnahme von Schwingungsspektren genutzt werden.

Abbildung 3.9 veranschaulicht schematisch die Vorgänge, die diesem Experiment zugrunde liegen: Variation der Frequenz der IR-Strahlung bewirkt eine unterschiedlich effektive IR-Anregung, da die Oszillatorstärke der mit der jeweiligen IR-Strahlung resonanten Schwingungsmoden im Cluster variiert. Das Ausmaß der vibronischen Anregung einer Spezies zeigt sich in Form einer erhöhten Signalintensität im Massenspektrum, da die Anzahl der Cluster steigt, deren IP unter die verwendete Photonenenergie sinkt. Liegt die verwendete Photonenenergie nur geringfügig oberhalb der EIE, kann das Massensignal durch IR-Anregung um einen Faktor von mehr als zwei erhöht werden. Dieser hohen Signalzunahme liegt nicht allein die in Abschnitt 3.2.2 beschriebene Verbesserung der Franck-Condon-Faktoren zugrunde. Simulierte Verteilungen der Ionisationsenergien natriumdotierter Methanolcluster von Forck et al. deuten auf das Vorliegen eines weiteren Mechanismus hin: Durch thermische Anregung wird die Geschwindigkeit der in kalten Clustern kinetisch gehinderten Solvatation des Natriumatoms und der damit verbundenen Delokalisation des auf das Wasserstoffbrückennetzwerk übertragenen 3s-Elektrons erhöht 236 . Im Falle von Wasserclustern laufen analoge Vorgänge ab 41|48|60|92. Die EIE natriumdotierter Wassercluster bleibt im gesamten, bisher untersuchten Größenbereich mit $N \geq 15$ nahezu konstant (vgl. Abschnitt 3.2.1). Als ungefähre Obergrenze für die Anwendbarkeit dieses Verfahrens lässt sich die Konstituentenzahl $N \approx 600$ angeben 54 . Im Größenbereich zwischen diesen beiden Werten können die Schwingungsspektren sämtlicher Wasseraggregate simultan unter Verwendung der gleichen Ionisationswellenlänge aufgenommen werden 4892 . Aufgrund dieses Vorteils erscheint das hier vorgestellte Verfahren zur Untersuchung von Wasseraggregaten besonders geeignet.

Das Ausmaß der beobachteten Signalzunahme hängt dabei nicht allein von der Energie der ionisierenden UV-Photonen ab, sondern auch von der Zeit, die zwischen der vibronischen Anregung der Cluster und der Ionisation vergangen ist 61|237 239]. Nach der IR-Anregung erfolgt eine instantane Signalzunahme, deren Maximum ca. 7 ns nach dem Eintreffen des IR-Pulsmaximums beobachtet wird? ${ }^{3}$. Darauf folgt ein exponentieller Abfall

\footnotetext{
${ }^{3}$ Es muss berücksichtigt werden, dass die Aufösung derart kurzer Zeitintervalle im Experiment durch
} die Pulslängen der eingesetzten Lasersysteme limitiert ist (vgl. Abschnitte 4.6 und 4.7) 
der Signalzunahme, der mit dem in Kapitel 4 beschriebenen Aufbau bisher über einen Zeitraum von ca. 250 ns verfolgt werden konnte. Daniel Becker konnte im Rahmen seiner Bachelorarbeit zeigen, dass dieser Abfall zum überwiegenden Teil auf die Signalabnahme detektierbarer, amorpher Cluster zurückzuführen ist und durch ein exponentielles Geschwindigkeitsgesetz erster Ordnung beschrieben werden kann. Unter Bedingungen und in Größenbereichen, in denen kristalline Nanopartikel die überwiegende Fraktion der ionisierbaren Spezies stellen, fällt der exponentielle Signalabfall deutlich geringer aus. Die Lebensdauern der Partikel, deren Signal abnimmt, liegen abhängig von der Größe und den Expansionsbedingungen im Bereich von $3 \cdot 10^{-8} \mathrm{~s}$ bis $9 \cdot 10^{-8} \mathrm{~s}$ 61238. Als Ursache für diesen Signalabfall kommen zwei Prozesse infrage: Der Erste besteht in der Evaporation von Konstituenten aufgrund der IR-induzierten Temperaturerhöhung, der Zweite in einer Erhöhung des IP, sofern das solvatisierte Elektron letztlich einen thermodynamisch stabileren Bindungszustand einnimmt. Die Relaxation eines solvatisierten Elektrons sollte auf einer wesentlich kürzeren Zeitskala im Femtosekundenbereich (bzw. maximal im Pikosekundenbereich) stattfinden, sofern der Cluster eine thermodynamische Senke enthält, die das Elektron aufnehmen kann $99|207| 224|225| 240$. Daher könnte die Einnahme eines stärker gebundenen Zustands (wie z. B. in einer lokalen Kavität) durch das solvatisierte Elektron als Ursache für den langsamen Signalabfall nur infrage kommen, sofern dessen Ausbildung kinetisch gehindert ist. Für die Evaporation sagen die meisten experimentellen und theoretischen Arbeiten hingegen Lebensdauern voraus, die die von Becker ermittelten Werte um mehrere Größenordnungen übertreffen $34|90| 235 \mid 241$. Zwei theoretische Arbeiten berichten jedoch Lebensdauern, die den von Becker bestimmten Werten ähneln. So fanden Klots et al. Werte von $\tau \approx 2 \cdot 10^{-8} \mathrm{~s}\left(k \approx 5 \cdot 10^{7} \mathrm{~s}^{-1}\right)$ für ungeladene Wassercluster mit $N=100$ bei Temperaturen um 230 K 189 . Somit wird als Ursache des Signalabfalls eine Erhöhung der EIE infolge der evaporativen Kühlung angenommen.

In den vergangenen Jahren wurde ein hoher Aufwand betrieben, um den Einfluss eventuell störender Faktoren auf das Experiment zu quantifizieren. Der Fokus lag dabei hauptsächlich auf zwei Fragen:

1. In welchem Ausmaß können evtl. im Zuge der Natriumaddition und -solvatation, der thermischen Anregung oder der Photoionisation auftretende Fragmentierungsbzw. Evaporationsprozesse die ermittelte Clustergröße verfälschen?

2. In welchem Ausmaß beeinflusst das angelagerte Natriumatom die Topologie des Wasserstoffbrückennetzwerks?

Der Verlust von $\mathrm{H}_{2} \mathrm{O}$-Monomeren aufgrund der Kollision mit Na-Atomen bzw. im Zuge der Photoionisation wurde in den Abschnitten 3.2.1 und 3.2.2 diskutiert und als vernachlässigbar eingeschätzt. Die Arbeiten von Schulz et al. legen nahe, dass in kleinen Clustern ein großer Teil der im Zuge der Solvatation des Natriumions frei werdenden Bindungsenergie aufgewendet wird, um die potentielle Energie des solvatisierten Elektrons zu erhöhen 199. Nach Jortner entspricht die Solvatationsenthalpie genau der adiabatischen Bindungsenergie des Elektrons 26 . Ein Verlust einzelner Monomere aufgrund der Solvatationsenergie ist nicht auszuschließen, auch dieser Effekt wird jedoch als gering eingeschätzt $\underline{36|196| 242}$. Auch die durch den IR-Laser eingetragene Energie trägt zur Temperaturerhöhung des Clusters bei und kann die Evaporation von Monomeren bewirken. Daher muss die Pulsenergie so gewählt werden, dass dieser Effekt minimiert wird. Einen 


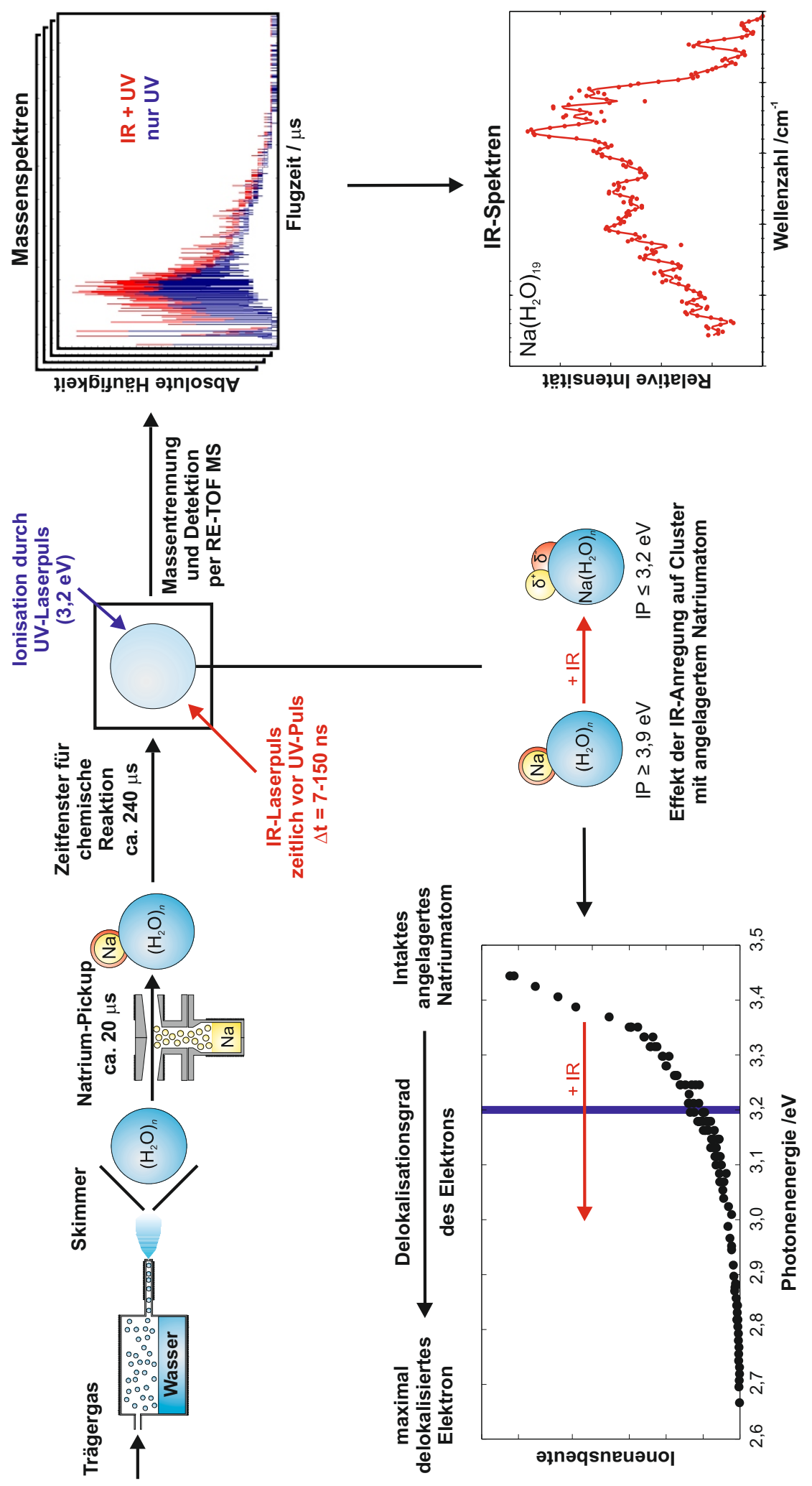

Abbildung 3.9.: Graphische Darstellung der Vorgänge im Experiment: Die IR-Anregung bewirkt eine Solvatation des Natrium-3s-Elektrons im $\mathrm{H}_{2} \mathrm{O}_{\mathrm{N}}$-Cluster, die mit einer IESenkung einhergeht. Durch größenselektive Detektion der ionisierten Cluster in Abhängigkeit von der eingestrahlten IR-Wellenlänge werden Massenspektren aufgenommen, aus denen IR-Spektren generiert werden; verändert nach Ref. [48]. 
Anhaltspunkt für die Änderung der Größenverteilung durch die Vorgänge, die nach IRAnregung der Cluster ausgelöst werden, bietet der Vergleich einer Verteilung, die ohne IRAnregung aufgenommen wurde, mit einer Verteilung, die durch nachträgliche thermische Anregung ionisierter Cluster erhalten wurde. Hier sind die oben diskutierten Zunahmeeffekte unterdrückt, sodass allein die zahlenmäßige Zunahme kleinerer Cluster auf Kosten der Abundanz größerer Aggregate betrachtet werden kann 61.

Pradzynski et al. sowie Dierking et al. zeigten, dass der größte Teil der Aggregate im Molekularstrahl eine hohe Ionisationsenergie besitzt. Hierbei handelt es sich um Strukturen, in denen das Natriumion nicht solvatisiert und über eine oder zwei Bindungen an dessen Oberfläche gebunden ist $41 \mid 60$. Pradzynski et al. nahmen darüber hinaus einen Vergleich der IR-Spektren des natriumdotierten Clusters $\mathrm{Na}\left(\mathrm{H}_{2} \mathrm{O}\right)_{19}$ und des phenoldotierten Clusters Phenol- $\left(\mathrm{H}_{2} \mathrm{O}\right)_{19}$ vor. Die hohe Übereinstimmung dieser Spektren legt nahe, dass ein angelagertes Natriumatom die Topologie des Wasserstoffbrückennetzwerks ab dieser Clustergröße nicht stärker stört, als eine Phenylgruppe, die aufgrund ihrer geringen Polarität auf der Clusteroberfläche lokalisiert ist ${ }_{41]}$. Die zusätzlich gebildeten $\mathrm{O}$-Na-Bindungen erzeugen allenfalls eine weitere IR-Bande um $3640 \mathrm{~cm}^{-153]}$.

Eine entsprechende Vergleichbarkeit ionischer Cluster der Zusammensetzung $\mathrm{Na}\left(\mathrm{H}_{2} \mathrm{O}\right)_{\mathrm{N}}{ }^{+}$bzw. $\mathrm{I}\left(\mathrm{H}_{2} \mathrm{O}\right)_{\mathrm{N}}{ }^{-}$konnte bisher lediglich oberhalb von $N=250 \pm 5$ gezeigt werden: Das IR-Spektrum neutraler, natriumdotierter Wassercluster mit $\bar{N}=225$ $(N=200-250$, bei $T=70 \mathrm{~K})$ von Pradzynski et al. 41$]$ zeigt gute Übereinstimmung mit den Spektren ionischer Cluster mit $N \approx 250$ aus Referenz [34]. Sowohl die Einhüllende als auch die subtile Bandenstruktur im Bereich der gebundenen $\mathrm{OH}$-Bande sind fast identisch, obwohl beide Spektren unter Ausnutzung stark unterschiedlicher Effekte (Natriumdotierung bzw. Ensemble-IRPD) erhalten wurden. Unterschiede zeigen sich allerdings bei der Absorption der ungebundenen OH-Oszillatoren auf der Clusteroberfläche, deren Bande sich in Abhängigkeit von der Ladung linear verschiebt $34|48| 71$.

Wird die Photoionisation schwellennah (d.h. mit möglichst niedriger Photonenenergie) durchgeführt, lässt sich der Mechanismus, der der Signalverstärkung zugrunde liegt, aus vier Gründen besonders effizient ausnutzen: Erstens minimiert die schwellennahe Photoionisation das Auftreten von Fragmentierungsereignissen, zweitens maximiert die hohe Abundanz von Isomeren mit hoher Ionisationsenergie die zur Ausnutzung des Zunahmeeffekts verfügbare Absorbanz. Drittens minimiert die geringe Abundanz von Clustern mit niedriger Ionisationsenergie spektrale Artefakte durch Strukturen mit bereits solvatisierten Elektronen. Viertens wird die IR-induzierte Signalzunahme vermutlich durch den großen UV-Absorptionsquerschnitt der schwach gebundenen, delokalisierten Elektronen verstärkt 41448 .

\section{Schwingungsfrequenzen unterschiedlich koordinierter Wassermoleküle}

Das Schwingungsspektrum von verfestigtem Wasser kann in vier Regionen unterteilt werden: Einen Nahinfrarotbereich, der die Oberton- und Kombinationsbanden enthält; den Bereich der OH-Streckschwingungen um $\tilde{\nu}_{\mathrm{IR}} \approx 2800 \mathrm{~cm}^{-1}-3800 \mathrm{~cm}^{-1}$; den Bereich der fundamentalen Biegeschwingung um $1650 \mathrm{~cm}^{-1}$ (ca. 6,5 $\mu \mathrm{m}$ ) sowie den Bereich des fernen Infrarot, in dem Gitterschwingungen sowie bei ca. $800 \mathrm{~cm}^{-1}$ (ca. $12 \mu \mathrm{m}$ ) die Librationsmoden zu beobachten sind 79 . Der Fokus dieser Arbeit liegt auf der Untersuchung der $\mathrm{OH}-S t r e c k s c h w i n g u n g e n$, insbesondere der durch die freien $\mathrm{OH}$-Oszillatoren auf der Oberfläche verursachten Bande bei ca. $3700 \mathrm{~cm}^{-1}$. 
Das Wassermonomer besitzt zwei fundamentale Streckschwingungen: Eine Symmetrische bei einer Wellenzahl von $3657 \mathrm{~cm}^{-1}$ und die antisymmetrische Streckschwingung bei $3756 \mathrm{~cm}^{-1}$. Innerhalb einer Wasserstoffbrückenbindung wird eine Rotverschiebung der OH-Streckschwingungsfrequenz beobachtet, wobei aufgrund des Symmetrieverlustes nicht mehr zwischen den beiden Fundamentalschwingungen unterschieden werden kann. Der Grund für diese Rotverschiebung ist eine im Zuge der Ausbildung einer H-Brückenbindung auftretende Verlängerung der OH-Bindungslänge bei gleichzeitiger Verkürzung der zugehörigen Wasserstoffbrücke. Diese bewirkt wiederum eine Zunahme der Anziehung zwischen dem vom Donormolekül stammenden H-Atom und dem O-Atom des Akzeptormoleküls durch attraktive elektrostatische, induktive und dispersive Wechselwirkungen. Die Folge ist eine Absenkung des Potentialminimums der OH-Bindung im vibronisch angeregten Zustand 40 . Der Gradient der Potentialhyperfläche entlang der O-H . . O-Koordinate und folglich auch die Schwingungsfrequenz der OH-Bindung stehen somit in reziprokem Zusammenhang zur Stärke der zugehörigen H-Brückenbindung. Deren Stärke wiederum wird hauptsächlich durch zwei Faktoren beeinflusst:

- Die Kooperativität der Wasserstoffbrücken: Die mittlere Stärke aller Wasserstoffbrücken eines Netzwerkes nimmt mit dessen Größe zu. Hauptgrund ist der Beitrag von induktiven Wechselwirkungen zwischen den Wassermolekülen, der bei größeren Clustern umso stärker ausfälltt5162 .

- Die Koordinationsgeometrie der Wassermoleküle: Die Stärke einer Wasserstoffbrückenbindung zeigt eine starke Abhängigkeit von den Winkeln, die zwischen den Bindungen eines Wassermoleküls bestehen, und von den Abständen zwischen den Sauerstoffatomen, zwischen denen die H-Brücke ausgebildet ist. Insbesondere in kleinen Clustern und in der Nähe der Clusteroberfläche kollidieren die Bestrebungen, die potentielle Energie der Struktur einerseits durch maximale Wasserstoffbrückenzahl und andererseits durch optimale Koordinationsgeometrie zu erreichen, sodass viele Moleküle hier nur schwache Wasserstoffbrücken ausbilden können 51|62|143|243.

Diese beiden Beiträge können nicht unabhängig voneinander betrachtet werden, da die kooperativen Wechselwirkungen sich bei optimaler Koordinationsgeometrie der beteiligten Wassermoleküle maximieren. Die resultierende Rotverschiebung der $\mathrm{OH}-$ Streckschwingungen kann bis zu $800 \mathrm{~cm}^{-1}$ betragen, sodass die Schwingungsfrequenz der OH-Bindung ein direktes Maß für die Stärke der assoziierten H-Brücke darstellt $40[143]$.

Als Folge der zahlreichen Wasserstoffbrücken besitzt flüssiges Wasser eine breite Bande mit einem Maximum um $3450 \mathrm{~cm}^{-1}$, die den gebundenen OH-Oszillatoren der vorwiegend vierfach koordinierten Moleküle im Inneren der flüssigen Phase entspringt 40[143]. Die Ableitung des Dipolmoments entlang der O-H... O-Bindung wird im Vergleich zum Monomer stark verändert, da das Dipolmoment aus Symmetriegründen verschwinden muss, sofern sich das H-Atom in der Mitte zwischen den O-Atomen befindet 244. Im Zuge einer $\mathrm{OH}-\mathrm{Schwingung}$ der darin enthaltenen $\mathrm{OH}$-Bindung ändert es sich folglich derart stark, dass diese Bande eine um den Faktor 17 erhöhte Intensität im Vergleich zur $\mathrm{OH}$ Streckschwingung des Wassermonomers besitzt 2 .

Verfestigtes Wasser besitzt im Vergleich zum Monomer eine um das ca. 28-fache erhöhte Intensität der gebundenen OH-Streckschwingungen 62 77/79|244|245], wobei die Bandenintensitäten amorpher und kristalliner Phasen sich nicht merklich unterscheiden 245 . 
Der Absorptionsbereich dieser Oszillatoren ist ebenfalls stark verbreitert. Die Ursache sind die in festphasigen Systemen auftretenden, intermolekularen Kopplungen der Übergangsdipolmomente. Als Folge lassen sich in Abhängigkeit von der Struktur Bandenmaxima zwischen $3000 \mathrm{~cm}^{-1}$ und $3400 \mathrm{~cm}^{-1}$ beobachten 79 . Weiterhin tragen die in Abschnitt 2.3.4 beschriebene Protonenunordnung 77 und die sogenannte Fermi-Resonanz zur Verbreiterung der Absorptionsbanden bei. Letztere entsteht durch die Kopplung des Obertons der OH-Biegeschwingung mit der Fundamentalfrequenz der symmetrischen OH-Streckschwingung: In isolierten Wassermolekülen liegt die Wellenzahl des ersten Obertons der OH-Biegeschwingung um mehr als $500 \mathrm{~cm}^{-1}$ unterhalb der Fundamentalen der symmetrischen Streckschwingung. Werden die Schwingungsfrequenzen von $\mathrm{OH}-$ Oszillatoren durch kooperative Wechselwirkungen gesenkt, verringert sich der energetische Abstand zwischen beiden Schwingungsmoden, die in der Folge in Resonanz treten können $2|155| 156 \mid 246]$.

Im optimal relaxierten Eiskristall führen die ausgeprägten kooperativen Wechselwirkungen zahlreicher H-Brücken zwischen nahezu ideal tetraedrisch koordinierten Wassermolekülen zu einer deutlichen Rotverschiebung der gebundenen $\mathrm{OH}$-Oszillatoren und zur Ausbildung einer charakteristischen IR-Bande bei ca. $3220 \mathrm{~cm}^{-1}$, die den Kristall vom amorphen Feststoff und von flüssigem Wasser unterscheidbar macht 62 143.

Die IR-Spektren kleiner Wassercluster hingegen werden durch den Einfluss der Oberfläche auf die lokale Umgebung der Wassermoleküle bestimmt: Moleküle, die an der Oberfläche liegen, verfügen zwangsläufig nicht alle über die notwendigen Partner, um vier Wasserstoffbrücken auszubilden. Hier dominieren dreifach koordinierte Wassermoleküle, die in Abhängigkeit von der koordinierenden Funktionalität als zweifacher Donor oder zweifacher Akzeptor als DDA- bzw. DAA-koordiniert bezeichnet werden (vgl. Abschnitt 2.3.2 4 , Abbildung 3.10 liefert einen qualitativen Überblick über die Beiträge unterschiedlich koordinierter Wassermoleküle zum IR-Spektrum eines Wasserclusters: In der IR-Bande der gebundenen OH-Oszillatoren zeigt sich der Beitrag unterkoordinierter Wassermoleküle in den Randbereichen. Daher besitzen kleine Cluster ein breites Absorptionsspektrum $40 \mid 79$. DDA-koordinierte Moleküle dominieren dabei den Hochfrequenzbereich dieser Bande, da die Ausbildung zweier Wasserstoffbrücken als Donoren meist mit einer Deformation des $\mathrm{H}-\mathrm{O}-\mathrm{H}-$ Winkels einhergeht. Die Folge sind im Mittel schwächere H-Brückenbindungen. DAA-koordinierte Moleküle hingegen besitzen eine freie OH-Gruppe. Als Folge geringerer Deformation des $\mathrm{H}-\mathrm{O}-\mathrm{H}-$ Winkels 40$]$ und der über die doppelte Donor-Koordination abfließenden Elektronendichte 51 absorbieren sie am niederfrequenten Ende der Bande. Vierfach koordinierte Moleküle absorbieren im Zentrum der Bande der gebundenen $\mathrm{OH}-$ Streckschwingungen 62. In den IR-Spektren amorpher Cluster zeigt sich die wachsende Anzahl vierfach koordinierter Moleküle im Clusterinneren als Zunahme der relativen Bandenintensität im Bereich zwischen $3300 \mathrm{~cm}^{-1}$ und $3400 \mathrm{~cm}^{-151]}$ auf Kosten der Randbereiche, wobei sich das Maximum amorpher, DDAA-koordinierter Moleküle aufgrund der zunehmenden Kooperativität von $3400 \mathrm{~cm}^{-1}$ langsam zu kleineren Wellenzahlen verschiebt 166.

IR-Spektren kristalliner Nanopartikel weisen im Bereich der OH-Streckschwingungen eine für kristallines Eis charakteristische, zentrale Bande mit zwei Schultern auf, die einer kollektiven, gleichphasigen antisymmetrischen Streckschwingungsmode entspringt 77 . Die

${ }^{4}$ DA-koordinierte Wassermoleküle kommen nur in sehr kleinen Clustern vor (vgl. Abschnitt 2.3.5) und sind auf der Oberfläche größerer Cluster und Nanopartikel nur bei Temperaturen unterhalb von $80 \mathrm{~K}$ stabil[15. 


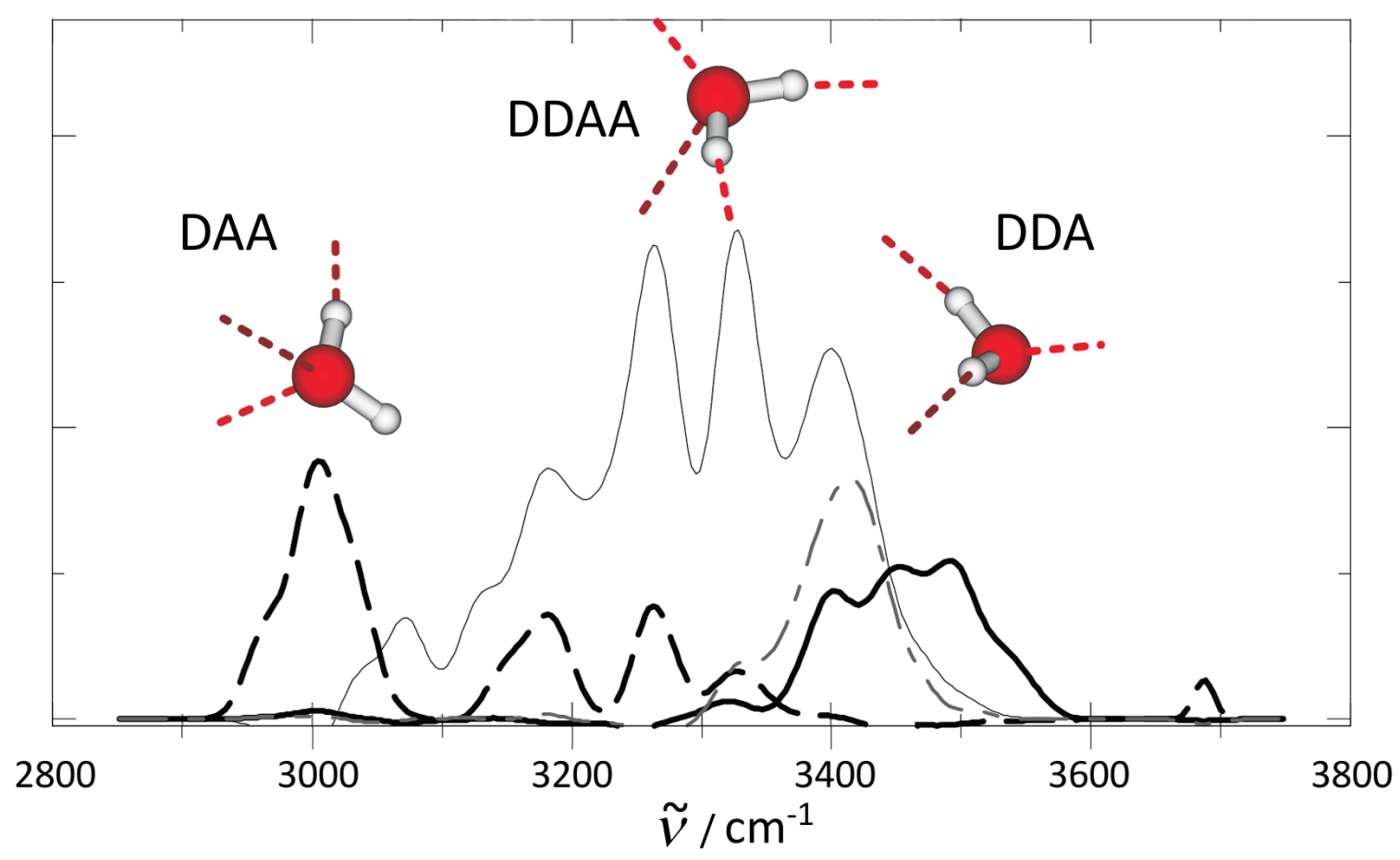

Abbildung 3.10.: Berechnetes Schwingungsspektrum des Clusters $\left(\mathrm{H}_{2} \mathrm{O}\right)_{48}$ nach Ref. [62], aufgeteilt nach den Beiträgen unterschiedlich koordinierter $\mathrm{H}_{2} \mathrm{O}$-Moleküle: Dünne, durchgezogene Linie: DDAA-Koordination; dicke, durchgezogene Linie: DDAkoordinierte Moleküle (freies Elektronenpaar am Sauerstoffatom); schwarze, unterbrochene Linie: DAA-koordinierte Moleküle (freies H-Atom); graue, unterbrochene Linie: Beitrag DDAA-koordinierter Moleküle, die als Donoren eine Wasserstoffbrücke mit einem DDAkoordinierten Molekül ausgebildet haben; entnommen aus Ref. [239].

kleinsten Partikel mit kristallartigem Kern zeigen eine Bande um $3250 \mathrm{~cm}^{-1} 75$. Mit zunehmender Partikelgröße zeigt sich eine graduelle Rotverschiebung auf ca. $3220 \mathrm{~cm}^{-1}$ bei einem Radius von ca. $5 \mathrm{~nm} 20$. Buch et al. interpretierten diesen Punkt als einen Schwellenwert, oberhalb dem das Bestreben der Oberfläche zur Maximierung der Wasserstoffbrückenzahl dem Energiegewinn des relaxierenden kristallinen Kerns unterliegt 62. Da die Eigenfrequenz einer Schwingung von der lokalen Koordinationsumgebung des Wassermoleküls abhängt, die in Eis $\mathrm{I}_{\mathrm{c}}$, Eis $\mathrm{I}_{\mathrm{h}}$, und Eis $\mathrm{I}_{\mathrm{sd}}$ identisch ist, können diese Modifikationen durch IR-spektroskopische Methoden nicht unterschieden werden 62 .

Im Zuge der Kristallisation findet folglich ein Übergang der Bandenintensität hin zu kleineren Wellenzahlen statt. Im Gegensatz zu den oben beschriebenen, graduellen größenund temperaturabhängigen Verschiebungen der Bandenmaxima tritt hier ein isosbestischer Punkt bei $\tilde{\nu}_{\mathrm{IR}} \approx 3370 \mathrm{~cm}^{-1}$ auf, der auf eine stöchiometrische Umwandlung ohne intermediär auftretende Übergangsformen hindeutet 75 . 
Um $3700 \mathrm{~cm}^{-1}$ findet sich die Bande der frei schwingenden OH-Oszillatoren DAAkoordinierter Moleküle auf der Clusteroberfläche, die in den kleinsten Clustern mit zweistelliger Konstituentenzahl bei $3710 \mathrm{~cm}^{-1}$ verortet ist und sich mit zunehmender Partikelgröße zu Werten von $3692 \mathrm{~cm}^{-162}$ in kristallinem Eis I bzw. $3696 \mathrm{~cm}^{-1} 15$ in amorphen Filmen verschiebt $51|52| 243$. Die Ursache dieser Rotverschiebung ist der Abzug von Elektronendichte aus der $\mathrm{OH}$-Bindung durch die benachbarten, als $\mathrm{H}$-Brückendonor fungierenden Wassermoleküle. Dieser Effekt nimmt mit der Stärke der H-Brücken in unmittelbarer Umgebung der frei schwingenden OH-Gruppe zu $15|40| 51|62| 79]$.

In IR-Spektren von Wasserclustern mit dreidimensionalen Wasserstoffbrückennetzwerken werden die Linienbreiten und -formen durch ein Wechselspiel aus der Verteilung der Bindungsstärken und den dynamischen Kopplungen der Schwingungsmoden zwischen verschiedenen OH-Bindungen bestimmt. Diese Kopplungen führen darüber hinaus zur Anregung kollektiver Schwingungsmoden mit Phasenbeziehungen, die durch die jeweils angewandte Messmethode begünstigt sein können. Daher spiegelt die Intensität einer Bande nicht notwendigerweise die bei einer bestimmten Anregungsfrequenz vorliegende Oszillatordichte wider. Eine Ausnahme bilden die freien OH-Gruppen auf der Clusteroberfläche: Diese treten nicht mit den übrigen Schwingungsmoden in Resonanz, sodass die Intensität ihrer Bande allein Funktion ihrer Anzahl und ihres Übergangsdipolmoments ist 62. Fujii et al. beobachteten eine Abnahme der relativen Bandenintensität der freien OH-Streckschwingung entsprechend dem sinkenden Anteil oberflächlich lokalisierter Wassermoleküle mit zunehmender Clustergröß 51 .

Auch die Tatsache, dass die Stärke der IR-Absorption durch Methoden der Wirkungsspektroskopie nur indirekt bestimmt wird, kann das gemessene Schwingungsspektrum beeinflussen: Die von den verschiedenen wirkungsspektroskopischen Methoden ausgenutzten Effekte (IR-induzierte Photodissoziation 34 52 bzw. IR-anregungsmodulierte Photoionisation 41[53] könnten nach Anregung oberflächennaher Wassermoleküle, die IRPhotonen höherer Energie absorbieren, mit gesteigerter Effizienz auftreten. Dies könnte zu einer leichten Verzerrung des gesamten Spektrums im Vergleich zu direkten Absorptionsmessungen führen 54 .

Unterschiedliche Messmethoden mit verschiedenen systematischen Fehlern und unterschiedlich akkurate Verfahren der Größenselektion führen bereits in den IR-Spektren kleiner Cluster zu widersprüchlichen Literaturangaben bezüglich der Schwingungswellenzahlen: So fanden Buck et al. für die gebundene OH-Streckschwingung DA-koordinierter Moleküle bei $N=4$ einen Wert von $3416 \mathrm{~cm}^{-1}$ 143|247], wohingegen Otto et al. einen Wert von $3401 \mathrm{~cm}^{-1}[155$ ermittelten. Weitere Angaben in diesen Referenzen zeigen einen systematischen Versatz der publizierten Wellenzahlen von $5 \mathrm{~cm}^{-1}$. Die Wellenzahl der freien OH-Streckschwingung von $N=8$ wurde von Buck et al. mit $3727 \mathrm{~cm}^{-1}$ angegeben. Gruenloh fand an benzoldotierten Clustern einen Wert von $3713,5 \mathrm{~cm}^{-1} 49$. Daher können die Wellenzahlen unterschiedlich koordinierter Moleküle nur in Form allgemeiner Tendenzen und Richtwerte angegeben werden, die in Tabelle 3.1 zusammengefasst sind.

Wie oben erwähnt, führt eine Wasserstoffbrückenbindung zur Abnahme der Schwingungswellenzahl der zugehörigen OH-Bindung. Die Stärke der H-Brücke ist dabei nicht nur von der lokalen Koordinationsgeometrie und vom Ausmaß der kooperativen Wechselwirkungen, sondern auch von der Temperatur der Partikel abhängig: Bei niedriger Temperatur nimmt die Intensität in den Randbereichen der Bande der gebundenen OH-Oszillatoren ab, sodass das Bandenmaximum in deren Zentrum schärfer hervortritt. 


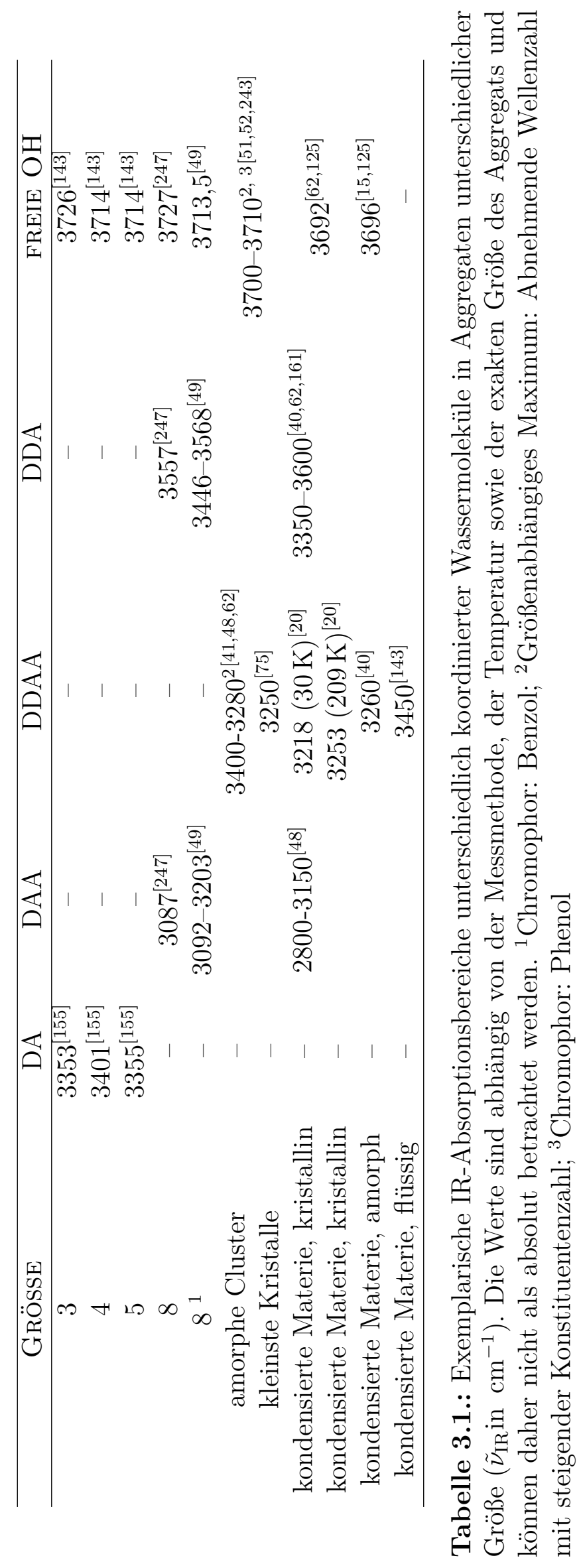


Weiterhin erfolgt eine Rotverschiebung des Maximums. Für dieses Verhalten können drei Ursachen ausgemacht werden: Erstens führt eine Verminderung der Temperatur zu einer reduzierten Population hochfrequenter Schwingungsmoden. Zweitens bewirkt eine Kontraktion des Kristallgitters eine Stärkung der intermolekularen Wasserstoffbrückenbindungen unter Verringerung der Bindungslänge, die mit einer weiteren Schwächung der zugehörigen $\mathrm{OH}$-Bindungen einhergeht. Drittens führt die thermisch induzierte Gitterkontraktion zusätzlich zu einer verstärkten Modenkopplung, wodurch die Rotverschiebung des Bandenmaximums weiter begünstigt wird. Insgesamt ergeben diese Effekte eine lineare Abnahme der Schwingungswellenzahl von $0,20 \mathrm{~cm}^{-1} \mathrm{~K}^{-1} 20 \mid 79$.

\subsubsection{Flugzeitbasierte Massenselektion}

Die Methode der Massenspektrometrie wird angewandt, um geladene Partikel in der Gasphase anhand des Quotienten $(\mathrm{m} / z)$ aus ihrer Masse $m$ und ihrer Ladungszahl $z \mathrm{zu}$ trennen. Diese Trennung erfolgt dadurch, dass geladene Teilchen beim Durchqueren eines elektrischen oder magnetischen Feldes eine Kraft erfahren, die sich proportional zu ihrer Ladung verhält. Die daraus resultierende Beschleunigung ist umgekehrt proportional zur Masse dieser Partikel, sodass als Folge eine räumliche Trennung von Ionen mit verschiedenen $m / z$-Werten eintritt248.

Der Vorzug der Flugzeitmassenspektrometrie (engl. time-of-flight mass spectrometry, kurz TOF-MS) besteht darin, dass in Abhängigkeit vom apparativen Aufbau ein weiter Massenbereich simultan erfasst wird. Eine Massentrennung über Massenfilter, die eine sequentielle Selektion von Ionen verschiedener $m / z$-Verhältnisse vornehmen, geht zwangsläufig mit einem hohen Signalverlust einher, was insbesondere bei einer diskontinuierlichen Ionenquelle die Messzeit vervielfacht, da jede Ionenmasse separat erfasst werden muss. Daher stellt ein TOF-Massenspektrometer eine sinnvolle Kombination mit einer gepulsten Ionenquelle dar, wie sie in der vorliegenden Arbeit verwendet wird 249 .

Die gleichzeitige Erfassung aller in der Quelle gebildeten Ionen wird erreicht, indem all diesen Teilchen zum gleichen Zeitpunkt die gleiche kinetische Energie zugeführt wird. Dies geschieht, indem diese gleichzeitig einem elektrischen Feld ausgesetzt werden. Die kinetische Energie $E_{\text {kin }}$, die auf ein Ion mit der Ladung $q=z \cdot e$ übertragen wird, das am Ort $x_{0}$ einem elektrischen Feld der Spannungsdifferenz $\Delta U$ ausgesetzt wird, beträgt:

$$
E_{\text {kin }}=q \cdot \Delta U\left(x_{0}\right)=\frac{1}{2} m v_{\mathrm{D}}^{2}
$$

Dabei stellt $\Delta U\left(x_{0}\right)$ die Spannungsdifferenz zwischen dem Ort $x_{0}$ und der Beschleunigungselektrode in Abzugsrichtung dar, $z$ bezeichnet die Ladungszahl und $e$ die Elementarladung. Die Zeitspanne $t_{\mathrm{D}}$, die ein Ion mit der Driftgeschwindigkeit $v_{\mathrm{D}}$ benötigt, um die Driftstrecke der Länge $\Delta x$ zurückzulegen, wird als Driftzeit bezeichnet. Sie berechnet sich aus der Geschwindigkeit nach $v_{\mathrm{D}}=\frac{\Delta x}{t_{\mathrm{D}}}$. Die Kenntnis des Zusammenhangs zwischen dem $m / z$-Verhältnis und der Zeitspanne, die zwischen Ionisation und Detektion eines Ions vergeht, ist Voraussetzung für die Bestimmung von dessen Masse. Sie setzt sich additiv aus dieser Driftzeit im feldfreien Bereich, seiner Beschleunigungszeit und der Zeitspanne zusammen, die es im Reflektron verbringt. Die letzteren Beiträge fallen kaum ins Gewicht. Für die Flugzeit im feldfreien Bereich ergibt sich aus Gleichung 3.29: 
(a)

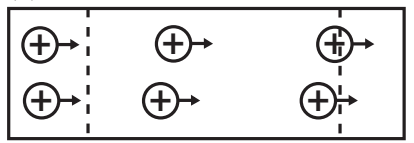

(c)

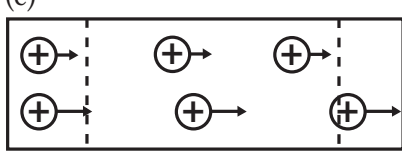

(b)

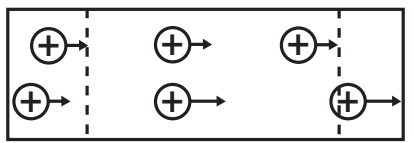

(d)

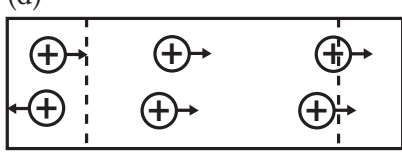

Abbildung 3.11.: Einflüsse, die eine Verminderung der Massenauflösung bewirken: (a): Ionisation $\mathrm{zu}$ unterschiedlichen Zeitpunkten; (b): variierende Anfangspositionen; (c): abweichende Ausgangsgeschwindigkeiten; (d): unterschiedliche Flugrichtungen vor der Ionisation; entnommen aus Referenz 250].

$$
\frac{m}{z}=\frac{2 \cdot e \cdot \Delta U\left(x_{0}\right)}{(\Delta x)^{2}}\left(t_{\mathrm{D}}\right)^{2}
$$

Die Zeitspanne $t_{\mathrm{D}}$ beginnt an einem Zeitpunkt $t_{0}$, der aufgrund des veränderlichen Strahlwegs des UV-Lasers und der Signallaufzeiten in den elektronischen Schaltungen variiert und somit aus einer Regressionsrechnung bestimmt werden muss. Aufgrund von Inhomogenitäten der elektrischen Felder und der sich daraus von diesen vereinfachten Annahmen ergebenden Abweichungen der Ionenflugbahnen im Reflektron ist Gleichung 3.30 insgesamt nur näherungsweise gültig. Der Quotient aus Ladung, Spannung und Flugstrecke wird in eine Apparatekonstante $c$ einbezogen, sodass sich der Zusammenhang zwischen Masse und Flugzeit eines Ions als Funktion der beiden Regressionsparameter $t_{0}$ und $c$ darstellen lässt, die nach Aufnahme einer Größenverteilung aus deren Maxima durch eine Regressionsrechnung bestimmt werden können 251:

$$
\frac{m}{z}=\frac{\left(t_{\mathrm{D}}-t_{0}\right)^{2}}{c}
$$

Die entscheidende Kenngröße eines Massenspektrometers ist sein Auflösungsvermögen. Die Unterscheidbarkeit zweier Ionen unterschiedlicher Masse mit der Massendifferenz $\Delta m$ beruht auf dem relativen Unterschied ihres Verhaltens im Massenanalysator. Diese Größe verhält sich umgekehrt proportional zur absoluten Ionenmasse. Daher wird die gerade noch unterscheidbare Massendifferenz $\Delta m$ zur absoluten Masse ins Verhältnis gesetzt 248.

$$
R_{\mathrm{m}}=\frac{m}{\Delta m}
$$

Die Signalbreiten und somit die im Experiment erzielbare Auflösung werden durch eine Reihe von Faktoren beeinflusst, die in Abbildung 3.11 dargestellt sind:

- Der von der Quelle ausgehende Ionenpuls besitzt grundsätzlich eine endliche Dauer, die sich im vorliegenden Fall aus der Länge des Laserpulses ergibt. Daher beginnt die Beschleunigung der entstandenen Ionen zu unterschiedlichen Zeitpunkten.

- Die räumliche Ausdehnung des Ionisationsbereichs bedingt unterschiedliche Anfangspunkte der Beschleunigungsstrecke. Ionen, die somit einen längeren Weg durch 
das Beschleunigungsfeld zurücklegen, besitzen anschließend eine höhere kinetische Energie und legen die Driftstrecke in kürzerer Zeit zurück.

- Wie in Abschnitt 3.1.2 beschrieben, besitzen die Aggregate zum Zeitpunkt ihrer Ionisation nicht alle die gleiche kinetische Energie, wodurch sich die Geschwindigkeitsvarianz weiter vergrößert.

- Die Varianz der Flugrichtung, bis hin zu der Möglichkeit, dass sich einzelne Partikel zum Zeitpunkt der Ionisation in Gegenrichtung bewegen und Zeit benötigen, um ihre Flugrichtung zu ändern, bewirkt ebenfalls eine Verbreiterung der Massensignale. Die hierfür benötigte Zeitspanne wird als turn-around-Zeit bezeichnet. Dieser Einfluss fällt im vorliegenden Experiment jedoch kaum ins Gewicht.

\section{Fokussierung der erzeugten Ionen}

Um den oben beschriebenen Einflüssen entgegenzuwirken und die Massenauflösung zu erhöhen, wird eine Fokussierung der untersuchten Ionen bezüglich des Ortes und der kinetischen Energie vorgenommen. Teilchen gleicher Masse, die an verschiedenen Orten der Ionisationszone ionisiert wurden, verlassen das Beschleunigungsfeld zu unterschiedlichen Zeitpunkten. Dieser Effekt wird jedoch kompensiert, da Ionen in Abhängigkeit vom Ort ihrer Entstehung aufgrund des Feldgradienten zwischen Repeller- und Extraktorelektrode unterschiedlich stark beschleunigt werden. Es existiert folglich ein Punkt, an dem die Ionen, die das Beschleunigungsfeld zu einem späteren Zeitpunkt, aber mit höherer Geschwindigkeit verlassen haben, die Ionen einholen, die eine geringere Geschwindigkeit aufweisen. Dieser Punkt wird Ortsfokus 1. Ordnung genannt. Wird an diesem Ort der Detektor positioniert, kann die aus der Startortvarianz resultierende Signalverbreiterung minimiert und die Auflösung verbessert werden. Dieser Punkt liegt jedoch nah bei der Ionenquelle, sodass Ionen mit unterschiedlicher Masse auf dieser kurzen Driftstrecke kaum separiert werden. Daher bleibt das Auflösungsvermögen einer solchen Anordnung niedrig.

Wird das Beschleunigungsfeld um ein weiteres elektrisches Feld (das sogenannte Abzugsfeld) ergänzt, kann durch Variation von dessen Stärke der Ortsfokus erster Ordnung verschoben und somit die Massenauflösung verbessert werden. Ein TOFMassenspektrometer dieser Bauart wird als Wiley-McLaren-Flugzeitmassenspektrometer bezeichnet252. Das Abzugsfeld besitzt jedoch keinen Einfluss auf die turn-around-Zeit, sodass der Fokussierung der Ionen durch Variation des Abzugsfeldes Grenzen gesetzt sind.

Eine weitere Erhöhung des Auflösungsvermögens kann durch den Einsatz eines Reflektrons erzielt werden. Hier findet eine Fokussierung von Ionen gleicher Masse statt, deren kinetische Energie variiert. Ein Reflektron besteht aus einer Anordnung von mehreren elektrischen Feldern, deren Richtung dem Beschleunigungsfeld entgegengesetzt ist. Diese Felder vermindern somit zunächst die Geschwindigkeit der Ionen, während diese in das Reflektron eintreten. Da die Feldstärken des Reflektrons so gewählt sind, dass die kinetische Energie der Ionen nicht ausreicht, um das Reflektron vollständig zu durchqueren, werden diese anschließend erneut beschleunigt, wobei sie ihren anfänglichen Impuls zurückerhalten. Der Winkel des Reflektrons ist dabei so gewählt, dass eine Umlenkung der Ionen um ca. $180^{\circ}$ erreicht wird 253 . Da Ionen mit hoher Geschwindigkeit weiter in die elektrischen Felder des Reflektrons eindringen und somit einen längeren Weg zurückle- 


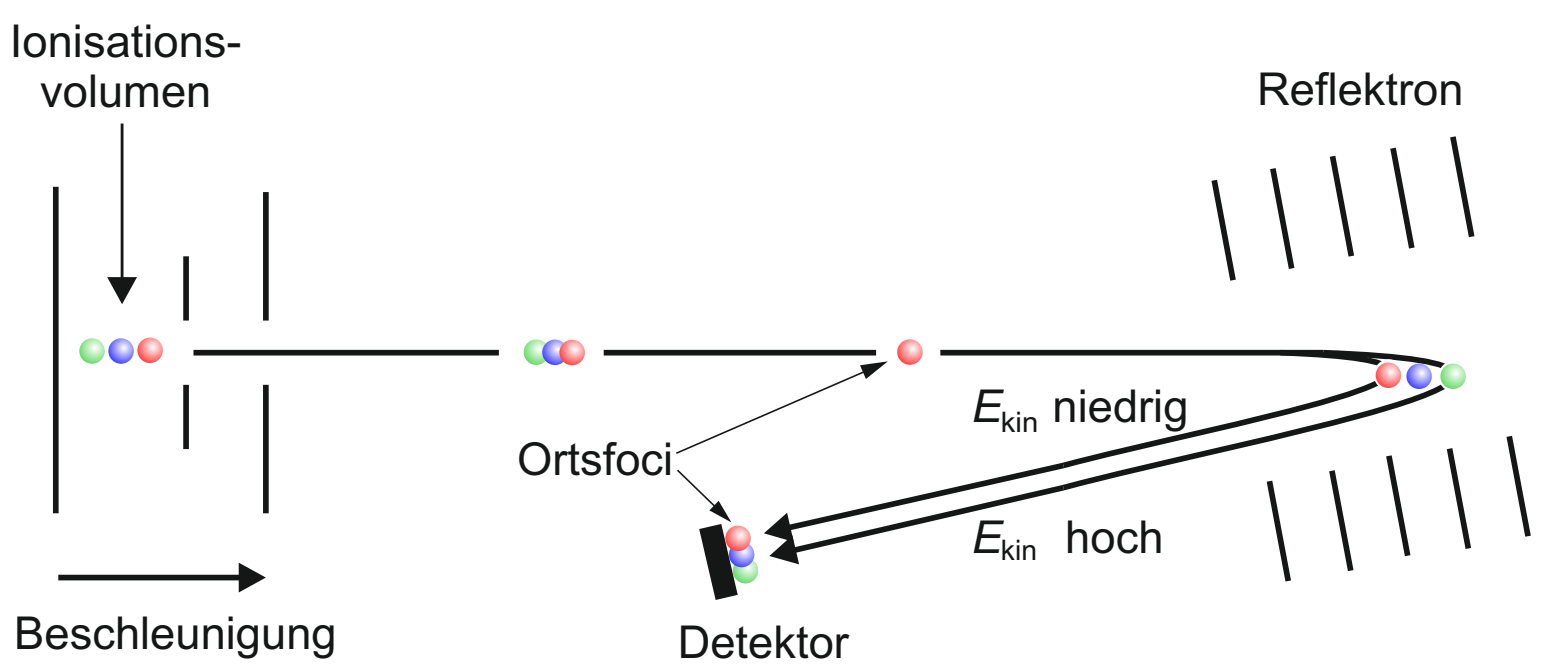

Abbildung 3.12.: Funktionsweise eines Reflektrons, verändert nach Referenz 251].

gen müssen, werden Unterschiede in der kinetischen Energie dieser Teilchen ausgeglichen (vgl. Abbildung 3.12). Es entsteht ein weiterer Ortsfokus, der sich durch Variation der Spannung an den Elektroden des Reflektrons auf den Ort des Detektors projizieren lässt.

Findet auf der Driftstrecke zwischen Extraktorelektrode und Reflektron eine Fragmentierung statt, so besitzen sämtliche Fragmente eines Teilchens die gleiche Geschwindigkeit wie zuvor das unfragmentierte Ion. Da die Fragmente jedoch aufgrund ihrer geringeren Masse einen geringeren Impuls besitzen, werden sie im Reflektron zu einem früheren Zeitpunkt zurückgeworfen. Im Massenspektrum erscheint ein sogenanntes metastabiles Signal, das von den Signalen mit ganzzahligen $m / z$-Werten separiert ist. Dieses Signal ist zwischen dem Signal des Fragments und dem Signal des unfragmentierten Ions zu finden und weist in der Regel eine größere Nähe zum Signal des Fragments auf. Somit ermöglicht ein Reflektron den Nachweis einer auf der Driftstrecke stattfindenden Fragmentierung, sofern diese vor der Passage des Reflektrons erfolgt ist.

\subsubsection{Detektion}

Einzelne Ionen können durch einen Sekundärelektronenvervielfacher (SEV) nachgewiesen werden. Die Ionen treffen hier auf die sogenannte Konversionsdynode, die ein stark negatives Potential besitzt, und lösen aufgrund ihrer kinetischen Energie Elektronen aus deren Oberfläche heraus. Dieser Elektronenstrom wird durch ein elektrisches Feld beschleunigt und setzt beim Auftreffen auf eine weitere Elektrode bei einem negativen Potential mit niedrigerem Betrag wiederum eine größere Ladungsmenge frei. Eine Kombination derartiger Elektroden, die hintereinander angeordnet sind und jeweils ein leicht niedrigeres, negatives Potential aufweisen, bewirkt somit eine kaskadenartige Verstärkung des resultierenden Elektronenstroms. Die Bündelung einer großen Zahl solcher Elemente ergibt sogenannte Mikrokanalplatten (engl. microchannel plates, MCP), die neben der zeitlichen Auflösung auch eine Ortsauflösung des auftreffenden Signals ermöglichen. Hier sind die einzelnen Elektroden, die beim SEV als separate Bauteile vorhanden sind, durch eine hochohmige Platte ersetzt, die an beiden Flächen leitende Beschichtungen trägt, zwischen denen eine Spannung von 1 bis $2 \mathrm{kV}$ anliegt (vgl. Abb. 3.13). Die Verstärkung des Elek- 

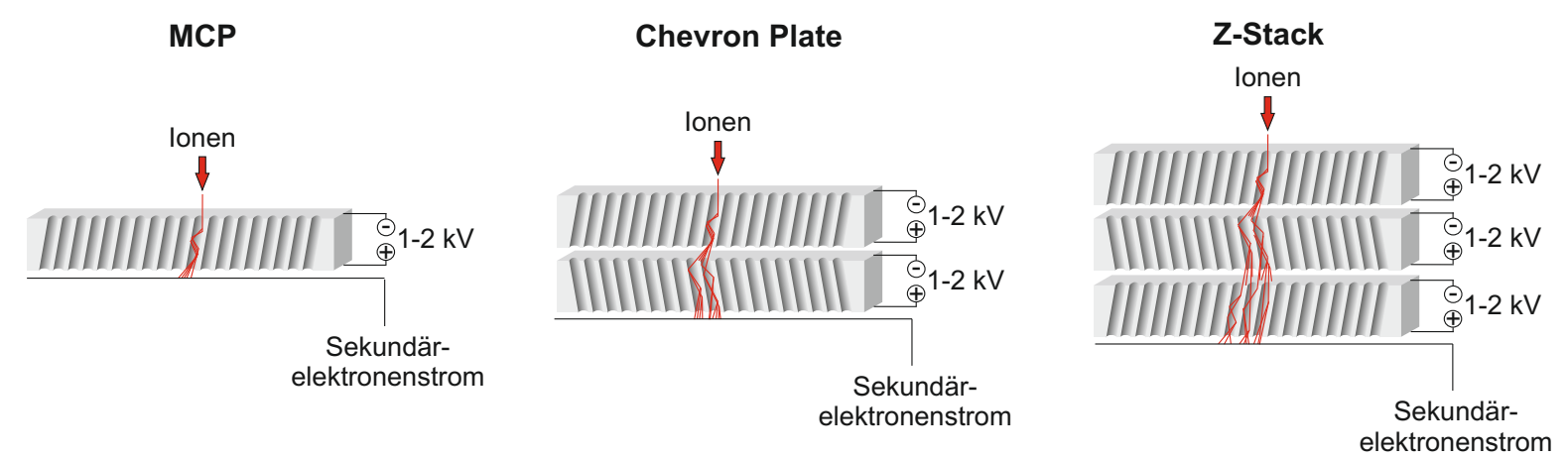

Abbildung 3.13.: Unterschiedliche Bauweisen von MCP-Detektoren; nach Ref. 248.

tronenstroms findet an den Innenwänden dünner Bohrungen statt, die in einem Winkel von ca. $10^{\circ}$ in die Platte eingebracht sind, sodass eintreffende Ionen mit Sicherheit auf deren Innenwand treffen.

Eine zusätzliche Verstärkung des emittierten Elektronenstroms kann durch die Kombination zweier bzw. dreier MCP erreicht werden. Eine solche Anordnung wird als Chevron Plate bzw. z-Stack bezeichnet, da die Achsen der Bohrungen benachbarter Platten jeweils um $180^{\circ}$ gegeneinander verkippt sind, um ein Auftreffen der Elektronen auf die inneren Wandflächen der Bohrungen sicherzustellen.

Die Elektronenausbeute nach dem Auftreffen eines Ions und damit die Detektionseffizienz eines MCP ist abhängig von der Geschwindigkeit der Auftreffenden Teilchen 254. Folglich werden schwerere Ionen weniger effizient detektiert, als leichte Ionen 18. Eine entsprechende Korrekturfunktion wurde von Schläppi et al. vorgeschlagen 208 . 


\section{Versuchsaufbau und experimentelle Methoden}

\section{1. Übersicht}

Alle Messdaten, auf denen die Ergebnisse der vorliegenden Arbeit beruhen, wurden mit Hilfe der Molekularstrahlapparatur P11 erhoben. Diese wurde in den 1990er Jahren im Rahmen der Promotion von Dr. M. Hobein 255 in der Arbeitsgruppe von Prof. Dr. Udo Buck am Max-Planck-Institut für Strömungsforschung in Göttingen konzipiert. Mitte der 90er Jahre führte Dr. S. Schütte im Rahmen seiner Promotion 256 die ersten erfolgreichen Messungen an Edelgasclustern durch. Ende 2004 wurde die Apparatur an das Institut für Physikalische Chemie der Universität Göttingen übergeben. Die Apparatur sowie daran vorgenommene Modifikationen wurden darüber hinaus in den Publikationen 71, 96, 179, 209, 237, 251, 257, 258 näher beschrieben. Eine Übersicht der im Rahmen dieser Arbeit eingesetzten Chemikalien findet sich in Anhang B.

Dieser Abschnitt enthält zunächst eine kurz gefasste Beschreibung der unverändert übernommenen Apparaturkomponenten. Die im Rahmen dieser Arbeit vorgenommenen Modifikationen des Messmodus und der elektronischen Steuerung sowie die hierfür entwickelte Software werden in Abschnitt 4.8 ausführlich erläutert.

Die Versuchsapparatur besteht aus drei Vakuumkammern. Diese sind aus Edelstahl gefertigt und durch kleinlumige Öffnungen miteinander verbunden, sodass sie differentiell gepumpt, durch Ventile getrennt und zu Wartungszwecken separat belüftet werden können. Der in ihrem Inneren propagierende Molekularstrahl gelangt somit sukzessive in Abschnitte zunehmend feineren Vakuums. Abbildung 4.1 zeigt eine schematische Übersicht dieses Aufbaus.

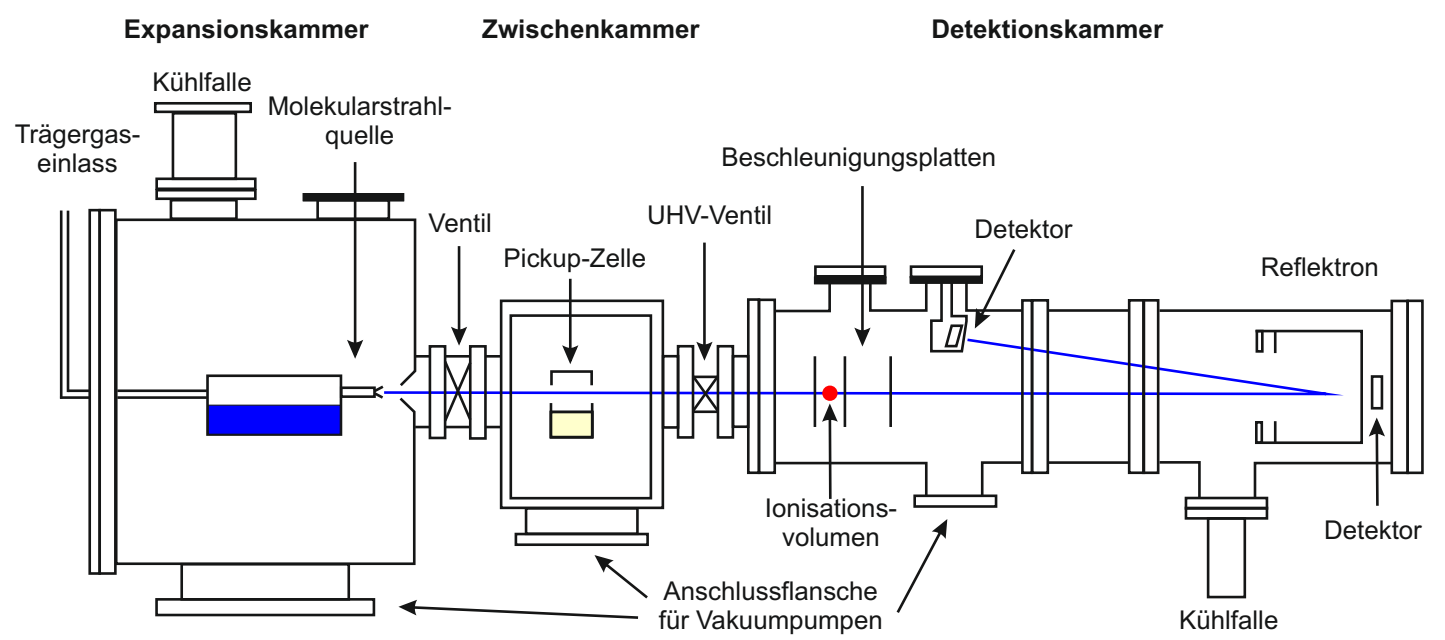

Abbildung 4.1.: Schematischer Aufbau der Apparatur P11, verändert nach Ref. 251]. 
Im Einzelnen besteht die Apparatur P11 aus folgenden Kompartimenten:

- Die Expansionskammer: Die erste Kammer beherbergt das beheizbare Substanzreservoir. Hier werden die zu untersuchenden Partikel in einer adiabatischen Überschallexpansion erzeugt, wobei der gasförmige Analyt durch eine Düse in die umgebende Vakuumkammer expandiert wird, während der in der Kammer herrschende Druck möglichst niedrig gehalten wird (vgl. Abschn. 3.1).

- Die Zwischenkammer: Die zweite Kammer beherbergt die Pickup-Zelle. Diese besteht aus einem beheizbaren Reservoir, das mit elementarem Natrium gefüllt ist, sowie einer darüber angeordneten Dotierungskammer mit Durchlass für den Molekularstrahl. In dieser Kammer bildet sich eine natriumdampfhaltige Atmosphäre aus, die vom Molekularstrahl passiert wird. Hier findet die Dotierung der zuvor erzeugten Cluster mit Natriumatomen statt, um diese später in Abhängigkeit von ihrer Temperatur ionisieren zu können (vgl. Abschn. 3.2.1).

- Die Detektionskammer: Die dritte Kammer besteht im Kern aus einem Flugzeitmassenspektrometer. In die Außenwand dieser Kammer sind vier Fenster eingelassen, um die zuvor erzeugten Partikel mittels IR- bzw. UV-Laserstrahlung zu erwärmen bzw. zu ionisieren. Weiterhin sind darin Elektroden zur Beschleunigung der ionisierten Partikel sowie ein MCP-Detektor angebracht, der auftreffende Teilchen in elektrische Signale umwandelt. Die dotierten Cluster werden hier mittels durchstimmbarer IR-Laserstrahlung erwärmt, durch UV-Strahlung ionisiert, anhand ihres $m / z$-Verhältnisses separiert und größenselektiv erfasst.

\subsection{Molekularstrahlquelle}

Die Molekularstrahlquelle der Apparatur P11 ist nicht gepulst, d.h. der Analyt wird kontinuierlich ins Vakuum expandiert. Sie ist in Form eines aus Edelstahl bestehenden, beheizbaren Hohlzylinders (dem Reservoir) mit einem Fassungsvermögen von ca. $150 \mathrm{~mL}$ ausgeführt 251], der an einem Ende einen Einlass für den Analyten sowie für das Trägergas besitzt, das während der Messung zugeführt wird. Am anderen Ende ist ein separat beheizbares Messingrohr (die Düsenhalterung) aufgesetzt, auf dessen Ende die Düse in Form einer runden Edelstahlscheibe mit konischer Bohrung durch sechs Schrauben fixiert ist 251. Die Übergänge zwischen diesen Bauteilen sind mit Dichtringen aus Fluorkarbonkautschuk (FKM, Viton) gegen die umgebende Vakuumkammer abgedichtet 258. Die Position der Molekularstrahlquelle innerhalb der Expansionskammer lässt sich bei geschlossener Kammer über Stellräder in allen drei Raumrichtungen variieren 251. Um die optimale Position des Reservoirs auffinden und reproduzierbar ansteuern zu können sind an den Stellrädern Schrittzähler angebracht. Der Aufbau der Quelle ist schematisch in Abbildung 4.2 dargestellt.

Die Temperaturen von Reservoir und Düse können über zwei separate Steuerungseinheiten geregelt werden. Zur Temperaturmessung sind an beiden Bauteilen Thermoelemente angebracht. Somit lässt sich der Dampfdruck des Analyten über die Temperatur des Reservoirs einstellen. Die Temperatur der Düse wird stets um mindestens $10^{\circ} \mathrm{C}$ höher eingestellt, als die Temperatur des Reservoirs, um eine teilweise oder vollständige Verstopfung der Düse durch Kondensation des Analyten zu vermeiden. 


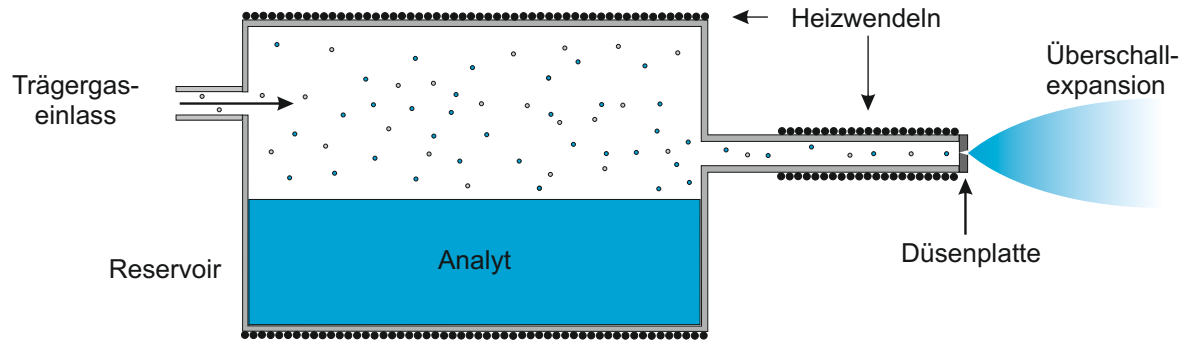

Abbildung 4.2.: Schematischer Aufbau der Molekularstrahlquelle, verändert nach [251].

Die in dieser Arbeit verwendete konische Düse wurde im Rahmen der Masterarbeit von Daniel Becker 239 erneut vermessen, nachdem dies zuletzt während der Promotion von Dr. Ingo Dauster 251] geschehen war. Sie besitzt nunmehr einen Innendurchmesser von ca. $75 \mu \mathrm{m}$ an der engsten Stelle. Der Öffnungswinkel kann weiterhin auf $40^{\circ}$ abgeschätzt werden und der Innendurchmesser der Öffnung am Auslass beträgt ca. 1,45 mm. Die gesamte Düse besitzt eine Länge von $2 \mathrm{~mm}$ (Dicke der Düsenplatte).

Im Abstand von ca. 2,5 cm nach der Düsenöffnung ist ein Skimmer angebracht, dessen Öffnung einen Durchmesser von 0,6 mm aufweist. Dieser besitzt drei Funktionen: Erstens begrenzt er den Teilchenstrom aus der Expansionskammer in die Zwischenkammer insgesamt, um die Erzeugung eines Druckgradienten zwischen den beiden Kammern zu ermöglichen. Der Abstand des Skimmers von der Düse ist so gewählt, dass er kürzer ist als der Abstand, nach dem sich durch Angleichung des Druckes der Expansion an den in der Kammer herrschenden Hintergrunddruck eine Schockwelle ausbilden kann (vgl. Abschnitt 3.1.1). Bei der Durchquerung einer solchen Zone würden die Cluster durch Stöße aufgeheizt und abgelenkt, was erstens zur Evaporation von Konstituenten und zweitens zu einer starken Abnahme des Messsignals führt. Der Skimmer stellt somit sicher, dass die Cluster in einen Bereich niedrigeren Hintergrunddruckes geleitet werden, bevor Stöße mit Bestandteilen der Gasphase in der Kammer auftreten können. Zweitens selektiert er den Teil der Expansion, in dem die Cluster die größte durchschnittliche Masse besitzen. Dies ist in der Zentralachse der Expansion der Fall, da die transversale Teilchenbewegung der Cluster umso schwächer ist, je schwerer sie sind 176. Drittens begrenzt er die Winkeldivergenz des Molekularstrahls und vermindert Artefakte durch Variation in der Flugrichtung der Teilchen, die das Auflösungsvermögen des Flugzeitmassenspektrometers beeinträchtigen würden.

Die Methode der Überschallexpansion bietet nicht die Möglichkeit, die Anzahl $N\left(\mathrm{H}_{2} \mathrm{O}\right)$ der Konstituenten der erzeugten Cluster genau vorherzubestimmen. Vielmehr werden ausschließlich Cluster erzeugt, deren Konstituentenzahl sich über einen breiteren Bereich verteilt. Durch die Geometrie der Düse sowie durch Auswahl einer geeigneten Kombination der Parameter Reservoirtemperatur $\left(T_{\text {Reservoir }}\right)$, Düsentemperatur ( $\left.T_{\text {Düse }}\right)$ Stagnationsdruck $\left(p_{\text {Gas }}\right)$ und Zusammensetzung des Trägergases lassen sich die Breite dieser Größenverteilung sowie die Lage ihres Maximums beeinflussen. Letztere Parameter beeinflussen ebenfalls die Geschwindigkeit der Abkühlung der Cluster im Inneren der Düse, von der die Wahrscheinlichkeit abhängt, dass diese ihre thermodynamisch günstigste Topologie einnehmen. Als Trägergase stehen die Edelgase Helium, Neon und Argon zur Auswahl. 


\subsection{Pickup-Zelle}

Die Pickup-Zelle besteht aus einer mit Natriumdampf gefüllten Gaszelle, die vom Molekularstrahl durchquert wird. In Abhängigkeit vom Dampfdruck des Natriums nehmen die Cluster dabei eines oder mehrere Natriumatome auf.

Diese Zelle wurde von Christof Steinbach im Rahmen seiner Promotion konstruiert 209|210]. Sie besteht aus zwei vertikal angeordneten, miteinander verschraubten Edelstahlkomponenten, deren Innenräume durch eine Öffnung miteinander verbunden sind: Der untere Teil dient als Reservoir, das mit metallischem Natrium gefüllt ist. Durch Erwärmen auf Temperaturen von 220 bis $240^{\circ} \mathrm{C}$ gehen Natriumatome in die Gasphase über. Der obere Teil der Pickup-Zelle besteht aus einem 5,4 cm langen Kanal, in dem der Molekularstrahl mit den Natriumatomen in Kontakt gebracht wird. Dieser Bereich wird ebenfalls beheizt, um ein Verstopfen des Kanals durch kondensierendes Natrium zu vermeiden. Die Temperatur wird jedoch jeweils um $40^{\circ} \mathrm{C}$ niedriger eingestellt, als die des Reservoirs, um die Kondensation

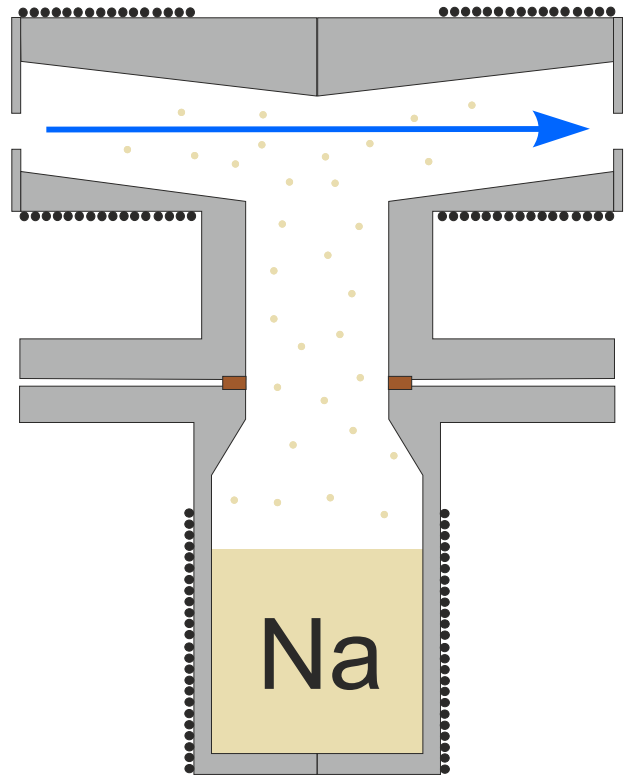

Abbildung 4.3.: Schematischer Aufbau der Natrium-Pickup-Zelle, verändert nach Referenz 251. überschüssiger Natriumatome in diesem Bereich zu ermöglichen und die Menge aus der Gaszelle austretender Natriumatome möglichst gering zu halten. Hierzu wird weiterhin die Größe der Eintritts- und Austrittsöffnungen für den Molekularstrahl durch Blendenbleche begrenzt. Die Wandungen des Kanals sind so gestaltet, dass ein Gefälle in Richtung des Durchgangs zum Reservoir entsteht, sodass an den Kanalwandungen kondensiertes Natrium in selbiges zurückfließen kann.

Reservoir und Gaszelle sind durch $1 \mathrm{~mm}$ starke Thermokoaxial-Heizdrähte separat beheizbar. Durch zwei Thermoelemente, die außen an der Unterseite des Reservoirs und seitlich in der Mitte der Gaszelle angebracht sind, erfolgt eine Rückmeldung der Temperaturen an die Heizungssteuerung. Durch Wahl der Temperatur kann die Anzahl der durchschnittlich aufgenommenen Natriumatome pro Cluster eingestellt werden. Der schematische Aufbau der Pickup-Zelle ist in Abbildung 4.3 dargestellt.

Die Pickup-Zelle ist innerhalb der Zwischenkammer an einer Mechanik angeschraubt, die zu Justagezwecken eine Variation ihrer Position in horizontaler und vertikaler Richtung jeweils senkrecht zum Molekularstrahl ermöglicht. Die Positionsänderung kann während des Betriebs über Stellräder an der Außenseite der Zwischenkammer vorgenommen werden. Diese sind mit Schrittzählern versehen, um die Positionen reproduzieren zu können. Um die Mechanik und das Kammergehäuse thermisch gegen die beheizte Zelle zu isolieren, sind zwei ca. $5 \mathrm{~mm}$ starke Keramikplatten zu beiden Seiten der Befestigungslasche an der Pickup-Zelle zwischen den verschraubten Metallteilen eingesetzt. 


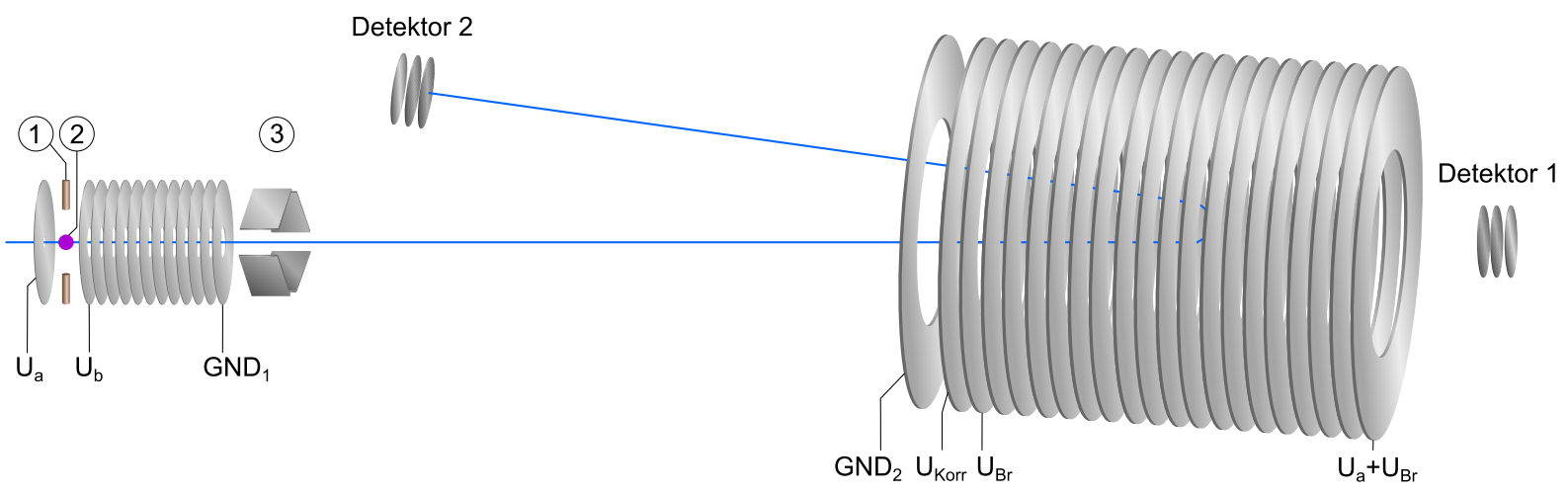

Abbildung 4.4.: Aufbau des Re-TOF-Massenspektrometers, bestehend aus Ionenquelle (links) und Reflektron (rechts). 1: Kupferelektroden; 2: Optimale Ionisationszone; 3: Ablenkkondensatoren; U: Anliegende Gleichspannungen; GND: Masse (ground)

\subsection{Reflektron-Flugzeitmassenspektrometer}

Die Ionisation der natriumdotierten Cluster, deren Separation nach ihrem $m / z$-Verhältnis sowie deren Erfassung finden in der Detektionskammer statt. Der Aufbau dieses Kompartiments ist schematisch in Abbildung 4.4 dargestellt.

Im Zuge der Detektion werden die Anzahl der erzeugten Ionen sowie deren Masse erfasst. Die zu untersuchenden Cluster werden in der Molekularstrahlquelle kontinuierlich erzeugt. Auch die Dotierung der Cluster in der Pickup-Zelle stellt einen kontinuierlichen Prozess dar. Die zur Bereitstellung der benötigten Laserstrahlung im IR- und UV-Bereich erforderliche Strahlungsdichte lässt sich jedoch nur in gepulsten Lasersystemen mit vertretbarem Aufwand realisieren. Die Ionisation der Cluster erfolgt durch Photonen in dem violett dargestellten Bereich der Ionenquelle (Abb. 4.4, links).

Da somit eine gepulste Ionenquelle zur Verfügung steht, bietet sich aus ökonomischen Gründen die Kombination mit einem Flugzeitmassenspektrometer (engl. time of flight, TOF) an. Die Massentrennung erfolgt anhand der verschiedenen Flugzeiten von Ionen mit unterschiedlichem $m / z$-Verhältnis bei annähernd gleicher kinetischer Energie. Am Ende der Flugbahn werden die Ionen durch einen MCP-Detektor 259 registriert.

In Tabelle 4.1 sind die Längen der Wegstrecken zusammengefasst, die der Molekularstrahl in den elektrischen Feldern der jeweiligen Komponenten zurücklegt.

\begin{tabular}{l|l|l}
\hline Ionenquelle & Abzugsfeld & $10 \mathrm{~mm}$ \\
& Beschleunigungsfeld & $44 \mathrm{~mm}$ \\
\hline Reflektron & Reflektorfeld & $216 \mathrm{~mm}$ \\
& Bremsfeld & $6 \mathrm{~mm}$ \\
& Korrekturfeld & $8 \mathrm{~mm}$ \\
\hline Driftstrecke & linear (ohne Reflektron) & $1330 \mathrm{~mm}$ \\
& mit Reflektron & $1820 \mathrm{~mm}$ \\
\hline
\end{tabular}

Tabelle 4.1.: Maße der Komponenten des Reflektron-Flugzeitmassenspektrometers, entnommen aus 71 


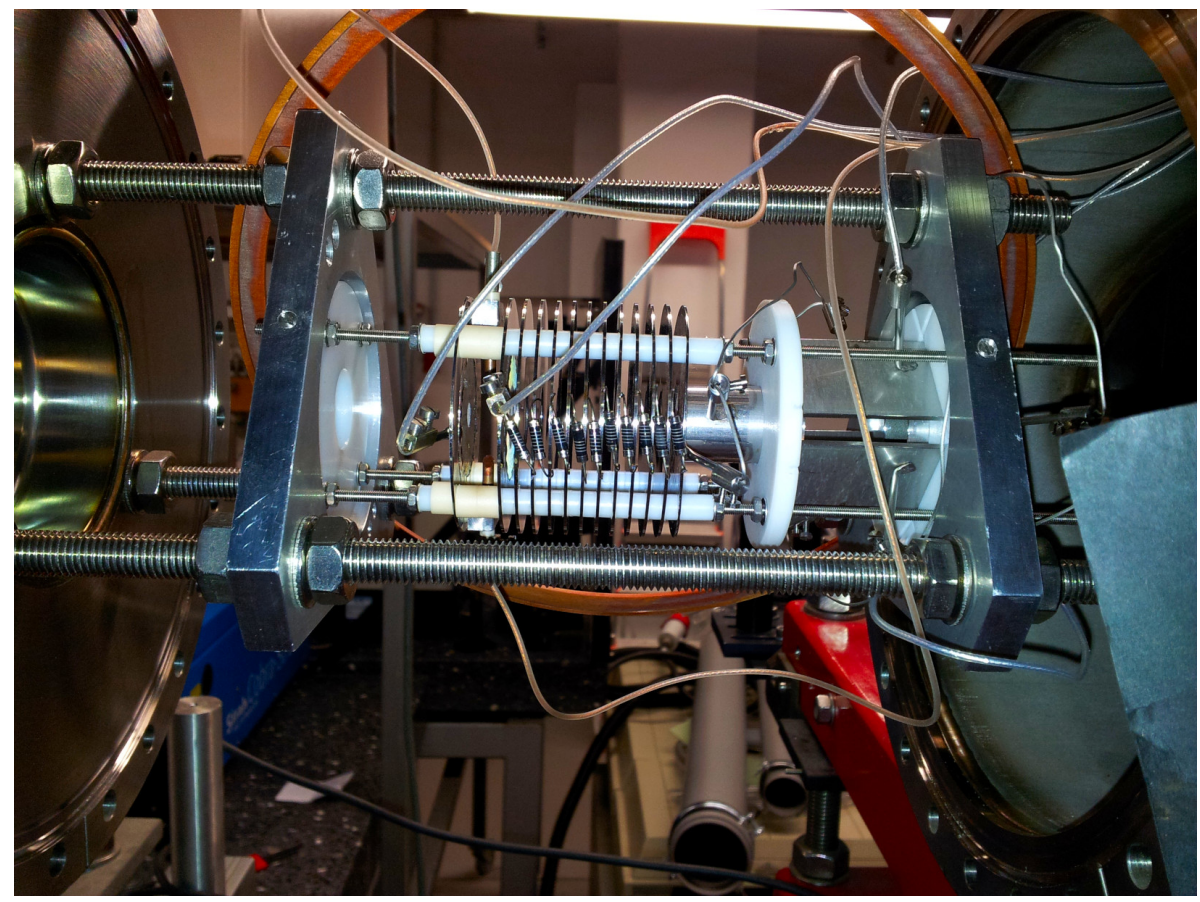

Abbildung 4.5.: Foto der Ionenquelle, aufgenommen im Juli 2013. Der Eintritt des Molekularstrahls erfolgt von der linken Seite.

\subsubsection{Aufbau der Ionenquelle}

Die natriumdotierten Wassercluster gelangen durch ein Ultrahochvakuum- (UHV)-Ventil aus der Zwischenkammer in die Detektionskammer. Dort passieren sie eine ringförmige Lochblende aus Edelstahl, an der eine Spannung von ca. $1210 \mathrm{~V}\left(U_{\mathrm{a}}\right)$ anliegt. Deren zentrale Öffnung ist mit einem Netz aus feinem Nickeldraht bespannt, das eine Transmission von $90 \%$ aufweist. In dem daran anschließenden Bereich erfolgt die Ionisation der Cluster durch das in Abschnitt 4.7 beschriebene UV/VIS-Lasersystem. Die hierbei emittierten Elektronen werden über zwei oberhalb und unterhalb der Ionisationszone angeordnete Kupferanoden abgeleitet. Diese besitzen einen Durchmesser von $3 \mathrm{~mm}$ und werden mit der gleichen Spannung versorgt. Im Anschluss an die Ionisationszone folgt im Strahlweg eine Anordnung von 12 über Widerstände mit dem Nennwert $1 \mathrm{M} \Omega$ elektrisch miteinander verbundene Lochblenden, von denen an der Ersten eine Spannung von $U_{\mathrm{b}}=1000 \mathrm{~V}$ anliegt. Die letzte Blende im Strahlweg ist auf Erdpotential gelegt $\left(\mathrm{GND}_{1}\right.$ in Abbildung 4.4), sodass sich entlang der 12 Blenden ein Potentialgradient ausbildet. Da die Blende bei $U_{\mathrm{a}}$ auf stärker positivem Potential liegt als die Blende bei $U_{\mathrm{b}}$, erfolgt nach dem UV-Laserpuls zunächst eine Beschleunigung der erzeugten Kationen in Richtung der letzteren Blende. Eine weitere Beschleunigung erfahren die Cluster beim Durchqueren der Potentialdifferenz zwischen den Blenden auf den Potentialen $U_{\mathrm{b}}$ und $\mathrm{GND}_{1}$. Die hierfür notwendige Homogenität des zwischen den Elektroden aufgebauten elektrischen Feldes wird durch Bespannung der Lochblenden bei $U_{\mathrm{b}}$ und $\mathrm{GND}_{1}$ mit Nickelnetzen sowie durch zehn weitere dazwischen angeordnete Blenden gewährleistet. Die Flugrichtung der beschleunigten Cluster kann über zwei diagonal angeordnete Ablenkkondensatoren mit $40 \mathrm{~mm}$ Länge und $25 \mathrm{~mm}$ Breite optimiert werden, die nach dem Durchtritt durch Blende GND $_{1}$ passiert werden, um sie in Richtung des Detektor zu lenken. 
Auch die Ionisation der Cluster durch Elektronenstöße ist möglich. Hierzu muss die elektrische Verschaltung der oben beschriebenen Komponenten wie in Referenz [256] beschrieben erfolgen. Die elektrischen Felder der Ionenquelle werden in diesem Betriebsmodus gepulst und mit der Emission des UV-Lasers synchronisiert. Im Moment des Laserpulses liegt eine Potentialdifferenz zwischen den Kupferelektroden an, während die Beschleunigungsfelder abgeschaltet sind. Durch die UV-Strahlung werden Elektronen aus der unteren Kupferelektrode herausgelöst. Die kinetische Energie der Elektronen ist dabei abhängig von der Potentialdifferenz zwischen oberer und unterer Kupferelektrode zum Zeitpunkt des Laserpulses. In der Folge treffen die Elektronen im Ionisationsvolumen auf den Molekularstrahl und lösen Elektronen aus dessen Konstituenten heraus. Nach dem UV-Laserpuls wird die Spannung der unteren Elektrode innerhalb von 27 ns der Spannung der oberen Elektrode angeglichen, um die entstandenen Ionen nicht durch ein zusätzliches elektrisches Feld senkrecht zur Flugrichtung abzulenken. Die Beschleunigungsspannungen $U_{\mathrm{a}}$ und $U_{\mathrm{b}}$ sowie die Spannungen der Ablenkkondensatoren werden nach dem Laserpuls mit einer Anstiegszeit von 30 ns aufgebaut.

Die dem Ionisationsvolumen nachgeordneten Blenden weisen einen Außendurchmesser von $60 \mathrm{~mm}$ und einen Innendurchmesser von $15 \mathrm{~mm}$ auf. Die davor angeordnete Blende bei $U_{\mathrm{a}}$ besitzt einen geringeren Innendurchmesser von $6 \mathrm{~mm}$. Die Ionenquelle ist über M10-Gewindestangen mit der Wandung der Detektionskammer verbunden, sodass ihre Position nur mit großem Aufwand verändert werden kann. Die elektrischen Zuleitungen sind durch einen DN 100-Flansch geführt, der oberhalb der Ionenquelle angebracht ist. Zu Wartungszwecken wird der DN 200-Flansch zwischen Detektions- und Zwischenkammer geöffnet.

\subsubsection{Aufbau des Reflektrons}

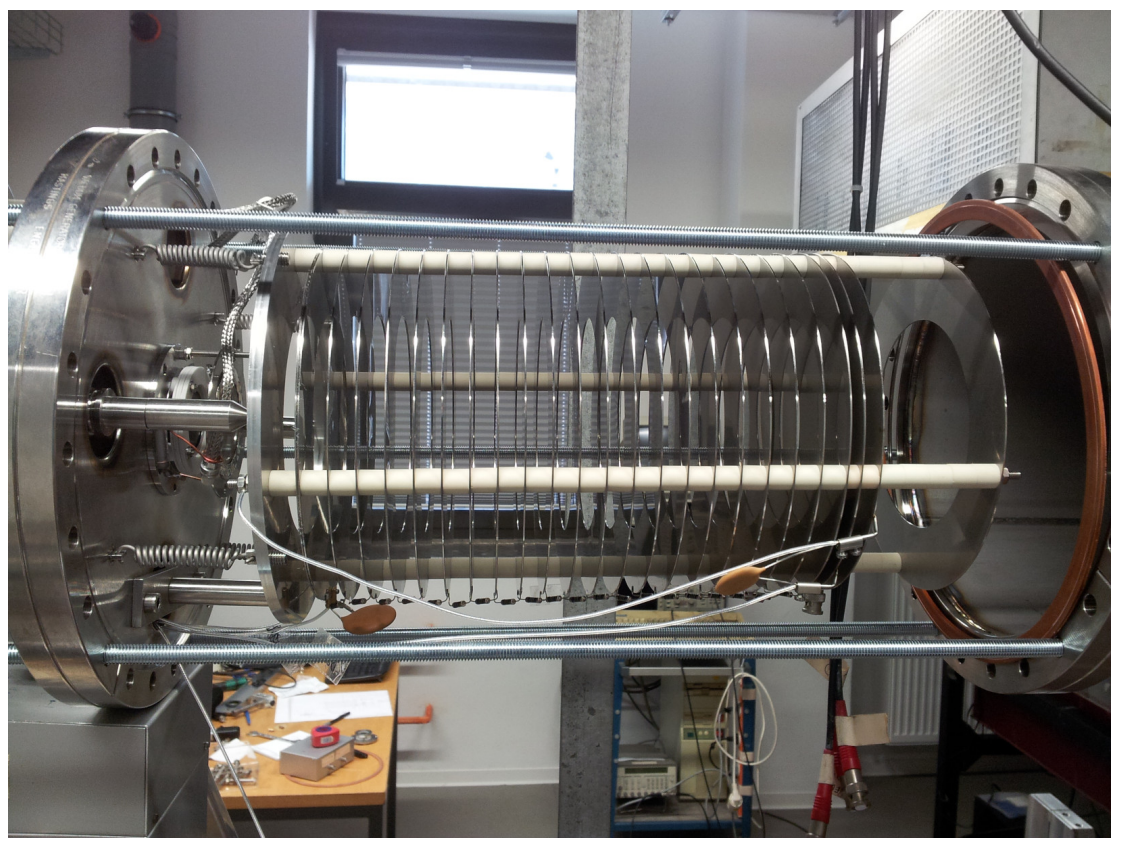

Abbildung 4.6.: Foto des ausgebauten Reflektrons, aufgenommen im Juli 2013. 


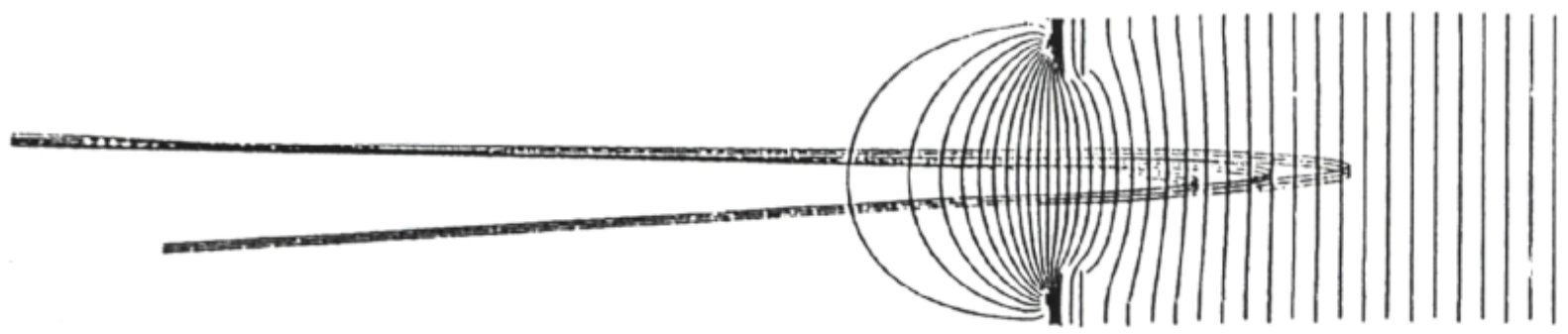

Abbildung 4.7.: Simulation von Ionentrajektorien bei drei verschiedenen Impulswerten im netzlosen Reflektron nach Risch 260 , entnommen aus 255.

Die am Reflektron eintreffenden, kationischen Cluster werden durch drei elektrische Felder unterschiedlicher Stärke abgebremst, fokussiert und schließlich in Richtung des MCPDetektors Nr. 2 beschleunigt. Für deren Erzeugung werden wie in der Ionenquelle Lochblenden aus Edelstahl verwendet (siehe Abbildung 4.6). Unter optimalen Bedingungen lässt sich mit dieser Anordnung ein Auflösungsvermögen von $R_{\mathrm{m}}=1600$ erreichen 53 .

Die Blenden besitzen einen Außendurchmesser von $180 \mathrm{~mm}$ und einen Innendurchmesser von $100 \mathrm{~mm}$. Anders als in der Ionenquelle ist lediglich die letzte Platte bei $U_{\mathrm{a}}+U_{\mathrm{Br}}$ mit einem Netz bespannt. Im Inneren entsteht daher ein inhomogenes elektrisches Feld, das in Richtung der Blende bei $U_{\mathrm{a}}+U_{\mathrm{Br}}$ zunehmend homogen wird (vgl. Abb. 4.7). Die Platte $\mathrm{GND}_{2}$ liegt auf Erdpotential. Das elektrische Feld zwischen $\mathrm{GND}_{2}$ und $\mathrm{U}_{\text {Korr }}$ dient der besseren Fokussierung des Clusterstrahls. Das nachfolgende Feld (bis zur Blende $\mathrm{U}_{\mathrm{Br}}$ ) wird als Bremsfeld bezeichnet. Während der Passage dieser Zone verlieren die Ionen zwei Drittel ihrer kinetischen Energie. Das dritte elektrische Feld wird als Reflektorfeld bezeichnet. Es wird, ähnlich dem zweiten Beschleunigungsfeld der Ionenquelle, über 18, in Abbildung 4.4 grau dargestellte Lochblenden erzeugt, die über Widerstände mit Nennwerten von $300 \mathrm{k} \Omega$ mit den jeweils angrenzenden Blenden leitend verbunden sind.

Die Lochblenden sind auf vier M4-Gewindestangen montiert, wobei sie durch keramische Hülsen und Rohre (Degussit) in Position gehalten und elektrisch gegen die Metallstangen isoliert werden. Die Gewindestangen sind auf einer $5 \mathrm{~mm}$ starken Grundplatte aus Aluminium montiert. Die gesamte Anordnung ist über vier Federn und drei Metalldorne auf einem DN 200-Blindflansch angebracht. Zwei der drei Dorne können über Vakuumdurchführungen in der Länge variiert werden, sodass die Neigung des Reflektrons in horizontaler und vertikaler Ebene verändert werden kann.

\subsubsection{Aufbau der Detektoreinheit}

Der Nachweis der kationischen Cluster erfolgt mit Hilfe von MCP-Detektoren 259] (vgl. Abschn. 3.2.5). Die Platten der hier eingesetzten Instrumente bestehen aus einem Grundkörper aus Bleiglas, der auf beiden Seiten eine metallische Beschichtung aufweist. In diese Platten sind zahlreiche, mikroskopisch kleine Kanäle mit einem Durchmesser von $10 \mu \mathrm{m}$ eingebracht, deren Ausrichtung um ca. $10^{\circ}$ gegen den Normalvektor Plattenoberfläche geneigt ist. Die Innenwände der Kanäle sind mit einem Halbleitermaterial ausgekleidet. Zwischen den Seiten einer jeden Platte liegt eine Spannung in Höhe von maximal $1 \mathrm{kV}$ an. Trifft ein Teilchen mit ausreichender Geschwindigkeit auf die Innenwand eines solchen Kanals, so werden Elektronen aus der Wand herausgelöst und in Richtung des 


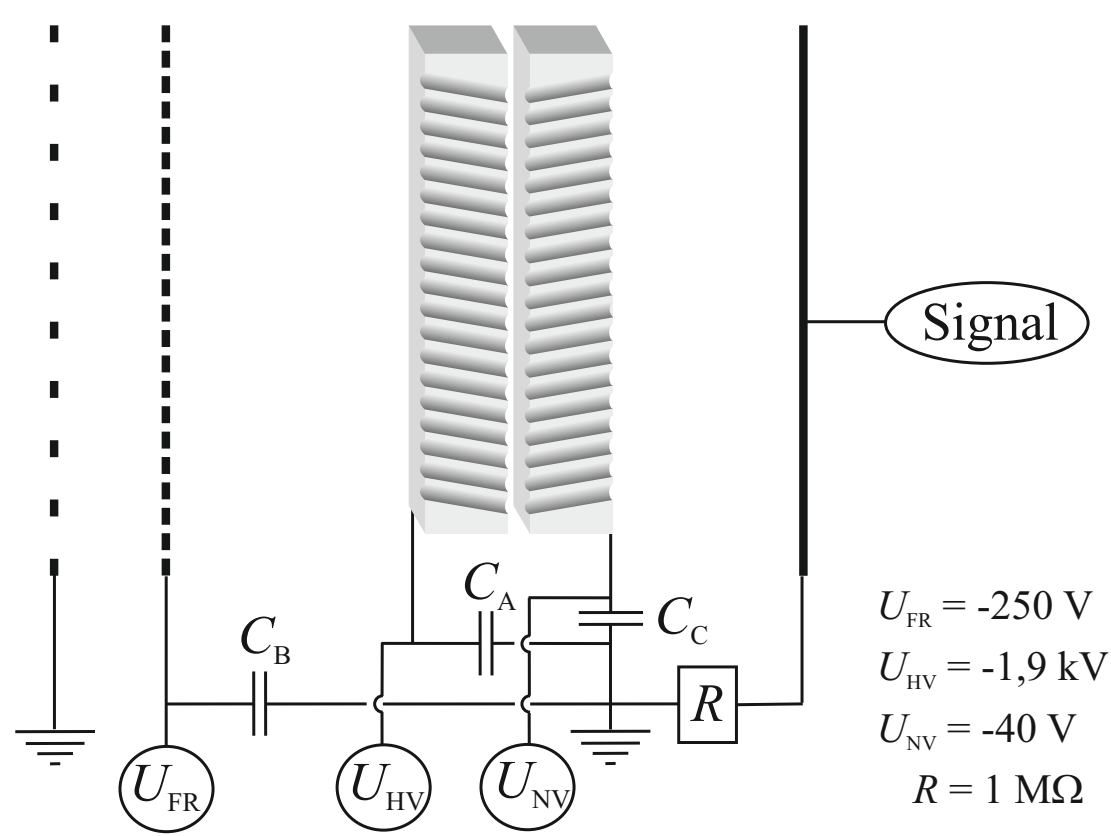

Abbildung 4.8.: Beschaltung des MCP-Detektors Nr. 2. Die Bezeichnungen $C_{\mathrm{A}}, C_{\mathrm{B}}$ und $C_{\mathrm{C}}$ repräsentieren Kondensatoren; verändert nach Referenz 71

elektrischen Feldes im Inneren der Platte beschleunigt. Durch die Neigung der Kanäle treffen die herausgelösten Elektronen erneut auf der Kanalinnenwand auf und lösen die Emission weiterer Elektronen aus. Die so erwirkte Signalverstärkung ist dabei abhängig von der an der Platte anliegenden Spannung. Darüber hinaus kann sie gesteigert werden, indem zwei (chevron) oder drei (z-stack) MCP derart hintereinander angeordnet werden, dass die Neigungsrichtung ihrer Kanäle um $180^{\circ}$ relativ zueinander rotiert ist.

In der Detektionskammer sind zwei chevron-MCP-Detektoren eingebaut: Detektor 1 ist direkt hinter dem Reflektron angeordnet und wird nur zu Wartungszwecken verwendet, um mögliche Defekte am Reflektron zu identifizieren. Detektor 2 ist oberhalb der Ionenquelle angebracht (vgl. Abb. 4.4) und wird im regulären Messbetrieb eingesetzt.

Die eingesetzten MCP-Detektoren besitzen einen Durchmesser von $25 \mathrm{~mm}$. Als Detektor 1 wird ein Chevron MCP (Modell: Double MCP; B035VA) der Firma El-Mul verwendet, Detektor 2 wurde von der Firma Tectra (Modell: MCP-25-D-S-A) hergestellt.

Die Beschaltung von Detektor 2 ist in Abbildung 4.8 dargestellt. Der Detektor wird durch ein Edelstahlgehäuse nach außen abgeschirmt, an dessen Vorderseite sich eine mit einem Netz bespannte Öffnung befindet, durch die der Ionenstrahl eintreten kann. Um die Empfindlichkeit des Detektors auf große Cluster zu erhöhen, ist im Inneren des Gehäuses ein weiteres Kupfernetz eingebracht, das eine Transmission von $50 \%$ aufweist. Diese Anordnung dient dazu, schwere Ionen zu fragmentieren und nachzubeschleunigen, da die Empfindlichkeit des MCP-Detektors nicht von der kinetischen Energie, sondern von der Geschwindigkeit der auftreffenden Partikel abhängt 2566. Diese ist im TOFMassenspektrometer bei großen Ionen naturgemäß niedriger. Die Kondensatoren dienen dazu, Ladungsträger nachzuliefern um einem kurzzeitigen Abfall der Detektorspannungen zu begegnen 255. Die am MCP-Detektor anliegende Hochspannung $U_{\mathrm{HV}}$ wird je nach zu erwartender Signalstärke auf einen Wert zwischen $-1,7 \mathrm{kV}$ und $-1,9 \mathrm{kV}$ eingestellt. Die 
maximal zulässige Spannung, die an den verwendeten Chevron-MCP-Detektor angelegt werden darf, beträgt $2 \mathrm{kV}$. Zur Erhöhung der Lebensdauer des Bauteils wird diese Maximalspannung nur zu Testzwecken angelegt. Eine statische Aufladung der Anode wird durch einen Massewiderstand mit einem Wert von $1 \mathrm{M} \Omega$ verhindert. Trifft ein Ion auf den Detektor, so tritt an der Anode ein Spannungspuls von ca. $4 \mathrm{mV}$ mit einer Dauer von 2-3 ns auf, der über ein mit Bayonet Neill-Concelman- (BNC)-Steckverbindungen versehenes Koaxialkabel an die im Messrechner befindliche Zählerkarte übertragen wird und dort über einen $50 \Omega$-Widerstand abfällt.

Die Beschaltung von Detektor 1 weicht von dieser Beschreibung ab. Er wird lediglich durch eine geerdete, mit einem Netz bespannte Blende gegen die elektrischen Felder der Driftstrecke abgeschirmt. Auf ein Fragmentierungsnetz wurde hier aus Platzgründen verzichtet. Da die Versorgung mit Hilfe einer Hochspannungsquelle realisiert wird, die über einen Gleichspannungswandler die Spannungen $U_{\mathrm{HV}}$ und $U_{\mathrm{NV}}$ bereitstellt, kann hier außerdem auf die Kondensatoren verzichtet werden. $U_{\mathrm{HV}}$ ist dabei direkt abhängig von der angelegten Spannung, $U_{\mathrm{NV}}$ wird ebenfalls auf $-40 \mathrm{~V}$ eingestellt.

\subsection{Vakuumsystem}

\subsubsection{Aufbau des Vakuumsystems}

Die drei Kompartimente der Apparatur werden durch separate Vakuumsysteme evakuiert, um in ihnen unterschiedliche Enddrücke einzustellen. Eine schematische Übersicht über die in der P11 eingesetzten Pumpentypen und deren Anordnung ist in Abb. 4.9 dargestellt. Eine Übersicht über die Spezifikationen der verwendeten Pumpen findet sich in Tabelle 4.3 .

\subsubsection{Vakuumpumpen}

Zur Vakuumerzeugung sind für jede Kammer mindestens zwei Pumpen hintereinander angeordnet. Eine Drehschieberpumpe stellt jeweils das Vorvakuum bereit, das für den Betrieb einer Hochvakuumpumpe erforderlich ist. Als Hochvakuumpumpen kommen in der Expansionskammer und in der Zwischenkammer je eine Öldiffusionspumpe zum Einsatz, da dieser Pumpentyp keine beweglichen Teile enthält und daher kaum anfällig für Störungen ist. Als Treibmittel für die Öldiffusionspumpen wird Leybonol LVO 500 verwendet. Aufgrund der hohen Gaslast in der Expansionskammer ist hier eine Wälzkolbenpumpe zwischen Drehschieber- und Öldiffusionspumpe angeordnet, um den Durchsatz des Pumpensystems zu erhöhen.

Zur Erzeugung des Vorvakuums der Expansionskammer können zwei unterschiedliche Pumpstände verwendet werden. Gewöhnlich wird Pumpstand 1 betrieben, der wie oben beschrieben aufgebaut ist. In diesem werden alternierend zwei Drehschieberpumpen (Modell Leybold D60A oder Leybold D65BCS) eingesetzt. Der Austausch erfolgt, sobald die im Einsatz befindliche Pumpe einer Wartung bedarf. Pumpstand 2 dient als Reservesystem für den Fall eines Defektes in Pumpstand 1. Hier wird das Vorvakuum über eine Sperrschieberpumpe erzeugt. Als Zwischenstufe kommen zwei parallel geschaltete Wälzkolbenpumpen zum Einsatz (siehe Tabelle 4.3). 


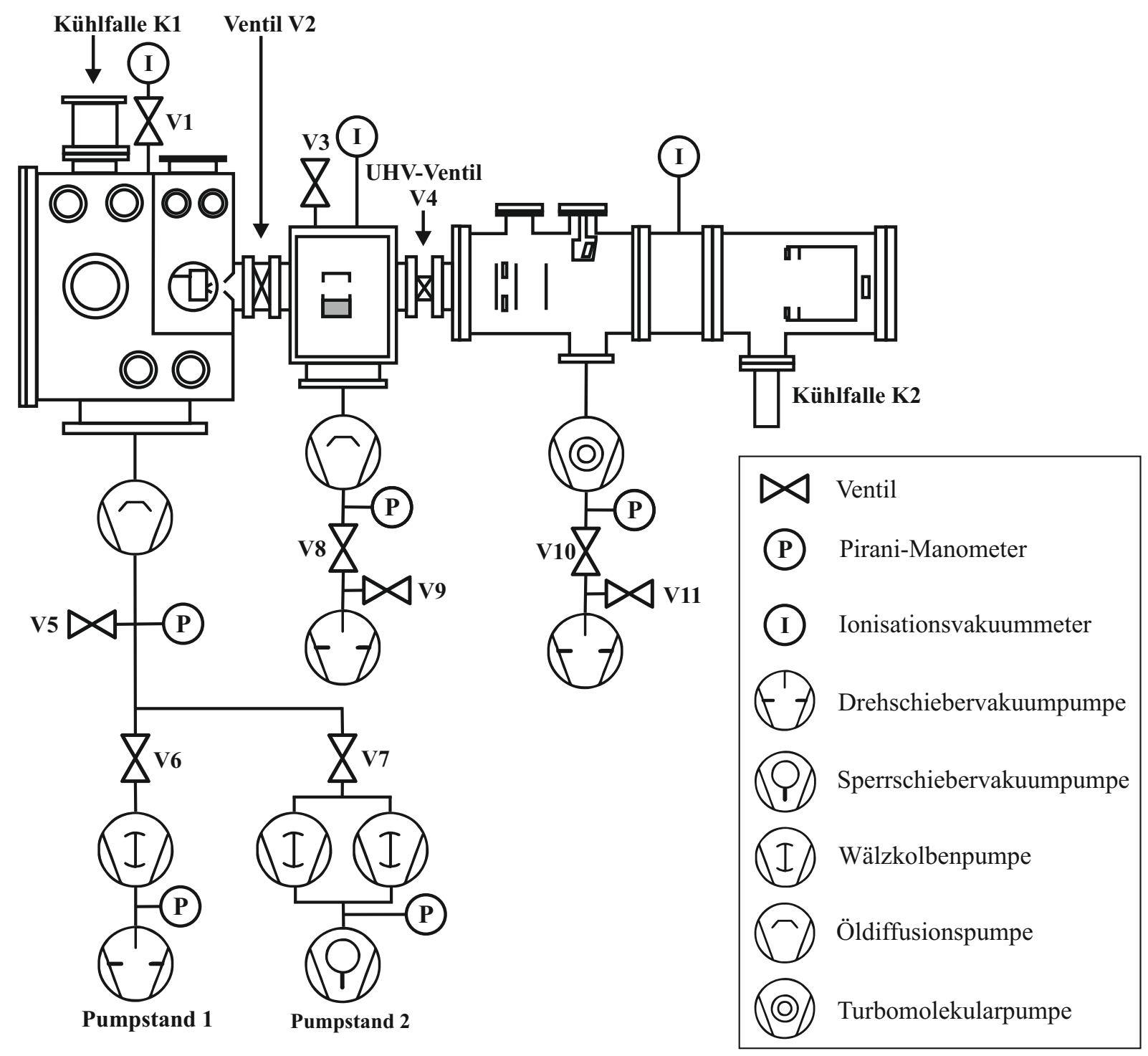

Abbildung 4.9.: Übersicht über das Vakuumsystem. Verändert nach Referenz 251] 
Zur Verbesserung des Vakuums in der Expansionskammer ist an deren Oberseite eine Kühlfalle angebracht, die mit flüssigem Stickstoff befüllt wird und durch Kondensation Bestandteile des Analyten sowie des Pumpenöls aus der Gasphase in der Kammer entfernt.

In der Detektionskammer herrschen besonders hohe Anforderungen an die Qualität des Vakuums, da hier die mittlere freie Weglänge der Cluster möglichst lang sein sollte, um Kollisionen mit anderen Bestandteilen der Gasphase zu vermeiden. Daher wird hier eine Turbomolekularpumpe eingesetzt, die verbliebene Gasbestandteile nicht durch einen Strom von Ölmolekülen, sondern durch einen mit Schaufeln besetzten Rotor aus dem Kammervolumen entfernt. Diese Pumpe besitzt lediglich auf der Hochdruckseite ein Reservoir mit Schmiermittel für das Lager der Rotorachse, sodass die Evakuierung dieser Kammer möglichst kontaminationsarm erfolgt. Stromversorgung und Steuerung der Turbomolekularpumpe erfolgen über ein separates Steuergerät (Modell TCP 310 der Firma Pfeiffer Vacuum). Die Detektionskammer verfügt ebenfalls über eine Kühlfalle, deren Verwendung jedoch keine erkennbare Verbesserung des Vakuums zur Folge hat.

\subsubsection{Druckmessung}

In der Expansionskammer sowie in der Zwischenkammer erfolgt die Druckmessung über Kaltkathoden-Ionisationsvakuummeter (Modell: Leybold Ionivac IE 20 - 163 14). In der Detektionskammer kommt hierfür ein Glühkathoden-Ionisationsvakuummeter nach Bayard und Alpert zum Einsatz. Die zwischen den Pumpstufen herrschenden Drücke werden mit Hilfe von Pirani-Vakuummetern überwacht.

\subsubsection{Betriebsparameter}

Bei voller Pumpenleistung und inaktiver Molekularstrahlquelle beträgt der Hintergrunddruck in der Expansionskammer ca. $1,3 \cdot 10^{-5} \mathrm{mbar}$ (ca. $10^{-5} \mathrm{mmHg}$ ). Während der Messung steigt dieser Wert auf ca. 2,6 $10^{-4} \mathrm{mbar}$ (ca. $2 \cdot 10^{-4} \mathrm{mmHg}$ ) an. Ein weiterer Anstieg des Hintergrunddruckes auf Werte oberhalb von $5 \cdot 10^{-3}$ mbar führt zum Zusammenbruch der Expansion, da die mittlere freie Weglänge der Gasmoleküle in der Expansionskammer zu kurz wird. Folgende Druckwerte sollten im Leerlauf bzw. im Betriebszustand erreicht werden:

\begin{tabular}{c|c|c}
\hline KomPARTIMENT & $p$ (Leerlauf) & $p$ (Betrieb) \\
\hline Expansionskammer & $<2 \cdot 10^{-5} \mathrm{mbar}$ & $<5 \cdot 10^{-3} \mathrm{mbar}$ \\
Zwischenkammer & $<1,2 \cdot 10^{-7} \mathrm{mbar}$ & $<1 \cdot 10^{-5} \mathrm{mbar}$ \\
Detektionskammer & $<1,3 \cdot 10^{-6} \mathrm{mbar}$ & $<2 \cdot 10^{-6} \mathrm{mbar}$ \\
\hline
\end{tabular}

Tabelle 4.2.: Richtwerte der in den drei Kompartimenten herrschenden Druckwerte im Leerlauf bzw. im Betriebszustand 


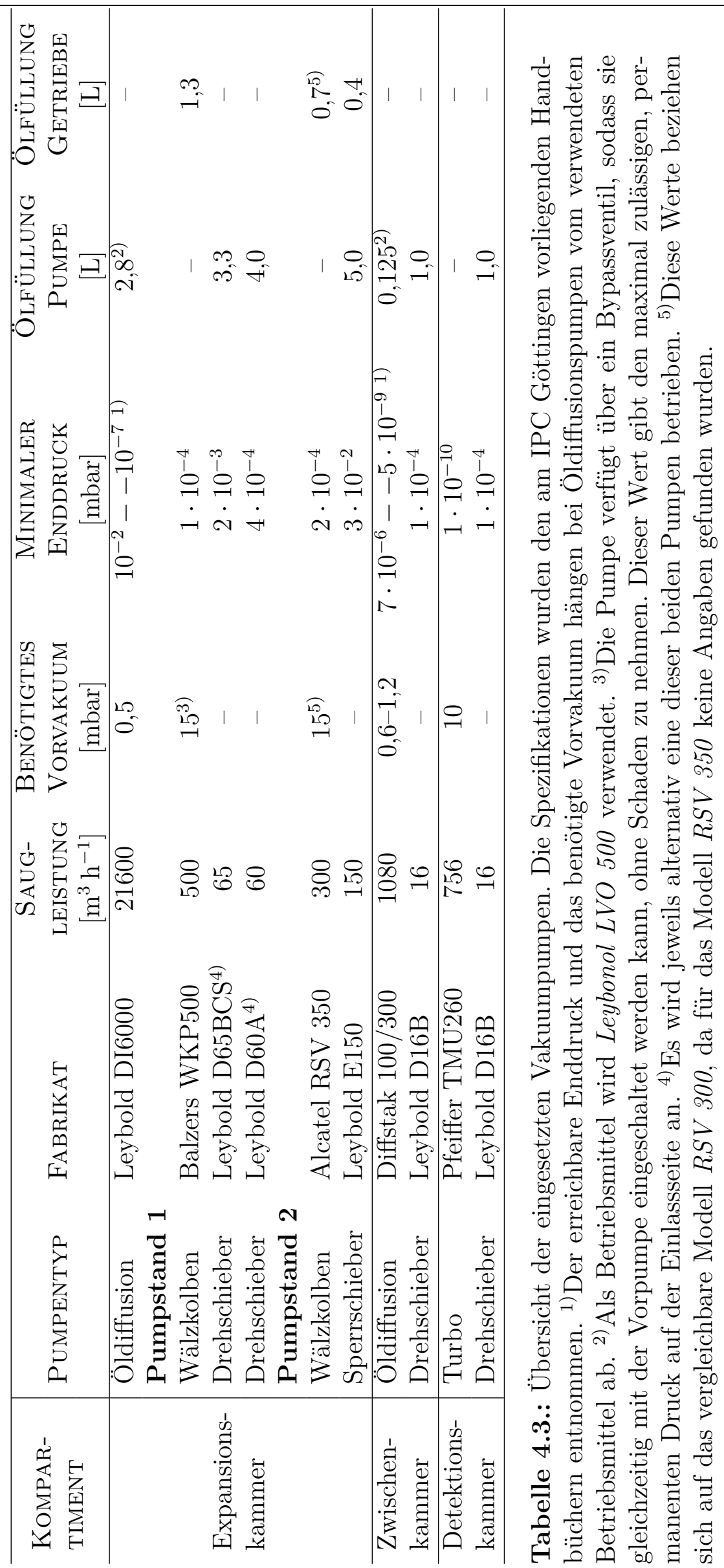




\subsection{Infrarot-Lasersystem}

Das Infrarot-Lasersystem besteht aus einem Nd:YAG-Festkörperlaser (Nd:YAG: neodymdotierter Yttrium-Aluminium-Granat; Modell: Powerlite Precision II 8000) der Firma Continuum, der Strahlung der Wellenlänge $1064 \mathrm{~nm}$ emittiert. Dieser wird zum optischen Pumpen eines OPO/OPA-Systems (OPO: optical parametric oscillator; OPA: optical parametric amplifier) der Firma LaserVision verwendet. Letzteres erzeugt durchstimmbare Laserstrahlung mit Wellenlängen im Bereich zwischen $710 \mathrm{~nm}$ und $885 \mathrm{~nm}$ sowie zwischen $1.35 \mu \mathrm{m}$ und $5 \mu \mathrm{m}$. Mit Hilfe dieses Lasersystems ist es möglich, sowohl OH- als auch CH- Streckschwingungen im Spektralbereich von $2600 \mathrm{~cm}^{-1}$ bis $3800 \mathrm{~cm}^{-1} \mathrm{zu}$ untersuchen. Die Spezifikationen dieses Systems sind in Tabelle 4.4 zusammengefasst, die dem Benutzerhandbuch 261 der Firma LaserVision entnommen ist.

\subsubsection{Nd:YAG-Pumplaser Powerlite Precision II 8000}

Als Pumplaser für das OPO/OPA-System kommt ein Nd:YAG-Festkörperlaser (Modell: Powerlite Precision II 8000 der Firma Continuum) zum Einsatz. Dieser emittiert Strahlung mit einer Wellenlänge von $1064 \mathrm{~nm}\left(\right.$ ca. $\left.9400 \mathrm{~cm}^{-1}\right)$. Abbildung 4.10 veranschaulicht schematisch den Aufbau des Gerätes.

Als aktive Medien werden in diesem Lasersystem stabförmige Kristalle aus YttriumAluminium-Granat $\left(\mathrm{Y}_{3} \mathrm{Al}_{5} \mathrm{O}_{12}\right)$ eingesetzt, die mit dreiwertigen Neodymionen $\left(\mathrm{Nd}^{3+}, \mathrm{An}-\right.$ teil ca. $0,6 \%$ bis 1,4\%) dotiert sind. Der Laser ist mit zwei Laserköpfen ausgestattet, in denen sich jeweils ein durch Xenon-Blitzlampen gepumpter Nd:YAG-Kristall befindet: Hauptaufgabe des ersten Laserkopfes im Inneren des Oszillators ist die Erzeugung eines möglichst kurzen Laserpulses mit homogenem Strahlprofil und geringer spektraler Bandbreite. Den größten Anteil seiner Strahlungsenergie erhält der Laserpuls während der Passage des zweiten Laserkopfes, der als Verstärker fungiert. Während Länge, Strahlprofil und spektrale Breite des Laserpulses durch den Laserkopf im Oszillator vorgegeben werden, wird die Pulsenergie durch den Grad der Besetzungsinversion im größervolumigen Verstärker zum Durchtrittszeitpunkt des Laserpulses bestimmt. Die eingesetzten Nd:YAG-Kristalle besitzen eine Länge von $115 \mathrm{~mm}$ und einen Durchmesser von $6 \mathrm{~mm}$ (Oszillator) bzw. $9 \mathrm{~mm}$ (Verstärker). An den Enden weisen sie eine antireflexive Beschichtung auf, um die Energieausbeute zu erhöhen.

Der Nd:YAG-Laser arbeitet nach einem Vier-Niveau-Schema: Durch die von den Blitzlampen emittierte Strahlung werden die $\mathrm{Nd}^{3+}$-Ionen aus ihrem elektronischen Grundzustand $\left({ }^{4} \mathrm{I}_{9 / 2}\right)$ angeregt. Aus dem zunächst populierten Zustand $\left({ }^{4} \mathrm{~F}_{5 / 2}\right)$ erfolgt ein rascher, strahlungsloser Übergang in das metastabile ${ }^{4} \mathrm{~F}_{3 / 2}$-Niveau. In diesem Zustand besitzen die Ionen eine hohe mittlere Lebensdauer von ca. $230 \mu \mathrm{s}$. Von hier aus erfolgt der Laserübergang unter Emission von Strahlung. Der mit der höchsten Wahrscheinlichkeit (ca. $60 \%$ ) auftretende Übergang erfolgt in das ${ }^{4} \mathrm{I}_{11 / 2}$-Niveau, wobei Strahlung Wellenlänge von $1064 \mathrm{~nm}$ emittiert wird. Aus diesem Zustand findet wiederum ein strahlungsloser Übergang in den Grundzustand statt 262 .

Da das Pumpen optisch nichtlinearer Bauteile neben einer geringen Bandbreite und einem homogenen Strahlprofil insbesondere eine hohe Intensität der eingehenden Laserstrahlung erfordert, ist der Laser mit einer Güteschaltung (engl. Q-switch) ausgestattet, um die Dauer des Laserpulses zu minimieren und so eine maximale Strahlungsintensität zu 


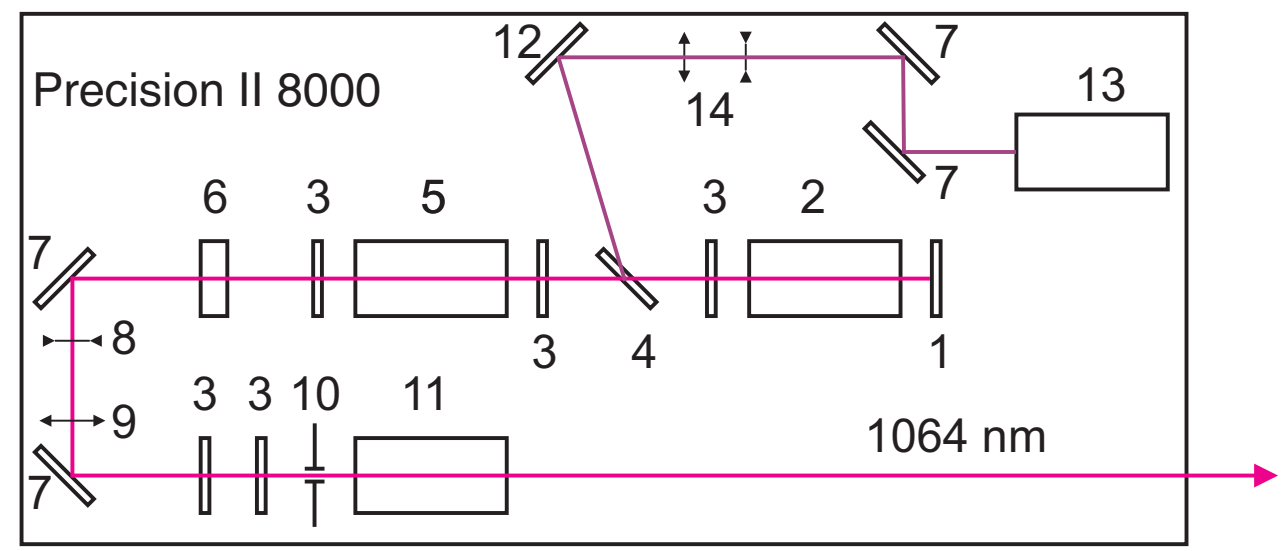

Abbildung 4.10.: Schema des Powerlite Precision II 8000 258|263.

1. Rückwärtiger Spiegel

2. Pockels-Zelle

3. $\lambda / 4$-Platte

4. Dielektrischer Polarisator

5. Laserkopf (Resonator)

6. Auskoppelspiegel

7. Umlenkspiegel, $45^{\circ}$
8. Divergente Linse

9. Konvergente Linse

10. Lochblende

11. Laserkopf (Verstärker)

12. Umlenkspiegel, $32^{\circ}$

13. Seeding Laser

14. Seeding Laser-Teleskop

erreichen. Durch diese Technik wird eine maximale Besetzungsinversion im Lasermedium erzielt, indem eine vorzeitige stimulierte Emission und die damit verbundene Abnahme der Anzahl angeregter $\mathrm{Nd}^{3+}$-Ionen im Lasermedium verhindert wird. Hierzu wird die Güte des Resonators zunächst so weit herabgesetzt, dass spontan emittierte Photonen nicht mehr verstärkt werden und somit kein Lasing-Prozess mehr stattfindet. Durch eine instantane Verbesserung der Resonatorgüte in dem Moment, in dem eine maximale Besetzungsinversion im Lasermedium vorliegt, wird ein intensiver, extrem kurzer Laserpuls erzeugt.

Die Güteschaltung enthält als aktives Element eine Pockels-Zelle, die mit einem Dünnschichtpolarisator und einer $\lambda / 4$-Platte kombiniert ist. Die Pockels-Zelle stellt einen elektrooptischen Schalter dar, der transparent ist, solange an ihm keine Spannung anliegt. Wird eine Spannung von ca. $3,6 \mathrm{kV}$ angelegt, verhält sich die Pockels-Zelle wie eine $\lambda / 4$-Platte. In diesem Laser enthält die Pockels-Zelle einen Kaliumdihydrogenphosphat(KDP)-Kristall als aktives Medium. Der Hochspannungspuls wird durch eine Marx-Bank mit einer Anstiegszeit von 20 ns erzeugt. Der Dünnschichtpolarisator wiederum ist nahezu transparent für horizontal polarisierte Strahlung (Transmission $>95 \%$ ) und fast gänzlich undurchlässig für vertikal polarisierte Strahlung (Reflexion >99\%). Entsprechend der Darstellung in Abbildung 4.11 können im Betrieb die beiden Fälle geschlossener Kavität (spannungsfreie Pockels-Zelle) und geöffneter Kavität (Hochspannung liegt an der Pockels-Zelle an) unterschieden werden: 
1. Geschlossene Kavität: Ist die Pockels-Zelle spannungsfrei, wird sie von der durch das Lasermedium emittierten Strahlung passiert, ohne dass sich beim Durchgang deren Polarisationsvektor ändert. Der Dünnschichtpolarisator erlaubt nur horizontal polarisierter Strahlung den Durchtritt vom Lasermedium in Richtung des rückwärtigen Resonatorspiegels. Diese Strahlung wird bei einem Durchlauf durch die $\lambda / 4$-Platte im ersten Schritt in zirkular polarisierte Strahlung umgewandelt. Am Resonatorspiegel findet eine Reflexion statt und es erfolgt ein zweiter Durchgang durch die Pockels-Zelle sowie durch die $\lambda / 4$-Platte, welche die zirkular polarisierte Strahlung in linear polarisierte Strahlung mit nunmehr vertikalem Polarisationsvektor umwandelt, sodass im Ergebnis eine Drehung des Polarisationsvektors um $90^{\circ}$ erfolgt. Diese vertikal polarisierte Strahlung wird vom Polarisator reflektiert und aus dem Resonator entfernt. Folglich kann keine Strahlung im Resonator umlaufen.

2. GeÖffnete Kavität: Wird ein Hochspannungspuls von $3,6 \mathrm{kV}$ an die Pockels-Zelle angelegt, so fungiert diese als weitere $\lambda / 4$-Platte, die im Zuge zweier Strahlungsdurchläufe eine Drehung der Polarisationsebene um $90^{\circ}$ bewirkt. In Verbindung mit der durch die invariante $\lambda / 4$-Platte erfolgten Drehung um weitere $90^{\circ}$ resultiert eine Rotation der Polarisationsebene um 180: Zunächst Horizontal polarisierte Strahlung wird im ersten Schritt zirkular und dann vertikal polarisiert, um im zweiten Schritt wiederum zirkular und letztlich abermals horizontal polarisiert zu werden. Da horizontal polarisierte Strahlung den Dünnschichtpolarisator ungehindert passieren kann, wird die Güte des Resonators binnen kürzester Zeit soweit verbessert, dass Strahlung darin umlaufen und sich ein Laserpuls aufbauen kann.

\subsubsection{Seeding-Laser}

Eine Verringerung der spektralen Bandbreite des Pumplasers und somit auch der vom nachgelagerten OPO/OPA-System emittierten Strahlung kann durch den Einsatz eines Seeding-Lasers (auch: Injection Seeder; Modell: Continuum SI-2000) erreicht werden.

Bei Raumtemperatur ist die Emissionsbande der $\mathrm{Nd}^{3+}$-Ionen des Lasermediums durch thermische Gitterschwingungen verbreitert, da diese Schwingungen eine zeitlich veränderliche Feldstärke an den Positionen dieser Ionen bewirken. Innerhalb dieser Bandbreite liegen mehrere Lasermoden vor, die die Randbedingungen der Resonatorkavität erfüllen, sodass Strahlungspakete mit mehreren diskreten Wellenzahlen beim Umlauf durch den Resonator verstärkt werden.

Mit Hilfe des Seeding-Lasers kann diese Verbreiterung vermindert werden, indem Strahlung einer dieser Moden ausgewählt und auf Kosten der Intensität der anderen Moden verstärkt wird. Der Seeding-Laser ist ein Faserlaser, der kontinuierlich im Spektralbereich zwischen $1030 \mathrm{~nm}$ und $1080 \mathrm{~nm}$ durchgestimmt werden kann. Im vorliegenden Aufbau wird er mit einer Wellenlänge von 1064,169 nm betrieben. Die Strahlungsintensität des SeedingLasers ist um ca. sechs Größenordnungen höher als die spontan vom Nd:YAG-Kristall emittierte Strahlung und so schmalbandig, dass sie nur die Emission einer einzigen Lasermode stimuliert. Da diese Strahlung aufgrund der Intensität des Seeding-Lasers schneller verstärkt wird als die spontan emittierte Strahlung der übrigen Moden, wird die stimulierte Emission aller anderen Moden zugunsten der durch den Seeding-Laser ausgewählten Mode unterdrückt und die Bandbreite der emittierten Strahlung somit verringert. 


\section{1: Geschlossene Kavität:}

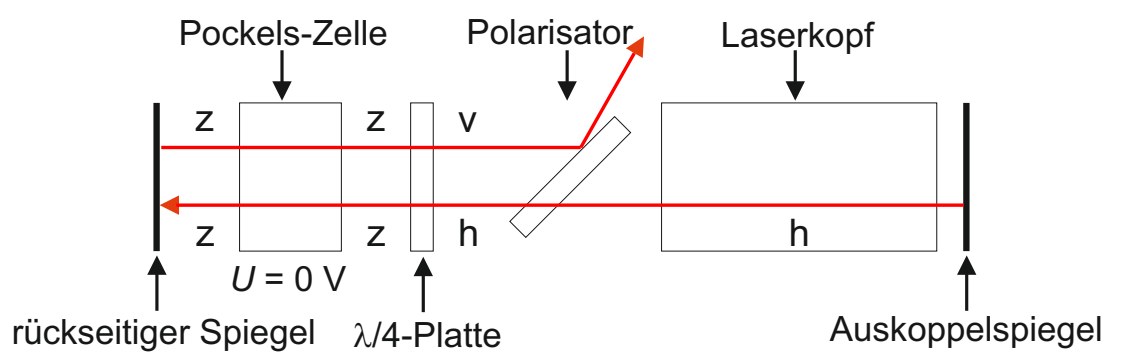

\section{2: Geöffnete Kavität:}

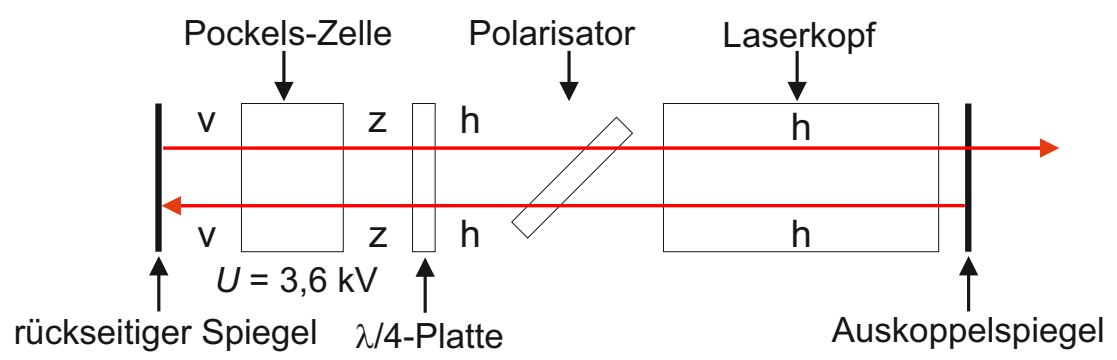

Abbildung 4.11.: Funktionsprinzip der Güteschaltung; 1: Geschlossene Kavität; 2: Geöffnete Kavität; h: horizontale Polarisation, v: vertikale Polarisation, z : zirkulare Polarisation; verändert nach Ref. 258.

Die gewählte Mode sollte diejenige mit der höchsten Intensität darstellen. Die Einstellung der Wellenlänge erfolgt über das Computerprogramm SI-2000. Als Kriterium zur Auswahl der am besten geeigneten Mode kann die verstrichene Zeit zwischen dem Auslösen der Güteschaltung und der Emission des Laserpulses herangezogen werden. Diese ist umso kürzer, je höher der Anteil der durch den Seeding-Laser verstärkten Mode an der Gesamtenergie des Laserpulses ist. Die Verminderung dieser Zeitspanne gegenüber dem ungeseedeten Laser wird als Q-switch buildup reduction time bezeichnet. Diese ist bei optimaler Einstellung des Seeding-Lasers maximal und sollte ca. 11 ns betragen.

Der Seeding-Laser verfügt über einen internen Regelkreis, der den Verstärkungsprozess während des Betriebs kontinuierlich optimiert. Hierzu wird die Resonatorlänge des Hauptlasers durch eine Steuerungselektronik im Seeding-Laser über einen Piezoaktor variiert, der am hinteren Resonatorspiegel eingebaut ist, und somit auf die Wellenlänge des Seeding-Lasers abgestimmt. Als Kriterium dient ebenfalls die Q-switch buildup reduction time. $\mathrm{Zu}$ deren Messung verfügt der Seeding-Laser über einen BNC-Eingang, über den ein Spannungspuls die zeitliche Information über die Aktivierung des Q-switch des Nd:YAGLasers liefert, sowie über einen Lichtleiter, über den vom rückwärtigen Resonatorspiegel durchgelassene Strahlung des in der Folge emittierten Laserpulses detektiert wird. Nähere Einzelheiten zur Bedienung sind dem Handbuch der Firma Continuum 264 zu entnehmen. 


\subsubsection{OPO/OPA-System}

\begin{tabular}{c|c|c}
\hline KENNGRÖSSE & SPEZIFIKATION & SPEKTRALBEREICH \\
\hline Wellenlängenbereich & $710-885 \mathrm{~nm} ; 1.35-5 \mu \mathrm{m}$ & \\
Energie (Pumplaser) & $550 \mathrm{~mJ}$ & $1064 \mathrm{~nm}$ \\
Polarisation (Pumplaser) & horizontal & \\
Pulsenergie & $12 \mathrm{~mJ}$ & $1.5-3.5 \mu \mathrm{m}$ \\
& $4 \mathrm{~mJ}$ & $4.0 \mu \mathrm{m}$ \\
Repetitionsrate & $0.5 \mathrm{~mJ}$ & $4.9 \mu \mathrm{m}$ \\
& $\leq 10 \mathrm{~Hz}$ &
\end{tabular}

Tabelle 4.4.: Spezifikationen des OPO/OPA-Lasersystems der Firma LaserVision, entnommen aus Referenz [261].

Die dem Nd:YAG-Laser entstammende, horizontal polarisierte Strahlung mit einer konstanten Wellenlänge von $1064 \mathrm{~nm}$ ist für die infrarotspektroskopische Untersuchung von Wasserstoffbrückenbindungen noch nicht geeignet, da hierfür durchstimmbare Strahlung im Spektralbereich zwischen $2632 \mathrm{~nm}$ und $3571 \mathrm{~nm}$ (entsprechend $3800 \mathrm{~cm}^{-1}$ bis $2800 \mathrm{~cm}^{-1}$ ) benötigt wird. Diese Strahlung wird daher für das optische Pumpen eines OPO/OPA-Systems der Firma Laser Vision genutzt, das in der Lage ist, unter Ausnutzung nichtlinearer optischer Effekte der darin verbauten Kristalle durchstimmbare Strahlung mit der benötigten Wellenlänge zu erzeugen. Der Aufbau dieses Systems ist in Abbildung 4.12 schematisch dargestellt. Derartige Lasersysteme ermöglichen die Erzeugung durchstimmbarer Laserstrahlung in Spektralbereichen, die durch Farbstofflasersysteme bislang nicht zugänglich sind $265 \mid 266$.

Nach dem Eintritt der Strahlung aus dem Pumplaser in das System durch die Eingangsiris (1) durchquert sie ein Teleskop (2), das ihren Durchmesser den Anforderungen des OPO/OPA-Systems entsprechend vermindert, und trifft anschließend auf einen Strahlteiler (3). Dieser reflektiert ein Drittel der Strahlung in Richtung des OPO und lässt zwei Drittel der Strahlung in Richtung des OPA passieren. Der in Richtung des OPO geleitete Anteil durchläuft eine verstellbare $\lambda / 2$-Platte (4), durch die im Betrieb der Polarisationsvektor der Strahlung um $45^{\circ}$ gedreht wird, sodass im Anschluss sowohl deren elektrischer als auch deren magnetischer Feldvektor um $45^{\circ}$ gegenüber der kristalloptischen Achse des folgenden Verdopplungskristalls (5) rotiert sind. Dieser besteht aus KTP (Kaliumtitanylphosphat) und verdoppelt anschließend die Frequenz der eingehenden Strahlung. Hierbei entsteht aus zwei $1064 \mathrm{~nm}$-Photonen ein Photon mit einer Wellenlänge von $532 \mathrm{~nm}$. Durch die Rotation der $\lambda / 2$-Platte (4) lässt sich die Effizienz dieses Prozesses zu Justagezwecken variieren. Über zwei Umlenkspiegel (6) und durch eine weitere Iris (8) gelangt der frequenzverdoppelte und nunmehr vertikal polarisierte Anteil der Strahlung zum Resonator des OPO, in den er über den Spiegel (9) eingekoppelt wird. Residuale $1064 \mathrm{~nm}$-Strahlung wird von den dichroitisch beschichteten Spiegeln (6), deren Beschichtung auf die Reflexion von Strahlung einer Wellenlänge von $532 \mathrm{~nm}$ optimiert ist, nicht reflektiert und im Strahlfänger (7) absorbiert. In der Resonatorkavität des OPO, die durch den Rückspiegel (10) und den Auskoppelspiegel (12) gebildet wird, befinden sich zwei ebenfalls aus KTP bestehende Kristalle (11), die durch die in der Kavität umlaufende Strahlung optisch gepumpt werden. Die beiden Kristalle des OPO sind auf Schrittmotoren 


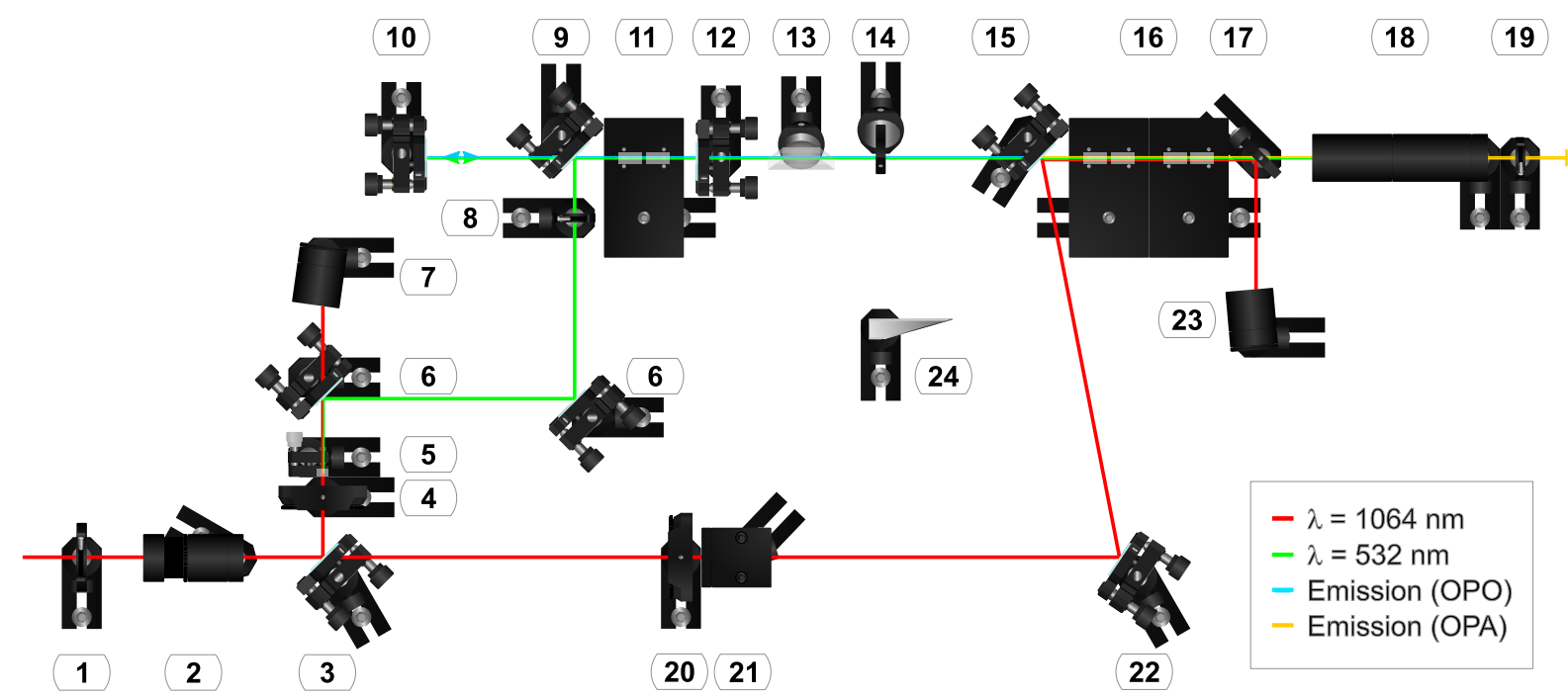

Abbildung 4.12.: Schematische Darstellung des OPO/OPA-Systems der Firma LaserVision; verändert nach Ref. 261

1. Eingangsiris

2. Teleskop

3. Strahlteiler $(1 / 3$ zu 2/3)

4. $\lambda / 2$-Platte, variabel

5. Verdopplungskristall (KTP)

6. Umlenkspiegel (dichroitisch beschichtet, $\lambda=532 \mathrm{~nm})$

7. Strahlfänger

8. Irisblende

9. Einkoppelspiegel des OPO

10. Hinterer Spiegel des OPO

11. OPO-Kristalle (KTP)

12. Auskoppelspiegel des OPO

13. Dove-Prisma
14. $\lambda / 2$-Platte, fixiert

15. Umlenkspiegel (dichroitisch beschichtet, $\lambda=1064 \mathrm{~nm})$

16. OPA-Kristalle (KTA)

17. Separatorspiegel (dichroitisch beschichtet, $\lambda=1064 \mathrm{~nm}$ )

18. Brewster-Stapel

19. Ausgangsiris

20. $\lambda / 2$-Platte, variabel

21. Dünnschicht-Polarisator

22. Umlenkspiegel (dichroitisch beschichtet, $\lambda=1064 \mathrm{~nm})$

23. Strahlfänger

24. Siliciumfilter 
montiert, sodass sie gegenläufig rotiert werden können. Durch Rotation der Kristalle wird deren Stellung im Strahlengang der OPO-Kavität und somit die vom System emittierte Wellenlänge verändert. Die Stellung des ersten Kristalls ist hierbei maßgeblich für die emittierte Wellenlänge, während der gegenläufig rotierte, zweite Kristall dazu dient, den durch Drehung des ersten Kristalls entstehenden Strahlversatz auszugleichen.

Im OPO wird ein $532 \mathrm{~nm}$-Photon in zwei Photonen mit unterschiedlicher Wellenlänge aufgeteilt, die als Signal- bzw. Idler-Photonen bezeichnet werden. Das Photon mit der höheren Frequenz trägt laut Konvention die Bezeichnung Signal. Der für die Erzeugung von IR-Strahlung der gewünschten Wellenlänge benötigte Anteil ist in diesem Falle der Idler, dessen Wellenlänge im Bereich zwischen $710 \mathrm{~nm}$ und $845 \mathrm{~nm}$ variiert werden kann. Dieser ist, wie die Pumpstrahlung, vertikal polarisiert. Er weist eine Halbwertsbreite von $3 \mathrm{~cm}^{-1}$ bei ungeseedetem Pumpstrahl und von $0,9 \mathrm{~cm}^{-1}$ bei geseedetem Pumpstrahl auf 261 .

Die so erzeugte Strahlung verlässt die Kavität durch den Auskoppelspiegel (12). In diesem System sind die Phase Matching-Bedingungen bei von der Resonatorachse abweichendem Strahlungsvektor in horizontaler Ebene schneller verletzt, als in der vertikalen Ebene, sodass die vom OPO ausgehende Strahlung in der horizontalen Ebene stärker kollimiert ist und eine Ellipse bildet, deren lange Achse vertikal ausgerichtet ist. Aus technischen Gründen, die den Aufbau des OPA betreffen (siehe unten), ist es jedoch sinnvoll, diesen mit Strahlung zu speisen, die in vertikaler Richtung stärker gebündelt ist. Daher wird die vom OPO ausgehende Strahlung durch ein Dove-Prisma (13) um 90 gedreht. Dem Dove-Prisma schließt sich eine verstellbare $\lambda / 2$-Platte aus Magnesiumfluorid (14) an, die den vertikal polarisierten Idler horizontal polarisiert.

Im OPA befinden sich vier identische Kristalle (16), die ebenfalls auf Schrittmotoren montiert sind und jeweils paarweise gegenläufig rotiert werden, um einen Strahlversatz auszugleichen. Aufgrund seiner höheren Transparenz im mittleren IR-Bereich kommt an dieser Stelle KTA (Kaliumtitanylarsenat) als optisch aktives Medium zum Einsatz. Die Wellenlänge des OPA kann im Bereich zwischen $1350 \mathrm{~nm}$ und $5000 \mathrm{~nm}\left(7460 \mathrm{~cm}^{-1}\right.$ bis $2000 \mathrm{~cm}^{-1}$ ) durchgestimmt werden. Die Montage der OPA-Kristalle auf Schrittmotoren mit vertikal verlaufenden Achsen und Rotation der Kristalle in horizontaler Ebene ist technisch leichter zu realisieren als deren Rotation in vertikaler Ebene und bietet darüber hinaus eine höhere Betriebssicherheit, da von den Kristalloberflächen reflektierte Strahlung das Gehäuse nicht verlassen kann. Da die Phase Matching-Bedingungen bei diesem Aufbau ebenfalls in horizontaler Ebene strenger gelten und folglich die nunmehr in horizontaler statt in vertikaler Ebene divergente Strahlungskomponenten der aus dem OPO eingehenden Strahlung supprimiert werden, kann auf diese Weise die Divergenz der vom System abgegebenen Strahlung minimiert werden.

Auf dem Weg in Richtung des OPA passiert die Fundamentale des Nd:YAG-Lasers eine nach dem Strahlteiler (3) positionierte, drehbare $\lambda / 2$-Platte (20), die deren Polarisationsvektor um $90^{\circ}$ rotiert. Nach diesem Bauteil ist ein Dünnschichtpolarisator (21) angeordnet, ausschließlich für vertikal polarisierte Strahlung transparent ist, während horizontal polarisierte Strahlung an dessen innere Gehäusewand reflektiert und dort absorbiert wird. In Kombination mit der davor angebrachten $\lambda / 2$-Platte wird durch diese Anordnung erstens die zum Betrieb des OPA mit horizontal rotierbaren Kristallen erforderliche, vertikale Polarisation der Strahlung erzielt und zweitens eine Verminderung der Intensität der zum OPA gelangenden Strahlung zu Justagezwecken ermöglicht. 
Durch die Wahl der Position des Spiegels (22), über den die nunmehr vertikal polarisierte Strahlung in den OPA eingekoppelt wird, wird diese soweit verzögert, dass sie beim Zusammenlaufen beider Strahlen in Spiegel (15) kohärent mit der dem OPO entstammenden Strahlung ist, die diesen Spiegel gleichzeitig durchtritt.

Im OPA wird der Idler aus dem OPO zum Signal-Puls. Durch Differenzfrequenzmischung des Idler mit dem zuvor durch den Strahlteiler (3) abgetrennten, nicht frequenzverdoppelten Anteil der Fundamentalen des Nd:YAG-Lasers wird ein neuer Idler-Puls erzeugt und gleichzeitig verstärkt.

Ein dichroitisch beschichteter Spiegel (17) reflektiert restliche, im Ausgangssignal des OPA vorhandene Strahlungsanteile mit einer Wellenlänge von $1064 \mathrm{~nm}$ in Richtung eines Strahlfängers (23). Ein Stapel aus im Brewster-Winkel angeordneten Siliciumplatten (18) dient dazu, entweder die vertikal polarisierte Idler-Strahlung oder die horizontal polarisierte Signal-Strahlung aus der den OPA verlassenden Strahlung abzutrennen. Nach Passage der Ausgangsiris (19) verlässt die Strahlung das System.

Zu Justagezwecken kann eine Siliciumfilter (24) zwischen den Komponenten (14) und (15) in den Strahlengang eingebracht werden, der als Tiefpassfilter fungiert und die vom OPO emittierte Signal-Strahlung sowie residuale Pumpstrahlung absorbiert.

Der Winkel, um den die Kristalle rotiert werden, kann über einen PC variiert werden, der mit Steuerkarten für die Schrittmotoren sowie der benötigten Software ausgestattet ist. Die Steuerung des Systems erfolgt mit Hilfe des auf dem Betriebssystem Windows 7 basierenden Computerprogramms Motor Controls der Firma LaserVision.

Die Bandbreite der erzeugten Strahlung beträgt ca. $1.7 \mathrm{~cm}^{-1}$ bei Verwendung des Seeding Lasers und ca. $3.7 \mathrm{~cm}^{-1}$, sofern dieser nicht zum Einsatz kommt 267. Da die Verwendung des Seeding Lasers hohe Schwankungen der zudem hierdurch stark verminderten IR-Pulsenergie zur Folge hat und die Datenqualität verschlechtern würde, wurde während der hier vorgestellten Messungen auf dessen Einsatz verzichtet. Aufgrund der breiten Banden in den gemessenen IR-Spektren und mit Blick auf den Fokus der hier vorgenommenen Auswertung ist hierdurch kein relevanter Informationsverlust zu erwarten.

Da die tatsächlich vom OPO/OPA-System emittierte Wellenzahl um einen konstanten Wert von dem im Programm eingestellten Wert abweicht, wird nach jeder Kalibrierung des Systems eine Referenzmessung an einer mit Chlorwasserstoffgas gefüllten Küvette oder auch anhand der Absorptionsbanden der Wassermoleküle in der Umgebungsluft durchgeführt. Durch Vergleich der gemessenen Absorptionsspektren mit Literaturdaten kann ein Korrekturwert ermittelt werden $239 \mid 258$. .

Die Einkopplung der IR-Strahlung in die Versuchsapparatur erfolgt über zwei Goldspiegel, die im Wellenlängenbereich von $1 \mu \mathrm{m}$ bis $5 \mu \mathrm{m}$ eine Reflexivität von über $97 \%$ besitzen. Die im OPA erzeugte Strahlung kann durch Bestandteile in der Umgebungsluft absorbiert und stark abgeschwächt werden, sofern diese eine Schwingungsmode besitzen, die mit Strahlung der im OPA erzeugten Frequenz wechselwirken kann. Im vorliegenden Experiment wird dieses Phänomen insbesondere durch Wassermoleküle verursacht, deren Absorptionsbanden sich zum Teil mit dem Absorptionsbereich der untersuchten Wassercluster überschneiden. Um derartige Energieverluste und daraus resultierende spektrale Artefakte durch Intensitätsschwankungen der IR-Strahlung zu minimieren, wurden Vorrichtungen konstruiert, die es ermöglichen, im Bereich des OPA sowie im gesamten Strahlengang zwischen dem Gehäuse des OPO/OPA-Systems und der Versuchsapparatur die Umgebungsluft durch wasserfreies Stickstoffgas zu verdrängen. 


\subsection{UV/VIS-Lasersystem}

Die der vorliegenden Arbeit zugrundeliegenden Experimente wurden mit einem UV/VISLasersystem durchgeführt, das aus den folgenden drei Komponenten besteht:

1. Pumplaser: Nd:YAG-Laser der Firma Continuum (Modell: Powerlite DLS 9010) mit SHG- (engl.: Second Harmonic Generation) und THG- (engl.: Third Harmonic Generation)-Option

2. Durchstimmbares OPO-System: Panther EX OPO der Firma Continuum

\section{Durchstimmbarer Farbstofflaser: Cobra-Stretch der Firma Sirah}

Der Pumplaser kann hierbei wahlweise verwendet werden, um den Farbstofflaser oder das OPO-System zu pumpen. Der Farbstofflaser besitzt den Vorzug, Strahlung im Spektralbereich des eingesetzten Laserfarbstoffes mit hoher Intensität und weitgehend homogenem Profil abzugeben, während das OPO-System die Möglichkeit eröffnet, die emittierte Wellenlänge innerhalb kurzer Zeit über einen großen Spektralbereich durchzustimmen. Sämtliche in dieser Arbeit vorgestellten Messungen wurden unter Verwendung des Farbstofflasers durchgeführt, der auf die Emission von UV-Strahlung der Wellenlänge von 385 nm eingestellt war. Als Farbstoff wurde hierfür Exalite 389 verwendet. Da der Aufbau des OPO-Systems für die Ergebnisse dieser Arbeit nicht relevant ist, werden im Folgenden nur der Pumplaser und der Farbstofflaser näher beschrieben. Eine eingehende Beschreibung des OPO-Systems ist dem Handbuch der Firma Continuum zu entnehmen 268.

\subsubsection{Nd:YAG-Pumplaser Powerlite 9010}

Dieses Lasersystem arbeitet nach den gleichen Funktionsprinzipien wie das in 4.6.1 vorgestellte Gerät. Es generiert ebenfalls Strahlung mit einer Wellenlänge von $1064 \mathrm{~nm}$. Deren Frequenz wird im Anschluss zunächst verdoppelt, sodass Strahlung mit einer Wellenlänge von $532 \mathrm{~nm}$ entsteht. Durch Mischung dieser frequenzverdoppelten Strahlung mit kohärent verlaufender Strahlung der Fundamentalwellenlänge wird bei Bedarf Strahlung der Wellenlänge von $355 \mathrm{~nm}$ erzeugt, sodass für das optische Pumpen weiterer Lasersysteme energiereiche Laserpulse mit diesen drei Wellenlängen zur Verfügung stehen. Der Aufbau des Pumplasers ist in Abbildung 4.13 schematisch dargestellt.

Als aktives Medium kommen wie im Pumplaser des IR-Lasersystems Nd:YAG-Kristalle zum Einsatz. Die im Powerlite 9010 verwendeten Kristalle besitzen ebenfalls eine Länge von $115 \mathrm{~mm}$, ihr Durchmesser beträgt $6 \mathrm{~mm}$ im Oszillator bzw. $9 \mathrm{~mm}$ in den beiden Verstärkern und auch hier sind die Enden der Kristalle mit einer antireflexiven Beschichtung versehen. Für die Erzeugung der frequenzverdoppelten oder frequenzverdreifachten Fundamentalwellenlänge werden Kristalle aus Kaliumdideuteriumphosphat $\left(\mathrm{KD}_{2} \mathrm{PO}_{4}\right)$ verwendet. Zur Frequenzverdopplung stehen zwei verschiedene Kristalle zur Verfügung, deren Verwendung zur Erzeugung von Strahlung unterschiedlicher Polarisationsrichtung führt. Der Kristall mit der Bezeichnung DS-1 SHG 1 wird zur Erzeugung vertikal polarisierter Strahlung verwendet, während der mit $D S S H G 2$ bezeichnete Kristall Strahlung mit horizontaler Polarisation erzeugt. Die Polarisation der Fundamentalen wird zuvor bei Bedarf über eine zwischen dem Oszillator und dem ersten Verstärker angeordnete $\lambda / 2$-Platte 


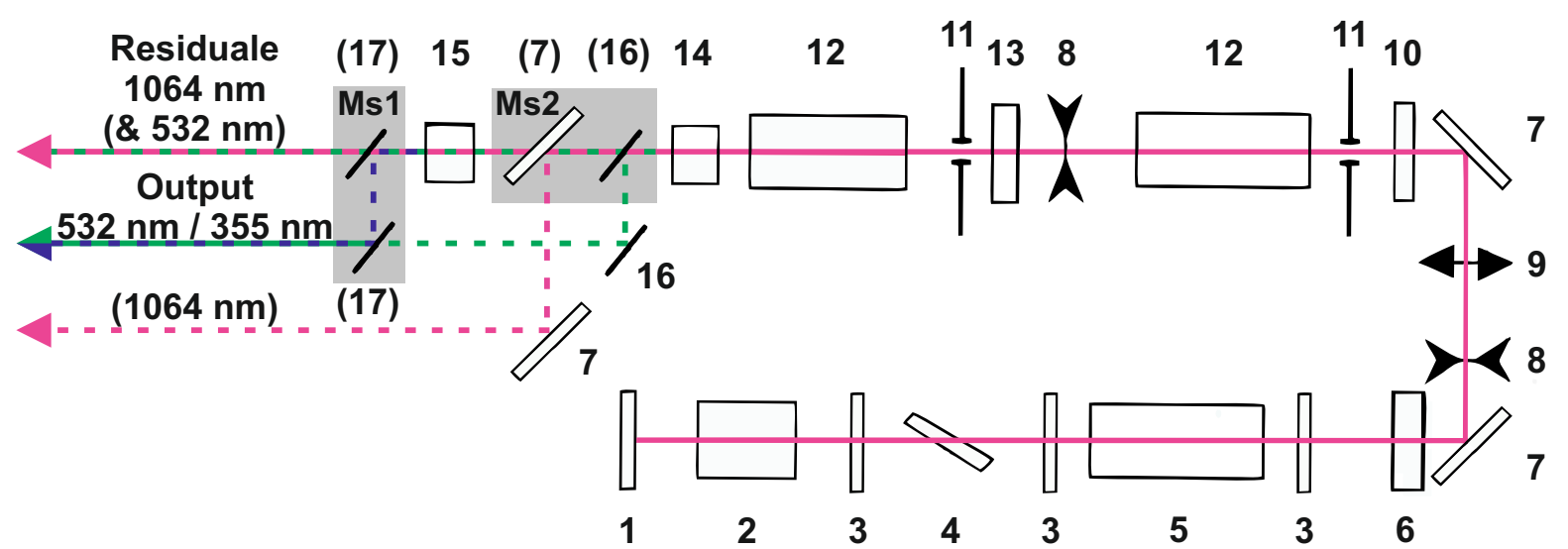

Abbildung 4.13.: Schematische Darstellung des Continuum Powerlite DLS 9010Lasersystems, verändert nach [269]. Die Spiegel mit eingeklammerten Nummern sind auf zwei mobilen mirror stages (Ms1 und Ms2) installiert, die alternativ eingesetzt werden.

1. Rückspiegel des Resonators

2. Pockels-Zelle

3. $\lambda / 4$-Platte

4. Dielektrischer Polarisator

5. Laserkopf (Resonator)

6. Auskoppelspiegel

7. Umlenkspiegel, $45^{\circ}(1064 \mathrm{~nm})$

8. Divergente Linse

9. Konvergente Linse
10. $\lambda / 2$-Platte

11. Lochblende

12. Laserkopf (Verstärker)

13. Quarz-Rotator

14. SHG-Kristall

15. THG-Kristall

16. Dichroitischer Spiegel (532 nm)

17. Dichroitischer Spiegel (355 nm)

angepasst. Der Erstere (Bez.: DS-1 SHG 1) besitzt eine im Vergleich um $25 \%$ niedrigere Effizienz bezogen auf die frequenzverdoppelte Strahlung. Er wird in Kombination mit einem weiteren $\mathrm{KD}_{2} \mathrm{PO}_{4}$-Kristall (Bezeichnung TS THG) zur Erzeugung von frequenzverdreifachter Strahlung $(\lambda=355 \mathrm{~nm})$ durch Summenfrequenzmischung mit der horizontal polarisierten Strahlung $(\lambda=1064 \mathrm{~nm})$ des Nd:YAG-Kristalls eingesetzt.

Die Auskopplung der Pumpstrahlung erfolgt jeweils entlang des Mittleren der drei Strahlengänge in Abb. 4.13. Da das OPO-System nur minimale Abweichungen des Strahlengangs toleriert, darf die optische Achse der Strahlung mit $\lambda=355 \mathrm{~nm}$ (blau dargestellt) nicht verändert werden. Die benötigte Wellenlänge wird daher allein durch den alternativen Einsatz einer von zwei mobilen, mit dichroitisch beschichteten Spiegeln bestückten mirror stages ausgewählt (Ms1 und Ms2 in Abb. 4.13). Bei Verwendung von Ms1 wird die frequenzverdreifachte Strahlung $(\lambda=355 \mathrm{~nm})$ emittiert, der Einsatz von Ms2 bewirkt die Emission der frequenzverdoppelten Fundamentalwellenlänge $(\lambda=532 \mathrm{~nm})$. 


\subsubsection{Farbstofflaser Cobra-Stretch}

Die frequenzverdoppelte bzw. -verdreifachte Strahlung des Powerlite 9010 wird über einen für die jeweilige Wellenlänge ausgelegten, dichroitisch beschichteten Spiegel, der auf einem eigens angefertigten flip mount zwischen Panther EX OPO und Pumplaser angebracht ist, in den Farbstofflaser eingekoppelt. Dessen Aufbau ist schematisch in Abbildung 4.14 dargestellt.

Die Laserstrahlung wird in diesem System im Inneren zweier Glasküvetten erzeugt. Als Lasermedium dienen durch zwei Schlauchsysteme zirkulierende Farbstofflösungen mit verschiedenen Konzentrationen, die diese beiden Küvetten durchströmen. Die Pumpstrahlung wird durch ein System von Strahlteilern und Spiegeln auf die Küvetten aufgeteilt.

Der Laser ist auf vertikal polarisierte Pumpstrahlung ausgelegt. Die vom Powerlite 9010 emittierte Strahlung der Wellenlänge $532 \mathrm{~nm}$ besitzt in der vorliegenden Konfiguration (vgl. Abschn. 4.7.1) die notwendige Polarisation. Die frequenzverdreifachte Fundamentalwellenlänge $(\lambda=355 \mathrm{~nm})$ ist allerdings horizontal polarisiert, sodass ihr Feldvektor mittels einer in den Strahlengang eingebrachten $\lambda / 2$-Platte angepasst werden muss.

Eine rechteckige Küvette aus Quarzglas (4) mit einer Länge von $20 \mathrm{~mm}$ dient als Lasermedium im Oszillator, der einerseits durch den Auskoppelspiegel (5) und andererseits durch den Aluminiumspiegel (1) begrenzt ist. Der im Oszillator erzeugte Laserpuls wird an anderer Stelle nochmals durch die Küvette geleitet, um ihn erstmals zu verstärken. Die Küvette stellt somit nicht nur den Oszillator, sondern auch einen Vorverstärker dar. Aufgabe dieser Anordnung ist jedoch nicht die Erzeugung eines möglichst energiereichen Laserpulses, sondern eines Pulses mit möglichst homogenem Profil, der zugleich weitgehend frei von spontan emittierter Strahlung mit abweichender Wellenlänge und Polarisation ist.

Die Auswahl der emittierten Wellenlänge erfolgt über ein $60 \mathrm{~mm}$ langes Gitter mit einer Gitterkonstanten von 2400 Gitterstegen pro Millimeter und einen beschichteten Aluminiumspiegel im Resonator des Lasers, deren Positionen im Strahlengang über eine elektronische Steuerung so eingestellt werden, dass die Resonanzbedingung für die jeweilige Wellenlänge erfüllt ist.

Der vorverstärkte Laserpuls wird durch ein Teleskop (6) geleitet, das den Strahldurchmesser an die Küvette im anschließenden Hauptverstärker anpasst. Im Hauptverstärker wurde für die vorliegende Arbeit eine runde, stabförmige Kapillarküvette (12) mit einem Durchmesser von $6 \mathrm{~mm}$ und einer Länge von $40 \mathrm{~mm}$ verwendet. Diese wird in Kombination mit einem Teleskop eingesetzt, das aus zwei konkaven zylindrischen Linsen und einer konvexen sphärischen Linse besteht und eine separate Anpassung des Strahldurchmessers in horizontaler und vertikaler Ebene ermöglicht.

Die Kapillarküvette bietet im Vergleich zu einer optional einsetzbaren, rechteckigen Küvette im Hauptverstärker den Vorteil eines homogeneren Strahlprofils, was zu vergleichsweise geringeren Fluktuationen der Ionisationswahrscheinlichkeit der Cluster und zu einer verbesserten Datenqualität führt. Für den optionalen Einsatz der nachgeschalteten Verdopplungseinheit ist die Verwendung der Kapillarküvette unerlässlich, da hierfür ein gleichmäßiges Strahlprofil Voraussetzung ist. Als Nachteil ist ein Verlust des Wirkungsgrades zu nennen, da der durch den Pumpstrahl überstrahlte Bereich größer gewählt wird, als die Seitenfläche der Kapillarküvette, um deren gleichmäßige Ausleuchtung zu gewährleisten. Da bei Verwendung der Kapillarküvette die Pumpenergie auf bis zu 1,5 J erhöht werden kann, lässt sich der Energieverlust jedoch leicht kompensieren. 


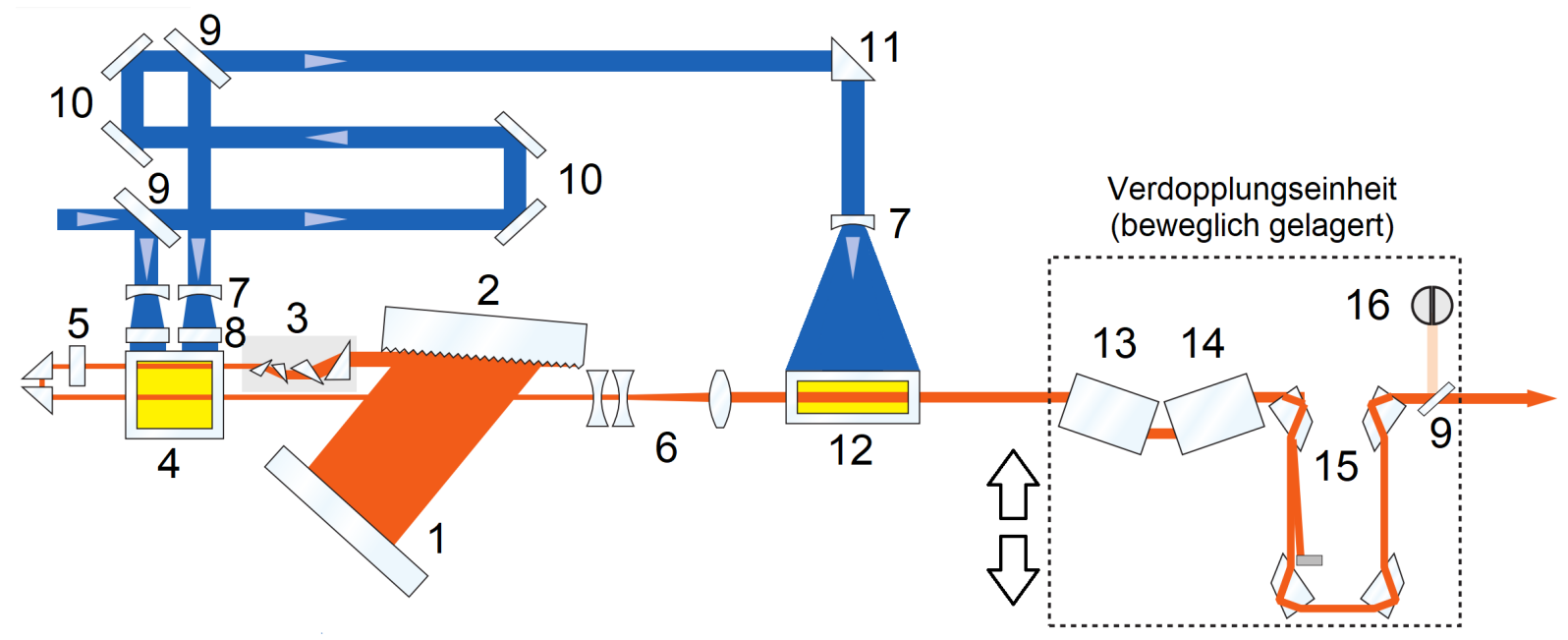

Abbildung 4.14.: Schematische Darstellung des Sirah Cobra-Stretch samt optionaler Verdopplungseinheit in der verwendeten Konfiguration, verändert nach [270]. Die Pumpstrahlung ( $355 \mathrm{~nm}$ bzw. $532 \mathrm{~nm})$ ist in blauer Farbe dargestellt, die erzeugte Laserstrahlung in Orange. Die Verdopplungseinheit kam nicht zum Einsatz.

1. Aluminiumspiegel

2. Gitter

3. Prismen-Strahlaufweiter

4. $20 \mathrm{~mm}-$ Küvette

5. Auskoppelspiegel

6. Teleskop

7. Zylindrische Linsen, divergent

8. Zylindrische Linsen, konvergent
9. Strahlteiler

10. Umlenkeinheit ( $355 \mathrm{~nm}$ bzw. $532 \mathrm{~nm})$

11. Prisma

12. $40 \mathrm{~mm}$-Kapillarküvette, $\varnothing=6 \mathrm{~mm}$

13. Verdopplungskristall

14. Kompensatorkristall

15. Pellin-Broca-Prismen

16. Pyroelektrischer Detektor

Durch optisches Pumpen unterschiedlicher Laserfarbstoffe mit Strahlung einer Wellenlänge von $355 \mathrm{~nm}$ ist UV-Strahlung im Wellenlängenbereich oberhalb von $372 \mathrm{~nm}$ direkt zugänglich. Zur Erzeugung kürzerer Wellenlängen wird durch Pumpen entsprechender Farbstoffe mit Strahlung der Wellenlänge von $532 \mathrm{~nm}$ zunächst Strahlung im sichtbaren Bereich erzeugt, deren Frequenz anschließend verdoppelt werden kann. Hierzu verfügt der Laser über eine auf Schienen horizontal beweglich gelagerte Verdopplungseinheit (eingerahmter Bereich in Abb. 4.14), die bei Bedarf in den Strahlengang eingebracht werden kann. Zur Frequenzverdopplung kann ein $\beta$-Bariumboratkristall (13) verwendet werden, mit dessen Hilfe der Wellenlängenbereich zwischen $280 \mathrm{~nm}$ und $450 \mathrm{~nm}$ abgedeckt werden kann. Ein Kompensatorkristall (14) gleicht den durch die Rotation des Verdopplungskristalls entstehenden Strahlversatz aus. Durch eine Anordnung aus vier einander kompensierende Pellin-Broca-Prismen (15) wird die verdoppelte Strahlung von der residualen, unverdoppelten Strahlung abgetrennt. 
Die Einstellung der Wellenlänge erfolgt entweder über das mitgelieferte Interface oder per USB-Schnittstelle gesteuert durch einen PC mit Windows XP als Betriebssystem, auf dem das Programm Sirah Controls installiert ist. Die Steuerung über einen PC ist zur Verwendung der Verdopplungseinheit notwendig, da nur das PC-basierte Kontrollprogramm die Aufnahme einer Kalibrierkurve unterstützt, mit deren Hilfe die Drehwinkel des Verdopplungskristalls sowie des Kompensatorkristalls relativ zur Strahlachse beim Durchstimmen der Wellenlänge automatisch angepasst werden können. Hierfür werden im Wellenlängenbereich des eingesetzten Farbstoffes zunächst Kalibrierkurven aufgenommen, wobei die optimalen Orientierungen der beiden Kristalle für jede Wellenlänge ermittelt werden. Die Aufnahme der Kalibrierkurven erfolgt voll automatisch. Die hierfür notwendigen Informationen werden durch einen zweiteiligen pyroelektrischen Detektor (16) gesammelt, der an der Gehäuseinnenwand der Verdopplungseinheit angebracht ist. Kurz bevor die Laserstrahlung das Gehäuse verlässt, fällt die sie durch einen im Winkel von $45^{\circ}$ angebrachten Strahlteiler aus Quarzglas. Dieses reflektiert ca. 1\% der Strahlung in Richtung des Detektors. Dieser kann erstens die Gesamtenergie der verdoppelten Strahlung erfassen, da der optimale Drehwinkel des Kristalls eine Funktion der Wellenlänge ist. Zweitens erfasst er über die Differenz der an beiden Detektorhälften gemessenen Energieportionen die Position des Strahls im Verhältnis zu seiner eigenen Mittellinie, sodass die Orientierung des Kompensatorkristalls dahingehend optimiert werden kann, dass beim automatisierten Wechsel der Wellenlänge kein Versatz der Strahlachse entsteht.

Der Wirkungsgrad des Farbstofflasers ist abhängig vom verwendeten Laserfarbstoff. In Tabelle 4.5 sind die Leistungsdaten als Richtwerte zusammengefasst. Die Energieausbeute der mit einer Wellenlänge von $355 \mathrm{~nm}$ gepumpten Farbstoffe liegt bei ca. 15\% $\pm 5 \%$ im Maximum ihres Emissionsbereiches. Die Emissionsbereiche verschiedener Laserfarbstoffe decken einen Wellenlängenbereich von $372 \mathrm{~nm}$ bis $910 \mathrm{~nm}$ ab, wobei die von ihnen emittierte Wellenlänge grundsätzlich länger ist, als die Wellenlänge der verwendeten Pumpstrahlung. Das in diesem Aufbau verwendete Gitter kann zur Auswahl von Wellenlängen im Spektralbereich zwischen $370 \mathrm{~nm}$ und $760 \mathrm{~nm}$ verwendet werden. Die mit $532 \mathrm{~nm}$ gepumpten Farbstoffe ermöglichen eine höhere Energieausbeute von ca. $25 \% \pm 5 \%$. Zudem besitzt der Pumplaser bei dieser Wellenlänge eine höhere Pulsenergie, da bei der Frequenzvervielfachung weniger Verluste auftreten. Da mit $532 \mathrm{~nm}$ gepumpte Laserfarbstoffe keiner UV-Strahlung ausgesetzt sind, besitzen diese eine deutlich längere Lebensdauer. Nähere Einzelheiten sind dem Benutzerhandbuch 270 des Herstellers zu entnehmen.

\begin{tabular}{c|c|c}
\hline KENNGRÖSSE & SPEZIFIKATION & KOMMENTAR \\
\hline Wellenlänge & $280-760 \mathrm{~nm}$ & \\
Repetitionsrate & $\leq 50 \mathrm{~Hz}$ & \\
Pumpstrahl & $400 \mathrm{~mJ}$ bei $355 \mathrm{~nm}$ & vertikal polarisiert \\
Pumpstrahl & $600 \mathrm{~mJ}$ bei $532 \mathrm{~nm}$ & vertikal polarisiert \\
Pulsenergie & $25 \mathrm{~mJ} \pm 10 \mathrm{~mJ}$ & $280-372 \mathrm{~nm}$ (verdoppelt) \\
Pulsenergie & $60 \mathrm{~mJ} \pm 20 \mathrm{~mJ}$ & $372-540 \mathrm{~nm}$ \\
Pulsenergie & $150 \mathrm{~mJ} \pm 30 \mathrm{~mJ}$ & $540-760 \mathrm{~nm}$
\end{tabular}

Tabelle 4.5.: Spezifikationen des Cobra-Stretch mit Verdopplungseinheit. Die kursiv gedruckten Einträge beziehen sich auf das optische Pumpen bei $\lambda=355 \mathrm{~nm}$; verändert nach Referenzen 71,270 . 


\subsubsection{Relay Imaging Teleskop}
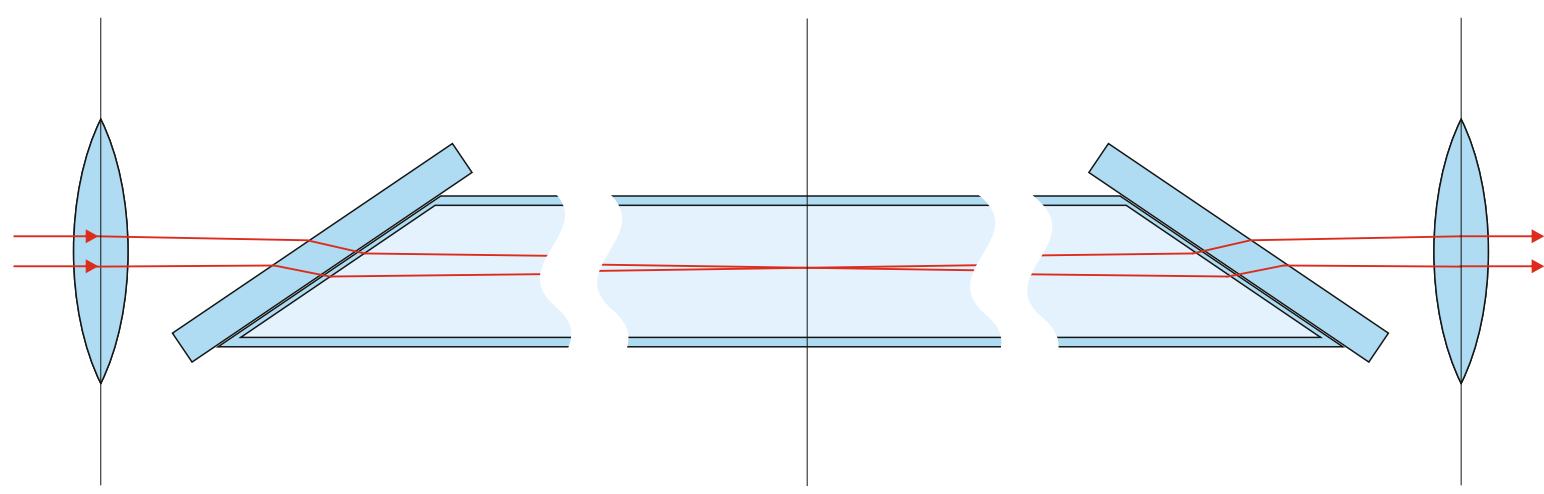

Abbildung 4.15.: Schematische Darstellung des Relay Imaging-Teleskops.

Um Fluktuationen der Ionisationswahrscheinlichkeit durch Positionsänderungen des UV-Laserstrahls zu minimieren, ist ein homogenes Strahlprofil Voraussetzung. Das Profil des am Gehäuse austretenden Laserstrahls (im sogenannten Nahfeld) erfüllt diese Anforderung. Infolge der freien Propagation durch den Raum wird die Intensitätsverteilung des Strahlprofils jedoch zunehmend Gaußförmig (Fernfeld). Weiterhin treten konzentrisch angeordnete Interferenzmuster auf, sodass die Ionisationswahrscheinlichkeit eines Clusters stark von dessen Position innerhalb des UV-Pulses abhängt.

Es erwies sich als notwendig, die freie Propagation des Laserstrahls zu unterbinden. Hierfür wurde ein Verfahren namens Relay Imaging angewendet, das es ermöglicht, ein Hologramm des homogenen Strahlprofils im Nahfeld auf den Clusterstrahl in der Apparatur abzubilden und den Einfluss durch Störungen des Laserstrahls, die z. B. durch Staub auf den Optiken und die Raumluft im Strahlengang hervorgerufen werden, zu minimieren 271.

Daher wurde in Kooperation mit der institutseigenen Glaswerkstatt ein Relay ImagingTeleskop konstruiert. Dieses ist in Abbildung 4.15 schematisch dargestellt. Da eine Anpassung des Strahldurchmessers nicht erforderlich war, wurde die Anordnung als $4 f$-Teleskop ausgelegt, d.h. das Teleskop wurde aus zwei Linsen gleicher Brennweite aufgebaut. Die Brennweite der Linsen sollte einerseits so groß wie möglich gewählt werden, um Abbildungsfehler zu minimieren, sodass das Teleskop idealerweise den größten Anteil der $2 \mathrm{~m}$ langen Wegstrecke zwischen dem Gehäuse des Farbstofflasers und der Apparatur überspannt. Andererseits sollte der Fokus möglichst auf der Mitte der Wegstrecke zwischen dem Ausgang der Kapillarküvette (Objektebene) und dem Molekularstrahl (Bildebene) liegen, sodass das Teleskop im Idealfall zu beiden Punkten den gleichen Abstand besitzt. Es wurden daher als Kompromiss zwei konvexe Linsen der Brennweite $f=500 \mathrm{~mm}$ gewählt, sodass die Gesamtlänge des Teleskops ca. $1 \mathrm{~m}$ beträgt.

Da in der Brennebene des Teleskops durch die hohe Energiedichte der fokussierten Laserstrahlung Elektronen aus den Luftmolekülen herausgelöst werden und kurzzeitig ein Plasma entsteht, was zu Intensitätsverlusten führt, wurde eine Vakuumkammer konstruiert, die aus einem evakuierten Glasrohr von $50 \mathrm{~cm}$ Länge besteht. Auf dessen Enden wurden mit Hilfe eines vakuumgeeigneten und UV-beständigen Zweikomponentenklebers 
Quarzglasfenster mit einer Dicke von $10 \mathrm{~mm}$ aufgeklebt. Die Enden des Glasrohres sind beidseitig so abgeschrägt, dass der Laserstrahl im Brewster-Winkel $\left(55,58^{\circ}\right)$ durch die Fenster fällt. Diese Bauweise ermöglicht es, den durch Reflexion an den Oberflächen der Fenster auftretenden Strahlungsverlust zu minimieren, sofern die Vakuumkammer derart ausgerichtet wird, dass die Einfallsebene der Strahlung in die Quarzfenster parallel zum elektrischen Feldvektor der Laserstrahlung verläuft.

\subsection{Steuerung des Messablaufs}

Im Rahmen dieser Arbeit wurde die elektronische Steuerung der Molekularstrahlapparatur P11 vollständig umgestellt. Die vorgenommenen Änderungen sollen daher in diesem Kapitel ausführlich beschrieben werden, um nachfolgenden ExperimentatorInnen den neuen Steuerungsaufbau zu veranschaulichen. Dieser umfangreiche Eingriff in den Aufbau des Experimentes wurde vorgenommen, da er zahlreiche Vorteile versprach. Hierzu zählen eine bessere Datenqualität durch Eliminierung von Fehlerquellen und ein erheblich verringerter Bedienungsaufwand sowie die Aufnahme größerer Datenmengen in der gleichen Zeit durch die Automatisierung des Messbetriebs, da einerseits Leerlaufzeiten zwischen den Messungen minimiert und andererseits die Betriebszeiten insgesamt deutlich verlängert werden konnten.

Zuvor waren über einen Zeitraum von je 100 Sekunden im Wechsel Massenspektren mit alleiniger UV-Ionisation (zur Bestimmung des Hintergrundsignals) und mit kombinierter IR-Anregung und UV-Ionisation (zur Ermittlung der Signalzunahme) aufgenommen worden. Dabei war der IR-Laserstrahl in jeder vierten Messung manuell blockiert und die mutmaßliche Stärke des Hintergrundsignals zum Zeitpunkt der Messung der Signalzunahme durch Interpolation zweier Hintergrundmessungen unter Verwendung eines gewichteten Mittelwertes bestimmt worden. Die IR-induzierte Signalzunahme war somit stets durch den Vergleich von Messwerten ermittelt worden, die mit zeitlichem Abstand aufgenommen worden waren und daher anfällig für Einflüsse durch veränderliche Messbedingungen waren. Hierzu zählen Intensitätsschwankungen des Molekularstrahls, periodische Änderungen der Laserenergien oder die kontinuierliche Abnahme der Detektorempfindlichkeit im Laufe eines Messtages. Sämtliche Tätigkeiten im Messbetrieb (manuelles Blockieren des IR-Laserstrahls, Messung der Pulsenergien, Einstellung einer neuen IR-Wellenzahl, Eintragung von Wellenzahl und IR-Pulsenergie in die Metadaten sowie das Starten einer neuen Messung) waren manuell und weitestgehend zwischen den Messungen durchgeführt worden, was den Messbetrieb verzögerte. Da die zur Aufnahme der Massenspektren verwendete Software MCDWIN der Firma FAST ComTec GmbH kein akustisches Signal bei Ende der Messung ausgibt, erforderte dieser Betriebsmodus die ständige Aufmerksamkeit mindestens einer Person. Idealerweise wurde die Bedienung der Apparatur durch zwei Personen übernommen, um in vertretbarer Zeit die Messung eines Infrarotspektrums fertigzustellen.

Die neue Steuerung ermöglicht zunächst die simultane Aufnahme der Massenspektren des Hintergrundsignals und der IR-induzierten Signalzunahme, indem der Clusterstrahl abwechselnd je einem UV-Laserpuls und einem kombinierten IR- und UV-Laserpuls ausgesetzt wird (siehe Abschnitt 4.8.1). Mögliche Störungen durch die oben beschriebenen Einflüsse werden somit minimiert. Da ein manuelles Blockieren des IR-Laserstrahls nunmehr obsolet ist, konnte zudem der komplette Messablauf automatisiert werden. Zu diesem 
Zweck wurde ein Computerprogramm auf Basis der Programmiersprache $\mathrm{C}++$ entwickelt, das in der Lage ist, sämtliche oben aufgeführte Tätigkeiten automatisiert durchzuführen und, soweit möglich, einige häufig auftretende Fehler in der Bedienung sowie der Software MCDWIN abzufangen (siehe Abschnitt 4.8.2). Die Apparatur arbeitet fortan während der Messung weitgehend autark und muss lediglich überwacht werden.

Dennoch ist in größeren Abständen ein manuelles Eingreifen in den Betrieb erforderlich. So muss die Einkopplung des IR-Lasers in die Detektionskammer bei der Durchstimmung der IR-Wellenzahl um mehr als $40 \mathrm{~cm}^{-1}$ überprüft und gegebenenfalls angepasst werden, da die Strahlposition sich ändern kann. Die neue Software ermöglicht zu diesem Zweck die Unterbrechung der Messung unter Ausgabe eines akustischen Signals in festgelegten Abständen, sodass die bedienenden Personen ihre Aufmerksamkeit während der Messung anderen Aufgaben widmen können. Auch die Befüllung der Kühlfalle oberhalb der Expansionskammer sowie eine Erhöhung der Pulsenergie des UV-Pumplasers zum Ausgleich des durch Abbau des Laserfarbstoffes auftretenden Energieabfalls im Farbstofflaser ist in größeren Abständen notwendig.

Der neue Messmodus wurde von Sabine Wolff im Rahmen ihrer Masterarbeit 237 hinsichtlich der Datenqualität im Vergleich zum alten Messmodus evaluiert. Es zeigte sich, dass die Standardabweichung der erhobenen Daten im neuen Messmodus je nach Umfang des ausgewählten Größenbereiches um den Faktor zwei bis sieben geringer ist. Dieser Gewinn in der Datenqualität wird zunächst mit einer um $50 \%$ verlängerten Messzeit erkauft, da zur Bestimmung des Hintergrundsignals nunmehr ebenso viele Messungen durchgeführt werden, wie zur Bestimmung der Signalzunahme. Dieser Nachteil wird jedoch durch die Automatisierung überkompensiert, da erstens die Leerlaufzeiten zwischen den Messungen auf ein Minimum reduziert werden und zweitens die absolute Messzeit nicht mehr durch die Konzentrationsfähigkeit der ExperimentatorInnen limitiert ist.

\subsubsection{Synchronisation der Lasersysteme und des Massenspektrometers}

Wie in Kapitel 4.4 dargelegt, müssen Ionisation und Detektion der Cluster trotz ihrer kontinuierlichen Erzeugung diskontinuierlich erfolgen. Das an der P11 durchgeführte Experiment erfordert somit den wiederholten Ablauf der folgenden drei Schritte im Laufe eines elektronisch getakteten Steuerungszyklus. Eine zuvor festgelegte Anzahl von Wiederholungen dieses Zyklus wird zu einer Messung zusammengefasst:

- IR-Laserpuls zur thermischen Anregung der Cluster (in jedem zweiten Zyklus)

- UV-Laserpuls zur Ionisation der Cluster

- Start der Flugzeitmessung zur Detektion der erzeugten Ionen

Im Unterschied zum alten Messmodus soll nunmehr jeder zweite IR-Laserpuls zuverlässig unterdrückt werden. Gleichsam zuverlässig benötigt die Zählerkarte, die die Signale des MCP-Detektors erfasst (siehe Abschnitt 5.1), die Information, ob die vom MCP-Detektor eingehenden Spannungspulse dem Signal- oder dem Hintergrundspektrum zuzuordnen sind. Dies geschieht durch Anlegen einer Spannung an den TAG-Eingang der Zählerkarte (siehe unten). 
Der zeitliche Abstand zwischen IR- und UV-Laserpuls soll dabei mit einer Genauigkeit von $\Delta(\Delta t) \leq \pm 1$ ns bekannt und im Rahmen dieser Messgenauigkeit stabil sein. Um diese Bedingung zu erfüllen, wird die Taktung des Messablaufs durch einen Delaygenerator (DG535 der Firma Stanford Research) realisiert. Je nach Fragestellung wird diese Signalverzögerung (das sogenannte Delay) im Messbetrieb auf Werte zwischen -20 ns und +150 ns eingestellt. Positive Delaywerte bezeichnen per Konvention eine Einstellung, bei der ein IR-Puls dem UV-Puls zeitlich vorausgeht ${ }^{1}$. Die Messung der Flugzeiten der durch einen UV-Laserpuls erzeugten Ionen soll simultan mit dessen Auftreffen auf den Molekularstrahl beginnen. Das Eintreffen des TAG-Signals an der Zählerkarte soll dem Start der Flugzeitmessung zuverlässig um eine Zeitspanne von wenigen Nanosekunden vorausgehen.

Da die Massenspektren, die das Hintergrundsignal enthalten, mit denjenigen, in denen die Signalzunahme erfasst wurde, exakt vergleichbar sein müssen, darf der Zeitpunkt, zu dem diese beiden Signale an der Zählerkarte eintreffen, sich weder durch Änderung von Delayparametern noch durch Temperaturänderungen elektrischer Bauteile, wie sie im Laufe eines Messtages auftreten können, verschieben. Zuvor waren die Flugzeitmessungen durch das Signal einer Photodiode ausgelöst worden, die gestreute Strahlung des UV-Laserpulses detektierte. Da der Aufbau der neuen Steuerungselektronik mit der Implementierung eines neuen UV/VIS-Lasersystems auf Basis eines Festkörperlasers zusammenfiel, das abseits des Laserpulses kaum Streustrahlung emittiert, erwies sich der bisherige Aufbau nunmehr als unzuverlässig. Daher wurde auch für das Starten der Flugzeitmessungen eine elektronische Lösung implementiert.

Zur externen Triggerung eines Pumplasers werden zwei Signale benötigt: Ein Triggerpuls für die Blitzlampen sowie ein weiterer Puls, der die Güteschaltung auslöst. Der zweite Puls muss hierbei in Abhängigkeit vom verwendeten Lasermodell um ca. 300 ps gegenüber dem Ersten verzögert sein, damit sich die Besetzungsinversion im Lasermedium vollständig aufbauen kann. Zur Steuerung beider Laser werden folglich vier Signale benötigt.

Sowohl der Pumplaser des UV-Lasersystems als auch derjenige des IR-Lasersystems sind auf den Betrieb mit einer Pulsfrequenz von $10 \mathrm{~Hz}$ ausgelegt. Durch den Betrieb der Blitzlampen mit dieser Frequenz wird sichergestellt, dass sich in beiden Geräten nach einer Aufwärmperiode von ca. 30 Minuten ein thermisches Gleichgewicht einstellt. Der Betrieb der Blitzlampen mit einer höheren Frequenz könnte zu Schäden an den Laserstäben führen, wohingegen bei einer niedrigeren Frequenz die Stabilität des Strahlprofils und der Energie der emittierten Laserpulse abnimmt. Dies würde zu einer Zunahme des statistischen Rauschens führen und die Qualität der Messergebnisse beeinträchtigen. Die Pulsfrequenz zum Betrieb der Blitzlampen darf daher nicht verändert werden.

Der IR-Pumplaser soll nur während jedes zweiten Triggerzyklus einen Puls emittieren. Die Emission eines Laserpulses wird allein durch die Auslösung der Güteschaltung veranlasst. Indem diese nur bei jedem zweiten Triggerzyklus ausgelöst wird, kann die erforderliche Pulsfolge zuverlässig implementiert werden.

\footnotetext{
${ }^{1}$ Kurz nach Implementierung des hier beschriebenen Messmodus zeigte sich, dass auch die IR-induzierte thermische Anregung zuvor erzeugter Kationen bei einigen Clustergrößen eine Signalzunahme bewirkt. Bei solchen, mit umgekehrter Pulsreihenfolge durchgeführten Messungen wird der Delaywert mit einem negativen Vorzeichen versehen.
} 
In Kooperation mit der institutseigenen Elektronikwerkstatt wurden drei auf die bereits vorhandenen elektronischen Komponenten abgestimmte logische Schaltungen entwickelt, um die Kommunikation zwischen diesen Komponenten zu gewährleisten:

1. Die sogenannte Pulsbox enthält vier identisch aufgebaute monostabile Kippstufen, die die vier logischen Übergänge des Delaygenerators in positive TTL-Pulse mit einer Dauer von $10 \mu \mathrm{s}$ umwandeln. Diese sind auf eine Eingangsimpedanz von $50 \Omega$ ausgelegt. Somit kann die Taktung sämtlicher Ereignisse über einen einzigen Delaygenerator erfolgen.

2. Die sogenannte Triggerbox leitet jeden zweiten am Eingang IN 1 eintreffenden Puls des Delaygenerators zur Triggerung des Q-switch an den Ausgang $Q$ Switch IR weiter, der mit dem Eingang $Q$ SWITCH TRIG IN des IR-Pumplasers verbunden ist. Sofern ein Puls weitergeleitet wurde, gibt sie einen positiven Spannungspuls mit einer Amplitude von $4 \mathrm{~V}$ und einer Dauer von $10 \mathrm{~ms}$ am Ausgang TAG Out aus, der an den $T A G$-Eingang der Zählerkarte $\mathrm{P}^{7} 88^{7} 7 \mathrm{im}$ Messrechner weitergeleitet wird. Die Ausgabe dieses TAG-Pulses wird jedoch verzögert, bis am Eingang IN 2 das Signal des $V S Y N C D E L A Y$ des UV-Pumplasers detektiert wird, das die Abgabe eines UVPulses anzeigt, um das synchrone Eintreffen von START-Signal und TAG-Signal an der Zählerkarte bei variierendem Delay zwischen den Laserpulsen zu gewährleisten. Der Schaltplan der Triggerbox ist im Anhang C dargestellt.

3. Die sogenannte Startbox verzögert das Signal des Q SWITCH SYNC OUT des UVPumplasers um 190 ns und wandelt das zunächst positive Signal am BNC-Ausgang des Lasers in einen negativen TTL-Puls mit einer Amplitude von $5 \mathrm{~V}$ und einer Dauer von 5 us um, das der Zählerkarte als Signal für den Start der Flugzeitmessung dient. Somit beginnt die Flugzeitmessung synchron mit dem Auftreffen des UVPulses auf den Clusterstrahl.

Ein schematischer Überblick über die Komponenten der Steuerungselektronik ist in Abbildung $4.16 \mathrm{zu}$ finden. Der Delaygenerator löst zehnmal pro Sekunde die Messung der im Molekularstrahl vorliegenden Clusterabundanz aus, indem er mit einer Frequenz von $10 \mathrm{~Hz}$ jeweils vier logische Übergänge in konstanten zeitlichen Abständen an den BNC-Ausgängen A bis D auf seiner Vorderseite ausgibt. Diese Signale werden in die Eingänge der Pulsbox eingespeist und dort in positive TTL-Signale mit einer Dauer von 10 us umgewandelt. Die am DG535 eingestellten Verzögerungen für die Signale an den jeweiligen Eingängen A bis D relativ zum Startzeitpunkt $\mathrm{T}_{0}$ eines Triggerzyklus werden im Folgenden als Delay $A$ bis Delay D bezeichnet.

Die Ausgänge A und B liefern die Triggersignale für die Blitzlampen sowie die Güteschaltung des IR-Pumplasers, während die Ausgänge C und D Blitzlampen und Güteschaltung des UV-Pumplasers auslösen. Tabelle 4.6 gibt die am DG535 eingestellten Verzögerungszeiten für ein Delay von 100 ns zwischen den beiden Laserpulsen an. Über Delay $A$ lässt sich die Verzögerung des IR-Lasers verstellen, während über Delay $D$ der UV-Laser eingestellt wird. Der zur Triggerung des Lasersystems jeweils nötige, zweite Puls (Delay $B$ bzw. Delay $C$ ) ist auf Delay $A$ bzw. Delay $D$ bezogen, sodass die zeitliche Konstanz zwischen den Delays A und B sowie C und D bei Änderung des Zeitintervalls zwischen den Laserpulsen gewährleistet ist. In der Praxis ist Delay A so eingestellt, dass 
die letzten drei Ziffern des Wertes von Delay D auf dem Display des DG535 der tatsächlichen Verzögerung des UV-Laserpulses an der Apparatur in Nanosekunden entsprechen und das Delay bequem eingestellt werden kann. Da sowohl die Laufzeiten der elektrischen Signale in den Koaxialkabeln als auch die Laufzeit des Laserstrahls durch das Labor in der Größenordnung von Nanosekunden liegen, hängen die einzustellenden Werte von den Längen der verwendeten BNC-Kabel und der räumlichen Anordnung der Apparaturkomponenten ab.

\begin{tabular}{c|c}
\hline KANAL & VERZÖGERUNG \\
\hline $\mathrm{A}$ & $\mathrm{T}_{0}+301 \mathrm{~ns}$ \\
$\mathrm{~B}$ & $\mathrm{~A}+320000 \mathrm{~ns}$ \\
$\mathrm{C}$ & $\mathrm{D}-283000 \mathrm{~ns}$ \\
$\mathrm{D}$ & $\mathrm{T}_{0}+320100 \mathrm{~ns}$
\end{tabular}

Tabelle 4.6.: Delay-Parameter für die externe Triggerung von IR- und UV/VISLasersystem mit Hilfe eines Stanford DG535 Delay Generators bei einer zeitlichen Verzögerung der Laserpulse von $100 \mathrm{~ns}$ an der Apparatur. $\mathrm{T}_{0}$ bezeichnet den Startpunkt eines Triggerzyklus des DG535.

Die Zählerkarte P7887 im Messrechner erfasst vom MCP-Detektor eingehende Signale in einem gesonderten Histogramm, sofern gleichzeitig eine Spannung an ihrem TAGEingang anliegt. Folglich nimmt die Zählerkarte simultan zwei separate Massenspektren in Form von Histogrammen auf, zu denen die vom MCP-Detektor eingehenden Signale einer zuvor festgelegten Anzahl von Einzelmessungen aufaddiert werden.

Der Eingangszeitpunkt des TAG-Signals relativ zum Zeitpunkt der Emission eines Laserpulses kann in der Steuerung des UV-Pumplasers durch Anpassung des Parameters VAR SYNC DELAY in einem Zeitintervall von $\pm 190 \mathrm{~ns}$ variiert werden, sodass über die Anpassung dieses Parameters die Synchronisation des TAG-Signals mit dem Beginn einer Flugzeitmessung erreicht werden kann.

Der Seeding Laser benötigt ebenfalls Triggersignale, die der Pulsemission des IRPumplasers zeitlich vorausgehen müssen. Eine zeitliche Verzögerung des Triggersignals für den Pumplaser von ca. 60 ns gegenüber dem Seeding Laser ist nach mündlicher Auskunft des Servicemitarbeiters der Firma Amplitude (ehemals Continuum) optimal. Diese Signale dienen nicht der Auslösung eines Laserpulses (der Seeding Laser emittiert Strahlung im Dauerstrichmodus), sondern der Optimierung der vom Seeding Laser emittierten Wellenlänge auf die Hauptmode des Pumplasers (siehe Kapitel 4.6.2). Der im Manual der Firma Continuum 263 beschriebene und bei der Installation des Lasersystems umgesetzte Anschluss des Seeding Lasers an den VAR SYNC OUT-Ausgang an der Laserbank des Pumplasers erwies sich im vorliegenden Aufbau als ungeeignet, da dieser Ausgang bei externer Triggerung des Lasersystems keine Signale ausgibt. Es zeigte sich jedoch, dass die Ausgangssignale des FIXED SYNC OUT trotz ihres geringen Vorlaufs von 24 ns gegenüber der Aktivierung der Pockels-Zelle zur Aufrechterhaltung der Feedback-Schleife des Seeding Lasers geeignet sind. 


\section{Delaygenerator DG535}

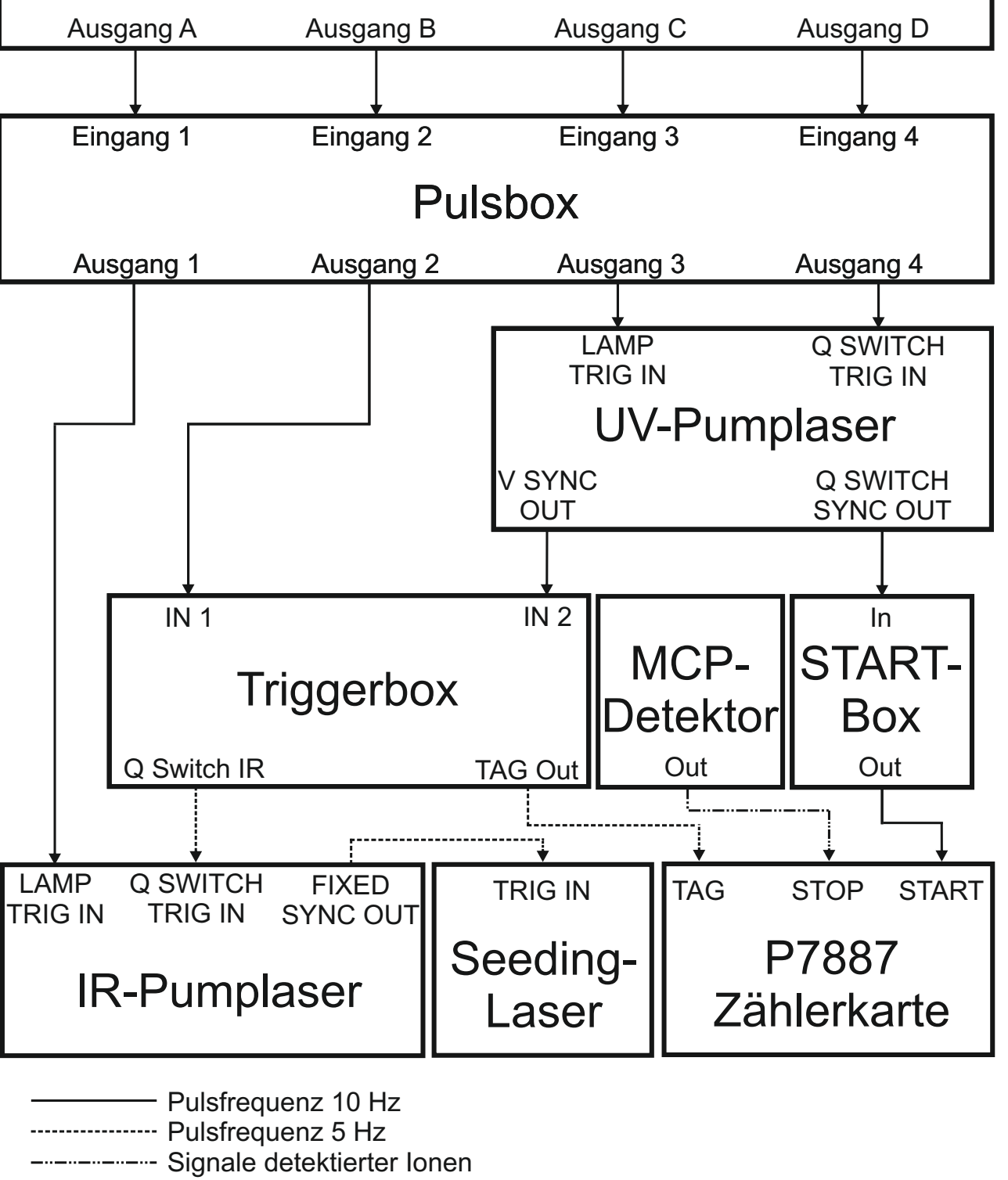

Abbildung 4.16.: Blockschaltbild der Steuerungskomponenten. 


\subsubsection{Automatisierung des Messablaufs}

Um die sich während einer Messung periodisch wiederholenden Abläufe zu automatisieren, wurde ein Programm auf Basis der Programmiersprache $\mathrm{C}++$ entwickelt, das imstande ist, mit der Steuerungssoftware der Zählerkarte (P7887 Server), das auf demselben PC installiert ist, sowie mit der Software des OPO/OPA-Systems (Motor Controls) zu kommunizieren. Letztere ist auf einem separaten PC installiert, der ebenfalls die Treiberkarten für die Schrittmotoren der Kristalle (vgl. Abschn. 4.6.3) beherbergt. Beide PC-Systeme arbeiten auf Basis des Betriebssystems Windows 7 und sind über ein lokales Netzwerk miteinander verbunden. Das Programm wurde unter Nutzung der Open Source IDE-Plattform Code::Blocks entwickelt. Da es während des Messbetriebs kontinuierlich evaluiert und verbessert wurde und außerdem die Anschaffung neuer Hardware geplant ist, die auch Anpassungen des Quellcodes erfordern wird, wurde es bisher nicht als unveränderliche, ausführbare Datei genutzt. Vielmehr wurden zur Änderung der Messparameter direkt die Werte der Variablen im Quelltext angepasst und das Programm vor jeder Messreihe durch den GCC Compiler 6.2 erneut kompiliert. Der komplette Quelltext ist im Anhang $\mathrm{D}$ wiedergegeben.

Die Kommunikation mit der Software Motor Controls erfolgt über eine von der Firma LaserVision bereitgestellte, ausführbare Datei namens MotorSendCmd.exe, die zur Fernsteuerung des OPO/OPA-Systems über ein lokales Netzwerk entwickelt wurde. Diese kann unter Übergabe einer Zeichenkette aufgerufen werden, die die IP-Adresse des betreffenden PCs, den von Motor Controls überwachten Netzwerkport sowie einen der vorgegebenen Befehle enthält (siehe hierzu die Dokumentation der Firma LaserVision 267). Die automatische Erfassung der IR-Pulsenergie erfolgt ebenfalls über Motor Controls. Die Software erfasst das analoge Ausgangssignal des IR-Energiemonitors (Modell MAESTRO der Firma Gentec, kombiniert mit einem pyroelektrischen Energiemesskopf vom Typ QE25SP$S-M B-D 0)$, dessen Amplitude sich proportional zur gemessenen IR-Pulsenergie verhält, über ein an den IR-Steuerungsrechner angeschlossenes USB-Voltmeter (Modell T3 der Firma Digital Measure).

Die Kommunikation mit der Software P7887 Server gestaltet sich schwieriger, da der Hersteller zur Integration der Zählerkarte in einen größeren apparativen Kontext optional erhältliche Software vorsieht, die nicht zur Verfügung stand. Da die vorhandene Software jedoch aus zwei Komponenten besteht (dem Serverprogramm P7887 Server, das die Zählerkarte steuert, sowie der Benutzeroberfläche $M C D W I N)$, die durch den Austausch von Windows Messages miteinander kommunizieren, lässt sich auf diesem Wege die Steuerung des Programms Pr887 Server erreichen, indem die von MCDWIN ausgehenden Windows Messages zunächst ausgelesen und bei Bedarf von anderer Stelle an das Server-Programm gesendet werden. So lässt sich bei Kenntnis des bei jedem Programmstart neu vergebenen window handle der Server-Anwendung durch die Übermittlung zweier aufeinander folgender Messages an das betreffende Fenster die Aufnahme einer Messung auslösen:

\begin{tabular}{c|c|c|c}
\hline Message Nr. & wParam & lParam & Methode \\
\hline 273 & 141 & 0 & send \\
273 & 109 & 0 & post
\end{tabular}

Tabelle 4.7.: Parameter der Windows Messages zum Starten einer Messung. 
Nach Ende einer Messung erzeugt das Programm P8778 Server zwei Textdateien, in denen die erhobenen Daten abgelegt werden. Diese Dateien besitzen den gleichen Namen, der sich aus einem vom Anwender vor Beginn der Messreihe vorgegebenen Präfix sowie einer dreistelligen, von $\mathrm{P}^{7887}$ Server fortlaufend vergebenen Laufnummer zusammensetzt, weisen jedoch unterschiedliche Endungen auf:

1. Eine Datei mit der Endung .asc, in der die beiden während der Messung simultan aufgenommenen Histogramme (die Messdaten) abgelegt werden.

2. Eine Datei mit der Endung .887, in der die Metadaten der betreffenden Messung (die Messbedingungen) erfasst sind.

Das Vorliegen der 887-Datei mit dem anfangs festgelegten Präfix und der anhand der Anzahl seit dem Programmstart bereits durchgeführter Messungen vorhergesagten Laufnummer im Dateinamen wird als Indikator für den Abschluss der betreffenden Messung herangezogen.

Vor der Programmausführung ist die Aktualisierung folgender Parameter notwendig:

- In einem Datenfeld (Array) namens IRWellenzahl werden durch Kommata getrennt sämtliche Wellenzahlen eingetragen, die im Laufe der aktuellen Messreihe angesteuert werden sollen.

- Der Dateipfad des Verzeichnisses, in dem die Software P7887 Server die Messdaten ablegt, in der Variable Speicherort

- Der Teil des Dateinamens, der anfänglich vorgegeben und von Pr887 Server nicht verändert wird, in der Variable praefix

- Die Laufnummer der Datei, deren Erzeugung nach Ende der ersten automatisch ausgelösten Messung erwartet wird, in der Variable laufnummer. Diese Variable wird vom Programm anschließend automatisch inkrementiert.

- Ggf. eine Anpassung der Variable maximale_startzahl. Diese sollte größer sein, als die im Laufe einer Messung durchgeführten Flugzeitmessungen. Sie dient dem Fehlerabfang, falls eine der drei obigen Eingaben nicht korrekt war, die erzeugte 887Datei nicht gefunden und das Ende der Messung nicht erkannt wird.

- Ggf. eine Anpassung der bool-Variablen pausieren. Erhält diese den Wert 1, unterbricht das Programm nach einer in der Variablen modulozahl festgelegten Anzahl von Messungen die Messreihe, um manuelle Änderungen der Messbedingungen zu erlauben. Nach Betätigung einer Taste auf der Tastatur wird die Messung fortgesetzt. Wird der Variable pausieren der Wert 0 zugewiesen, läuft das Programm ohne Unterbrechung durch.

Nach dem Programmstart werden innerhalb eines Schleifenkörpers in der Hauptfunktion main() unter Inkrementierung einer Variablen namens index, die der Indexnummer des aktuellen Elements im Array IRWellenzahl entspricht, für jede Messung folgende Operationen ausgeführt (siehe $\mathrm{Abb}$. 4.17): 
1. Bereitstellung einer Zeichenkette (String), die den Dateipfad der 887-Datei enthält, deren Erzeugung nach Ende der nächsten Messung erwartet wird, durch Konkatenierung der Strings Speicherort, praefix und der in einen String umgewandelten Variable laufnummer

2. Abfrage der eingestellten IR-Wellenlänge und ggf. deren Anpassung

3. Kontrolle, ob die Datei mit dem in Schritt 1 erzeugten Dateipfad bereits vorhanden ist. Existiert diese bereits, wird eine Fehlermeldung ausgegeben. Ein Tastendruck führt zum Ende des Programms, um ein Überschreiben bereits vorhandener Daten auszuschließen.

4. Falls die Variable pausieren den Wert 1 enthält, eine Unterbrechung des Programmablaufs zu Justagezwecken

5. Start einer Messung durch Übermittlung zweier Windows Messages an das Programm P7887 Server (siehe Tabelle 4.7)

6. Während einer Messung: Abfrage der aktuellen IR-Pulsenergie und Prüfung auf Existenz der nach Abschluss erzeugten 887-Datei in Intervallen von ca. $200 \mathrm{~ms}$

7. Nach dem Abschluss einer Messung: Prüfung, ob die Messung aus bestimmten Gründen erkennbar fehlgeschlagen ist (siehe unten). Falls kein Fehler erkennbar ist, wird die Variable index inkrementiert, andernfalls wird abermals das gleiche Element des Arrays IRWellenzahl abgerufen und die Messung somit wiederholt.

8. Aktualisierung von IR-Energie und Wellenzahl in der gerade erzeugten 887-Datei

9. Inkrementierung der Laufnummer zur Bereitstellung des Dateipfads der folgenden 887-Datei

Jeder Durchlauf dieser Schleife beginnt mit einem Aufruf der Funktion DateinamenBasteln(). Diese stellt durch Konkatenierung der Strings Speicherort, praefix und der in einen String umgewandelten Variable laufnummer einen String bereit, der den vollständigen Dateipfad der 887-Datei enthält, die nach Ende der nächsten Messung erzeugt wird. Diese Information wird vom Programm später benötigt, um erstens den Zeitpunkt zu ermitteln, zu dem die Messung abgeschlossen ist, und zweitens die während der Messung eingestellte IR-Wellenzahl sowie die gemittelte IR-Pulsenergie in diese Datei einzutragen.

Die Abfrage der eingestellten IR-Wellenzahl wird von der Funktion IRchecken() übernommen. Diese berechnet zunächst aus der im Array IRWellenzahl abgelegten Wellenzahl die Idler-Wellenlänge, die im OPO/OPA-System eingestellt sein sollte, um Strahlung dieser Wellenzahl zu erzeugen. Anschließend ruft sie das Programm MotorSendCmd.exe unter Übergabe eines Befehlsstrings auf, der die Rückgabe der im OPO eingestellten Idler-Wellenlänge bewirkt. Beim anschließenden Vergleich dieser beiden Werte unterscheidet die Funktion drei Fälle:

1. Fall 1: Beide Werte unterscheiden sich um weniger als 0,03 nm. In diesem Fall weicht die eingestellte Wellenzahl um weniger als $0,5 \mathrm{~cm}^{-1}$ von der Benötigten ab und die Funktion kehrt ohne weitere Operationen zurück. 


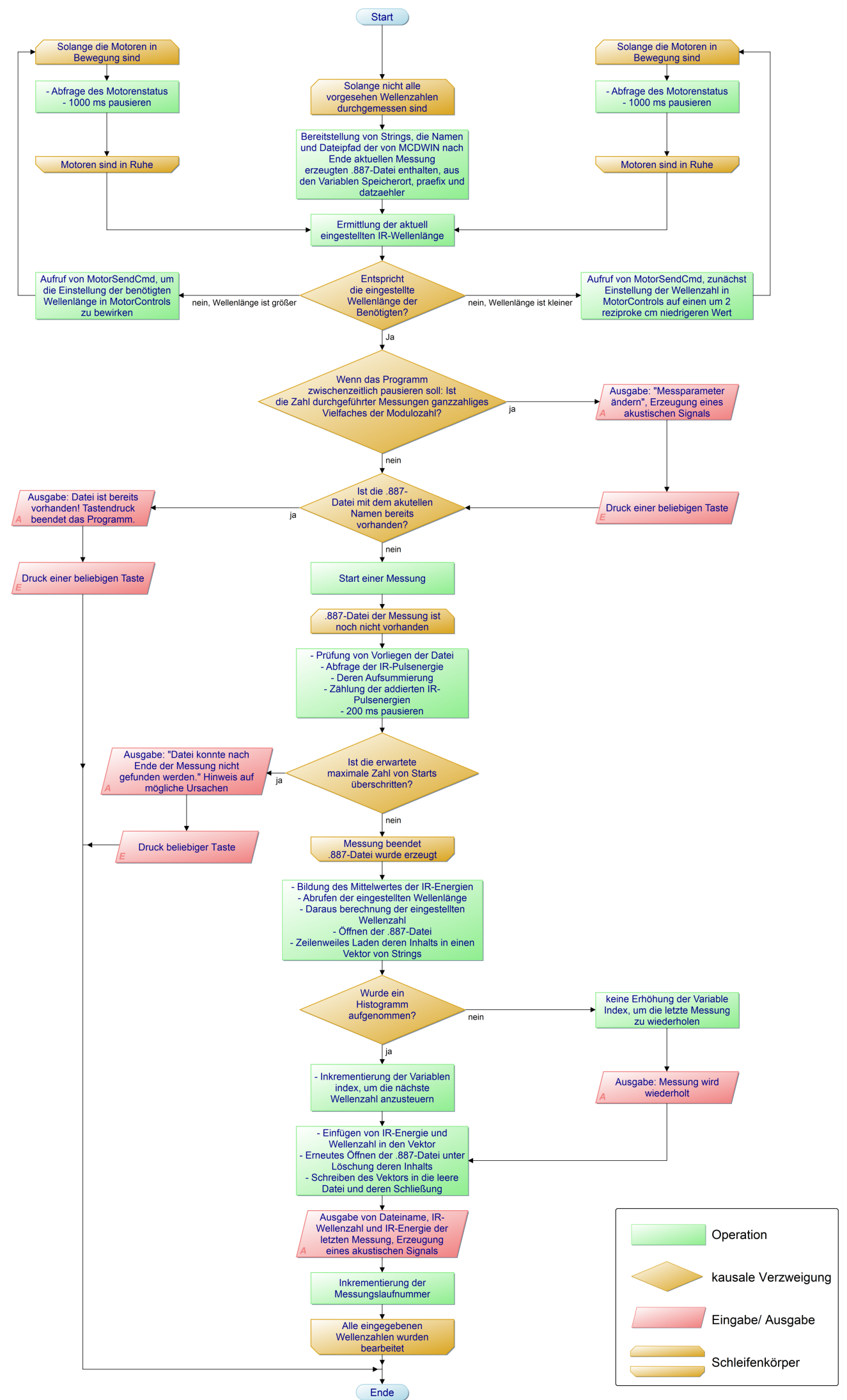

Abbildung 4.17.: Programmablaufplan des Quelltextes zur Automatisierung des Messablaufs. 
2. Fall 2: Die am OPO/OPA-System eingestellte Idler-Wellenlänge ist größer als die Erforderte, d. h. die eingestellte Wellenzahl ist zu niedrig. In diesem Fall ruft sie die Funktion IREinstellen() auf, um die benötigte Wellenzahl einzustellen. Diese ruft abermals die Funktion IRchecken() auf, die nach erfolgreicher Neueinstellung der Wellenzahl mit Fall 1 enden sollte.

3. Fall 3: Die am OPO/OPA-System eingestellte Idler-Wellenlänge ist kleiner als die Benötigte, d.h. die eingestellte Wellenzahl ist zu hoch. In diesem Fall ruft sie die Funktion IREinstellen() unter Übergabe einer Wellenzahl auf, die um $2 \mathrm{~cm}^{-1}$ niedriger ist, als die Benötigte. Anschließend ruft diese analog zu Fall 2 die Funktion IRchecken() auf, die nun mit Fall 2 enden sollte, sodass im folgenden Schritt die benötigte Wellenzahl eingestellt wird. Die Einstellung erfolgt nunmehr aus der Richtung niedrigerer Wellenzahlen.

Aufgrund des Spiels zwischen den Getriebebauteilen der Schrittmotoren weisen die gemessenen IR-Banden einen Versatz von ca. $0,8 \mathrm{~cm}^{-1}$ relativ zueinander auf, wenn sie unter Anlaufen der Kristallpositionen im OPO/OPA-System aus unterschiedlichen Richtungen aufgenommen wurden. Durch diesen rekursiven Algorithmus wird erstens die oben angesprochene Fehlerquelle ausgeschaltet und zweitens sichergestellt, dass die eingestellte IR-Wellenlänge in jedem Fall überprüft wird, bevor eine Messung gestartet wird.

Die Funktion IReinstellen() erhält als Übergabewert die aktuell benötigte Ausgangswellenzahl. Diese muss zunächst in die am OPO/OPA-System einzustellende IdlerWellenlänge umgerechnet und in einen String eingebettet werden, der an MotorSendCmd übergeben werden kann. Diese Aufgaben werden von der Funktion kommandozeileBasteln() übernommen, die von IReinstellen() aufgerufen wird und den benötigten String zurückliefert. Dieser wird über MotorSendCmd an Motor Controls übergeben.

Die Änderung der IR-Wellenzahl wird durch Verkippung der auf Schrittmotoren montierten KTP- und KTA-Kristalle im Gehäuse des OPO/OPA-Systems erreicht. Da nach Absenden des Befehls an Motor Controls keine Rückmeldung über den Abschluss dieses mehrere Sekunden andauernden Vorgangs erfolgt, muss dieser Zeitpunkt durch eine wiederholte Abfrage ermittelt werden. Hierzu wird aus der Funktion IReinstellen() die Funktion RuhezustandAbwarten() aufgerufen, die in Intervallen von $1000 \mathrm{~ms}$ den Zustand der Schrittmotoren des OPO/OPA-Systems abfragt. Auf diese Abfrage liefert Motor Controls einen Hexadezimalwert zurück, in dem die Bewegungsrichtungen sämtlicher im System eingebauten Motoren verschlüsselt ist. Dieser Wert ist von Null verschieden, sofern der Einstellvorgang andauert und sich einer der Motoren in Bewegung befindet. Ist der Rückgabewert Null, kehrt die Funktion RuhezustandAbwarten() zurück und aus der Funktion IReinstellen() wird abermals die Funktion IRchecken() aufgerufen, um den Erfolg des Einstellvorgangs zu kontrollieren.

Nach erfolgreicher Einstellung der IR-Wellenzahl wird das Programm P7887 Server zur Aufnahme eines Histogramms veranlasst, indem die Funktion MessungStarten() aufgerufen wird, die wie oben beschrieben zwei Windows Messages an dessen Fenster übermittelt. Zuvor prüft diese Funktion, ob die 887-Datei, deren Erzeugung erst nach Abschluss der nun folgenden Messung erwartet wird, aufgrund einer fehlerhaften Eingabe eventuell bereits vorhanden ist. In diesem Falle gibt sie eine Fehlermeldung aus und beendet das Programm nach einem Tastendruck, um bereits vorhandene Messdaten nicht zu überschreiben. 
Nach dem Start der Messung wird aus der Hauptfunktion die Funktion HistogrammAufnehmen() aufgerufen. Diese Prüft im Intervall von $200 \mathrm{~ms}$ das Vorliegen der 887Datei, die im Anschluss an eine Messung vom Programm P7887 Server erzeugt wird. Ihr Vorhandensein dient als Signal für den Abschluss der Messung. Sofern die Datei noch nicht vorhanden ist, wird nach jeder Prüfung von Motor Controls die zuletzt vom IREnergiemesskopf gemessene IR-Pulsenergie abgefragt, um nach Abschluss der Messung den Mittelwert aller Pulsenergien zu bilden. Liegt die Datei vor, wird sie zunächst wieder geschlossen. Dann wird der Mittelwert der gemessenen IR-Pulsenergien gebildet, abermals die eingestellte IR-Wellenzahl abgefragt und diese beiden Informationen in einer Zeichenkette vereint, die als neue Zeile 14 in die eben erzeugte 887-Datei eingesetzt werden soll. Anschließend wird die 887-Datei abermals geöffnet und ihr Inhalt zeilenweise in einen Vektor geladen. Die neue Zeile 14 wird an Indexposition 13 in den Vektor eingefügt. Die 887-Datei wird geleert und ihr Inhalt wird durch die Elemente des Vektors ersetzt, in dem die betreffende Zeile ausgetauscht wurde.

Ein gelegentlich (ca. alle 500 bis 1000 Messungen) auftretendes Fehlerbild äußert sich darin, dass das Programm Pr887 Server die START-Signale vom UV-Pumplaser nicht korrekt verarbeitet. Es wird kein Histogramm aufgenommen und das Programm $M C D$ WIN zeigt keine Inkrementierung der Anzahl bereits erfolgter STARTS an. Trotzdem wird die Messung nach Eingang der vorgesehenen Anzahl von STARTS unter Erzeugung der beiden Ausgabedateien beendet. Die asc-Datei enthält jedoch kein Histogramm und die 887-Datei weist in Zeile 7, die die Anzahl der START-Pulse angibt, den Wert Null aus. Dieser Fehler tritt sowohl bei manueller als auch bei automatischer Bedienung des Messprogramms auf. Um eine Beeinträchtigung der Datenqualität durch einen solchen Ausfall einzelner Messwerte zu vermeiden, wird ebenfalls die betreffende Zeile der 887Datei ausgelesen. Falls keine Aufnahme eines Histogramms erfolgt ist, wird die Variable index, die der Position der aktuellen IR-Wellenlänge im Array IRWellenzahl entspricht, nicht erhöht, sodass die letzte Messung wiederholt wird. 


\section{Datenverarbeitung}

In diesem Kapitel soll die Gewinnung interpretierbarer Datensätze aus den vom MCPDetektor erzeugten Spannungspulsen erläutert werden. Die Erhebung von Rohdaten und deren Speicherung in Histogrammen folgt weitgehend dem Modus früherer Arbeiten 258. Der veränderte Messmodus erforderte hingegen die Entwicklung eines neuen Algorithmus zur softwarebasierten Datenauswertung, der in Abschnitt 5.2 ausführlich dargelegt werden soll, um vollständige Transparenz bezüglich der Ergebnisse dieser Arbeit herzustellen und zukünftigen AnwenderInnen die Möglichkeit zu geben, diesen Algorithmus ebenfalls zu nutzen, bei Bedarf nachzuvollziehen und ggf. den Anforderungen zukünftiger Fragestellungen entsprechend zu modifizieren.

\subsection{Erhebung und Speicherung von Rohdaten}

Die vom MCP-Detektor erzeugten Spannungspulse werden durch einen Vielkanalzähler in Form einer Zählerkarte (Modell P7887 der Firma FAST ComTec GmbH) erfasst und anhand der zeitlichen Verzögerung ihres Eintreffens an deren STOP-Eingang gegenüber dem am START-Eingang eintreffenden Signal der Startbox in ein Histogramm eingeordnet, auf dessen Abszissenachse die Kanalnummer aufgetragen ist. Hierfür verfügt die Zählerkarte über $2^{38}$ Kanäle, die fortlaufend aktiviert werden und jeweils für ein Zeitfenster von 250 ps geöffnet sind. Die Schwelle, oberhalb derer ein Spannungspuls als Signal erfasst wurde, wurde so gewählt, dass der Quotient aus der Anzahl registrierter Zählereignisse und dem Signal-Rausch-Verhältnis optimiert wurde.

Da die Spannungspulse des MCP-Detektors jeweils eine Dauer von 2-3ns besitzen, werden die Kanäle zu Gruppen von jeweils 32 Stück zusammengefasst, sodass die Zeitauflösung der Zählerkarte auf 8 ns herabgesetzt und die erzeugte Datenmenge reduziert wird. Zur weiteren Datenreduktion wurde die Anzahl der in einem Histogramm erfassten Kanalgruppen (die sogenannte range) auf die Anzahl von 147456 Gruppen beschränkt, sodass nach dem Eintreffen eines Startsignals jeweils ein Zeitfenster von 1,18 ms erfasst wurde, was zur vollständigen Erfassung der erzeugten Cluster in einem TOF-Massenspektrum ausreichend war. Aus der Position eines Signals im Histogramm kann somit zunächst die Flugzeit des Ions und daraus dessen Masse, sowie im hier vorliegenden Falle natriumdotierter Wassercluster, die Konstituentenzahl $N\left(\mathrm{H}_{2} \mathrm{O}\right)$ berechnet werden.

Die Zählerkarte verfügt über eine sogenannte TAG-Option, die eine simultane Aufnahme von mehreren Histogrammen gestattet. Wie in Abschnitt 4.8 beschrieben, wurden jeweils zwei Histogramme gleichzeitig aufgenommen, von denen eines das Hintergrundsignal und eines das IR-anregungsmodulierte Signal enthält. Sofern eine Spannung am $T A G$-Anschluss anliegt, werden die währenddessen eintreffenden Signale dem letzteren Spektrum zugeordnet. Meist wurde die Anzahl der STARTS, die zu einer Messung zusammengefasst wurden, auf einen Wert von 2000 eingestellt. Dieser Wert entspricht gleichzeitig der Anzahl der während einer Messung abgegebenen UV-Laserpulse, wobei jedem 
zweiten UV-Puls ein IR-Laserpuls vorausging. Die resultierenden Histogramme werden von der Software P7887 Server in einer gemeinsamen ASCII-Datei mit der Endung .asc abgelegt, in der die Zählereignisse sämtlicher Kanalgruppen zeilenweise angeordnet sind. Die erste Hälfte der Datei enthält das Hintergrundhistogramm, während die zweite Hälfte das Histogramm mit den anregungsmodulierten Signalen enthält. Die Anzahl der Zeilen in dieser Datei entspricht somit dem doppelten Wert der range, die von der Software P7887 Server erfasst wird.

Vor Beginn einer Messung wurden sämtliche manuell eingestellten Versuchsparameter in die Benutzeroberfläche $M C D W I N$ eingetragen. Diese Informationen werden von P7887 Server gemeinsam mit weiteren Metadaten in einer separaten Textdatei mit gleichem Namen und der Endung .887 abgelegt. Die während der Messung durch die in Abschnitt 4.8.2 beschriebene Automatisierungssoftware eigenständig veränderten Parameter wurden jeweils nach Erzeugung der 887-Datei automatisch darin ergänzt (siehe dort). Die Daten einer Messreihe wurden jeweils in einem Ordner abgelegt, dessen Name dem sechsstelligen Datum der Messung in absteigender Reihenfolge (Jahr-Monat-Tag) entspricht. Die Namen der von Pr887 Server erstellten Dateien bestehen aus einem Präfix, das sich aus der Datumsangabe in gleicher Form sowie einem „a“ zusammensetzt, gefolgt von einer dreistelligen Laufnummer.

\subsection{Automatisierte Datenauswertung}

Im Zuge der Verbesserung des Messmodus wurde eine neue Auswertungssoftware entwickelt, die in diesem Abschnitt ausführlich vorgestellt werden soll. Die Software basiert auf der Programmiersprache Python 3.4 und erfordert zur Ausführung die Installation einer geeigneten Python-Distribution sowie der Module NumPy, SciPy und Matplotlib.

Python wurde gewählt, da diese Programmiersprache im Gegensatz zur zuvor genutzten, proprietären Software Matlab einer Open Source-Lizenz unterliegt. Als Interpretersprach $£^{1}$ erlaubt Python jederzeit Zugriff auf den Quelltext. Dieser fällt darüber hinaus um den Faktor 2 bis 10 kürzer aus, als der eines vergleichbaren, z. B. in der Compilersprache ${ }^{2} C$ verfassten Programms. Die Kürze und die geringere Komplexität eines in Python verfassten Quelltextes vereinfachen weiterhin dessen Wartung, vermindert die Wahrscheinlichkeit von Programmierfehlern und ermöglicht zukünftigen AnwenderInnen eine schnellere Einarbeitung sowie die einfache Anpassung des Codes an die jeweiligen Erfordernisse 272]. Die Software übernimmt folgende Aufgaben:

- Sie ermittelt eigenständig die Positionen $t_{\text {Peak, } N}^{\prime}$ der Massensignalmaxima (Peaks) sowie von deren Grenzen $t_{\text {Anfang, } N}^{\prime}$ und $t_{\text {Ende, } N}^{\prime}$, wobei sie zuverlässig den Größenbereich von $N_{\min }=4$ bis $N_{\max }>100$ abdeckt.

- Sie bestimmt die Parameter einer quadratischen Regressionsfunktion, mit deren Hilfe auch Clustergrößen $N$ im Größenbereich oberhalb von $N_{\max }$ für die Auswertung zugänglich werden.

\footnotetext{
${ }^{1}$ d. h. der Quelltext wird erst während der Programmausführung in Maschinensprache übersetzt

${ }^{2}$ Die Übersetzung in Maschinensprache (Kompilieren) erfolgt einmalig bei Fertigstellung des Programms
} 
- Die Ergebnisse dieses ersten Auswertungsschrittes werden in zwei Diagrammen visualisiert, sodass eventuell fehlerhaft bestimmte Integrationsgrenzen und Regressionsparameter auf einen Blick erkennbar sind.

- Sie berechnet entsprechend der Vorgaben bezüglich der auszuwertenden Einzelgrößen und Größenbereiche die Summen $\Sigma_{N}$ der Zählraten und deren Zunahme $I_{N}$ der vorgegebenen Clustergrößen $N$ und stellt die Ergebnisse in Kombination mit den zugehörigen Metadaten in einer csv-Datei zusammen, sodass weitere Auswertungsschritte mittels eines Tabellenkalkulationsprogramms durchgeführt werden können.

- Falls gewünscht, erstellt sie nach der Auswertung eine zweidimensionale Auftragung, in der zwei beliebige Parameter der Messreihe einander gegenübergestellt werden. Dabei bietet sie die Möglichkeit, eine Auswahl aus den zuvor ausgewerteten Größenbereichen zu treffen, um das Verhalten von Clustern unterschiedlicher Größe bereits während der Aufnahme einer Messreihe zu vergleichen.

\subsubsection{Bedienung der Auswertungssoftware}

Das Skript ist so programmiert, dass es als Konsolenprogramm bedient werden kann. Somit können die erforderlichen Eingaben ohne Kenntnis des Quelltextes getätigt werden. Die Eingabe des Wortes „Hilfe“ bewirkt die Anzeige eines Hilfetextes, in dem sämtliche Optionen der Software erläutert werden. Die Eingabe von „Stop“ beendet das Programm. Zur Übergabe von Anweisungen kann eine Zeichenkette eingegeben werden, die optional aus den unten aufgeführten Befehlen zusammengesetzt ist. Mehrere Befehle werden dabei durch einen doppelten Bindestrich (--) getrennt.

- Hilfe: Bewirkt die Ausgabe des Hilfetextes.

- Stop: Beendet das Programm.

- - -Pfad: Gibt den Dateipfad des Verzeichnisses an, in dem die auszuwertenden Dateien gespeichert sind. Der Wert wird der Variablen Dateipfad zugewiesen. Standardwert: Der Speicherort der Skriptdatei (wird vom Programm selbstständig erkannt). Beispiel: - -Pfad C: \P7887\170904

- - -Uverz: Sind die Dateien in einem Unterverzeichnis des Verzeichnisses abgelegt, in dem die Skriptdatei gespeichert ist, kann dessen Dateipfad ausgehend vom Speicherort der Skriptdatei hiermit angegeben werden. Dieser Wert wird an den in der Variablen Dateipfad abgelegten String angehängt. Beispiel: - -Uverz 170904\170904

- - -Praefix: Gibt den Teil des Dateinamens an, der allen auszuwertenden Dateien gemeinsam ist. Der Wert wird der Variablen Dateipraefix zugewiesen. Standardwert: Letzte 6 Zeichen des Dateipfads, gefolgt von einem „a“; Beispiel: - -Praefix 170904a

- - -Dateibereich: Gibt, getrennt durch einen Bindestrich, die Laufnummern der ersten und der letzten in die Auswertung einzubeziehenden Datei an, falls nicht alle Dateien im gewählten Ordner ausgewertet werden sollen. Die Anweisung wird in zwei Ganzzahlen aufgetrennt, deren Werte den Variablen erste_Datei und letzte_Datei zugewiesen werden. Standardwert: 1-400; Beispiel: --Dateibereich 20-130 
- - -Einzelgroessen: Größenbereich, in dem jede Clustergröße $N$ einzeln ausgewertet werden soll, getrennt durch einen Bindestrich; diese Auswertung kann nur für Clustergrößen im Bereich $N_{\min } \leq N \leq N_{\max }$ erfolgen. Der Wert wird der Variablen Einzelgroessen zugewiesen. Beispiel: --Einzelgroessen 13-100

- - -Groessenbereiche: Bereiche, in denen mehrere Clustergrößen zusammengefasst werden sollen, getrennt durch einen Bindestrich. Mehrere Angaben werden durch Doppelpunkte separiert. Der Wert wird der Variablen Groessenbereiche zugewiesen. Beispiel: - -Groessenbereiche 10-19:20-29:30-39:40-49

- - Kanalbereiche: Auszuwertende Bereiche zwischen Kanälen im Histogramm, getrennt durch einen Bindestrich. Mehrere Bereiche können durch Doppelpunkte getrennt angegeben werden. Der Wert wird der Variablen Kanalbereiche zugewiesen. Beispiel: - Kanalbereiche 3300-3400:6500-80000

- - -findePeaks: Wird das Programm zum ersten Mal in einem Verzeichnis ausgeführt, ermittelt es zunächst die Peakpositionen, -grenzen sowie die Regressionsparameter und legt diese in Dateien namens Peakstarts.txt, Peakends.txt, Peaks.txt und Fitparameter.txt ab. Sind diese Dateien beim Programmstart vorhanden, wird ihr Inhalt geladen und die Bestimmung der Signalpositionen wird nicht erneut ausgeführt. Bei Eingabe dieses Befehls wird der bool-Variablen finde_Peaks der Wert 1 zugewiesen und das Programm führt in jedem Fall eine erneute Berechnung dieser Daten durch. Standardwert: 0; Beispiel: --findePeaks

- - -Plot: Bewirkt eine graphische Darstellung der Auswertungsergebnisse in einer Auftragung im Format (x-Achse):(y-Achse):(Clustergrößen- bzw. Kanalbereiche). Die Angabe der auf den Achsen aufzutragenden Größen erfolgt durch Eingabe von Nummern. Es kann unter folgenden Parametern gewählt werden: 1: ROI-Summe, 2: Starts, 3: Trägergasdruck $\left(p_{\text {Gas }}\right)$, 4: MCP-Spannung $\left(U_{\mathrm{HV}}\right)$, 5: IR-Wellenzahl $\left(\tilde{\nu}_{\mathrm{IR}}\right)$, 6: IR-Energie $\left(E_{\mathrm{IR}}\right)$, 7: UV-Wellenlänge $\left(\lambda_{\mathrm{UV}}\right)$, 8: UV-Energie $\left(E_{\mathrm{UV}}\right)$, 9: Delay $(\Delta t)$, 10: Startkanal, 11: Endkanal, 12: (nicht vergeben), 13: Signalstärke ohne IR-induzierte Anregung $\left(\Sigma_{\mathrm{UV}, N}\right), 14$ : Signalstärke mit IR-induzierter Anregung $\left(\Sigma_{\mathrm{IR}+\mathrm{UV}, N}\right)$, 15: Signalzunahme $\left(I_{N}=\Sigma_{\mathrm{IR}+\mathrm{UV}, N} / \Sigma_{\mathrm{UV}, N}\right), 16$ : Auf IR-Energie normierte Signalzunahme $\left(I_{N}\right.$, normiert $\left.=\left(I_{N}-1\right) / E_{\mathrm{IR}}\right)$. Der eingegebene String wird der Variablen Plotparameter als Wert zugewiesen. Beispiel: - -Plot 5:15 trägt die Signalzunahme über der IR-Wellenzahl auf. Sofern bei der Auswertung mehrere Größenbereiche angegeben wurden, kann aus diesen eine Auswahl für die Auftragung getroffen werden. Dies geschieht durch Eingabe der gewünschten Bereiche, getrennt durch weitere Doppelpunkte. Beispiel: - -Plot 5:16:20:22:30-39:40-49 bewirkt die Auftragung der auf die IR-Energie normierten Signalzunahme über der IR-Wellenzahl für Cluster der Größenbereiche $N=20,22,30-39$ und $40-49$.

Es ist leicht möglich, die voreingestellten Auswertungsparameter in der Kopfzeile der Funktion Eingabe() anzupassen, sodass diese nicht bei jeder Nutzung erneut eingegeben werden müssen. Unter Windows besteht ferner die Möglichkeit, eine vorbereitete Skriptdatei in das Verzeichnis mit den Messdaten zu kopieren und dort auszuführen. Vorteil dieser Vorgehensweise ist, dass eine Kopie des Skriptes in der die für die Auswertung 
verwendeten Form in diesem Verzeichnis verbleiben kann und eine lückenlose Dokumentation der Datenverarbeitung sichergestellt wird. Diese Arbeitsweise wurde im Rahmen der vorliegenden Arbeit gewählt.

\subsubsection{Funktionsweise des Algorithmus}

In Abbildung 5.1 ist die Arbeitsweise des Auswertungsskriptes als Programmablaufplan dargestellt. Der vollständige, kommentierte Quelltext ist in Anhang E wiedergegeben.

Nach Ausführung des Skriptes wird zunächst die Funktion Eingabe() aufgerufen, die zur optionalen Eingabe von 'Hilfe', 'Stop' oder einer Kommandozeile auffordert, die einige der in Abschnitt 5.2.1 beschriebenen Befehle enthält. Daraufhin wird versucht, die Anwendereingabe aufzulösen. Parameter, die erkannt werden, werden den entsprechenden Variablen als Werte zugewiesen. Wurde ein Parameter nicht gesetzt oder nicht erkannt oder konnte die Eingabe aufgrund falscher Syntax nicht verarbeitet werden, kommen die in der Kopfzeile der Funktion Eingabe() vorgegebenen Werte zum Einsatz.

Nach Anpassung der Variablenwerte, unter denen sich auch die Angaben über Speicherort, Dateipräfix und Laufnummern der auszuwertenden Dateien befinden, werden diese Parameter an die Funktion lade_daten() übergeben. Diese iteriert über die eingegebenen Laufnummern, konkateniert dabei jeweils Dateipfad, Präfix und die fortlaufende Nummer zu einem String und versucht, die betreffende 887-Datei zu öffnen. Ist die 887-Datei vorhanden, werden daraus die Metadaten ausgelesen. Kann sie nicht geöffnet werden, geht die Funktion zur nächsten Laufnummer über.

Liegt die 887-Datei vor, wird ebenfalls versucht, die gleichnamige asc-Datei zu öffnen. Bei Erfolg wird deren Inhalt zeilenweise eingelesen und in einem NumPy-Array abgelegt. Dieser Datentyp ermöglicht die effiziente Handhabung großer Datenmengen unter Python.

Falls auch die zugehörige asc-Datei gefunden wurde, werden die aus der 887-Datei extrahierten Metadaten an entsprechende Listen angehängt, andernfalls werden sie unter Ausgabe eines Hinweises verworfen. Somit ist sichergestellt, dass trotz eventueller Fehler beim Löschen oder Verschieben von Dateien eine richtige Zuordnung von Messdaten und Metadaten erfolgt.

Im nächsten Schritt wird die Funktion ermittele_Parameter() aufgerufen. Diese stellt für die folgende Auswertung die Kanalnummern von $t_{\text {Anfang, } N}^{\prime}, t_{\text {Ende, } N}^{\prime}$ und $t_{\text {Peak }, N}^{\prime}$ sowie die Regressionsparameter $t_{0}^{\prime}, c^{\prime}$ und $b$ der Ausgleichskurve bereit. Wurde das Skript bereits einmal in dem Verzeichnis ausgeführt, in dem es gespeichert ist, sind diese Werte bereits in Form von Textdateien namens Peakstarts.txt, Peakends.txt, Peaks.txt und Fitparameter.txt hinterlegt. Falls jede dieser Dateien vorliegt, wird deren Inhalt ausgelesen. Der Inhalt der ersten drei Dateien wird in Listen mit gleichem Namen gespeichert, die Werte der Regressionsparameter werden den Variablen t0, $c$ und $b$ zugewiesen. Diese Informationen werden an die Funktion Eingabe() zurückgegeben.

Sofern eine der vier Dateien nicht vorliegt oder die Eingabe - -findePeaks erfolgt ist, werden die Parameter neu ermittelt. Hierzu wird aus der Funktion ermittele_Parameter() die Funktion suche_clusterpeaks() aufgerufen.

Die Funktion suche_clusterpeaks() koordiniert die Auswertungsschritte zur Ermittlung der Positionen von Maxima $\left(t_{\text {Peak, } N}^{\prime}\right)$ und Grenzen $\left(t_{\text {Anfang, } N}^{\prime}, t_{\text {Ende, } N}^{\prime}\right)$ der Massen- 


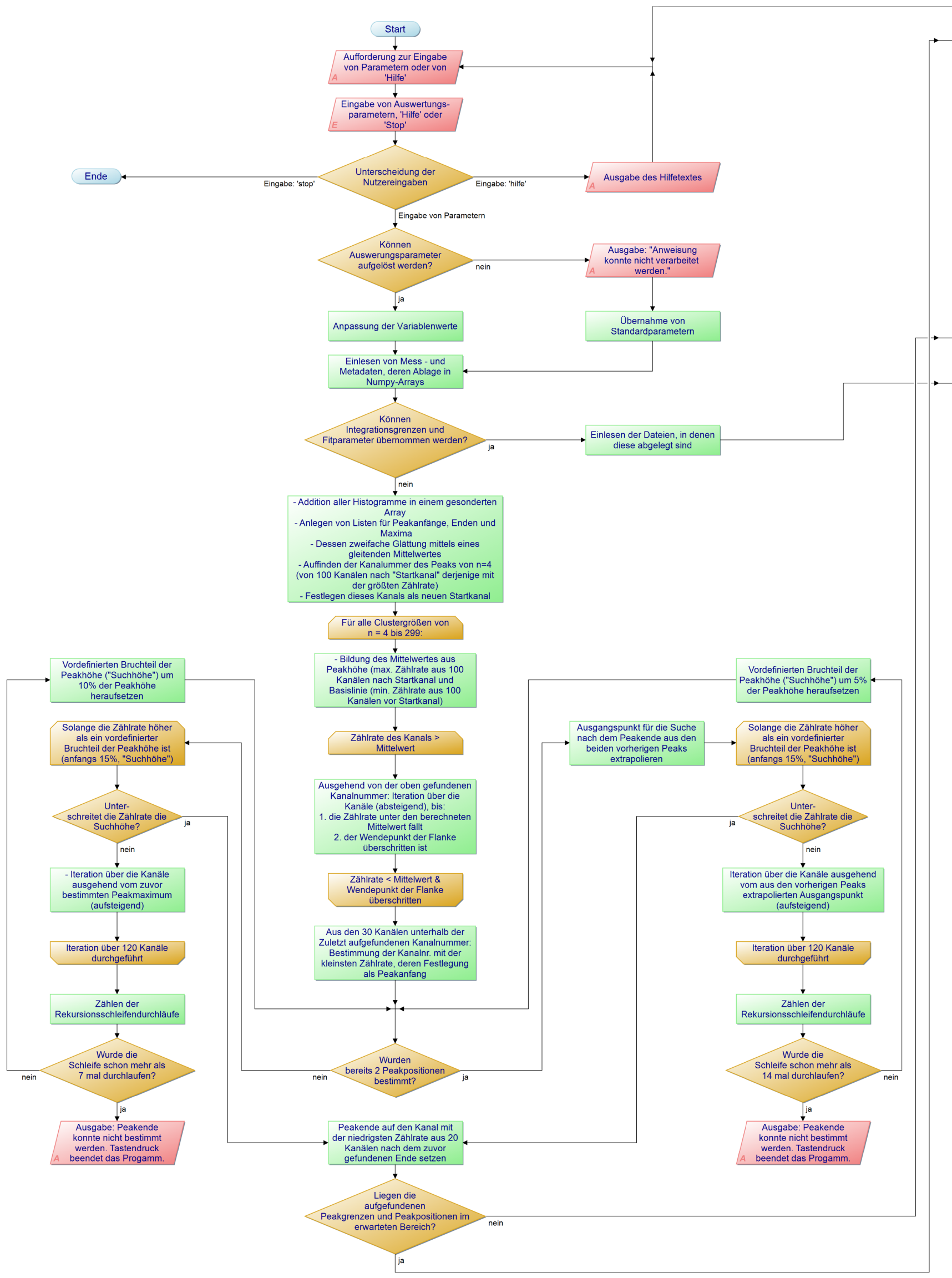

Abbildung 5.1.: Programmablaufplan des Auswertungsskripts. 





signale sowie der Regressionsparameter:

- Die Erzeugung eines NumPy-Arrays, das die Summe aller Histogramme der Messreihe enthält, um für die Bestimmung der Peakpositionen eine maximale Signalstärke $\mathrm{zu}$ erreichen durch Aufruf der Funktion addiere_massenspektren().

- Eine zweimalige Glättung des eben erzeugen Arrays, die durch zweimaligen Aufruf der Funktion moving_average() erreicht wird. Hierdurch wird das statistische Rauschen soweit minimiert, dass die Positionen der Massensignale zuverlässig ermittelt werden können.

- Das Auffinden der Peakmaxima $t_{\text {Peak, } N}^{\prime}$ sowie deren Grenzen ( $t_{\text {Anfang, } N}^{\prime}$ und $\left.t_{\text {Ende, } N}^{\prime}\right)$ in einem möglichst weiten Größenbereich durch Aufruf der Funktion finde_maxima ()

- Die Bestimmung der Regressionsparameter aus zwei von der Funktion finde_maxima() erzeugten Listen mit den Peakpositionen $t_{\text {Peak, } N}^{\prime}$ und den zugehörigen Konstituentenzahlen $\mathrm{N}\left(\mathrm{H}_{2} \mathrm{O}\right)$ durch Aufruf der Funktion bestimme_Fitparameter()

- Erzeugung zweier Auftragungen zur Visualisierung der Ergebnisse aus den letzten Schritten. Erstere zeigt ein durch Addition der Histogramme aller in die Auswertung eingeflossenen Einzelmessungen erzeugtes Histogramm mitsamt der aufgefundenen Peaks sowie deren Grenzen in Form vertikaler Geraden. Letztere zeigt eine Auftragung der Clustergröße $N\left(\mathrm{H}_{2} \mathrm{O}\right)$ über den Kanalnummern $t_{\text {Peak, } N}^{\prime}$ der Peaks mitsamt der durch bestimme_Fitparameter() ermittelten Regressionskurve. Diese erlaubt die sofortige Feststellung eventueller fehlerhaft bestimmter Peakpositionen.

- Ablegen der Werte in Dateien namens Peakstarts.txt, Peakends.txt, Peaks.txt und Fitparameter.txt, um diese bei einem erneuten Programmaufruf nutzen zu können.

Die Funktion finde_maxima() übernimmt die Bestimmung der Peakpositionen sowie der Grenzen der Massensignale. Der hierfür verwendete Algorithmus wurde so gewählt, dass er die bei dem vorliegenden apparativen Aufbau und in dem durchgeführten Experiment auftretenden spezifischen Schwierigkeiten durch Ausnutzung der sich in diesem speziellen Fall bietender Vorteile überwindet. Folgende Eigenschaften der erhobenen Messdaten wurden bei der Entwicklung in Betracht gezogen:

Die Signale sind mit denen von Reaktionsprodukten mehrfach dotierter Cluster assoziiert, mit denen sie sich meist derart überschneiden, dass diese nur noch als Schultern der Hauptsignale erkennbar sind (vgl. Abb. 5.2, Teil b). Hier soll eine bestmögliche Trennung unter gleichzeitiger Minimierung der hierbei verworfenen Signalintensität erreicht werden.

Die Auflösung des Massenspektrometers ist limitiert, sodass im Größenbereich um $N\left(\mathrm{H}_{2} \mathrm{O}\right)=50$ auch die Massensignale beginnen, sich zu überschneiden. Folge ist eine kontinuierliche Anhebung der observablen Grundlinie, die sich der gleichzeitig abnehmenden Signalamplitude annähert (siehe Abb. 5.2, Teil a). Im gleichen Zuge nimmt das Signal-Rausch-Verhältnis in diesem Bereich des Histogramms ab, sodass die Bestimmung der Peakpositionen $t_{\text {Peak, } N}^{\prime}$ ungenau wird (siehe Abb. 5.2, Teil c). Dennoch sollten diese 

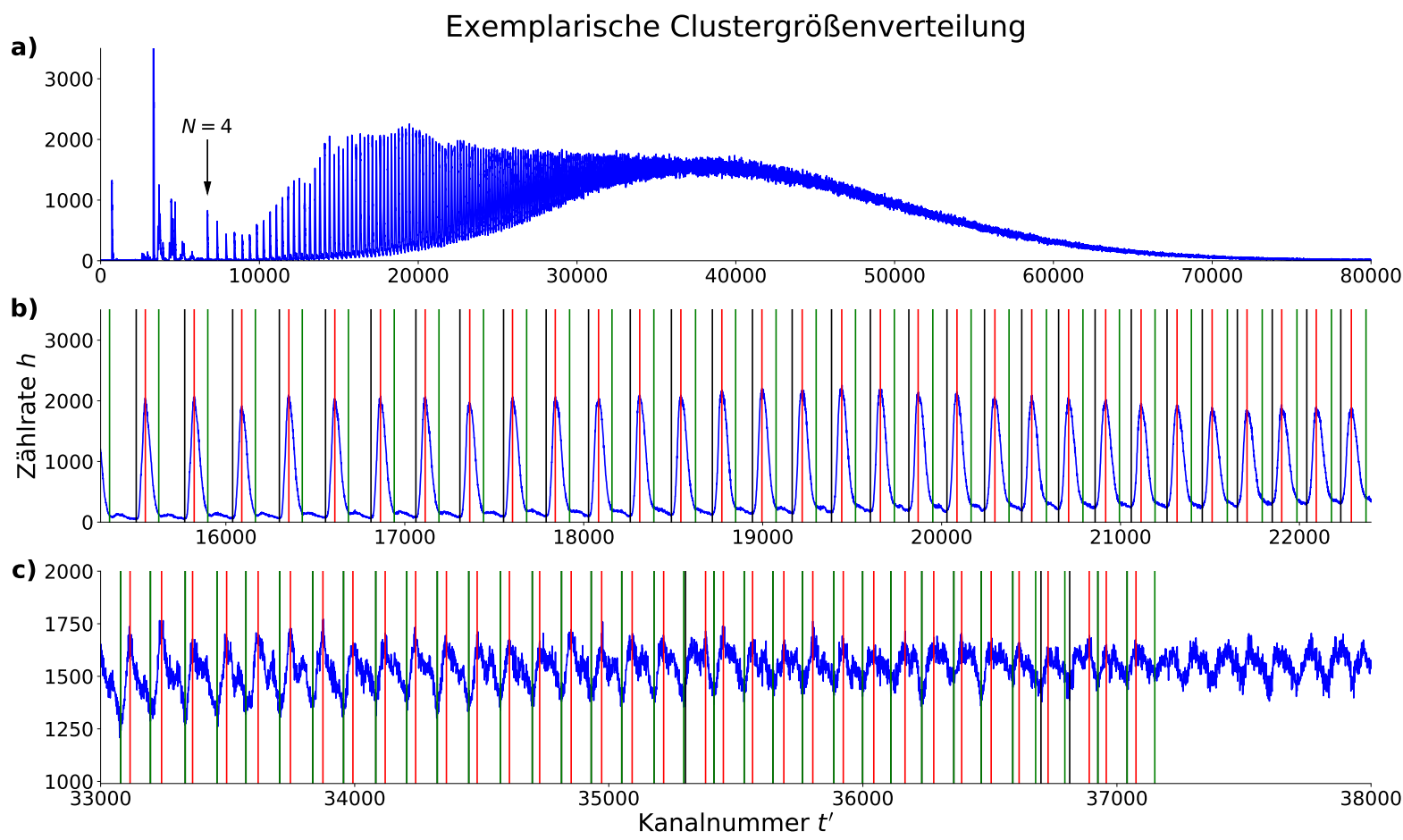

Abbildung 5.2.: Exemplarische Clustergrößenverteilung. Das Histogramm wurde durch Bildung der Summe über 110 Messungen erzeugt, die je 2000 UV-Laserpulse umfassten. Auftragung a) zeigt die vollständige Verteilung, Auftragung b) zeigt den Größenbereich $N\left(\mathrm{H}_{2} \mathrm{O}\right)=27-57$, Auftragung c) zeigt den Größenbereich $N\left(\mathrm{H}_{2} \mathrm{O}\right)=128-169$. Die vertikalen Linien stellen die durch den Algorithmus ermittelten Integrationsgrenzen $t_{\text {Anfang }}^{\prime}$ (schwarz) und $t_{\text {Ende }}^{\prime}$ (grün) sowie die Signalmaxima $t_{\text {Peak }}^{\prime}$ (rot) dar. In Teil c) sind die schwarzen Linien größtenteils durch grüne Linien überdeckt, da die Positionen von $t_{\text {Anfang }}^{\prime}$ (schwarz) und $t_{\text {Ende }}^{\prime}$ hier weitgehend zusammenfallen. Messung vom 22.08.2017; $p(\mathrm{Ne})=$ 2,3 bar, $T_{\text {Reservoir }}=100^{\circ} \mathrm{C}, T_{\text {Düse }}=110^{\circ} \mathrm{C}, \lambda_{\mathrm{UV}}=385 \mathrm{~nm}$

in einem möglichst weiten Größenbereich bestimmt werden, um die Genauigkeit der Regressionskurve zu maximieren, die zur Ermittlung der Signalpositionen größerer Cluster mit $N>N_{\max }$ herangezogen wird.

Wichtig ist in diesem Zuge das Auffinden einer Kombination von Kriterien, anhand derer zuverlässig entschieden werden kann, ob ein Triple von neu aufgefundenen Kanalnummern $t_{\text {Anfang, } N}^{\prime}, t_{\text {Peak, } N}^{\prime}$ und $t_{\text {Ende, } N}^{\prime}$ ein Massensignal beschreibt, das sich physikalisch sinnvoll in die Größenverteilung einordnet, um die Auswertung nicht durch falsch bestimmte Signalpositionen zu verfälschen.

Vorteilhaft ist hierbei, dass die Positionen $t^{\prime}$ der Massensignale zueinander in einer Beziehung stehen und sich zudem zwischen den einzelnen Messreihen nur geringfügig ändern. Es gilt näherungsweise Gleichung 5.1, wobei die Abstände zwischen den Signalmaxima mit steigender Clustergröße abnehmen:

$$
t_{N+1}^{\prime}-t_{N}^{\prime} \approx t_{N}^{\prime}-t_{N-1}^{\prime}
$$


Die auftretenden Positionsänderungen sind durch Temperaturschwankungen der elektronischen Bauteile und daraus folgende minimale Variationen in den eingestellten Verzögerungszeiten zu erklären, da die Kapazitäten der verbauten Kondensatoren sich geringfügig ändern. Eine erneute Bestimmung der Integrationsgrenzen ist daher bei der Auswertung jeder Messreihe sinnvoll.

Als praktikabel erwies sich ein Algorithmus, der beginnend bei dem Massensignal der kleinsten im Massenspektrum beobachteten Clustergröße $(N=4)$ die Positionen der Signalgrenzen und Maxima bestimmt und iterativ unter Inkrementierung der Konstituentenzahl $N\left(\mathrm{H}_{2} \mathrm{O}\right)$ jeweils zum Signal der nächsthöheren Clustergröße fortschreitet. Die im Zuge der Positionsbestimmung der zuletzt aufgefundenen Signale gewonnenen Informationen über die Höhe der Grundlinie, die zu erwartende Amplitude sowie über den Bereich, in dem die Positionen von Maximum und Grenzen des folgenden Massensignals zu erwarten sind, werden bei dessen Untersuchung ausgenutzt. Diese Iteration findet innerhalb der Funktion finde_maxima() statt.

Darüber hinaus prüft diese Funktion die ermittelten Positionen auf deren Plausibilität und beendet die Suche nach den Maxima weiterer Clustergrößen, sofern die Signale nicht mehr ausreichend separiert sind.

Zunächst werden ausgehend von einer in der Variablen Startkanal vorgegebenen Kanalnummer $t_{\text {Start }}^{\prime}$ die Position $t_{\text {Peak }, N=4}^{\prime}$ und die Signalhöhe $h_{\text {Peak, } N=4}$ des ersten Massensignals der Größenverteilung bestimmt. Dessen Maximum wird im Bereich von 99 Kanälen oberhalb von $t_{\text {Start }}^{\prime}$ erwartet. Der Wert der Variablen Startkanal zu Beginn des Programmablaufs ist dem derzeitigen Aufbau entsprechend auf die Kanalnummer $t^{\prime}=6700$ festgelegt.

Im folgenden Schritt wird die niedrigste Zählrate $h$ aus 100 Kanälen vor dem Kanal $t_{\text {Peak, } N=4}^{\prime}$ als Höhe der Basislinie $h_{\text {Basis }}$ angenommen. Ferner wird angenommen, dass die Höhe des nächsten Maximums in jedem Fall einen zwischen diesen beiden Werten liegenden Schwellenwert $h_{\text {Schwelle, Start }}$ überschreitet, der sich nach Gleichung 5.2 berechnet:

$$
h_{\text {Schwelle, Start }}=h_{\text {Basis }}+\left(h_{\text {Peak, } N}-h_{\text {Basis }}\right) \cdot P_{\text {Schwelle, Start }}
$$

Der Wert $P_{\text {Schwelle, Start }}$ ist der Variablen schwelle_startkanal zugewiesen und auf den Betrag von 0,5 festgelegt. Somit entspricht der Wert von $h_{\text {Schwelle, Start }}$ dem von diesen beiden Werten gebildeten Mittelwert, d.h. der halben Peakhöhe oberhalb der Basislinie. Dieser Wert wird der Variablen schwellenwert zugewiesen.

Die Breite der Massensignale variiert im Bereich zwischen 80 und 150 Kanalnummern. Als Peakanfang $\left(t_{\text {Anfang, } N}^{\prime}\right)$ wird der Kanal mit der niedrigsten Nummer bezeichnet, der dem Signal der Clustergröße $N$ zugeordnet wird. Um diesen Punkt zu ermitteln, wird aus der Funktion finde_maxima() heraus die Funktion bestimme_Peakanfang() aufgerufen. Diese bestimmt die Kanalnummer $t_{\text {Anfang, } N}^{\prime}$ in drei Schritten: Im ersten Schritt wird die Kanalnummer $t^{\prime}$ ausgehend von $t_{\text {Start }}^{\prime}$ dekrementiert, bis die Zählrate $h_{t^{\prime}}$ den Wert von $h_{\text {Schwelle, Start }}$ unterschreitet und die aufsteigende Flanke des Peaks erreicht ist. Im zweiten Schritt wird $t^{\prime}$ weiter dekrementiert, bis die Steigung der aufsteigenden Flanke abnimmt und deren Wendepunkt unterschritten ist. Im dritten Schritt wird der Kanal mit der niedrigsten Zählrate aus den 30 Kanälen unterhalb dieses Punktes als $t_{\text {Anfang, } N}^{\prime}$ festgelegt und dessen Nummer an die Funktion finde_maxima() zurückgegeben. 
Nach der Bestimmung des Peakanfangs wird als Peakende $\left(t_{\text {Ende, } N}^{\prime}\right)$ der Kanal mit der höchsten Nummer ermittelt, die dem Peak der Clustergröße $N$ zugeordnet wird. Hierfür wird aus der Funktion finde_maxima() heraus die rekursive Funktion bestimme_Peakende() aufgerufen. Diese iteriert in aufsteigender Reihenfolge über die Kanäle

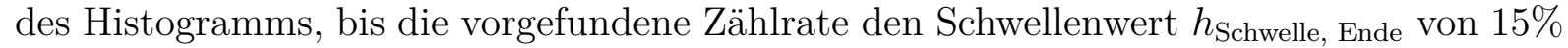
der Peakhöhe oberhalb der Basislinie unterschreitet. Dieser Wert berechnet sich nach Glei-

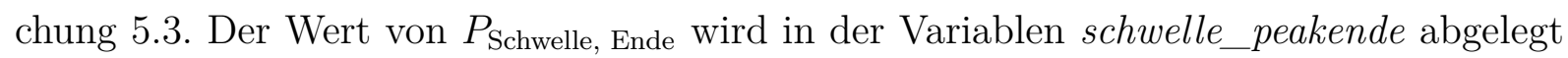
und ist zu Beginn des Programmdurchlaufs auf den Wert 0,15 eingestellt:

$$
h_{\text {Schwelle, Ende }}=h_{\text {Anfang, } N}+\left(h_{\text {Peak, } N}-h_{\text {Anfang, } N}\right) \cdot P_{\text {Schwelle, Ende }}
$$

Der Betrag $h_{\text {Schwelle, Ende }}$ wird nicht explizit in einer Variablen abgelegt. Anfangs- und Endpunkt dieser Iteration ( $t_{\text {Anfangspunkt }}^{\prime}$ und $\left.t_{\text {Endpunkt }}^{\prime}\right)$ werden in Abhängigkeit von der Anzahl bisher aufgefundener Clusterpeaks gewählt:

Fall 1: Falls bisher nur die Position eines einzigen Massensignals $\left(t_{\mathrm{Peak}, N=4}^{\prime}\right)$ ermittelt wurde, wird als Anfangspunkt das Maximum dieses Signals gewählt und der Bereich innerhalb von 119 Kanälen oberhalb dieses Punktes untersucht.

Fall 2: Falls bereits die Positionen von zwei oder mehr Signalen bestimmt wurden, ist es möglich, aus den letzten beiden Positionen den Bereich zu extrapolieren, in dem das Ende des nächsten Signals zu erwarten ist, und so den Suchbereich einzuschränken. In diesem Fall wird zunächst jeweils der Mittelwert von Peakanfang und Peakende der beiden zuletzt gefundenen Signale gebildet und deren Differenz ermittelt. Die Differenz dieser beiden Signalmittelpunkte wird auf den arithmetischen Signalmittelpunkt des zuletzt gefundenen Signals addiert:

$$
\begin{aligned}
& \bar{t}_{N-2}=\frac{t_{\text {Anfang, } N-2}^{\prime}+t_{\text {Ende, } N-2}^{\prime}}{2} \\
& \bar{t}_{N-1}^{\prime}=\frac{t_{\text {Anfang, } N-1}^{\prime}+t_{\text {Ende, } N-1}^{\prime}}{2} \\
& \Delta \bar{t}_{N-2, N-1}={\overline{t^{\prime}}}_{N-1}-{\overline{t^{\prime}}}_{N-2} \\
& t_{\text {Anfangspunkt }}^{\prime}=\bar{t}_{N-1}^{\prime}+\Delta \bar{t}_{N-2, N-1}^{\prime}
\end{aligned}
$$

Dieser Wert wird als Anfangspunkt des Suchbereichs festgelegt. Der Endpunkt wird bestimmt, indem die Differenz der Signalmittelpunkte mit dem Faktor 1,1 multipliziert und auf die Position des arithmetischen Mittelpunktes des zuletzt gefundenen Signals addiert wird:

$$
t_{\text {Endpunkt }}^{\prime}=\bar{t}_{N-1}^{\prime}+\Delta \bar{t}_{N-2, N-1}^{\prime} \cdot 1,1
$$

Wird der Wert von $h_{\text {Schwelle, Ende }}$ innerhalb dieser Grenzen nicht unterschritten, so wird der Faktor $P_{\text {Schwelle, Ende }}$ im Fall 1 um den Betrag von 0,1 bzw. im Fall 2 um den Betrag von 0,05 erhöht und die Funktion bestimme_Peakende() aus sich selbst heraus aufge- 
rufen, um mit dem modifizierten Schwellenwert abermals die Suche nach dem Peakende zu beginnen. Die Anzahl der rekursiven Funktionsaufrufe wird überwacht. Falls die Rekursionstiefe den willkürlich festgesetzten Wert von 14 Aufrufen überschreiten sollte, würde eine Fehlermeldung ausgegeben und das Programm nach Betätigung der Eingabetaste beendet, um einem Absturz vorzubeugen. Als Ergebnis der Suche innerhalb der Funktion bestimme_Peakende() wird eine Kanalnummer an die Funktion finde_maxima() zurückgegeben, die geringfügig niedriger ist, als das Ende der absteigenden Signalflanke. Die endgültige Bestimmung von $t_{\text {Ende, } N}^{\prime}$ erfolgt innerhalb von finde_maxima() durch Auffinden des Kanals mit der niedrigsten Zählrate aus 19 Kanälen nach dem von bestimme_Peakende() zurückgelieferten Wert.

Um festzustellen, ob die Signalqualität für die Bestimmung der Integrationsgrenzen ausreichend war, werden die Werte des zuletzt bestimmten Massensignals auf ihre Plausibilität geprüft. Zunächst wird der arithmetische Mittelpunkt des Signals bestimmt und dessen Abstand zum arithmetischen Mittelpunkt des vorherigen Signals berechnet:

$$
\begin{gathered}
{\overline{t^{\prime}}}_{N}=\frac{t_{\text {Anfang, } N}^{\prime}+t_{\text {Ende }, N}^{\prime}}{2} \\
\Delta{\overline{t^{\prime}}}_{N, N-1}={\overline{t^{\prime}}}_{N}-{\overline{t^{\prime}}}_{N-1}
\end{gathered}
$$

Weiterhin wird der Quotient aus den Differenzen der arithmetischen Mittelpunkte der drei zuletzt aufgefundenen Massensignale gebildet:

$$
Q_{N}=\frac{\Delta \bar{t}_{N, N-1}}{\Delta \bar{t}_{N-1, N-2}^{\prime}}
$$

Im nächsten Schritt wird eine Reihe von Bedingungen geprüft, um festzustellen, ob die ermittelten Positionen von $t_{\text {Anfang, } N}^{\prime}, t_{\text {Peak, } N}^{\prime}$ und $t_{\text {Ende, } N}^{\prime}$ ein Signal beschreiben, das sich physikalisch sinnvoll in die Größenverteilung einordnen lässt:

$$
\begin{gathered}
t_{\text {Ende, } N}^{\prime}-t_{\text {Anfang, } N}^{\prime}>\Delta t_{\text {min }}^{\prime} \\
t_{\text {Ende, } N}^{\prime}-t_{\text {Anfang, } N}^{\prime}<\Delta t_{\text {max }}^{\prime} \\
t_{\text {Anfang, } N}^{\prime}<t_{\text {Peak, } N}^{\prime}<t_{\text {Ende, } N}^{\prime} \\
Q_{\text {min }}<Q_{N}<Q_{\max }
\end{gathered}
$$

Die Grenzwerte $Q_{\min }=0,58$ und $Q_{\max }=1,61$ sind das Resultat einer empirischen Optimierung des Algorithmus anhand einer großen Anzahl von Messreihen. Treffen all diese Bedingungen $\mathrm{zu}$, wird das soeben gefundene Signal als korrekt erkannt angenommen und die zuletzt bestimmten Werte von $t_{\text {Anfang, } N}^{\prime}, t_{\text {Peak, } N}^{\prime}$ und $t_{\text {Ende, } N}^{\prime}$ sowie die Konstituentenzahl $\mathrm{N}\left(\mathrm{H}_{2} \mathrm{O}\right)$ werden an entsprechende Listen namens Peakstarts, Peaks, Peakends und $n$ Wasser angehängt. Andernfalls wird die Suche nach weiteren Massensignalen eingestellt und die oben beschriebenen Listen namens Peakstarts, Peaks, Peakends und nWasser werden in NumPy-Arrays umgewandelt und an die Funktion suche_clusterpeaks() zurückgegeben. 
Um die Suche nach weiteren Signalen fortzusetzen, wird der Wert von $t_{\text {Start }}^{\prime}$ in der Variablen Startkanal geändert, sodass von dieser Position aus die Grenzen des nächsten Massensignals ermittelt werden können. Hierfür wird aus der Funktion finde_maxima() die ebenfalls rekursive Funktion bestimme_startkanal() aufgerufen. Diese führt wiederum eine aufsteigende Iteration über die Kanäle des Histogramms durch, bis die Zählrate $h$ eines Kanals den Wert von $h_{\text {Schwelle, Start }}$ überschreitet. Der Anfangspunkt dieser Iteration liegt in jedem Fall fünf Kanäle oberhalb des Endpunktes des letzten Signals. Der Endpunkt dieser Iteration wird wiederum abhängig von der Anzahl bereits bestimmter Massenpeaks gewählt:

Fall 1: Falls bisher nur die Position eines einzigen Massenpeaks bestimmt wurde, wird der gesamte Bereich des Histogramms untersucht, da noch keine Aussage über die Position des nächsten Signals getroffen werden kann.

Fall 2: Falls bereits die Positionen von zwei oder mehr Signalen bestimmt wurden, wird der Endpunkt der Suche nach dem neuen Startkanal $t_{\text {Start }}^{\prime}$ gemäß Gleichung 5.16 festgelegt, da das Maximum des folgenden Massensignals $t_{\text {Peak, } N+1}^{\prime}$ im Bereich unterhalb dieses Kanals zu erwarten ist:

$$
t_{\text {Endpunkt }}^{\prime}=\bar{t}_{N}+\Delta \bar{t}_{N-1, N}
$$

Wird im Fall 1 kein zweites Signalmaximum gefunden, gibt das Programm eine Fehlermeldung aus. In diesem Fall muss entweder der Parameter $t_{\text {Start }}^{\prime}$ oder der Parameter $P_{\text {Schwelle, Start }}$ angepasst werden, der den Bruchteil des Signalmaximums oberhalb der Grundlinie angibt, den die Zählrate des nächsten Signals überschreiten muss, um als solches erkannt zu werden. Dieser Fall ist aufgrund der hohen Signalstärke im Größenbereich um $N=5$ rein hypothetischer Natur. Ein Wechsel des untersuchten Clustersystems würde hingegen eine Änderung dieser Parameter erforderlich machen.

Im Fall 2 wird der Wert von $h_{\text {Schwelle, Start }}$ in der Variablen schwellenwert gemäß Gleichung 5.17 vermindert und die Funktion bestimme_startkanal() rekursiv aufgerufen, um die Suche nach dem folgenden Massensignal mit dem veränderten Schwellenwert erneut durchzuführen.

$$
h_{\text {Schwelle, Start }}=h_{\text {Basis }}+\left(h_{\text {Schwelle, Start }}-h_{\text {Basis }}\right) \cdot 0,9
$$

Dieser Fall tritt aufgrund der mit zunehmender Clustergröße abnehmenden Signalqualität regelmäßig ein. Die maximale Rekursionstiefe wird wiederum begrenzt und willkürlich auf den Wert 14 festgesetzt, um einem Absturz des Programms vorzubeugen.

Die physikalische Plausibilität der neu aufgefundenen Position $t_{\text {Start, } N+1}^{\prime}$ wird gemäß Gleichung 5.18 geprüft. Trifft auch diese Bedingung zu, wird die Konstituentenzahl um den Betrag von 1 inkrementiert und im Rahmen eines weiteren Schleifendurchlaufs wird versucht, die Grenzen des im Bereich um $t_{\text {Start, } N+1}^{\prime}$ vermuteten Massensignals zu ermitteln.

$$
\frac{t_{\text {Start, } N+1}^{\prime}-\bar{t}_{N}^{\prime}}{\bar{t}_{N}-\bar{t}_{N-1}^{\prime}}<2
$$

Falls eine der in Gleichungen 5.12, 5.13, 5.14, 5.15 und 5.18 angegebenen Bedingungen nicht zutrifft, wird die Suche nach weiteren Massensignalen eingestellt und die 
oben beschriebenen Listen namens Peakstarts, Peaks, Peakends und nWasser werden in NumPy Arrays umgewandelt und an die Funktion suche_clusterpeaks() zurückgegeben. Diese übergibt die Arrays namens Peaks und nWasser an die Funktion bestimme_Fitparameter(), um die Parameter der Regressionsfunktion (Gleichung 5.19) zu bestimmen, die in der Funktion Fitkurve() definiert ist und eine Erweiterung von Gleichung 3.31 um einen dritten Regressionsparameter $b$ darstellt:

$$
N\left(\mathrm{H}_{2} \mathrm{O}\right)=\frac{\frac{\left(t^{\prime}-t_{0}^{\prime}\right)^{2}}{c^{\prime}}-M_{\text {Dotand }}}{M_{\text {Analyt }}}+b
$$

Das Kürzel $M_{\text {Analyt }}$ bezeichnet hier die molare Masse des Analyten (im vorliegenden Falle Wasser); $M_{\text {Dotand }}$ bezeichnet die molare Masse des Dotanden (hier: Natrium); die Angabe erfolgt jeweils in $\mathrm{g} \mathrm{mol}^{-1}$.

Schließlich werden die ermittelten Werte in Dateien namens Peakstarts.txt, Peakends.txt, Peaks.txt und Fitparameter.txt gespeichert, um bei einem erneuten Programmstart auf diese zurückgreifen zu können.

Sofern die oben beschriebenen Auswertungsschritte durchgeführt wurden, werden deren Ergebnisse in Form zweier Auftragungen graphisch dargestellt: Eine der Darstellung in Abbildung 5.2 entsprechende Auftragung zeigt die Summe über alle in die Auswertung einbezogenen Histogramme. Die ermittelten Positionen von $t_{\text {Anfang, } N}^{\prime}, t_{\text {Ende, } N}^{\prime}$ sowie $t_{\text {Peak, } N}^{\prime}$ sind für alle erkannten Massensignale der Clustergrößen $N$ durch unterschiedlich eingefärbte, vertikale Linien markiert.

Ein zweites Diagramm entsprechend Abbildung 5.3 stellt eine Auftragung der Konstituentenzahl $N\left(\mathrm{H}_{2} \mathrm{O}\right)$ aller erkannten Massensignale über der zugehörigen Kanalnummer $t_{\text {Peak, } N}^{\prime}$ der auf deren Basis berechneten Regressionskurve gegenüber. Diese ermöglicht auf einen Blick, eventuell fehlerhaft bestimmte Signalmaxima zu erkennen. Eine korrekt durchgeführte Auswertung ist daran erkennbar, dass sämtliche Positionen aufgefundener Signalmaxima auf der Regressionskurve liegen. Im Falle einer fehlerhaften Auswertung wäre eine Streuung der zugehörigen Punkte um den Graphen der Regressionskurve im oberen Clustergrößenbereich zu beobachten. Sollte diese Auftragung Punkte enthalten, die nicht auf der Regressionskurve liegen, müssten die in Gleichungen 5.12, 5.13, 5.14, 5.15 und 5.18 angegebenen Bedingungen zur Evaluierung der gefundenen Massensignale enger gefasst werden, indem deren Parameter geändert werden. Die in diesen Bedingungen enthaltenen Parameter sind jedoch bewusst eng gefasst, um diesen Fall möglichst auszuschließen. Sämtliche in dieser Arbeit dargestellten Ergebnisse wurden unter Verwendung des gleichen Satzes von Parametern erhalten.

Die NumPy-Arrays namens Peakstarts, Peaks, Peakends und $n$ Wasser sowie die Variablen $t 0, c$ und $b$ werden an die Funktion ermittele_Parameter() und von dieser an die Funktion Eingabe() zurückgegeben, von der die Funktion Auswertung() aufgerufen wird. Diese ermittelt aus den Anwendereingaben die auszuwertenden Clustergrößen und koordiniert entsprechend dieser Vorgaben die Integration der Zählraten zwischen den vorgegebenen Bereichsgrenzen.

Die Summation der Zählraten selbst wird innerhalb der Funktion integriere_groessenbereich() vorgenommen, die als Argumente unter anderem die Konstituentenzahlen $N\left(\mathrm{H}_{2} \mathrm{O}\right)$ des kleinsten sowie des größten Clusters im auszuwertenden Bereich des Massenspektrums erhält. Sie liefert die Summen $\Sigma_{\mathrm{IR}+\mathrm{UV}}, \Sigma_{\mathrm{UV}}$ sowie die Werte von $t_{\text {Anfang, } N}^{\prime}$ und $t_{\text {Ende, } N}^{\prime}$ des integrierten Bereiches zurück. Im Falle einer auszuwertenden 


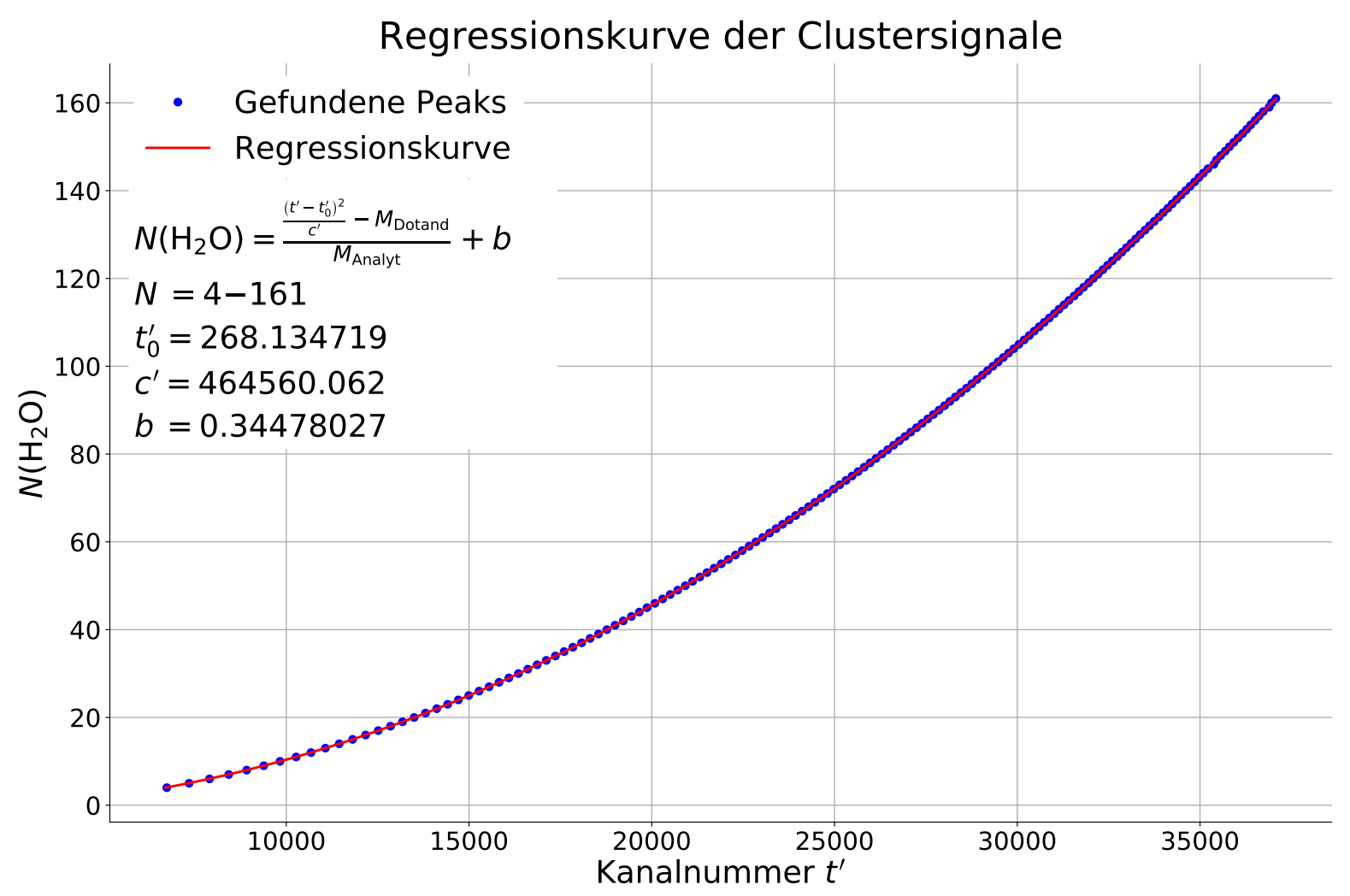

Abbildung 5.3.: Ausgabe der Auswertungssoftware: Auftragung der Konstituentenzahl $N\left(\mathrm{H}_{2} \mathrm{O}\right)$ über der zugehörigen Kanalnummer $t_{\text {Peak, } N}^{\prime}$. Gefundene Signalmaxima sind in Form blauer Punkte dargestellt, die Regressionskurve liegt als rote Linie darüber. Weiterhin werden automatisch deren Formel sowie die Regressionsparameter im Diagramm angegeben. (Messdaten vom 22.08.2017)

Einzelgröße sind die beiden an die Funktion integriere_groessenbereich() übergebenen Konstituentenzahlen identisch. Die Integrationsgrenzen $t_{\text {Anfang, } N}^{\prime}$ und $t_{\text {Ende, } N}^{\prime}$ werden aus den Arrays Peakstarts, und Peakends ausgelesen. Die Übergabe einer einzelnen Clustergröße $N$ zur Auswertung an die Funktion integriere_groessenbereich() erfolgt allerdings nur für $N$ im Bereich $N_{\text {min }} \leq N \leq N_{\text {max }}$.

Bei der Bündelung mehrerer Einzelgrößen zu einem gemeinsamen Spektrum für einen Größenbereich wird zu jeder Einzelgröße $N$ die Summe der Zählraten über den zwischen den Kanälen $t_{\text {Anfang, } N}^{\prime}$ und $t_{\text {Ende, } N}^{\prime}$ liegenden Bereich des Histogramms gebildet, sofern deren Signalgrenzen zuvor ermittelt werden konnten. Die Summation dieser Werte liefert die Signalintensitäten $\Sigma_{\mathrm{UV}}$ und $\Sigma_{\mathrm{IR}+\mathrm{UV}}$ des auszuwertenden Größenbereiches. Im Größenbereich $N>N_{\max }$ geht die Funktion integriere_groessenbereich() dazu über, das Ende bzw. Anfang und Ende des Integrationsbereiches durch Übergabe der Konstituentenzahl der betreffenden Clustergröße an die Funktion berechne_kanalnummer() vorzunehmen, die anhand der Regressionsgleichung 5.19 die Kanalnummer $t_{\text {Peak, } N}^{\prime}$ des zugehörigen Signalmaximums zurückgibt. Diese Werte werden dann als Integrationsgrenzen verwendet. Umfasst ein Auswertungsbereich die größte erkannte Einzelgröße $N_{\max }$, werden bis zu diesem Punkt ausschließlich die Bereiche innerhalb der Signalgrenzen in die Auswertung einbezogen. Oberhalb von $N_{\max }$ werden die Kanäle von $t_{\text {Ende }}^{\prime}$ der Clustergröße $N_{\max }$ 
bis zum Punkt $t_{\text {Peak }}^{\prime}$ der höchsten Clustergröße im aktuell betrachteten Größenbereich integriert. In dieser Weise werden sowohl die Signalstärken ohne IR-Anregung $\Sigma_{\mathrm{UV}, N}$ als auch die Signalstärken bei kombinierter UV- und IR-Anregung $\Sigma_{\mathrm{IR}+\mathrm{UV}, N}$ ermittelt. Die Signalzunahme $I_{N}$ wird durch Bildung des Quotienten berechnet:

$$
\begin{gathered}
I_{N}=\frac{\Sigma_{\mathrm{IR}+\mathrm{UV}, N}}{\Sigma_{\mathrm{UV}, N}} \\
I_{N, \text { normiert }}=\frac{\left(I_{N}-1\right)}{E_{\mathrm{IR}}}
\end{gathered}
$$

Diese Werte werden gemeinsam mit den Metadaten in einem Array namens Auswertung_aller_Parameter zusammengestellt, das spaltenweise folgende Informationen enthält: Spalte 1: Dateiname; Spalte 2: ROI-Summe; Spalte 3: Starts; Spalte 4: $p_{\text {Gas }}$; Spalte 5: $U_{\mathrm{HV}}$; Spalte 6: $\tilde{\nu}_{\mathrm{IR}}$; Spalte 7: $E_{\mathrm{IR}}$; Spalte 8: $\lambda_{\mathrm{UV}}$; Spalte 9: $E_{\mathrm{UV}}$; Spalte 10: $\Delta t$; Spalte 11: Startkanal (Anfang des Integrationsbereiches); Spalte 12: Endkanal (Ende des Integrationsbereiches); Spalte 13: Clustergröße bzw. Größen- oder Kanalbereich; Spalte 14: $\Sigma_{\mathrm{UV}, N}$; Spalte 15: $\Sigma_{\mathrm{IR}+\mathrm{UV}, N} ;$ Spalte 16: $I_{N}$; Spalte 17: $I_{N, \text { normiert }}$.

Zuletzt legt die Software das Ergebnis der Auswertung in Form zweier csv-Dateien in dem Verzeichnis ab, in dem auch die Skriptdatei selbst gespeichert ist. Die Datenwerte werden innerhalb dieser Dateien durch Semikola voneinander getrennt. Die beiden Dateien unterscheiden sich in Auswahl und Anordnung der Ergebnisse und sind jeweils für die weitere Auswertung mittels eines Tabellenkalkulationsprogramms im Hinblick auf unterschiedliche Fragestellungen optimiert:

Erstere wird unter dem Namen Ausgabe_IR_<Präfix $>$ abgelegt und weist die Ergebnisse jeder Messung in je einer Zeile aus, in der $\bar{\Delta} t$, die $\tilde{\nu}_{\mathrm{IR}}, E_{\mathrm{IR}}$ sowie jeweils in einer Spalte die Signalzunahmen der ausgewerteten Einzelgrößen bzw. Größenbereiche eingetragen sind. Diese Datei enthält somit sämtliche zur Anfertigung von IR-Spektren notwendigen Informationen in einer für diesen Zweck optimierten Anordnung.

Letztere erhält den Namen Ausgabe_<Präfix $>$. Die Anordnung der Daten entspricht dem Array Auswertung_aller_Parameter. Diese Datei enthält neben den durch die Auswertungssoftware berechneten Werten der Signalintensitäten und -zunahmen auch spaltenweise sämtliche Metadaten, die aus den 887-Dateien ausgelesen wurden. Sie ermöglicht die Auswertung im Hinblick auf weitere Fragestellungen, die z. B. Signalstärke und -zunahme in Abhängigkeit von den übrigen Messparametern betreffen.

Falls die Eingabe eines Plot-Befehls erfolgt ist, wird eine entsprechende Auftragung angefertigt und ausgegeben. Die numerisch kodierte Angabe der auf den Achsen aufzutragenden Größen innerhalb des Plot-Befehls (vgl. Abschnitt 5.2.1) entspricht dabei der (bei 0 beginnenden) Indexnummer der Spalten im Array Auswertung_aller_Parameter. 


\section{Ergebnisse und Diskussion}

Die Frage, wie viele Moleküle ein Wassercluster mindestens enthalten muss, um eine kristallartige Struktur auszubilden, wurde trotz ihrer Bedeutung (vgl. Kap. 1) bis heute nicht endgültig geklärt. In bisherigen Arbeiten konnte der fragliche Größenbereich zunehmend eingegrenzt werden. Als alleiniges Kriterium wurde hierbei das Erscheinen einer Bande um $\tilde{\nu}_{\mathrm{IR}} \approx 3200 \mathrm{~cm}^{-1}$ im IR-Spektrum herangezogen, die kristallartige von amorphen Strukturen unterscheidbar macht (vgl. Abschnitt 2.3.5). Die Festsetzung der beobachteten kritischen Clustergröße beruhte folglich auf einem Vergleich mit den IR-Spektren kleinerer Cluster im gleichen Experiment. Sie erfordert eine entsprechend deutliche Ausprägung der betreffenden Bande, da diese sicher von der in Abschnitt 3.2 .3 beschriebenen, größenabhängigen Rotverschiebung des Bandenmaximums amorpher Cluster unterschieden werden muss. Weiterhin ist davon auszugehen, dass die Kristallisation nicht in sämtlichen im Experiment vorliegenden Aggregaten stattfindet, in denen sie thermodynamisch möglich ist 48. Im Größenbereich knapp oberhalb von $N^{*}$ sollten kristallartige Partikel daher nur einen kleinen Anteil der beobachteten Cluster dieser Größen darstellen das IR-Spektrum nur minimal verändern.

Es soll daher zunächst versucht werden, einen direkten Vergleich der fraglichen Spektralbereiche mutmaßlich kristallartiger Aggregate mit den entsprechenden Wellenzahlen garantiert amorpher Cluster gleicher Größe vorzunehmen. Unter der Prämisse, dass das Vorliegen kristallartiger Strukturen sich im IR-Spektrum durch die Ausbildung einer Bande um ca. $3200 \mathrm{~cm}^{-1}$ nachweisen lässt, sollte der Quotient aus der Signalzunahme bei dieser IR-Wellenzahl und dem um $3400 \mathrm{~cm}^{-1}$ verorteten Absorptionsmaximum amorpher Cluster abnehmen, sofern ein Teil der Cluster in der betreffenden Größenfraktion kristallisiert. Hierdurch wird die zeitaufwendige Aufnahme eines vollständigen IR-Spektrums zu diesem Zweck überflüssig. Stattdessen können die Vorteile des in Kapitel 4 beschriebenen, automatisierten Messmodus ausgenutzt werden, um durch eine hohe Signalstärke bei nur wenigen Messpunkten die statistisch bedingte Messunsicherheit zu minimieren.

Aus den Ausführungen in Abschnitt 3.2.3 ergibt sich, dass auch die freie $\mathrm{OH}$ Streckschwingung als Indikator für kristallartige Strukturen infrage kommt, da ihre Position sensibel auf die Stärke der benachbarten Wasserstoffbrücken reagiert. Nachdem der Punkt, ab dem die Kristallisation unter den vorliegenden Expansionsbedingungen einsetzt, auf Basis des oben beschriebenen Kriteriums bestimmt worden ist, sollen daher im nächsten Schritt mit der Kristallisation verbundene Änderungen der freien OH-Bande untersucht werden. Zur Klärung der Frage, ob die gefundenen Änderungen auf bloßer Koinzidenz beruhen, oder ob stattdessen ein kausaler Zusammenhang vorliegt, werden die experimentellen Ergebnisse mit den Vorhersagen parallel durchgeführter MD-Simulationen kooperierender, theoretisch ausgerichteter Gruppen verglichen.

Die in den Abschnitten 6.2 und 6.3 vorgestellten Ergebnisse waren in Teilen ebenfalls Bestandteil der Masterarbeit von Daniel Becker ${ }^{239}$, der die zugrundeliegenden Messungen gemeinsam mit dem Autor durchführte. 


\begin{tabular}{r|c|c}
\hline PARAMETER & NiEDRIGE KüHLRATE & HohE KÜHLRATE \\
\hline Trägergas & Argon & Helium \\
$p_{0}[$ bar $]$ & $1.05 \pm 0.05$ & $5,8 \pm 0.05$ \\
$T_{\text {Düse }}\left[{ }^{\circ} \mathrm{C}\right]$ & 70 & 130 \\
$T_{\text {Reservoir }}\left[{ }^{\circ} \mathrm{C}\right]$ & 70 & 120 \\
$p_{\text {eq }}\left(\mathrm{H}_{2} \mathrm{O}\right)[\mathrm{bar}]$ & $\approx 0,3$ & $\approx 2,0$ \\
$X\left(\mathrm{H}_{2} \mathrm{O}\right)$ & $\approx 0,3$ & $\approx 0,3$ \\
$\lambda_{\mathrm{UV}}[\mathrm{nm}]$ & 385 & 385 \\
$E_{\mathrm{UV}}[\mathrm{mJ}]$ & $\approx 6,0$ & $\approx 6,0$ \\
$E_{\mathrm{IR}}(3420 \mathrm{~nm})[\mathrm{mJ}]$ & $\approx 10,5$ & $\approx 10,5$ \\
$E_{\mathrm{IR}}(3180 \mathrm{~nm})[\mathrm{mJ}]$ & $\approx 9,7$ & $\approx 9,7$
\end{tabular}

Tabelle 6.1.: Experimentelle Parameter zur Erzeugung hoher bzw. niedriger Kühlraten

\subsection{Wahl und Beschreibung der Expansionsbedingungen}

Die Gesamtschau der bisherigen Arbeiten zeigt, dass die experimentell bestimmte kritische Clustergröße stark von den Versuchsbedingungen abhängt 41|48|59. Da der hohe Laplace-Druck im Inneren kleiner Cluster zu einer Absenkung der homogenen Nukleationstemperatur führt (vgl. Abschnitt 2.2.1), stellt sich zunächst die Frage, ob ein Zusammenhang mit der im Experiment erreichbaren Endtemperatur der erzeugten Cluster besteht. Dies gilt insbesondere für Studien, in denen freie Überschallexpansionen zur Clustererzeugung angewandt wurden, da eine akkurate Bestimmung der Endtemperatur hier große Schwierigkeiten bereitet. Der Phasenübergang sollte in den kleinsten Clustern bei Temperaturen knapp unterhalb von $200 \mathrm{~K}$ (und damit ca. $30 \mathrm{~K}$ unterhalb von $T_{\mathrm{H}}$ ) eintreten (vgl. Abschnitt 2.3.3 75. Diese Temperatur wird im vorliegenden Experiment höchstwahrscheinlich unterschritten. Buck et al. zeigten, dass eine Erhöhung des Trägergasdrucks die Nukleation in den kleineren Clustern unterdrückt, obwohl die Endtemperatur hierdurch verringert wird (im beschriebenen Fall von ca. $70 \mathrm{~K}$ auf ca. $50 \mathrm{~K}$ ) 48 . Die Tatsache, dass trotz dieser Erhöhung der thermodynamischen Triebkraft die Phasenänderung in kleineren Clustern nicht mehr beobachtet werden konnte, deutet auf den Einfluss eines kinetischen Faktors hin: Die Erhöhung des Stagnationsdruckes bewirkt neben der Absenkung der Endtemperatur eine Steigerung der Strahlgeschwindigkeit und der Kühlrate im gesamten durchlaufenen Temperaturbereich (vgl. Abschnitt 3.1.5). Nach heutigem Kenntnisstand stellt die Ausbildung eines kritischen Nukleus ein stochastisches Ereignis dar, dessen Wahrscheinlichkeit sowohl mit der Anzahl der im Cluster enthaltenen Wassermoleküle als auch mit der Länge des hierfür zur Verfügung stehenden Zeitfensters abnimmt 975 (siehe auch Abschnitt 2.3.3). Letztere ergibt sich aus der Geschwindigkeit, mit der ein Aggregat das Temperaturintervall durchläuft, in dem die Phasenänderung einerseits thermodynamisch begünstigt und andererseits kinetisch möglich ist.

Die exakte Bezifferung der in freien Überschallexpansionen erzielten Kühlraten erfordert einen hohen Rechenaufwand. Gimelshein et al. führten eine solche Rechnung für Bedingungen mit $X\left(\mathrm{H}_{2} \mathrm{O}\right) \approx 0,3$ und Neon als Trägergas durch [59, dessen Atommasse zwischen den Massen von Helium und Argon liegt. Es ergaben sich Werte zwischen ca. $1 \mathrm{Kns}^{-1}$ kurz nach Passage des Düsenhalses und $0,5 \mathrm{Kns}^{-1}$ bei $T \approx 200 \mathrm{~K}$, sodass die Kühlraten in den vorliegenden Experimenten darüber bzw. darunter liegen dürften. Die 

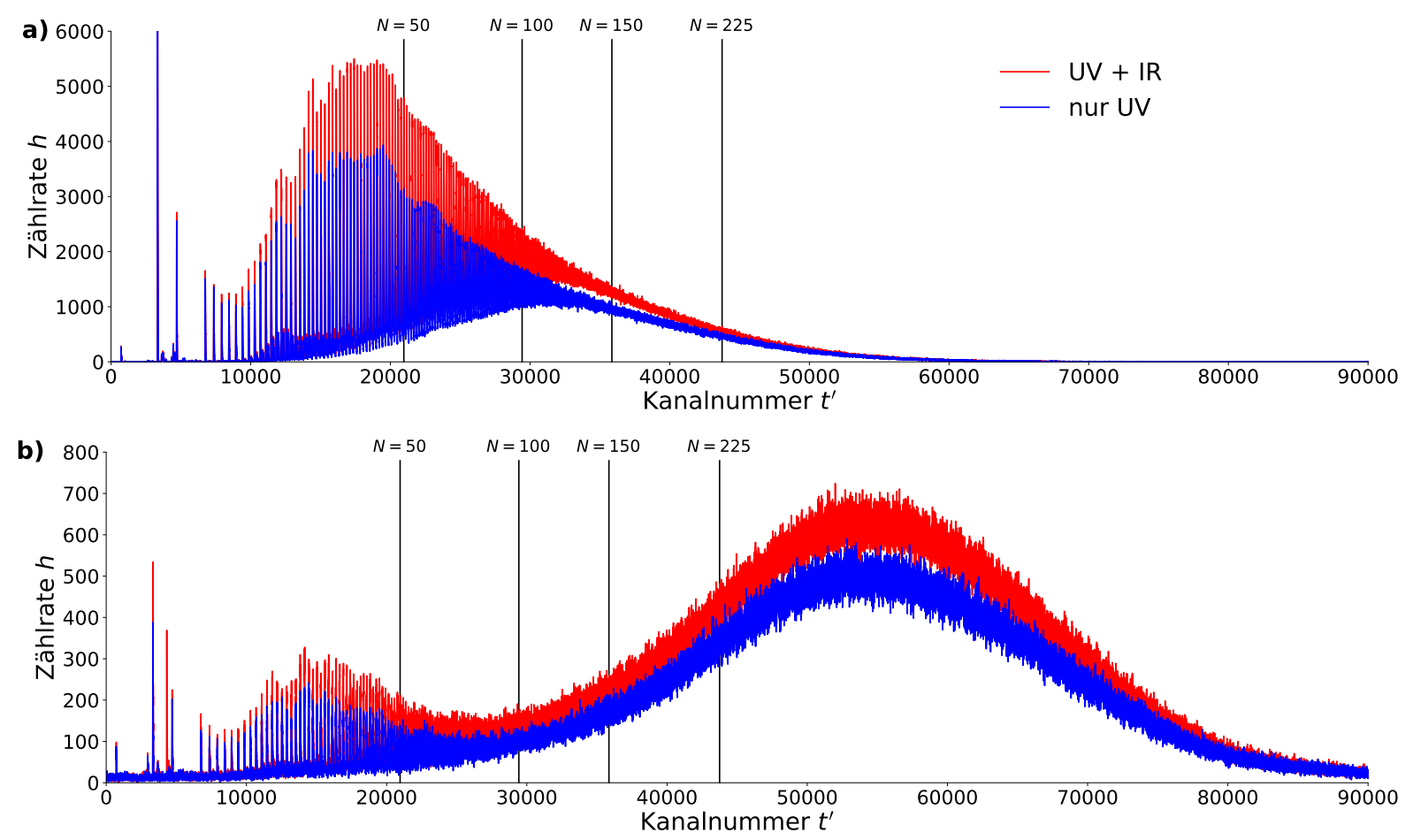

Abbildung 6.1.: Exemplarische Größenverteilungen nach alleiniger Photoionisation (blau) bzw. mit vorheriger IR-Anregung (rot); Teil a): Niedrige Kühlrate (Argon; $p(\mathrm{Ar})=1,05 \pm 0,05$ bar); Messung vom 01.11.2017; Teil b): Hohe Kühlrate (Helium; $p(\mathrm{He})=5,80 \pm 0,05$ bar $)$; Messung vom 13.11.2017. Summe über jeweils 130 Einzelmessungen mit 1000 UV-Laserpulsen und identischen IR-Wellenzahlen.

unterschiedlichen Versuchsbedingungen sowie die resultierenden Größenverteilungen werden einander in Tab. 6.1 sowie in Abb. 6.1 gegenübergestellt. Eine Übersicht über die Versuchsbedingungen sämtlicher Messungen, denen die in diesem Kapitel vorgestellten Ergebnisse zugrunde liegen, findet sich in Anhang A.

\subsubsection{Clustererzeugung unter optimalen Kristallisationsbedingungen}

Um optimale Bedingungen für die Nukleation zu schaffen, sollte die Kühlrate der Cluster während der Passage des fraglichen Temperaturfensters zwischen $150 \mathrm{~K}$ und $200 \mathrm{~K}$ so niedrig wie möglich gehalten werden. Gleichzeitig war eine ausreichend niedrige Endtemperatur sicherzustellen, um die adsorbierten Natriumatome kinetisch zu stabilisieren. Weiterhin war ein hinreichend hoher Anteil von Wasser in der expandierten Gasmischung notwendig, um ein Messsignal von akzeptabler Intensität zu erhalten. Eine Verminderung der Kühlrate bei gleichzeitig niedriger Endtemperatur konnte durch den Einsatz von Argon als Trägergas erzielt werden, das aufgrund seiner hohen Atommasse eine niedrige Strömungsgeschwindigkeit gewährleistet (vgl. Kap. 3.1.5). Dies gilt umso mehr, da auch der Stagnationsdruck und die Reservoirtemperatur niedriger gewählt werden können. Ein Wert von $p_{0}=1,05 \pm 0,10$ bar genügte, um eine ausreichende Kühlung der gebildeten Aggregate zu gewährleisten und die kinetische Stabilisierung der Na-Atome auf den Clusteroberflächen sicherzustellen. So führt die Kollision mit einem Na-Atom bei einer Strahl- 


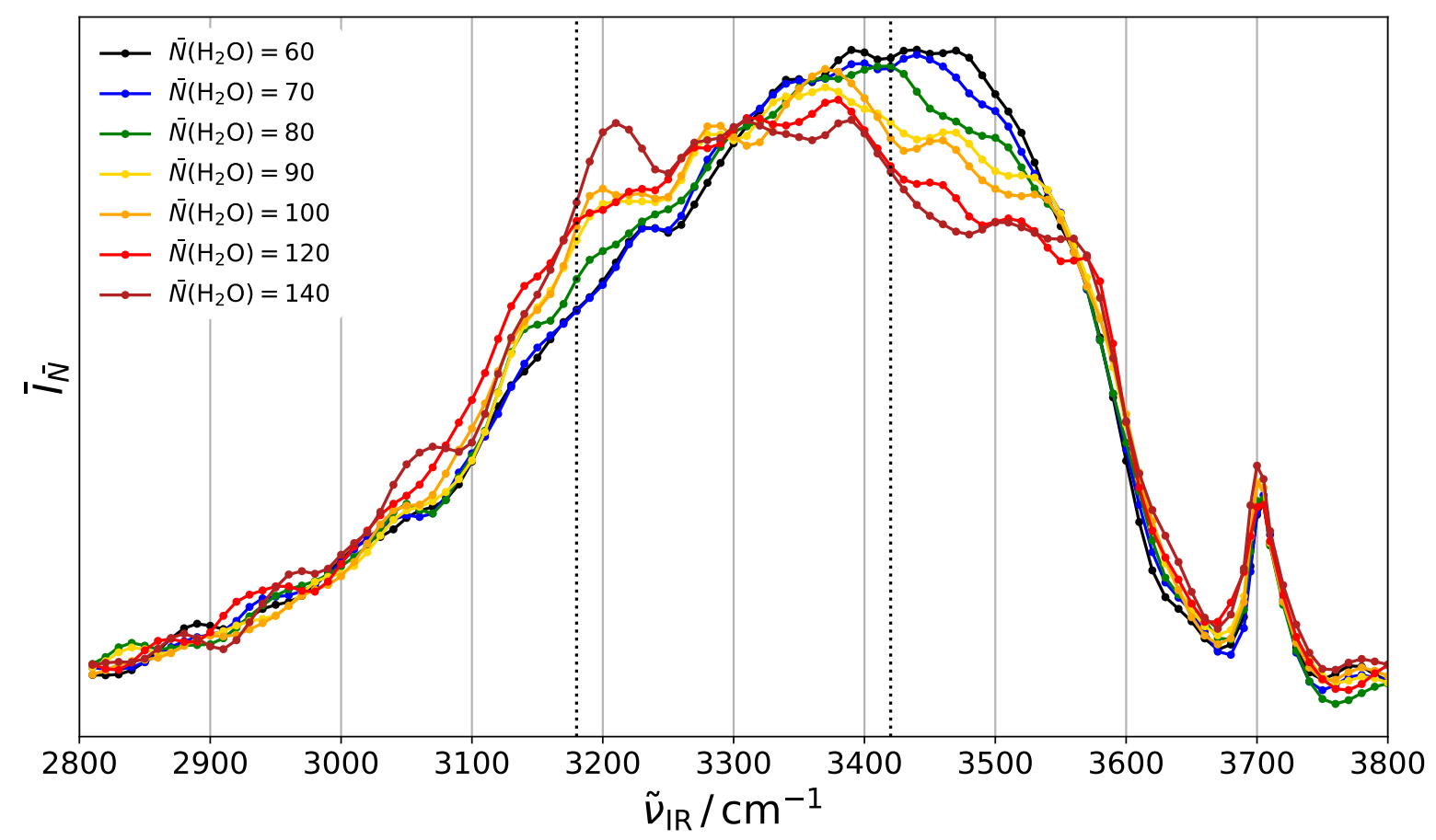

Abbildung 6.2.: Exemplarische IR-Spektren natriumdotierter Wassercluster; Trägergas: Argon $\left(p_{0} \approx 1,05\right.$ bar $)$; Aufgetragen ist die Mittelung über 20 Clustergrößen sowie drei Spektren, die zuvor einer zweifachen Dreiecksglättung der Gewichtung 25:50:25 unterzogen wurden. Die Normierung erfolgte auf das Gesamtintegral der gebundenen OH-Streckschwingung; Messungen vom 11.04., 12.04., sowie vom 18. und 19.04.2017; aufgenommen von Christoph Dierking. Die punktierten Linien entsprechen den in Abschnitt 6.2 verwendeten IR-Wellenzahlen. Auf Basis dieser Daten wurde die Größe von $N^{*}$ auf $N \approx 90 \pm 10$ eingegrenzt 61 .

geschwindigkeit von maximal $1000 \mathrm{~m} \mathrm{~s}^{-1}$ zu einem Energieeintrag von ca. $1,92 \cdot 10^{-20} \mathrm{~J} 208$. Die Solvatation setzt einen Energiebetrag von maximal 3,204 $\cdot 10^{-19} \mathrm{~J}$ frei. Ein Cluster mit $N=100\left(C_{V} \approx 9,654 \cdot 10^{-21} \mathrm{~J}\right.$ bei $C_{V} \approx 9,654 \cdot 10^{-2} \mathrm{~J}$ pro Monomer $\left.{ }^{[273}\right)$ erwärmt sich hierdurch um maximal $35 \mathrm{~K}$. Um einen ausreichend hohen Stoffmengenanteil gasförmigen Wassers (hier: $\left.X\left(\mathrm{H}_{2} \mathrm{O}\right) \approx 0,3\right)$ im expandierten Gasgemisch zu erreichen, genügte eine Reservoirtemperatur von $70^{\circ} \mathrm{C}(343 \mathrm{~K})$.

Abbildung 6.2 zeigt eine Auswahl exemplarischer Spektren, die unter vergleichbaren Bedingungen aufgenommen wurden. Erkennbar ist die stetige Abnahme der Intensität im Bereich um $\tilde{\nu}_{\mathrm{IR}}=3300-3550 \mathrm{~cm}^{-1}$. Die Spektren kleinerer Cluster $(\bar{N}=60, \bar{N}=70)$ weisen im Bereich um $\tilde{\nu}_{\text {IR }}=3200 \mathrm{~cm}^{-1}$ kaum einen Unterschied auf. Ab $\bar{N}=90$ bildet sich in diesem Bereich eine Bande aus, die auf das Vorliegen kristallartiger Strukturen schließen lässt. Ein isosbestischer Punkt bei $\tilde{\nu}_{\mathrm{IR}} \approx 3300 \mathrm{~cm}^{-1}$ ist erkennbar.

Die Endtemperatur der erzeugten Cluster kann nicht exakt ermittelt werden. Eine konservative Schätzung ergibt einen Temperaturbereich zwischen $100 \mathrm{~K}$ und $150 \mathrm{~K}$ : Gimelshein et al. berechneten die Endtemperatur von Clustern, die mit Neon $\left(p_{0}=2,3\right.$ bar) erzeugt wurden, auf ca. $100 \mathrm{~K}$. Weiterhin zeigten sie, dass die Temperatur des Gasge- 

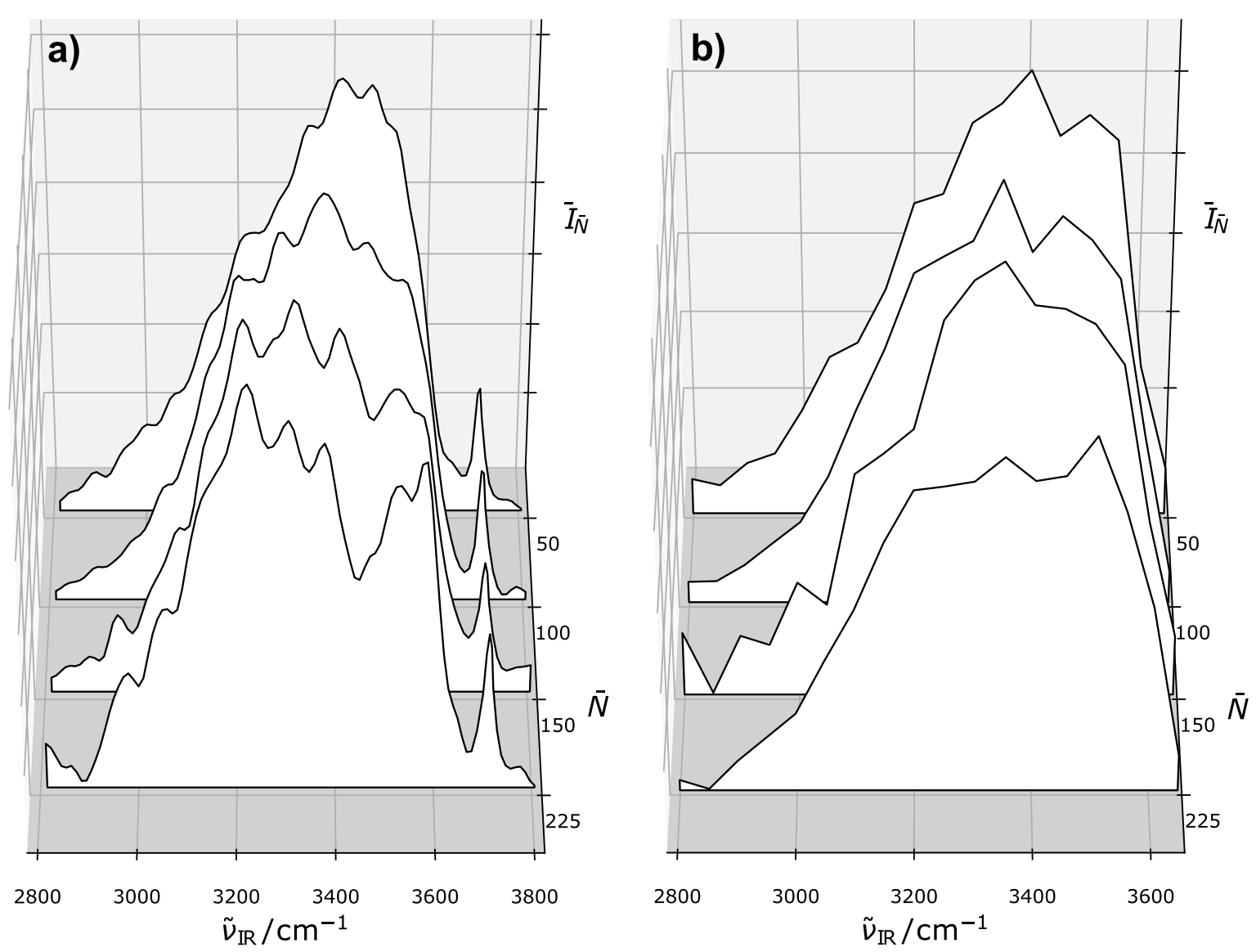

Abbildung 6.3.: Gegenüberstellung exemplarischer Größenscheiben der komplementären Expansionsbedingungen; Paneel a): Argon (wie Abb. 6.2); Paneel b): Helium, Messung vom 03.09.2018. Aufgrund eines technischen Defekts konnte hier das Spektrum unterhalb von $\tilde{\nu}_{\mathrm{IR}}=3650 \mathrm{~cm}^{-1}$ aufgenommen werden. Die erhobenen Daten waren jedoch für die beabsichtigte Auswertung hinreichend.

misches vor der Expansion nur geringen Einfluss auf die final erreichbaren Clustertemperaturen hat 59. Da der Argon-Druck in der vorliegenden Expansion unter dem Wert von 2,3 bar lag, kann dieser Temperaturwert mit einiger Vorsicht als Untergrenze der erreichbaren Temperatur angesetzt werden, wenngleich Argon unter vergleichbaren Expansionsbedingungen eine effektivere Kühlung der Schwingungsfreiheitsgrade gebildeter Wassercluster bewirkt $48 \mid 195$. Hock et al. bestimmten die Temperatur, oberhalb der die Cluster auf einer Sekundenzeitskala zerfallen $\left(T_{\text {evap }}\right)$, zu $136 \pm 6 \mathrm{~K}\left(N\left(\mathrm{H}_{2} \mathrm{O}\right)=48\right)$ bzw. $144 \pm 6 \mathrm{~K}\left(N\left(\mathrm{H}_{2} \mathrm{O}\right)=118\right) \underline{170}$. Auf der deutlich kürzeren Zeitskala des hier vorgestellten Experiments (Partikel mit $N\left(\mathrm{H}_{2} \mathrm{O}\right)=100$ erreichen den Detektor ca. $240 \mu$ s nach der Ionisation) können allerdings auch höhere Temperaturen auftreten, ohne dass signifikante Evaporationsprozesse zu erwarten sind. So ergab eine von Buck durchgeführte Abschätzung der Endtemperatur auf Basis der Umwandlung thermischer Energie in kinetische Energie einen Wert von $T \approx 150 \mathrm{~K}$ vor der Passage der Pickup-Zelle 274 . 


\subsubsection{Unterdrückung der Kristallisation}

Zur Erzeugung amorpher Cluster für den direkten Vergleich konnte auf Erfahrungen zurückgegriffen werden, die in der Vergangenheit mit Helium als Trägergas gesammelt worden waren 41/48. Durch Erhöhung des Stagnationsdruckes auf 5, 80 \pm 0,1 bar wurde die Kühlrate maximiert. Um den Stoffmengenanteil von Wasser wiederum auf einen Wert von ca. 0,3 einzustellen, musste die Reservoirtemperatur auf einen Wert von $120^{\circ} \mathrm{C}$ (393 K) erhöht werden. Eine Abschätzung der Clustertemperatur basierend auf dem von Buck entwickelten Verfahren ergab eine finale Temperatur der gebildeten Cluster von ca. $50 \mathrm{~K} 274$. Die niedrigere Endtemperatur bei hohem Stagnationsdruck zeigt sich zudem in der Ausbildung einer bimodalen Größenverteilung mit deutlich erhöhter Abundanz großer Aggregate, die in der Expansion mit niedrigem Stagnationsdruck aufgrund unzureichender Ableitung der Kondensationswärme nicht stabilisiert werden können (vgl. Abb. 6.1). Neben der höheren Kühlrate ist somit auch eine niedrigere Endtemperatur dieser Cluster sichergestellt. Dieser Unterschied ist deshalb von Bedeutung, da sich das Absorptionsmaximum der gebundenen $\mathrm{OH}$-Streckschwingung bei niedrigeren Temperaturen eher zu kleineren IR-Wellenzahlen verschiebt (vgl. Kap. 3.2.3). Da die langsam gekühlten Cluster eine höhere Endtemperatur besitzen, dürfte der Einfluss der Temperatur die hier diskutierte, strukturbedingte Änderung des IR-Spektrums teilweise kompensieren und den daraus abgeleiteten Anfangspunkt der Kristallisation eher zu höheren Größenwerten verschieben. Somit kann ausgeschlossen werden, dass die hier allein an zwei Punkten des IR-Spektrums beobachtete, relative Rotverschiebung der Absorptionsbande der langsamer abgekühlten Cluster die Folge der niedrigeren Endtemperatur ist.

In Abbildung 6.3 sind exemplarische IR-Spektren unter diesen komplementären Expansionsbedingungen erzeugter Wassercluster einander gegenübergestellt. Die Messungen mit Argon als Trägergas zeigen wiederum einen Übergang des Bandenmaximums von $\tilde{\nu}_{\mathrm{IR}}>3400 \mathrm{~cm}^{-1}$ hin $\mathrm{zu} \tilde{\nu}_{\mathrm{IR}} \approx 3200 \mathrm{~cm}^{-1}$, der auf einen zunehmenden Anteil von Wassermolekülen in kristallartiger Koordinationsumgebung hinweist. Das Minimum bei $\tilde{\nu}_{\mathrm{IR}} \approx 3400 \mathrm{~cm}^{-1} \mathrm{im}$ Spektrum der Größenscheibe $\bar{N}=225$ ist auf eine stärkere Evaporation der größten Cluster nach vibronischer Anregung durch IR-Strahlung dieser Energie zurückzuführen. Diese Partikel können aufgrund ihres abweichenden Bildungsmechanismus eine höhere Ausgangstemperatur besitzen, als kleinere Aggregate. Der Temperatursprung im Moment der IR-Einstrahlung kann die Verflüssigung eines kristallartigen Partikels dieser Größe innerhalb weniger Nanosekunden bewirken 68, wodurch dessen Absorptionswahrscheinlichkeit für Photonen mit $\tilde{\nu}_{\mathrm{IR}} \approx 3400 \mathrm{~cm}^{-1}$ schlagartig erhöht wird. Die Aufnahme weiterer Photonen führt zu einer verstärkten Evaporation. Es sei darauf hingewiesen, dass der im Argon-Fall eingestellte Stagnationsdruck nicht geeignet ist, um Aggregate dieser Größe zu präparieren (vgl. hierzu auch die Position in der Größenverteilung in Abb. 6.1). Diese Größenscheibe wurde dennoch aufgetragen, da sie das größte präsentable IR-Spektrum der Argon-Expansion darstellt, sodass sich im direkten Vergleich zeigen lässt, dass eine vergleichbare Entwicklung in den Experimenten mit hohem Stagnationsdruck bei dieser Größenscheibe allenfalls beginnt. Daher dürften die aufgefundenen Bedingungen geeignet sein, um amorphe und kristalline Cluster im relevanten Größenbereich um $N \approx 100$ direkt zu vergleichen. 


\subsection{Die gebundene $\mathrm{OH}$-Streckschwingung als Kriterium der Phasenänderung}

Um das erstmalige Auftreten kristallartiger Strukturen mit ausreichender Signifikanz auf einen möglichst kleinen Größenbereich eingrenzen zu können, wurden unter den beiden oben beschriebenen, komplementären Expansionsbedingungen die Signalzunahme bei $\tilde{\nu}_{\mathrm{IR}}=3180 \mathrm{~cm}^{-1}$ und bei $\tilde{\nu}_{\mathrm{IR}}=3420 \mathrm{~cm}^{-1}$ ermittelt. Hierdurch sollten möglichst selektiv amorphe bzw. kristalline Strukturen vibronisch angeregt werden. Es wurden jeweils 120 Einzelmessungen vorgenommen, von denen jede eine Summe über je 500 UV-Laserpulse mit und ohne vorherige IR-Anregung darstellt, die alternierend aufgenommen wurden.

Die Arbeiten von Sabine Wolff 237, Daniel Becker 238|239] und Christoph Dierking [61] zur Abhängigkeit der Signalzunahme von der Delayzeit zwischen beiden Laserpulsen zeigten, dass dieser Parameter die Kontrolle des Anteils amorpher Spezies an der Gesamtmenge der ionisierten Partikel ermöglicht. Diese sind für einen Großteil der Signalzunahme bei kurzen Delayzeiten verantwortlich. Der durch amorphe Cluster verursachte Anteil der Signalzunahme nimmt allerdings auf einer Nanosekundenzeitskala exponentiell ab, sodass die beobachtete Signalzunahme mit zunehmender Delayzeit ebenfalls exponentiell abfällt. Oberhalb von $\Delta t=100 \mathrm{~ns}$ ist dieser Prozess weitgehend abgeschlossen, sodass die Signalstärke sich beim Wechsel auf $\Delta t=150 \mathrm{~ns}$ nur noch unwesentlich ändert. Diese Beobachtung legt nahe, dass die Zusammensetzung der ionisierten Partikelfraktion sich ab diesem Zeitpunkt ebenfalls nur noch geringfügig ändert. Wird das Zeitintervall zwischen beiden Laserpulsen auf einen Wert von ca. 100 ns eingestellt, lässt sich folglich der Beitrag amorpher Cluster zur Signalzunahme vermindern und die Sensitivität des Experiments bezogen auf kristallartige Strukturen erhöhen.

Um den Einfluss der Delayzeit auf das Resultat der vorliegenden Analyse bemessen zu können, wurde ebenfalls ein Vergleich zu Bedingungen vorgenommen, unter denen auch die Argon-geseedete Expansion noch einen hohen Anteil amorpher Cluster enthalten sollte. Hierzu wurde die spektrale Intensität auch bei $\Delta t=7 \mathrm{~ns}$ vermessen ${ }^{1}$. Es wurden ebenfalls je 120 Messungen gleicher Dauer vorgenommen.

Die vier möglichen Kombinationen aus den Trägergasen Argon bzw. Helium sowie den beiden Delayzeit wurden vergleichend betrachtet: Zunächst wurden jeweils gleitende Mittelwerte $I_{\bar{N} \pm 3}$ der Signalzunahmen $I_{N}$ über sieben Größenbereiche gebildet. Der Wert der Signalzunahme $I_{N}$ entspricht allerdings nicht der absoluten Spektralintensität. Vielmehr nimmt er als Quotient der Signalstärken $\Sigma_{\mathrm{UV}, N}$ und $\Sigma_{\mathrm{IR}+\mathrm{UV}, N}$ einen Wert von 1 an, sofern die gemessene Spektralintensität auf die Grundlinie abfällt (vgl. Glg. 5.20). Daher wurde die absolute, gemittelte Spektralintensität $\bar{I}_{\bar{N} \pm 3 \text {,Abs }}$ berechnet, indem zunächst die Mittelwerte über alle Signalzunahmen $I_{\bar{N}}$ der jeweiligen Größenfraktionen aus allen vergleichbaren Messungen gebildet wurden. Von diesen Werten wurde anschließend der Betrag 1 abgezogen, um die absolute Zunahme $\bar{I}_{\bar{N} \text {,Abs }}$ zu erhalten. Als Maß für die relative Änderung dieser absoluten Spektralintensitäten wurde der Quotient $Q_{\text {IR }}$ gebildet:

\footnotetext{
${ }^{1}$ Ursprünglich war ein Wert von $\Delta t=5 \mathrm{~ns}$ bzw. $100 \mathrm{~ns}$ eingestellt worden, eine nachträgliche Überprüfung ergab allerdings einen zeitlichen Versatz von $2 \mathrm{~ns}$
} 


$$
\begin{aligned}
& \bar{I}_{\bar{N}, \tilde{\nu}_{\mathrm{IR}}} \equiv \frac{\sum I_{\bar{N} \pm 3, \tilde{\nu}_{\mathrm{IR}}}}{N_{\text {Messungen }}} \\
& \bar{I}_{\bar{N}, \mathrm{Abs}, \tilde{\nu}_{\mathrm{IR}}} \equiv \bar{I}_{\bar{N}, \tilde{\nu}_{\mathrm{IR}}}-1 \\
& Q_{\mathrm{IR}} \equiv \bar{I}_{\bar{N}, \mathrm{Abs}, 3420} \\
& \bar{I}_{\bar{N}, \mathrm{Abs}, 3180}
\end{aligned}
$$

Der Wert von $Q_{\mathrm{IR}}$ wird vermindert, sofern die Signalintensität im Spektralbereich um $\tilde{\nu}_{\mathrm{IR}}=3180 \mathrm{~cm}^{-1}$ sich im Vergleich zum Absorptionsmaximum amorpher Cluster erhöht. Ein kristalliner Cluster sollte sich folglich durch einen niedrigeren Wert von $Q_{\mathrm{IR}}$ von einem amorphen Cluster unterscheiden.

Als Fehler $\Delta \bar{I}_{\bar{N}}, \tilde{\nu}_{\mathrm{IR}}$ der gemittelten Signalzunahmen wurde die einfache Standardabweichung über 120 Messwerte zugrunde gelegt. Der Fehler des Quotienten $Q_{\mathrm{IR}}$ wurde durch eine Fehlerfortpflanzung nach Gauß ermittelt:

$$
\Delta Q_{\mathrm{IR}}=\sqrt{\left(\frac{\partial Q_{\mathrm{IR}}}{\partial \bar{I}_{\bar{N}, \mathrm{Abs}, 3420}} \Delta \bar{I}_{\bar{N}, \mathrm{Abs}, 3420}\right)^{2}+\left(\frac{\partial Q_{\mathrm{IR}}}{\partial \bar{I}_{\bar{N}, \mathrm{Abs}, 3180}} \Delta \bar{I}_{\bar{N}, \mathrm{Abs}, 3180}\right)^{2}}
$$

Die Ergebnisse dieser Messreihe sind in Abbildung 6.4 dargestellt: Entsprechend der generell beobachteten Rotverschiebung des Absorptionsmaximums mit zunehmender Clustergröße (vgl. Abschnitt 3.2.3) fallen die Werte von $Q_{\mathrm{IR}}$ in allen vier Experimenten ab. Die kürzeren Fehlerbalken der in blauer Farbe dargestellten Graphen sind auf eine ca. um den Faktor 10 erhöhte Signalstärke im Falle von Helium als Trägergas ( $p_{0} \approx 6$ bar) zurückzuführen (vgl. Abb. 6.1). Der vergleichsweise stärkere Signalabfall amorpher Cluster, deren Absorptionsmaximum um $\tilde{\nu}_{\mathrm{IR}}=3400 \mathrm{~cm}^{-1}$ liegt, äußert sich in generell niedrigeren Werten von $Q_{\mathrm{IR}}$ nach langer Delayzeit. Oberhalb eines bestimmten Größenwertes unterschreiten die Werte von $Q_{\mathrm{IR}}$ der langsam abgekühlten Cluster die Werte aus der Vergleichsmessung mit hoher Kühlrate, die nach der gleichen Delayzeit aufgenommen wurde. Dieser Punkt sollte die Größenscheibe markieren, ab der in den Clustern, denen bei der Erzeugung mehr Zeit für die Nukleation zur Verfügung stand, erste kristallartige Strukturen auftreten.

Nach langer Delayzeit ist dieser Befund bereits bei kleineren Clustern sichtbar, da die Signalzunahme kristallartiger Partikel im zeitlichen Verlauf langsamer abfällt, als die amorpher Partikel. Dieser Umstand ist durch mehrere Faktoren begründet: Das Netzwerk aus stärkeren Wasserstoffbrücken führt hier nicht nur zu einer niedrigeren potentiellen Energie, sondern bewirkt auch eine um das ca. achtfache erhöhte Wärme- und Temperaturleitfähigkeit im Vergleich zu amorphen Clustern 62 135. Somit kann eine lokal begrenzt eingetragene Energiemenge in einem kristallartigen Wasserstoffbrückennetzwerk schneller über sämtliche Schwingungsfreiheitsgrade verteilt werden. Konkurrierende Prozesse wie die Ablösung von Monomeren bzw. Oligomeren (Letztere wurde von Andersson et al. ausschließlich an amorphen Clustern beobachtet 166] werden daher unterdrückt. Darüber hinaus ist die Fähigkeit kristalliner Cluster zur Energieaufnahme durch IR-Strahlung eingeschränkt: Sobald durch die resultierende Temperaturerhöhung eine Phasenänderung eintritt, verändert sich die Lage des Absorptionsmaximums. Daher kann diesen Partikeln bei IR-Anregung am Absorptionsmaximum weniger Energie zugeführt werden, als amor- 
phen Clustern gleicher Größe. Folglich verschiebt sich die Isomerenzusammensetzung nach $\Delta t=102$ ns oberhalb von $N^{*}$ zugunsten von Partikeln mit kristallartigem Kern.

Die Unterschiede in den Werten von $Q_{\mathrm{IR}}$ bei $\Delta t=7 \mathrm{~ns}$ dürften durch die stark verschiedenen Kühlraten und Temperaturen bedingt sein: Den schnell abgekühlten Clustern steht generell weniger Zeit zur Verfügung, um eine möglichst günstige, lokale Minimumstruktur auszubilden. So zeigt in der kälteren Expansion die Signalzunahme bei $\tilde{\nu}_{\mathrm{IR}}=3180 \mathrm{~cm}^{-1}$ einen Abfall zwischen den Messungen bei $\Delta t=7 \mathrm{~ns}$ und $\Delta t=102 \mathrm{~ns}$, während die Bandenintensität sich in der wärmeren Expansion kaum ändert. Als Folge unterscheiden sich die Werte von $Q_{\text {IR }}$ für kleine Cluster mit $\bar{N} \leq 76$ bei $\Delta t=7$ ns in Abbildung 6.4 .

Bemerkenswert ist hingegen der nahezu deckungsgleiche Verlauf der beiden Graphen von $Q_{\mathrm{IR}}$ für unterschiedlich schnell abgekühlte Cluster bei $\Delta t=102 \mathrm{~ns}$ im Größenbereich $50 \leq \bar{N} \leq 76$ : Entsprechend den Ausführungen in Abschnitt 2.2.1 bilden kleine Cluster auch bei langsamer Abkühlung keinen kristallartigen Kern aus, sondern weisen eine amorphe Struktur auf. Der hier vorgestellte Befund kann als Bestätigung dieser Annahme aufgefasst werden. Die in diesem Größenbereich annähernd identischen Werte von $Q_{\mathrm{IR}}$ in beiden Experimenten deuten auf eine ähnliche Verteilung der Bindungsstärken in den nach 102 ns ionisierten Clustern und damit eine vergleichbare Güte der Relaxation dieser Strukturen hin. Nach dieser Zeitspanne sind überwiegend solche Aggregate vorhanden, die vor der IR-Anregung sowie der Natriumsolvatation eine ausreichend niedrige innere Energie besaßen, um durch den mit diesen Vorgängen verbundenen Energieeintrag keine bzw. nur wenige Evaporationsereignisse zu verzeichnen.

Im Größenbereich oberhalb von $\bar{N}=76 \pm 3$ beginnen die Graphen der unterschiedlich schnell gekühlten Cluster, zu divergieren: Die Fraktion der langsamer abgekühlten Cluster gewinnt im Spektralbereich um $3200 \mathrm{~cm}^{-1}$ an relativer Intensität. Dieser Gewinn kann nicht durch DAA-koordinierte Moleküle verursacht worden sein, da deren relativer Anteil mit zunehmender Clustergröße abnehmen sollte $52[62$. Als wahrscheinliche Ursache kommen damit die DDAA-koordinierten Wassermoleküle im Kern des Clusters in Betracht, deren Absorption im Spektralbereich um $3200 \mathrm{~cm}^{-1}$ zunimmt, sofern sie stärkere H-Brücken ausbilden. Voraussetzung hierfür ist eine möglichst optimale Koordinationsgeometrie. Ein zunehmender Anteil weitgehend tetraedrisch koordinierter Wassermoleküle im Clusterinneren bewirkt folglich den stärkeren Abfall von $Q_{\mathrm{IR}}$ in den langsam abgekühlten Clustern. Zwar nimmt auch die Bindungsstärke der H-Brücken in amorphen Clustern mit zunehmender Größe des H-Brückennetzwerkes zu (vgl. Abschnitt 3.2.3), was durch den generell beobachteten Abfall von $Q_{\mathrm{IR}}$ mit zunehmender Clustergröße in Abbildung 6.4 ebenfalls ersichtlich wird. Entscheidend ist allerdings der Unterschied zwischen den mit unterschiedlicher Geschwindigkeit abgekühlten Clustern:

Die Datenpunkte bei $\bar{N}=88 \pm 3$ zeigen eine weitgehende Überschneidung der Fehlerbalken, während die spektralen Signaturen der nächstgrößeren Fraktion $\bar{N}=90 \pm 3$ sich bereits deutlich unterscheiden. Oberhalb von $\bar{N}=94 \pm 3$ wird der Unterschied zwischen beiden Experimenten größer als die doppelte Standardabweichung. Daher besteht ein Signifikanzniveau von $<5 \%$ für die Hypothese, dass diese Fraktion Isomere mit kristallartigem Kern enthielt und somit Aggregate mit $N\left(\mathrm{H}_{2} \mathrm{O}\right) \leq 97$ imstande sind, eine Phasenänderung zu vollziehen. Die Messung nach kurzer Delayzeit zeigt ein entsprechendes Resultat erst ab der Größenscheibe $\bar{N}=98 \pm 3$. Diese Ergebnisse sind konsistent mit den Vorhersagen von Bandow und Hartke, denen zufolge kristallartige Strukturen ab $N \gtrsim 90$ thermodynamisch mit amorphen Clustern konkurrieren können[70] sowie mit 
den Messungen von Christoph Dierking (vgl. Abb. 6.2), anhand derer das Einsetzen der Kristallisation auf $N \approx 90 \pm 10$ eingegrenzt wurde 61 .

Da die Endtemperaturen der untersuchten Partikel sich aufgrund der verschiedenen Stagnationsbedingungen stark unterscheiden, ist die Frage zu klären, ob die obige Interpretation zulässig ist bzw. in welchem Umfang die hieraus erwachsenden Unterschiede im IR-Spektrum das Messergebnis beeinträchtigt haben könnten. Aus Abb. 6.3 geht hervor, dass Partikel mit $\bar{N}=225$ nach IR-Anregung im Bereich um $\tilde{\nu}_{\mathrm{IR}} \approx 3400 \mathrm{~cm}^{-1}$ verstärkt Evaporationsprozessen unterliegen. Daher soll im Folgenden besonders deren Einfluss betrachtet werden:

Ein überproportional starker Verlust von Clusterkonstituenten um $\tilde{\nu}_{\text {IR }} \approx 3400 \mathrm{~cm}^{-1}$ kann den Wert des Quotienten $Q_{\mathrm{IR}}$ nach unten verfälschen, sodass bereits kleinere Cluster zu kristallisieren scheinen, wenn dieser Einfluss nicht in beiden Experimenten gleichermaßen auftritt. Das Resultat der vorliegenden Messreihe würde folglich ungünstig beeinträchtigt, sofern erstens größere Cluster verstärkt evaporieren, diese Prozesse zweitens bei $\tilde{\nu}_{\mathrm{IR}}=3400 \mathrm{~cm}^{-1}$ überproportional auftreten, sodass ihr Einfluss bei der Bildung von $Q_{\mathrm{IR}}$ nicht ausgeglichen wird, und drittens der Wert des Quotienten $Q_{\mathrm{IR}}$ der kälteren Heliumcluster hierdurch nicht in gleichem Maße vermindert wird.

Die Berechnungen von Gimelshein et al. zeigten, dass alle Cluster im fraglichen GröBenbereich $(50 \lesssim \bar{N} \lesssim 100)$ annähernd die gleiche Temperatur aufweisen 59 . Sowohl die Wärmekapazität 270 der Clusters als auch die Absorptionswahrscheinlichkeit für IRPhotonen verhalten sich näherungsweise proportional zur Konstituentenzahl $N$. Aufgrund der höheren Wärmekapazität größerer Cluster sollte bei diesen die durch Adsorption und Solvatation der Natriumatome bedingte Temperaturerhöhung geringer ausfallen.

Die Ausführungen in Abschnitt 2.2.2 legen nahe, dass die Kristallisation, die im makroskopischen Maßstab einen Phasenübergang erster Ordnung darstellt, diesen Charakter in kleinen Clustern verliert, da die Differenz der freien Energien beider möglicher Zustände mit abnehmender Clustergröße sinkt. Aus diesen Überlegungen ergibt sich, dass die exakte Festlegung der kritischen Clustergröße nicht nur experimentell unmöglich ist, da sie einen sehr langen (theoretisch einen unendlich langen) Beobachtungszeitraum erfordert, sondern dass ein diskreter Wert für $N^{*}$ durch die in kleinen Clustern verschwindenden Unterschiede zwischen verschiedenen phasenartigen Zuständen nicht existieren dürfte. Vielmehr dürften sich strukturelle (vgl. Abschnitt 2.3.5) und energetische Unterschiede zwischen zwei möglichen, phasenartigen Zuständen in Abhängigkeit von der Clustergröße und Umgebungsbedingungen ausbilden. Es entsteht ein Größenbereich, in dem zwischen beiden Zuständen ein Gleichgewicht herrscht, dessen Lage abhängig von den Bedingungen ist, unter denen der Cluster vorliegt.

Die im vorliegenden Experiment beobachtete Abhängigkeit der Anzahl kristalliner Cluster von der Kühlrate ist auch konsistent mit den Ergebnissen bisheriger experimenteller und theoretischer Studien, die eine Phasenänderung als Folge eines stochastisch auftretenden Nukleationsereignisses auffassen (vgl. Abschnitt 2.3.3). Demnach ist die Wahrscheinlichkeit, mit der in einem Cluster gegebener Größe ein Nukleationsereignis auftritt, abhängig von seinen Umgebungsbedingungen (d.h. von der Temperatur, theoretisch auch vom Druck und auch von der Stärke eines evtl. vorliegenden elektrischen Feldes ${ }^{172}$ ) und steigt mit der Zeit, die für eine Nukleation zur Verfügung steht. Diese Überlegungen sowie der deckungsgleiche Verlauf beider Graphen kleinerer Cluster bei $\Delta t=102 \mathrm{~ns}$ und deren graduelles Auseinanderlaufen oberhalb von $N \approx 80$ legen die Vermutung nahe, dass auch 


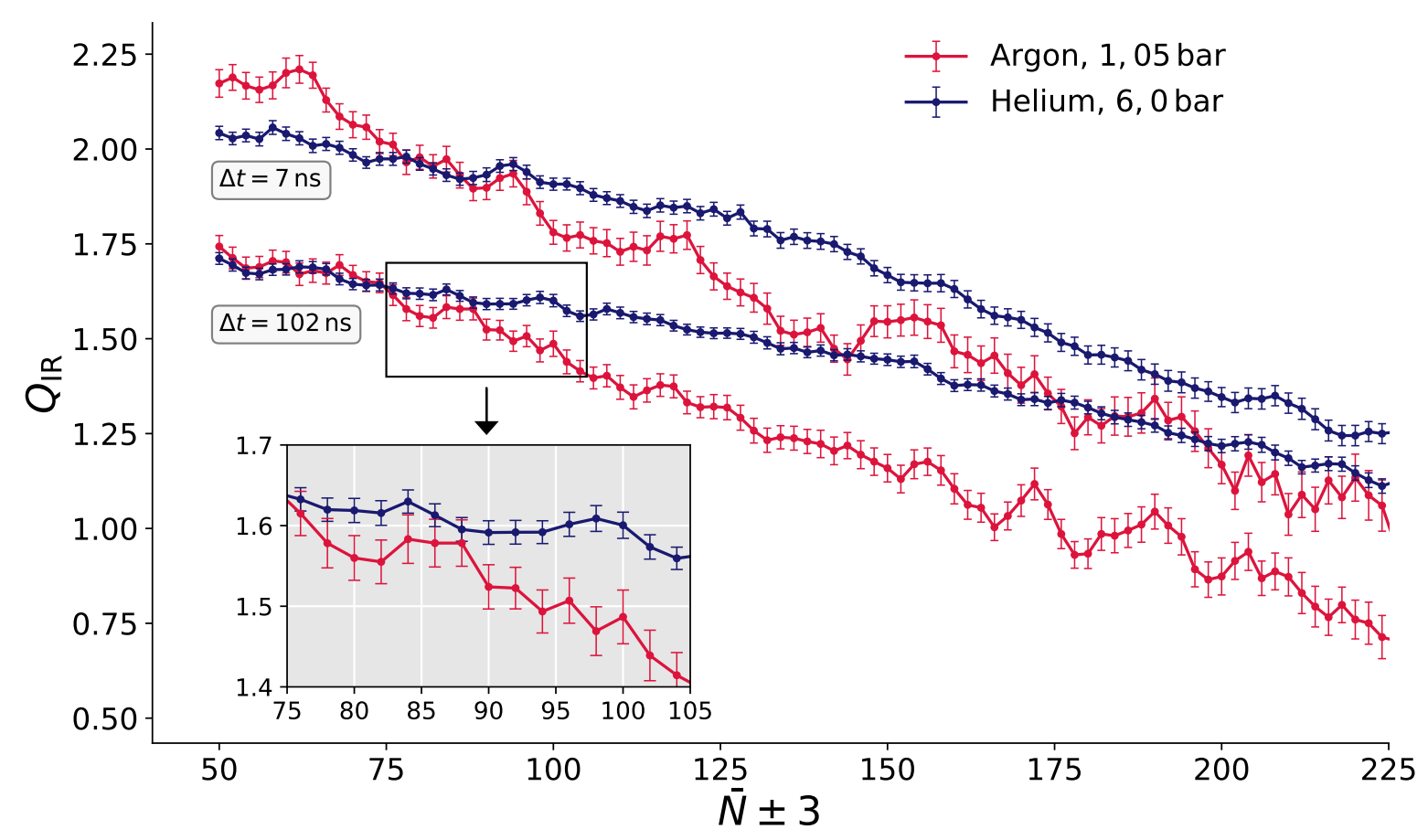

Abbildung 6.4.: Größenabhängige Entwicklung des Quotienten der Signalintensitäten bei $\tilde{\nu}_{\mathrm{IR}}=3420 \mathrm{~cm}^{-1}$ und $\tilde{\nu}_{\mathrm{IR}}=3180 \mathrm{~cm}^{-1}$ bei schneller (Helium) und langsamer Abkühlung (Argon) sowie kurzer (obere Kurven) und langer Delayzeit (untere Kurven). Aufgetragen wurde der gleitende Mittelwert über sieben Clustergrößen. Die Fehlerbalken entsprechen einer einfachen Standardabweichung. Der vergrößerte Einsatz zeigt den Bereich, in dem die Abweichung der spektralen Signaturen signifikant zutage tritt.

Cluster im Größenbereich $N<90$ zur Ausbildung eines kristallartigen Kerns in der Lage sein könnten, sodass die untere Grenze für das Auftreten einer Phasenänderung auf Basis der vorliegenden Ergebnisse mit aller Vorsicht bei $N \approx 80$ angesetzt werden kann.

\subsection{Der Einfluss der Kristallisation auf die Bande der freien $\mathrm{OH}-$ Oszillatoren}

Die Bindungsstärke der freien OH-Gruppen auf der Clusteroberfläche variiert stark in Abhängigkeit von ihrer Koordinationsumgebung sowie der Größe 32|62 und der Ladung 34 des Partikels (vgl. Abschnitt 3.2.3). Die Position ihrer gut separierten Schwingungsbande bewegt sich dabei im Bereich zwischen $\tilde{\nu}_{\mathrm{IR}}=3680 \mathrm{~cm}^{-1}$ und $\tilde{\nu}_{\mathrm{IR}}=3720 \mathrm{~cm}^{-1}$. In den kleinsten, neutralen Wasserclustern mit zweistelliger Konstituentenzahl ist das Bandenmaximum bei $3710 \mathrm{~cm}^{-1}$ verortet 32 . Es verschiebt sich mit zunehmender Partikelgröße zu Werten von $3692 \mathrm{~cm}^{-162}$ in kristallinem Eis I bzw. $3696 \mathrm{~cm}^{-1} 15$ in Filmen aus amorph verfestigtem Wasser. Diese Verschiebung allein dürfte als Indikator nicht in Betracht kommen, da die maximale Differenz zwischen den Bandenpositionen amorph und kristallin koordinierter Wassermoleküle von $4 \mathrm{~cm}^{-1}$ nur in der kondensierten Materie beobachtet wird. In kleineren Partikeln verringert sich der Unterschied in der Bandenposition entsprechend. 
Da die freien OH-Gruppen keinen Kopplungen mit den gebundenen OH-Oszillatoren im Clusterinneren unterliegen, sollte ihre Signalstärke sich allein proportional zu ihrem Übergangsdipolmoment und ihrer Anzahl verhalten 62. Ihre Bandenintensität sollte folglich gemäß Gleichung 2.5 mit $N^{-1 / 3}$ skalieren, sofern die Beschaffenheit des Clusters sich nicht ändert. Ein entsprechendes Verhalten wurde an protonierten Wasserclustern ${ }^{32}$ sowie an natriumdotierten Wasserclustern 275 bereits beobachtet.

Da die in Abschnitt 6.2 vorgestellten experimentellen Ergebnisse in hohem Maße mit den Vorhersagen von Bandow und Hartke ${ }^{70}$ übereinstimmen, erscheint ein Blick auf die von diesen Autoren gefundenen Strukturen lohnend. Eine genauere Inspektion der freien OH-Gruppen der Clustergröße $N=90$ ergab einen deutlichen Unterschied zwischen amorphen Clustern und solchen, die einen kristallinen Kern aufweisen: Die gefundene globale Minimumstruktur eines amorphen Clusters weist 16 freie OH-Gruppen auf der Oberfläche auf, während die Clusterstrukturen, die durch Optimierung von Ausschnitten kristalliner Netzwerke erhalten wurden, 22 (Eis $\mathrm{I}_{\mathrm{h}}$ ) bzw. 23 (Eis $\mathrm{I}_{\mathrm{c}}$ ) freie OH-Gruppen aufweisen 276]. Dieser Unterschied sollte sich in einer gesteigerten Bandenintensität der freien OH-Streckschwingung in Relation zur Bande der gebundenen OH-Oszillatoren äußern, sofern sich ein kristalliner Kern ausbildet.

Um den Unterschied zwischen kristallartigen und amorphen Clustern zu erfassen, wurden wiederum Cluster verglichen, die verschiedenen Kühlraten ausgesetzt waren. Unter den in Abschnitt 6.1 vorgestellten Expansionsbedingungen $(\Delta t=100 \mathrm{~ns})$ wurden Spektren der freien $\mathrm{OH}-$ Streckschwingung aufgenommen, die anschließend auf die Bande der gebundenen OH-Oszillatoren normiert wurden. Hierzu wurden jeweils die in Abbildung 6.3 vorgestellten Spektren verwendet. Der Spektralbereich, auf den die Normierung erfolgt, muss allerdings mit Bedacht gewählt werden: Es ist nicht auszuschließen, dass die IRAnregung der gebundenen OH-Oszillatoren im Wellenzahlbereich um $\tilde{\nu}_{\mathrm{IR}}=3400 \mathrm{~cm}^{-1}$ eine verstärkte Dissoziation der Cluster zur Folge hat. Die hieraus folgende Abschwächung der gebundenen $\mathrm{OH}$-Bande hätte zwangsläufig einen scheinbaren, relativen Intensitätsgewinn der freien OH-Streckschwingung zur Folge. Wird jedoch der Bereich um $\tilde{\nu}_{\mathrm{IR}}=3400 \mathrm{~cm}^{-1}$ aus der Normierung ausgenommen, wird die relative Entwicklung der gebundenen $\mathrm{OH}$-Bande durch den Intensitätsgewinn der Bande kristalliner Strukturen dominiert. Da dieser Anstieg aufgrund des hohen Übergangsdipolmoments kristalliner H-Brückennetzwerke deutlich größer ausfällt, als die zu erwartende Zunahme der freien $\mathrm{OH}-$ Streckschwingung, muss ein Spektralbereich gefunden werden, der sich durch die Kristallisation möglichst nicht verändert. Dies gelingt nicht vollständig: Da kristalline Strukturen ebenfalls Banden um $3000 \mathrm{~cm}^{-1}$ und $3100 \mathrm{~cm}^{-1}$ ausbilden (siehe Abbildung 6.2) 48], kämen nur die äußersten Randbereiche der gebundenen OH-Bande für eine solche Normierung infrage. Die Gesamtintensität dieser Bereiche ist jedoch zu gering, sodass die Ergebnisse mit großen statistischen Fehlern behaftet wären.

Daher wurden zwei Auswertungen durchgeführt: Erstere enthält eine Normierung auf die gesamte, gebundene OH-Bande im Bereich von $2850 \mathrm{~cm}^{-1}$ bis $3650 \mathrm{~cm}^{-1}$. Die Ergebnisse sind als Konturplots in Abbildung 6.5 dargestellt. Aufgetragen wurde jeweils der Mittelwert über 20 Clustergrößen. Aus den oben angeführten Gründen wird ein relativer Intensitätsgewinn der freien OH-Bande möglicherweise bereits bei kleineren Clustern beobachtet, als dies tatsächlich der Fall ist. Diese Auswertung kann daher als ein möglicherweise zu früh anzeigender Indikator für die Kristallisation aufgefasst werden. Eine weitere Auswertung wurde unter Normierung Auf den Wellenzahlenbereich von $2850 \mathrm{~cm}^{-1}$ 
bis $3150 \mathrm{~cm}^{-1}$ vorgenommen. Da die Ausbildung eines kristallartigen Kerns einen geringen Intensitätszuwachs in diesem Spektralbereich zur Folge hat und auch gebundene $\mathrm{OH}-$ Gruppen DAA-koordinierter Moleküle mit freier OH-Gruppe hier absorbieren, kann diese Auswertung als mit Sicherheit später Indikator für die Kristallisation aufgefasst werden. Die Resultate dieser Auswertung sind in Abbildung 6.6 dargestellt.

Deutlich ist der Unterschied zwischen den langsam abgekühlten Clustern (jeweils in der oberen Seitenhälfte) und den schnell abgekühlten Clustern (jeweils in der unteren Seitenhälfte) erkennbar: Während letztere in beiden Fällen die bereits zuvor beschriebene relative Intensitätsabnahme mit zunehmender Clustergröße zeigen, durchlaufen die relativen Intensitäten der freien OH-Banden ersterer ein Minimum: Unterhalb einer bestimmten Größenscheibe wird ebenfalls ein Abfall beobachtet, der entsprechend dem abnehmenden Verhältnis von Oberfläche und Volumen zu erwarten war. Oberhalb dieses Wertes verzeichnet die freie $\mathrm{OH}$-Bande eine relative Intensitätszunahme. Diese ist im Falle der Normierung auf die gesamte gebundene $\mathrm{OH}$-Bande deutlich ausgeprägter und bei den größten Clustern sicherlich durch Evaporation verstärkt. Das Minimum tritt hier bei $\bar{N}=90$ auf. Auch die konservative Auswertung in Abbildung 6.6 zeigt eine relative Zunahme der freien OH-Schwingung, obwohl dieser Befund durch die Wahl des Normierungsbereiches mit Sicherheit abgeschwächt ist. Dementsprechend tritt hier das Minimum erst relativ spät bei $\bar{N}=120$ auf. Der relative Anstieg mit wachsender Partikelgröße verläuft hier moderat und mündet oberhalb von $\bar{N}=140$ in ein Plateau. Ein Grund könnte sein, dass dieser Effekt durch zunehmende Beiträge kristallartiger Strukturen in dem für die Normierung verwendeten Bereich der gebundenen $\mathrm{OH}$-Bande gedämpft wird.

Das Maximum der freien OH-Streckschwingung liegt in amorphen Clustern mit $\bar{N}=50$ in allen Messungen bei $\tilde{\nu}_{\mathrm{IR}}=3703 \mathrm{~cm}^{-1}$ und verschiebt sich zu $\tilde{\nu}_{\mathrm{IR}}=3699 \mathrm{~cm}^{-1}$ in kristallinen bzw. $\tilde{\nu}_{\mathrm{IR}}=3701 \mathrm{~cm}^{-1}$ in amorphen Nanopartikeln. Dieses Resultat ist konsistent mit den oben erwähnten Messungen von Buch und Devlin an großen, kristallinen Nanopartikeln bzw. amorphen Filmen, nach denen die Bande in beiden Fällen eine Rotverschiebung aufweisen sollte, die jedoch bei kristallinen Nanopartikeln stärker ausgeprägt ist 15|62. Ebenfalls besteht Konsistenz zu den Messungen von Mizuse et al., die eine Rotverschiebung des Maximums von $3709 \mathrm{~cm}^{-1}(N=11)$ bis zu $3704.5 \mathrm{~cm}^{-1}(N=49)$ an neutralen, phenoldotierten Clustern fanden 243. Die vorliegenden Messungen knüpfen somit an die Ergebnisse beider Studien an und schließen eine Lücke in dem Größenbereich, in dem keine dieser beiden Methoden Ergebnisse liefern konnte.

Vergleichbare experimentelle Studien, die sich mit Phasenänderungen in wässrigen Nanopartikeln befassen, sind rar. An neutralen Clustern wurde dieses Thema bisher ausschließlich mit Hilfe der in der vorliegenden Arbeit verwendeten Methode größenselektiv untersucht. Devlin und Buch untersuchten den Einfluss von Adsorbaten auf kristalline Nanopartikel. Sie fanden, dass nach Adsorption von Molekülen, die starke HBrückenbindungen ausbilden können (z. B. $\mathrm{SO}_{2}$ und $\mathrm{NH}_{3}$ ), der kristalline Anteil eines Partikels zunimmt, während die Dicke der amorphen Suboberfläche sich vermindert. Daraus folgerten sie, dass solche Adsorbate durch Insertion in oberflächennahe H-Brücken und deren Spaltung in der Lage sind, dort Spannungen abzubauen, sodass das Wachstum des kristallinen Kerns energetisch begünstigt wird. Eine Betrachtung der Bandenintensität der betreffenden OH-Gruppen führten sie nicht durch, da bereits bekannt war, dass Adsorbate diese stark beeinflussen können $79 \mid 277$. 

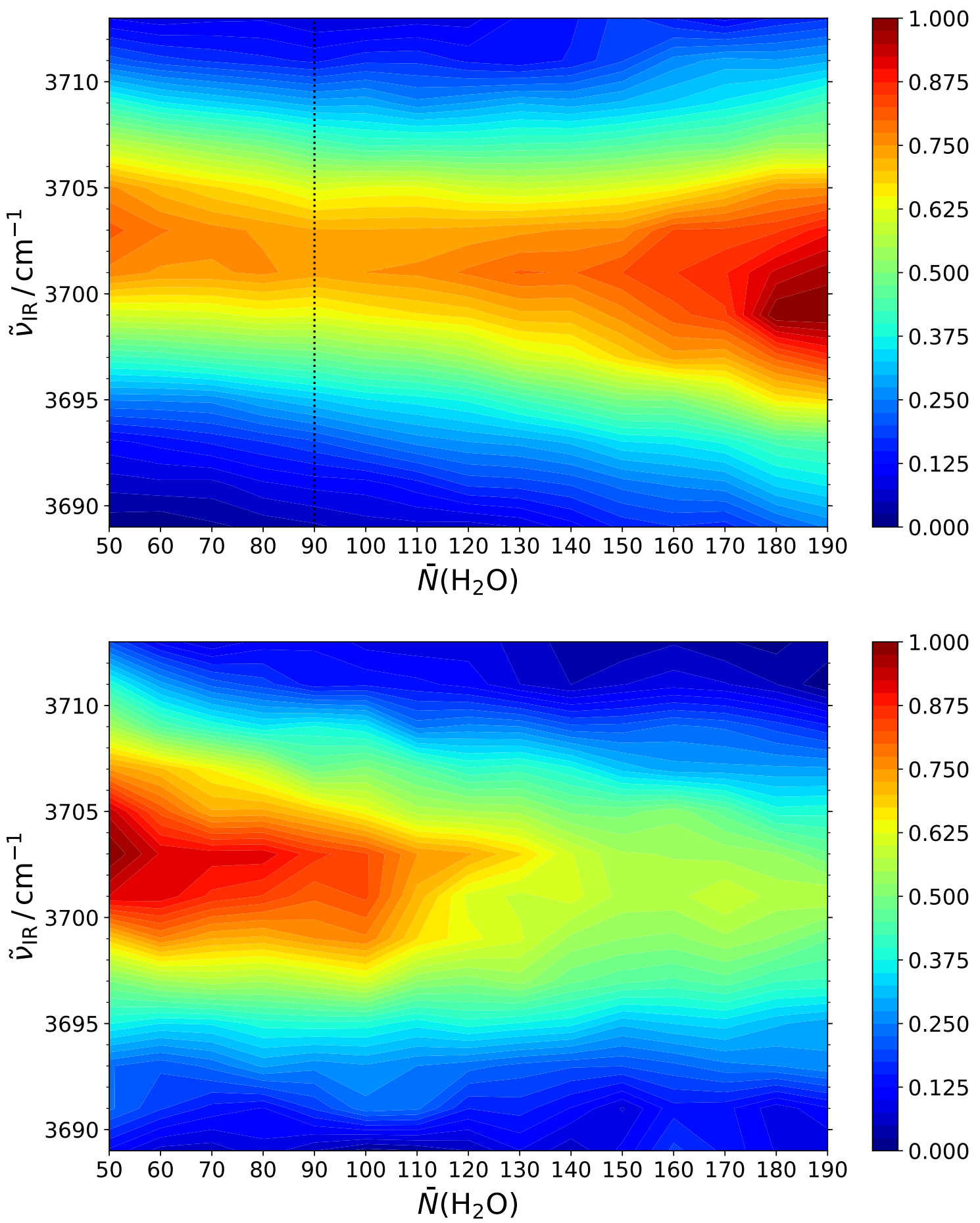

Abbildung 6.5.: Größenabhängige Entwicklung der freien OH-Streckschwingung, normiert auf den Spektralbereich von $2850 \mathrm{~cm}^{-1}$ bis $3650 \mathrm{~cm}^{-1}$ der Bande der gebundenen OH-Oszillatoren; Oben: Niedrige Kühlrate (Argon, $p_{0}=1,05$ bar; Unten: Hohe Kühlrate (Helium, $p_{0}=5,80$ bar); Mittelung über 20 Clustergrößen; die vertikale Linie markiert die Position des Minimums. 

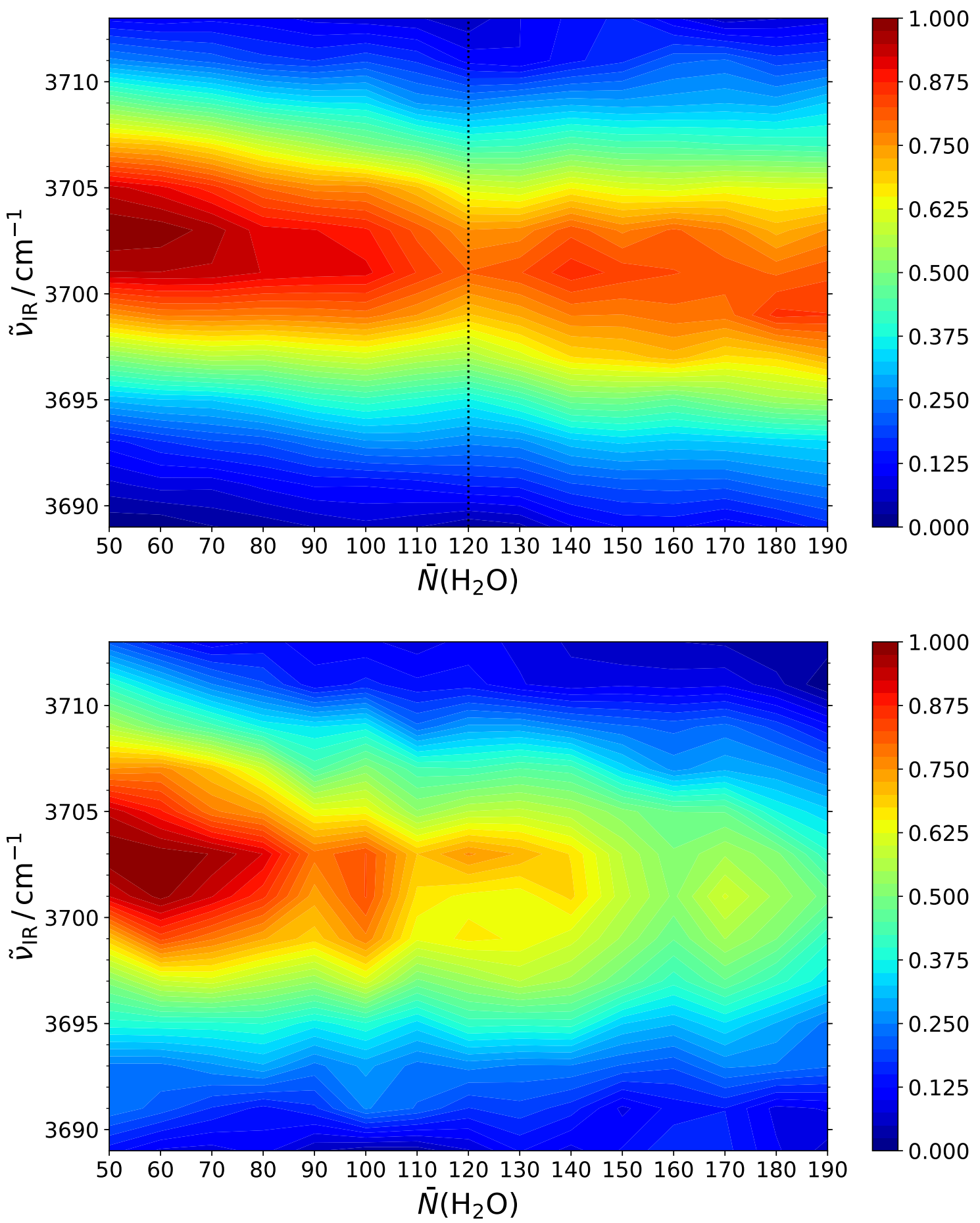

Abbildung 6.6.: Größenabhängige Entwicklung der freien OH-Streckschwingung, normiert auf den Spektralbereich von $2850 \mathrm{~cm}^{-1}$ bis $3150 \mathrm{~cm}^{-1}$ der Bande der gebundenen OH-Oszillatoren; Oben: Niedrige Kühlrate (Argon, $p_{0}=1,05$ bar; Unten: Hohe Kühlrate (Helium, $p_{0}=5,80$ bar); Mittelung über 20 Clustergrößen; die vertikale Linie markiert die Position des Minimums. 
Die Ergebnisse der vorliegenden Arbeit können als komplementäre Beobachtung des gleichen Effektes aufgefasst werden: Da oberflächlich lokalisierte Wassermoleküle nicht in der Lage sind, eine optimal tetraedrische Koordinationsgeometrie auszubilden, entstehen Spannungen, die sich über den gesamten Cluster verteilen (siehe Abschnitt 2.3.5). Das Gleichgewicht zwischen den Bindungsstärken oberflächlich und zentral lokalisierter H-Brückenbindungen verschiebt sich im Zuge der Kristallisation zugunsten der letzteren, da diese sich durch die bei tetraedrischer Koordination maximierten kooperativen Effekte (siehe Abschnitt 2.3.2) maximal verstärken. Die Koordinationsgeometrie der im kristallartigen Kern lokalisierten und durch stärkere H-Brücken verbundenen Wassermoleküle wird auf Kosten der Koordinationsgeometrie oberflächennaher Moleküle optimiert. Diese Prozesse führen nicht nur zur Schwächung oberflächennaher OH-Bindungen, die sich in der niederfrequenten Schwingungsbande kristallinen Eises äußert, sondern auch zum Abbau der nunmehr in den oberflächennahen Bereichen konzentrierten Spannungen durch Spaltung der schwächeren H-Brücken auf der Partikeloberfläche. Die Folge ist ein zahlenmäßiger Zuwachs ungebundener OH-Gruppen, der sich im IR-Spektrum in einer relativen Zunahme der IR-Bandenintensität niederschlägt. Dieser Mikroeffekt, der als unmittelbare Folge der Strukturänderung im Zuge der Kristallisation auftritt, konnte in der vorliegenden Arbeit erstmals direkt im Experiment beobachtet werden.

Weiterhin existieren zwei Studien an kationischen Aggregaten, die für einen Vergleich infrage kommen: Mizuse et al. fanden Hinweise auf Kristallisation in $\mathrm{H}^{+}\left(\mathrm{H}_{2} \mathrm{O}\right)_{N^{-A g g r e g a t e n}}$ mit $N \gtrsim 10032$. Cooper et al. beobachteten den verzögerten Beginn der Kristallisation in Nanopartikeln der Zusammensetzung $\mathrm{La}^{3+}\left(\mathrm{H}_{2} \mathrm{O}\right)_{N}$ mit $N \approx 37532$. Beiden gemeinsam ist, dass sie eine starke Verschiebung des Bandenmaximums der freien OH-Streckschwingung zu niedrigeren Wellenzahlen mit abnehmender Clustergröße beobachten.

Diese Beobachtung steht im Gegensatz zu dem Verhalten neutraler, natriumdotierter 54 und phenoldotierter ${ }^{243}$ Cluster, in denen eine entgegengesetzte Verschiebung beobachtet wird. Die Ursache hierfür liegt in einer Stärkung des H-Brückennetzwerkes durch die zusätzliche positive Ladung. Als Folge wird verstärkt Elektronendichte aus den Bindungen der freien OH-Gruppen abgezogen. Mit zunehmender Clustergröße nimmt dieser Effekt ab 32 . Mizuse et al. schlossen daraus, dass ein überzähliges Proton die Struktur des HBrückennetzwerkes bis zum Größenbereich um $N=100$ beeinflusst 32 .

Folglich sind Ergebnisse, die an ionischen Spezies gewonnen wurden, nur eingeschränkt mit denen neutraler Spezies vergleichbar. Dennoch soll eine entsprechende Betrachtung versucht werden: Während Mizuse et al. die relative Bandenintensität nicht näher quantifizierten, führten Cooper et al. eine genauere Auswertung durch. Ebenso wie Mizuse et al. 32 berichteten sie einen Abfall mit zunehmender Partikelgröße, wobei sie einen proportionalen Zusammenhang mit $N^{-1 / 3}$ berichteten $[76$. Somit kann ein Vergleich der Werte der vorliegenden Arbeit mit den Ergebnissen dieser Autoren vorgenommen werden:

Die größenabhängige Entwicklung der relativen Bandenintensitäten ist in Abbildung 6.7 dargestellt. Die Paneele a und b enthalten die Ergebnisse der vorliegenden Arbeit, die sich in der Wahl des Normierungsbereiches unterscheiden. Paneel c gibt die Entwicklung in $\mathrm{La}^{3+}$-dotierten Clustern von Cooper et al. wieder. Die Größenscheiben, die oberhalb des jeweils anhand der gebundenen OH-Streckschwingung festgestellten Einsatzpunktes der Kristallisation liegen, sind in blauer Farbe dargestellt. In beiden Studien zeigt sich die erwartete relative Abnahme der Bandenintensität, sofern die Clustergröße zu gering ist, um eine Phasenänderung zu vollziehen. Die Umkehr dieses Trends mit dem 

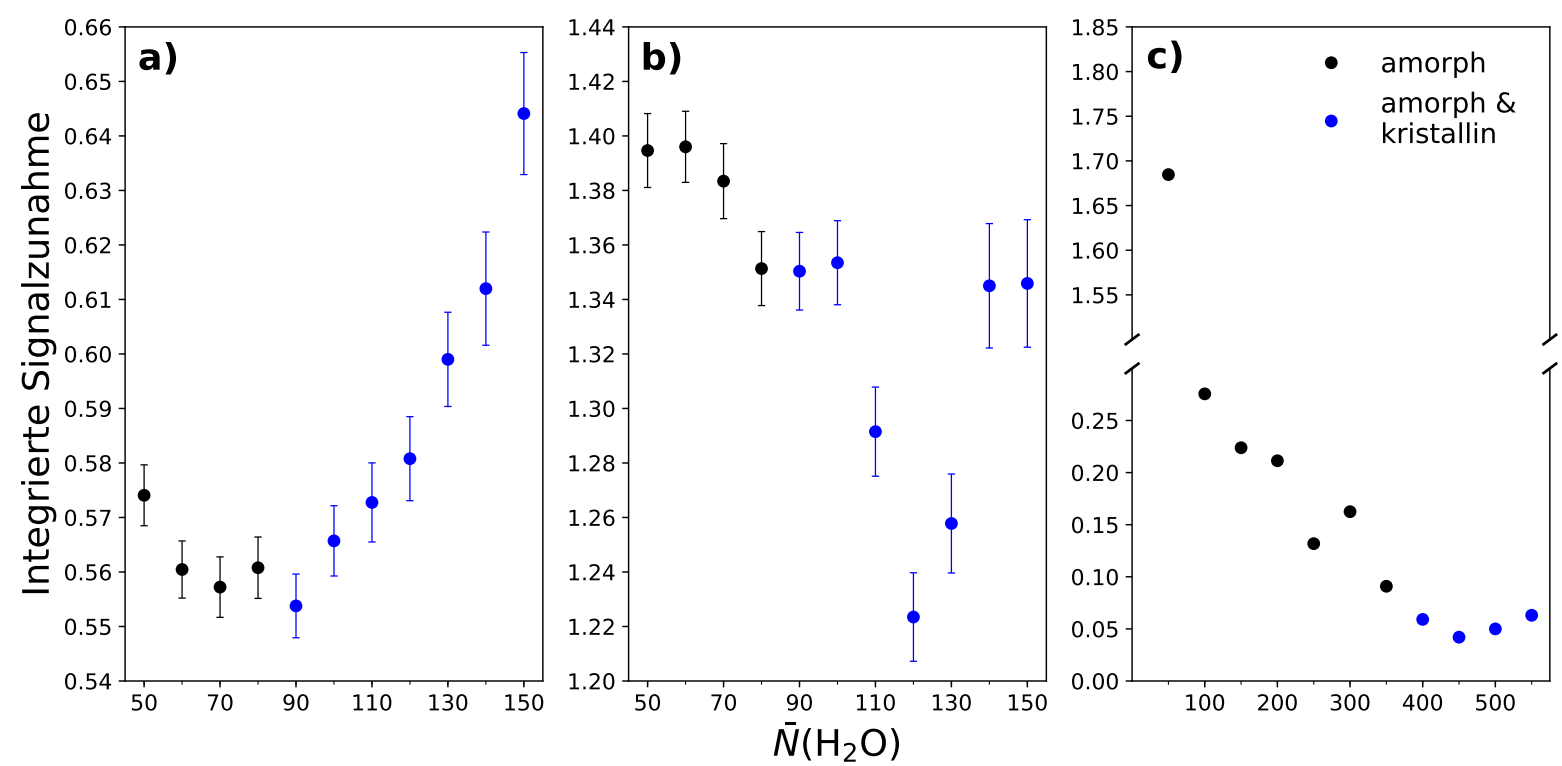

Abbildung 6.7.: Gesamtintensität der freien OH-Streckschwingung; Paneel a: Vorliegende Arbeit, normiert auf den Spektralbereich von $2850 \mathrm{~cm}^{-1}$ bis $3650 \mathrm{~cm}^{-1}$ der gebundenen OH-Bande, Mittelung über 20 Clustergrößen; Paneel b: Vorliegende Arbeit, normiert auf den Spektralbereich von $2850 \mathrm{~cm}^{-1}$ bis $3150 \mathrm{~cm}^{-1}$ der gebundenen OH-Bande, Mittelung über 20 Clustergrößen; Die Fehlerbalken entsprechen jeweils einem Konfidenzintervall von $2 \sigma$; Paneel c: IRPD-Studien an $\mathrm{La}^{3+}$-dotierten Clustern von Cooper et al. ${ }^{76}$, normiert auf den Spektralbereich von $3000 \mathrm{~cm}^{-1}$ bis $3650 \mathrm{~cm}^{-1}$. Die Partikelfraktionen, die jeweils als (z. T.) kristallin klassifiziert wurden, sind durch blaue Markierungen dargestellt.

Einsatz der Kristallisation ist in den Paneelen a und b deutlich erkennbar. In Paneel a, das die relative Intensität nach Normierung auf den gesamten Bereich der gebundenen OH-Streckschwingung wiedergibt, liegt das Minimum der Bandenintensität bei $\bar{N}=90$ und somit in dem Bereich, in dem bereits von Dierking die kritische Clustergröße verortet wurde. Wie oben erwähnt, ist die starke Zunahme der Intensität in den größten Clustern bei dieser Normierung mit hoher Wahrscheinlichkeit auf Evaporationsprozesse zurückzuführen. Bei Normierung allein auf den niederfrequenten Teil der gebundenen $\mathrm{OH}-$ Bande erscheint das Minimum entsprechend später (Paneel b). Der in Paneel a sichtbare, starke Anstieg bleibt hingegen aus. In den $\mathrm{La}^{3+}$-dotierten Clustern (Paneel c) ist eine relative Zunahme der Bandenintensität ebenfalls als Tendenz erkennbar, dieser Befund wird allerdings im Vergleich zum in dieser Arbeit berichteten Einsatzpunkt der Kristallisation bei $N=375$ erst mit Verspätung sichtbar.

O'Brien et al. zeigten, dass eine in den Partikel eingebrachte Ladung nicht nur eine näherungsweise zu deren Größe proportionale Verschiebung der freien OH-Bande verursacht 34 . Weiterhin berichteten sie, dass die relative Bandenintensität der freien $\mathrm{OH}-$ Streckschwingung eine starke Abhängigkeit von der Ladung eines eingebrachten Kations zeigt. So nimmt dieser Wert ca. um den Faktor 1,4 zu, sofern dessen Ladungszahl von +1 auf +3 erhöht wird. Bei Dotierung mit Anionen zeigt sich ein entgegengesetzter Effekt. Als Grund führten die Autoren den strukturierenden Einfluss des Kations auf das 
Wasserstoffbrückennetzwerk an, der sich vom Zentrum (in dessen Nähe das Ion mit der höchsten Wahrscheinlichkeit vermutet wird) bis zur Oberfläche fortsetzt und dort die Anzahl frei schwingender OH-Gruppen erhöht $33 \mid 34$. Einen starken Einfluss der Ladung auf die Übergangsdipolmomente der freien OH-Gruppen schlossen sie aus, da die Änderung der Bandenintensitäten zwischen den Ladungszuständen -1 und +1 im Vergleich geringer ausgeprägt ist 34 .

Eine Studie zur Kristallisation an kationischen Clustern mit geringerer Ladungsdichte wäre aus diesem Grund wünschenswert. Es ist seit Langem bekannt, dass unterschiedliche Ionen die Strukturen von Wasserstoffbrückennetzwerken beeinflussen und auch die $\mathrm{Nu}$ kleation flüssigen Wassers stören können 104|278. Fast ebenso alt ist die Erkenntnis, dass durch das Anlegen eines elektrischen Feldes die Nukleation unterkühlten Wassers induziert werden kann 172]. Möglicherweise war der Einsatz eines dreifach geladenen Kations an dieser Stelle notwendig, um durch dessen hohe Feldstärke die im gleichen Zuge beobachtete Störung des H-Brückennetzwerkes im gesamten Nanotropfen zu kompensieren und letztendlich eine Phasenänderung beobachten zu können. Die Ergebnisse von Mizuse et al. ${ }^{32}$ legen nahe, dass ein überschüssiges Proton im Gegensatz zu einem Metallkation eine weitaus geringere Störung des H-Brückennetzwerkes induziert, wenngleich auch diese Autoren im Größenbereich um $N=100$ einen deutlichen Unterschied zwischen protonierten und elektrisch neutralen Wasserclustern konstatieren.

Weiterhin muss der Einfluss des von Cooper et al. ausgenutzten Wirkungsmechanismus betrachtet werden: Die Spektren wurden in IRPD-Experimenten erhalten. Da die Spaltung von oberflächennahen H-Brücken zu im Mittel schwächer gebundenen Wassermolekülen auf der Oberfläche führt, dürfte der Effekt hier verstärkt auftreten, da in IRPD-Experimenten (im Gegensatz zur hier verwendeten Methode) aus dem Anteil dissoziierter Moleküle die Spektralintensität berechnet wird. Darüber hinaus weisen deren Daten im fraglichen Größenbereich eine deutliche Streuung auf, sodass deren Messgenauigkeit hier stark herabgesetzt gewesen sein dürfte.

Auf theoretischer Seite besteht Konsistenz mit MD-Simulationen, die von Francesco Paesani und Mitarbeitern auf Basis des MB-Pol-Potentials 72 durchgeführt wurden. Diese griffen im Größenbereich $N \leq 120$ auf die Strukturen von Bandow und Hartke zurück. Auch die auf Basis dieser Simulationen berechneten Spektren zeigen eine relative Zunahme der freien $\mathrm{OH}-$ Bande kristallartiger Partikel im Vergleich zu amorphen Aggregaten gleicher Größe. Die Auswertung der relativen Bandenintensitäten amorpher und kristalliner Cluster für $N=90$ ergab für kristalline Partikel eine um den Faktor 1,49 $(T=150 \mathrm{~K})$ bzw. 1,34 $(T=100 \mathrm{~K})$ vergrößerte freie OH-Bande im Vergleich zu amorphen Clustern. Die Anzahl freier OH-Gruppen im Cluster mit $N=90$ steigt von 16 (amorph) auf 22 (Eis $\mathrm{I}_{\mathrm{h}}$ ) bzw. 23 (Eis $\mathrm{I}_{\mathrm{c}}$ ), was einem Zuwachs um den Faktor 1,38 (Eis $\mathrm{I}_{\mathrm{h}}$ ) bzw. 1,44 (Eis $I_{c}$ ) entspricht. Nach diesem Modell entspricht der relative Intensitätszuwachs der freien $\mathrm{OH}-$ Streckschwingung weitgehend der zahlenmäßigen Zunahme der Oszillatoren und die Übergangsdipolmomente freier $\mathrm{OH}-$ Oszillatoren in amorphen und kristallinen Umgebungen sind näherungsweise gleich, obwohl die Elektronendichte in diesen Gruppen abnimmt. Hier besteht Übereinstimmung mit den Ergebnissen von O'Brien et al., die eine starke Abhängigkeit des Übergangsdipolmoments vom Ladungszustand 34 und somit von der mittleren Stärke des H-Brückennetzwerkes und dessen Elektronendichte 32 ausschlossen. Es ist daher unwahrscheinlich, dass der beobachtete Effekt allein auf eine 

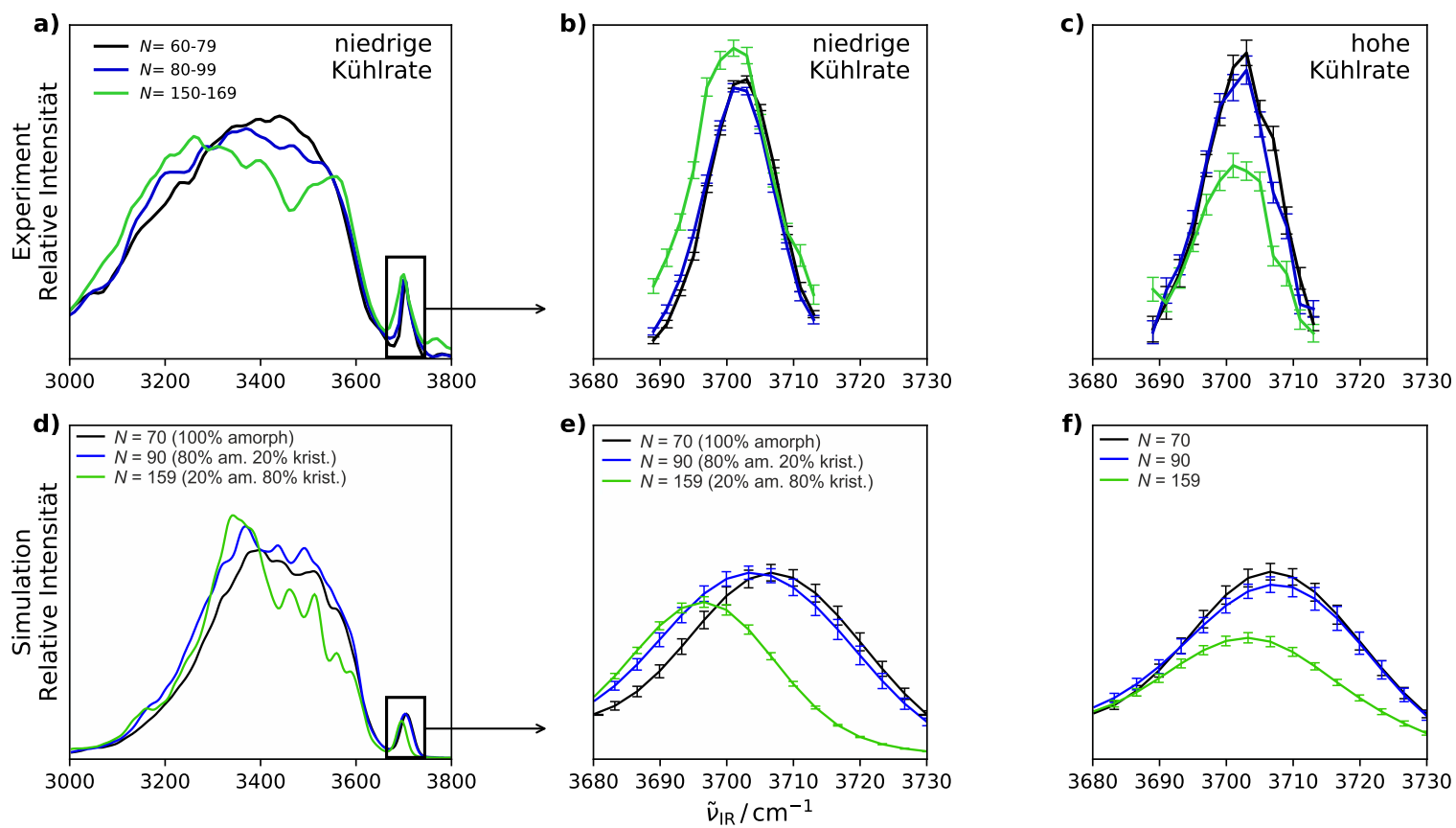

Abbildung 6.8.: Vergleich der experimentellen Ergebnisse (obere Paneele (a bis c), aufgetragen ist jeweils der Mittelwert über die gleichen 20 Clustergrößen; die Spektren in Paneel a wurden einer dreifachen Dreiecksglättung der Gewichtung 25:50:25 unterzogen und auf das Gesamtintegral normiert) mit den Simulationen von Paesani und Mitarbeitern (untere Paneele d bis f) 274. Paneele a und d: Gesamter Spektralbereich der $\mathrm{OH}-$ Streckschwingungen; Paneele $\mathrm{b}$ und e zeigen den zugehörigen Ausschnitt der freien OH-Streckschwingung jeweils bei niedriger Kühlrate, normiert auf den Spektralbereich von $2850 \mathrm{~cm}^{-1}$ bis $3650 \mathrm{~cm}^{-1}$. Paneele c und f zeigen im Gegensatz die freie $\mathrm{OH}-$ Streckschwingung bei hoher Kühlrate (Helium, gleiche Normierung). Die simulierten Spektren der Clustergrößen $N=90$ und $N=109$ in den Paneelen e und f stellen gewichtete Linearkombinationen der IR-Spektren amorpher und kristalliner Cluster dar. Die Fehlerbalken entsprechen einem Konfidenzintervall von $1 \sigma$.

Änderung des Übergangsdipolmoments der freien OH-Gruppen zurückzuführen ist. Eine größenabhängige Rotverschiebung der freien $\mathrm{OH}-\mathrm{Bande}$, die bei kristallinen Partikeln stärker ausgeprägt ist, als bei amorphen Partikeln, geht aus den Berechnungen von Francesco Paesani und Mitarbeitern ebenfalls hervor.

Die experimentellen Ergebnisse sind in Abbildung 6.8 den theoretischen Vorhersagen gegenübergestellt. Der Einsatz der Phasenänderung bei langsamer Abkühlung im GröBenbereich $\bar{N} \lesssim 100$ (blaue Graphen) unter den vorliegenden Expansionsbedingungen (Argon, $p_{0} \approx 1,05$ bar; vgl. Abschnitt 6.1) ergibt sich aus der erhöhten Intensität um $\tilde{\nu}_{\mathrm{IR}}=3200 \mathrm{~cm}^{-1}$ (Paneel a) bzw. $\tilde{\nu}_{\mathrm{IR}}=3300 \mathrm{~cm}^{-1}$ (Paneel d). Die Abweichung zwischen Theorie und Experiment ist durch die Fermi-Resonanz 155 erklärbar, die durch das zur Berechnung des Spektrums in Paneel d verwendete MB-Pol-Potential nicht akkurat wiedergegeben wird ${ }^{74]}$. Als Folge unterschätzt dieses Modell die spektralen Auswirkungen der in regelmäßigen Sechsringen auftretenden Kooperativität und verortet unter anderem das Bandenmaximum kristallartiger Strukturen bei höheren Wellenzahlen, als dies 
im Experiment beobachtet wird. Das gemessene Spektrum erstreckt sich hingegen weiter in den Bereich niedriger IR-Wellenzahlen. Unter den gleichen Expansionsbedingungen zeigt die freie OH-Streckschwingung (Paneel b) eine leichte Rotverschiebung sowie eine beginnende Intensitätszunahme, die vom Modell mit guter Übereinstimmung vorhergesagt wird, sofern eine gewichtete Linearkombination der Spektren amorpher (80\%) und kristalliner Cluster (20\%) zugrunde gelegt wird (siehe Paneele d und e). Im Vergleich zum experimentellen Befund (Paneel b) ist der vorhergesagte Effekt hier bei $N=90$ stärker ausgeprägt, wohingegen bei $N=159$ der experimentelle Befund die Vorhersage der Theorieseite übertrifft.

Die Paneele e und f zeigen im Gegensatz hierzu die Intensitätsentwicklung bei schneller Abkühlung, wie sie im Experiment durch Helium $\left(p_{0} \approx 5,8\right.$ bar $)$ erreicht wird. Hier ist die Rotverschiebung schwächer ausgeprägt. Die relative Intensität nimmt mit zunehmender Clustergröße ab, es besteht somit kein Hinweis auf eine Phasenänderung.

Abbildung 6.9 zeigt die Spektren der freien OH-Streckschwingung im Größenbereich $70 \leq \bar{N} \leq 130$. Die aufgetragenen Fehlerbalken entsprechen einem Konfidenzintervall von $2 \sigma$. Im niederfrequenten Bereich von Paneel a bei $\tilde{\nu}_{\mathrm{IR}}=3693 \mathrm{~cm}^{-1}-3697 \mathrm{~cm}^{-1}$ zeigt sich eine signifikante Zunahme der Signalstärke. Die hohe Datenqualität, die durch eine große Anzahl durchgeführter Messungen erreicht wurde, sowie die Übereinstimmung der experimentellen Ergebnisse mit theoretischen Vorhersagen auf Basis aktueller und hoch entwickelter Modelle lässt den Schluss zu, dass auch durch Betrachtung der freien $\mathrm{OH}-$ Streckschwingung der Einsatz der Kristallisation im Größenbereich $N \leq 100$ nachgewiesen werden kann.

Die Konsistenz der auf Basis der in dieser Arbeit vorgestellten Ansätze gefundenen Werte für $N^{*}$ mit den Arbeiten von Dierking 61 und den Vorhersagen von Paesani lässt vermuten, dass dieses Ergebnis nur geringfügig durch selektiv bei größeren, wärmeren, amorphen Clustern und um $\tilde{\nu}_{\mathrm{IR}}=3420 \mathrm{~cm}^{-1}$ auftretende Evaporation verfälscht ist. Auch der in Abbildung 6.2 gut erkennbare isosbestische Punkt weist darauf hin, dass im betrachteten Größenbereich nur eine moderate Verfälschung der gemessenen Spektren durch selektiv auftretende Evaporation stattfinden kann. Allerdings kann auch der von Dierking gewählte Ansatz, das gesamte Spektrum zu vermessen, geringfügig durch um $3400 \mathrm{~cm}^{-1}$ verstärkt auftretende Evaporationseffekte beeinträchtigt sein, da auch hier die Relation der spektralen Intensitäten um $\tilde{\nu}_{\mathrm{IR}} \approx 3400 \mathrm{~cm}^{-1}$ und $\tilde{\nu}_{\mathrm{IR}}=3200 \mathrm{~cm}^{-1}$ als Indikator für die Kristallisation dient.

Damit kann zwischen den vielfältigen theoriebasierten Vorhersagen entschieden werden, die bisher zum Beginn der Kristallisation in Wasserclustern getroffen wurden. Die in Tabelle 2.1 aufgeführten theoretischen Arbeiten verwenden drei unterschiedliche Kriterien zur Bestimmung der Größe von $N^{*}$ : Kazimirski und Buch führten die thermodynamische Stabilität der Kerntopologie einer kristallinen Ausgangsstruktur in MD-Simulationen an 69 . Dieses Kriterium ermöglicht allerdings keine Aussage darüber, ob die Überwindung der Nukleationsbarriere thermodynamisch möglich ist und eine Trajektorie existiert, entlang der der kristallartige Zustand auf der Zeitskala des Experiments erreichbar ist (vgl. Abschnitt 2.3.3. Die Bedeutung dieses Nachteils dürfte dadurch relativiert werden, dass die Erreichbarkeit des kristallinen Zustands auf einer bestimmten Zeitskala von der Initialstruktur der Nanotröpfchen abhängt und sich daher unter den unzähligen, im Experiment gebildeten Tröpfchen immer auch solche finden werden, in denen ein Phasenübergang stattfindet, sofern er thermodynamisch möglich ist. 

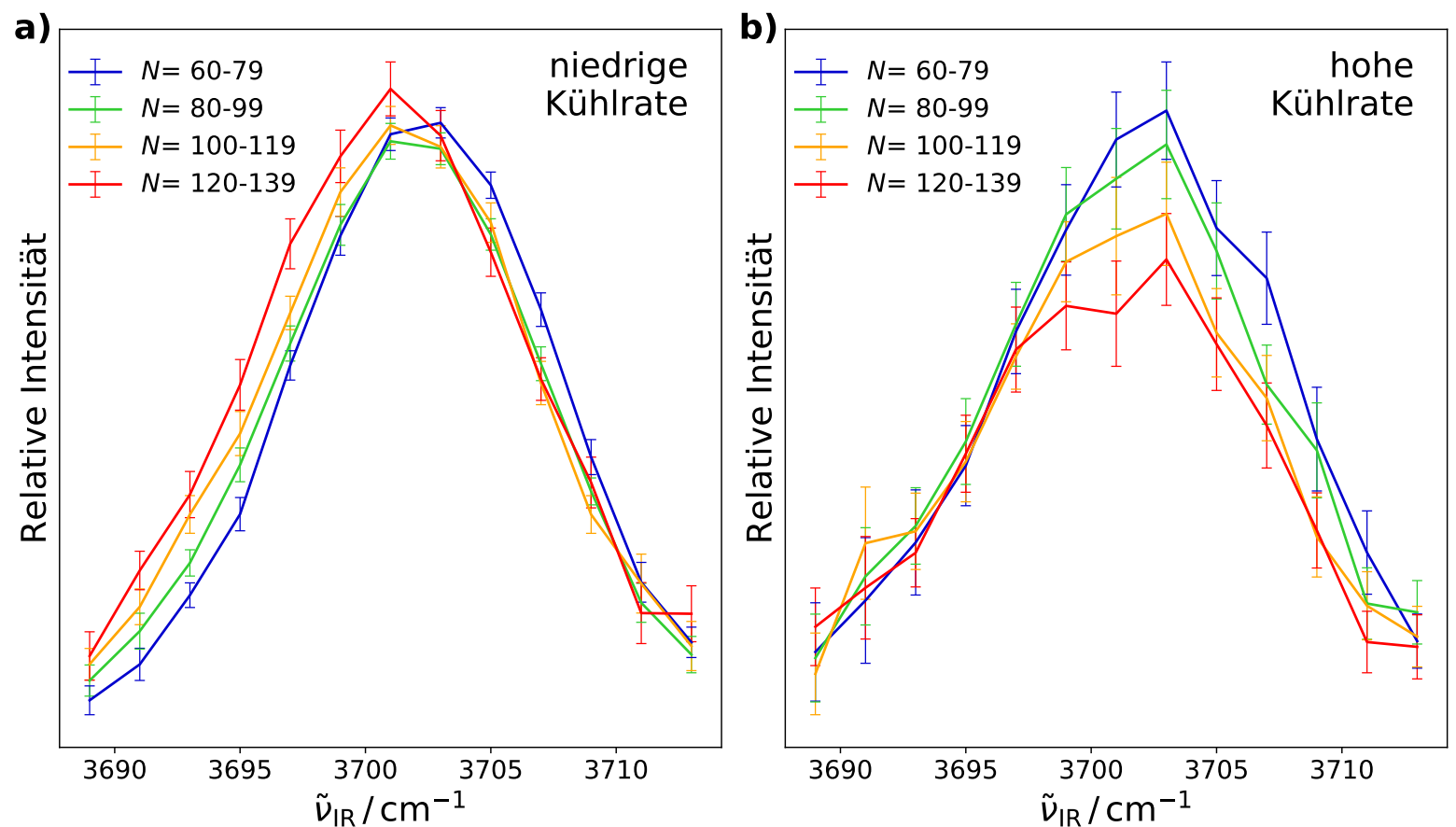

Abbildung 6.9.: Größenabhängige Entwicklung der freien OH-Streckschwingung im Bereich der kritischen Clustergröße; Paneel a: Niedrige Kühlrate (Argon, $p_{0} \approx 1.05$ bar); Paneel b: Hohe Kühlrate (Helium, $p_{0} \approx 5,8$ bar); normiert auf den Spektralbereich von $2850 \mathrm{~cm}^{-1}$ bis $3650 \mathrm{~cm}^{-1}$. Die Fehlerbalken entsprechen einem Konfidenzintervall von $2 \sigma$.

Ein weiterer Nachteil dieser Arbeit ist allerdings die ungenaue Potentialhyperfläche auf TIP4P-Basis, deren Verwendung aus Gründen der Rechenökonomie notwendig war, und die möglicherweise den Energiebeitrag der oberflächennahen H-Brücken zu hoch gewichtete. So trat in MD-Simulationen von Partikeln mit $N=123$ ein Verlust der kristallartigen Kernstruktur zugunsten einer günstigeren Koordinationsgeometrie oberflächennaher Wassermoleküle auf, die vermutlich bei Verwendung eines akkurateren Potentials nicht beobachtet worden wäre.

Weiterhin geht aus Abschnitt 2.2.2 hervor, dass kristalline Strukturen nicht zwingend erst ab der Konstituentenzahl auftreten können, ab der sie das globale Minimum darstellen, sondern bereits zum Gesamtbild beitragen könnten, sobald sie energetisch mit amorphen Strukturen konkurrieren können. Sofern der Energiebeitrag der zusätzlichen Oberfläche zwischen amorpher und kristalliner Phase durch die Anzahl der in die letztere übergegangenen Wassermoleküle nicht vollständig kompensiert wird, könnte ein kurzzeitig gebildeter, kristallartiger Partikel sogar eine geringfügig höhere potentielle Energie besitzen, als eine amorphe Struktur gleicher Größe. Somit kann aus der von Buch et al. in MD-Simulationen beobachteten Umwandlung einer anfangs kristallinen Struktur mit $N=123$ in einen gänzlich amorphen Partikel nicht die thermodynamische Unmöglichkeit kristallartiger Strukturen in Partikeln dieser Größe abgeleitet werden.

Das Kriterium von Johnston und Molinero war die Beobachtung eines Nukleationsereignisses in einer MD-Simulation. Dieses Kriterium besitzt einen hohen Realitätsbezug, krankt aber ebenfalls daran, dass zur Umsetzung das vereinfachte mW-Wassermodell 279. zum Einsatz kam, das nur kurzreichweitige Wechselwirkungen berücksichtigt. Die generel- 
le Schwierigkeit bei der Behandlung von Wasser in MD-Simulationen, die sichere Erfüllung der Ergodizitätsforderung in diesem hochkomplexen Phasenraum, stellt für Theoretiker eine große Herausforderung dar. Wenngleich die Beobachtung eines Nukleationsereignisses unter Wassermolekülen vor diesem Hintergrund als großer Erfolg zu werten ist, kann aus diesem Kriterium ebenfalls nur ein oberer Grenzwert abgeleitet werden, da die Nukleation mit abnehmender Clustergröße unwahrscheinlicher wird und deren Ausschluss einen theoretisch unendlich langen Beobachtungszeitraum erfordert 906775.

Das von Bandow und Hartke angewandte Kriterium besteht im Vergleich der Energiegehalte amorpher und kristalliner Strukturen gleicher Konstituentenzahl. Dieses hat wie das Kriterium von Kazimirski und Buch den Nachteil, dass es nicht das Vorliegen einer Trajektorie aufzeigt, entlang der eine Phasenänderung stattfinden kann 70. Die Autoren führen aus, dass es trotz intensiver Anstrengungen nicht gelungen ist, ein Nukleationsereignis in Aggregaten zu beobachten, in denen die Kristallisation aus energetischer Sicht möglich wäre. Die Tatsache, dass es in numerischen Simulationen nicht gelungen ist, eine Trajektorie aufzufinden, die zu einer Phasenänderung führt, schließt deren Existenz jedoch nicht aus (siehe oben). Weiterhin führen die Autoren an, dass die von ihnen berechneten Energiegehalte absolute Obergrenzen darstellen und die reale innere Energie der Cluster mit hoher Wahrscheinlichkeit darunter liegt. Der Bereich um $N \approx 90$ stellt nach Bandow und Hartke den Bereich dar, in dem amorphe und kristalline Strukturen annähernd isoenergetisch werden. Es ist daher unwahrscheinlich, dass in Partikeln dieser Größe eine hohe thermodynamische Triebkraft vorliegt, die die Kristallisation begünstigt 70 . Berechnungen der freien Energien amorpher und kristallartiger Aggregate auf Basis der Normalschwingungen ergaben im Größenbereich $90 \lesssim N \lesssim 150$ sehr kleine Unterschiede zwischen Aggregaten mit und ohne kristallartigen $\operatorname{Kern}\left(\Delta F \leq 0,5 \mathrm{~kJ} \mathrm{~mol}^{-1}\right.$ pro Molekül ${ }^{274}$.

Die Abnahme der thermodynamischen Triebkraft hat Auswirkungen auf die Größe des kritischen Nukleus $N^{*}$ : Da ein kleiner kristallartiger Nukleus eine höhere Grenzflächenenergie besitzt, müssen im Bild der klassischen Nukleationstheorie entsprechend mehr Monomereinheiten zu einer solchen Struktur zusammenkommen, bevor diese die kritische Größe überschreitet und ein weiteres Wachstum thermodynamisch begünstigt ist (vgl. Abschnitt 2.3.3). Bei der Beschreibung der kleinsten Cluster wird dieses klassische Bild aber zunehmend nutzlos, da insbesondere die größenabhängige Entwicklung der Grenzflächenenergie schwer zu berechnen ist 64|108. Theoretische Vorhersagen von Buch et al. legen nahe, dass die kleinsten kristallartigen Strukturen zunehmende Abweichungen von der optimalen, tetraedrischen Koordinationsgeometrie aufweisen 62 [79. Daher liegt die Vermutung nahe, dass die Grenzflächenenergie zwischen solchen Aggregaten und der diese umgebenden, amorphen bzw. flüssigen Phase hier niedriger ausfällt, als in der kondensierten Phase.

Dies dürfte insbesondere im Temperaturbereich knapp oberhalb der (vom LaplaceDruck abhängigen) homogenen Nukleationstemperatur der Fall sein, da hier laut Moore et al. eine weitere strukturelle Annäherung der Flüssigkeit an den Kristall stattfindet. MD-Simulationen auf Basis des mW-Wassermodells ergaben hier Hinweise auf sehr niedrige Energiewerte für die Grenzflächen zwischen Bereichen mit kristallartiger Struktur und amorphen Bereichen bei $T \gtrsim T_{\mathrm{H}}$. Die in diesen Simulationen auftretenden kritischen Nuclei weisen stark unterschiedliche Gerade der Kompaktheit auf. Dies lässt vermuten, dass die Strukturen flüssigen und kristallinen Wassers sich nahe $T_{\mathrm{H}}$ soweit annähern, dass die Grenzflächenspannung nahezu verschwindet 64 . 
Die Energiebarriere, die zur Bildung eines kritischen Nukleus überwunden werden muss, erwächst aus der Summe der Grenzflächenenergien, die einerseits zwischen kristallinem Kern und amorpher Oberfläche und andererseits zwischen der Oberfläche und der Gasphase ausgebildet werden. Die Spaltung der schwächeren H-Brücken an der Oberfläche ermöglicht eine geringere Schichtdicke der amorphen Oberfläche. Während Buch et al. eine Schichtdicke von ca. $5 \AA$ annahmen, die ca. einer Doppellage von Wassermolekülen entspricht 62. weisen neuere experimentelle und theoretische Studien auf Werte zwischen $2,6 \AA 280$ und 2,8 $\AA$ 68 hin. Diese niedrigeren Werte entsprechen ca. einer Monolage aus Wassermolekülen, wodurch sich der Anteil der ungeordneten Oberfläche auf den minimal möglichen Wert reduziert. Daher folgt aus dieser Betrachtungsweise, dass diese Strukturänderung der flüssigen Phase sowie der Spannungsabbau an der Grenzfläche zur Gasphase die homogene Nukleation in derart kleinen Partikeln erst ermöglichen. Die akkurate Vorhersage der Dicke der amorphen Oberfläche ist allerdings maßgeblich für die korrekte Vorhersage der kritischen Clustergröße. Nach einer Überschlagsrechnung unter Annahme eines molekularen Volumens von $3,35 \cdot 10^{-2} \mathrm{~nm}^{3}[2]$ stehen in einem Cluster mit $N=90$, in dem die Ausbildung eines kristallartigen Kerns nach Bandow und Hartke energetisch möglich ist 70, bei einer Schichtdicke von 2,6 , nur ca. 30 bis 40 Wassermoleküle im Clusterkern für Ausbildung einer kristallartigen Struktur zur Verfügung. Diese Größe liegt jedoch unterhalb der von Moore et al. auf Basis des mW-Modells vorhergesagten Größe kritischer Nuclei von $N_{\text {krit }}=90[64$ bei $T \approx 200 \mathrm{~K}$ sowie des von Huang und Bartell durch eine Abschätzung auf Basis der klassischen Nukleationstheorie gefundenen Größenbereiches von $N_{\text {krit }}=52-65128$.

Wie in Abschnitt 2.3.3 dargelegt, erfolgt die Bildung eines kristallinen Partikels im fraglichen Temperaturbereich $(T \approx 150-200 \mathrm{~K})$ grundsätzlich durch Nukleation eines unterkühlten, flüssigkeitsartigen Clusters $75|78| 128$. Nach den Gesetzen der Thermodynamik bedingt ein solcher, mit endlicher Geschwindigkeit stattfindender und nicht vollständig ablaufender Übergang zwischen zwei Zuständen mit endlicher Differenz der freien Energien zwangsläufig ebenfalls den Ablauf des gegenläufigen Übergangs (siehe Abschnitt 2.2.2): Es stellt sich ein Gleichgewicht zwischen den beiden phasenartigen Zuständen ein.

Ein kritischer Cluster dieser kleinen Größenordnung erfordert im Bild der klassischen Nukleationstheorie ein äußerst niedriges Verhältnis von Oberflächenenergie und freier Phasenübergangsenthalpie, um thermodynamisch stabil zu sein. Da diese Größen nicht mit hinreichender Genauigkeit bekannt sind bzw. in diesem Größenbereich ihre physikalische Bedeutung verlieren, kann keine Aussage über die thermodynamische Stabilität eines derart kleinen Eiskristalls gemacht werden.

Die experimentellen Resultate legen jedoch nahe, dass die Bildung kristallartiger Cluster auch in Abwesenheit einer starken thermodynamischen Triebkraft stattfindet, sodass das Gleichgewicht dieses Prozesses in den kleinsten Clustern, in denen er Auftritt, bei weitem nicht vollständig auf Seiten der kristallartigen Struktur liegt. Trotz der geringen Energieunterschiede zwischen amorphen und kristallartigen Clustern im Größenbereich $90 \lesssim N \lesssim 150$ belegen die in dieser Arbeit vorgestellten experimentellen Ergebnisse das Vorliegen kristalliner Strukturen in Nanopartikeln dieser Größe auf Basis zweier unabhängiger Kriterien. Die IR-Spektren der Größenscheiben, in denen kristallartige Strukturen nachweisbar sind, zeigen allerdings gleichzeitig hohe Intensität um $\tilde{\nu}_{\mathrm{IR}}=3400 \mathrm{~cm}^{-1}$ und somit deutliche Anzeichen für das simultane Vorliegen amorpher Cluster gleicher Größe neben solchen Partikeln, in denen eine Nukleation stattgefunden hat. Die Gewichtung 
des Anteils amorpher Strukturen, die zu einer bestmöglichen Übereinstimmung zwischen Theorie und Experiment führt, nimmt mit zunehmender Größe ab, sodass sich das Verhältnis beim Übergang vom Größenbereich um $N=90$ zum Bereich um $N=159$ annähernd umkehrt.

Diese Befunde lassen es plausibel erscheinen, dass trotz der vergleichsweise geringen thermodynamischen Triebkraft im Größenbereich $N \lesssim 150$ Phasenänderungen auftreten können. Weiterhin erscheint es plausibel, dass die im fraglichen Temperaturbereich vorhandene Nukleationsbarriere nicht in allen Aggregaten überwunden wird. Vor diesem theoretischen Hintergrund erscheint es angemessen, die im Experiment erhaltenen Spektren, die sowohl Banden amorpher als auch kristallartiger Isomere enthalten, einer Mischung aus beiden Strukturen zuzuordnen, wobei letztere mit zunehmender Partikelgröße die Oberhand gewinnen.

Die Frage, ob sich dieses Gleichgewicht im gleichzeitigen Vorliegen kristallartiger und amorpher Bereiche in ein und demselben Partikel oder in Form zeitlicher Oszillationen der Clusterstruktur äußert, lässt sich nur entscheiden, sofern eine verlässliche Aussage über das Vorliegen einer Barriere möglich ist, die im Zuge der Phasenumwandlung überwunden werden muss. Eine Barriere in Form eines thermodynamisch ungünstigen Übergangszustands kann aus dem Energiebeitrag der Grenzfläche zwischen diesen beiden Bereichen erwachsen: Sofern die Grenzflächenspannung einen endlichen Wert besitzt, steigt deren Energiebedarf mit der Oberfläche der kristallartigen Phase an und es entsteht eine Barriere endlicher Höhe, die die beiden möglichen Phasenzustände (vollständig amorph bzw. überwiegend kristallartig) voneinander trennt und in einem bestimmten Zeitintervall mit einer endlichen Wahrscheinlichkeit überwunden wird. Auch der in Abschnitt 2.2.2 erwähnte „Flaschenhals“ zwischen den Phasenräumen des flüssigkeitsartigen und des feststoffartigen Zustands kann eine solche Barriere darstellen. Ein zeitlich oszillierender Wechsel zwischen Phasenzuständen wurde in MD-Simulationen von Wasserclustern mit $N=137[68$ sowie von einer räumlich begrenzten Doppelschicht aus Wassermolekülen ${ }^{121}$ beobachtet.

Der direkte experimentelle Nachweis zeitlich oszillierender Phasenzustände erweist sich als schwierig, da ein solches Experiment erstens die gleichzeitige Kontrolle der Größe und der Temperatur der beobachteten Aggregate erfordert ${ }^{164}$, zweitens beide Phasen in einem Temperaturbereich oberhalb von $T \approx 150 \mathrm{~K}$ metastabil gegenüber der Gasphase werden können $129[170$ und drittens die Struktur der Aggregate beprobt werden müsste, ohne deren Temperatur zu verändern. Die hier vorgestellten Ergebnisse weisen jedoch deutlich darauf hin, dass der Phasenübergang zwischen flüssigem Wasser und Eis in ausreichend kleinen Aggregaten seinen singulären Charakter verliert. 


\section{Zusammenfassung}

Die vorliegende Arbeit hatte zum Ziel, mittels der infrarotanregungsmodulierten Photoionisationsspektroskopie die Bande der freien OH-Streckschwingung natriumdotierter Wassercluster mit bisher nicht erreichter Genauigkeit zu vermessen. Weiterhin sollte deren Verhalten in Abhängigkeit vom Phasenzustand dieser Cluster untersucht werden. Hierzu erwies es sich als notwendig, zunächst einige Verbesserungen an der vorhandenen Molekularstrahlapparatur vorzunehmen:

Im ersten Schritt wurde ein neues UV-Lasersystem in den Aufbau integriert, da das zuvor eingesetzte Gerät aufgrund seines Alters zahlreiche Defekte aufwies. Zur Verbesserung des Strahlprofils war die Konstruktion eines Relay Imaging-Teleskops notwendig. Der Bau erfolgte in Zusammenarbeit mit den institutseigenen Werkstätten im Bereich der Glastechnik sowie der Feinmechanik.

Im zweiten Schritt wurde ein neuer Messmodus implementiert, der die simultane Aufnahme von Signal- und Hintergrundspektren ermöglichte. Hierzu wurde die elektronische Steuerung neu aufgebaut: In Kooperation mit der institutseigenen Elektronikwerkstatt wurden logische Schaltungen entworfen, um die elektronischen Steuerungssignale des Delaygenerators in erforderlicher Weise zu modifizieren, zu verzögern und an die verschiedene Komponenten des Aufbaus bis hin zur Zählerkarte weiterzuleiten. Eine Evaluierung des neuen Messmodus ergab eine erhebliche Verbesserung der Datenqualität 237. Die geschickte Integration des Delaygenerators ermöglicht seither die bequeme Variation der Verzögerungszeit zwischen IR- und UV-Laserpuls. Die nunmehr mögliche Zeitauflösung erweiterte die erzeugbaren Messdaten um eine weitere Dimension, deren Einfluss in parallel stattfindenden Projekten ausführlich untersucht wurde. Aus der Betrachtung der Zeitabhängigkeit der Signalverstärkung ergaben sich entscheidende Hinweise für das Verständnis der im Experiment ablaufenden Vorgänge 61|238|239.

Die veränderte Datenstruktur erforderte die Entwicklung einer neuen Auswertungssoftware. Diese wurde in der modernen und quelloffenen Skriptsprache Python 3 umgesetzt. Sie ermöglicht eine zeitnahe Vorauswertung der erhobenen Daten zur Kontrolle auch während der Aufnahme einer Messreihe und kann zudem leicht an zukünftig auftretende Fragestellungen angepasst werden.

Im dritten Schritt wurde eine Automatisierung des Messmodus erreicht. Hierzu wurden die Steuerungsrechner über ein lokales Netzwerk verbunden. Eine Software auf Basis der Programmiersprache $\mathrm{C}++$ wurde entwickelt, die die Steuerung des IR-Lasersystems sowie der Software zur Datenerhebung übernimmt. Dies ermöglichte eine Aufnahme größerer Datenmengen in der gleichen Zeit sowie eine insgesamt verlängerte Betriebszeit, da die Versuchsapparatur nunmehr autark arbeitet und lediglich überwacht werden muss. Hierdurch wird die automatisierte, vielfache Aufnahme von Messwerten mit anschließender statistischer Datenauswertung ermöglicht. Statistische Fehler können nunmehr in dem Maße reduziert werden, in dem die Fragestellung es erfordert. Dies war zuvor durch den hohen personellen Aufwand während der Messung nicht möglich. 
Die hier vorgestellten apparativen Verbesserungen änderten zwar nicht das Funktionsprinzip der Apparatur, waren aber Voraussetzung für die experimentelle Bearbeitung der in der vorliegenden Arbeit behandelten Fragestellungen:

Es wurde erstmals anhand zweier komplementärer Indikatoren nachgewiesen, dass bereits weniger als 100 Wassermoleküle ausreichen, um einen Cluster mit kristallartigem Kern zu bilden. Als Indikator kam zunächst das Verhältnis der IR-Banden der Wassermoleküle in kristallartiger und amorpher Umgebung bei $\tilde{\nu}_{\mathrm{IR}}=3180 \mathrm{~cm}^{-1}$ und bei $\tilde{\nu}_{\text {IR }}=3420 \mathrm{~cm}^{-1}$ zum Einsatz. Hierfür wurden erstmals die IR-Spektren mutmaßlich kristallartiger Cluster direkt mit denen amorpher Cluster gleicher Größe verglichen.

Als weiterer Indikator konnte die Bande der freien OH-Streckschwingung natriumdotierter Cluster herangezogen werden. Diese Bande wurde unter verschiedenen experimentellen Bedingungen mit zuvor in neutralen Clustern nicht erreichter Präzision vermessen. In Kooperation mit den Forschungsgruppen von Valeria Molinero und Francesco Paesani gelang der Nachweis eines subtilen Mikroeffektes, der im Zuge der Phasenänderung auftritt: Das energetische Gleichgewicht zwischen den Wasserstoffbrückenbindungen einerseits im Inneren und andererseits an der Oberfläche lokalisierter Wassermoleküle verschiebt sich während der Kristallisation zugunsten ersterer, sodass schwächere, an der Oberfläche gelegene Bindungen aufbrechen und die Anzahl ungebundener OH-Oszillatoren erhöht wird. Die Folge ist eine Zunahme der relativen Bandenintensität der freien OH-Streckschwingung um $\tilde{\nu}_{\mathrm{IR}}=3700 \mathrm{~cm}^{-1}$. Es zeigte sich, dass dieser Effekt ebenfalls im Größenbereich zwischen 90 und 100 Wassermolekülen auftritt.

Die Kombination dieser komplementären experimentellen Befunde mit den theoretischen Vorhersagen der Forschungsgruppen von Valeria Molinero und Francesco Paesani ergibt ein konsistentes Bild, das es gestattet, zwischen den in der Vergangenheit publizierten Vorhersagen über die Größe der kleinstmöglichen kristallartigen Eisstrukturen zu entscheiden. Diese Frage ist von hoher Relevanz für Forschungen auf dem Gebiet der Atmosphärenchemie.

Die akkurate Modellierung des oben beschriebenen Effektes stellt ebenso wie die korrekte Vorhersage der kritischen Clustergröße einen Prüfstein für die mathematische Beschreibung der Wechselwirkungen zwischen Wassermolekülen dar, der theoretisch arbeitenden Forschungsgruppen einen besseren Feinabgleich der von ihnen entwickelten insilico-Modelle ermöglichen kann. 


\section{Ausblick}

Zur weiteren Verbesserung der Apparatur ist für die Zukunft der Einbau eines Strahlstabilisierungssystems für den IR-Laserstrahl geplant, da die beobachtete Signalzunahme empfindlich auf geringe Positionsänderungen der Laserstrahlen reagiert. Von dieser Maßnahme kann auch eine Erweiterung des vollautomatischen Messbetriebes erhofft werden, da der frequenzabhängige Strahlversatz bei großen Änderungen der IR-Wellenzahl für diesen Betriebsmodus derzeit den limitierenden Faktor darstellt. Weiterhin ist die Implementierung einer neuen Steuerungselektronik für das OPO/OPA-System geplant, die den Steuerungsrechner ersetzen soll. Diese Maßnahme dient der Systemstabilität und der Zukunftssicherheit, da das bisher verwendete Rechnersystem sich als anfällig erwiesen hat.

Die Gesamtschau der in den letzten Jahren vorgenommenen Forschungsarbeiten legt nahe, dass eine optimale Relaxation von Clustern unter maximal verminderter Kühlrate in dem Bereich geschieht, in dem die Konstituenten ihre Mobilität verlieren. Gleichzeitig könnte eine möglichst niedrige Endtemperatur die eventuell durch Natriumdotierung induzierte Evaporation einiger Konstituenten minimieren. Die Konstruktion einer längeren Düse mit kleinerem Öffnungswinkel hätte eine bessere Relaxation durch eine niedrigere Kühlrate sowie eine stärkere Abnahme der Endtemperatur durch eine längere Verweilzeit zur Folge und sollte in Erwägung gezogen werden, wenngleich die Herstellung eines solchen Werkstückes eine Herausforderung darstellt. Weiterhin ist durch die stärkere Kollimation der Expansion eine höhere Teilchendichte in deren Zentralachse bei gleichbleibendem Gasdurchsatz zu erwarten. Die Reduktion der Anzahl unterschiedlich gut relaxierter Isomere im Molekularstrahl ermöglicht eine bessere Strukturaufklärung im Bereich kleinerer Cluster. Auch hier ergeben sich interessante Implikationen für die Zusammenarbeit mit theoretisch arbeitenden Forschungsgruppen.

In der Literatur finden sich zahlreiche Hinweise darauf, dass die Lebensdauer eines Clusters durch evaporative Kühlung erhöht wird. Dies gilt insbesondere für Strukturen mit hoher thermodynamischer Stabilität, die sich durch eine hohe Energiebarriere bezogen auf die Dissoziation eines Monomers auszeichnen 189 . Mit der Variation der Delayzeit steht nun ein Werkzeug zur Verfügung, das geeignet ist, die stabilsten Isomere zu selektieren. Durch Aufnahme von Schwingungsspektren mit variierender Delayzeit und Subtraktion der Spektren der stabilsten Isomere könnten sukzessive die Spektren von weniger stabilen Strukturen erhalten werden.

Auch bei langen, positiven Delayzeiten von z. B. $150 \mathrm{~ns}$ (d. h. sofern die Photoionisation nach der IR-Anregung erfolgte) wird, anders als in den mit umgekehrter Pulsfolge vorgenommenen IRMPD-Messungen, am oberen Ende der Größenverteilung kein Abfall der Zählrate im Massenspektrum nach kombinierter IR- und UV-Anregung unter die Zählrate nach alleiniger UV-Anregung beobachtet. In bisherigen delayzeitabhängigen Messungen der Signalzunahme wurde lediglich die Anzahl ionisierbarer Cluster unterhalb einer bestimmten Ionisationsenergie untersucht. Sofern der auf einer Nanosekundenzeitskala beobachtete exponentielle Abfall der Signalzunahme nicht allein im Zerfall der Cluster, 
sondern in einer IP-Erhöhung über die Energie der UV-Photonen begründet ist, könnte sich durch Einbezug der Photonenenergie als Parameter die IP-Zunahme der Cluster als Funktion der Zeit auflösen lassen.

Die akkurate und störungsfreie Bestimmung von Partikelgrößen stellt in vielen Bereichen eine Herausforderung dar. Die Anwendungsmöglichkeiten der Natriumdotierung erschöpfen sich höchstwahrscheinlich nicht in der Charakterisierung von Clustern der bisher untersuchten Substanzen. Vielmehr könnte diese Methode auch an anderen Systemen, die für die Atmosphärenchemie sowie diverse industrielle Prozesse relevant sind, Anwendung finden. Bisher wurden nur wenige Fälle berichtet, in denen die Natriumdotierung nicht anwendbar ist. Eine weitere Auslotung der Möglichkeiten dieses schonenden und kostengünstigen Verfahrens an unterschiedlichen Substanzgruppen erscheint lohnend.

In jedem Falle ist auch in Zukunft eine enge Kooperation mit theoretisch ausgerichteten Forschungsgruppen angestrebt, da nur der kombinierte Ansatz aus Experiment und Theorie einen Erkenntnisgewinn an diesem komplexen Forschungsgegenstand ermöglicht. Als Beispiel für mögliche Erweiterungen des MB-Pol-Potentials sei die Einbeziehung des Verlustes der molekularen Integrität von Wasser in realen Elektrolytlösungen genannt 281.

Die Kombination einer akkuraten Potentialhyperfläche wie MB-Pol mit einer Methode, die in der Lage ist, zuverlässig alle Isomere einer Clustergröße aufzuspüren und mit einem Algorithmus wie dem local monomer-Verfahren, das die Vorhersage von IR-Spektren mit hoher Genauigkeit erlaubt, würde letztlich den Einsatz von deep learning-Methoden ermöglichen. In der Folge könnten Algorithmen auf Basis neuronaler Netzwerke entwickelt werden, die zur automatischen Klassifikation unter Nichtgleichgewichtsbedingungen gemessener Schwingungsspektren (z. B. in Überschallexpansionen) in der Lage sind 156 . Erste vielversprechende Arbeiten auf diesem Gebiet wurden bereits durchgeführt 282].

Neuere Forschungen ergeben interessante Parallelen zwischen den in der vorliegenden Arbeit beschriebenen Beobachtungen und Vorgängen in biologischen Systemen: Thoke et al. beobachteten eine Kopplung zwischen der glykolytischen Aktivität in mehreren Stämmen von Saccharomyces cerevisiae und dem Phasenzustand des Wassers, das in deren Cytoplasma zwischen Membranen, Proteinen und anderen Biomolekülen eingeengt ist 283. Durch Modulation des Polarisationsgrades dieser intrazellulären Wassermoleküle, die über induktive Wechselwirkungen mit verschiedenen Proteinen vermittelt wird, können diese Wassermoleküle in einen Zustand höherer Ordnung und herabgesetzter Mobilität überführt werden 284. Diese Resultate legen nahe, dass der Temperaturbereich, in dem der Phasenzustand eingeengter Wassermengen leicht zwischen einer flüssigkeitsartigen und einer feststoffartigen Form wechseln kann, unter bestimmten Bedingungen in den für physiologische Prozesse lebender Zellen höchst relevanten Temperaturbereich zwischen $25^{\circ} \mathrm{C}$ und $35^{\circ} \mathrm{C}$ fällt. Kastelowitz et al. konnten in ihren MD-Simulationen durch Modulation der Wechselwirkungen mit der Wand die Schmelztemperatur einer eingeengten Doppelschicht aus Wassermolekülen im Temperaturbereich oberhalb von $0^{\circ} \mathrm{C}$ variieren 121 . Diese konsistenten experimentellen und theoretischen Ergebnisse lassen vermuten, dass lebende Zellen die dynamischen Eigenschaften des Mediums Wasser bzw. deren Änderungen ausnutzen können, um unter anderem auf diesem Wege ihre Stoffwechselaktivität zu regulieren. 


\section{A. Experimentelle Parameter der vorgestellten Messungen}

\begin{tabular}{cccccc}
\hline DATUM & $\begin{array}{c}T_{\text {Reservoir }} / T_{\text {Düse }} \\
{\left[{ }^{\circ} \mathrm{C}\right]}\end{array}$ & $\begin{array}{c}T_{\mathrm{O}} / T_{\mathrm{U}} \\
{\left[{ }^{\circ} \mathrm{C}\right]}\end{array}$ & $\begin{array}{c}\mathrm{GAS}, p_{0}{ }^{\mathrm{a}} \\
{[\mathrm{bar}]}\end{array}$ & $\begin{array}{c}\tilde{\nu}_{\mathrm{IR}}{ }^{\mathrm{b}} \\
{\left[\mathrm{cm}^{-1}\right]}\end{array}$ & $\begin{array}{c}\Delta t \\
{[\mathrm{~ns}]}\end{array}$ \\
\hline $11.04 .17^{\mathrm{c}}$ & $60 / 70$ & $180 / 230$ & $\mathrm{Ar}, 1,05$ & $2800-3800$ & 100 \\
$12.04 .17^{\mathrm{c}}$ & $60 / 70$ & $180 / 230$ & $\mathrm{Ar}, 1,05$ & $2800-3800$ & 100 \\
$18.04 .17^{\mathrm{c}}$ & $60 / 70$ & $180 / 230$ & $\mathrm{Ar}, 1,05$ & $2800-3110^{\mathrm{d}}$ & 100 \\
$19.04 .17^{\mathrm{c}}$ & $60 / 70$ & $180 / 230$ & $\mathrm{Ar}, 1,05$ & $3120-3800^{\mathrm{d}}$ & 100 \\
01.11 .17 & $60 / 70$ & $180 / 230$ & $\mathrm{Ar}, 1,05$ & $3689-3709$ & 100 \\
02.11 .17 & $60 / 70$ & $180 / 230$ & $\mathrm{Ar}, 1,05$ & $3689-3709$ & 100 \\
03.11 .17 & $60 / 70$ & $180 / 230$ & $\mathrm{Ar}, 1,05$ & $3689-3709$ & 100 \\
07.11 .17 & $60 / 70$ & $180 / 230$ & $\mathrm{Ar}, 1,05$ & $3689-3709$ & 100 \\
08.11 .17 & $60 / 70$ & $180 / 230$ & $\mathrm{Ar}, 1,05$ & $3689-3709$ & 100 \\
30.11 .17 & $60 / 70$ & $180 / 230$ & $\mathrm{Ar}, 1,05$ & $3709-3713$ & 100 \\
13.12 .17 & $60 / 70$ & $180 / 230$ & $\mathrm{Ar}, 1,05$ & $3709-3713$ & 100 \\
10.04 .18 & $57 / 70$ & $180 / 230$ & $\mathrm{Ar}, 1,05$ & $3420 ; 3180$ & $7 ; 102$ \\
11.04 .18 & $57 / 70$ & $180 / 230$ & $\mathrm{Ar}, 1,05$ & $3420 ; 3180$ & $7 ; 102$ \\
12.04 .18 & $57 / 70$ & $180 / 230$ & $\mathrm{Ar}, 1,05$ & $3420 ; 3180$ & $7 ; 102$ \\
04.06 .18 & $120 / 130$ & $170 / 230$ & $\mathrm{He}, 5,80$ & $3420 ; 3180$ & $7 ; 102$ \\
06.06 .18 & $120 / 130$ & $170 / 230$ & $\mathrm{He}, 5,80$ & $3420 ; 3180$ & $7 ; 102$ \\
13.06 .18 & $120 / 130$ & $170 / 230$ & $\mathrm{He}, 5,80$ & $3688-3714$ & 150 \\
21.06 .18 & $120 / 130$ & $170 / 230$ & $\mathrm{He}, 5,80$ & $3688-3714$ & 150 \\
03.09 .18 & $120 / 130$ & $170 / 230$ & $\mathrm{He}, 5,80$ & $2800-3650$ & 102 \\
\hline
\end{tabular}

Tabelle A.1.: Übersicht über die im Rahmen dieser Arbeit vorgestellten Messreihen.

${ }^{a}$ Die exakten Werte von $p_{0}$ wurden auf der Grundlage von Christoph Dierking durchgeführten Kalibrierung des verwendeten Manometers nachträglich neu berechnet. Nähere Einzelheiten finden sich in dessen Dissertation 61.

b Die exakten Werte von $\tilde{\nu}_{\mathrm{IR}}$ wurden nach jeder Kalibrierung des OPO anhand der bekannten IR-Banden von HCl-Gas sowie des in der Laborluft enthaltenen Wassers von neuem bestimmt. Die Wellenzahlen wurden mit einem entsprechenden Versatz angesteuert und nachträglich korrigiert. Einzelheiten hierzu finden sich in der Masterarbeit von Daniel Becker 239.

c Von Christoph Dierking durchgeführte Messungen 61.

d Das Spektrum wurde aus technischen Gründen an zwei verschiedenen Tagen aufgenommen. Zur Sicherstellung der Konsistenz wurde der Spektralbereich $\tilde{\nu}_{\mathrm{IR}}=3060-3110 \mathrm{~cm}^{-1}$ an beiden Tagen vermessen. Die Tabelle gibt die jeweils in die Auswertung eingeflossenen Messungen an. 


\section{B. Liste verwendeter Chemikalien}

Bidestilliertes Wasser wurde unter Nutzung der hauseigenen Bidestille des IPC Göttingen mit der freundlichen Unterstützung von Herrn Michael Schlote aus demineralisiertem Wasser hergestellt. Alle weiteren verwendeten Chemikalien wurden im kommerziellen Handel bezogen und ohne weitere Aufbereitung eingesetzt.

\begin{tabular}{c|c|c|c|c}
\hline SuBSTANZ & FORMEL & CAS-NR. & REINHEITSGRAD & LIEFERANT \\
\hline Natrium & $\mathrm{Na}$ & $7440-23-5$ & $\geq 99,99 \%$ & Merck \\
Wasser & $\mathrm{H}_{2} \mathrm{O}$ & $7732-18-5$ & (bidestilliert) & IPC Göttingen \\
Helium & $\mathrm{He}$ & $7440-59-7$ & $\geq 99,996 \%$ & Linde \\
Argon & $\mathrm{Ar}$ & $7440-37-1$ & $\geq 99,998 \%$ & Air Liquide \\
Stickstoff & $\mathrm{N}_{2}$ & $7727-37-9$ & $\geq 99,999 \%$ & Air Liquide \\
1,4-Dioxan & $\mathrm{C}_{4} \mathrm{H}_{8} \mathrm{O}_{2}$ & $123-91-1$ & $\geq 99,5 \%$ & Carl Roth \\
Exalite 389 & (nicht angegeben) & (nicht angegeben) & (nicht angegeben) & Radiant Dyes \\
\hline
\end{tabular}

Tabelle B.1.: Übersicht der verwendeten Chemikalien 


\section{Schaltplan der Triggerbox}

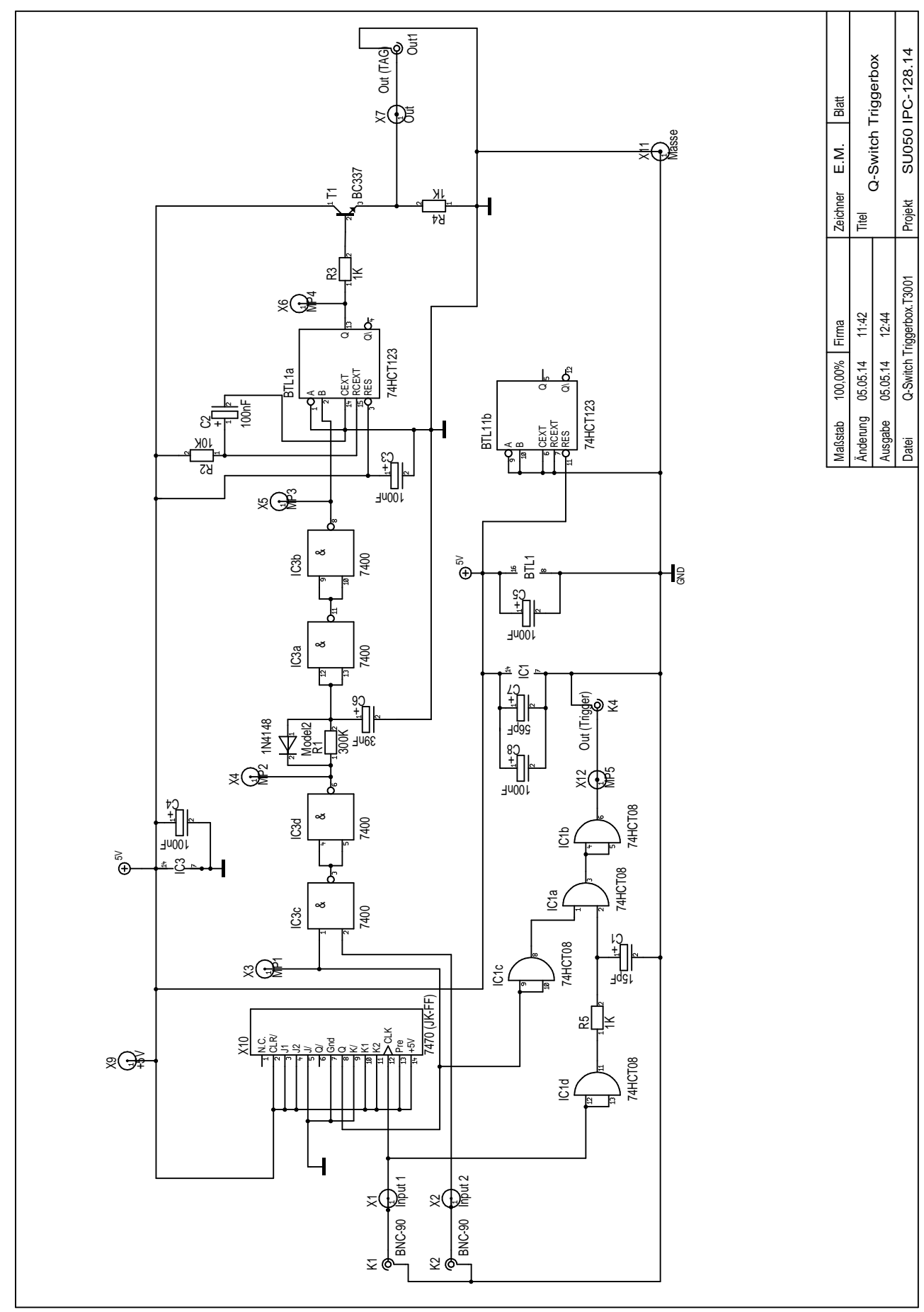




\section{Quelltext zur Automatisierung}

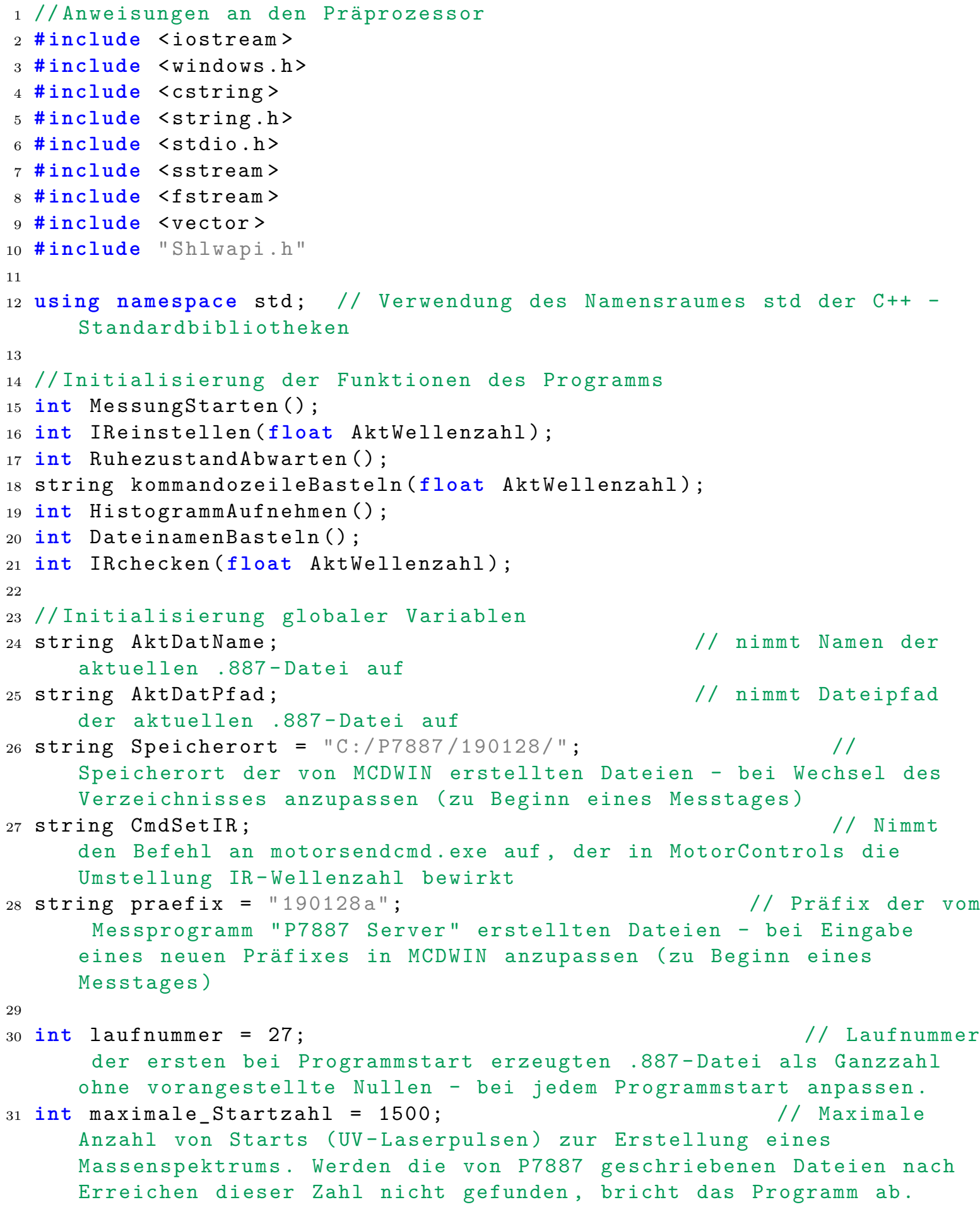


32 int GemIREnergie $=0$;

// Arithmetisches

Mittel der Energie sämtlicher während einer Messung abgegebener IR - Laserpulse in $\mathrm{m} J$

33 unsigned int index $=0$

// Indexnummer des

Elements im Array IRWellenzahl[] mit der aktuellen Wellenzahl

34 float IRWellenlaenge $=0$;

// Aus der im

gerade abgerufenen, an der Position "index" befindlichen Feld des Arrays IRWellenzahl[] abgelegten IR-Wellenzahl berechnete IR-

Wellenlänge in $\mathrm{nm}$

35 float Ermittelte_Wellenzahl = 0

// Aus der von

MotorControls abgefragten, momentan eingestellten IR-Wellenlänge berechnete IR-Wellenzahl in reziproken $\mathrm{cm}$

36 bool pausieren $=0$;

ob der Programmablauf nach der in der Variable modulozahl

festgelegten Anzahl von Messungen unter Erwartung eines

Tastendruckes unterbrochen werden soll (0: Programm pausiert, 1: Programm läuft durch)

37 int EIR_Offset $=0$;

// Ermöglicht die

Eingabe eines Offset-Wertes, falls der vom IR-Energiedetektor

übermittelte Wert bei blockiertem IR-Laser von 0 abweicht.

38 int EIR_Skalierungsfaktor $=1$;

// Faktor,

durch den die gemittelte IR-Energie geteilt wird, damit sie nicht mehr als 3 Stellen vor dem Komma besitzt, da das

Auswertungsprogramm analyse.exe von S. Wolff nicht mehr Stellen verarbeiten kann

39 int wartezeit $=170$;

$$
\text { // }
$$

Zeitintervall, das waehrend der Messung zwischen zwei Abfragen der IR-Energie vergehen soll, abzüglich der vom Programm für die Abfrage und weitere in der gleichen Schleife anfallende Aufgaben benötigt. Das Gesamtintervall soll 200 ms betragen.

40 int modulozahl = 12 ;

// Anzahl

der Messungen, nach der das Programm bei pausieren = 1 (wahr)

pausieren und auf einen Tastendruck warten soll

41

42 float IRWellenzahl[]\{

// Nimmt eine durch

Kommata getrennte Liste der Wellenzahlen auf, die zur Aufnahme eines spektrums in dieser Reihenfolge angesteuert werden sollen

3700 ,

443702 ,

453704

46 \};

47

48 //Initialisierung einer Konstanten, die die Anzahl der im Array

IRWellenzahl abgelegten Werte, d. h. der Anzahl durchzuführender Messungen enthält

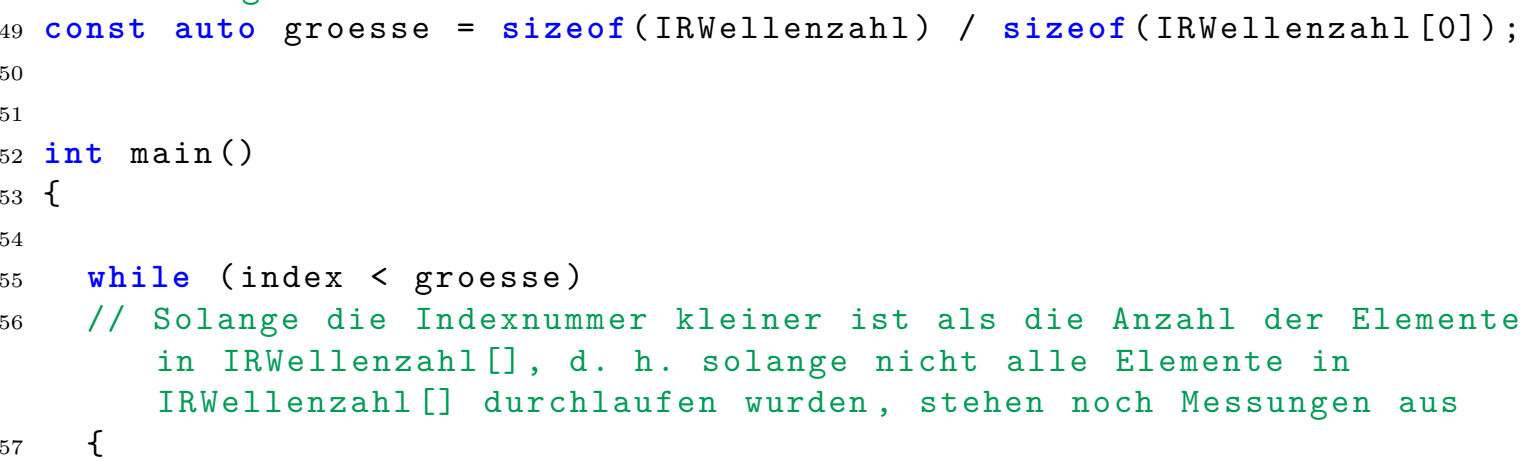


Strings, die Namen und Dateipfad der von MCDWIN nach Ende aktuellen Messung erzeugten .887-Datei enthalten, aus den Variablen Speicherort, praefix und laufnummer
MessungStarten ( ) ;

eines Massenspektrums

HistogrammAufnehmen (); erwarten, währenddessen regelmäßig IR-Pulsenergie messen und IR-Laserpulse zählen

cout $<" \backslash t "<" \backslash t "<<$ "Dateiname: " $<$ AktDatName $<" \backslash t "<<"$ IR-Wellenzahl: " $<$ Ermittelte_Wellenzahl $<$ " \t" $<$ "IREnergie: " < GemIREnergie $<$ "\a" $<$ endl; // Ausgabe von gemittelter IR-Energie und Wellenzahl nach Ende jeder Messung // Inkrementierung der Variablen laufnummer, um die Zusammensetzung des Dateinamens der nächsten .887-Datei zu ermöglichen, auf deren Erzeugung dieses Programm wartet

Ermittelte_Wellenzahl = 0 ;

\}

cout $<" \backslash a "<$ endl;

// Ausgabe eines

akustischen Signals am Ende der Messkampagne return 0 ;

$$
\text { . }
$$
(1)

// Initialisierung eines Stringstreams, Übergabe der Ganzzahl laufnummer an diesen und Umwandlung der Zahl in einen String namens DatNameEnde

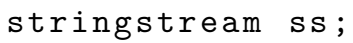

// Konkatenierung der Strings AktDatName und DatNameEnde. Abhängig von der Stellenzahl von laufnummer werden Nullen eingefügt, da laufnummer ++; 


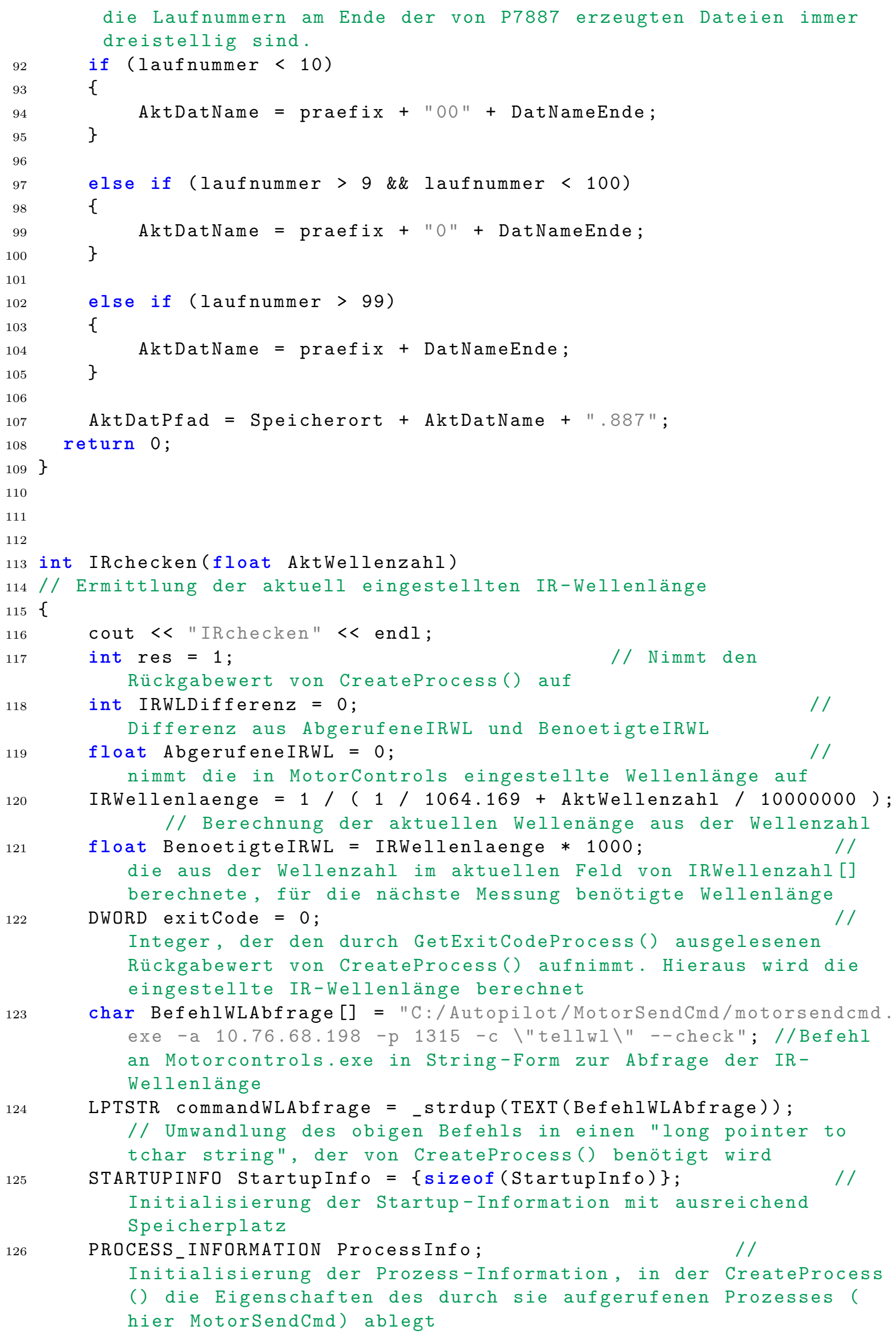


res = CreateProcess (

Aufruf von CreateProcess(). Diese fragt über MotorSendCmd.exe die in Motor Controls eingestellte Wellenlänge ab NULL , commandWLAbfrage,

// Verweis auf den NULL , Zeiger mit dem Befehlsstring zur Wellenlängenabfrage

NULL ,

TRUE ,

0 ,

NULL,

NULL ,

\& StartupInfo,

\&Processinfo // Verweis auf den

Speicherort der Prozessinformation. Hier soll die

eingestellte IR-Wellenlänge abgelegt werden.

) ;

if (!res)

// Fehlerabfang - Falls CreateProcess() fehlgeschlagen ist, beträgt ihr Rückgabewert 0 . Eine Fehlermeldung wird ausgegeben.

\section{\{}

std: cout $<<$ "Wellenlaenge konnte nicht ermittelt werden" $<<$ "\a" $<$ std: : endl;

system("pause");

\}

else

// Im Falle eines erfolgreichen Durchlaufs von CreateProcess()

\{

res = WaitForSingleObject (

Verfügbartkeit der Prozessinformation, um durch

GetExitCodeProcess abgerufen zu werden

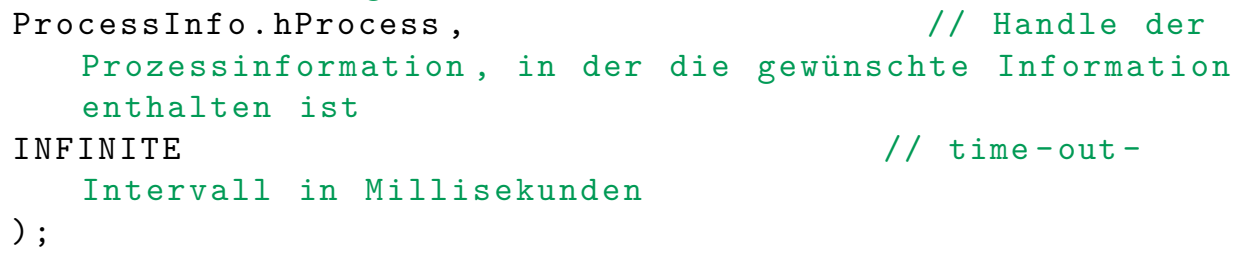

GetExitCodeProcess (ProcessInfo.hProcess, \&exitCode);

Ablegen der IR-Wellenlänge aus der von CreateProcess()

angelegten Prozessinformation über MotorSendCmd.exe in der

Variable exitCode

AbgerufeneIRWL = exitCode / 100 ;

// Umwandlung

der in exitCode abgelegten Zahlenfolge in einen Wert in der

Einheit Nanometer durch Verschieben des Dezimaltrennzeichens

IRWLDifferenz = AbgerufeneIRWL - BenoetigteIRWL; //

Berechnung der Differenz aus aktuell benötigter und

eingestellter Wellenlänge

cout $<$ "Abgerufene IR-Wellenlaenge: " $<$ AbgerufeneIRWL $<$ endl

cout $<$ "Benoetigte IR-Wellenlaenge: " $<$ BenoetigteIRWL $<$ endl ; 


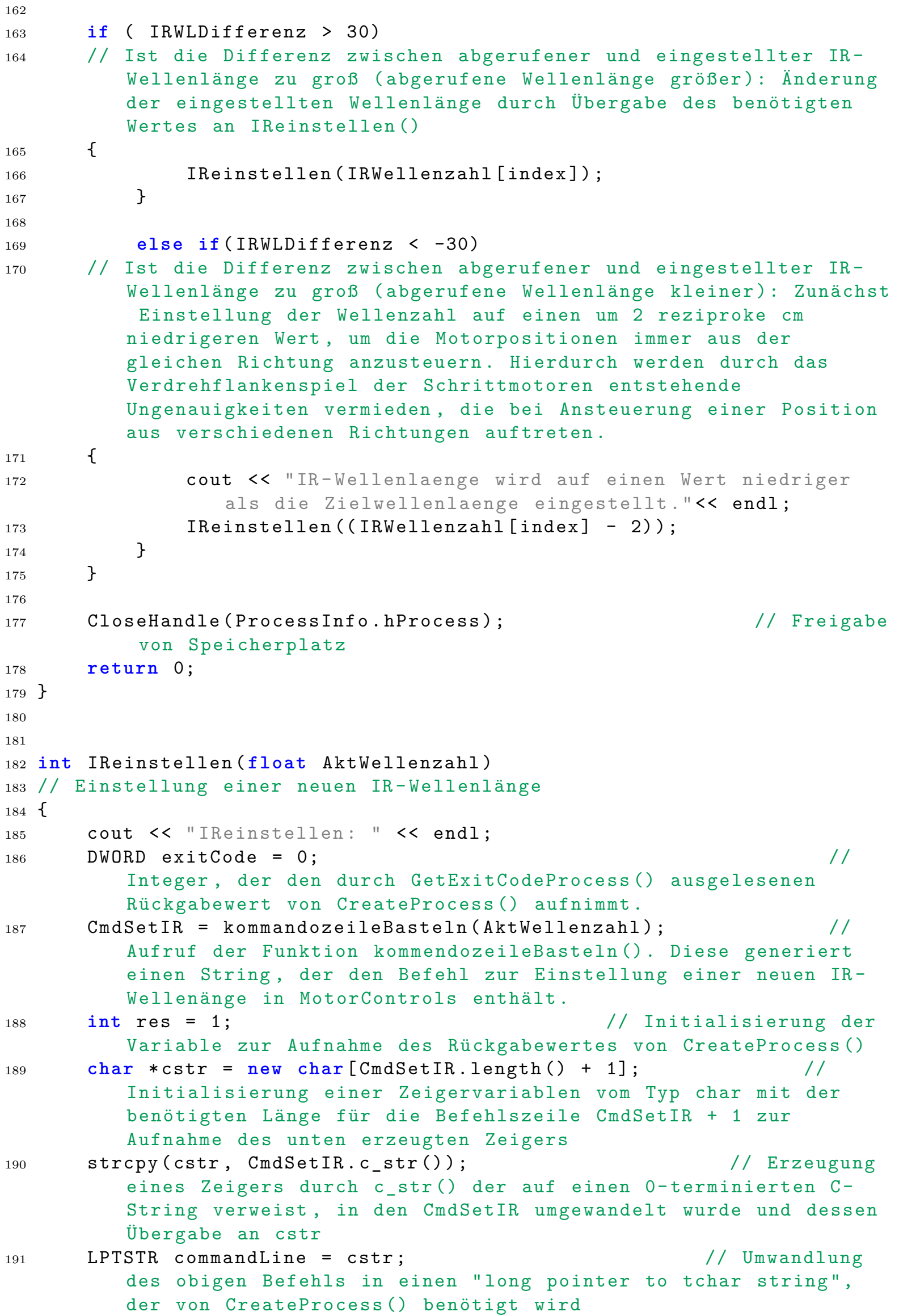


Initialisierung der Startup-Information mit ausreichend Speicherplatz

\section{PROCESS_INFORMATION ProcessInfo;}

Initialisierung der Prozess-Information, in der CreateProcess () die Eigenschaften des durch sie aufgerufenen Prozesses ( hier MotorSendCmd) ablegt

\section{res $=$ CreateProcess (}

NULL ,

commandLine,

// Verweis auf den

Zeiger, der den Befehlsstring zur Einstellung der neuen Wellenlänge enthält

NULL ,

NULL ,

TRUE ,

0 ,

NULL ,

NULL ,

\& StartupInfo,

\&Processinfo

// Verweis auf den

Speicherort der Prozessinformation. Hier soll der rückgemeldete Motorenstatus abgelegt werden.

$$
) \text {; }
$$

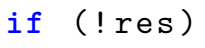

// Fehlerabfang - Falls CreateProcess() fehlgeschlagen ist, beträgt ihr Rückgabewert 0 . Eine Fehlermeldung wird ausgegeben .

\{

std: cout $<$ "Wellenlaenge konnte nicht eingestellt werden" $<" \backslash a "<$ std: : endl;

\}

system ("pause");

else

// Falls der Befehl zur Einstellung der IR-Wellenlänge übermittelt werden konnte, wird auf den Rückgabewert gewartet und dieser in der Variable exitCode abgelegt. Andernfalls könnte er in der Funktion RuzeztustandAbwarten() als Rückgabewert interpretiert werden, was zu einem vorzeitigen Start der Messung führen könnte

\{

res = WaitForSingleObject (

gartkeit der Prozessinformation, um durch

GetExitCodeProcess abgerufen zu werden

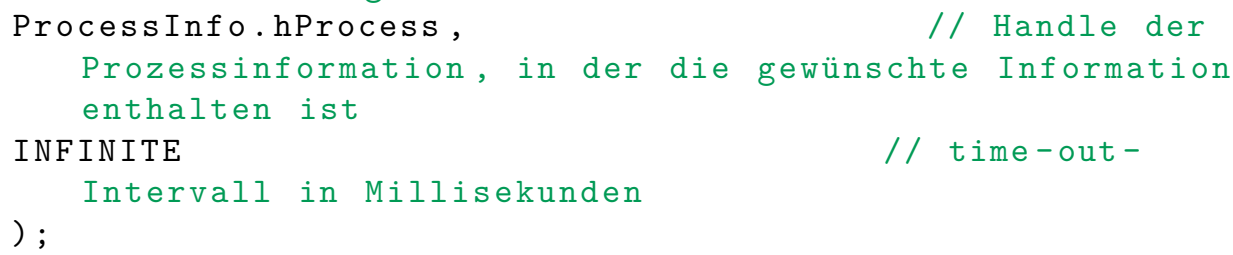

GetExitCodeProcess (ProcessInfo.hProcess, \&exitCode);

Ablegen des Rückgabewertes aus der von CreateProcess()

angelegten Prozessinformation über MotorSendCmd.exe in der Variable exitCode 


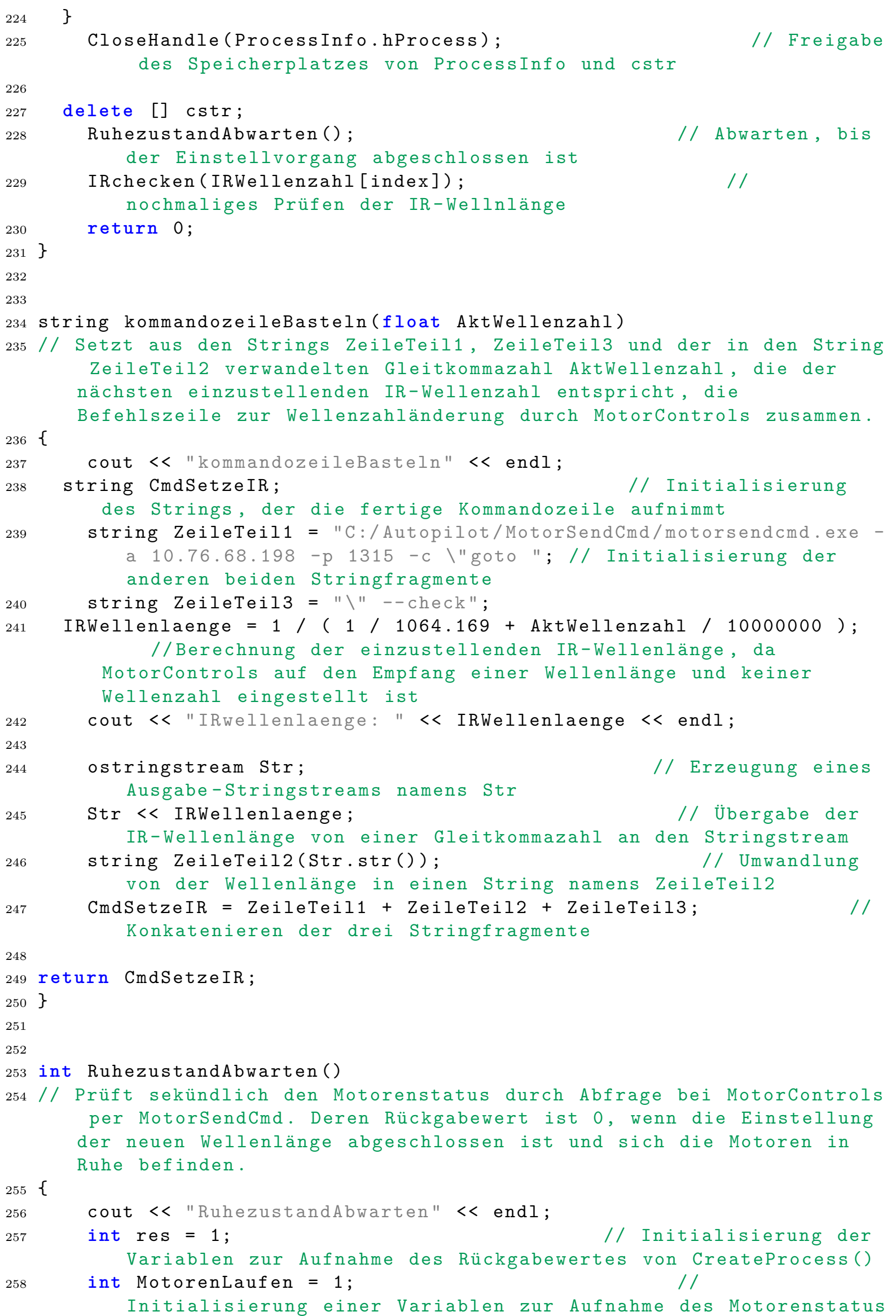


mit einer Zahl ungleich 0, um die Bedingung für die

Positionsabfrage anfänglich zu erfüllen. DWORD exitCode $=0$;

durch GetExitCodeProcess() ausgelesenen Returnwert von CreateProcess () aufnimmt. Ist dieser 0, befinden sich die Motoren des OPO/OPA-Systems in Ruhe

STARTUPINFO StartupInfo $=\{$ sizeof (StartupInfo) $\}$;

Initialisierung der Startup-Information mit ausreichend Speicherplatz

\section{PROCESS_INFORMATION Processinfo:}

\section{//}

Initialisierung der Prozess-Information, in der CreateProcess () die Eigenschaften des durch sie aufgerufenen Prozesses ( hier MotorSendCmd) ablegt

char BefehlStatAbfrage[] = "C:/Autopilot/MotorSendCmd/ motorsendcmd.exe -a 10.76.68.198 -p 1315 -c \"tellstat\" -check"; // Befehlszeile für MotorSendCmd zur Abfrage des Status

LPTSTR commandStatAbfrage = _strdup (TEXT(BefehlStatAbfrage)); // Umwandlung des obigen Befehls in einen "long pointer to tchar string", der von CreateProcess() benötigt wird

while (MotorenLaufen $\quad !=0$ )

//Solange die Motoren in Bewegung sind, wird deren Status wiederholt Abgefragt.

\{

\section{res $=$ CreateProcess (}

NULL,

commandStatAbfrage, $\quad$ // Verweis auf den Zeiger, der den Befehlsstring zur Abfrage des Motorenstatus

NULL ,

NULL ,

TRUE ,

0 ,

NULL ,

NULL ,

\&StartupInfo,

\&Processinfo

);

if (!res)

// Fehlerabfang - CreateProcess() ist fehlgeschlagen. Ihr eigener Rückgabewert ist dann 0 . Rückmeldung an den Anwender \{

std: : cout $<$ "Motorenstatus konnte nicht ermittelt werden $"<" \backslash a "<$ std: : endl;

\}

system("pause");

else

// Im Falle eines erfolgreichen Durchlaufs von CreateProcess() \{

res = WaitForSingleObject

Verfügbarkeit der Prozessinformation abwarten, um durch GetExitCodeProcess() abgerufen zu werden 


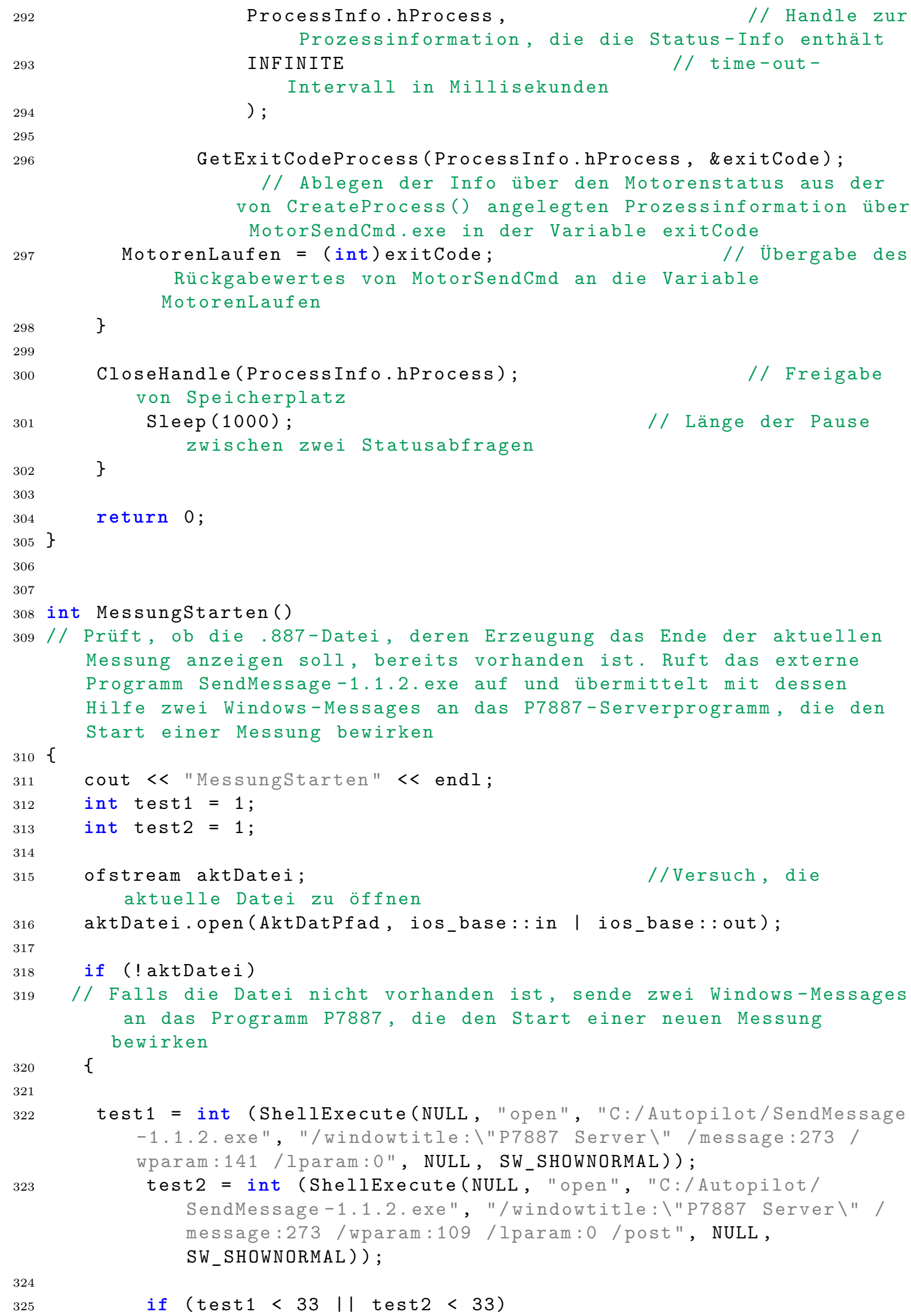

test1 = int (ShellExecute(NULL, "open", "C:/Autopilot/SendMessage -1.1.2.exe", "/windowtitle:\"P7887 Server\" /message:273 / wparam:141 /lparam:0", NULL, SW_SHOWNORMAL)) ; test2 = int (ShellExecute(NULL, "open", "C:/Autopilot/ SendMessage-1.1.2. exe", "/windowtitle: "P7887 Server\" / message:273 /wparam:109 /lparam:0 /post", NULL, SW_SHOWNORMAL ) ) ;

if (test $1<33||$ test $2<33$ ) 

nach Ende der Messung geschrieben wird, mittlerweile vorliegt. Ihr Vorhandensein dient als Signal für den Abschluss der Messung. Sofern die Datei noch nicht vorhanden ist, wird nach jeder Prüfung die zuletzt vom IR-Energiemesskopf gemessene IR-Pulsenergie abgefragt, um nach Ende der Messung den Mittelwert aller Pulsenergien zu bilden.

348\{

// Fehlerabfang: Falls eine der Windows-Messages nicht gesendet werden konnte, wird eine Fehlermeldung ausgegeben und eine Tastatureingabe abgewartet

$$
\{
$$

cout $<$ "Messung konnte nicht gestartet werden." $<$ "\a" $<<$ endl;

system ("pause");

\}

\section{else}

// Falls die Datei, deren Erzeugung nach Ende der vorgesehenen Messung erwartet wird, bereits vorhanden ist, wird eine Fehlermeldung ausgegeben und das Programm nach Tastendruck beendet.

$$
\{
$$

cout $<$ "Datei " $<$ AktDatPfad $<$ " ist bereits vorhanden!" $<<$ endl $<$ "Tastendruck beendet das Programm." $<<" \backslash a "<<$ endl;

system ( "pause");

\}

exit (0);

aktDatei.close ();

Filestreams aktDatei

// Schließung des

return 0 ;

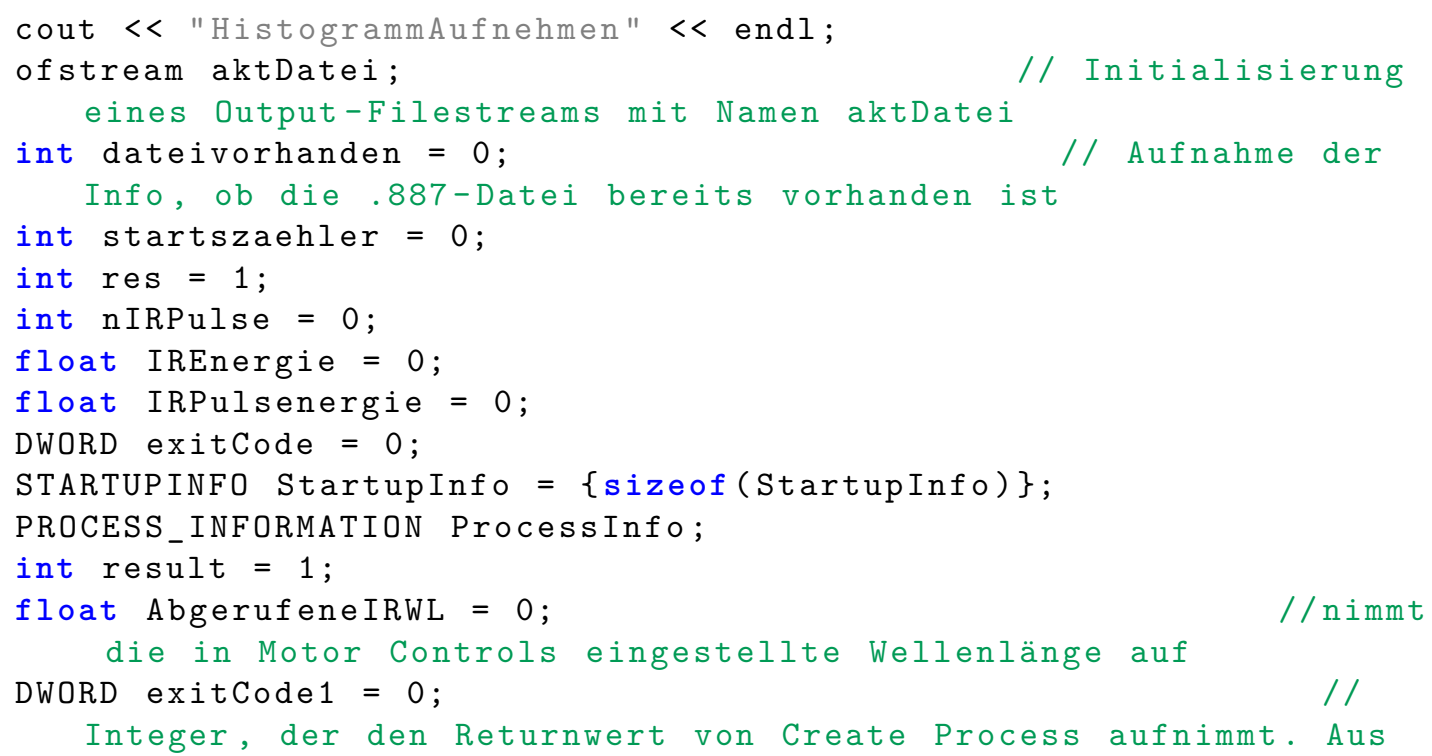


363

364

365

366

367

368

369

370

371

372

373

374

375

376

377

378

379

380

381

382

383

384

385

386

387

388

389

390

391

392

393

394

395

396

397

398

399

diesem wird die eingestellte IR-Wellenlänge berechnet char BefehlWLAbfrage [] = "C:/Autopilot/MotorSendCmd/motorsendcmd. exe -a 10.76.68.198-p 1315 - c "tellwl】" - -check"; //Befehl

an Motorcontrols.exe zur Rückgabe der IR-Wellenzahl als String LPTSTR commandWLAbfrage = _strdup (TEXT(BefehlWLAbfrage));

STARTUPINFO StartupInfo1 $=\{$ sizeof (StartupInfo) $\}$;

PROCESS_INFORMATION ProcessInfo1;

char BefehlEnergieMessen [] = "C:/Autopilot/MotorSendCmd/

motorsendcmd. exe -a 10.76.68.198 -p 1315 - c \"tellpwr】" --

check";

LPTSTR cmdEnergieMessen = _strdup(TEXT(BefehlEnergieMessen));

while ('dateivorhanden \&\& startszaehler $<=$ (maximale_Startzahl + 10) )

// Solange die erwartete .887-Datei noch nicht gefunden wurde, versuche sie zu öffnen, um zu prüfen, ob sie inzwischen geschrieben wurde. Falls sie gefunden wurde, füge die gemittelte IR-Energie und die aktuelle Wellenzahl in diese ein. Falls sie nicht vorliegt, obwohl sie längst hätte erzeugt werden sollen, gib eine Fehlermeldung aus .

aktDatei.open(AktDatPfad, ios_base::in | ios_base::out);

if (! aktDatei)

// Gelingt es nicht die die Datei zu öffnen (weil sie fehlt), frage die zuletzt vom IR-Energiemesskopf gemessene IRPulsenergie ab

\{

aktDatei.close ();

Datei zunächst wieder

// Schließe die

startszaehler = startszaehler +2 ;

/ /

Setze die Zahl der in dieser Messung durchlaufenen

Starts um 2 herauf, weil während jedes

Schleifendurchlaufs zwei Starts stattfinden

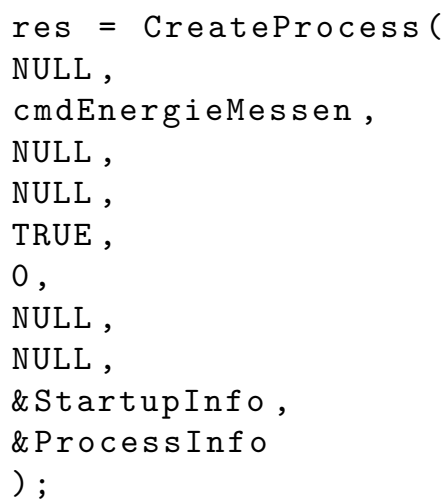




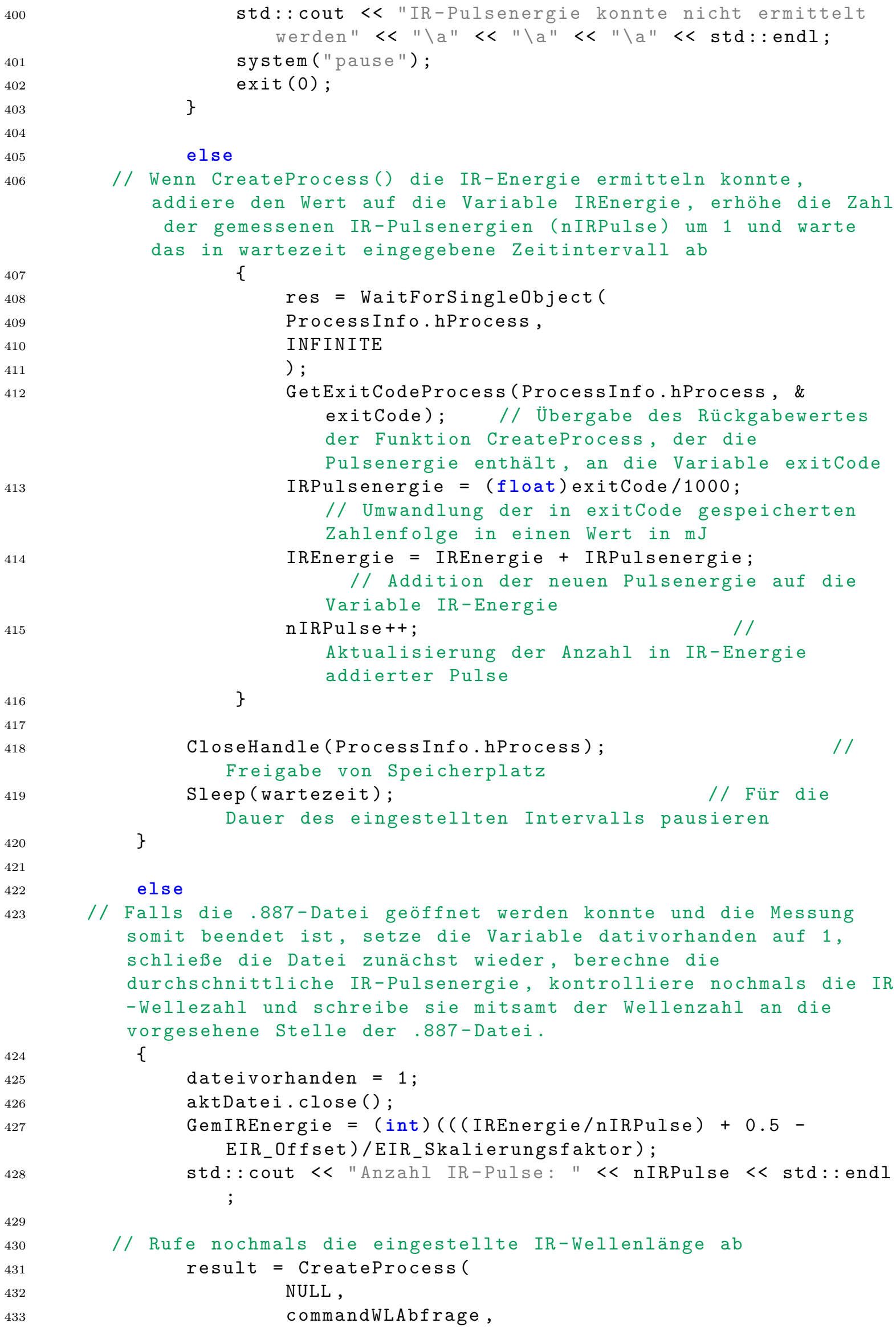




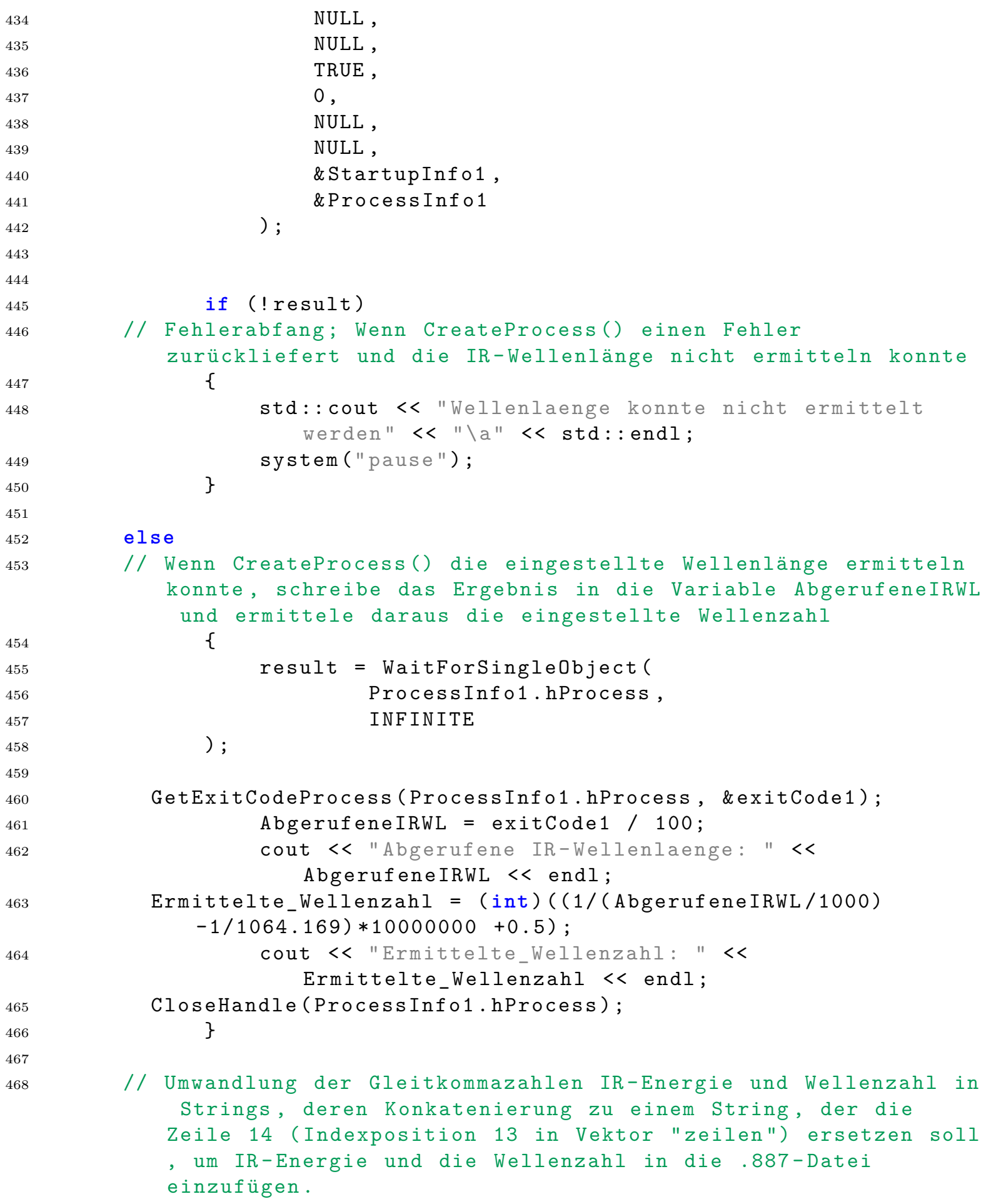

469 470

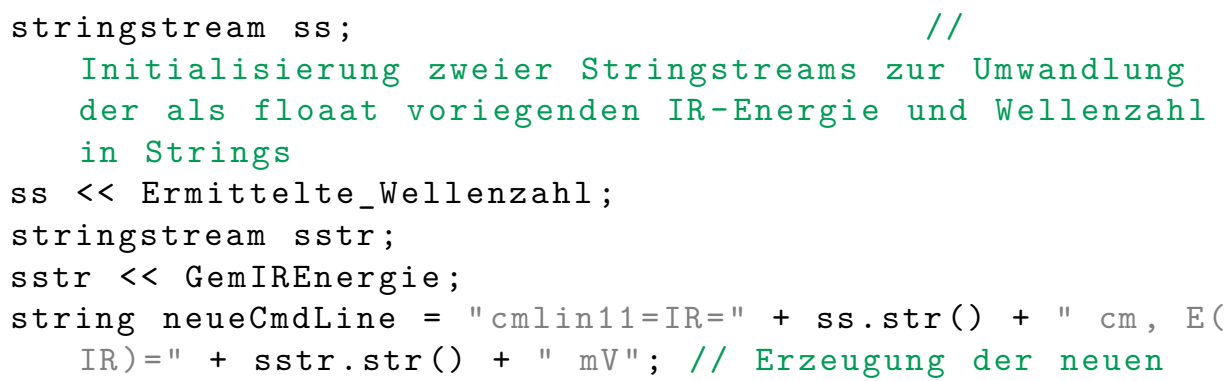


Zeile 12 der.887-Datei ifstream infile (AktDatPfad);

Erzeugung eines Input-Filestreams mit dem Dateipfad der zu modifizierenden .887-Datei

\section{vector<string> zeilen;}

\section{//}

Initialisierung eines Vektors von Strings namens " zeilen" zur zeilenweisen Aufnahme der .887-Datei string zeile; //

Initialisierung eines Strings namens "zeile" zur Aufnahme einer Zeile der .887-Datei

while (infile.good ())

// Solange die Datei Zeilen enthält, werden diese eingelesen. \{

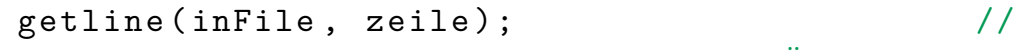

Übergabe des Inhalts von "zeile" an den Vektor "

\} zeilen"

string startzeile = zeilen[6];

Auslesen von Element Nr. 7 (Indexposition 6) des

Vektors "zeilen", Prüfung ob die Zählerkarte

Startsignale detektiert hat.

if (startzeile $==$ "STARTS: 0")

// Falls die Zählerkarte aus irgendewelchen Gründen keine Startsignale Detektiert hat, wird eine Fehlermeldung ausgegeben und die Variable index nicht inkrementiert, sodass die letzte Messung, deren IR-Wellenzahl an Position " index" im Array IRWellenzahl[] abgelegt ist, wiederholt wird

\{

\}

cout $\ll$ "Messung bei WZ " $<$ IRWellenzahl[index] $<"$ in Datei " $<$ AktDatName $<$ " bekam keine Starts und wird wiederholt." $<<$ endl;

else

\{

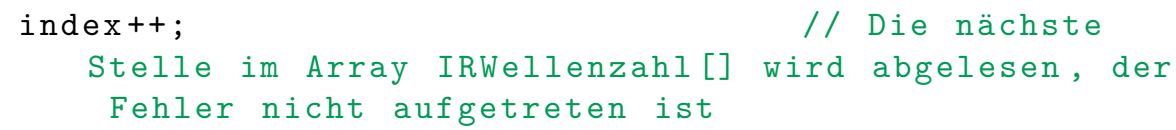


Datei.

502

503

504

505

506

507

508

509

510

511

512

513

514

515

516

517

518

$519\}$

outFile.close();

\}

if (startszaehler $>=$ (maximale_Startzahl + 10))

// Falls die maximale Startzahl erreicht ist und die .887-Datei fehlt, obwohl sie längst hätte geschrieben werden sollen, wird eine Fehlermeldung ausgegeben und das Programm nach Tastendruck beendet.

\{

cout $\ll$ "Datei " $<$ AktDatName $<$ " konnte nach Ende der Messung nicht gefunden werden." $<<" \backslash a "<$ endl;

cout $<$ "Ist der Dateipfad " $<$ AktDatPfad $<$ " korrekt?" $<$ endl;

cout $<$ "Entspricht der Dateiname " $<$ AktDatName $<$ "korrekt? $"<$ endl;

cout $\ll$ "Entspricht die Laufnummer " $<$ laufnummer $<$ " der in MCDWIN Angezeigten?" $<$ endl;

cout < "Ist der Wert 'Starts Preset' in MCDWIN kleiner als " $<$ maximale_Startzahl < "?" < endl;

exit (0) ;

system ("pause");

\}

\}

return 0 ; 


\section{E. Auswertungsskript}

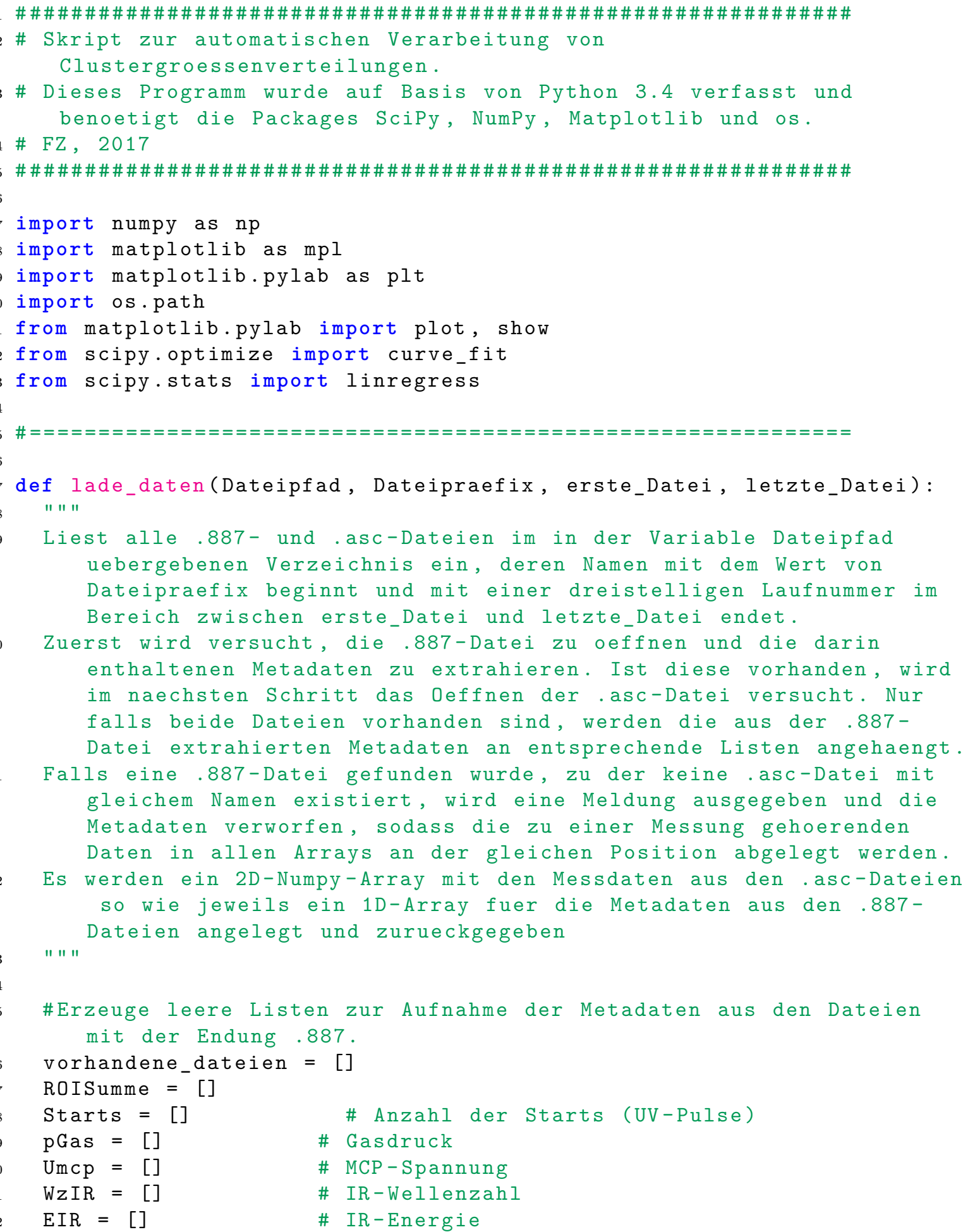




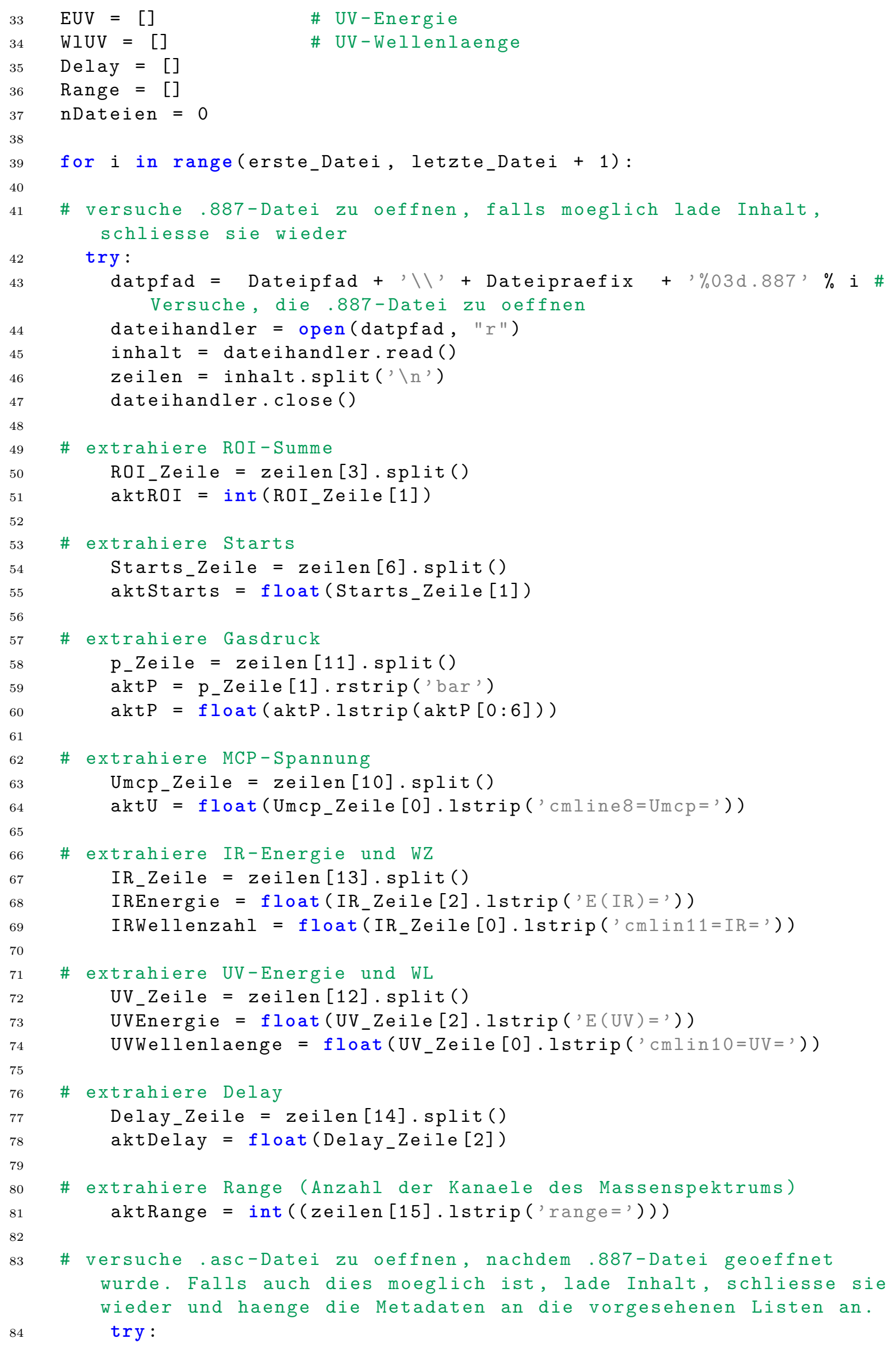




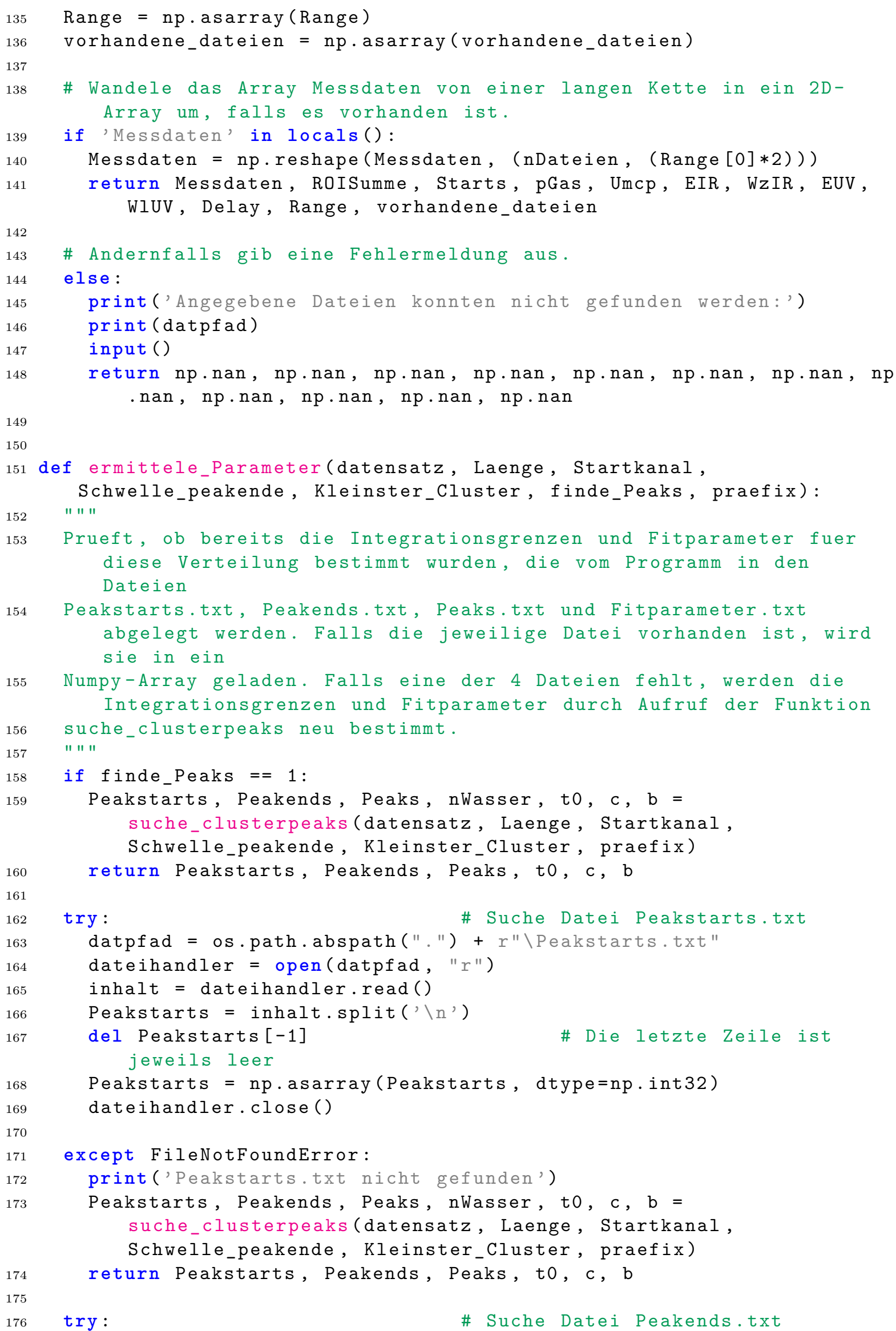




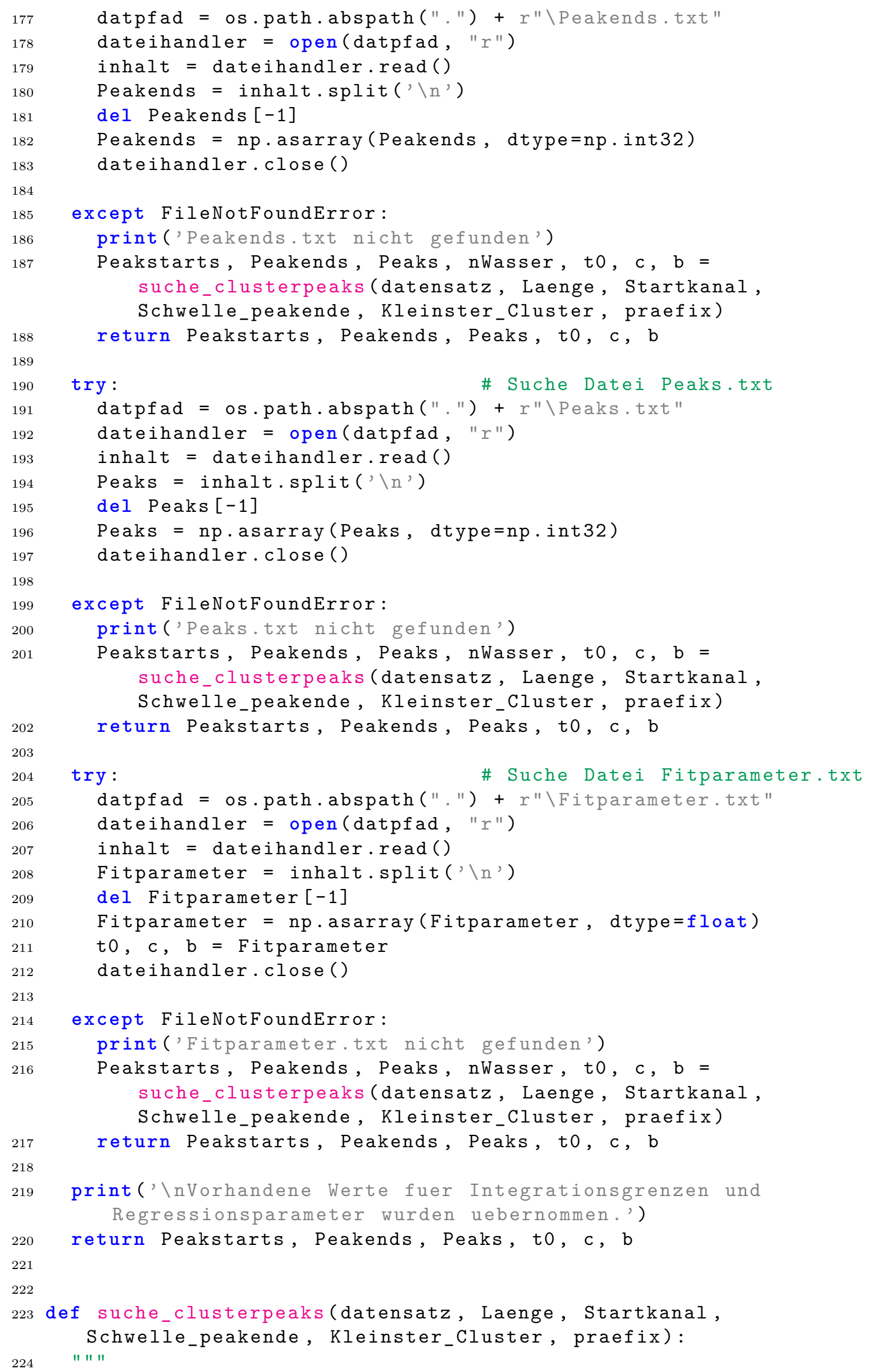


Addiert alle Massenspektren in datensatz zu einem einzigen Spektrum mit dem Namen spektrensumme, das dann zweimal einer Glaettung durch die Funktion moving_average unterzogen wird. Dieses geglaettete Spektrum wird im Array spektrenglaettung gespeichert und

von der Funktion finde maxima benutzt, um die Positionen der Peaks $\mathrm{zu}$ bestimmen.

Durch die Funktion bestimme_Fitparameter werden anschliessend die Fitparameter der Ausgleichskurve bestimmt.

Um diese Parameter sowie die Grenzen der Clusterpeaks nicht bei jedem Programmstart erneut bestimmen zu muessen, werden diese in Dateien namens

Peakstarts.txt, Peakends.txt, Peaks.txt und Fitparameter.txt gespeichert.

np. savetxt produziert Werte in wissenschaftlicher Notation mit so vielen Stellen des Wertes 0, dass diese von np.astype nicht mehr als Integer erkannt werden. Daher wurde zum Speichern die Methode des Fileobjekts gewaehlt.

Zuletzt werden zwei Auftragungen erstellt, die der Kontrolle der Auswertung dienen: Eine zeigt die addierten Massenspektren ( Array spektrensumme) zusammen mit den markierten Kanalnummern fuer Peakanfang, Peakmaximum und Peakende als schwarze, rote und gruene vertikale Linien. Die Andere stellt die Kanalnummern der gefundenen Peaks der mit Hilfe der Fitparameter berechneten Ausgleichskurve gegenueber.

print (' \nSignalmaxima und Integrationsgrenzen werden neu ermittelt. )

spektrensumme = addiere_massenspektren(datensatz, Laenge)

print ('Berechne gleitenden Mittelwert')

spektrenglaettung = moving_average(spektrensumme, 9, 'mirror')

spektrenglaettung = moving_average( spektrenglaettung, 9, 'mirror')

print ('Bestimme Peakpositionen')

Peakstarts, Peakends, Peaks, nWasser = finde_maxima( spektrenglaettung, Laenge, Startkanal, Schwelle_peakende, Kleinster_Cluster)

Fitparameter = bestimme_Fitparameter (nWasser, Peaks)

to, $c, b=$ Fitparameter

fobj = open ('Peakstarts.txt', 'w')

for wort in Peakstarts :

fobj.write (str(wort) + '\n')

fobj.close ()

$f \circ b j=$ open ('Peakends.txt', ' $w$ ')

for wort in Peakends:

fobj.write (str (wort) + '\n')

fobj.close ()

$f o b j=$ open ('Peaks.txt', 'w')

for wort in Peaks :

fobj.write (str (wort) + '\n')

fobj.close () 


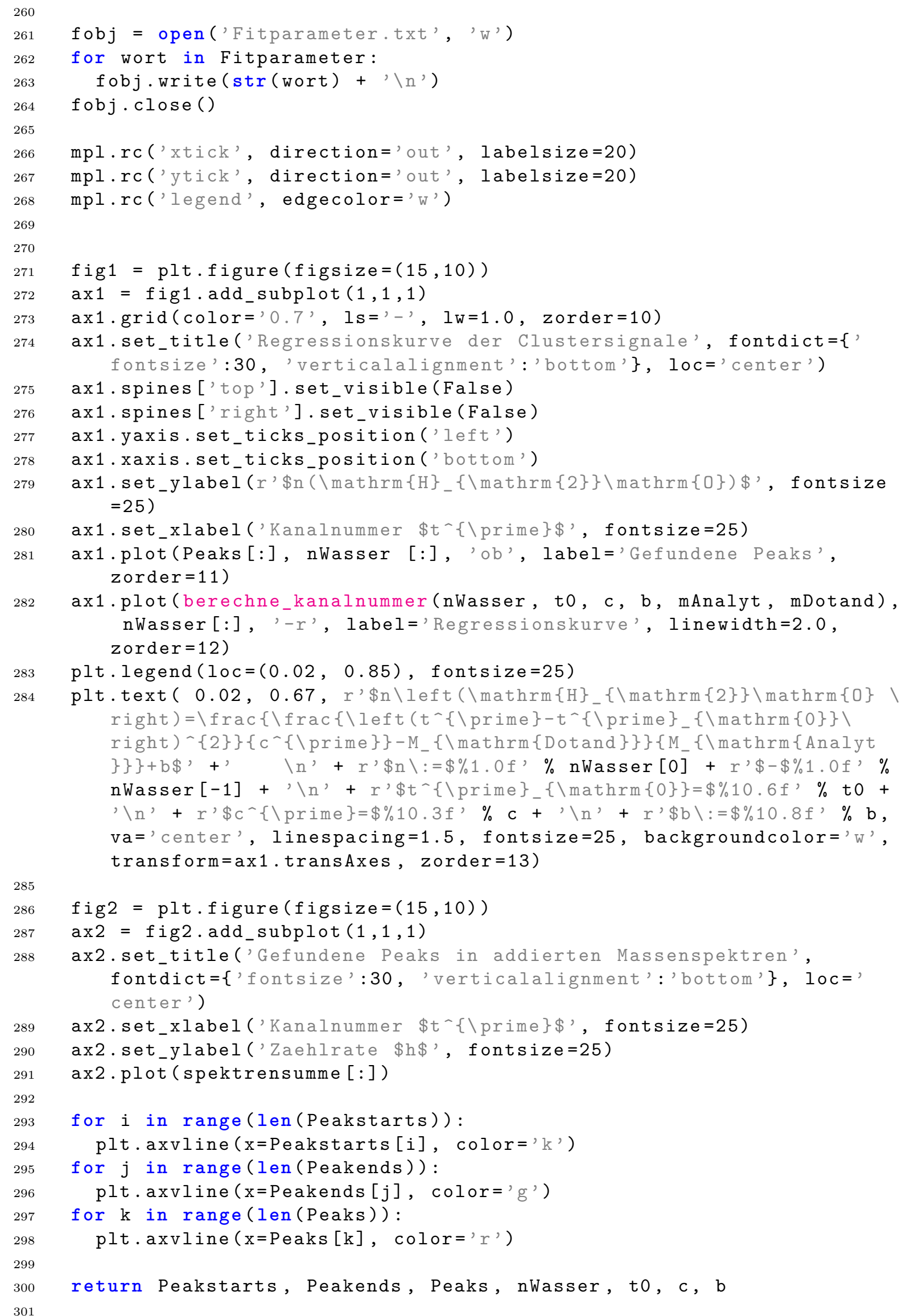




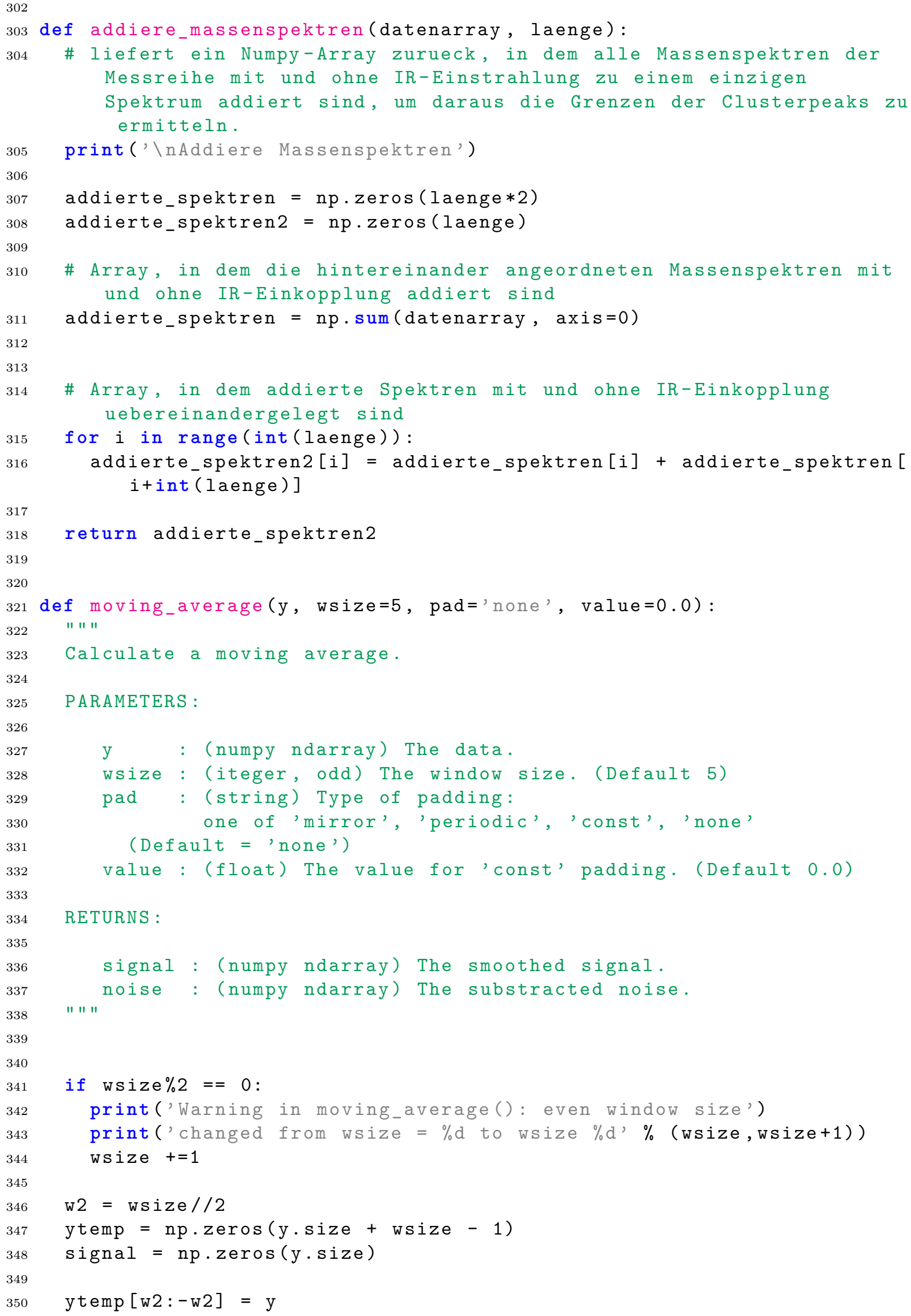




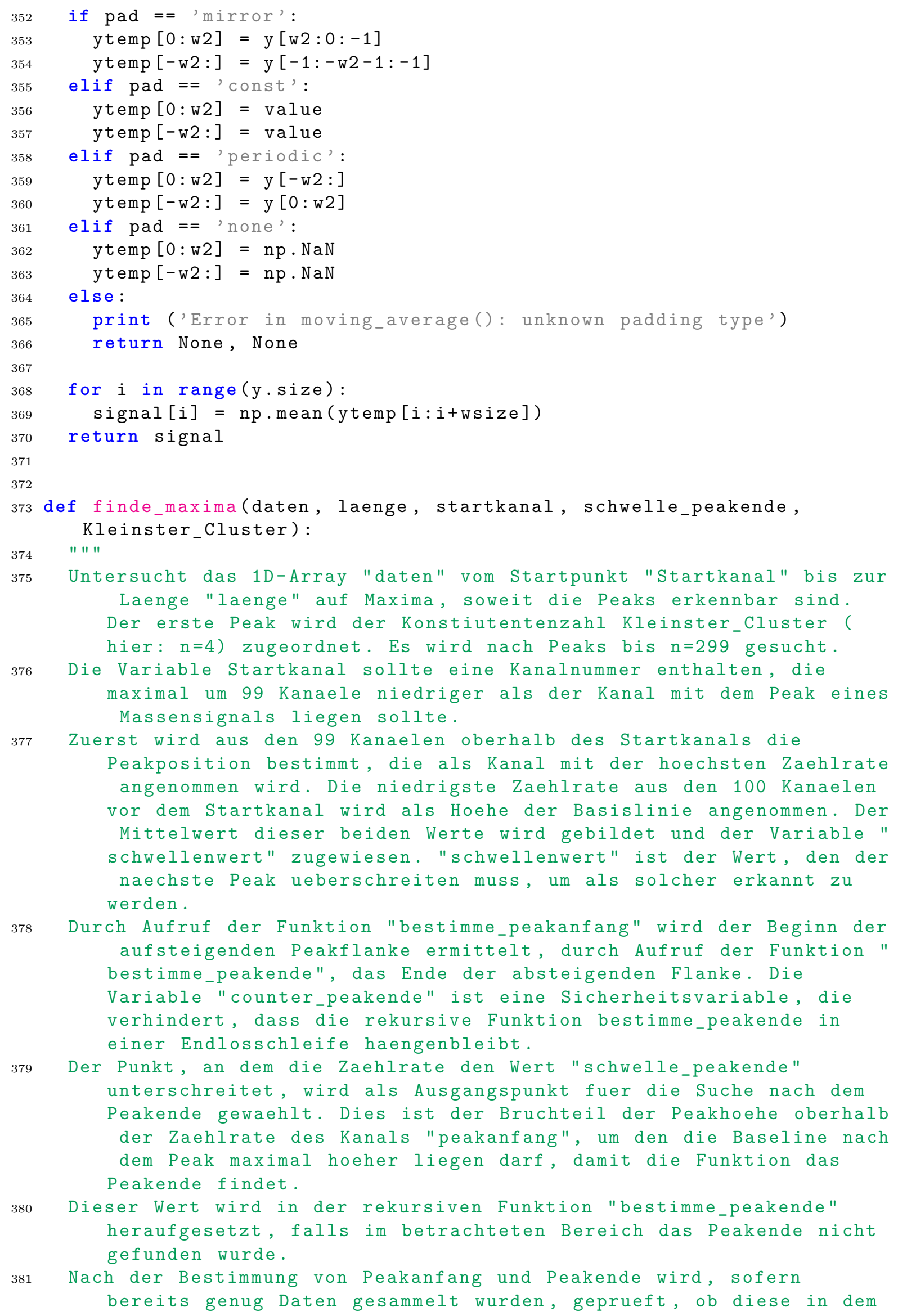


Bereich liegen, in dem die Integrationsgrenzen erwartet werden . Ist dies der Fall, werden die Werte in Listen gespeichert und durch Aufruf von "bestimme_startkanal" der naechste Startkanal ermittelt. Auch dieser Wert wird, falls schon moeglich, geprueft

Ist eine der Pruefbedingungen nicht erfuellt, beendet die Funktion die Suche.

Die Funktion gibt 4 Arrays mit Peakanfaengen, Peakenden, Peakmaxima und der zugehoerigen Anzahl der Wassermolekuele im Cluster zurueck. 


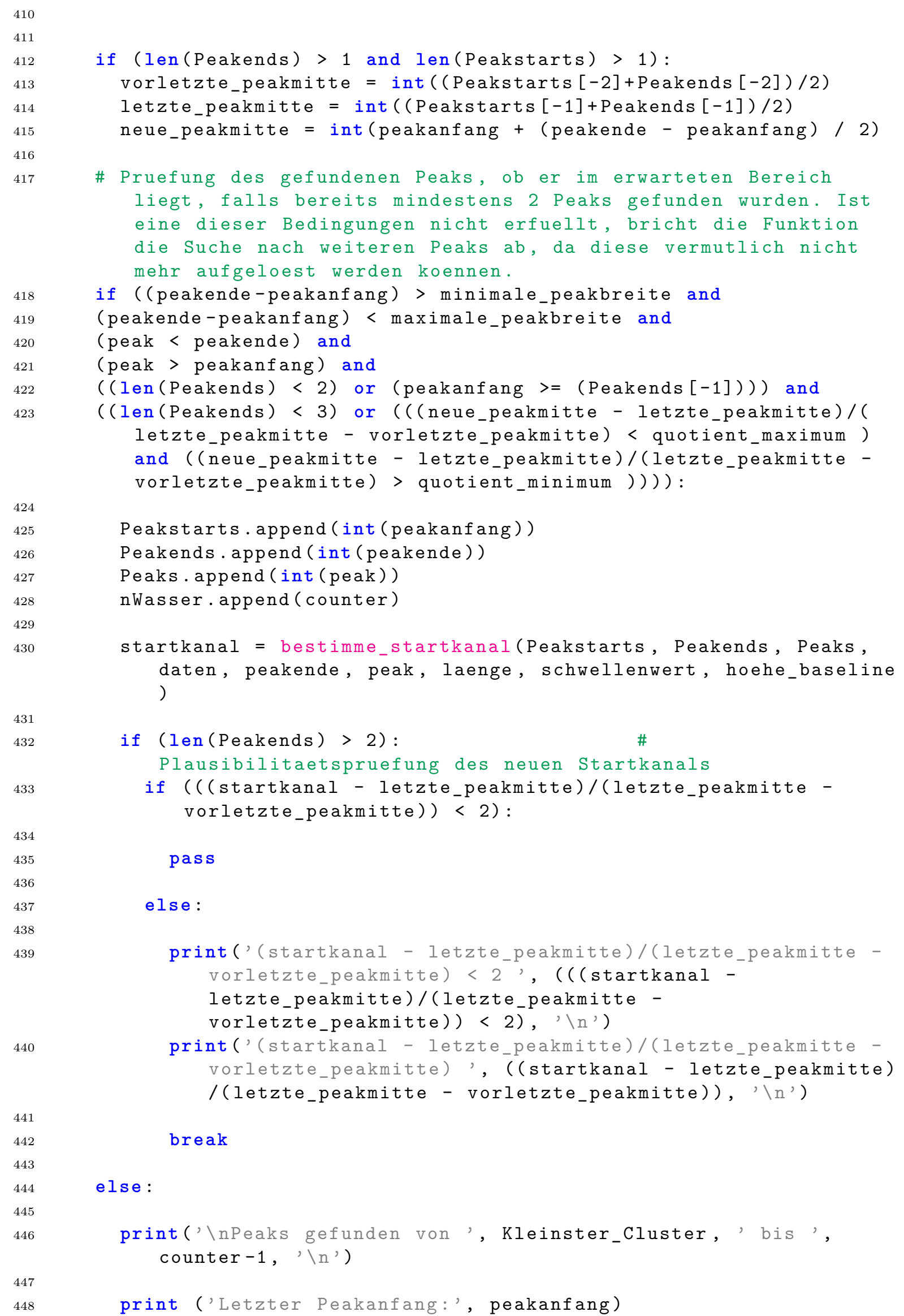




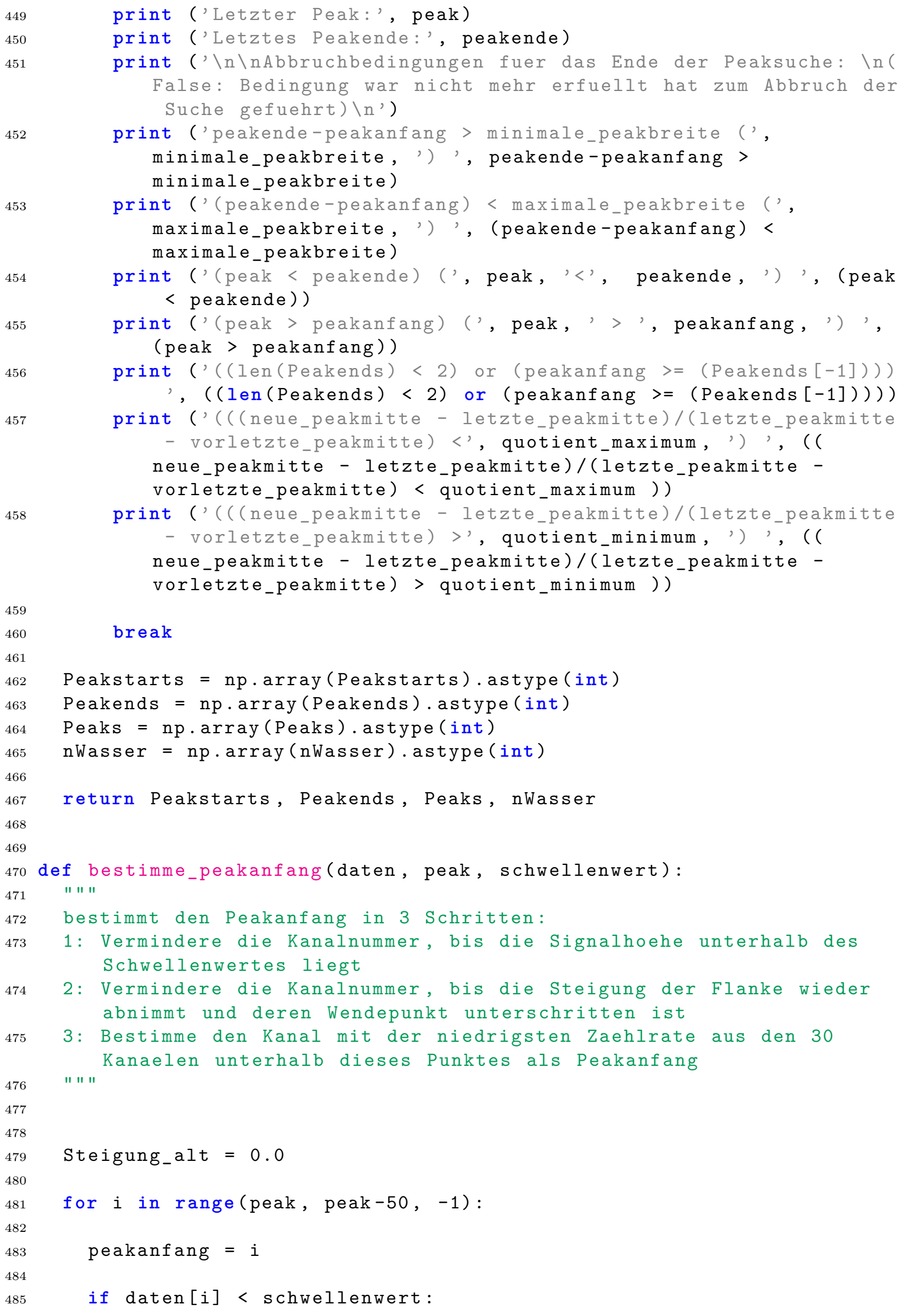




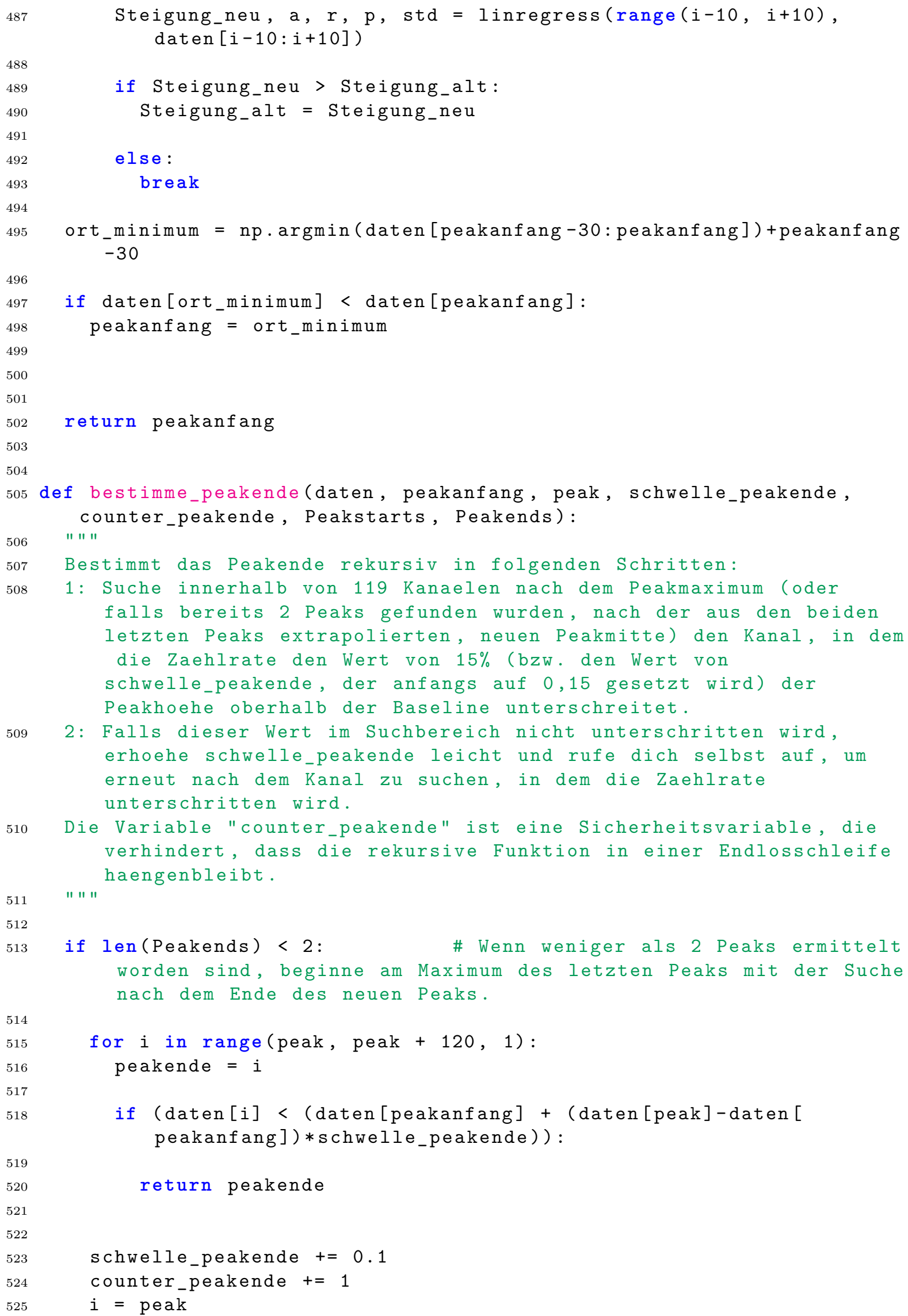

501

504

505

506

507

508

509

Steigung_neu, a, r, p, std = linregress $(\operatorname{range}(i-10, i+10)$, daten $[i-10: i+10])$

if Steigung_neu > Steigung_alt:

Steigung_alt = Steigung_neu

else:

break

ort_minimum = np.argmin (daten[peakanfang-30:peakanfang]) +peakanfang $-30$

if daten[ort_minimum] < daten[peakanfang]: peakanfang $=$ ort_minimum

return peakanfang

def bestimme_peakende(daten, peakanfang, peak, schwelle_peakende, counter_peakende, Peakstarts, Peakends): " " "

Bestimmt das Peakende rekursiv in folgenden Schritten:

1: Suche innerhalb von 119 Kanaelen nach dem Peakmaximum (oder falls bereits 2 Peaks gefunden wurden, nach der aus den beiden letzten Peaks extrapolierten, neuen Peakmitte) den Kanal, in dem die Zaehlrate den Wert von $15 \%$ (bzw. den Wert von schwelle_peakende, der anfangs auf 0,15 gesetzt wird) der Peakhoehe oberhalb der Baseline unterschreitet.

2: Falls dieser Wert im Suchbereich nicht unterschritten wird, erhoehe schwelle peakende leicht und rufe dich selbst auf, um erneut nach dem Kanal zu suchen, in dem die Zaehlrate unterschritten wird.

Die Variable "counter_peakende" ist eine Sicherheitsvariable, die verhindert, dass die rekursive Funktion in einer Endlosschleife haengenbleibt. " " "

if len (Peakends) $<2$ :

\# Wenn weniger als 2 Peaks ermittelt worden sind, beginne am Maximum des letzten Peaks mit der Suche nach dem Ende des neuen Peaks.

for $i$ in range (peak, peak $+120,1)$ : peakende $=i$

if (daten [i] < (daten[peakanfang] + (daten[peak]-daten [ peakanfang])*schwelle_peakende)) :

return peakende

schwelle_peakende $+=0.1$

counter_peakende $+=1$

$i=$ peak 


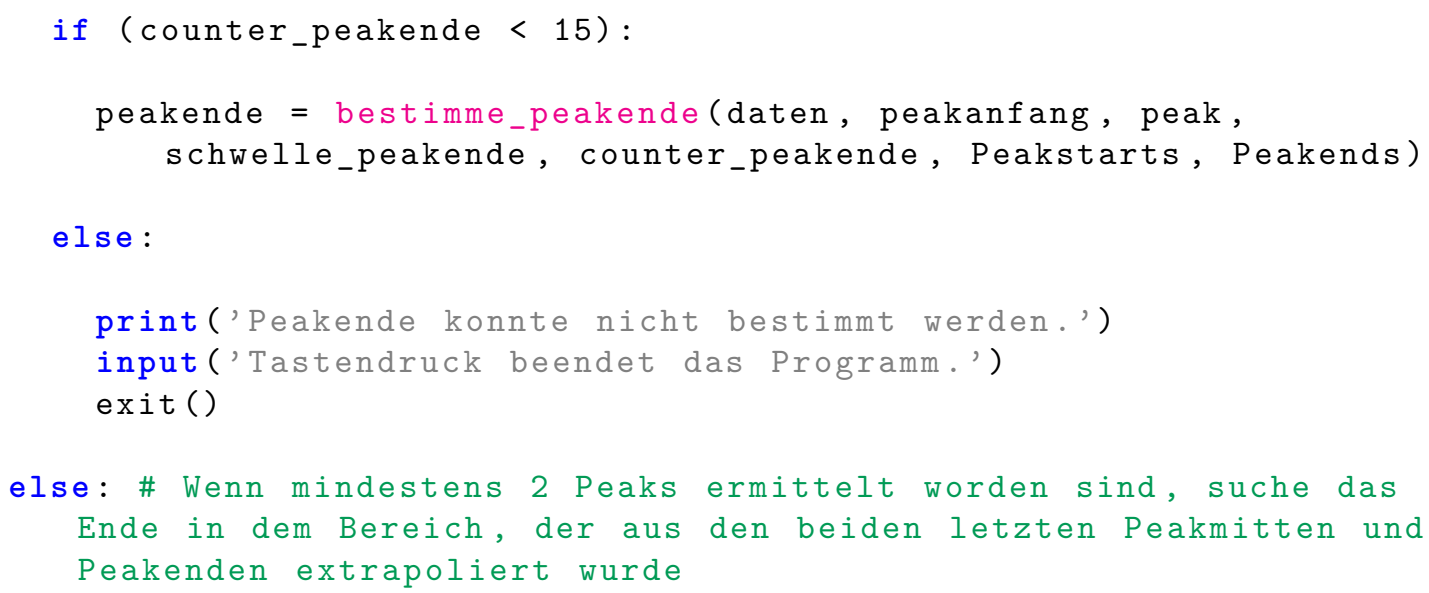


Findest du keinen Kanal mit Zaehlrate oberhalb des Schwellenwertes, vermindere diese um 10\% und rufe dich selbst auf, um abermals den Bereich abzusuchen. 


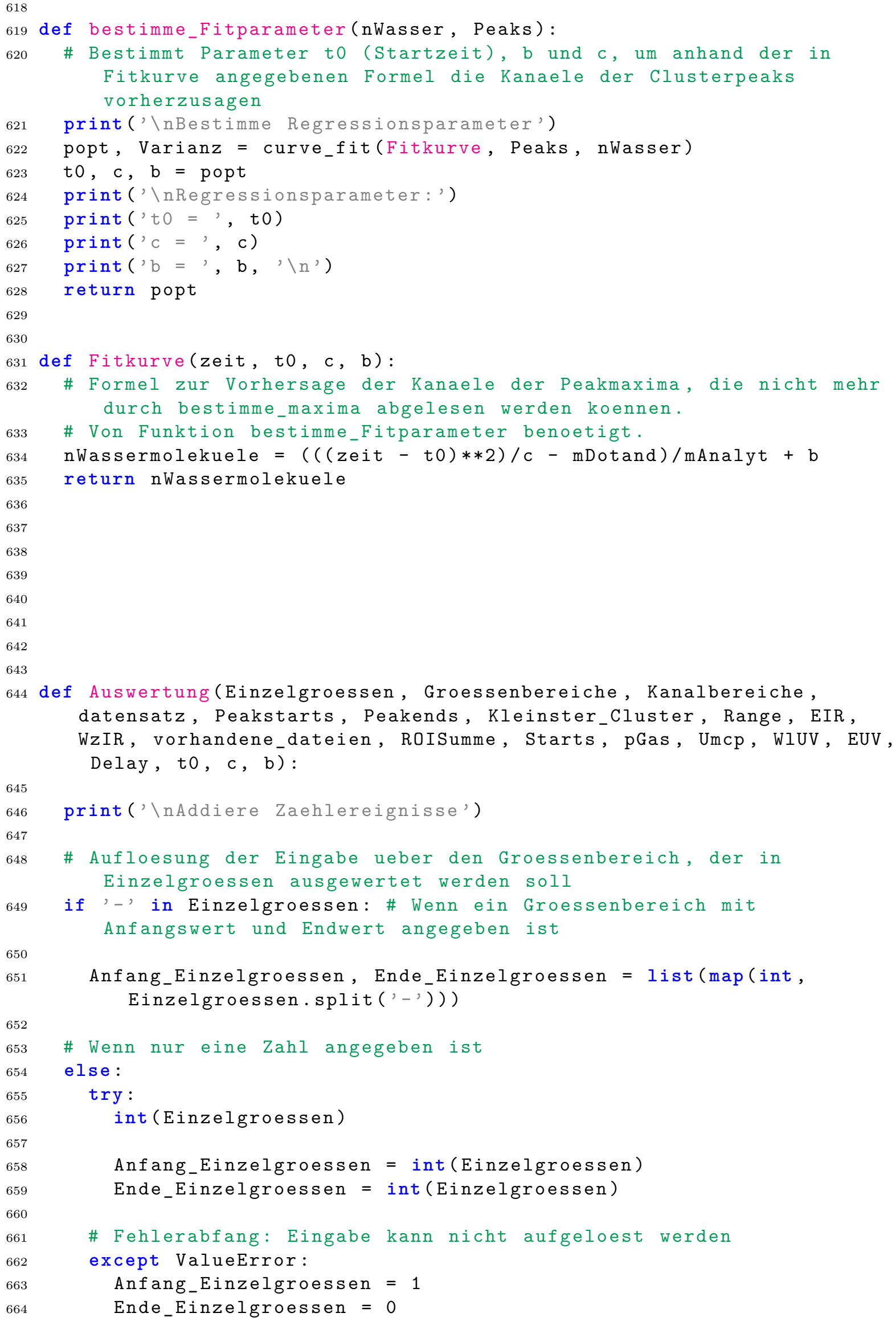




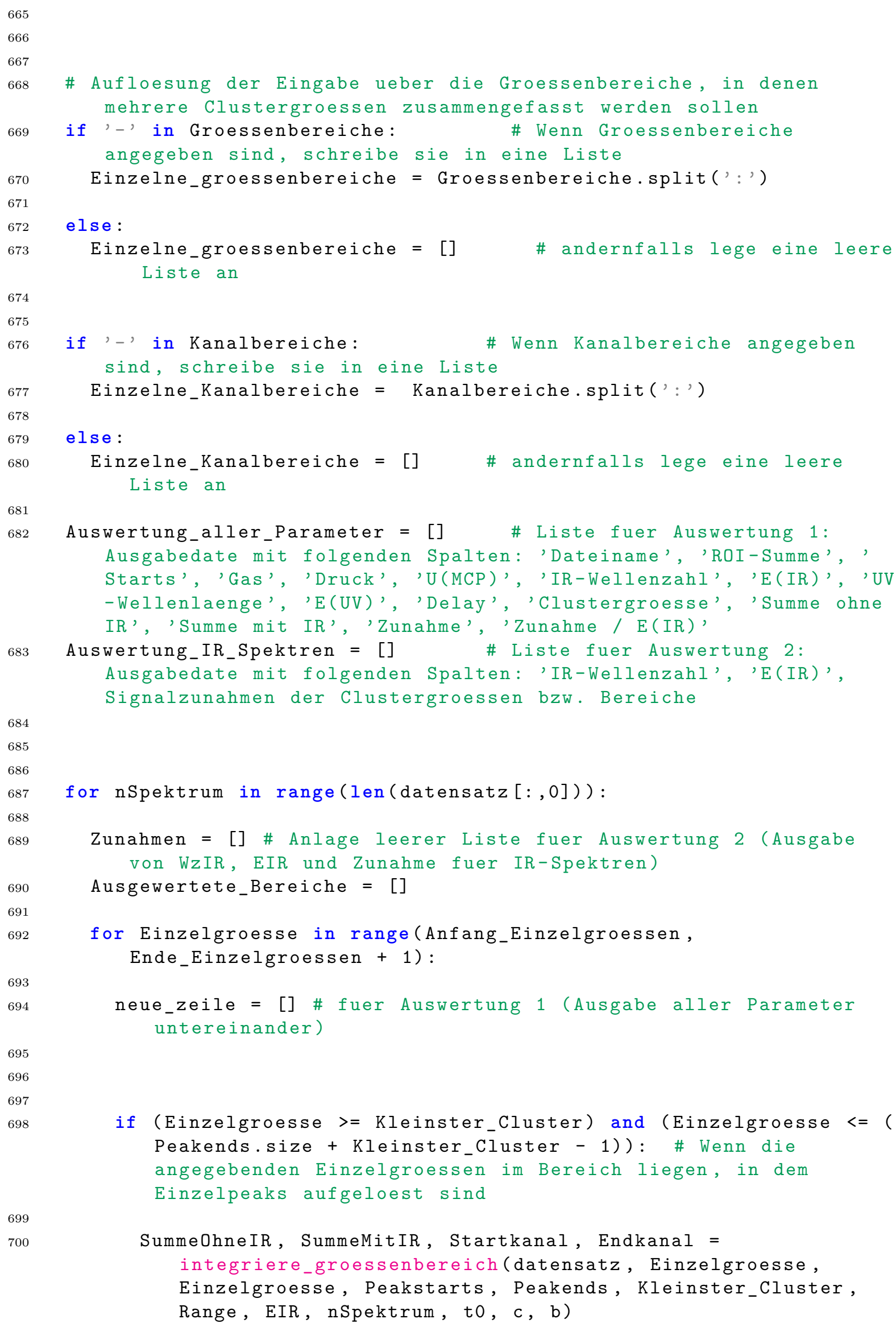

\# Aufloesung der Eingabe ueber die Groessenbereiche, in denen mehrere Clustergroessen zusammengefasst werden sollen

for nSpektrum in range(len (datensatz $[:, 0]))$ :

Zunahmen $=[]$ \# Anlage leerer Liste fuer Auswertung 2 (Ausgabe von WzIR, EIR und Zunahme fuer IR-Spektren)

Ausgewertete_Bereiche = []

for Einzelgroesse in range (Anfang_Einzelgroessen, Ende_Einzelgroessen +1 ):

neue_zeile $=[$ ] \# fuer Auswertung 1 (Ausgabe aller Parameter untereinander)

if (Einzelgroesse >= Kleinster_Cluster) and (Einzelgroesse <= ( Peakends.size + Kleinster_Cluster - 1)): \# Wenn die angegebenden Einzelgroessen im Bereich liegen, in dem Einzelpeaks aufgeloest sind

SummeOhneIR, SummeMitIR, Startkanal, Endkanal = integriere_groessenbereich (datensatz, Einzelgroesse, Einzelgroesse, Peakstarts, Peakends, Kleinster_Cluster, Range, EIR, nSpektrum, to, c, b) 


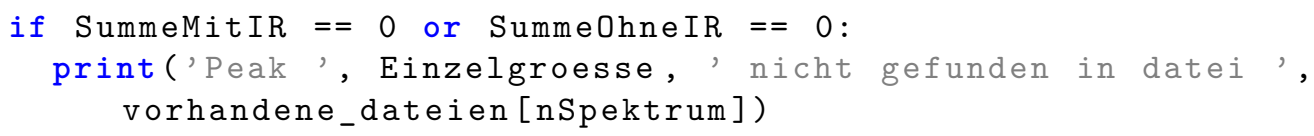




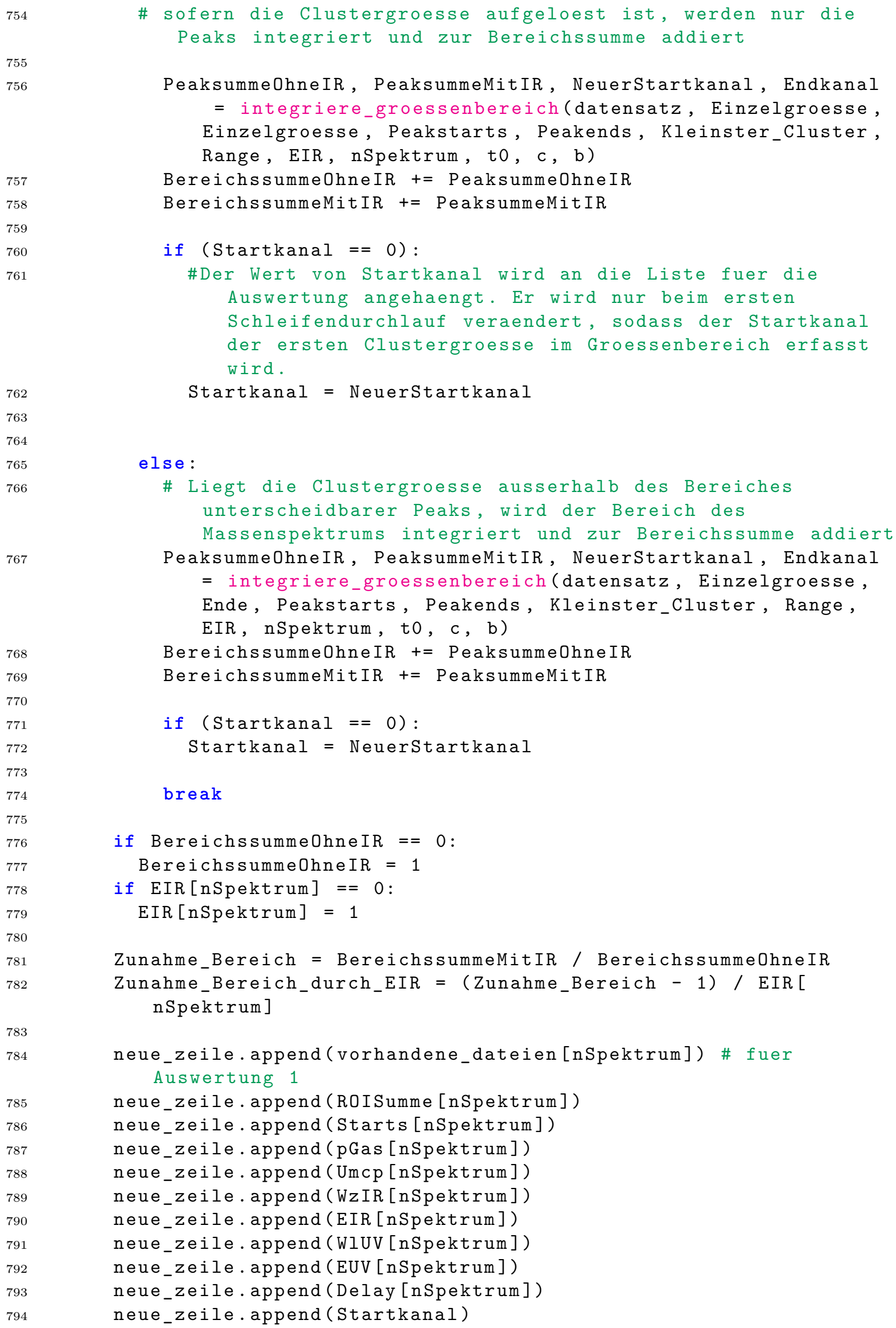

PeaksummeOhneIR, PeaksummeMitIR, NeuerStartkanal, Endkanal = integriere_groessenbereich (datensatz, Einzelgroesse, Einzelgroesse, Peakstarts, Peakends, Kleinster_Cluster, Range, EIR, nSpektrum, to, c, b)

BereichssummeOhneIR $+=$ PeaksummeOhneIR

BereichssummeMitIR $+=$ PeaksummeMitIR

if (Startkanal $==0)$ :

\#Der Wert von Startkanal wird an die Liste fuer die Auswertung angehaengt. Er wird nur beim ersten Schleifendurchlauf veraendert, sodass der Startkanal der ersten Clustergroesse im Groessenbereich erfasst wird.

Startkanal $=$ NeuerStartkanal

else:

\# Liegt die Clustergroesse ausserhalb des Bereiches unterscheidbarer Peaks, wird der Bereich des Massenspektrums integriert und zur Bereichssumme addiert PeaksummeOhneIR, PeaksummeMitIR, NeuerStartkanal, Endkanal = integriere_groessenbereich (datensatz, Einzelgroesse, Ende, Peakstarts, Peakends, Kleinster_Cluster, Range, EIR, nSpektrum, to, c, b)

BereichssummeOhneIR $+=$ PeaksummeOhneIR

BereichssummeMitIR $+=$ PeaksummeMitIR

if (Startkanal == 0):

Startkanal = NeuerStartkanal

break

if BereichssummeOhneIR $==0$ :

BereichssummeOhneIR $=1$

if $\operatorname{EIR}[$ nSpektrum $]==0$ :

$\operatorname{EIR}[$ nSpektrum $]=1$

Zunahme_Bereich = BereichssummeMitIR / BereichssummeOhneIR Zunahme_Bereich_durch_EIR = (Zunahme_Bereich - 1) / EIR [ nSpektrum ] 


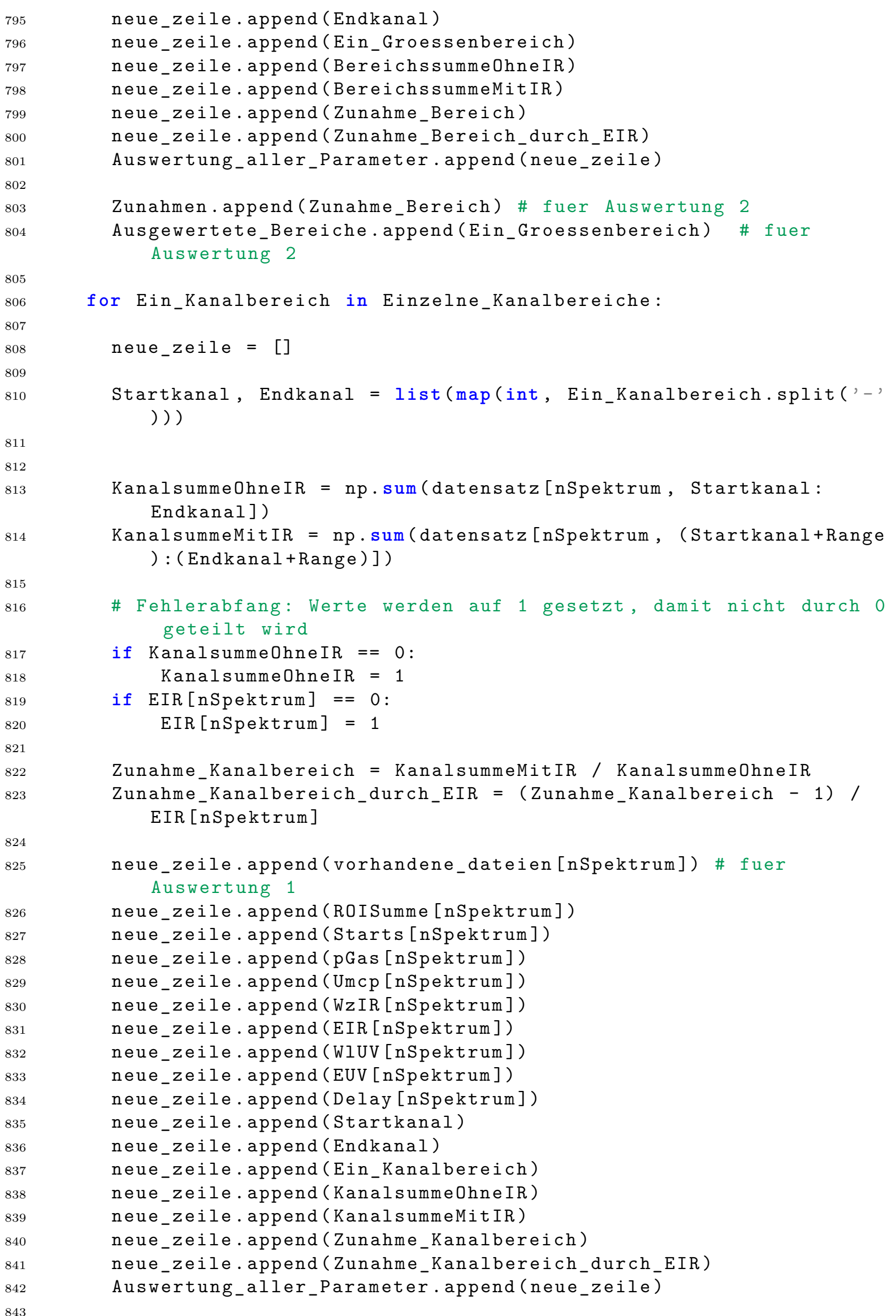


Zunahmen. append (Zunahme_Kanalbereich) \# fuer Auswertung 2 Ausgewertete_Bereiche.append(Ein_Kanalbereich) \# fuer Auswertung 2

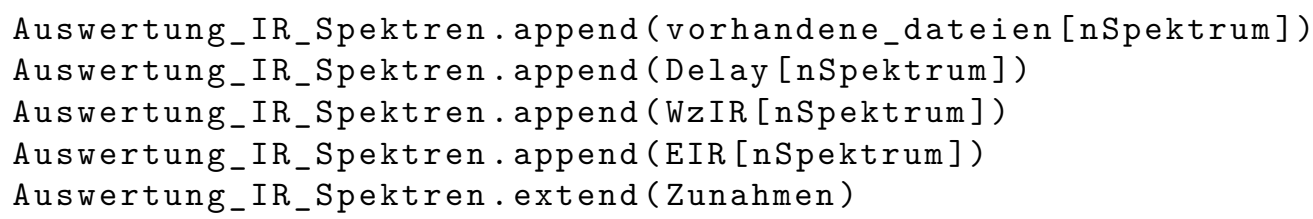




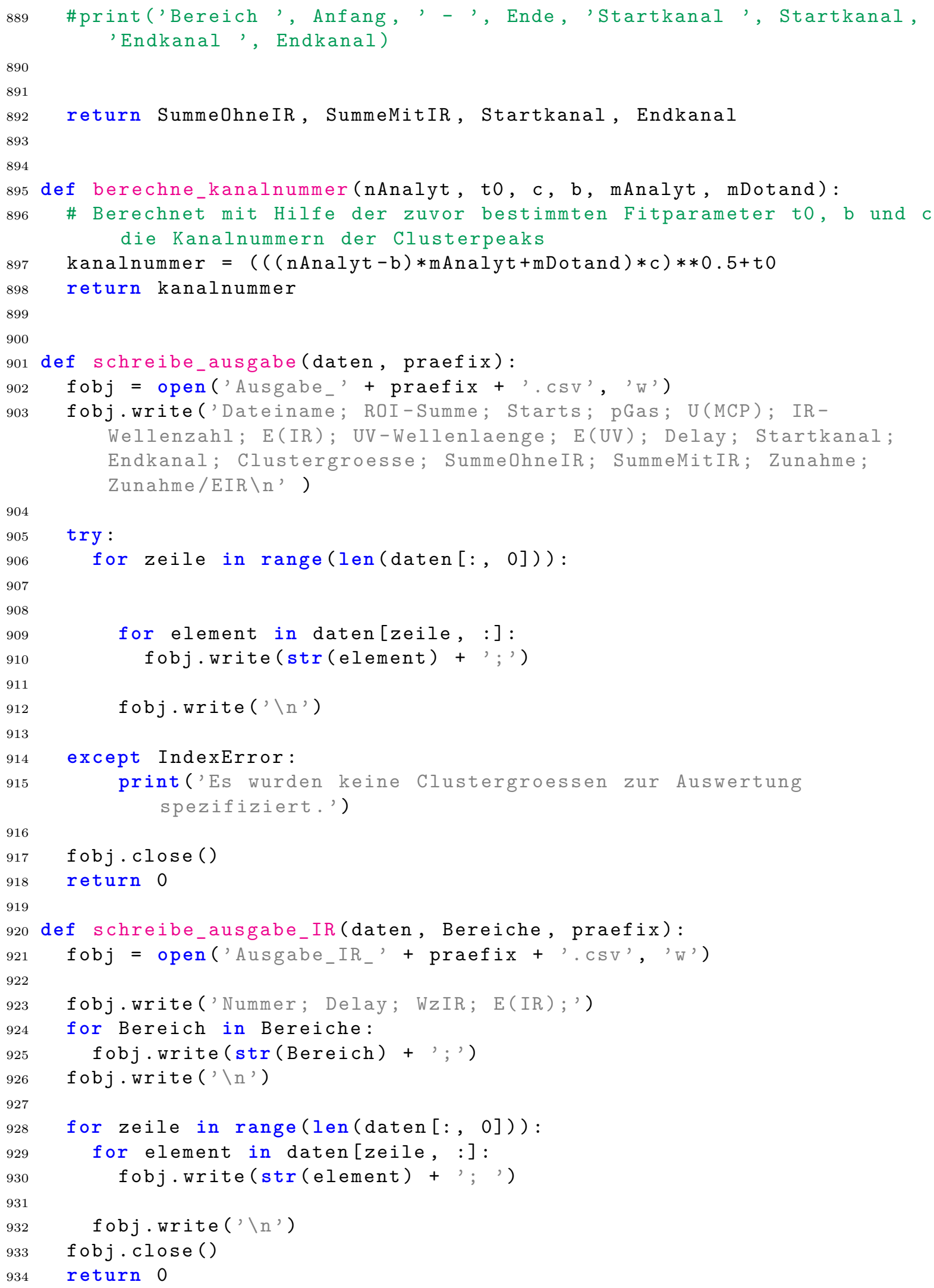




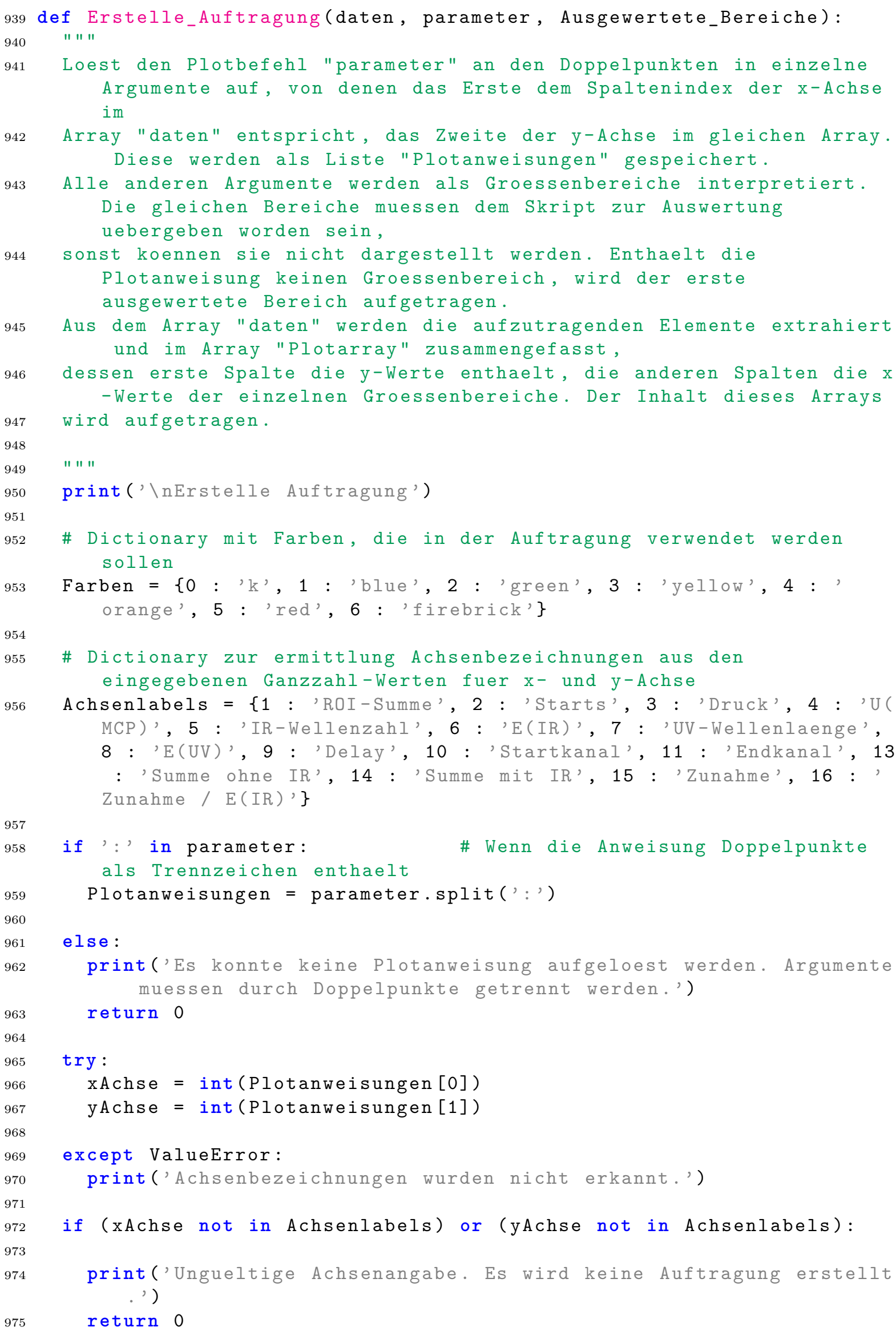

943

944

945

946

947

948

949

950

951

952

953

954

955

956

957

958

Alle anderen Argumente werden als Groessenbereiche interpretiert. Die gleichen Bereiche muessen dem Skript zur Auswertung uebergeben worden sein,

sonst koennen sie nicht dargestellt werden. Enthaelt die Plotanweisung keinen Groessenbereich, wird der erste ausgewertete Bereich aufgetragen.

Aus dem Array "daten" werden die aufzutragenden Elemente extrahiert und im Array "Plotarray" zusammengefasst,

dessen erste Spalte die y-Werte enthaelt, die anderen Spalten die $x$ -Werte der einzelnen Groessenbereiche. Der Inhalt dieses Arrays wird aufgetragen.

" " "

print ('\nErstelle Auftragung')

\# Dictionary mit Farben, die in der Auftragung verwendet werden sollen

Farben $=\left\{0:\right.$ ' $^{\mathrm{k}}$ ', 1 : 'blue', 2 : 'green', 3 : 'yellow', 4: ' orange', 5 : 'red', 6 : 'firebrick'\}

\# Dictionary zur ermittlung Achsenbezeichnungen aus den eingegebenen Ganzzahl-Werten fuer $x$ - und $y$-Achse

Achsenlabels $=\{1$ : 'ROI-Summe', 2 : 'Starts', 3 : 'Druck', 4 : 'U( MCP)', 5 : 'IR-Wellenzahl', 6 : 'E(IR)', 7 : 'UV-Wellenlaenge', 8 : 'E(UV)', 9 : 'Delay', 10 : 'Startkanal', 11 : 'Endkanal', 13 : 'Summe ohne IR', 14 : 'Summe mit IR', 15 : 'Zunahme', 16 : ' Zunahme / $E(I R)$,

if ': ' in parameter:

\# Wenn die Anweisung Doppelpunkte

als Trennzeichen enthaelt

Plotanweisungen = parameter.split (': ')

else:

print ('Es konnte keine Plotanweisung aufgeloest werden. Argumente muessen durch Doppelpunkte getrennt werden.') 


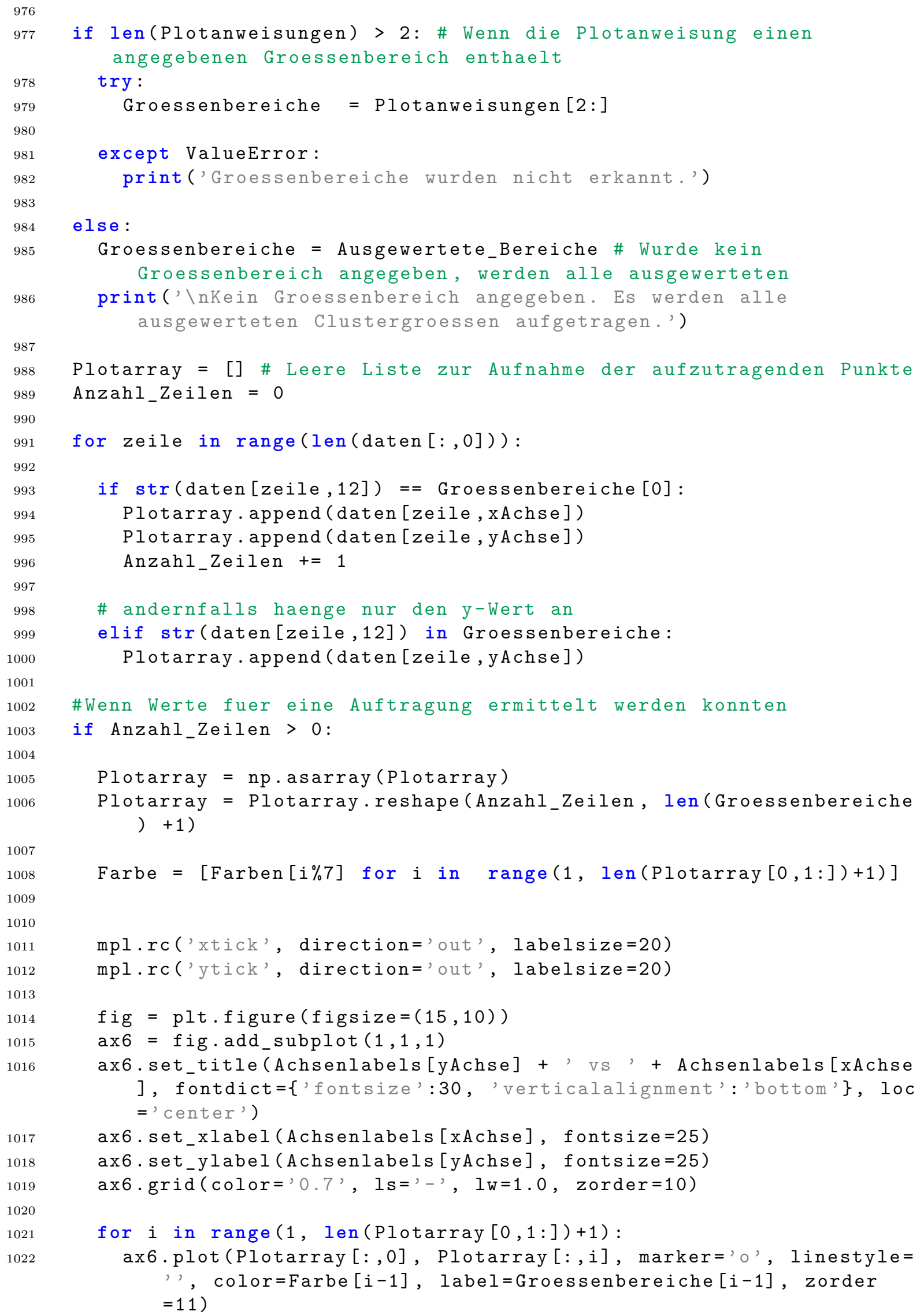




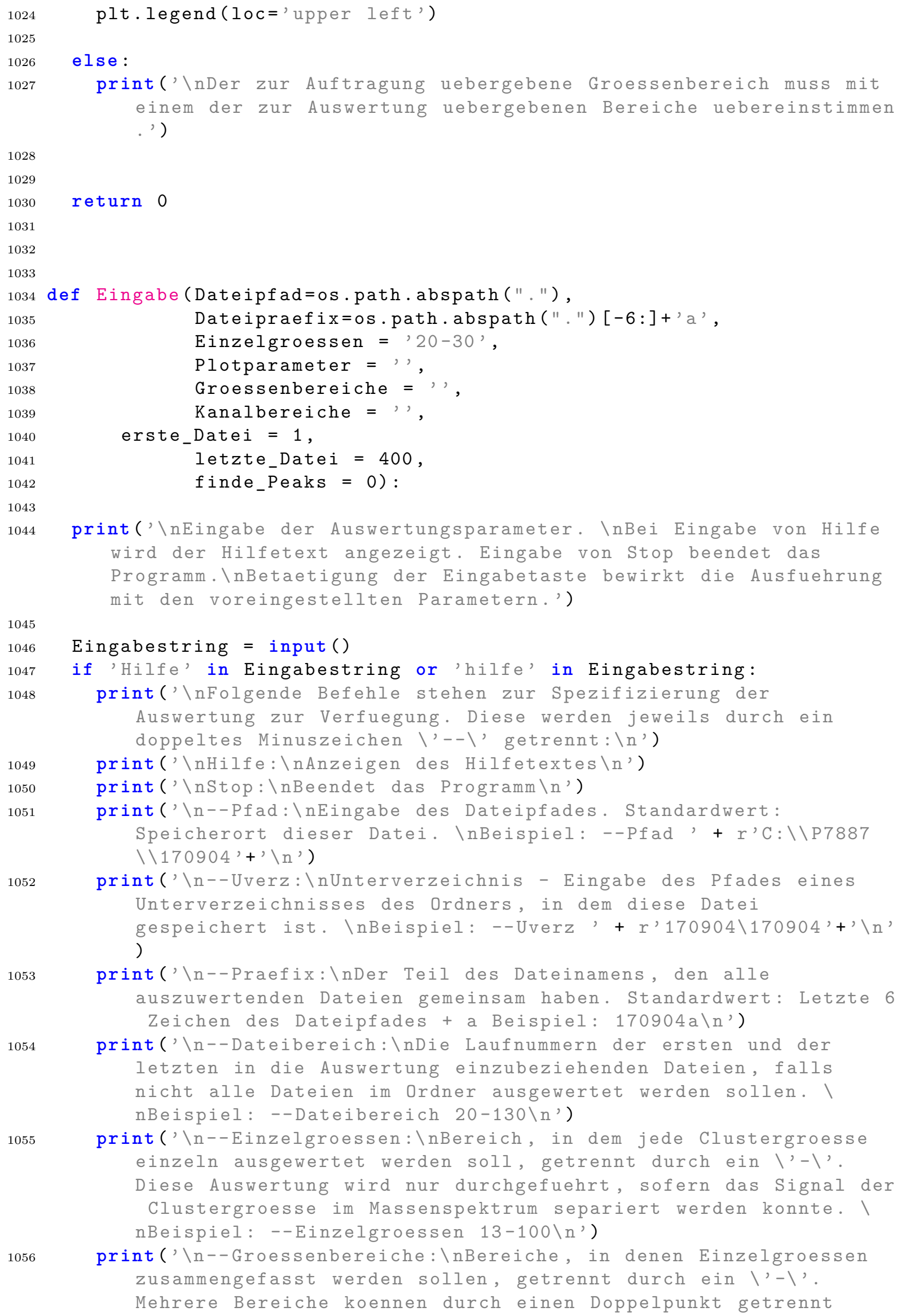

print(' \nEingabe der Auswertungsparameter. \nBei Eingabe von Hilfe wird der Hilfetext angezeigt. Eingabe von Stop beendet das Programm. \nBetaetigung der Eingabetaste bewirkt die Ausfuehrung mit den voreingestellten Parametern.' )

\section{Eingabestring $=$ input ()}

if 'Hilfe' in Eingabestring or 'hilfe' in Eingabestring:

print(' $\backslash n$ Folgende Befehle stehen zur Spezifizierung der Auswertung zur Verfuegung. Diese werden jeweils durch ein doppeltes Minuszeichen \'--\' getrennt: \n') print('\nHilfe: \nAnzeigen des Hilfetextes \n')

print (' \nstop: \nBeendet das Programm \n')

print(' \n--Pfad: \nEingabe des Dateipfades. Standardwert: Speicherort dieser Datei. \nBeispiel: --Pfad' + r'C: \\P7887 $\left.\backslash \backslash 170904^{\prime}+{ }^{\prime} \backslash n^{\prime}\right)$

print(' \n--Uverz: \nUnterverzeichnis - Eingabe des Pfades eines Unterverzeichnisses des Ordners, in dem diese Datei gespeichert ist. \nBeispiel: --Uverz ' + r'170904\170904'+' \n' )

print(' \n--Praefix: \nDer Teil des Dateinamens, den alle auszuwertenden Dateien gemeinsam haben. Standardwert: Letzte 6 Zeichen des Dateipfades + a Beispiel: 170904a\n')

print(' $\backslash n--D a t e i b e r e i c h: \backslash n D i e$ Laufnummern der ersten und der letzten in die Auswertung einzubeziehenden Dateien, falls nicht alle Dateien im Ordner ausgewertet werden sollen. I nBeispiel: --Dateibereich 20-130\n')

print(' \n--Einzelgroessen: \nBereich, in dem jede Clustergroesse einzeln ausgewertet werden soll, getrennt durch ein \'- $\backslash$ '. Diese Auswertung wird nur durchgefuehrt, sofern das Signal der Clustergroesse im Massenspektrum separiert werden konnte. । nBeispiel: --Einzelgroessen 13-100\n' )

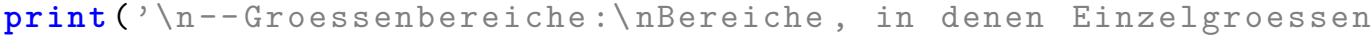
zusammengefasst werden sollen, getrennt durch ein \'- $\backslash^{\prime}$. Mehrere Bereiche koennen durch einen Doppelpunkt getrennt 
angegeben werden. \nBeispiel: --Groessenbereiche $\left.10-19: 20-29: 30-39: 40-49 \backslash n^{\prime}\right)$

1057

1058

1059

1060

1061

1062

1063

1064

1065

1066

1067

1068

1069

1070

1071

1072

1073

1074

1075

1076

1077

print(' \n--Kanalbereiche: \nBereiche von Kanaelen im

Massenspektrum, getrennt durch ein \'-\'. Mehrere Bereiche

koennen durch einen Doppelpunkt getrennt angegeben werden.

nBeispiel: --Kanalbereiche 3300-3400:6500-80000\n')

print(' \n--findePeaks: \nWird das Programm zum ersten Mal in einem

Verzeichnis aufgerufen, speichert es die Parameter der

Groessenverteilung in Dateien namens Peakstarts.txt, Peakends.

txt, Peaks.txt und Fitparameter.txt')

print(' \nSind diese Dateien beim Programmstart vorhanden, wird

ihr Inhalt geladen und die Bestimmung von Peakgrenzen wird

nicht erneut durchgefuehrt. ')

print ('findePeaks Bewirkt in jedem Fall eine erneute Untersuchung des Massenspektrums mit Bestimmung der Grenzen der

Massenpeaks und der Fitparameter $\backslash \mathrm{n}^{\prime}$ )

print(' \n--Plot: \nBewirkt Darstellung der Auswertungsergebnisse in einer Auftragung im Format x-Achse:y-Achse:Groessen-bzw.

Kanalbereiche.' )

print('Die Angabe der auf der Achse aufutragenden Groesse

erfolgt durch eine Nummer. \n\n1: ROI-Summe, \n2: Starts, \n3:

Traegergasdruck, \n4: MCP-Spannung, \n5: IR-Wellenzahl, \n6:

IR-Energie, \n7: UV-Wellenlaenge, \n8: UV-Energie, \n9: Delay,

$\backslash n 10$ : Startkanal, \n11: Endkanal, \n13: Summe ohne IR-Laser,

$\backslash n 14:$ Summe mit IR-Laser, \n15: Signalzunahme, \n16:

Signalzunahme-1)/IR-Energie')

print(' $\backslash n$ Die Werte der Parameter 1-9 werden aus den Comment-

Dateien extrahiert, Parameter 11-13 vom Programm berechnet.

Die Nummern entsprechen den Spaltennummern der Groessen in der

Datei Ausgabe.csv, beginnend bei 0. InBeispiel: --Plot 5:15

traegt die Zunahme ueber der IR-Wellenzahl auf.')

print(' \nSofern bei der Auswertung mehrere Groessenbereiche bzw.

Kanalbereiche angegeben wurden, kann hieraus fuer die

Auftragung eine Auswahl getroffen werden. Dies geschieht durch

Eingabe der Bereiche getrennt durch weitere Doppelpunkte. I

nBeispiel: --Plot 5:16:20:21:30-39:40-49 traegt die auf IR-

Energie normierte Signalzunahme ueber der IR-Wellenzahl fuer

Cluster der Groesse $n=20,22,30-39$ und 40-49 auf. In')

print(' $\backslash n$ Folgende Parameter sind derzeit als Standardparameter eingestellt: $\left.\backslash n^{\prime}\right)$

print ('\nDateipfad: ', Dateipfad)

print ('Praefix: ', Dateipraefix, ' (letzte 6 Zeichen des

Dateipfads + a)')

print ('Dateibereich: ', erste_Datei, , - ', letzte_Datei)

print ('Einzelgroessen: ', Einzelgroessen)

print ('Groessenbereiche: ', Groessenbereiche)

print ('Plotparameter: ', Plotparameter)

print ('Kanalbereiche: ', Kanalbereiche)

print(' $\backslash$ nDieses Program wurde in Python 3.4 verfasst und benoetigt die Packages os, SciPy, NumPy und Matplotlib.\n')

Dateipfad, Dateipraefix, Einzelgroessen, Groessenbereiche, erste_Datei, letzte_Datei, finde_Peaks, Plotparameter = Eingabe ()

elif 'Stop' in Eingabestring or 'stop' in Eingabestring: 


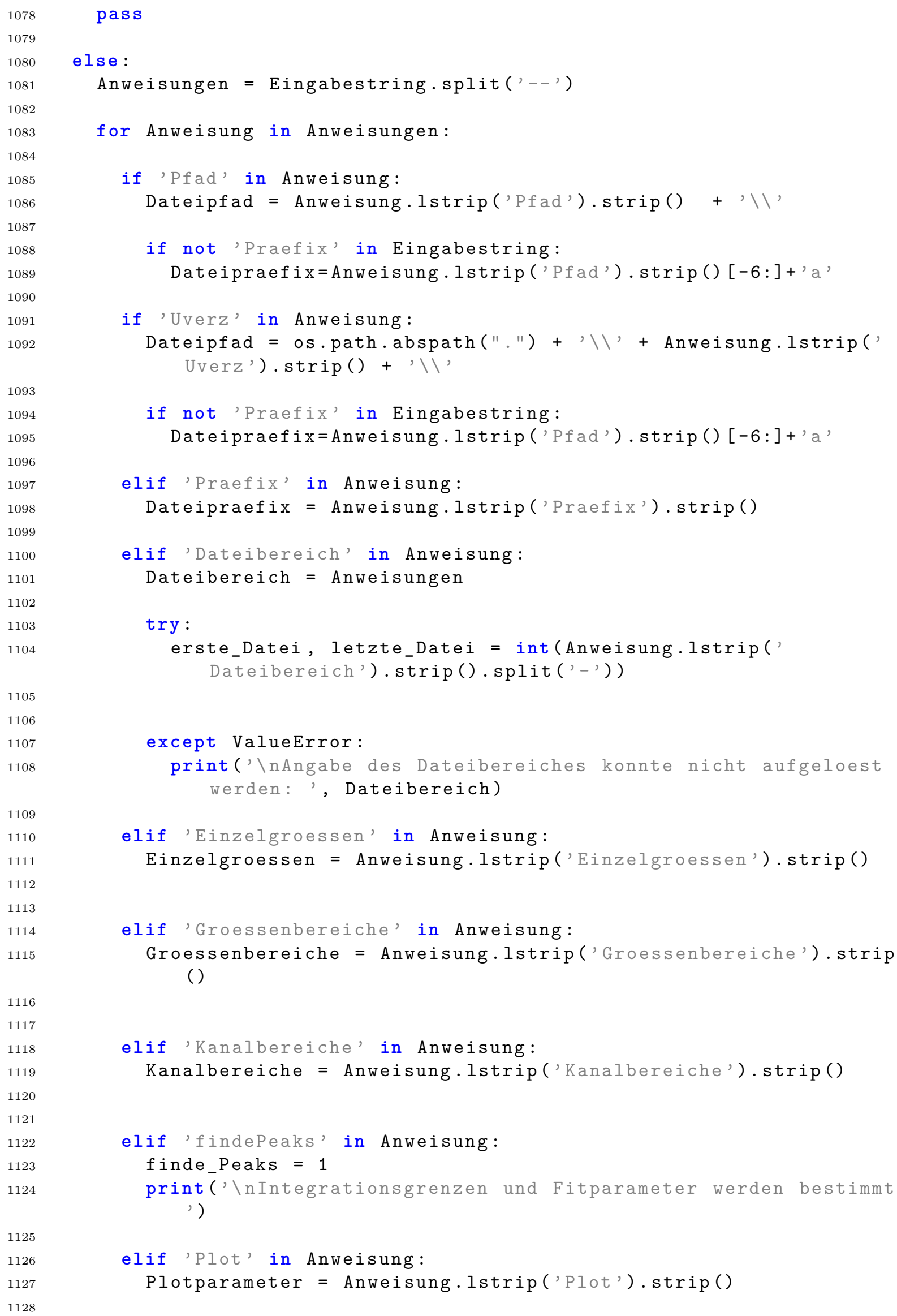




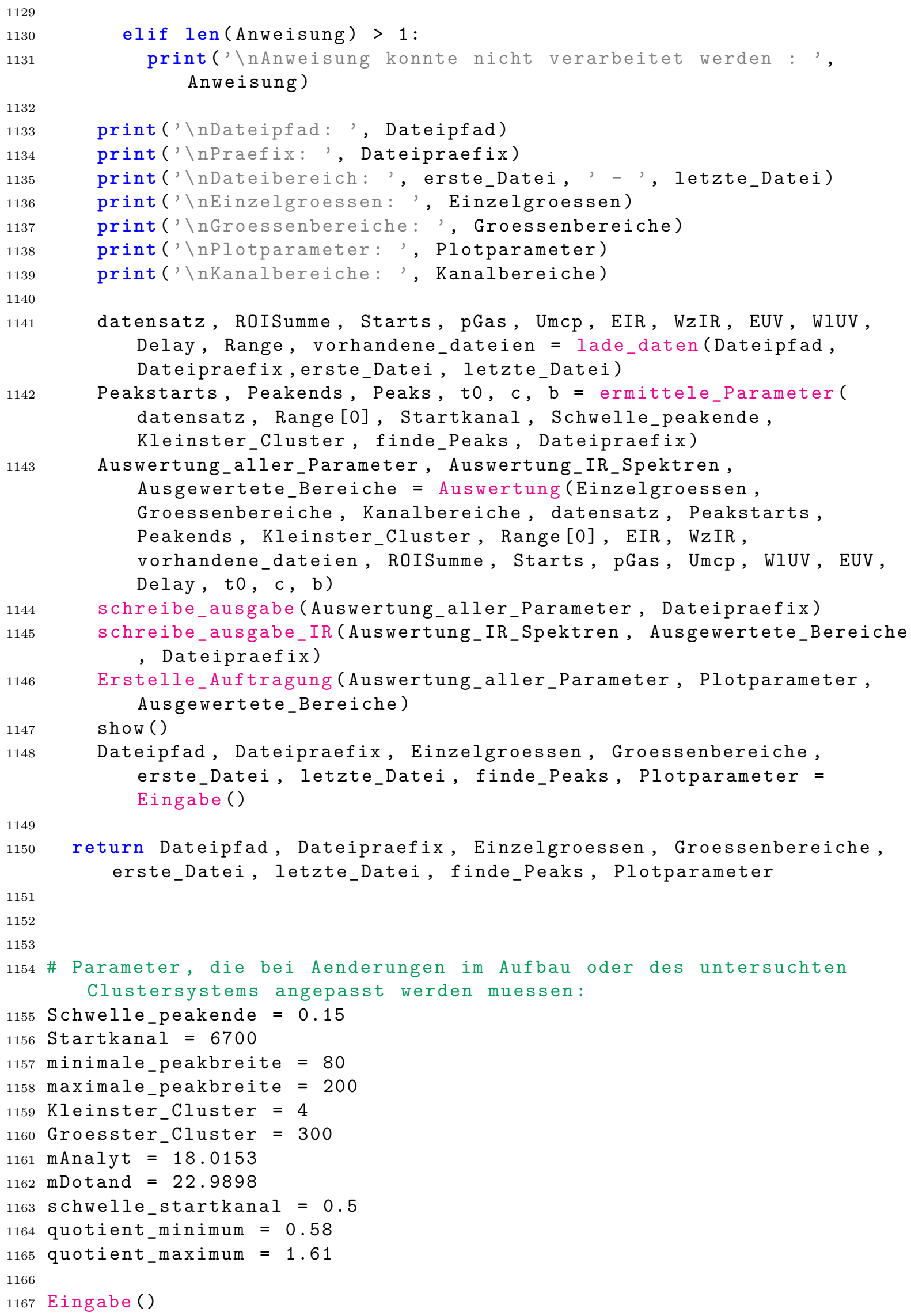





\section{Liste der verwendeten Symbole}

$A$ Fläche.

a lokale Schallgeschwindigkeit.

b Parameter der Regressionsgleichung 5.19 .

$C$ Kapazität eines Kondensators.

$C_{p}$ Wärmekapazität bei konstantem Druck.

$C_{V}$ Wärmekapazität bei konstantem Volumen.

c Apparatekonstante des Massenspektrometers.

$c_{p}$ spezifische Wärmekapazität bei konstantem Druck.

$c^{\prime}$ Parameter der Regressionsgleichung 5.19.

$c_{V}$ spezifische Wärmekapazität bei konstantem Volumen.

$D_{\text {eq }}$ Maßzahl der Verteilung zwischen zwei Zuständen.

d Dicke einer Schicht bzw. eines Flüssigkeitsfilms.

$d_{\mathrm{D}}$ Düsendurchmesser.

$d_{\mathrm{D}, \mathrm{eq}}$ äquivalenter Düsendurchmesser nach Hagena.

$E_{\mathrm{IR}}$ Pulsenergie der Infrarotlaserstrahlung.

$E_{\text {kin }}$ kinetische Energie.

$E_{\text {pot }}$ potentielle Energie.

$E_{\mathrm{UV}}$ Pulsenergie der ultravioletten Laserstrahlung.

e Elementarladung.

F Freie Energie . $^{\ddagger}$

$f$ Anzahl der Bewegungsfreiheitsgrade eines Moleküls.

$G$ Freie Enthalpie ${ }^{\ddagger}$

$g$ molare freie Enthalpie ${ }^{\ddagger}$. 
H Elementsymbol des chemischen Elements Wasserstoff.

$H$ Enthalpie ${ }^{\ddagger}$.

$h_{\text {Schwelle,Start }}$ Schwellenwert, den die Zählrate eines Kanals überschreiten muss, um als Ausgangspunkt für die Suche nach Maximum und Anfang Massensignals zu dienen; d. h. die Höhe eines Massensignals muss diesen Wert überschreiten, um als solches erkannt zu werden.

$h_{\text {Basis }}$ Höhe der Grundlinie des Histogramms (Massenspektrums); näherungsweise bestimmt durch Ermittlung der minimalen Zählrate innerhalb eines ausgewählten Kanalbereiches.

$h_{\text {Peak }, N}$ Höhe eines Massensignals; Maximale, einem Kanal innerhalb von dessen Grenzen zugeordnete Zählrate.

$h_{\text {Schwelle,Ende }}$ Schwellenwert, den die Zählrate eines Kanals unterschreiten muss, um als Ausgangspunkt für die Suche nach dem Ende eines Massensignals zu dienen.

$h_{t^{\prime}}$ Zählrate; Summe der Zählereignisse, die einem Kanal $t^{\prime}$ zugeordnet werden.

I Elementsymbol des chemischen Elements Iod.

$I_{N}$ Infolge der Anregung durch Infrarotstrahlung induzierte Zunahme der Signalstärke; $I_{N}=\Sigma_{\mathrm{IR}+\mathrm{UV}, N} / \Sigma_{\mathrm{UV}, N}$.

$I_{N, \mathrm{Abs}}$ Absolute Spektralintensität; $I_{N, \mathrm{Abs}}=I_{N}-1$.

$I_{N, \text { normiert }}$ Absolute Spektralintensität, normiert auf die Energie der eingestrahlten Infrarotstrahlung; $I_{N \text {, normiert }}=I_{N, \mathrm{Abs}} / E_{\mathrm{IR}}$.

$K_{\text {eq }}$ Gleichgewichtskonstante.

$k$ Geschwindigkeitskonstante erster Ordnung.

$k_{\mathrm{B}}$ Boltzmann-Konstante.

$M_{\text {Analyt }}$ Molare Masse des Analyten (hier: Wasser).

$M$ lokale Machzahl.

$M_{\text {Dotand }}$ Molare Masse des Dotanden (hier: Natrium).

$m$ Masse eines Atoms bzw. Moleküls.

N Elementsymbol des chemischen Elements Stickstoff.

$N, N\left(\mathrm{H}_{2} \mathrm{O}\right)$ Anzahl der in einem Cluster bzw. Nanopartikel enthaltenen Wassermoleküle.

$N^{*}$ Anzahl der zur Ausbildung eines Nanopartikels mit kristallartigem Kern notwendigen Konstituenten. 
$N_{\text {krit }}$ Anzahl der zur Ausbildung eines kritischen Nukleus notwendigen Wassermoleküle.

$N_{\max }$ Höchste in den durch die Auswertungssoftware P7887 erstellten Histogrammen (Massenspektren) separierbare Clustergröße.

$N_{\text {Messungen }}$ Anzahl durchgeführter bzw. berücksichtigter Messungen.

$N_{\text {min }}$ Niedrigste in den durch die Auswertungssoftware Pr887 erstellten Histogrammen (Massenspektren) separierbare Clustergröße.

$N_{\mathrm{O}}$ Zahl der Konstituenten an der Clusteroberfläche.

$n$ Teilchendichte in der Gasphase.

$n_{0}$ Initiale Teilchendichte einer Gasphase zu Beginn eines Expansionsprozesses; Stagnationsparameter.

$\mathrm{Na}$ Elementsymbol des chemischen Elements Natrium.

O Elementsymbol des chemischen Elements Sauerstoff.

$p$ Druck $^{\ddagger}$.

$p^{*}$ Standarddruck $=1$ bar.

$p_{\mathrm{ch}}$ charakteristischer Druck einer Substanz nach Hagena.

$p_{\text {eq }}$ Gleichgewichtsdampfdruck einer Substanz.

$p_{\text {Gas }}$ Im Experiment eingestellter Druck des koexpandierten Trägergases.

$p_{0}$ Initialer Druck einer Gasphase zu Beginn eines Expansionsprozesses; Stagnationsparameter.

$p_{1}$ Hintergrunddruck in der Expansionskammer der Molekularstrahlapparatur.

$Q_{\mathrm{IR}}$ Quotient der absoluten Spektralintensitäten bei $\tilde{\nu}_{\mathrm{IR}}=3420 \mathrm{~cm}^{-1}$ und $\tilde{\nu}_{\mathrm{IR}}=3180 \mathrm{~cm}^{-1}$.

$Q_{N}$ Quotient der Differenzen der arithmetischen Mittelpunkte der dreier aufeinander folgender Massensignale der Clustergrößen $N-2, N-1$, und $N$.

$q$ Ladung eines Ions.

$R$ allgemeine Gaskonstante (Kap. 2); elektrischer Widerstand (Kap. 4).

$R_{\mathrm{m}}$ Auflösungsvermögen eines Massenspektrometers.

$\vec{R}$ Generalisierte Ortskoordinate.

$r_{\text {ch }}$ charakteristischer Radius einer Substanz nach Hagena.

$r_{\mathrm{K}}$ Radius des Kerns eines Nanopartikels. 
$r^{*}$ Kritischer Radius im Modell der klassischen Nukleationstheorie.

$r_{\text {mono }}$ Effektiver Radius eines Atoms bzw. Moleküls.

$r$ Radius eines Clusters bzw. Nanopartikels.

$S$ Entropie ${ }^{\ddagger}$.

$S_{\mathrm{FC}, v^{\prime \prime}, v^{\prime}}^{2}$ Franck-Condon-Faktor eines elektronischen Übergangs.

$T$ Temperatur ${ }^{\ddagger}$

$T_{\|}$Auf die Besetzung der Translationsfreiheitsgrade bezogene Temperatur.

$T_{\text {ch }}$ charakteristische Temperatur einer Substanz nach Hagena.

$T_{\text {Düse }}$ Im Experiment eingestellte Temperatur der Düsenplatte der Molekularstrahlquelle.

$T_{\text {eq }}$ Temperatur bei Phasenkoexistenz.

$T_{\text {evap }}$ Evaporationstemperatur eines evaporativen Ensembles.

$T_{\mathrm{f}}$ Untere Grenze des Temperaturbereiches möglicher Phasenkoexistenz nach Berry.

$T_{\mathrm{G}}$ Glasübergangstemperatur.

$T_{\mathrm{H}}$ Homogene Nukleationstemperatur.

$T_{\mathrm{m}}$ Obere Grenze des Temperaturbereiches möglicher Phasenkoexistenz nach Berry.

$T_{\mathrm{O}}$ Im Experiment eingestellte Temperatur des Oberteils der Pickup-Zelle.

$T_{\text {Reservoir }}$ Im Experiment eingestellte Reservoirtemperatur der Molekularstrahlquelle.

$T_{\mathrm{U}}$ Temperatur des Unterteils der Pickup-Zelle.

$t^{\prime}$ Nummer des Kanals der Zählerkarte, dem das Signal eines am Detektor auftreffenden Teilchens zugeordnet wurde.

$t_{\text {Anfang, } n}^{\prime}$ Der Kanal mit der niedrigsten Nummer, der dem Signal der Clustergröße $N$ in den durch die Auswertungssoftware P7887 erstellten Histogrammen (Massenspektren) zugeordnet wird.

$t_{\mathrm{D}}$ Driftzeit eines Ions im Massenspektrometer.

$t_{\text {Ende, } n}^{\prime}$ Der Kanal mit der höchsten Nummer, der dem Signal der Clustergröße $N$ in den durch die Auswertungssoftware P7887 erstellten Histogrammen (Massenspektren) zugeordnet wird.

$\bar{t}_{N}$ Arithmetischer Mittelpunkt des Massensignales der Clustergröße $N$; berechnet als Mittelwert von $t_{\text {Anfang, } N}^{\prime}$ und $t_{\text {Ende, } N}^{\prime}$. 
$\bar{t}_{N}$ Arithmetischer Mittelwert der Grenzen $t_{\text {Start, } N}^{\prime}$ und $t_{\text {Ende, } N}^{\prime}$ des Massensignals der Clustergröße $N$.

$t_{0}$ Anfangszeitpunkt der Driftzeit $t_{\mathrm{D}}$ der Ionen im Flugzeitmassenspektrometer.

$t_{0}^{\prime}$ Parameter der Regressionsgleichung 5.19.

$t_{\text {Peak }, N}^{\prime}$ Nummer des Kanals mit der höchsten Zählrate innerhalb eines Signalbereiches (d.h. Position des Peaks) in den durch die Auswertungssoftware Pr8877 erstellten Histogrammen (Massenspektren).

$t_{\text {Start }, N}^{\prime}$ Nummer des Kanals, von dem ausgehend die Auswertungssoftware Maximum und Grenzen des im Bereich um diesen Kanal vorliegenden Massensignals ermittelt.

$U$ innere Energie eines Systems.

$U_{\text {el }}$ elektrische Spannung.

$U_{\mathrm{HV}}$ Am MCP-Detektor anliegende Hochspannung im Experiment.

$V$ Volumen ${ }^{\ddagger}$

$v_{\mathrm{D}}$ Driftgeschwindigkeit eines Ions im Massenspektrometer.

$v_{\mathrm{F}}$ Lokale Strömungsgeschwindigkeit eines Gases.

$\bar{v}_{\mathrm{F}}$ Mittlere, lokale Strömungsgeschwindigkeit eines Gases.

$v_{\mathrm{m}}$ Molares Volumen einer Substanz ${ }^{\ddagger}$.

$v_{\text {mono }}$ Effektives Volumen eines Atoms bzw. Moleküls.

$W_{\text {rev }}$ reversible Arbeit im Sinne der klassischen Thermodynamik.

$X$ Stoffmengenanteil ${ }^{\ddagger}$.

$x$ absoluter Abstand von der Düsenengstelle.

$x_{0}$ Ort der Ionisation eines Teilchens in der Ionenquelle.

$x_{\text {red }}$ reduzierter Abstand von der Düsenengstelle.

Z mikrokanonische Zustandssumme.

$z$ Ladungszahl eines Ions.

$\alpha$ Relativgeschwindigkeit der Konstituenten eines Molekularstrahls.

$\Gamma$ Skalierungsparameter nach Hagena.

$\Gamma^{*}$ reduzierter Skalierungsparameter nach Hagena.

$\gamma$ Mobilitätsparameter nach Berry (Abschnitt 2.2.2); Quotient $C_{p} / C_{V}$ (Abschnitt 3.1). 
$\Delta_{\mathrm{m}} H$ Schmelzenthalpie ${ }^{\ddagger}$.

$\Delta_{\mathrm{m}} S$ Schmelzentropie ${ }^{\ddagger}$.

$\Delta t$ (engl. delay) Zeitlicher Abstand zwischen IR- und UV-Laserpuls.

$\delta_{\mathrm{T}}$ Tolman-Länge.

$\Delta x$ von einem Ion im Massenspektrometer zurückgelegter Weg.

$\lambda_{\mathrm{M}}$ Mittlere freie Weglänge eines Teilchens in der Gasphase.

$\lambda_{\mathrm{UV}}$ Wellenlänge der ultravioletten Laserstrahlung.

$\mu$ chemisches Potential ${ }^{\ddagger}$.

$\tilde{\nu}_{\mathrm{IR}}$ Wellenzahl der Infrarotlaserstrahlung; Einheit: Reziproke Zentimeter $\left(\mathrm{cm}^{-1}\right)$.

$\Phi$ Übersättigungsverhältnis.

$\Psi$ Schwingungseigenfunktion eines elektronischen Zustands.

$\psi$ Skalierungsparameter nach Hagena.

$\psi^{*}$ reduzierter Skalierungsparameter nach Hagena.

$\rho$ Dichte.

$\rho_{0}$ Dichte der Gasphase im Substanzreservoir, Stagnationsparameter.

$\Sigma_{N}$ Signalintensität eines Massensignals; Summe über alle in den Kanälen $t^{\prime}$ erfassten Zählereignisse, die dem Massensignal der Clustergröße $N$ zugeordnet werden.

$\Sigma_{\mathrm{UV}, N}$ Signalintensität eines Massensignals ohne Anregung durch IR-Laserstrahlung; Summe über alle in den Kanälen $t^{\prime}$ erfassten Zählereignisse, die dem Massensignal der Clustergröße $N$ zugeordnet werden.

$\Sigma_{\text {IR+UV,N }}$ Signalintensität eines Massensignals nach kombinierter Anregung durch IRund UV-Strahlung; Summe über alle in den Kanälen $t^{\prime}$ erfassten Zählereignisse, die dem Massensignal der Clustergröße $N$ zugeordnet werden.

$\sigma$ Oberflächenspannung $\ddagger$.

$\sigma_{\mathrm{T}}$ Nach Tolman korrigierte Oberflächenspannung.

$\sigma_{\mathrm{PI}}$ Photoionisationsquerschnitt.

$\sigma_{\mathrm{s}}$ Stoßquerschnitt.

$\tau$ Lebensdauer einer Spezies.

$\varnothing$ Durchmesser.

¥ Diese Größe kann durch folgende Indices näher spezifiziert sein: Tiefgestellte Indices: l (Flüssigkeit), s (Feststoff), v (gasförmiger Zustand), i (Phase bzw. Komponente i); das Symbol ${ }^{\circ}$ kennzeichnet den Wert der Größe unter Standardbedingungen. 


\section{Abkürzungsverzeichnis}

AIE adiabatische Ionisationsenergie.

BNC Bayonet Neill-Concelman.

CT (engl. Charge Transfer) Ladungstransfer.

DA Donor-Akzeptor-Koordination eines Wassermoleküls.

DAA Donor-Akzeptor-Akzeptor-Koordination eines Wassermoleküls.

DDA Donor-Donor-Akzeptor-Koordination eines Wassermoleküls.

DN (frz. diamètre nominale) Nennweite.

EIE Erscheinungsionisationsenergie.

FKM Fluorkarbonkautschuk.

GND Erdpotential (engl. ground).

H-Atom Wasserstoffatom.

H-Brücke Wasserstoffbrückenbindung.

ICR (engl. Ion Cyclotron Resonance) Ionen-Zyklotron-Resonanz.

IP Ionisationspotential.

IR Infrarot.

IRPD (engl. Infrared Induced Photodissociation) Infrarot-induzierte Photodissoziation.

IUPAC International Union of Pure and Applied Chemistry.

IVR Interne, vibronische Relaxation.

KDP Kaliumdihydrogenphosphat.

KTA Kaliumtitanylarsenat.

KTP Kaliumtitanylphosphat.

M10 Metrisches ISO-Gewinde, Nenndurchmesser $10 \mathrm{~mm}$. 
MCP (engl. Microchannel Plate) Mikrokanalplatte.

MS Massenspektrometer.

Nd:YAG Neodymdotierter Yttrium-Aluminium-Granat.

O-Atom Sauerstoffatom.

OPA Optical Parametric Amplifier.

OPO Optical Parametric Oscillator.

PC (engl. Personal Computer) Einzelplatzrechner.

PSC (engl. Polar Stratospheric Clouds), polare, stratosphärische Wolken.

RE-TOF MS Reflektron-time-of-flight Massenspektrometer.

SEV Sekundärelektronenvervielfacher.

SHG (engl. Second Harmonic Generation) Frequenzverdopplung.

SIE Sättigungsionisationsenergie.

SMPS Scanning Mobility Particle Sizing.

THG (engl. Third Harmonic Generation) Frequenzverdreifachung.

TOF (engl. time of flight) Flugzeit.

UHV Ultrahochvakuum.

UV Ultraviolett.

VIE vertikale Ionisationsenergie.

VIS (engl. visible) sichtbarer Bereich des elektromagnetischen Spektrums.

VRT engl. Vibration-Rotation Tunneling Spectroscopy, Vibrations-Rotations-Tunnelspektroskopie. 


\section{Literatur}

[1] R. Ludwig, Angewandte Chemie 2001, 113, 1856-1876.

[2] Y. Maréchal, The hydrogen bond and the water molecule: The physics and chemistry of water, aqueous and bio media, 1st ed., Elsevier, Amsterdam und Boston, 2007.

[3] Water: A Comprehensive Treatise, (Hrsg.: F. Franks), Plenum Press, New York, 1972.

[4] H. Tanaka, K. Koga in Water in confining geometries, (Hrsg.: V. Buch, J. P. Devlin), Physics and astronomy online library, Springer, Berlin, 2003.

[5] A. Falenty, T. C. Hansen, W. F. Kuhs, Nature 2014, 516, 231-233.

[6] H.-C. Flemming in Chemie über den Wolken, (Hrsg.: R. Zellner), Sachbuch, WileyVCH Verlag GmbH \& Co. KGaA, Weinheim, 2011.

[7] D. Möller, J. Feichter, H. Herrmann in Chemie über den Wolken, (Hrsg.: R. Zellner), Sachbuch, Wiley-VCH Verlag GmbH \& Co. KGaA, Weinheim, 2011, S. 128-139.

[8] H. R. Pruppacher, Journal of the Atmospheric Sciences 1995, 52, 1924-1933.

[9] H. R. Pruppacher, J. D. Klett, Microphysics of Clouds and Precipitation: Second revised and enlarged edition with an introduction to cloud chemistry and cloud electricity, Kluwer, Dordrecht, 1997.

[10] B. J. Murray, T. W. Wilson, S. Dobbie, Z. Cui, S. M. R. K. Al-Jumur, O. Möhler, M. Schnaiter, R. Wagner, S. Benz, M. Niemand, H. Saathoff, V. Ebert, S. Wagner, B. Kärcher, Nature Geoscience 2010, 3, 233-237.

[11] B. J. Murray, E. J. Jensen, Journal of Atmospheric and Solar-Terrestrial Physics 2010, 72, 51-61.

[12] Murphy, D. S. Thomson, Mahoney, Science 1998, 282, 1664-1669.

[13] J. P. Devlin, N. Uras, J. Sadlej, V. Buch, Nature 2002, 417, 269-271.

[14] A. B. Horn, J. R. Sodeau in Water in confining geometries, (Hrsg.: V. Buch, J. P. Devlin), Physics and astronomy online library, Springer, Berlin, 2003, S. 295-334.

[15] J. P. Devlin, V. Buch, The Journal of Physical Chemistry 1995, 99, 16534-16548.

[16] D. J. Donaldson, K. T. Valsaraj, Environmental science \& technology 2010, 44, 865-873.

[17] M. Fárník, V. Poterya, Frontiers in chemistry 2014, 2, 4.

[18] B. L. Yoder, J. H. Litman, P. W. Forysinski, J. L. Corbett, R. Signorell, The Journal of Physical Chemistry Letters 2011, 2, 2623-2628. 
[19] M. Kulmala, J. Kontkanen, H. Junninen, K. Lehtipalo, H. E. Manninen, T. Nieminen, T. Petäjä, M. Sipilä, S. Schobesberger, P. Rantala, A. Franchin, T. Jokinen, E. Järvinen, M. Äijälä, J. Kangasluoma, J. Hakala, P. P. Aalto, P. Paasonen, J. Mikkilä, J. Vanhanen, J. Aalto, H. Hakola, U. Makkonen, T. Ruuskanen, R. L. Mauldin, J. Duplissy, H. Vehkamäki, J. Bäck, A. Kortelainen, I. Riipinen, T. Kurtén, M. V. Johnston, J. N. Smith, M. Ehn, T. F. Mentel, K. E. J. Lehtinen, A. Laaksonen, V.-M. Kerminen, D. R. Worsnop, Science 2013, 339, 943-946.

[20] C. Medcraft, D. McNaughton, C. D. Thompson, D. R. T. Appadoo, S. Bauerecker, E. G. Robertson, Physical Chemistry Chemical Physics 2013, 15, 3630-3639.

[21] A. Y. Zasetsky, A. F. Khalizov, M. E. Earle, J. J. Sloan, The journal of physical chemistry. A 2005, 109, 2760-2764.

[22] M. J. Molina, T. L. Tso, L. T. Molina, F. C. Wang, Science 1987, 238, 1253-1257.

[23] B. J. Gertner, J. T. Hynes, Science 1996, 271, 1563-1566.

[24] B. J. Finlayson-Pitts, J. N. Pitts, Chemistry of the upper and lower atmosphere: Theory, experiments, and applications, [Nachdr.], Academic Press, San Diego, Calif., 1999.

[25] E. Illenberger in Linking the Gaseous and Condensed Phases of Matter, (Hrsg.: L. G. Christophorou, E. Illenberger, W. F. Schmidt), NATO ASI Series, Series B, Springer Science/Plenum Press, New York, 1994, S. 151-153.

[26] J. Jortner, The European Physical Journal D 1992, 24, 247-275.

[27] R. S. Berry, T. L. Beck, H. L. Davis in Evolution of size effects in chemical dynamics, (Hrsg.: I. Prigogine, S. A. Rice), Advances in chemical physics, J. Wiley, New York, 1988, S. 75-138.

[28] P. Jena, A. W. Castleman, Proceedings of the National Academy of Sciences of the United States of America 2006, 103, 10560-10569.

[29] F. Huisken, M. Kaloudis, A. Kulcke, The journal of chemical physics 1996, 104, $17-25$.

[30] J. V. Coe, International Reviews in Physical Chemistry 2001, 20, 33-58.

[31] L. Ma, K. Majer, F. Chirot, B. v. Issendorff, The journal of chemical physics 2009, 131, 144303.

[32] K. Mizuse, N. Mikami, A. Fujii, Angewandte Chemie (International ed. in English) 2010, 49, 10119-10122.

[33] J. S. Prell, J. T. O'Brien, E. R. Williams, Journal of the American Chemical Society 2011, 133, 4810-4818.

[34] J. T. O'Brien, E. R. Williams, Journal of the American Chemical Society 2012, 134, 10228-10236.

[35] T. D. Märk in Linking the Gaseous and Condensed Phases of Matter, (Hrsg.: L. G. Christophorou, E. Illenberger, W. F. Schmidt), NATO ASI Series, Series B, Springer Science/Plenum Press, New York, 1994, S. 155-182.

[36] C. Bobbert, S. Schütte, C. Steinbach, U. Buck, The European Physical Journal D 2002, 19, 183-192. 
[37] U. Buck, A fully size-resolved perspective on the crystallization of water clusters, Göttingen, 2012-09-20.

[38] G. Torchet, P. Schwartz, J. Farges, M. F. de Feraudy, B. Raoult, The journal of chemical physics 1983, 79, 6196-6202.

[39] L. S. Bartell, Y. G. Chushak in Water in confining geometries, (Hrsg.: V. Buch, J. P. Devlin), Physics and astronomy online library, Springer, Berlin, 2003, S. 399424.

[40] U. Buck, C. Steinbach in Water in confining geometries, (Hrsg.: V. Buch, J. P. Devlin), Physics and astronomy online library, Springer, Berlin, 2003, S. 53-77.

[41] C. C. Pradzynski, R. M. Forck, T. Zeuch, P. Slavíček, U. Buck, Science 2012, 337, 1529-1532.

[42] Clusters of Atoms and Molecules I: Theory, experiment, and clusters of atoms ; 14 tables, 1. ed., corr. 2. print, (Hrsg.: H. Haberland), Springer, Berlin, 1995.

[43] S. Bauerecker, M. Taraschewski, C. Weitkamp, H. K. Cammenga, The Journal of Physical Chemistry 2001, 72, 3946-3955.

[44] J. P. Devlin, C. Joyce, V. Buch, The journal of physical chemistry. A 2000, 104, 1974-1977.

[45] M. Kappes, S. Leutwyler in Atomic and molecular beam methods, (Hrsg.: G. Scoles), Oxford Univ. Press, New York, 1988, S. 380-415.

[46] R. Maisonny, M. Capron, S. Maclot, J. C. Poully, A. Domaracka, A. Méry, L. Adoui, P. Rousseau, B. A. Huber, Journal of Physics: Conference Series 2013, 438, 012007.

[47] I. Braud, S. Zamith, J.-M. L'Hermite, The Review of scientific instruments 2017, 88, 043102 .

[48] U. Buck, C. C. Pradzynski, T. Zeuch, J. M. Dieterich, B. Hartke, Physical chemistry chemical physics : PCCP 2014, 16, 6859-6871.

[49] C. J. Gruenloh, Science 1997, 276, 1678-1681.

[50] W. Roth, M. Schmitt, C. Jacoby, D. Spangenberg, C. Janzen, K. Kleinermanns, Chemical Physics 1998, 239, 1-9.

[51] A. Fujii, K. Mizuse, International Reviews in Physical Chemistry 2013, 32, 266307.

[52] T. Hamashima, K. Mizuse, A. Fujii, The journal of physical chemistry. A 2011, $115,620-625$.

[53] C. Steinbach, U. Buck, The journal of physical chemistry. A 2006, 110, 3128-3131.

[54] F. Zurheide, C. W. H. R. Dierking, C. C. Pradzynski, R. M. Forck, F. Flüggen, U. Buck, T. Zeuch, The journal of physical chemistry. A 2015, 119, 2709-2720.

[55] A. Borodin, O. Höfft, U. Kahnert, V. Kempter, Y. Ferro, A. Allouche, The journal of chemical physics 2004, 120, 8692-8697.

[56] J.-H. Kim, Y.-K. Kim, H. Kang, The Journal of Physical Chemistry C 2009, 113, $321-327$. 
[57] R. M. Forck, I. Dauster, Y. Schieweck, T. Zeuch, U. Buck, M. Ončák, P. Slavíček, The journal of chemical physics 2010, 132, 221102.

[58] R. M. Forck, J. M. Dieterich, C. C. Pradzynski, A. L. Huchting, R. A. Mata, T. Zeuch, Physical chemistry chemical physics : PCCP 2012, 14, 9054-9057.

[59] N. Gimelshein, S. Gimelshein, C. C. Pradzynski, T. Zeuch, U. Buck, The journal of chemical physics 2015, 142, 244305.

[60] C. W. H. R. Dierking, F. Zurheide, T. Zeuch, J. Med, S. Parez, P. Slavíček, The journal of chemical physics 2017, 146, 244303.

[61] C. W. H. R. Dierking, Dissertation, Georg-August-Universität Göttingen, Göttingen, 2019.

[62] V. Buch, S. Bauerecker, J. P. Devlin, U. Buck, J. K. Kazimirski, International Reviews in Physical Chemistry 2004, 23, 375-433.

[63] J. M. Anglada, G. J. Hoffman, L. V. Slipchenko, M. M. Costa, M. F. Ruiz-López, J. S. Francisco, The journal of physical chemistry. A 2013, 117, 10381-10396.

[64] E. B. Moore, V. Molinero, Nature 2011, 479, 506-508.

[65] M. Lippe, S. Chakrabarty, J. J. Ferreiro, K. K. Tanaka, R. Signorell, The journal of chemical physics 2018, 149, 244303.

[66] T. Koop, B. Luo, A. Tsias, T. Peter, Nature 2000, 406, 611-614.

[67] T. Li, D. Donadio, G. Galli, Nature communications 2013, 4, 1887.

[68] J. C. Johnston, V. Molinero, Journal of the American Chemical Society 2012, 134, 6650-6659.

[69] J. K. Kazimirski, V. Buch, The journal of physical chemistry. A 2003, 107, 97629775 .

[70] B. Bandow, B. Hartke, The journal of physical chemistry. A 2006, 110, 5809-5822.

[71] C. C. Pradzynski, Dissertation, Georg-August-Universität Göttingen, Göttingen, 2014.

[72] V. Babin, G. R. Medders, F. Paesani, Journal of chemical theory and computation 2014, 10, 1599-1607.

[73] S. K. Reddy, D. R. Moberg, S. C. Straight, F. Paesani, The journal of chemical physics 2017, 147, 244504.

[74] D. R. Moberg, S. C. Straight, F. Paesani, The journal of physical chemistry. B 2018, 122, 4356-4365.

[75] A. Manka, H. Pathak, S. Tanimura, J. Wölk, R. Strey, B. E. Wyslouzil, Physical chemistry chemical physics : PCCP 2012, 14, 4505-4516.

[76] R. J. Cooper, M. J. DiTucci, T. M. Chang, E. R. Williams, Journal of the American Chemical Society 2016, 138, 96-99.

[77] V. Buch, J. P. Devlin, The journal of chemical physics 1999, 110, 3437-3443.

[78] L. Delzeit, D. Blake, Journal of Geophysical Research: Planets 2001, 106, 3337133379 . 
[79] J. P. Devlin, V. Buch in Water in confining geometries, (Hrsg.: V. Buch, J. P. Devlin), Physics and astronomy online library, Springer, Berlin, 2003, S. 425-462.

[80] R. S. Berry, J. Jellinek, G. Natanson, Physical Review A 1984, 30, 919-931.

[81] R. S. Berry in Theory of atomic and molecular clusters, (Hrsg.: J. Jellinek), Springer Series in Cluster Physics, Springer, Berlin, 1999.

[82] J. Jortner, Berichte der Bunsengesellschaft für physikalische Chemie 1984, 88, 188-201.

[83] H. Pauly, Atom, Molecule, and Cluster Beams II: Cluster Beams, Fast and Slow Beams, Accessory Equipment and Applications, Springer, Berlin, 2000.

[84] C. F. Landes, M. Braun, M. A. El-Sayed, The Journal of Physical Chemistry B 2001, 105, 10554-10558.

[85] Van der Waals, Johannes Diderik, Doktorarbeit, Universität Leiden, Leiden, 1873.

[86] R. S. Berry in Linking the Gaseous and Condensed Phases of Matter, (Hrsg.: L. G. Christophorou, E. Illenberger, W. F. Schmidt), NATO ASI Series, Series B, Springer Science/Plenum Press, New York, 1994, S. 231-249.

[87] W. A. de Heer, Reviews of Modern Physics 1993, 65, 611-676.

[88] T. M. Soini, N. Rösch, Physical Chemistry Chemical Physics 2015, 17, 2846328483.

[89] M. Knapp, O. Echt, D. Kreisle, E. Recknagel, The Journal of Physical Chemistry 1987, 91, 2601-2607.

[90] T. Schindler, C. Berg, G. Niedner-Schatteburg, V. E. Bondybey, Chemical Physics Letters 1996, 250, 301-308.

[91] S. Kazachenko, A. J. Thakkar, The journal of chemical physics 2013, 138, 194302.

[92] T. Zeuch, U. Buck, Chemical Physics Letters 2013, 579, 1-10.

[93] R. N. Barnett, U. Landman, C. L. Cleveland, J. Jortner, Chemical Physics Letters 1988, 145, 382-386.

[94] R. N. Barnett, U. Landman, C. L. Cleveland, N. R. Kestner, J. Jortner, Chemical Physics Letters 1988, 148, 249-252.

[95] R. N. Barnett, U. Landman, Physical review letters 1993, 70, 1775-1778.

[96] C. Steinbach, U. Buck, The journal of chemical physics 2005, 122, 134301.

[97] J. R. R. Verlet, A. E. Bragg, A. Kammrath, O. Cheshnovsky, D. M. Neumark, Science 2005, 307, 93-96.

[98] S. Barth, M. Ončák, V. Ulrich, M. Mucke, T. Lischke, P. Slavíček, U. Hergenhahn, The journal of physical chemistry. A 2009, 113, 13519-13527.

[99] J. R. R. Verlet, A. Kammrath, G. B. Griffin, D. M. Neumark, The journal of chemical physics 2005, 123, 231102.

[100] I. V. Hertel, Hüglin, Nitsch, C. P. Schulz, Physical review letters 1991, 67, 17671770 . 
[101] R. N. Barnett, U. Landman, C. L. Cleveland, J. Jortner, Canadian Journal of Chemistry 1988, 88, 4421-4428.

[102] J. V. Coe, G. H. Lee, J. G. Eaton, S. T. Arnold, H. W. Sarkas, K. H. Bowen, C. Ludewigt, H. Haberland, D. R. Worsnop, The journal of chemical physics 1990, 92, 3980-3982.

[103] G. Kaptay, Journal of nanoscience and nanotechnology 2012, 12, 2625-2633.

[104] G. Wedler, Lehrbuch der physikalischen Chemie, 5., vollst. überarb. und aktualis. Aufl., 2. Nachdr, Wiley-VCH, Weinheim, 2010.

[105] J. H. Seinfeld, S. N. Pandis, Atmospheric chemistry and physics: From air pollution to climate change, Wiley, New York, 1998.

[106] J. Liu, C. E. Nicholson, S. J. Cooper, Langmuir : the ACS journal of surfaces and colloids 2007, 23, 7286-7292.

[107] R. C. Tolman, The journal of chemical physics 1949, 17, 333-337.

[108] S. M. A. Malek, P. H. Poole, I. Saika-Voivod, The journal of chemical physics 2019, 150, 234507.

[109] G. Tammann, Zeitschrift für Physikalische Chemie 1910, 68, 205.

[110] Frenken, J. F. van der Veen, Physical review letters 1985, 54, 134-137.

[111] J. G. Dash, H. Fu, J. S. Wettlaufer, Reports on Progress in Physics 1995, 58, 115-167.

[112] V. F. Petrenko, R. W. Whitworth, Physics of ice, 1., Oxford Univ. Press, Oxford, 1999.

[113] C. Gerthsen, D. Meschede, Physik, 23., überarb. Aufl., Springer, Berlin, 2006.

[114] R. Kofman, P. Cheyssac, A. Aouaj, Y. Lereah, G. Deutscher, T. Ben-David, J. M. Penisson, A. Bourret, Surface Science 1994, 303, 231-246.

[115] L. van Hove, Physica 1949, 15, 951-961.

[116] T. L. Hill, Thermodynamics of small systems, Dover Publications, New York, 2003.

[117] S. Gartenhaus, C. Schwartz, Physical Review 1957, 108, 482-490.

[118] F. H. Stillinger, T. A. Weber, The journal of chemical physics 1984, 81, 50955103.

[119] D. J. Wales, M. P. Hodges, Chemical Physics Letters 1998, 286, 65-72.

[120] B. Hartke, Zeitschrift für Physikalische Chemie 2000, 214, 1.

[121] N. Kastelowitz, V. Molinero, ACS nano 2018, 12, 8234-8239.

[122] J. J. Dannenberg, L. Haskamp, A. Masunov, The journal of physical chemistry. A 1999, 103, 7083-7086.

[123] E. Arunan, G. R. Desiraju, R. A. Klein, J. Sadlej, S. Scheiner, I. Alkorta, D. C. Clary, R. H. Crabtree, J. J. Dannenberg, P. Hobza, H. G. Kjaergaard, A. C. Legon, B. Mennucci, D. J. Nesbitt, Pure and Applied Chemistry 2011, 83, 1637-1641.

[124] C. N. R. Rao in Water: A Comprehensive Treatise, (Hrsg.: F. Franks), Plenum Press, New York, 1972. 
[125] S. C. Silva, J. P. Devlin, The Journal of Physical Chemistry 1994, 98, 1084710852 .

[126] J. E. Huheey, E. A. Keiter, R. L. Keiter, R. Steudel, Anorganische Chemie: Prinzipien von Struktur und Reaktivität, 3., durchges. Aufl., de Gruyter, Berlin, 2003.

[127] R. J. Speedy, C. A. Angell, The journal of chemical physics 1976, 65, 851-858.

[128] J. Huang, L. S. Bartell, The Journal of Physical Chemistry 1995, 99, 3924-3931.

[129] M. Schmidt, B. v. Issendorff, The journal of chemical physics 2012, 136, 164307.

[130] M. Matsumoto, S. Saito, I. Ohmine, Nature 2002, 416, 409-413.

[131] T. L. Malkin, B. J. Murray, C. G. Salzmann, V. Molinero, S. J. Pickering, T. F. Whale, Physical chemistry chemical physics : PCCP 2015, 17, 60-76.

[132] H. König, Zeitschrift für Kristallographie - Crystalline Materials 1943, 105, 279286.

[133] B. J. Murray, C. G. Salzmann, A. J. Heymsfield, S. Dobbie, R. R. Neely, C. J. Cox, Bulletin of the American Meteorological Society 2015, 96, 1519-1531.

[134] J. Sadlej, V. Buch, J. K. Kazimirski, U. Buck, The journal of physical chemistry. A 1999, 103, 4933-4947.

[135] O. Andersson, H. Suga, Solid State Communications 1994, 91, 985-988.

[136] T. L. Malkin, B. J. Murray, A. V. Brukhno, J. Anwar, C. G. Salzmann, Proceedings of the National Academy of Sciences of the United States of America 2012, 109, 1041-1045.

[137] H. Kiefte, M. J. Clouter, E. Whalley, The journal of chemical physics 1984, 81, 1419-1420.

[138] T. Takahashi, Journal of Crystal Growth 1982, 441-449.

[139] G. P. Johari, The journal of chemical physics 2005, 122, 194504.

[140] A. Y. Zasetsky, S. V. Petelina, I. M. Svishchev, Atmospheric Chemistry and Physics 2009, 9, 965-971.

[141] T. R. Dyke, J. S. Muenter, The journal of chemical physics 1974, 60, 2929-2930.

[142] M. F. Vernon, D. J. Krajnovich, H. S. Kwok, J. M. Lisy, Y. R. Shen, Y. T. Lee, The journal of chemical physics 1982, 77, 47-57.

[143] U. Buck, F. Huisken, Chemical reviews 2000, 100, 3863-3890.

[144] H. Kabrede, R. Hentschke, The Journal of Physical Chemistry B 2003, 107, 39143920 .

[145] S. Kazachenko, A. J. Thakkar, Molecular Physics 2010, 108, 2187-2193.

[146] T. R. Dyke, K. M. Mack, J. S. Muenter, The journal of chemical physics 1977, $66,498-510$.

[147] J. P. Devlin, J. Sadlej, V. Buch, The journal of physical chemistry. A 2001, 105, 974-983.

[148] N. Pugliano, R. J. Saykally, The journal of chemical physics 1992, 96, 1832-1839. 
[149] N. Pugliano, R. J. Saykally, Science 1992, 257, 1937-1940.

[150] J. D. Cruzan, L. B. Braly, K. Liu, M. G. Brown, J. G. Loeser, R. J. Saykally, Science 1996, 271, 59-62.

[151] K. Liu, M. G. Brown, J. D. Cruzan, R. J. Saykally, Science 1996, 271, 62-64.

[152] K. Liu, M. G. Brown, C. Carter, R. J. Saykally, J. K. Gregory, D. C. Clary, Nature 1996, 381, 501-503.

[153] K. Liu, J. D. Cruzan, R. J. Saykally, Science 1996, 271, 929-933.

[154] U. Buck, H. Meyer, Physical review letters 1984, 52, 109-112.

[155] K. E. Otto, Z. Xue, P. Zielke, M. A. Suhm, Physical chemistry chemical physics : PCCP 2014, 16, 9849-9858.

[156] S. E. Brown, A. W. Götz, X. Cheng, R. P. Steele, V. A. Mandelshtam, F. Paesani, Journal of the American Chemical Society 2017, 139, 7082-7088.

[157] C. Pérez, M. T. Muckle, D. P. Zaleski, N. A. Seifert, B. Temelso, G. C. Shields, Z. Kisiel, B. H. Pate, Science 2012, 336, 897-901.

[158] C. Steinbach, P. Andersson, M. Melzer, J. K. Kazimirski, U. Buck, V. Buch, Physical Chemistry Chemical Physics 2004, 6, 3320-3324.

[159] C. J. Tainter, J. L. Skinner, The journal of chemical physics 2012, 137, 104304.

[160] Nauta, Miller, Science 2000, 287, 293-295.

[161] U. Buck, I. Ettischer, M. Melzer, V. Buch, J. Sadlej, Physical review letters 1998, $80,2578-2581$.

[162] J. Brudermann, M. Melzer, U. Buck, J. K. Kazimirski, J. Sadlej, V. Buch, The journal of chemical physics 1999, 110, 10649-10652.

[163] U. Buck, The Journal of Physical Chemistry 1988, 92, 1023-1031.

[164] J. Brudermann, U. Buck, V. Buch, The journal of physical chemistry. A 2002, $106,453-457$.

[165] T. Watanabe, T. Ebata, S. Tanabe, N. Mikami, The journal of chemical physics 1996, 105, 408-419.

[166] P. Andersson, C. Steinbach, U. Buck, The European Physical Journal D 2003, 24, $53-56$.

[167] C. J. Burnham, S. S. Xantheas, Molecular Physics 2002, 116, 5115.

[168] A. Lagutschenkov, G. S. Fanourgakis, G. Niedner-Schatteburg, S. S. Xantheas, The journal of chemical physics 2005, 122, 194310.

[169] H. Haberland, C. Ludewigt, H.-G. Schindler, D. R. Worsnop, Surface Science 1985, $156,157-164$.

[170] C. Hock, M. Schmidt, R. Kuhnen, C. Bartels, L. Ma, H. Haberland, B. v. Issendorff, Physical review letters 2009, 103, 073401.

[171] B. Hartke, Physical Chemistry Chemical Physics 2003, 5, 275-284.

[172] W. Rau, Zeitschrift für Naturforschung A 1951, 6, 649-657. 
[173] G. P. Johari, G. Fleissner, A. Hallbrucker, E. Mayer, The Journal of Physical Chemistry 1994, 98, 4719-4725.

[174] E. W. Becker, K. Bier, W. Henkes, Z. Physik (Zeitschrift für Physik) 1956, 146, $333-338$.

[175] D. R. Miller in Atomic and molecular beam methods, (Hrsg.: G. Scoles), Oxford Univ. Press, New York, 1988, S. 15-53.

[176] P. Milani, S. Iannotta, Cluster Beam Synthesis of Nanostructured Materials, Springer, Berlin, Heidelberg, 1999.

[177] H. Pauly, Atom, Molecule, and Cluster Beams I: Basic theory, production, and detection of thermal energy beams, Springer, Berlin, 2000.

[178] G. D. Stein in Contribution of Clusters Physics to Materials Science and Technology, (Hrsg.: J. Davenas, P. M. Rabette), Springer Netherlands, Dordrecht, 1986.

[179] S. Schütte, U. Buck, International Journal of Mass Spectrometry 2002, 220, 183192.

[180] O. F. Hagena, The European Physical Journal D 1987, 4, 291-299.

[181] G. D. Stein, Surface Science 1985, 156, 44-56.

[182] P. L. Owen, M. A. Thornhill, The Flow in an Axially-Symmetric Supersonic Jet from a Nearly-Sonic Orifice into a Vacuum, London, 1948.

[183] W. Demtröder, H.-J. Foth, Physik Journal 1987, 43, 7-13.

[184] S. Iannotta, T. Toccoli, Journal of Polymer Science Part B: Polymer Physics 2003, 41, 2501-2521.

[185] J. B. Anderson, R. P. Andres, J. B. Fenn in Advances in Chemical Physics, (Hrsg.: J. Ross), Advances in chemical physics, John Wiley \& Sons, Inc, Hoboken, NJ, USA, 1966, S. 275-317.

[186] A. Kantrowitz, J. Grey, Review of Scientific Instruments 1951, 22, 328-332.

[187] R. E. Smalley, L. Wharton, D. H. Levy, Accounts of Chemical Research 1977, 10, 139-145.

[188] O. F. Hagena, Surface Science 1981, 106, 101-116.

[189] C. E. Klots, The journal of chemical physics 1985, 83, 5854-5860.

[190] C. E. Klots, Nature 1987, 327, 222-223.

[191] C. E. Klots, The European Physical Journal D 1991, 20, 105-109.

[192] C. E. Klots, The European Physical Journal D 1987, 5, 83-89.

[193] O. F. Hagena, W. Obert, The journal of chemical physics 1972, 56, 1793-1802.

[194] O. F. Hagena in Rarefied Gas Dynamics, (Hrsg.: L. Trilling, H. Y. Wachmann), Academic Press, New York, 1969, S. 1465-1468.

[195] R. M. Young, M. A. Yandell, S. B. King, D. M. Neumark, The journal of chemical physics 2012, 136, 094304.

[196] C. Huang, V. V. Kresin, A. Pysanenko, M. Fárník, The journal of chemical physics 2016, 145, 104304. 
[197] C. Hock, C. Bartels, S. Strassburg, M. Schmidt, H. Haberland, B. v. Issendorff, A. Aguado, Physical review letters 2009, 102, 043401.

[198] C. P. Schulz, R. Haugstätter, H.-U. Tittes, I. V. Hertel, Physical review letters 1986, 5\%, 1703-1706.

[199] C. P. Schulz, R. Haugstätter, H.-U. Tittes, I. V. Hertel, The European Physical Journal D 1988, 10, 279-290.

[200] F. Misaizu, K. Tsukamoto, M. Sanekata, K. Fuke, Chemical Physics Letters 1992, $188,241-246$.

[201] N. Okai, H. Ishikawa, K. Fuke, Chemical Physics Letters 2005, 415, 155-160.

[202] H.-D. Jakubke, Lexikon der Chemie, 1. Aufl., Spektrum Akademischer Verlag, Heidelberg, Berlin, 1998.

[203] E. Riedel, Anorganische Chemie, 4. Aufl., de Gruyter, Berlin, 1999.

[204] J. Lengyel, A. Pysanenko, P. Rubovič, M. Fárník, The European Physical Journal D 2015, 69, 23.

[205] J. H. Litman, B. L. Yoder, B. Schläppi, R. Signorell, Physical chemistry chemical physics : PCCP 2013, 15, 940-949.

[206] J. Lengyel, A. Pysanenko, J. Kočišek, V. Poterya, C. C. Pradzynski, T. Zeuch, P. Slavíček, M. Fárník, The Journal of Physical Chemistry Letters 2012, 3, 30963101.

[207] D. Šmídová, J. Lengyel, A. Pysanenko, J. Med, P. Slavíček, M. Fárník, The Journal of Physical Chemistry Letters 2015, 6, 2865-2869.

[208] B. Schläppi, J. J. Ferreiro, J. H. Litman, R. Signorell, International Journal of Mass Spectrometry 2014, 372, 13-21.

[209] C. Steinbach, Dissertation, Georg-August-Universität Göttingen, Göttingen, 2000.

[210] U. Buck, C. Steinbach, The journal of physical chemistry. A 1998, 102, 7333-7336.

[211] C. Steinbach, U. Buck, Physical Chemistry Chemical Physics 2005, 7, 986.

[212] J. Fedor, V. Poterya, A. Pysanenko, M. Fárník, The journal of chemical physics 2011, 135, 104305.

[213] R. Moro, R. Rabinovitch, V. V. Kresin, Review of Scientific Instruments 2005, 76,056104 .

[214] P. J. Campagnola, L. A. Posey, M. A. Johnson, The journal of chemical physics 1991, 95, 7998-8004.

[215] P. E. Mason, F. Uhlig, V. Vaněk, T. Buttersack, S. Bauerecker, P. Jungwirth, Nature chemistry 2015, 7, 250-254.

[216] L. Bewig, U. Buck, S. Rakowsky, M. Reymann, C. Steinbach, The journal of physical chemistry. A 1998, 102, 1124-1129.

[217] C. J. Mundy, J. Hutter, M. Parrinello, Journal of the American Chemical Society 2000, 122, 4837-4838.

[218] F. Mercuri, C. J. Mundy, M. Parrinello, The journal of physical chemistry. A 2001, $105,8423-8427$. 
[219] H. Shinohara, N. Nishi, N. Washida, The journal of chemical physics 1986, 84, 5561-5567.

[220] Ciocca, Burkhardt, Leventhal, Bergeman, Physical review. A Atomic molecular and optical physics 1992, 45, 4720-4730.

[221] R. Takasu, F. Misaizu, K. Hashimoto, K. Fuke, The journal of physical chemistry. A 1997, 101, 3078-3087.

[222] K. R. Siefermann, Y. Liu, E. Lugovoy, O. Link, M. Faubel, U. Buck, B. Winter, B. Abel, Nature chemistry 2010, 2, 274-279.

[223] K. R. Siefermann, B. Abel, Angewandte Chemie 2011, 123, 5374-5383.

[224] J. M. Herbert, M. P. Coons, Annual review of physical chemistry 2017, 68, 447472 .

[225] L. D. Jacobson, J. M. Herbert, Journal of the American Chemical Society 2011, 133, 19889-19899.

[226] A. Lietard, J. R. R. Verlet, The Journal of Physical Chemistry Letters 2019, 10, $1180-1184$.

[227] E. U. Condon, Physical Review 1928, 32, 858-872.

[228] M. Born, R. Oppenheimer, Annalen der Physik 1927, 389, 457-484.

[229] H. Mustroph, S. Ernst, Chemie in unserer Zeit 2011, 45, 256-269.

[230] E. A. Gislason, The journal of chemical physics 1973, 58, 3702-3707.

[231] H. Haberland, Surface Science 1985, 156, 305-312.

[232] U. Buck in The Chemical Physics of Atomic and Molecular Clusters, (Hrsg.: G. Scoles), North-Holland, Amsterdam und Oxford, 1990, S. 543-578.

[233] K. Hashimoto, K. Morokuma, Journal of the American Chemical Society 1994, $116,11436-11443$.

[234] L. Cwiklik, U. Buck, W. Kulig, P. Kubisiak, P. Jungwirth, The journal of chemical physics 2008, 128, 154306.

[235] F. Dong, S. Heinbuch, J. J. Rocca, E. R. Bernstein, The journal of chemical physics 2006, 124, 224319.

[236] R. M. Forck, C. C. Pradzynski, S. Wolff, M. Ončák, P. Slavíček, T. Zeuch, Physical chemistry chemical physics : PCCP 2012, 14, 3004-3016.

[237] S. Wolff, Masterarbeit, Georg-August-Universität Göttingen, Göttingen, 2014.

[238] D. Becker, Bachelorarbeit, Georg-August-Universität Göttingen, Göttingen, 2016.

[239] D. Becker, Masterarbeit, Georg-August-Universität Göttingen, Göttingen, 2018.

[240] A. E. Bragg, J. R. R. Verlet, A. Kammrath, O. Cheshnovsky, D. M. Neumark, Science 2004, 306, 669-671.

[241] G. Niedner-Schatteburg, V. E. Bondybey, Chemical reviews 2000, 100, 4059-4086.

[242] J. Lengyel, A. Pysanenko, V. Poterya, J. Kočišek, M. Fárník, Chemical Physics Letters 2014, 612, 256-261. 
[243] K. Mizuse, T. Hamashima, A. Fujii, The journal of physical chemistry. A 2009, 113, 12134-12141.

[244] E. Whalley, D. D. Klug, The journal of chemical physics 1986, 84, 4807-4809.

[245] E. Whalley, Canadian Journal of Chemistry 1977, 55, 3429-3441.

[246] F. Paesani, Accounts of Chemical Research 2016, 49, 1844-1851.

[247] U. Buck, I. Dauster, B. Gao, Z.-f. Liu, The journal of physical chemistry. A 2007, 111, 12355-12362.

[248] J. H. Gross, Mass spectrometry: A textbook; with tables, corr. 2nd printing Edition, Springer, Berlin, Heidelberg, 2004.

[249] H. Budzikiewicz, M. Schäfer, Massenspektrometrie: Eine Einführung, 6., vollst. überarb. und aktualisierte Aufl., Wiley-VCH, Weinheim, 2013.

[250] R. J. Cotter, Analytical chemistry 1992, 64, 1027A-1039A.

[251] I. Dauster, Dissertation, Georg-August-Universität Göttingen, Göttingen, 2008.

[252] W. C. Wiley, I. H. McLaren, Review of Scientific Instruments 1955, 26, 11501157.

[253] B. A. Mamyrin, V. I. Karataev, D. V. Shmikk, V. A. Zagulin, Sov. Phys. 1973, $37,45-48$.

[254] G. W. Fraser, International Journal of Mass Spectrometry 2002, 215, 13-30.

[255] M. Hobein, Dissertation, Georg-August-Universität Göttingen, Göttingen, 1994.

[256] S. Schütte, Dissertation, Georg-August-Universität Göttingen, Göttingen, 1996.

[257] C. C. Pradzynski, Masterarbeit, Georg-August-Universität Göttingen, Göttingen, 2010.

[258] R. M. Forck, Dissertation, Georg-August-Universität Göttingen, Göttingen, 2012.

[259] J. L. Wiza, Nuclear Instruments and Methods 1979, 162, 587-601.

[260] A. Risch, Dissertation, Universität Freiburg, Freiburg, 1991.

[261] D. R. Guyer, LaserVison Manual, Bellevue, WA, 2009.

[262] D. L. Andrews, Lasers in Chemistry, Third Edition, Springer, Berlin, Heidelberg, 1997.

[263] Continuum, Powerlite Precision II Scientific Laser System: Manual: Manual, (Hrsg.: Continuum), 2002.

[264] Continuum, SI-2000 Seeder System - Operation and Service Manual, (Hrsg.: Continuum), 2008.

[265] W. R. Bosenberg, D. R. Guyer, Applied Physics Letters 1992, 61, 387-389.

[266] W. R. Bosenberg, D. R. Guyer, Applied Physics Letters 1993, 10, 1716.

[267] D. R. Guyer, Broadband OPO/A System Manual, (Hrsg.: LaserVision), 2014.

[268] Continuum, EX OPO \& EX PLUS OPO - Operation and Maintenance, Manual, (Hrsg.: Continuum), 2011.

[269] Continuum, Powerlite ${ }^{\mathrm{TM}}$ DLS Laser System, Manual, (Hrsg.: Continuum), 2010. 
[270] Sirah, Pulsed dye laser, User Manual, (Hrsg.: Sirah), 2012.

[271] J. T. Hunt, J. A. Glaze, W. W. Simmons, P. A. Renard, Applied optics 1978, 17, 2053-2057.

[272] M. Harrison, Illustrated guide to Python 3: A complete walkthrough of beginning Python with unique illustrations showing how Python really works, hairysun.com, [Vereinigte Staaten], 2017.

[273] R. Jansen, I. Wysong, S. Gimelshein, M. Zeifman, U. Buck, The journal of chemical physics 2010, 132, 244105.

[274] D. R. Moberg, D. Becker, C. W. H. R. Dierking, F. Zurheide, B. Bandow, U. Buck, A. Hudait, V. Molinero, F. Paesani, T. Zeuch, Proceedings of the National Academy of Sciences of the United States of America 2019, DOI 10.1073/pnas. 1914254116.

[275] F. Flüggen, Bachelorarbeit, Georg-August-Universität Göttingen, Göttingen, 2013.

[276] Bandow Bernhard, : persönliche Mitteilung, 2019.

[277] N. Uras, V. Buch, J. P. Devlin, The Journal of Physical Chemistry B 2000, 104, 9203-9209.

[278] F. Hofmeister, Archiv für Experimentelle Pathologie und Pharmakologie 1888, 24, $247-260$.

[279] V. Molinero, E. B. Moore, The Journal of Physical Chemistry B 2009, 113, 40084016.

[280] D. W. Moreau, H. Atakisi, R. E. Thorne, IUCrJ 2019, 6, 346-356.

[281] G. A. Cisneros, K. T. Wikfeldt, L. Ojamäe, J. Lu, Y. Xu, H. Torabifard, A. P. Bartók, G. Csányi, V. Molinero, F. Paesani, Chemical reviews 2016, 116, 75017528 .

[282] J. Behler, Angewandte Chemie 2017, 129, 13006-13020.

[283] H. S. Thoke, S. Thorsteinsson, R. P. Stock, L. A. Bagatolli, L. F. Olsen, Scientific reports $\mathbf{2 0 1 7}$, 7, 16250.

[284] H. S. Thoke, L. A. Bagatolli, L. F. Olsen, Integrative Biology 2018, 10, 587-597. 



\section{Danksagung}

Während der Forschungsarbeiten für die vorliegende Dissertationsschrift wurde mir die Unterstützung von zahlreicher Menschen zuteil, die maßgeblich zum Gelingen dieses Vorhabens beigetragen haben:

Mein besonderer Dank gilt Herrn Prof. Dr. Thomas Zeuch für die Aufnahme in seinen Arbeitskreis und die Betreuung dieser Arbeit, für seine zahlreichen Hilfestellungen und für anregende Diskussionen, für die Freiheiten, die er mir gelassen sowie für das Vertrauen und für die Geduld, die er mir besonders in schwierigen Phasen entgegengebracht hat.

Herrn Prof. Dr. Burkhard Geil danke ich für die anregenden Gespräche und seine Unterstützung bei der Durchführung der Experimente sowie für seine Einführung in die Programmiersprache Python, die sich als wertvolles und vielseitiges Werkzeug erwies.

Herrn Prof. Dr. Dietmar Stalke danke ich für seine spontane Breitschaft, das Korreferat der vorliegenden Dissertationsschrift zu übernehmen.

Bei Herrn Dr. Christoph Pradzynski und Herrn Dr. Richard Forck bedanke ich mich für die Einweisung in die Bedienung der Molekularstrahlapparatur P11. Herrn Dr. Christoph Dierking danke ich für die kollegiale Zusammenarbeit und seine Unterstützung bei Reparaturarbeiten der Apparatur. Daniel Becker Sabine Wolff und Arne Sandmann die sich während meiner Promotionszeit für Bachelor- bzw. Masterarbeiten den an der P11 betriebenen Forschungen anschlossen danke ich für ihre Unterstützung und Mitwirkung bei den umfangreichen Wartungs- und Justagearbeiten, die der Messbetrieb mit sich bringt, ihre Mitarbeit während der langen Messtage sowie ihre Beiträge zur Auswertung des umfangreichen Datenmaterials, das wir an diesen Tagen erhoben haben. Herrn Dr. Christoph Dierking und Herrn Richard Cooper, Ph.D. danke ich außerdem für die freundliche Überlassung der Rohdaten ihrer Messergebnisse.

$\mathrm{Zu}$ großem Dank verpflichtet bin ich den MitarbeiterInnen der institutseigenen Werkstätten, namentlich der Feinmechanikwerkstatt unter der Leitung von Herrn Volker Meyer bzw. Mike Zippert und Herrn Reinhard Hildebrandt, der Elektronikwerkstatt unter der Leitung von Herrn Andreas Knorr sowie der glastechnischen Werkstatt und ihrer Leiterin Frau Annika von Roden. Ohne die Expertise und den Erfahrungsschatz dieser Menschen, ihre Flexibilität und Hilfsbereitschaft, bei Defekten der Apparatur schnell und unkompliziert Abhilfe zu schaffen, wären der Betrieb und die Weiterentwicklung eines derart komplexen und anfälligen Versuchsaufbaus nicht möglich.

Ein besonderer Dank geht auch an Herrn Sven Pioch für seinen Einsatz bei der Rettung unserer Diffusionspumpe. Danken möchte ich weiterhin Herrn Norbert Neisen 
für seine Unterstützung bei der Behebung von EDV-Problemen sowie Herrn Werner Noack, von dessen umfangreichem Erfahrungsschatz ich bei technischen Fragestellungen sehr profitiert habe. Herrn Michael Schlote danke ich für die Bereitstellung bidestillierten Wassers als Analyt. Herrn Clemens Heymann danke ich für seinen Rat und seine Unterstützung bei zahlreichen alltäglichen Handgriffen im Laborbetrieb, insbesondere bei der Entsorgung verbrauchter Farbstofflösungen und der Versorgung der Apparatur mit Betriebsgasen während des Messbetriebs.

Den Mitgliedern des AK Suhm danke ich für den anregenden Austausch über unsere Arbeitsfelder, für zahlreiche Ratschläge bezüglich der großen und kleinen Fallstricke des Forscherlebens, für die angenehme und kollegiale Atmosphäre und die vielen schönen Stunden im Rahmen von Feierlichkeiten und Ausflügen.

Frau Dr. Helga Jander und Herrn Dr. Dean Cáceres danke ich für das Korrekturlesen der vorliegenden Dissertationsschrift.

Der Deutschen Forschungsgemeinschaft danke ich für die finanzielle Unterstützung des Forschungsprojektes. Der Heinrich-Böll-Stiftung danke ich ebenfalls für die finanzielle Unterstützung sowie für die ideelle Förderung im Rahmen einer Reihe spannender und bereichernder Veranstaltungen, die ich während meiner Promotion besuchen durfte. 
„What we know is a drop, what we don't know is an ocean."

- Isaac Newton - 